\title{
Stereospecific Furanosylations Catalyzed by Bis-Thiourea Hydrogen-Bond Donors
}

\author{
Andrew B. Mayfield, ${ }^{\ddagger}$ Jan B. Metternich, ${ }^{\ddagger}$ Adam H. Trotta, and Eric N. Jacobsen* \\ Department of Chemistry \& Chemical Biology, Harvard University, Cambridge, Massachusetts 02138, United States. \\ ${ }^{\ddagger}$ These authors contributed equally. \\ Corresponding Author: jacobsen@chemistry.harvard.edu
}

- General Information S2

- Experimental Section S3

- General Information S3

- Catalyst Synthesis $\quad$ S4

- Donor Syntheses S11

- Hydrogen-bond-donor-catalyzed

Furanosylation Reactions $\quad \mathrm{S} 15$

- Reaction Optimization $\quad$ S29

- Furanose Phosphate

Optimization $\quad$ S29

- Catalyst Optimization $\quad$ S30

Reaction Conditions
Optimization $\quad$ S32

- Scope Limitations S35

- Mechanistic Investigation S37

- Effect of Donor Anomeric Purity S37

- Multi-pot Quench Kinetic Experiments S38

- Catalyst-induced Donor

Solubility and Resting State

Determination S62

- X-Ray Crystallography $\quad \mathrm{S70}$

- NMR Spectra of Key Compounds $\quad$ S75

- References S134 


\section{General Information}

\section{General Experimental Methods}

Unless otherwise noted, all reactions for the preparation of substrates and catalysts were performed in standard, dry glassware, under a nitrogen atmosphere. All catalytic experiments were performed in 0.5 dram and 20 dram vials. Reactions were monitored by thin-layer chromatography (TLC) on Silica Gel 60 F254 plates (EMD), visualized under UV light $(254 \mathrm{~nm})$ and/or ceric ammonium molybdate (CAM) stain, which developed upon heating. Unless stated otherwise, flash chromatography was performed using SiliaFlash P60 (230-400 mesh, SiliCycle). Automated column chromatography was performed on a Biotage Isolera One. Organic solutions were concentrated in vacuo on a Büchi rotary evaporator.

\section{Materials and Reagents}

All commercially available reagents were purchased from Sigma-Aldrich, Alfa Aesar, Matrix Scientific, Carbosynth, or $\mathrm{TCl}$ and used without purification, unless otherwise indicated. Extraction and chromatography solvents (EMD or $\mathrm{BDH}$ ) were reagent grade and used without purification. Reaction solvents, including acetonitrile, dichloromethane, tetrahydrofuran, diethyl ether, and 1,4-dioxane (PURE SOLV) were first dried by passage through columns of activated alumina. Di- $n$-propyl ether was filtered over activated basic alumina prior to use in the catalytic reactions. Triethylamine and $\mathrm{N}, \mathrm{N}$-diisopropylethylamine were distilled from calcium hydride or sodium metal under a nitrogen atmosphere prior to use. Deuterated solvents (namely $\mathrm{CDCl}_{3}, \mathrm{C}_{6} \mathrm{D}_{6}, \mathrm{DMSO}-d_{6}, \mathrm{CD}_{3} \mathrm{OD}$ and $\mathrm{CD}_{3} \mathrm{CN}$ (Cambridge Isotope Laboratories)) were used without purification. Molecular sieves $(4 \AA)$ were dried under vacuum at $130{ }^{\circ} \mathrm{C}$ for $18 \mathrm{~h}$ prior to use.

\section{Instrumentation}

Proton nuclear magnetic resonance ( ${ }^{1} \mathrm{H}$ NMR) spectra and proton-decoupled carbon nuclear magnetic resonance $\left({ }^{13} \mathrm{C}\right.$ NMR) spectra were recorded on a Varian Mercury Inova500 (500 MHz) or Inova $600(600 \mathrm{MHz})$ spectrometer at $25^{\circ} \mathrm{C}$. Phosphorus nuclear magnetic resonance $\left({ }^{31} \mathrm{P} \mathrm{NMR}\right)$ spectra were recorded on a Varian Mercury400B $(400 \mathrm{MHz})$ at $25^{\circ} \mathrm{C}$ unless otherwise indicated. All chemical shifts $(\delta)$ are reported in parts per million (ppm) downfield from tetramethylsilane and are referenced to the residual solvent signal of the NMR solvent. Data are represented as follows: chemical shift, multiplicity $(b r=$ broad, $s=$ singlet, $d=$ doublet, $t=$ triplet, $q=$ quartet,$\quad$ quint $=$ quintet,$\quad$ sext $=$ sextet,$\quad$ sept $=$ septet,$\quad m=$ multiplet , coupling constants $(\mathrm{J})$ in Hertz $(\mathrm{Hz})$, integration value. High-resolution mass spectrometric data were obtained on an Agilent 6210 electrospray ionization, time-of-flight HPLC/MS spectrometer (ESI-TOF) or a Waters Quattro micro GC/MS/MS spectrometer (EI). 


\section{Experimental Section}

\section{General Information}

(R)-2-((tert-Butoxycarbonyl)amino)-3,3-dimethylpent-4-enoic acid, ${ }^{1}$ 3,5-Di-O-benzyl-2fluoro- $\beta$-D-arbinofuranose ${ }^{2,3}$ 1-O-methyl-3,4-O-acetonide-6-O-TBDPS galactopyranose $2 \mathbf{2 e},{ }^{4} \quad$ 1-O-methyl-3,4-O-isoproylidene- $\alpha$-L-fucopyranose $2 \mathbf{2 f},{ }^{5}$ 1-Omethyl-2,3-O-isopropylidene- $\alpha$-L-rhamnopyranose $\mathbf{2 g},{ }^{6}$ 1-O-methyl-3-O-benzyl-4,5-Obenzylidene- $\alpha$-D-glucopyranose $\quad \mathbf{2 h},{ }^{7} \quad$ 1-O-methyl-2,3-di-O-allyl-6-O-benzyl- $\alpha-\mathrm{D}-$ glucopyranose 2i, ${ }^{8}$ 1-O-methyl-2,3-di-O-benzyl-D-xylopyranose $\mathbf{2 j},{ }^{9}$ 1-O-methyl-3,4-diO-benzyl-D-xylopyranose $\mathbf{2 k},{ }^{9}$ 1-O-methyl-3,5-di-O-benzyl- $\alpha$-D-xylofuranose 2 I, ${ }^{10,11,12}$ 1-O-methyl-3,5-di-O-benzyl- $\beta$-D-xylofuranose $2 \mathrm{~m},{ }^{10,11,12}$ 1-O-methyl-3,5-di-O-benzylD-arabinofuranose $\mathbf{2 0},{ }^{11,12} \mathrm{~N}$-Boc-D-threonine methyl ester $\mathbf{2} \mathbf{p},{ }^{13}$ and the catalysts $\mathbf{4 a}$, ent-4a, $\mathbf{4 c - 4 e},{ }^{14}$ and $\mathbf{4 g},{ }^{15}$ were synthesized according to literature procedures. The catalysts $\mathbf{4 b}, \mathbf{4 f}, \mathbf{4 h - 4 n}$, were synthesized according to the procedure reported herein for the catalyst ent-4i.

The anomeric assignment of products was performed by 2D-NMR (gHMBC, gHSQC, and NOESY) analysis of $\mathbf{3 a}, \mathbf{6 a}, \mathbf{6 b}, \mathbf{6 e}$, and $\mathbf{6 f}$. The stereochemistry of the remaining glycosylation products was assigned by analogy based on coupling constant and chemical shift of the anomeric hydrogen signal. 


\section{Catalyst Synthesis}
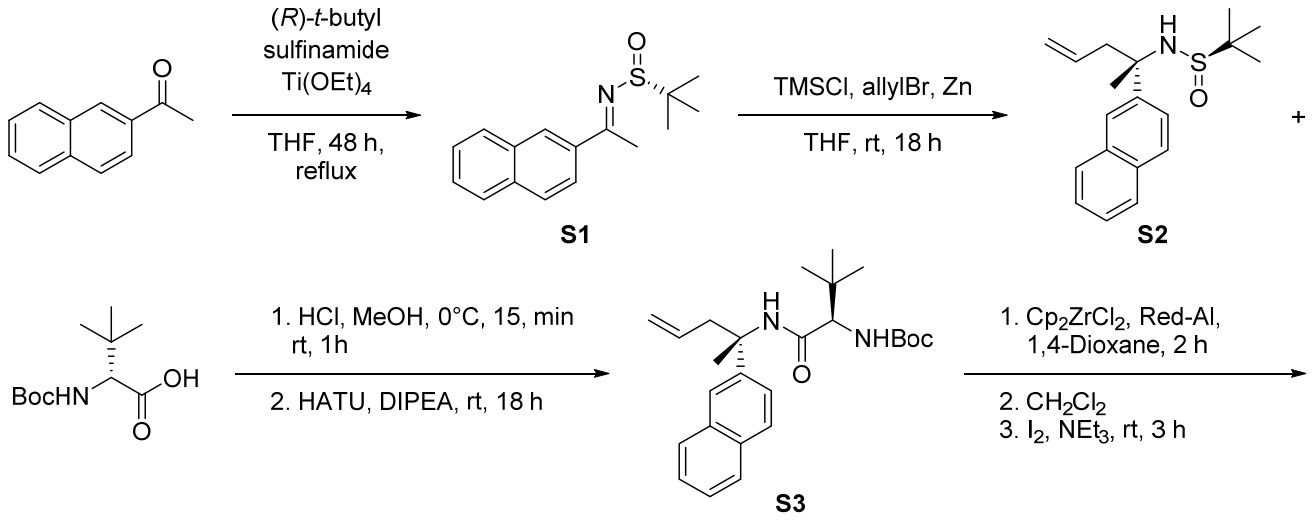<smiles>CCCCCNC(C(=O)N1CCC[C@H]1C)C(C)(C)C</smiles>

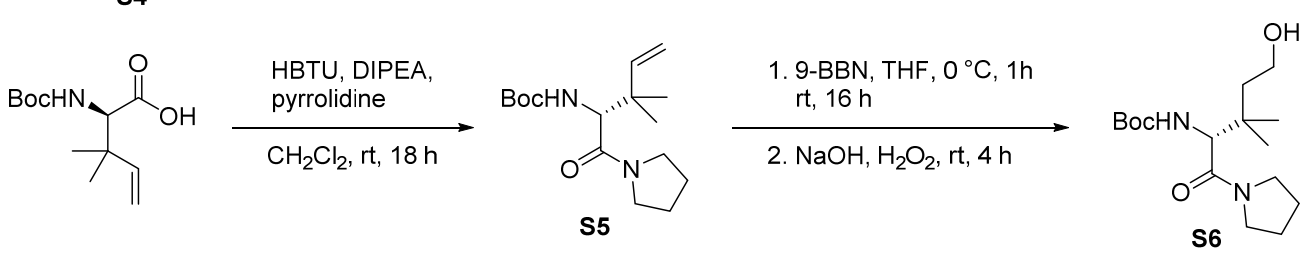

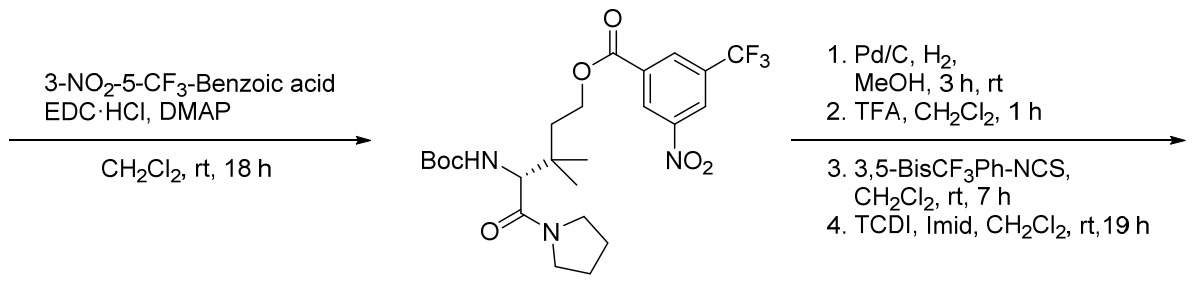<smiles></smiles>

s7

S10<smiles>CC(C)(C)OC(=O)N[C@@H](C(=O)N1CCC[C@]1(C)c1ccc2ccccc2c1)C(C)(C)C</smiles>

S4<smiles>CC(C)(C)[C@H](NC(=S)Nc1cc(C(=O)OCCC(C)(C)[C@H](NC(=S)Nc2cc(C(F)(F)F)cc(C(F)(F)F)c2)C(=O)N2CCCC2)cc(C(F)(F)F)c1)C(=O)N1CCC[C@]1(C)c1ccc2ccccc2c1</smiles>

ent-4i

Scheme S1 Synthesis of the Optimal Catalyst Scaffold. 


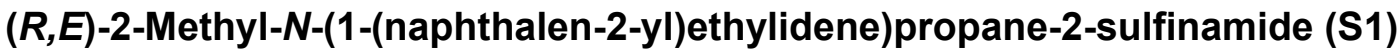

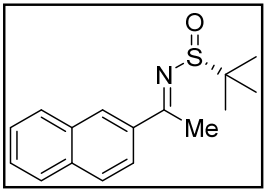

2-Acetonaphthone $(3.4 \mathrm{~g}, \quad 20.0 \mathrm{mmol}, \quad 1.0 \mathrm{eq}$.$) and (R)$-t-butyl sulfinamide ( $2.9 \mathrm{~g}, 24.0 \mathrm{mmol}, 1.2 \mathrm{eq}$.) were dissolved in THF (20 mL) in a flame-dried flask equipped with a reflux condenser and $\mathrm{Ti}(\mathrm{OEt})_{4}$ (10.0 g, $44.0 \mathrm{mmol}, 2.2 \mathrm{eq}$.) was added. The resulting solution was stirred at reflux for $48 \mathrm{~h}$ before the mixture was diluted with EtOAc and sat. $\mathrm{Na}_{2} \mathrm{SO}_{4}$ solution (aq.) was added. After filtration, the organic phase was concentrated in vacuo and purification by column chromatography $\left(\mathrm{SiO}_{2}, 5-30 \%\right.$ EtOAc/hexanes, Biotage) yielded the product $\mathbf{S 1}$ as a bright yellow solid $(4.5 \mathrm{~g}, 82 \%)$.

${ }^{1} \mathrm{H} \mathrm{NMR}\left(600 \mathrm{MHz}_{\mathrm{CDCl}}\right): \delta=8.30(\mathrm{~s}, 1 \mathrm{H}), 8.07(\mathrm{dd}, J=8.6,1.9 \mathrm{~Hz}, 1 \mathrm{H}), 7.95-7.90$ $(\mathrm{m}, 1 \mathrm{H}), 7.88-7.83(\mathrm{~m}, 2 \mathrm{H}), 7.60-7.50(\mathrm{~m}, 2 \mathrm{H}), 2.89(\mathrm{~d}, J=1.6 \mathrm{~Hz}, 3 \mathrm{H}), 1.36(\mathrm{~d}$, $J=1.5 \mathrm{~Hz}, 9 \mathrm{H}) \mathrm{ppm} ;{ }^{13} \mathrm{C} \mathrm{NMR}\left(126 \mathrm{MHz}, \mathrm{CDCl}_{3}\right): \delta=176.3,136.3,135.0,132.8$, 129.4, 128.3, 128.1, 127.8, 126.8, 124.0, 57.7, 22.7, 19.9 ppm; HR-ESI-MS: $\mathrm{m} / \mathrm{z}$ : 274.1256 $\left([\mathrm{M}+\mathrm{H}]^{+}\right.$, calcd. for $\mathrm{C}_{16} \mathrm{H}_{20} \mathrm{NOS}^{+}:$274.1260).

\section{(R)-2-Methyl-N-((S)-2-(naphthalen-2-yl)pent-4-en-2-yl)propane-2-sulfinamide} (S2)

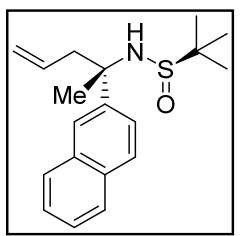

Zinc (powder, -100 mesh, $1.32 \mathrm{~g}, 20.1 \mathrm{mmol}, 1.1 \mathrm{eq}$.$) was suspended$ in dry THF $(75 \mathrm{~mL})$ and TMSCl $(0.12 \mathrm{~mL}, 0.9 \mathrm{mmol}, 0.05$ eq. $)$ was added. After stirring at rt for $5 \mathrm{~min}$, allyl bromide $(1.74 \mathrm{~mL}, 20.1 \mathrm{mmol}$, 1.1 eq.) was added and the resulting mixture was stirred at rt for $30 \mathrm{~min}$, before a solution of $\mathbf{S 1}(5.0 \mathrm{~g}, 18.3 \mathrm{mmol}, 1.0 \mathrm{eq}$.$) in dry THF (75 \mathrm{~mL})$ was added and the reaction was stirred at $\mathrm{rt}$ for $18 \mathrm{~h}$. The mixture was diluted with EtOAc $(50 \mathrm{~mL})$ and sat. $\mathrm{NaHCO}_{3}$ solution (aq., $\left.50 \mathrm{~mL}\right)$, and the resulting biphasic mixture was stirred at $\mathrm{rt}$ for $3 \mathrm{~h}$ before the aqueous layer was extracted with EtOAc (3x). The combined organic layers were washed with brine, dried over $\mathrm{MgSO}_{4}$ and concentrated in vacuo. Purification by column chromatography $\left(\mathrm{SiO}_{2}, 5-80 \%\right.$ $\mathrm{Et}_{2} \mathrm{O} /$ hexanes, Biotage) yielded the product $\mathbf{S 2}$ as a pale yellow oil $(3.73 \mathrm{~g}, 66 \%)$.

${ }^{1} \mathrm{H}$ NMR $\left(600 \mathrm{MHz}, \mathrm{CDCl}_{3}\right): \delta=7.87(\mathrm{~d}, \mathrm{~J}=2.0 \mathrm{~Hz}, 1 \mathrm{H}), 7.85-7.81(\mathrm{~m}, 3 \mathrm{H}), 7.58$ (dd, $J=8.7,2.0 \mathrm{~Hz}, 1 \mathrm{H}), 7.52-7.45(\mathrm{~m}, 2 \mathrm{H}), 5.59$ (ddt, $J=17.4,10.1,7.4 \mathrm{~Hz}, 1 \mathrm{H}), 5.20$ (ddt, $J=17.1,2.3,1.3 \mathrm{~Hz}, 1 \mathrm{H}), 5.13$ (ddd, $J=10.1,1.9,0.9 \mathrm{~Hz}, 1 \mathrm{H}$ ), $3.81(\mathrm{~s}, 1 \mathrm{H}), 2.82$ $-2.71(\mathrm{~m}, 2 \mathrm{H}), 1.90(\mathrm{~s}, 3 \mathrm{H}), 1.24(\mathrm{~s}, 9 \mathrm{H}) \mathrm{ppm} ;{ }^{13} \mathrm{C}$ NMR $\left(126 \mathrm{MHz}, \mathrm{CDCl}_{3}\right): \delta=142.7$, 133.2, 133.2, 132.5, 128.4, 128.1, 127.6, 126.3, 126.2, 125.5, 124.9, 120.5, 60.2, 56.4, 49.2, 27.8, 23.0 ppm; HR-ESI-MS: $\mathrm{m} / \mathrm{z}: 316.1725\left([\mathrm{M}+\mathrm{H}]^{+}\right.$, calcd. for $\mathrm{C}_{19} \mathrm{H}_{26} \mathrm{NOS}^{+}$: 316.1730).

tert-Butyl ((R)-3,3-dimethyl-1-(((S)-2-(naphthalen-2-yl)pent-4-en-2-yl)amino)-1oxobutan-2-yl)carbamate (S3)

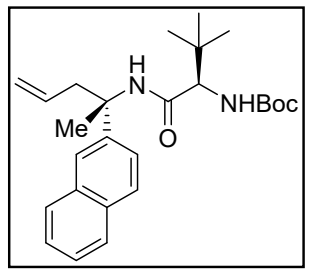

S2 (3.7 g, $11.7 \mathrm{mmol}, 1.0$ eq.) was dissolved in $\mathrm{MeOH}(45 \mathrm{~mL})$ and $\mathrm{HCl}$ (4.0 $\mathrm{M}$ in Dioxane, $14.7 \mathrm{~mL}, 58.6 \mathrm{mmol}, 5.0$ eq.) was added at $0^{\circ} \mathrm{C}$ before the solution was stirred at $0{ }^{\circ} \mathrm{C}$ for $1 \mathrm{~h}$. The mixture was sparged with $\mathrm{N}_{2}$ to remove excess $\mathrm{HCl}$, the solvent was removed in vacuo, and the product was dried under high vaccuum. The crude product was dissolved in dry $\mathrm{CH}_{2} \mathrm{Cl}_{2}(50 \mathrm{~mL})$, DIPEA $(12 \mathrm{~mL}$, $58.6 \mathrm{mmol}, 5.0$ eq.) was added at $0{ }^{\circ} \mathrm{C}$ and after stirring at $0^{\circ} \mathrm{C}$ for $10 \mathrm{~min}, \mathrm{~N}$-Boc-Dtert-Leucine (3.26 g, $14.1 \mathrm{mmol}, 1.2 \mathrm{eq}$.) and HATU (8.92 g, $23.5 \mathrm{mmol} .2 .0$ eq.) were 
added. The resulting mixture was gradually warmed to $\mathrm{rt}$ as the ice bath melted, stirring for $18 \mathrm{~h}$. $\mathrm{H}_{2} \mathrm{O}(20 \mathrm{~mL})$ was added, the aqueous phase was exctrated with $\mathrm{CH}_{2} \mathrm{Cl}_{2}(3 \mathrm{x})$, the combined organic phases were washed with brine, dried over $\mathrm{MgSO}_{4}$, and concentrated in vacuo. Purification by column chromatography $\left(\mathrm{SiO}_{2}, 10-75 \% \mathrm{Et}_{2} \mathrm{O} /\right.$ hexanes, Biotage) yielded the product $\mathbf{S} 3$ as an off-white solid (4.52 g, 87\%).

${ }^{1} \mathrm{H}$ NMR $\left(600 \mathrm{MHz}, \mathrm{CDCl}_{3}\right): \delta=7.82-7.73(\mathrm{~m}, 4 \mathrm{H}), 7.49-7.39(\mathrm{~m}, 3 \mathrm{H}), 6.14(\mathrm{~s}, 1 \mathrm{H})$, 5.62 (ddt, $J=17.3,10.1,7.3 \mathrm{~Hz}, 1 \mathrm{H}), 5.22-5.13(\mathrm{~m}, 3 \mathrm{H}), 3.84(\mathrm{~d}, J=9.4 \mathrm{~Hz}, 1 \mathrm{H})$, $3.80(\mathrm{~s}, 1 \mathrm{H}), 2.79(\mathrm{dd}, J=13.8,7.5 \mathrm{~Hz}, 1 \mathrm{H}), 2.59(\mathrm{dd}, J=13.8,7.2 \mathrm{~Hz}, 1 \mathrm{H}), 1.88(\mathrm{~s}$, $3 \mathrm{H}), 1.47(\mathrm{~s}, 9 \mathrm{H}), 1.02(\mathrm{~s}, 9 \mathrm{H}) \mathrm{ppm} ;{ }^{13} \mathrm{C} \mathrm{NMR}\left(126 \mathrm{MHz}, \mathrm{CDCl}_{3}\right): \delta=170.1,156.2$, 142.6, 133.4, 133.2, 132.4, 128.3, 128.2, 127.5, 126.0, 125.8, 124.0, 123.7, 119.9, 79.8, 62.9, 58.2, 47.8, 34.5, 28.5, 26.8, $21.6 \mathrm{ppm}$; HR-ESI-MS: $\mathrm{m} / \mathrm{z}: 425.2793\left([\mathrm{M}+\mathrm{H}]^{+}\right.$, calcd. for $\mathrm{C}_{26} \mathrm{H}_{37} \mathrm{~N}_{2} \mathrm{O}_{3}{ }^{+}$: 425.2799).

tert-Butyl ((R)-3,3-dimethyl-1-((S)-2-methyl-2-(naphthalen-2-yl)pyrrolidin-1-yl)-1oxobutan-2-yl)carbamate (S4)

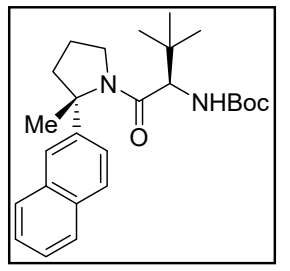

$\mathrm{Cp}_{2} \mathrm{ZrCl}_{2}$ (12.39 g, $42.4 \mathrm{mmol}, 4.0$ eq.) was suspended in 1,4dioxane $(100 \mathrm{~mL})$ and Red-Al $(60 \%$ wt. in toluene, $6.9 \mathrm{~mL}$, $21.2 \mathrm{mmol}, 2.0$ eq.) was slowly added over $5 \mathrm{~min}$. After stirring at $\mathrm{rt}$ for $2.5 \mathrm{~h}$, S3 (4.5 g, $10.6 \mathrm{mmol}, 1.0$ eq.) was added in $\mathrm{CH}_{2} \mathrm{Cl}_{2}(50 \mathrm{~mL})$ at $0{ }^{\circ} \mathrm{C}$. The resulting mixture was stirred at $\mathrm{rt}$ for $18 \mathrm{~h}$, before $\mathrm{I}_{2}$ (11.0 g, $43.5 \mathrm{mmol}, 4.1$ eq.) and NEt3 (7.4 mL, $53.0 \mathrm{mmol}, 5.0$ eq.) were added and the resulting suspension was stirred at $\mathrm{rt}$ for an additional $3 \mathrm{~h}$. The solution was adsorbed on $\mathrm{SiO}_{2}$, filtered over a $\mathrm{SiO}_{2}$ plug, and the product mixture was eluted with copious amounts of $\mathrm{Et}_{2} \mathrm{O}(\sim 1 \mathrm{~L})$. The resulting filtrate was concentrated in vacuo and purification by column chromatography $\left(\mathrm{SiO}_{2}, 5-50 \%\right.$ EtOAc/hexanes, Biotage) yielded the product $\mathbf{S 4}$ as an off-white solid after drying under high vaccuum $(2.48 \mathrm{~g}, 55 \%)$.

${ }^{1} \mathrm{H}$ NMR $\left(600 \mathrm{MHz}, \mathrm{CDCl}_{3}\right): \delta=7.78-7.73(\mathrm{~m}, 2 \mathrm{H}), 7.71(\mathrm{~d}, J=8.6 \mathrm{~Hz}, 1 \mathrm{H}), 7.61$ (d, $J=2.0 \mathrm{~Hz}, 1 \mathrm{H}), 7.43-7.36(\mathrm{~m}, 2 \mathrm{H}), 7.29(\mathrm{dd}, J=8.6,2.0 \mathrm{~Hz}, 1 \mathrm{H}), 5.15(\mathrm{~d}$, $J=10.1 \mathrm{~Hz}, 1 \mathrm{H}), 4.39(\mathrm{~d}, J=10.0 \mathrm{~Hz}, 1 \mathrm{H}), 4.27(\mathrm{dt}, J=9.7,6.4 \mathrm{~Hz}, 1 \mathrm{H}), 3.88$ (dt, $J=10.0,7.4 \mathrm{~Hz}, 1 \mathrm{H}$ ), 2.08 (hept, $J=7.2,6.8 \mathrm{~Hz}, 2 \mathrm{H}), 2.00(\mathrm{~s}, 3 \mathrm{H}), 1.92(\mathrm{p}, J=7.0 \mathrm{~Hz}$, $2 \mathrm{H}), 1.51(\mathrm{~s}, 9 \mathrm{H}), 1.04(\mathrm{~d}, J=1.7 \mathrm{~Hz}, 9 \mathrm{H}) \mathrm{ppm} ;{ }^{13} \mathrm{C}$ NMR $\left(126 \mathrm{MHz} \mathrm{CDCl}_{3}\right): \delta=170.2$, 156.5, 143.8, 133.4, 132.2, 128.2, 128.0, 127.5, 125.8, 125.4, 123.6, 123.3, 79.6, 67.4, 58.9, 50.2, 44.5, 35.0, 28.6, 26.6, 25.1, 22.8 ppm; HR-ESI-MS: m/z: $425.2793\left([M+H]^{+}\right.$, calcd. for $\mathrm{C}_{26} \mathrm{H}_{37} \mathrm{~N}_{2} \mathrm{O}_{3}{ }^{+}: 425.2799$ ).

tert-Butyl (R)-(3,3-dimethyl-1-oxo-1-(pyrrolidin-1-yl)pent-4-en-2-yl)carbamate (S5)

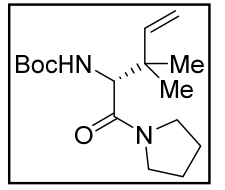

(R)-2-((tert-Butoxycarbonyl)amino)-3,3-dimethylpent-4-enoic $\quad$ acid $^{1}$ (1.00 g, $4.1 \mathrm{mmol}, 1.0$ eq.) was dissolved in dry $\mathrm{CH}_{2} \mathrm{Cl}_{2}(15 \mathrm{~mL})$ and pyrrolidine $(0.41 \mathrm{~mL}, 4.9 \mathrm{mmol}, 1.2$ eq. $)$, DIPEA $(2.3 \mathrm{~mL}, 13.2 \mathrm{mmol}$, 3.2 eq.), and HBTU (2.03 g, $5.3 \mathrm{mmol}, 1.3$ eq.) were added before the resulting mixture was stirred at $\mathrm{rt}$ for $18 \mathrm{~h}$. $\mathrm{H}_{2} \mathrm{O}$ was added, the aqueous layer was exctracted with $\mathrm{CH}_{2} \mathrm{Cl}_{2}$, the combined organic layers were dried over $\mathrm{MgSO}_{4}$, and concentrated in vacuo. Purification by column chromatography $\left(\mathrm{SiO}_{2}, 5-30 \%\right.$ EtOAc/hexanes, Biotage) yielded the product $\mathbf{S 5}$ as a colorless oil $(1.11 \mathrm{~g}, 91 \%)$. 
${ }^{1} \mathrm{H}$ NMR $\left(600 \mathrm{MHz}, \mathrm{CDCl}_{3}\right): \delta=5.95(\mathrm{dd}, J=17.3,10.9 \mathrm{~Hz}, 1 \mathrm{H}), 5.23(\mathrm{~d}, J=9.8 \mathrm{~Hz}$, $1 \mathrm{H}), 5.06(\mathrm{~d}, J=1.7 \mathrm{~Hz}, 1 \mathrm{H}), 5.03(\mathrm{dd}, J=9.2,1.2 \mathrm{~Hz}, 1 \mathrm{H}), 4.31(\mathrm{~d}, J=9.9 \mathrm{~Hz}, 1 \mathrm{H})$, $3.67(\mathrm{dt}, \quad J=10.2,6.7 \mathrm{~Hz}, 1 \mathrm{H}), \quad 3.51$ (tt, $J=9.9,7.1 \mathrm{~Hz}, 2 \mathrm{H}), 3.37$ (ddd, $J=11.8,7.6,5.9 \mathrm{~Hz}, 1 \mathrm{H}), 1.92(\mathrm{dqd}, J=9.6,6.6,6.0,3.8 \mathrm{~Hz}, 2 \mathrm{H}), 1.89-1.78(\mathrm{~m}, 2 \mathrm{H})$, 1.41 (s, 9H), 1.09 (s, 3H), $1.08(\mathrm{~s}, 3 \mathrm{H}) \mathrm{ppm} ;{ }^{13} \mathrm{C}$ NMR (126 MHz, $\left.\mathrm{CDCl}_{3}\right): \delta=169.9$, 155.8, 143.9, 113.4, 79.6, 58.1, 47.6, 45.8, 41.2, 28.50, 26.2, 24.4, 24.3, 23.1 ppm; HR-ESI-MS: m/z: $297.2168\left([M+H]^{+}\right.$, calcd. for $\mathrm{C}_{16} \mathrm{H}_{29} \mathrm{~N}_{2} \mathrm{O}_{3}{ }^{+}:$297.2173).

\section{tert-Butyl (R)-(5-hydroxy-3,3-dimethyl-1-oxo-1-(pyrrolidin-1-yl)pentan-2- yl)carbamate (S6)}

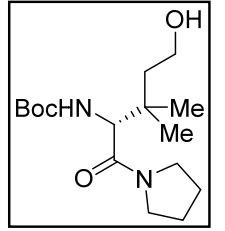

S5 $(1.10 \mathrm{~g}, 3.7 \mathrm{mmol}, 1.0$ eq.) was dissolved in dry THF $(37 \mathrm{~mL})$, a solution of 9-BBN (0.4 M in hexanes, $28 \mathrm{~mL}, 11.1 \mathrm{mmol}, 3$ eq.) was added and the resulting mixture was stirred at $\mathrm{rt}$ for $16 \mathrm{~h}$. The solution was cooled to $0{ }^{\circ} \mathrm{C}, \mathrm{NaOH}\left(2.0 \mathrm{M}, 17 \mathrm{~mL}, 34.1 \mathrm{mmol}, 9.2\right.$ eq.) and $\mathrm{H}_{2} \mathrm{O}_{2}$ ( $30 \%$ in $\mathrm{H}_{2} \mathrm{O}$ w/w, $14.8 \mathrm{~mL}, 145.1 \mathrm{mmol}, 39.1$ eq.) were added before the solution was stirred for $4 \mathrm{~h}$ at $\mathrm{rt}$. The resulting mixture was diluted with $\mathrm{H}_{2} \mathrm{O}(40 \mathrm{~mL})$ and EtOAc $(40 \mathrm{~mL})$, the aqueous phase was extracted with EtOAc (3x), the combined organic phases were washed with sat. $\mathrm{NH}_{4} \mathrm{Cl}$ solution (aq.), sat. $\mathrm{Na}_{2} \mathrm{~S}_{2} \mathrm{O}_{3}$ solution (aq.), dried over $\mathrm{MgSO}_{4}$, and concentrated in vacuo. Purification by column chromatography $\left(\mathrm{SiO}_{2}, 10-100 \%\right.$ EtOAc/hexanes, Biotage) yielded the product $\mathbf{S} 6$ as a pale yellow oil (918 $\mathrm{mg}, 79 \%)$.

${ }^{1} \mathrm{H}$ NMR $\left(600 \mathrm{MHz}, \mathrm{CDCl}_{3}\right): \delta=6.33(\mathrm{~d}, J=10.2 \mathrm{~Hz}, 1 \mathrm{H}), 4.57(\mathrm{~d}, J=10.2 \mathrm{~Hz}, 1 \mathrm{H})$, 3.77 (dq, $J=11.5,4.6 \mathrm{~Hz}, 2 \mathrm{H}$ ), 3.70 (ddd, $J=11.4,10.0,3.7 \mathrm{~Hz}, 1 \mathrm{H}$ ), 3.53 (ddt, $J=21.4,12.2,7.0 \mathrm{~Hz}, 2 \mathrm{H}), 3.41$ (ddd, $J=12.6,7.7,5.5 \mathrm{~Hz}, 1 \mathrm{H}), 1.98-1.91(\mathrm{~m}, 2 \mathrm{H})$, 1.87 (dddd, $J=17.9,14.7,9.8,6.4 \mathrm{~Hz}, 3 \mathrm{H}$ ), 1.42 (s, 9H), 1.37 (dt, $J=15.1,4.2 \mathrm{~Hz}$, $1 \mathrm{H}), 1.04(\mathrm{~s}, 3 \mathrm{H}), 1.01(\mathrm{~s}, 3 \mathrm{H}) \mathrm{ppm} ;{ }^{13} \mathrm{C}$ NMR $\left(126 \mathrm{MHz}, \mathrm{CDCl}_{3}\right): \delta=171.1,156.4$, 79.7, 59.0, 57.7, 47.8, 46.0, 41.6, 37.2, 28.5, 26.8, 26.2, 24.4, 24.2 ppm; HR-ESI-MS: $\mathrm{m} / \mathrm{z}: 315.2274\left([\mathrm{M}+\mathrm{H}]^{+}\right.$, calcd. for $\left.\mathrm{C}_{16} \mathrm{H}_{31} \mathrm{~N}_{2} \mathrm{O}_{4}^{+}: 315.2278\right)$.

(R)-4-((tert-Butoxycarbonyl)amino)-3,3-dimethyl-5-oxo-5-(pyrrolidin-1-yl)pentyl 3-nitro-5-(trifluoromethyl)benzoate (S7)

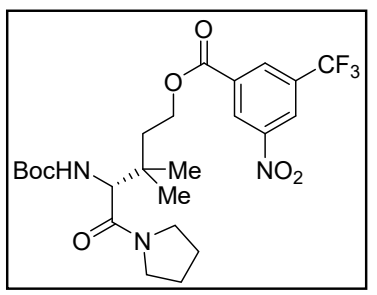
concentrated in vacuo. Purification by column chromatography $\left(\mathrm{SiO}_{2}, 10-50 \%\right.$ EtOAc/hexanes, Biotage) yielded the product $\mathbf{S 7}$ as a pale yellow oil (1.32 $\mathrm{g}, 86 \%)$.

${ }^{1} \mathrm{H}$ NMR $\left(600 \mathrm{MHz}, \mathrm{CDCl}_{3}\right): \delta=9.01(\mathrm{t}, J=1.8 \mathrm{~Hz}, 1 \mathrm{H}), 8.66(\mathrm{t}, J=2.1 \mathrm{~Hz}, 1 \mathrm{H}), 8.60$ $(\mathrm{d}, J=1.7 \mathrm{~Hz}, 1 \mathrm{H}), 5.32(\mathrm{~d}, J=9.8 \mathrm{~Hz}, 1 \mathrm{H}), 4.53(\mathrm{t}, J=7.4 \mathrm{~Hz}, 2 \mathrm{H}), 4.40$ (d, $J=9.9 \mathrm{~Hz}, 1 \mathrm{H}), 3.73(\mathrm{dt}, J=10.1,6.5 \mathrm{~Hz}, 1 \mathrm{H}), 3.53(\mathrm{ddt}, J=13.8,10.0,7.2 \mathrm{~Hz}, 2 \mathrm{H})$, 3.41 (ddd, $J=12.6,7.6,5.5 \mathrm{~Hz}, 1 \mathrm{H}), 2.01-1.82(\mathrm{~m}, 6 \mathrm{H}), 1.42(\mathrm{~s}, 9 \mathrm{H}), 1.10(\mathrm{~s}, 3 \mathrm{H})$, 1.09 (s, 3H) ppm; $\left.{ }^{13} \mathrm{C} \mathrm{NMR} \mathrm{(126} \mathrm{MHz,} \mathrm{CDCl}_{3}\right): \delta=169.9,163.2,155.7,148.5,133.5$, $132.8\left(q, J_{C F}=34.8 \mathrm{~Hz}\right), 131.9\left(q, J_{C F}=3.7 \mathrm{~Hz}\right), 127.5,124.4\left(q, J_{C F}=3.8 \mathrm{~Hz}\right), 122.4$ $\left(q, J_{C F}=273.1 \mathrm{~Hz}\right), 79.8,63.6,57.9,47.6,45.8,37.5,36.7,28.3,26.1,24.3,23.6$, 
$23.3 \mathrm{ppm} ;{ }^{19} \mathrm{~F} \mathrm{NMR}\left(471 \mathrm{MHz}, \mathrm{CDCl}_{3}\right): \delta=-62.93 \mathrm{ppm}$; HR-ESI-MS: $\mathrm{m} / \mathrm{z}: 532.2256$ $\left([\mathrm{M+H}]^{+}\right.$, calcd. for $\mathrm{C}_{24} \mathrm{H}_{33} \mathrm{~F}_{3} \mathrm{~N}_{3} \mathrm{O}_{7}^{+}$: 532.2265).

\section{(R)-4-((tert-Butoxycarbonyl)amino)-3,3-dimethyl-5-oxo-5-(pyrrolidin-1-yl)pentyl} 3-amino-5-(trifluoromethyl)benzoate (S8)

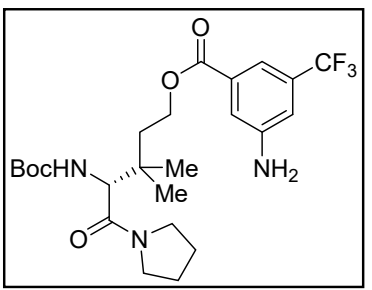

S8 as an off-white solid (892 $\mathrm{mg}, 88 \%$ ).

$\mathbf{S 7}$ (1.31 g, $2.5 \mathrm{mmol}, 1.0$ eq.) was dissolved in $\mathrm{MeOH}(25 \mathrm{~mL})$, $\mathrm{Pd} / \mathrm{C}\left(10 \% \mathrm{wt}, 524 \mathrm{mg}\right.$ ) was added under $\mathrm{N}_{2}$ and the flask was purged with $\mathrm{H}_{2}$ before the resulting suspension was stirred at $\mathrm{rt}$ under $\mathrm{H}_{2}$ for $3 \mathrm{~h}$. The $\mathrm{Pd}$ was removed by filtration over Celite, the product was eluted with EtOAc, and the solvent was removed in vacuo. Purification by column chromatography $\left(\mathrm{SiO}_{2}, 10-50 \%\right.$ EtOAc/hexanes, Biotage) yielded the product

${ }^{1} \mathrm{H}$ NMR $\left(600 \mathrm{MHz}, \mathrm{CDCl}_{3}\right): \delta=7.61(\mathrm{~d}, J=1.6 \mathrm{~Hz}, 1 \mathrm{H}), 7.52(\mathrm{t}, J=1.9 \mathrm{~Hz}, 1 \mathrm{H}), 7.04$ (t, $J=2.0 \mathrm{~Hz}, 1 \mathrm{H}), 5.32(\mathrm{~d}, J=9.9 \mathrm{~Hz}, 1 \mathrm{H}), 4.41$ (ddd, $J=8.4,5.6,1.9 \mathrm{~Hz}, 3 \mathrm{H}), 4.05$ (s, 2H), 3.71 (dt, $J=10.2,6.5 \mathrm{~Hz}, 1 \mathrm{H}$ ), 3.51 (ddt, $J=26.2,10.0,7.2 \mathrm{~Hz}, 2 \mathrm{H}$ ), 3.40 (ddd, $J=12.6,7.7,5.4 \mathrm{~Hz}, 1 \mathrm{H}), 1.96-1.79(\mathrm{~m}, 6 \mathrm{H}), 1.42(\mathrm{~s}, 9 \mathrm{H}), 1.08(\mathrm{~s}, 3 \mathrm{H}), 1.06(\mathrm{~s}$, 3H) ppm; ${ }^{13} \mathrm{C}$ NMR $\left(126 \mathrm{MHz}, \mathrm{CDCl}_{3}\right): \delta=170.2,165.7,155.9,147.2,132.3,132.1$ (q, $\left.J_{\mathrm{CF}}=32.7 \mathrm{~Hz}\right), 123.8\left(q, J_{\mathrm{CF}}=272.5 \mathrm{~Hz}\right), 118.8,116.1\left(q, J_{\mathrm{CF}}=3.6 \mathrm{~Hz}\right), 115.2(q$, $J_{\mathrm{CF}}=3.0 \mathrm{~Hz}$ ), 79.9, 62.5, 58.1, 47.7, 45.9, 37.5, 36.8, 28.5, 26.2, 24.4, 23.6, 23.5 ppm; ${ }^{19} \mathrm{~F}$ NMR (471 MHz, $\left.\mathrm{CDCl}_{3}\right): \delta=-63.06 \mathrm{ppm}$; HR-ESI-MS: $\mathrm{m} / \mathrm{z}: 592.2516\left([\mathrm{M}+\mathrm{H}]^{+}\right.$, calcd. for $\mathrm{C}_{24} \mathrm{H}_{35} \mathrm{~F}_{3} \mathrm{~N}_{3} \mathrm{O}_{5}{ }^{+}: 502.2523$ ).

(R)-4-(3-(3,5-Bis(trifluoromethyl)phenyl)thioureido)-3,3-dimethyl-5-oxo-5(pyrrolidin-1-yl)pentyl 3-amino-5-(trifluoromethyl)benzoate (S9)

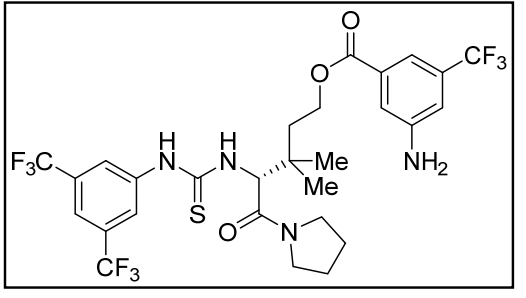

combined organic phases were dried over $\mathrm{MgSO}_{4}$ and the solvent was removed vacuo. The resulting off-white solid was redissolved in dry $\mathrm{CH}_{2} \mathrm{Cl}_{2}(15 \mathrm{~mL})$ and 3,5bis(trifluoromethyl)pheny isothiocyanate $(0.42 \mathrm{~mL}, 2.3 \mathrm{mmol}, 1.05 \mathrm{eq}$.) was added at $0{ }^{\circ} \mathrm{C}$ before the resulting mixture was stirred at $\mathrm{rt}$ for $18 \mathrm{~h}$. The solvent was removed in vacuo and purification by column chromatography $\left(\mathrm{SiO}_{2}, 10-50 \%\right.$ EtOAc/hexanes, Biotage) yielded the product $\mathbf{S 9}$ as an off-white solid (709 $\mathrm{mg}, 47 \%$ ).

${ }^{1} \mathrm{H}$ NMR $\left(600 \mathrm{MHz}, \mathrm{CDCl}_{3}\right): \delta=9.40(\mathrm{~s}, 1 \mathrm{H}), 7.94(\mathrm{~d}, J=1.6 \mathrm{~Hz}, 2 \mathrm{H}), 7.65-7.56(\mathrm{~m}$, $2 \mathrm{H}), 7.47(\mathrm{t}, J=1.8 \mathrm{~Hz}, 1 \mathrm{H}), 7.04(\mathrm{t}, J=2.0 \mathrm{~Hz}, 1 \mathrm{H}), 5.65(\mathrm{~d}, J=9.4 \mathrm{~Hz}, 1 \mathrm{H}), 4.45(\mathrm{t}$, $J=7.1 \mathrm{~Hz}, 2 \mathrm{H}), 4.18(\mathrm{ddd}, J=10.5,7.6,4.5 \mathrm{~Hz}, 1 \mathrm{H}), 4.05(\mathrm{~d}, J=15.2 \mathrm{~Hz}, 2 \mathrm{H}), 3.61$ (dt, $J=10.3,7.5 \mathrm{~Hz}, 1 \mathrm{H}), \quad 3.44$ (ddd, $J=12.2,7.3,5.1 \mathrm{~Hz}, 1 \mathrm{H}), \quad 3.25$ (dt, $J=12.0,7.7 \mathrm{~Hz}, \quad 1 \mathrm{H}), \quad 1.98 \quad(\mathrm{dtd}, \quad J=14.6,7.1,2.9 \mathrm{~Hz}, \quad 3 \mathrm{H}$ ), $1.90 \quad$ (tdd, $J=14.4,10.2,5.7 \mathrm{~Hz}, 1 \mathrm{H}), 1.85-1.75(\mathrm{~m}, 2 \mathrm{H}), 1.22(\mathrm{~s}, 3 \mathrm{H}), 1.18(\mathrm{~s}, 3 \mathrm{H}) \mathrm{ppm} ;{ }^{13} \mathrm{C}$ NMR $\left(126 \mathrm{MHz}, \mathrm{CDCl}_{3}\right): \delta=182.6,171.5,165.7,147.3,140.5,132.1,132.1$ (q, $\left.J_{C F}=32.4 \mathrm{~Hz}\right), \quad 131.7 \quad\left(q, \quad J_{C F}=33.5 \mathrm{~Hz}\right), \quad 124.9 \quad\left(d, \quad J_{C F}=4.1 \mathrm{~Hz}\right), \quad 123.8 \quad(q$, $\left.J_{\mathrm{CF}}=272.5 \mathrm{~Hz}\right), \quad 123.2\left(\mathrm{q}, J_{\mathrm{CF}}=272.8 \mathrm{~Hz}\right), 118.7,118.6,116.1-115.9$ (q, 
$\left.J_{\mathrm{CF}}=4.3 \mathrm{~Hz}\right), 115.4\left(\mathrm{q}, J_{\mathrm{CF}}=3.1 \mathrm{~Hz}\right), 62.3,62.3,48.8,47.0,38.1,37.6,25.9,24.3$, 24.2, $24.1 \mathrm{ppm} ;{ }^{19} \mathrm{~F}$ NMR (471 MHz, $\left.\mathrm{CDCl}_{3}\right): \delta=-62.98$ (s, 6F), - 63.06 (s, 3F) ppm; HR-ESI-MS: m/z: $673.1871\left([M+\mathrm{H}]^{+}\right.$, calcd. for $\mathrm{C}_{28} \mathrm{H}_{30} \mathrm{~F}_{9} \mathrm{~N}_{4} \mathrm{O}_{3} \mathrm{~S}^{+}:$673.1889).

(R)-4-(3-(3,5-Bis(trifluoromethyl)phenyl)thioureido)-3,3-dimethyl-5-oxo-5(pyrrolidin-1-yl)pentyl 3-isothiocyanato-5-(trifluoromethyl)benzoate (S10)

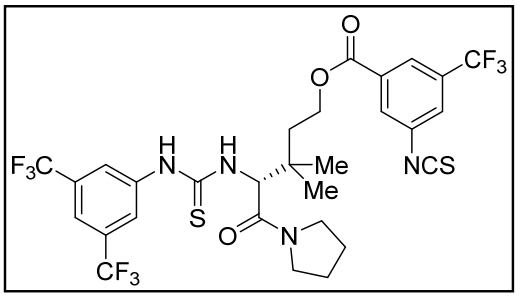
and purification by column chromatography $\left(\mathrm{SiO}_{2}, 10-70 \%\right.$ EtOAc/hexanes, Biotage $)$ yielded the product $\mathbf{S} 10$ as an off-white solid (290 $\mathrm{mg}, 39 \%)$.

${ }^{1} \mathrm{H}$ NMR $\left(600 \mathrm{MHz}, \mathrm{CDCl}_{3}\right): \delta=9.39(\mathrm{~s}, 1 \mathrm{H}), 8.16-8.12(\mathrm{~m}, 1 \mathrm{H}), 8.01(\mathrm{~d}, J=1.8 \mathrm{~Hz}$, $1 \mathrm{H}), 7.94(\mathrm{~d}, J=1.5 \mathrm{~Hz}, 3 \mathrm{H}), 7.65-7.59(\mathrm{~m}, 2 \mathrm{H}), 5.68(\mathrm{~d}, J=9.5 \mathrm{~Hz}, 1 \mathrm{H}), 4.52(\mathrm{t}$, $J=7.2 \mathrm{~Hz}, 2 \mathrm{H}), 4.19(\mathrm{ddd}, J=10.6,7.6,4.5 \mathrm{~Hz}, 1 \mathrm{H}), 3.62(\mathrm{dt}, J=10.4,7.5 \mathrm{~Hz}, 1 \mathrm{H}$ ), 3.44 (ddd, $J=12.2,7.2,5.2 \mathrm{~Hz}, 1 \mathrm{H}), 3.25(\mathrm{dt}, J=12.0,7.8 \mathrm{~Hz}, 1 \mathrm{H}), 2.06-1.96(\mathrm{~m}$, $3 \mathrm{H}), 1.96-1.88(\mathrm{~m}, 1 \mathrm{H}), 1.86-1.79(\mathrm{~m}, 2 \mathrm{H}), 1.25(\mathrm{~s}, 4 \mathrm{H}), 1.20(\mathrm{~s}, 3 \mathrm{H}) \mathrm{ppm} ;{ }^{13} \mathrm{C}$ NMR $\left(126 \mathrm{MHz} \mathrm{CDCl}_{3}\right): \delta=182.7,171.5,164.1,140.4,140.0,133.5,133.1,132.9$ (q, $\left.J_{C F}=33.9 \mathrm{~Hz}\right), 131.8\left(q, J_{C F}=33.5 \mathrm{~Hz}\right), 129.8,126.6\left(q, J_{C F}=3.0 \mathrm{~Hz}\right), 124.9(q$, $\left.J_{C F}=4.5 \mathrm{~Hz}\right), \quad 124.1 \quad\left(q, \quad J_{C F}=3.8 \mathrm{~Hz}\right), \quad 123.2 \quad\left(q, \quad J_{C F}=272.8 \mathrm{~Hz}\right), \quad 122.9 \quad(q$, $\left.J_{\mathrm{CF}}=272.9 \mathrm{~Hz}\right), 119.0-118.3(\mathrm{~m}), 63.0,62.1,48.8,47.0,38.1,37.7,25.9,24.3,24.2$, $24.1 \mathrm{ppm} ;{ }^{19} \mathrm{~F}$ NMR (471 MHz, $\left.\mathrm{CDCl}_{3}\right): \delta=-62.99$ (s, 6F), - 63.04 (s, 3F) ppm; HRESI-MS: $m / z$ : $737.1259\left([\mathrm{M}+\mathrm{Na}]^{+}\right.$, calcd. for $\mathrm{C}_{29} \mathrm{H}_{27} \mathrm{~F}_{9} \mathrm{~N}_{4} \mathrm{O}_{3} \mathrm{~S}_{2} \mathrm{Na}^{+}:$737.1273).

(R)-4-(3-(3,5-Bis(trifluoromethyl)phenyl)thioureido)-3,3-dimethyl-5-oxo-5(pyrrolidin-1-yl)pentyl 3-(3-((R)-3,3-dimethyl-1-((S)-2-methyl-2-(naphthalen-2yl)pyrrolidin-1-yl)-1-oxobutan-2-yl)thioureido)-5-(trifluoromethyl)benzoate (ent4i)

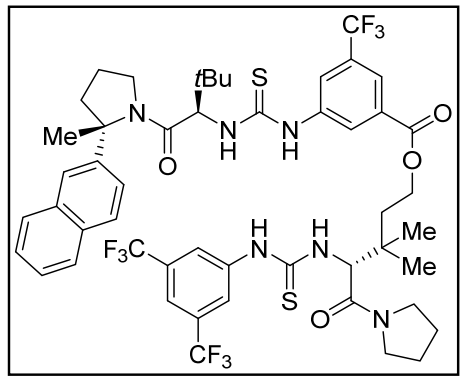

S4 (180 mg, $0.42 \mathrm{mmol}, 1.2$ eq.) was dissolved in dry $\mathrm{CH}_{2} \mathrm{Cl}_{2}(3 \mathrm{~mL})$ and TFA $(1.5 \mathrm{~mL})$ was added at $0{ }^{\circ} \mathrm{C}$ before the resulting solution was stirred at $0{ }^{\circ} \mathrm{C}$ for $1.5 \mathrm{~h}$. The reaction was diluted with $\mathrm{CH}_{2} \mathrm{Cl}_{2}(10 \mathrm{~mL})$, quenched by addition of sat. $\mathrm{Na}_{2} \mathrm{CO}_{3}$ solution (aq.), diluted with $\mathrm{H}_{2} \mathrm{O}$ and the aqueous phase was extracted with $\mathrm{CH}_{2} \mathrm{Cl}_{2}$. The combined organic phases were dried over $\mathrm{MgSO}_{4}$, the solvent was removed in vacuo, and the resulting colorless oil was dissolved in dry $\mathrm{CH}_{2} \mathrm{Cl}_{2}$. At $0{ }^{\circ} \mathrm{C}$, NEt3 $(0.05 \mathrm{~mL})$ was added and after stirring at $0{ }^{\circ} \mathrm{C}$ for $10 \mathrm{~min}, \mathbf{S} 10$ (256 mg, $0.36 \mathrm{mmol}, 1.0$ eq.) was slowly added to the mixture. The reaction was gradually warmed to $\mathrm{rt}$ and stirred for $18 \mathrm{~h}$, before the solvent was removed in vacuo and purification by column chromatography $\left(\mathrm{SiO}_{2}, 10-100 \% \mathrm{Et}_{2} \mathrm{O} /\right.$ hexanes, Biotage) yielded the product ent $-4 \mathbf{i}$ as an off-white solid (268 $\mathrm{mg}, 72 \%$ ).

${ }^{1} \mathrm{H}$ NMR $\left(600 \mathrm{MHz}_{\mathrm{CDCl}}\right): \delta=8.95(\mathrm{~d}, J=13.9 \mathrm{~Hz}, 2 \mathrm{H}), 8.33(\mathrm{t}, J=1.8 \mathrm{~Hz}, 1 \mathrm{H}), 8.14$ $(\mathrm{s}, 1 \mathrm{H}), 7.96(\mathrm{~d}, J=1.7 \mathrm{~Hz}, 3 \mathrm{H}), 7.69(\mathrm{~d}, J=9.1 \mathrm{~Hz}, 1 \mathrm{H}), 7.66(\mathrm{~d}, J=2.1 \mathrm{~Hz}, 2 \mathrm{H})$, 
$7.66-7.63(\mathrm{~m}, 2 \mathrm{H}), 7.63-7.60(\mathrm{~m}, 1 \mathrm{H}), 7.38(\mathrm{dd}, J=8.7,2.0 \mathrm{~Hz}, 1 \mathrm{H}), 7.33$ (ddd, $J=8.1,6.8,1.2 \mathrm{~Hz}, 1 \mathrm{H}), 7.27(\mathrm{~s}, 1 \mathrm{H}), 7.20(\mathrm{~d}, J=9.2 \mathrm{~Hz}, 1 \mathrm{H}), 5.34(\mathrm{t}, J=8.6 \mathrm{~Hz}, 2 \mathrm{H})$, 4.59 (ddd, $J=10.0,7.8,4.0 \mathrm{~Hz}, 1 \mathrm{H}$ ), 4.45 (td, $J=6.9,2.2 \mathrm{~Hz}, 2 \mathrm{H}$ ), 4.03 (ddd, $J=10.4,7.2,4.9 \mathrm{~Hz}, \quad 1 \mathrm{H}), \quad 3.90 \quad$ (ddd, $J=10.1,8.6,7.0 \mathrm{~Hz}, \quad 1 \mathrm{H}$ ), $3.56 \quad(\mathrm{dt}$, $J=10.4,7.3 \mathrm{~Hz}, 1 \mathrm{H}$ ), 3.32 (t, $J=6.9 \mathrm{~Hz}, 2 \mathrm{H}$ ), 2.09 (ddd, $J=12.6,10.1,6.4 \mathrm{~Hz}, 1 \mathrm{H}$ ), 2.00 (ddd, $J=12.6,6.3,4.3 \mathrm{~Hz}, 1 \mathrm{H}), 1.95(\mathrm{~m}, 3 \mathrm{H}), 1.93-1.69(\mathrm{~m}, 8 \mathrm{H}), 1.16(\mathrm{~s}, 3 \mathrm{H})$, $1.14(\mathrm{~s}, 3 \mathrm{H}), 1.09$ (s, 9H) ppm; ${ }^{13} \mathrm{C}$ NMR (126 MHz, $\left.\mathrm{CDCl}_{3}\right): \delta=182.8,182.7,171.1$, 170.6, 165.5, 144.3, 141.8, 141.5, 134.0, 132.8, 132.7, 131.8 (q, J JF $=33.3 \mathrm{~Hz}), 131.4$ $\left(q, J_{C F}=33.0 \mathrm{~Hz}\right), 128.6\left(q, J_{C F}=33.5 \mathrm{~Hz}\right), 128.1\left(q, J_{C F}=4.2 \mathrm{~Hz}\right), 128.1,127.5$, $126.7,126.3,124.9,124.7,124.7\left(\mathrm{q}, J_{\mathrm{CF}}=271.8 \mathrm{~Hz}\right), 124.3\left(\mathrm{q}, \mathrm{J}_{\mathrm{CF}}=272.0 \mathrm{~Hz}\right), 122.5$, $68.8,63.9,63.3,63.0,51.1,48.9,47.1,44.8,38.4,38.3,36.0,32.3,30.6,27.1,26.6$, 25.7, 24.7, 24.4, $\left.23.1 \mathrm{ppm} ;{ }^{19} \mathrm{~F} \mathrm{NMR} \mathrm{(471} \mathrm{MHz,} \mathrm{CDCl}_{3}\right): \delta=-63.26(\mathrm{~s}, 3 \mathrm{~F}),-63.44$ (s, 6F) ppm; HR-ESI-MS: m/z: $1039.3633\left([\mathrm{M}+\mathrm{H}]^{+}\right.$, calcd. for $\mathrm{C}_{50} \mathrm{H}_{56} \mathrm{~F}_{9} \mathrm{~N}_{6} \mathrm{O}_{4} \mathrm{~S}_{2}{ }^{+}$: 1039.3655). 


\section{Donor Synthesis}

\section{General Procedure A for the Synthesis of Furanose Phosphate Donors}

The furanose hemiacetal $\left(1.0 \mathrm{mmol}, 1.0\right.$ eq.) was dissolved in dry $\mathrm{CH}_{2} \mathrm{Cl}_{2}(10 \mathrm{~mL})$ under $\mathrm{N}_{2}$, before 2-chloro-5,5-dimethyl-1,3,2-dioxaphosphirane 2-oxide $(3.0 \mathrm{mmol}$, 3.0 eq.) and DMAP (3.0 mmol, 3.0 eq.) were added at rt. After stirring at rt for $18 \mathrm{~h}$, the reaction was diluted with $\mathrm{CH}_{2} \mathrm{Cl}_{2}$, washed with sat. $\mathrm{NaHCO}_{3}$ solution and concentrated in vacuo. The product was purified by column chromatography (Davisil Grade 643 $150 \AA$ Å pore size, $200-425$ mesh, 0-40-70\% Et 2 O/hexanes, Biotage).

\section{2-((2R,3S,4R,5R)-3,4-Bis(benzyloxy)-5-((benzyloxy)methyl)tetrahydrofuran-2- yl)oxy)-5,5-dimethyl-1,3,2-dioxaphosphinane 2-oxide (1c)}

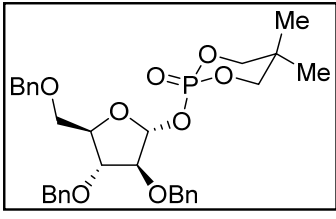

Prepared according to General Procedure A, 2,3,5-tri-O-benzyl$\beta$-D-arabinofuranose $(421 \mathrm{mg}, 1.0 \mathrm{mmol})$ was converted to $1 \mathrm{c}$, yielding the product as a white solid ( $\alpha: \beta 15: 1)$, which upgraded to pure $\alpha$-phosphate after storing as a pure solid at $4{ }^{\circ} \mathrm{C}$ for $24 \mathrm{~h}$ (aonly, $278 \mathrm{mg}, 49 \%$ ).

${ }^{1} \mathrm{H} \mathrm{NMR}\left(600 \mathrm{MHz}, \mathrm{CDCl}_{3}\right): \delta=7.39-7.22(\mathrm{~m}, 15 \mathrm{H}), 5.99(\mathrm{~d}, J=4.1 \mathrm{~Hz}, 1 \mathrm{H}), 4.68(\mathrm{~d}$, $J=11.9 \mathrm{~Hz}, 1 \mathrm{H}), 4.57(\mathrm{~d}, J=3.0 \mathrm{~Hz}, 2 \mathrm{H}), 4.52(\mathrm{~d}, J=11.8 \mathrm{~Hz}, 1 \mathrm{H}), 4.47(\mathrm{~d}$, $J=3.3 \mathrm{~Hz}, 2 \mathrm{H}), 4.44(\mathrm{q}, J=5.3 \mathrm{~Hz}, 1 \mathrm{H}), 4.22(\mathrm{~d}, J=1.6 \mathrm{~Hz}, 1 \mathrm{H}), 4.13$ (dd, $\left.J_{\mathrm{HH}}=11.0,2.4 \mathrm{~Hz}, 1 \mathrm{H}\right), 4.03(\mathrm{dd}, J=11.2,2.3 \mathrm{~Hz}, 1 \mathrm{H}), 3.96(\mathrm{dd}, J=4.7,1.6 \mathrm{~Hz}, 1 \mathrm{H})$, 3.80 (dddd, $J=32.5,21.8,10.9,2.9 \mathrm{~Hz}, 2 \mathrm{H}$ ), 3.66 (dd, $J=10.4,5.7 \mathrm{~Hz}, 2 \mathrm{H}$ ), 3.61 (dd, $J=10.4,5.4 \mathrm{~Hz}, 2 \mathrm{H}), 1.24(\mathrm{~s}, 3 \mathrm{H}), 0.68(\mathrm{~s}, 3 \mathrm{H}) \mathrm{ppm} ;{ }^{13} \mathrm{C} \mathrm{NMR}\left(126 \mathrm{MHz}, \mathrm{CDCl}_{3}\right)$ : $\delta=138.1,137.6,137.2,128.6,128.6,128.5,128.1,128.1,128.0,127.9,127.8,103.5$ $\left(\mathrm{d}, J_{\mathrm{CP}}=5.2 \mathrm{~Hz}\right), 87.1\left(\mathrm{~d}, J_{\mathrm{CP}}=7.6 \mathrm{~Hz}\right), 84.5,83.7,78.2\left(\mathrm{~d}, J_{\mathrm{CP}}=6.8 \mathrm{~Hz}\right), 78.0(\mathrm{~d}$, $\left.J_{\mathrm{CP}}=6.7 \mathrm{~Hz}\right), 73.6,72.3,72.2,69.9,32.1\left(\mathrm{~d}, \mathrm{~J}_{\mathrm{CP}}=5.8 \mathrm{~Hz}\right), 21.9,20.2 \mathrm{ppm} ;{ }^{31} \mathrm{P} N \mathrm{NMR}$ $\left(162 \mathrm{MHz}, \mathrm{CDCl}_{3}\right): \delta=-10.59 \mathrm{ppm}$; HR-ESI-MS: m/z: $569.2292\left([\mathrm{M}+\mathrm{H}]^{+}\right.$, calcd. for $\mathrm{C}_{31} \mathrm{H}_{38} \mathrm{O}_{8} \mathrm{P}_{1}^{+}$: 569.2299).

\section{2-(((2R,3S,4R,5R)-3,4-Bis(benzyloxy)-5-((S)-1-(benzyloxy)ethyl)tetrahydrofuran- 2-yl)oxy)-5,5-dimethyl-1,3,2-dioxaphosphinane 2-oxide (5a)}

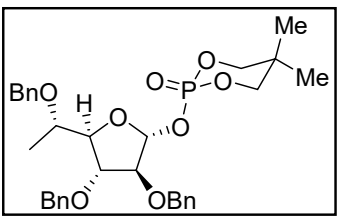

Prepared according to General Procedure A, 2,3,5-tri-O-benzylL-fucofuranose $(580 \mathrm{mg}, 1.4 \mathrm{mmol})$ was converted to phopshate $5 a$, yielding the product as a white solid (inseparable mixture, $\alpha: \beta$ $10: 1,302 \mathrm{mg}, 52 \%)$.

${ }^{1} \mathrm{H} \quad \mathrm{NMR} \quad\left(600 \mathrm{MHz}, \quad \mathrm{CDCl}_{3}\right): \delta=7.37 \quad(\mathrm{~d}, \quad J=4.3 \mathrm{~Hz}, \quad 4 \mathrm{H}), \quad 7.31 \quad$ (ddt, $J=22.4,11.9,7.0 \mathrm{~Hz}, 9 \mathrm{H}), 7.24-7.20(\mathrm{~m}, 2 \mathrm{H}), 6.02(\mathrm{~d}, J=4.3 \mathrm{~Hz}, 1 \mathrm{H}), 4.72(\mathrm{~d}$, $J=11.9 \mathrm{~Hz}, 1 \mathrm{H}), 4.65(\mathrm{~d}, J=11.8 \mathrm{~Hz}, 1 \mathrm{H}), 4.56(\mathrm{dd}, J=18.5,11.8 \mathrm{~Hz}, 2 \mathrm{H})$, $4.46-4.38(\mathrm{~m}, 2 \mathrm{H}), 4.27(\mathrm{t}, J=5.6 \mathrm{~Hz}, 1 \mathrm{H}), 4.22(\mathrm{~d}, J=1.8 \mathrm{~Hz}, 1 \mathrm{H}), 4.16$ (dd, $J=11.0,2.4 \mathrm{~Hz}, 1 \mathrm{H}), 4.06(\mathrm{dd}, J=10.8,2.3 \mathrm{~Hz}, 1 \mathrm{H}), 3.96(\mathrm{dd}, J=5.7,1.8 \mathrm{~Hz}, 1 \mathrm{H})$, 3.82 (tdd, $J=21.7,10.8,2.8 \mathrm{~Hz}, 2 \mathrm{H}), 3.76-3.73(\mathrm{~m}, 1 \mathrm{H}), 1.25$ (s, 3H), 1.22 (d, $J=6.4 \mathrm{~Hz}, 3 \mathrm{H}), 0.72(\mathrm{~s}, 3 \mathrm{H}) \mathrm{ppm} ;{ }^{13} \mathrm{C}$ NMR $\left(126 \mathrm{MHz}, \mathrm{CDCl}_{3}\right): \delta=138.7,137.6$, 137.3, $128.6\left(\mathrm{~d}, J_{\mathrm{CP}}=2.1 \mathrm{~Hz}\right), 128.5,128.5,128.2,128.1(2 \mathrm{C}), 128.1,128.0,127.7$, 103.4 (d, $\left.J_{\mathrm{CP}}=5.0 \mathrm{~Hz}\right), 87.6,87.4\left(\mathrm{~d}, J_{\mathrm{CP}}=7.3 \mathrm{~Hz}\right), 83.4,78.2\left(\mathrm{~d}, J_{\mathrm{CP}}=7.0 \mathrm{~Hz}\right), 78.0$ $\left(\mathrm{d}, \mathrm{J}_{\mathrm{CP}}=6.7 \mathrm{~Hz}\right), 74.1,72.3,72.2,71.6,32.2\left(\mathrm{~d}, \mathrm{~J}_{\mathrm{CP}}=5.9 \mathrm{~Hz}\right), 22.0,20.3,15.9 \mathrm{ppm}$; 
${ }^{31} \mathrm{P}$ NMR $\left(162 \mathrm{MHz}, \mathrm{CDCl}_{3}\right): \delta=-10.50 \mathrm{ppm}$; HR-ESI-MS: $\mathrm{m} / \mathrm{z}: 600.2737\left(\left[\mathrm{M}+\mathrm{NH}_{4}\right]^{+}\right.$, calcd. for $\left.\mathrm{C}_{32} \mathrm{H}_{39} \mathrm{O}_{8} \mathrm{PNH}_{4}^{+}: 600.2721\right)$.

2-(((2R,3R,4S,5S)-3,4-Bis(benzyloxy)-5-((R)-1,2-bis(benzyloxy)ethyl) tetrahydrofuran-2-yl)oxy)-5,5-dimethyl-1,3,2-dioxaphosphinane 2-oxide (5b)

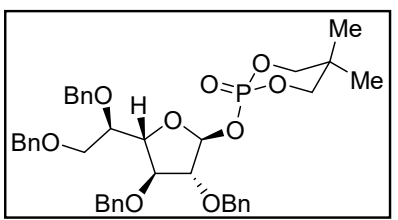

Prepared according to General Procedure A, 2,3,5,6-tetra-Obenzyl-D-galactofuranose $(540 \mathrm{mg}, 1.0 \mathrm{mmol})$ was converted to phosphate $\mathbf{5 b}$, yielding the product as a white solid (inseparable mixture, $\alpha: \beta 1: 7,321 \mathrm{mg}, 47 \%$ ).

${ }^{1} \mathrm{H}$ NMR $\left(600 \mathrm{MHz}, \mathrm{CDCl}_{3}\right): \delta=7.39-7.24(\mathrm{~m}, 18 \mathrm{H}), 7.22-7.17(\mathrm{~m}, 2 \mathrm{H}), 6.00(\mathrm{~d}$, $J=4.4 \mathrm{~Hz}, 1 \mathrm{H}), 4.73(\mathrm{~d}, J=11.8 \mathrm{~Hz}, 1 \mathrm{H}), 4.69(\mathrm{~d}, J=11.9 \mathrm{~Hz}, 1 \mathrm{H}), 4.59(\mathrm{~d}$, $J=11.8 \mathrm{~Hz}, 1 \mathrm{H}), 4.51-4.48(\mathrm{~m}, 2 \mathrm{H}), 4.41-4.36(\mathrm{~m}, 2 \mathrm{H}), 4.29(\mathrm{~d}, J=11.5 \mathrm{~Hz}, 1 \mathrm{H})$, $4.19(\mathrm{~d}, J=2.1 \mathrm{~Hz}, 1 \mathrm{H}), 4.10-4.00(\mathrm{~m}, 4 \mathrm{H}), 3.83-3.72(\mathrm{~m}, 3 \mathrm{H}), 3.67$ (qd, $J=10.2,5.7 \mathrm{~Hz}, 2 \mathrm{H}), 1.23(\mathrm{~s}, 3 \mathrm{H}), 0.66(\mathrm{~s}, 3 \mathrm{H}) \mathrm{ppm} ;{ }^{13} \mathrm{C} \mathrm{NMR}\left(126 \mathrm{MHz}, \mathrm{CDCl}_{3}\right)$ : $\delta=138.4,138.3,137.6,137.3,128.6,128.6,128.5,128.5,128.5,128.4,128.3,128.2$, 128.1, 128.0, 127.8, 127.7, 127.7, $103.4\left(\mathrm{~d}, \mathrm{~J}_{\mathrm{CP}}=5.4 \mathrm{~Hz}\right), 87.5\left(\mathrm{~d}, \mathrm{~J}_{\mathrm{CP}}=7.7 \mathrm{~Hz}\right), 84.7$, 82.8, 78.2 (d, $\left.J_{\mathrm{CP}}=7.1 \mathrm{~Hz}\right), 77.9\left(\mathrm{~d}, J_{\mathrm{CP}}=6.8 \mathrm{~Hz}\right), 76.7,73.5,73.4,72.3,72.1,70.3$, $32.1\left(\mathrm{~d}, \mathrm{~J}_{\mathrm{CP}}=5.9 \mathrm{~Hz}\right), 21.9,20.2 \mathrm{ppm} ;{ }^{31} \mathrm{P} \mathrm{NMR}\left(162 \mathrm{MHz}, \mathrm{CDCl}_{3}\right): \delta=-10.51 \mathrm{ppm}$; HR-ESI-MS: $\mathrm{m} / \mathrm{z}$ : $711.2703\left([\mathrm{M}+\mathrm{Na}]^{+}\right.$, calcd. for $\mathrm{C}_{39} \mathrm{H}_{45} \mathrm{O}_{9} \mathrm{PNa}^{+}:$711.2699).

\section{2-(((2R,3S,4R,5R)-4-(Benzyloxy)-5-((benzyloxy)methyl)-3-fluorotetrahydrofuran-} 2-yl)oxy)-5,5-dimethyl-1,3,2-dioxaphosphinane 2-oxide (5c)

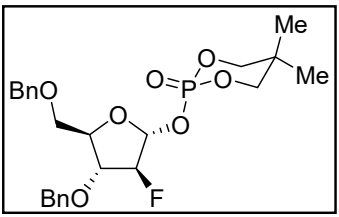

Prepared according to General Procedure A, 3,5-di-O-benzyl-2fluoro- $\beta$-D-arabinofuranose $(250 \mathrm{mg}, 0.75 \mathrm{mmol})$ was converted to phosphate $5 \mathbf{c}$, yielding the product as a white solid ( $\alpha$ only, $192 \mathrm{mg}, 53 \%$ ).

${ }^{1} \mathrm{H}$ NMR $\left(600 \mathrm{MHz}, \mathrm{CDCl}_{3}\right): \delta=7.38-7.28(\mathrm{~m}, 10 \mathrm{H}), 6.03(\mathrm{dd}, J=9.0,4.4 \mathrm{~Hz}, 1 \mathrm{H})$, $5.16(\mathrm{~d}, J=49.6 \mathrm{~Hz}, 1 \mathrm{H}), 4.65(\mathrm{~d}, J=11.5 \mathrm{~Hz}, 1 \mathrm{H}), 4.61-4.54(\mathrm{~m}, 3 \mathrm{H}), 4.47$ (q, $J=5.1 \mathrm{~Hz}, 1 \mathrm{H}), 4.14-4.07(\mathrm{~m}, 2 \mathrm{H}), 4.03(\mathrm{dd}, J=10.8,2.4 \mathrm{~Hz}, 1 \mathrm{H}), 3.83$ (dddd, $J=21.5,18.4,10.8,2.9 \mathrm{~Hz}, \quad 3 \mathrm{H}$ ), 3.68 (dd, $J=10.5,5.3 \mathrm{~Hz}, \quad 1 \mathrm{H}$ ), 3.60 (dd, $J=10.5,5.4 \mathrm{~Hz}, 1 \mathrm{H}), 1.23$ (s, 4H), 0.69 (s, 3H) ppm; ${ }^{13} \mathrm{C}$ NMR (126 MHz, CDCl 3 ): $\delta=137.9, \quad 137.1, \quad 128.7, \quad 128.6, \quad 128.3,128.1, \quad 127.9,127.8,102.5$ (dd, $\left.J_{C F / C P}=38.1,4.9 \mathrm{~Hz}\right), 98.0\left(\mathrm{ddd}, J_{\mathrm{CF} / \mathrm{CP}}=183.9,9.1,2.2 \mathrm{~Hz}\right), 84.9\left(\mathrm{~d}, \mathrm{~J}_{\mathrm{CP}}=1.9 \mathrm{~Hz}\right)$, $82.6\left(\mathrm{~d}, J_{\mathrm{CF}}=26.2 \mathrm{~Hz}\right), 78.3\left(\mathrm{~d}, J_{\mathrm{CP}}=6.8 \mathrm{~Hz}\right), 78.1\left(\mathrm{~d}, J_{\mathrm{CP}}=6.7 \mathrm{~Hz}\right), 73.7,72.7,69.4$, $32.1\left(\mathrm{~d}, J_{\mathrm{CP}}=5.9 \mathrm{~Hz}\right), 21.8,20.2 \mathrm{ppm}$; ${ }^{31} \mathrm{P}$ NMR $\left(162 \mathrm{MHz}, \mathrm{CDCl}_{3}\right): \delta=-10.99(\mathrm{~d}$, $\left.J_{\mathrm{PF}}=1.7 \mathrm{~Hz}\right) \mathrm{ppm} ;{ }^{19} \mathrm{~F}$ NMR $\left(471 \mathrm{MHz}, \mathrm{CDCl}_{3}\right): \delta=-187.86\left(\mathrm{~d}, J_{\mathrm{FP}}=3.3 \mathrm{~Hz}\right) \mathrm{ppm}$; HR-ESI-MS: $m / z$ : $498.2059\left(\left[M+\mathrm{NH}_{4}\right]^{+}\right.$, calcd. for $\mathrm{C}_{24} \mathrm{H}_{30} \mathrm{FO}_{7} \mathrm{PNH}_{4}^{+}:$498.2051).

\section{2-(((2S,3R,4S,5S)-3,4-Bis(benzyloxy)-5-((benzyloxy)methyl)tetrahydrofuran-2-} yl)oxy)-5,5-dimethyl-1,3,2-dioxaphosphinane 2-oxide (5d)

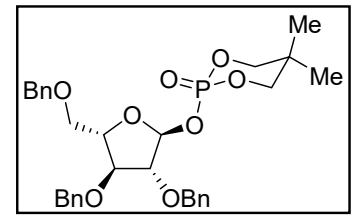

Prepared according to General Procedure A, 2,3,5-tri-O-benzyl$\beta$-L-arabinofuranose $(421 \mathrm{mg}, 1.0 \mathrm{mmol})$ was converted to phopshate $\mathbf{5 d}$, yielding the product as a white solid ( $\alpha: \beta$ 18:1), 
which upgraded to pure $\alpha$-phosphate ( $\alpha$ only) after storing as a pure solid at $4{ }^{\circ} \mathrm{C}$ for $24 \mathrm{~h}(264 \mathrm{mg}, 46 \%)$.

${ }^{1} \mathrm{H}$ NMR $\left(600 \mathrm{MHz}, \mathrm{CDCl}_{3}\right): \delta=7.39-7.22(\mathrm{~m}, 15 \mathrm{H}), 6.00(\mathrm{~d}, J=4.1 \mathrm{~Hz}, 1 \mathrm{H}), 4.68(\mathrm{~d}$, $J=11.8 \mathrm{~Hz}, 1 \mathrm{H}), 4.57(\mathrm{~d}, J=2.9 \mathrm{~Hz}, 2 \mathrm{H}), 4.53(\mathrm{~d}, J=11.9 \mathrm{~Hz}, 1 \mathrm{H}), 4.48(\mathrm{~d}$, $J=3.2 \mathrm{~Hz}, 2 \mathrm{H}), 4.45(\mathrm{q}, J=5.3 \mathrm{~Hz}, 1 \mathrm{H}), 4.23(\mathrm{~d}, J=1.6 \mathrm{~Hz}, 1 \mathrm{H}), 4.13$ (dd, $J=11.0,2.4 \mathrm{~Hz}, 1 \mathrm{H}), 4.04(\mathrm{dd}, J=10.9,2.3 \mathrm{~Hz}, 1 \mathrm{H}), 3.96(\mathrm{dd}, J=4.8,1.7 \mathrm{~Hz}, 1 \mathrm{H})$, 3.80 (dddd, $J=32.6,21.8,10.9,2.9 \mathrm{~Hz}, 2 \mathrm{H}$ ), 3.67 (dd, $J=10.4,5.7 \mathrm{~Hz}, 1 \mathrm{H}$ ), 3.61 (dd, $J=10.4,5.4 \mathrm{~Hz}, 1 \mathrm{H}), 1.24(\mathrm{~s}, 3 \mathrm{H}), 0.69$ (s, 3H) ppm; ${ }^{13} \mathrm{C}$ NMR $\left(126 \mathrm{MHz}, \mathrm{CDCl}_{3}\right)$ : $\delta=138.1,137.6,137.2,128.6,128.6,128.5,128.1,128.1,128.0,127.9,127.8,103.5$ $\left(\mathrm{d}, J_{\mathrm{CP}}=5.3 \mathrm{~Hz}\right), 87.1\left(\mathrm{~d}, J_{\mathrm{CP}}=7.6 \mathrm{~Hz}\right), 84.5,83.7,78.2\left(\mathrm{~d}, J_{\mathrm{CP}}=6.8 \mathrm{~Hz}\right), 78.0(\mathrm{~d}$, $\left.J_{\mathrm{CP}}=6.7 \mathrm{~Hz}\right), 73.6,72.3,72.2,69.9,32.1\left(\mathrm{~d}, J_{\mathrm{CP}}=5.8 \mathrm{~Hz}\right), 21.9,20.2 \mathrm{ppm} ;{ }^{31} \mathrm{P}$ NMR $\left(162 \mathrm{MHz}, \mathrm{CDCl}_{3}\right): \delta=-10.59 \mathrm{ppm}$; HR-ESI-MS: $\mathrm{m} / \mathrm{z}: 591.2126\left([\mathrm{M}+\mathrm{Na}]^{+}\right.$, calcd. for $\left.\mathrm{C}_{31} \mathrm{H}_{37} \mathrm{O}_{8} \mathrm{PNa}^{+}: 591.2124\right)$.

\section{2-(((2S,3R,4R,5R)-3,4-Bis(benzyloxy)-5-((benzyloxy)methyl)tetrahydrofuran-2- yl)oxy)-5,5-dimethyl-1,3,2-dioxaphosphinane 2-oxide (5e)}

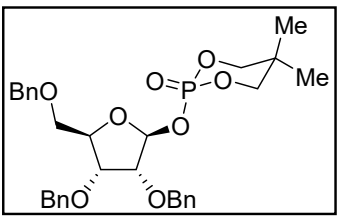

Prepared according to General Procedure A, 2,3,5-tri-O-benzyl$\beta$-D-ribofuranose $(421 \mathrm{mg}, 1.0 \mathrm{mmol})$ was converted to phosphate $\mathbf{5 e}$, yielding the product as a white solid (inseparable mixture, $\beta$ only, $202 \mathrm{mg}, 36 \%$ ).

${ }^{1} \mathrm{H}$ NMR $\left(600 \mathrm{MHz} \mathrm{CDCl}_{3}\right): \delta=7.43-7.38(\mathrm{~m}, 2 \mathrm{H}), 7.37-7.33(\mathrm{~m}, 2 \mathrm{H}), 7.33-7.29$ $(\mathrm{m}, 5 \mathrm{H}), 7.29-7.23(\mathrm{~m}, 6 \mathrm{H}), 5.84(\mathrm{~d}, J=5.2 \mathrm{~Hz}, 1 \mathrm{H}), 4.76(\mathrm{~d}, J=12.0 \mathrm{~Hz}, 1 \mathrm{H}), 4.64$ (d, J = $12.0 \mathrm{~Hz}, 1 \mathrm{H}), 4.55(\mathrm{~d}, J=11.8 \mathrm{~Hz}, 1 \mathrm{H}), 4.54(\mathrm{~d}, J=12.0 \mathrm{~Hz}, 1 \mathrm{H}), 4.49$ (d, $J=12.0 \mathrm{~Hz}, 1 \mathrm{H}), 4.41-4.37(\mathrm{~m}, 2 \mathrm{H}), 4.22(\mathrm{dd}, J=8.1,4.4 \mathrm{~Hz}, 1 \mathrm{H}), 4.10(\mathrm{~d}$, $J=4.5 \mathrm{~Hz}, 1 \mathrm{H}), 4.08(\mathrm{dd}, J=11.0,3.0 \mathrm{~Hz}, 1 \mathrm{H}), 3.87(\mathrm{dd}, J=10.9,2.8 \mathrm{~Hz}, 1 \mathrm{H}), 3.78$ (dd, $J=11.1,2.9 \mathrm{~Hz}, 1 \mathrm{H}$ ), 3.71 (dddd, $J=41.6,21.4,10.9,2.9 \mathrm{~Hz}, 2 \mathrm{H}$ ), 3.59 (dd, $J=11.1,4.4 \mathrm{~Hz}, 1 \mathrm{H}), 1.19(\mathrm{~s}, 3 \mathrm{H}), 0.64(\mathrm{~s}, 3 \mathrm{H}) \mathrm{ppm} ;{ }^{13} \mathrm{C}$ NMR $\left(126 \mathrm{MHz}, \mathrm{CDCl}_{3}\right)$ : $\delta=138.2,137.6,137.4,128.6,128.6,128.5,128.4,128.1,128.1,128.0,127.8,127.7$, $102.1\left(\mathrm{~d}, J_{\mathrm{CP}}=5.0 \mathrm{~Hz}\right), 81.9,79.8\left(\mathrm{~d}, J_{\mathrm{CP}}=6.4 \mathrm{~Hz}\right), 78.1\left(\mathrm{~d}, J_{\mathrm{CP}}=6.3 \mathrm{~Hz}\right), 77.8(\mathrm{~d}$, $\left.J_{\mathrm{CP}}=6.7 \mathrm{~Hz}\right), 76.1,73.4,72.6,72.5,69.6,32.1\left(\mathrm{~d}, J_{\mathrm{CP}}=6.1 \mathrm{~Hz}\right), 21.9,20.2 \mathrm{ppm}$; ${ }^{31} \mathrm{P}$ NMR (162 MHz, $\left.\mathrm{CDCl}_{3}\right): \delta=-10.56 \mathrm{ppm}$; HR-ESI-MS: $m / z: 591.2128\left([\mathrm{M}+\mathrm{Na}]^{+}\right.$, calcd. for $\left.\mathrm{C}_{31} \mathrm{H}_{37} \mathrm{O}_{8} \mathrm{PNa}^{+}: 591.2124\right)$.

\section{2-(((2S,3R,4S,5R)-3,4-Bis(benzyloxy)-5-((benzyloxy)methyl)tetrahydrofuran-2- yl)oxy)-5,5-dimethyl-1,3,2-dioxaphosphinane 2-oxide (5f)}

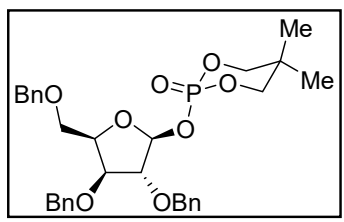

Prepared according to General Procedure A, 2,3,5-tri-O-benzyl$\beta$-D-xylofuranose $(421 \mathrm{mg}, \quad 1.0 \mathrm{mmol})$ was converted to phopshate $\mathbf{5 f}$, yielding the product as a white solid (inseparable mixture, $\alpha: \beta<1: 30,214 \mathrm{mg}, 38 \%)$.

${ }^{1} \mathrm{H}$ NMR $\left(600 \mathrm{MHz}, \mathrm{CDCl}_{3}\right): \delta=7.39-7.22(\mathrm{~m}, 15 \mathrm{H}), 5.94(\mathrm{~d}, J=4.1 \mathrm{~Hz}, 1 \mathrm{H}), 4.71(\mathrm{~d}$, $J=11.9 \mathrm{~Hz}, 1 \mathrm{H}), 4.63-4.59(\mathrm{~m}, 2 \mathrm{H}), 4.56(\mathrm{dd}, J=12.0,1.2 \mathrm{~Hz}, 2 \mathrm{H}), 4.53(\mathrm{~d}$, $J=11.5 \mathrm{~Hz}, 1 \mathrm{H}), 4.45(\mathrm{~d}, J=11.5 \mathrm{~Hz}, 1 \mathrm{H}), 4.28(\mathrm{~d}, J=1.5 \mathrm{~Hz}, 1 \mathrm{H}), 4.11-4.06(\mathrm{~m}$, $2 \mathrm{H}), 3.92-3.89(\mathrm{~m}, 1 \mathrm{H}), 3.87(\mathrm{dd}, J=10.3,5.4 \mathrm{~Hz}, 1 \mathrm{H}), 3.77(\mathrm{dd}, J=10.3,6.7 \mathrm{~Hz}$, 1H), 3.69 (dddd, $J=22.0,11.2,4.9,2.9 \mathrm{~Hz}, 2 \mathrm{H}), 1.20$ (s, 3H), 0.55 (s, 3H) ppm; ${ }^{13} \mathrm{C}$ NMR $\left(126 \mathrm{MHz}_{\mathrm{CDCl}}\right): \delta=138.2,137.7,137.2,128.7,128.6,128.5,128.2,128.0$, 
128.0, 127.8, 127.8, 103.0 (d, $\left.J_{\mathrm{CP}}=5.2 \mathrm{~Hz}\right), 85.5\left(\mathrm{~d}, J_{\mathrm{CP}}=7.6 \mathrm{~Hz}\right), 82.9,80.9,78.1(\mathrm{~d}$, $\left.J_{\mathrm{CP}}=6.8 \mathrm{~Hz}\right), 77.6\left(\mathrm{~d}, \mathrm{~J}_{\mathrm{CP}}=6.7 \mathrm{~Hz}\right), 73.5,72.4,72.3,68.9,31.9\left(\mathrm{~d}, J_{\mathrm{CP}}=5.7 \mathrm{~Hz}\right)$, 21.9, $20.1 \mathrm{ppm} ;{ }^{31} \mathrm{P}$ NMR (162 MHz, $\left.\mathrm{CDCl}_{3}\right): \delta=-10.61 \mathrm{ppm}$; HR-ESI-MS: $\mathrm{m} / \mathrm{z}$ : $569.2297\left([M+\mathrm{H}]^{+}\right.$, calcd. for $\mathrm{C}_{31} \mathrm{H}_{38} \mathrm{O}_{8} \mathrm{P}^{+}:$569.2299).

\section{2-(((4aR,6R,7S,7aR)-7-(Benzyloxy)-2,2-di-tert-butyltetrahydro-4H-furo[3,2- d][1,3,2]dioxasilin-6-yl)oxy)-5,5-dimethyl-1,3,2-dioxaphosphinane 2-oxide (5g)}

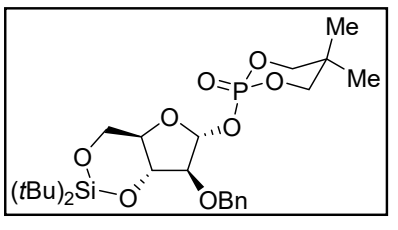

Prepared according to General Procedure A, 2-O-benzyl-3,5(di-tert-butylsilanediyl)- $\beta$-D-arabinofuranose $0.26 \mathbf{m m o l}$ ) was converted to phopshate $\mathbf{5 g}$, yielding the product as a white solid (inseparable mixture, $\alpha: \beta 1: 26,44 \mathrm{mg}$, $32 \%)$.

${ }^{1} \mathrm{H} \mathrm{NMR}\left(600 \mathrm{MHz}, \mathrm{CDCl}_{3}\right): \delta=7.39(\mathrm{~d}, J=7.2 \mathrm{~Hz}, 2 \mathrm{H}), 7.35(\mathrm{t}, J=7.5 \mathrm{~Hz}, 2 \mathrm{H}), 7.32$ $-7.29(\mathrm{~m}, 1 \mathrm{H}), 5.78(\mathrm{dd}, J=6.2,2.2 \mathrm{~Hz}, 1 \mathrm{H}), 4.79(\mathrm{~d}, J=12.0 \mathrm{~Hz}, 1 \mathrm{H}), 4.75(\mathrm{~d}$, $J=12.0 \mathrm{~Hz}, 1 \mathrm{H}), 4.38(\mathrm{dd}, J=9.3,5.0 \mathrm{~Hz}, 1 \mathrm{H}), 4.19-4.13(\mathrm{~m}, 2 \mathrm{H}), 4.10$ (dtd, $J=12.2,6.9,6.0,3.6 \mathrm{~Hz}, 1 \mathrm{H}), 4.05(\mathrm{ddd}, J=11.4,7.3,4.2 \mathrm{~Hz}, 2 \mathrm{H}), 3.98-3.90(\mathrm{~m}$, $3 \mathrm{H}), 1.24(\mathrm{~s}, 3 \mathrm{H}), 1.07$ (s, 9H), $1.01(\mathrm{~s}, 9 \mathrm{H}), 0.89$ (s, 3H) ppm; ${ }^{13} \mathrm{C} \mathrm{NMR}(126 \mathrm{MHz}$, $\left.\mathrm{CDCl}_{3}\right): \delta=137.5,128.5,128.1,128.0,104.6\left(\mathrm{~d}, \mathrm{~J}_{\mathrm{CP}}=5.8 \mathrm{~Hz}\right), 87.8\left(\mathrm{~d}, \mathrm{~J}_{\mathrm{CP}}=8.6 \mathrm{~Hz}\right)$, 81.2, 78.1 (d, JCP = 7.1 Hz), 78.0, 75.5, 72.1, 67.3, $32.3\left(\mathrm{~d}, \mathrm{~J}_{\mathrm{CP}}=6.0 \mathrm{~Hz}\right), 27.6,27.2$, 22.7, 21.8, 20.6, $20.2 \mathrm{ppm}$; ${ }^{31} \mathrm{P} \mathrm{NMR}\left(162 \mathrm{MHz}, \mathrm{CDCl}_{3}\right): \delta=-10.19 \mathrm{ppm}$; HR-ESIMS: $\mathrm{m} / \mathrm{z}$ : $546.2642\left(\left[\mathrm{M}+\mathrm{NH}_{4}\right]^{+}\right.$, calcd. for $\mathrm{C}_{25} \mathrm{H}_{45} \mathrm{NO}_{8} \mathrm{PSi}^{+}$: 546.2647$)$.

$(2 R, 3 R, 4 S, 5 S)-2-((B e n z o y l o x y)$ methyl)-5-((5,5-dimethyl-2-oxido-1,3,2dioxaphosphinan-2-yl)oxy)tetrahydrofuran-3,4-diyl dibenzoate (5h)

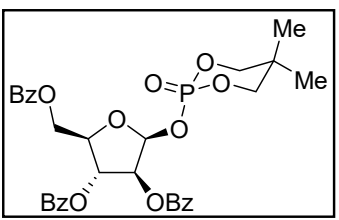

Prepared according to General Procedure A, 2,3,5-O-benzoyl- $\beta$ D-arabinofuranose $(230 \mathrm{mg}, \quad 0.5 \mathrm{mmol})$ was converted to phopshate $\mathbf{5 h}$, yielding the product as a white solid (inseparable mixture, $\alpha: \beta 13: 1,116 \mathrm{mg}, 38 \%)$.

${ }^{1} \mathrm{H} \mathrm{NMR}\left(600 \mathrm{MHz}, \mathrm{CDCl}_{3}\right): \delta=8.12-8.09(\mathrm{~m}, 2 \mathrm{H}), 8.05(\mathrm{td}, J=8.0,1.4 \mathrm{~Hz}, 4 \mathrm{H}), 7.62$ $-7.57(\mathrm{~m}, 2 \mathrm{H}), 7.56-7.52(\mathrm{~m}, 1 \mathrm{H}), 7.48-7.43(\mathrm{~m}, 4 \mathrm{H}), 7.41-7.36(\mathrm{~m}, 2 \mathrm{H}), 6.28(\mathrm{dd}$, $J=5.6,4.3 \mathrm{~Hz}, 1 \mathrm{H}), 5.95(\mathrm{dd}, J=6.7,5.4 \mathrm{~Hz}, 1 \mathrm{H}), 5.76(\mathrm{ddd}, J=6.8,4.3,1.5 \mathrm{~Hz}, 1 \mathrm{H})$, $4.79(\mathrm{dd}, \quad J=11.8,4.9 \mathrm{~Hz}, 1 \mathrm{H}), 4.74(\mathrm{dd}, J=11.8,6.8 \mathrm{~Hz}, 1 \mathrm{H}), 4.64$ (dt, $J=6.9,5.1 \mathrm{~Hz}, 1 \mathrm{H}), 3.98(\mathrm{dd}, J=11.0,3.4 \mathrm{~Hz}, 1 \mathrm{H}), 3.92(\mathrm{dd}, J=11.0,3.4 \mathrm{~Hz}, 1 \mathrm{H})$, 3.80 (ddd, $J=20.9,11.0,2.7 \mathrm{~Hz}, 1 \mathrm{H}$ ), 3.70 (ddd, $J=20.9,11.0,2.8 \mathrm{~Hz}, 1 \mathrm{H}$ ), 1.16 (s, $3 \mathrm{H}), 0.68(\mathrm{~s}, 3 \mathrm{H}) \mathrm{ppm} ;{ }^{13} \mathrm{C} \mathrm{NMR}\left(126 \mathrm{MHz}, \mathrm{CDCl}_{3}\right): \delta=166.3,165.9,165.6,133.9$, 133.8, 133.4, 130.2, 130.1, 129.9, 129.7, 128.7, 128.5, 98.0 (d, JCP = 4.8 Hz), 80.4, $78.1\left(\mathrm{~d}, J_{\mathrm{CP}}=7.1 \mathrm{~Hz}\right), 78.0\left(\mathrm{~d}, J_{\mathrm{CP}}=7.6 \mathrm{~Hz}\right), 75.5,65.4,32.1\left(\mathrm{~d}, J_{\mathrm{CP}}=6.1 \mathrm{~Hz}\right), 21.7$, $20.1 \mathrm{ppm} ;{ }^{31} \mathrm{P}$ NMR (162 MHz, $\left.\mathrm{CDCl}_{3}\right): \delta=-10.63 \mathrm{ppm}$; HR-ESI-MS: $\mathrm{m} / \mathrm{z}: 628.1936$ $\left(\left[M+\mathrm{NH}_{4}\right]^{+}\right.$, calcd. for $\left.\mathrm{C}_{31} \mathrm{H}_{35} \mathrm{NO}_{11} \mathrm{P}^{+}: 628.1942\right)$. 


\section{Hydrogen-bond-donor-catalyzed Furanosylation Reactions}

\section{General Procedure B for the Catalyzed Glycosylation of Furanose Phosphates}

The donor $(0.2 \mathrm{mmol}, 1.0$ eq.), the corresponding acceptor $(0.4 \mathrm{mmol}, 2.0$ eq. $)$, $4 \mathbf{i}$ or ent-4i (0.01 mmol, 0.05 eq.), 1,3,5-trimethoxybenzene (33.6 mg, $0.2 \mathrm{mmol}, 1.0$ eq.) and $4 \AA$ molecular sieves $(500 \mathrm{mg})$ were suspended in $n \mathrm{Pr}_{2} \mathrm{O}(5.0 \mathrm{~mL})$ and the resulting mixture was stirred at the given temperature for the given time. An aliquot $(0.25 \mathrm{~mL})$ was taken from the reaction, filtered through a syringe filter, concentrated in vacuo and analyzed by ${ }^{1} \mathrm{H}$ NMR to determine the NMR yield and $\beta / \alpha$ selectivity. The NMR sample was added to the crude mixture, which was concentrated in vacuo and purified by column chromatography $\left(\mathrm{SiO}_{2}, 0-15-30 \%\right.$ EtOAc/hexanes, Biotage).

\section{General Procedure C for the TMSOTf Induced Glycosylation of Furanose Phosphates}

The donor $(0.018 \mathrm{mmol}, 1.0$ eq. $)$ and the corresponding acceptor $(0.036 \mathrm{mmol}$, 2.0 eq.) were suspended in $\mathrm{CH}_{2} \mathrm{Cl}_{2}(0.5 \mathrm{~mL})$, and the resulting mixture was cooled to $-78{ }^{\circ} \mathrm{C}$ before TMSOTf $(0.003 \mathrm{~mL}, 0.018 \mathrm{mmol}, 1.0$ eq. $)$ was added. The resulting mixture was stirred at $-78{ }^{\circ} \mathrm{C}$ for $1 \mathrm{~h}$, sat. $\mathrm{NaHCO}_{3}$ solution was added, the aqueous phase was extracted with $\mathrm{Et}_{2} \mathrm{O}(3 \mathrm{x}), 1,3,5$-trimethoxybenzene $(3.0 \mathrm{mg}, 0.018 \mathrm{mmol}$, 1.0 eq.) was added in $0.1 \mathrm{~mL} \mathrm{CH} \mathrm{Cl}_{2}$ and the organic phases were concentrated in vacuo. The product yield and anomeric ratio were determined by ${ }^{1} \mathrm{H}$ NMR.

$(2 R, 3 R, 4 S, 5 R)-3,4-B$ is(benzyloxy)-2-((benzyloxy)methyl)-5(octyloxy)tetrahydrofuran (3c)

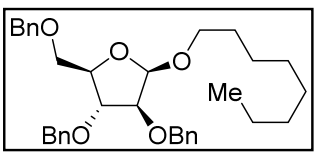

Prepared according to General Procedure B, 1c $(114 \mathrm{mg}$, $0.2 \mathrm{mmol}, 1.0$ eq.) and octanol $2 \mathrm{c}(63 \mu \mathrm{L}, 0.4 \mathrm{mmol}, 2.0$ eq.) were converted to $3 \mathrm{c}$ at $40{ }^{\circ} \mathrm{C}$ in $5 \mathrm{~h}$, yielding the product as a colorless oil $(78 \mathrm{mg}, 73 \%, \beta: \alpha 19: 1)$. The anomeric ratio was determined by integration of the arabinose anomeric signals of the $\beta$ - and $\alpha$-product: ${ }^{1} \mathrm{H}$ NMR $\left(600 \mathrm{MHz}, \mathrm{CDCl}_{3}\right): \delta=5.04\left(\mathrm{~s}, \mathrm{H}_{\alpha}\right), 4.88\left(\mathrm{~d}, J=4.2 \mathrm{~Hz}, \mathrm{H}_{\beta}\right) \mathrm{ppm}$, respectively.

Following General Procedure C, the glycosylation induced by TMSOTf was performed, yielding the desired product (35\% NMR yield, $\beta: \alpha$ 9:1).

${ }^{1} \mathrm{H} \mathrm{NMR}\left(600 \mathrm{MHz}, \mathrm{CDCl}_{3}\right): \delta=7.39-7.26(\mathrm{~m}, 15 \mathrm{H}), 4.88(\mathrm{~d}, J=4.2 \mathrm{~Hz}, 1 \mathrm{H}), 4.68(\mathrm{~d}$, $J=11.8 \mathrm{~Hz}, 1 \mathrm{H}), 4.65-4.57(\mathrm{~m}, 3 \mathrm{H}), 4.56(\mathrm{~s}, 2 \mathrm{H}), 4.14-4.09(\mathrm{~m}, 2 \mathrm{H}), 4.06$ (dd, $J=6.6,4.3 \mathrm{~Hz}, 1 \mathrm{H}), 3.66(\mathrm{dt}, J=9.7,6.8 \mathrm{~Hz}, 1 \mathrm{H}), 3.55(\mathrm{qd}, J=9.9,5.9 \mathrm{~Hz}, 2 \mathrm{H}), 3.32$ (dt, $J=9.7,6.9 \mathrm{~Hz}, 1 \mathrm{H}), 1.62-1.52(\mathrm{~m}, 2 \mathrm{H}), 1.33-1.24(\mathrm{~m}, 10 \mathrm{H}), 0.89(\mathrm{t}, J=7.0 \mathrm{~Hz}$, $3 \mathrm{H}) \mathrm{ppm} ;{ }^{13} \mathrm{C}$ NMR $\left(126 \mathrm{MHz}, \mathrm{CDCl}_{3}\right): \delta=138.4,138.3,137.9,128.5,128.5,128.5$, 128.2, 128.0, 127.9, 127.9, 127.8, 127.8, 127.7, 100.7, 84.4, 83.7, 80.3, 73.5, 72.9, 72.5, 72.4, 68.2, 32.0, 29.6, 29.6, 29.4, 26.4, 22.8, 14.3 ppm; HR-ESI-MS: $\mathrm{m} / \mathrm{z}$ : $550.3529\left(\left[M+\mathrm{NH}_{4}\right]^{+}\right.$, calcd. for $\left.\mathrm{C}_{34} \mathrm{H}_{48} \mathrm{NO}_{5}{ }^{+}: 550.3527\right)$. 
$(2 R, 3 R, 4 S, 5 R)-3,4-B$ is(benzyloxy)-2-((benzyloxy)methyl)-5isopropoxytetrahydrofuran (3d)

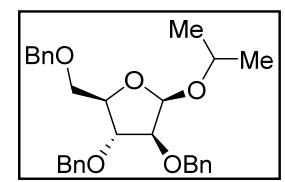

Prepared according to General Procedure B, 1c $(114 \mathrm{mg}, 0.2 \mathrm{mmol}$, 1.0 eq.) and $i \mathrm{PrOH} \mathbf{2 d}(31 \mu \mathrm{L}, 0.4 \mathrm{mmol}, 2.0$ eq.) were converted to $3 \mathrm{~d}$ at $40^{\circ} \mathrm{C}$ in $5 \mathrm{~h}$, yielding the product as a colorless oil $(86 \mathrm{mg}, 93 \%$, $\beta: \alpha>30: 1)$. The anomeric ratio was determined by integration of the arabinose anomeric signals of the $\beta$ - and $\alpha$-product: ${ }^{1} \mathrm{H}$ NMR $\left(600 \mathrm{MHz}, \mathrm{CDCl}_{3}\right)$ : $\delta=5.30\left(\mathrm{~s}, \mathrm{H}_{\alpha}\right), 5.03\left(\mathrm{~d}, J=4.3 \mathrm{~Hz}, \mathrm{H}_{\beta}\right) \mathrm{ppm}$, respectively.

Following General Procedure C, the glycosylation induced by TMSOTf was performed, yielding the desired product (23\% NMR yield, $\beta: \alpha$ 8:1).

${ }^{1} \mathrm{H} \mathrm{NMR}\left(600 \mathrm{MHz}, \mathrm{CDCl}_{3}\right): \delta=7.39-7.26(\mathrm{~m}, 15 \mathrm{H}), 5.03(\mathrm{~d}, J=4.3 \mathrm{~Hz}, 1 \mathrm{H}), 4.69(\mathrm{~d}$, $J=11.8 \mathrm{~Hz}, 1 \mathrm{H}), 4.66-4.59(\mathrm{~m}, 2 \mathrm{H}), 4.58-4.53(\mathrm{~m}, 3 \mathrm{H}), 4.12-4.08(\mathrm{~m}, 2 \mathrm{H}), 4.05$ (dd, $J=6.7,4.3 \mathrm{~Hz}, 1 \mathrm{H}$ ), 3.89 (hept, $J=6.2 \mathrm{~Hz}, 1 \mathrm{H}$ ), 3.57 (qd, $J=9.8,6.0 \mathrm{~Hz}, 2 \mathrm{H}$ ), $1.16(\mathrm{dd}, J=13.8,6.2 \mathrm{~Hz}, 6 \mathrm{H}) \mathrm{ppm} ;{ }^{13} \mathrm{C} \mathrm{NMR}\left(126 \mathrm{MHz}, \mathrm{CDCl}_{3}\right): \delta=138.5,138.3$, $137.9,128.6,128.5,128.5,128.3,128.0,127.9,127.8,127.8,127.7,98.9,84.1,83.8$, 80.1, 73.4, 73.1, 72.5, 72.4, 69.9, 23.6, 21.7 ppm; HR-ESI-MS: $\mathrm{m} / \mathrm{z}: 480.2743$ $\left(\left[\mathrm{M}+\mathrm{NH}_{4}\right]^{+}\right.$, calcd. for $\left.\mathrm{C}_{29} \mathrm{H}_{38} \mathrm{NO}_{5}{ }^{+}: 480.2744\right)$.

(3a $R, 5 R, 5 \mathrm{a} S, 8 \mathrm{a} S, 8 \mathrm{~b} R)-5-(((2 R, 3 S, 4 R, 5 R)-3,4-\mathrm{Bis}$ (benzyloxy)-5-((benzyloxy) methyl)tetrahydrofuran-2-yl)oxy)methyl)-2,2,7,7-tetramethyltetrahydro-5Hbis([1,3]dioxolo) [4,5-b:4',5'-d]pyran (3a)

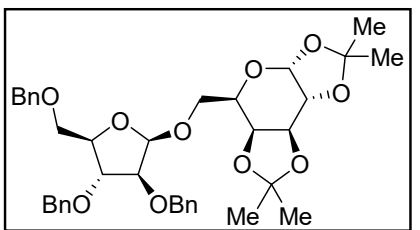

Prepared according to General Procedure B, 1c (114 mg, $0.2 \mathrm{mmol}, \quad 1.0$ eq.) and 1,2:3,4-di-O-isopropylidene- $\alpha$-Dgalactopyranose $2 \mathrm{a} \quad(88 \mathrm{mg}, \quad 0.4 \mathrm{mmol}, \quad 2.0$ eq.) were converted to $3 \mathrm{a}$ at $40{ }^{\circ} \mathrm{C}$ in $3 \mathrm{~h}$, yielding the product as a colorless oil $(115 \mathrm{mg}, 87 \%, \beta: \alpha 25: 1)$. The anomeric ratio was determined by integration of the arabinose anomeric signals of the $\beta$ - and $\alpha$-product: ${ }^{1} \mathrm{H} \mathrm{NMR}\left(600 \mathrm{MHz}, \mathrm{CDCl}_{3}\right): \delta=5.22\left(\mathrm{~d}, J=4.2 \mathrm{~Hz}, \mathrm{H}_{\beta}\right), 5.17\left(\mathrm{~d}, J=1.4 \mathrm{~Hz}, \mathrm{H}_{\alpha}\right) \mathrm{ppm}$, respectively.

Following General Procedure $C$, the glycosylation induced by TMSOTf was performed, yielding the desired product (50\% NMR yield, $\beta: \alpha 4: 1)$.

${ }^{1} \mathrm{H} \mathrm{NMR}\left(600 \mathrm{MHz}, \mathrm{CDCl}_{3}\right): \delta=7.43-7.39(\mathrm{~m}, 2 \mathrm{H}), 7.37-7.31(\mathrm{~m}, 6 \mathrm{H}), 7.31-7.24$ $(\mathrm{m}, 7 \mathrm{H}), 5.56(\mathrm{~d}, J=5.1 \mathrm{~Hz}, 1 \mathrm{H}), 5.22(\mathrm{~d}, J=4.2 \mathrm{~Hz}, 1 \mathrm{H}), 4.77(\mathrm{~d}, J=12.0 \mathrm{~Hz}, 1 \mathrm{H})$, $4.68(\mathrm{~d}, J=11.9 \mathrm{~Hz}, 1 \mathrm{H}), 4.62-4.55(\mathrm{~m}, 5 \mathrm{H}), 4.31(\mathrm{dd}, J=5.1,2.3 \mathrm{~Hz}, 1 \mathrm{H}), 4.18(\mathrm{dd}$, $J=7.9,1.9 \mathrm{~Hz}, 1 \mathrm{H}), 4.15-4.10(\mathrm{~m}, 2 \mathrm{H}), 4.07-4.04(\mathrm{~m}, 1 \mathrm{H}), 4.01(\mathrm{td}, J=5.9,1.9 \mathrm{~Hz}$, $1 \mathrm{H}), 3.76(\mathrm{~d}, J=5.9 \mathrm{~Hz}, 2 \mathrm{H}), 3.64-3.59(\mathrm{~m}, 1 \mathrm{H}), 3.59-3.54(\mathrm{~m}, 1 \mathrm{H}), 1.44(\mathrm{~s}, 3 \mathrm{H})$, $1.43(\mathrm{~s}, 3 \mathrm{H}), 1.32(\mathrm{~s}, 3 \mathrm{H}), 1.31(\mathrm{~s}, 3 \mathrm{H}) \mathrm{ppm} ;{ }^{13} \mathrm{C} \mathrm{NMR}\left(126 \mathrm{MHz}, \mathrm{CDCl}_{3}\right): \delta=138.4$, 138.4, 138.1, 128.5, 128.4, 128.1, 127.9, 127.8, 127.8, 127.7, 127.7, 109.4, 108.7, 101.1, 96.4, 84.0, 83.7, 80.5, 73.4, 72.9, 72.3, 72.0, 71.3, 70.8, 70.5, 68.4, 66.1, 26.2, 26.1, 25.1, 24.6 ppm; HR-ESI-MS: $m / z$ : $685.2967\left([M+N a]^{+}\right.$, calcd. for $\mathrm{C}_{38} \mathrm{H}_{46} \mathrm{O}_{10} \mathrm{Na}^{+}$: $685.2983)$. 
(((3aS,4R,6S,7R,7aS)-7-(((2S,3S,4R,5R)-3,4-Bis(benzyloxy)-5-((benzyloxy) methyl)tetrahydrofuran-2-yl)oxy)-6-methoxy-2,2-dimethyltetrahydro-4H[1,3]dioxolo[4,5-c]pyran-4-yl)methoxy)(tert-butyl)diphenylsilane (3e)

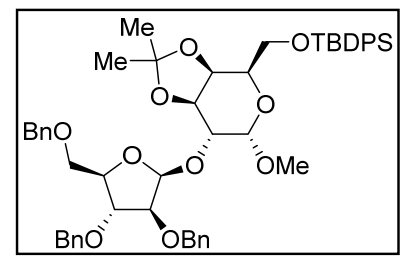

Prepared according to General Procedure B, 1c $(114 \mathrm{mg}$, $0.2 \mathrm{mmol}, 1.0$ eq.) and 1-O-methyl-3,4-O-acetonide-6-OTBDPS- $\alpha$-D-galactopyranose 2 e (189 $\mathrm{mg}, 0.4 \mathrm{mmol}, 2.0$ eq.) were converted to $3 \mathrm{e}$ at $40{ }^{\circ} \mathrm{C}$ in $18 \mathrm{~h}$, yielding the product as a colorless oil (156 mg, $90 \%, \beta: \alpha>30: 1$ ), which solidified in the fridge. The anomeric ratio was determined by integration of the arabinose anomeric signals of the $\beta$ - and $\alpha$-product: ${ }^{1} \mathrm{H}$ NMR $\left(600 \mathrm{MHz}, \mathrm{CDCl}_{3}\right)$ : $\delta=5.30\left(\mathrm{~d}, J=4.6 \mathrm{~Hz}, \mathrm{H}_{\beta}\right), 5.20\left(\mathrm{~d}, J=1.4 \mathrm{~Hz}, \mathrm{H}_{\alpha}\right) \mathrm{ppm}$, respectively.

Following General Procedure $\mathrm{C}$, the glycosylation induced by TMSOTf was performed, yielding the desired product (95\% NMR yield, $\beta: \alpha 5: 1)$.

${ }^{1} \mathrm{H}$ NMR $\left(600 \mathrm{MHz}, \mathrm{CDCl}_{3}\right): \delta=7.77(\mathrm{td}, J=7.8,1.5 \mathrm{~Hz}, 4 \mathrm{H}), 7.53-7.27(\mathrm{~m}, 21 \mathrm{H})$, $5.36(\mathrm{~d}, J=4.5 \mathrm{~Hz}, 1 \mathrm{H}), 4.86(\mathrm{~d}, J=11.4 \mathrm{~Hz}, 1 \mathrm{H}), 4.78(\mathrm{~d}, J=11.9 \mathrm{~Hz}, 1 \mathrm{H}), 4.77(\mathrm{~d}$, $J=3.5 \mathrm{~Hz}, 1 \mathrm{H}), 4.67(\mathrm{~d}, J=11.8 \mathrm{~Hz}, 1 \mathrm{H}), 4.60-4.55(\mathrm{~m}, 3 \mathrm{H}), 4.36$ (dd, $J=8.1,5.3 \mathrm{~Hz}, 1 \mathrm{H}), 4.33(\mathrm{dd}, J=5.3,2.5 \mathrm{~Hz}, 1 \mathrm{H}), 4.22(\mathrm{dd}, J=7.0,5.9 \mathrm{~Hz}, 1 \mathrm{H}), 4.17$ (q, $J=6.0 \mathrm{~Hz}, 1 \mathrm{H}), 4.12$ (dd, $J=7.0,4.5 \mathrm{~Hz}, 1 \mathrm{H}), 4.08(\mathrm{td}, J=6.6,2.4 \mathrm{~Hz}, 1 \mathrm{H}), 4.03$ (dd, $J=9.9,6.8 \mathrm{~Hz}, 1 \mathrm{H}), 3.94(\mathrm{dd}, J=9.9,6.4 \mathrm{~Hz}, 1 \mathrm{H}), 3.80(\mathrm{~d}, J=7.1 \mathrm{~Hz}, 2 \mathrm{H}), 3.63$ (qd, J = 9.8, 6.2 Hz, 2H), 3.28 (s, 3H), $1.57(\mathrm{~s}, 3 \mathrm{H}), 1.39$ (s, 3H), 1.13 (s, 10H) ppm; ${ }^{13} \mathrm{C}$ NMR $\left(126 \mathrm{MHz} \mathrm{CDCl}_{3}\right): \delta=138.5,138.2,138.0,135.8,135.7,133.7,133.6$, $129.8,128.5,128.5,128.4,128.4,127.8,127.8,127.8,127.8,127.7,127.6,109.0$, 102.6, 99.5, 83.7, 83.4, 80.2, 78.0, 75.6, 73.6, 73.3, 72.6, 72.4, 71.8, 67.7, 63.1, 55.4, 55.1, 53.5, 26.9, 19.3 ppm; HR-ESI-MS: $\mathrm{m} / \mathrm{z}: 897.3985\left([\mathrm{M}+\mathrm{Na}]^{+}\right.$, calcd. for $\mathrm{C}_{52} \mathrm{H}_{62} \mathrm{O}_{10} \mathrm{SiNa}^{+}:$897.4004).

(3aR,4S,6R,7S,7aR)-7-(((2S,3S,4R,5R)-3,4-Bis(benzyloxy)-5-((benzyloxy)methyl) tetrahydrofuran-2-yl)oxy)-6-methoxy-2,2,4-trimethyltetrahydro-4H[1,3]dioxolo[4,5-c]pyran (3f)

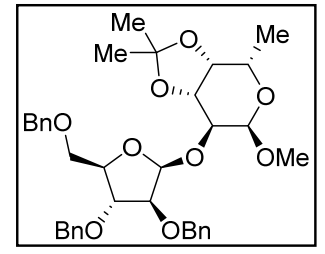

Prepared according to General Procedure B, 1c $(114 \mathrm{mg}$, $0.2 \mathrm{mmol}, \quad 1.0$ eq.) and 1-O-methyl-3,4-O-isoproylidene- $\alpha-\mathrm{L}-$ fucopyranose $2 \mathrm{f}$ ( $87 \mathrm{mg}, 0.4 \mathrm{mmol}, 2.0$ eq.) were converted to $\mathbf{3 f}$ at $40{ }^{\circ} \mathrm{C}$ in $18 \mathrm{~h}$, yielding the product as a colorless oil $(64 \mathrm{mg}, 52 \%$, $\beta: \alpha>30: 1)$, which solidified in the fridge. The anomeric ratio was determined by integration of the arabinose anomeric signals of the $\beta$ - and $\alpha$-product: ${ }^{1} \mathrm{H}$ NMR $\left(600 \mathrm{MHz}, \mathrm{CDCl}_{3}\right): \delta=5.19\left(\mathrm{~d}, J=4.3 \mathrm{~Hz}, \mathrm{H}_{\beta}\right), 5.14$ (d, $\left.J=0.9 \mathrm{~Hz}, \mathrm{H}_{\alpha}\right) \mathrm{ppm}$, respectively.

Following General Procedure C, the glycosylation induced by TMSOTf was performed, yielding the desired product (37\% NMR yield, $\beta: \alpha 4: 1)$.

${ }^{1} \mathrm{H}$ NMR $\left(600 \mathrm{MHz} \mathrm{CDCl}_{3}\right): \delta=7.41-7.25(\mathrm{~m}, 15 \mathrm{H}), 5.21(\mathrm{~d}, J=4.3 \mathrm{~Hz}, 1 \mathrm{H}), 4.79(\mathrm{~d}$, $J=3.5 \mathrm{~Hz}, 1 \mathrm{H}), 4.73(\mathrm{dd}, J=16.5,11.6 \mathrm{~Hz}, 2 \mathrm{H}), 4.65(\mathrm{~d}, J=11.9 \mathrm{~Hz}, 1 \mathrm{H}), 4.62-4.53$ $(\mathrm{m}, 3 \mathrm{H}), 4.31(\mathrm{t}, J=6.3 \mathrm{~Hz}, 1 \mathrm{H}), 4.21(\mathrm{dd}, J=7.1,5.8 \mathrm{~Hz}, 1 \mathrm{H}), 4.15(\mathrm{q}, J=6.1 \mathrm{~Hz}$, $1 \mathrm{H}), 4.13$ (qd, $J=6.7,2.3 \mathrm{~Hz}, 1 \mathrm{H}), 4.09$ (dd, $J=7.1,4.3 \mathrm{~Hz}, 1 \mathrm{H}$ ), 4.00 (dd, $J=5.9,2.3 \mathrm{~Hz}, 1 \mathrm{H}), 3.92(\mathrm{dd}, J=6.8,3.5 \mathrm{~Hz}, 1 \mathrm{H}), 3.73-3.65(\mathrm{~m}, 2 \mathrm{H}), 3.40(\mathrm{~s}, 3 \mathrm{H})$, 
$1.48(\mathrm{~s}, 3 \mathrm{H}), 1.31(\mathrm{~m}, 6 \mathrm{H}) \mathrm{ppm} ;{ }^{13} \mathrm{C}$ NMR (126 MHz, $\left.\mathrm{CDCl}_{3}\right): \delta=138.5,138.4,138.1$, $128.5,128.5,128.4,128.1,127.9,127.8,127.7,127.7,127.6,109.1,99.3,97.5,84.2$, 83.6, 80.5, 76.0, 74.6, 73.3, 73.2, 72.9, 72.4, 72.0, 64.1, 55.4, 27.9, 26.2, 16.4 ppm; HR-ESI-MS: $m / z$ : $643.2863\left([M+N a]^{+}\right.$, calcd. for $\mathrm{C}_{36} \mathrm{H}_{44} \mathrm{O}_{9} \mathrm{Na}^{+}:$643.2878).

(3aR,4R,6S,7S,7aR)-7-(((2S,3S,4R,5R)-3,4-Bis(benzyloxy)-5-((benzyloxy)methyl) tetrahydrofuran-2-yl)oxy)-4-methoxy-2,2,6-trimethyltetrahydro-4H$[1,3]$ dioxolo[4,5-c]pyran $(3 \mathrm{~g})$

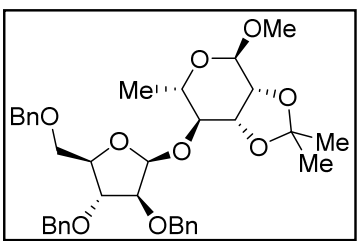

Prepared according to General Procedure B, 1c (114 mg, $0.2 \mathrm{mmol}, 1.0$ eq.) and 1-O-methyl-2,3-O-isoproylidene- $\alpha-\mathrm{L}-$ rhamnopyranose $2 \mathrm{~g}(87 \mathrm{mg}, 0.4 \mathrm{mmol}, 2.0$ eq.) were converted to $3 \mathrm{~g}$ at $40{ }^{\circ} \mathrm{C}$ in $18 \mathrm{~h}$, yielding the product as a colorless oil (82 $\mathrm{mg}, 66 \%, \beta: \alpha>30: 1$ ), which solidified in the fridge. The anomeric ratio was determined by integration of the arabinose anomeric signals of the $\beta$ - and $\alpha$-product: ${ }^{1} \mathrm{H}$ NMR $\left(600 \mathrm{MHz}, \mathrm{CDCl}_{3}\right): \delta=5.55$ (d, $\left.J=3.7 \mathrm{~Hz}, \mathrm{H}_{\beta}\right), 5.44\left(\mathrm{~s}, \mathrm{H}_{\alpha}\right)$ ppm, respectively.

Following General Procedure $\mathrm{C}$, the glycosylation induced by TMSOTf was performed, yielding the desired product (70\% NMR yield, $\beta: \alpha 6: 1)$.

${ }^{1} \mathrm{H}$ NMR $\left(600 \mathrm{MHz}, \mathrm{CDCl}_{3}\right): \delta=7.42-7.39(\mathrm{~m}, 2 \mathrm{H}), 7.39-7.35(\mathrm{~m}, 2 \mathrm{H}), 7.35-7.27$ $(\mathrm{m}, 11 \mathrm{H}), 5.60-5.56(\mathrm{~m}, 1 \mathrm{H}), 4.87(\mathrm{~s}, 1 \mathrm{H}), 4.79(\mathrm{dd}, J=25.0,11.7 \mathrm{~Hz}, 2 \mathrm{H}), 4.62$ (dd, $J=18.0,11.7 \mathrm{~Hz}, 2 \mathrm{H}), 4.59-4.50(\mathrm{~m}, 2 \mathrm{H}), 4.17(\mathrm{dd}, J=7.3,5.7 \mathrm{~Hz}, 1 \mathrm{H}), 4.10(\mathrm{~d}$, $J=6.1 \mathrm{~Hz}, 4 \mathrm{H}), 3.66(\mathrm{dq}, J=10.1,6.2 \mathrm{~Hz}, 1 \mathrm{H}), 3.60-3.53(\mathrm{~m}, 3 \mathrm{H}), 3.36(\mathrm{~s}, 3 \mathrm{H}), 1.57$ (s, 3H), $1.37(\mathrm{~s}, 3 \mathrm{H}), 1.24(\mathrm{~d}, J=6.2 \mathrm{~Hz}, 3 \mathrm{H}) \mathrm{ppm} ;{ }^{13} \mathrm{C}$ NMR $\left(126 \mathrm{MHz}, \mathrm{CDCl}_{3}\right)$ : $\delta=138.4,138.1,137.9,128.5,128.5,128.4,128.1,127.9,127.9,127.8,127.8,127.7$, 109.5, 100.0, 98.0, 98.0, 83.7, 82.6, 79.5, 79.3, 78.6, 76.1, 73.4, 72.6, 72.3, 72.3, 64.1, 54.8, 28.1, 26.5, $18.1 \mathrm{ppm}$; HR-ESI-MS: $\mathrm{m} / \mathrm{z}: 643.2864\left([\mathrm{M}+\mathrm{Na}]^{+}\right.$, calcd. for $\mathrm{C}_{36} \mathrm{H}_{44} \mathrm{O}_{9} \mathrm{Na}^{+}:$643.2878).

(4aR,6S,7R,8S,8aR)-8-(Benzyloxy)-7-(((2S,3S,4R,5R)-3,4-bis(benzyloxy)-5((benzyloxy)methyl)tetrahydrofuran-2-yl)oxy)-6-methoxy-2-phenylhexahydropyrano[3,2-d][1,3]dioxine $(3 \mathrm{~h})$

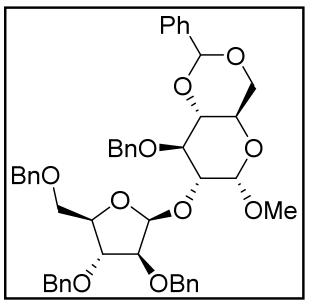

Prepared according to General Procedure B, 1c $(114 \mathrm{mg}$, $0.2 \mathrm{mmol}, 1.0$ eq.) and 1-O-methyl-3-O-benzyl-4,5-O-benzylidene$\alpha$-D-glucopyranose $\mathbf{2 h}$ (149 $\mathrm{mg}, 0.4 \mathrm{mmol}, 2.0$ eq.) were converted to $3 \mathrm{~h}$ at $40{ }^{\circ} \mathrm{C}$ in $48 \mathrm{~h}$, yielding the product as a colorless oil $(104 \mathrm{mg}, 67 \%, \beta: \alpha 23: 1)$, which solidified in the fridge. The anomeric ratio was determined by integration of the arabinose anomeric signals of the $\beta$ - and $\alpha$-product: ${ }^{1} \mathrm{H}$ NMR $(600 \mathrm{MHz}$, $\left.\mathrm{CDCl}_{3}\right): \delta=5.30\left(\mathrm{~d}, J=4.5 \mathrm{~Hz}, \mathrm{H}_{\beta}\right), 5.25\left(\mathrm{~s}, \mathrm{H}_{\alpha}\right) \mathrm{ppm}$, respectively.

Following General Procedure C, the glycosylation induced by TMSOTf was performed, yielding the desired product (73\% NMR yield, $\beta: \alpha$ 8:1).

${ }^{1} \mathrm{H}$ NMR $\left(600 \mathrm{MHz}, \mathrm{CDCl}_{3}\right): \delta=7.46(\mathrm{dd}, J=7.5,2.3 \mathrm{~Hz}, 2 \mathrm{H}), 7.41-7.15(\mathrm{~m}, 23 \mathrm{H})$, $5.58(\mathrm{~s}, 1 \mathrm{H}), 5.27(\mathrm{~d}, J=4.5 \mathrm{~Hz}, 1 \mathrm{H}), 4.98(\mathrm{~d}, J=11.7 \mathrm{~Hz}, 1 \mathrm{H}), 4.87(\mathrm{~d}, J=3.7 \mathrm{~Hz}$, $1 \mathrm{H}), 4.74-4.70(\mathrm{~m}, 2 \mathrm{H}), 4.69(\mathrm{~d}, J=3.5 \mathrm{~Hz}, 1 \mathrm{H}), 4.62(\mathrm{~d}, J=11.8 \mathrm{~Hz}, 1 \mathrm{H}), 4.58$ (s, 
2H), $4.40(\mathrm{~d}, \quad J=12.2 \mathrm{~Hz}, 1 \mathrm{H}), \quad 4.31$ (dd, $J=10.1,4.6 \mathrm{~Hz}, 1 \mathrm{H}$ ), 4.19 (dd, $J=7.1,6.0 \mathrm{~Hz}, 1 \mathrm{H}), 4.12(\mathrm{q}, J=6.2 \mathrm{~Hz}, 1 \mathrm{H}), 4.08-4.02(\mathrm{~m}, 2 \mathrm{H}), 3.85$ (td, $J=9.9,4.6 \mathrm{~Hz}, 1 \mathrm{H}), 3.78(\mathrm{t}, J=10.3 \mathrm{~Hz}, 1 \mathrm{H}), 3.74(\mathrm{dd}, J=9.4,3.7 \mathrm{~Hz}, 1 \mathrm{H}), 3.69(\mathrm{t}$, $J=9.3 \mathrm{~Hz}, 1 \mathrm{H}), 3.61$ (qd, $J=9.8,6.2 \mathrm{~Hz}, 2 \mathrm{H}), 3.29$ (s, 3H) ppm; ${ }^{13} \mathrm{C} \mathrm{NMR}(126 \mathrm{MHz}$, $\left.\mathrm{CDCl}_{3}\right): \delta=139.0,138.3,138.1,137.8,137.5,129.0,128.6,128.4,128.4,128.3$, $127.9,127.9,127.9,127.8,127.8,127.7,127.7,127.7,127.4,127.4,126.1,103.3$, 101.4, 100.1, 83.3, 83.2, 82.9, 80.0, 79.5, 77.8, 74.7, 73.3, 72.5, 72.4, 71.8, 69.3, 62.2, 54.7 ppm; HR-ESI-MS: m/z: $797.3284\left([M+N a]^{+}\right.$, calcd. for $\mathrm{C}_{47} \mathrm{H}_{50} \mathrm{O}_{10} \mathrm{Na}^{+}:$797.3296).

$(2 S, 3 R, 4 S, 5 R, 6 R)-3,4-B i s($ allyloxy)-6-((benzyloxy)methyl)-5-(((2S,3S,4R,5R)-3,4bis(benzyloxy)-5-((benzyloxy)methyl)tetrahydrofuran-2-yl)oxy)-2-methoxy tetrahydro-2H-pyran (3i)

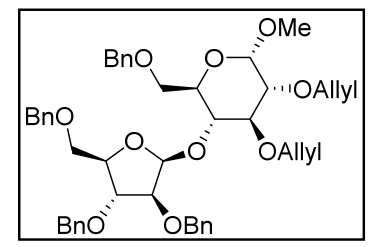

Prepared according to General Procedure B, 1c (114 mg, $0.2 \mathrm{mmol}, 1.0$ eq.) and 1-O-methyl-2,3-di-O-allyl-6-O-benzyl- $\alpha$ D-glucopyranose $2 \mathbf{i}(146 \mathrm{mg}, 0.4 \mathrm{mmol}, 2.0$ eq.) were converted to $3 \mathbf{i}$ at $40{ }^{\circ} \mathrm{C}$ in $48 \mathrm{~h}$, yielding the product as a colorless oil (117 $\mathrm{mg}, 76 \%, \beta: \alpha 24: 1$ ). The anomeric ratio was determined by integration of the arabinose anomeric signals of the $\beta$ - and $\alpha$ product: ${ }^{1} \mathrm{H}$ NMR $\left(600 \mathrm{MHz}, \mathrm{CDCl}_{3}\right): \delta=1 \mathrm{H} \mathrm{NMR}(600 \mathrm{MHz}$, Chloroform-d) $\delta 4.80(\mathrm{~d}$, $J=3.5 \mathrm{~Hz}, \mathrm{H}_{\beta}$ ), $4.78\left(\mathrm{~s}, \mathrm{H}_{\alpha}\right) \mathrm{ppm}$, respectively.

Following General Procedure $C$, the glycosylation induced by TMSOTf was performed, yielding the desired product ( $82 \%$ NMR yield, $\beta: \alpha$ 9:1).

${ }^{1} \mathrm{H}$ NMR $\left(600 \mathrm{MHz}, \mathrm{CDCl}_{3}\right): \delta=7.48-7.17(\mathrm{~m}, 20 \mathrm{H}), 6.06-5.99(\mathrm{~m}, 1 \mathrm{H}), 5.95$ (ddt, $J=16.2,10.4,5.9 \mathrm{~Hz}, 1 \mathrm{H}), 5.31(\mathrm{dq}, J=17.2,1.6 \mathrm{~Hz}, 1 \mathrm{H}), 5.28(\mathrm{~s}, 1 \mathrm{H}), 5.23-5.19$ (m, 1H), $5.13(\mathrm{dt}, J=10.5,1.6 \mathrm{~Hz}, 1 \mathrm{H}), 4.80(\mathrm{~d}, J=3.5 \mathrm{~Hz}, 1 \mathrm{H}), 4.66(\mathrm{~d}, J=11.9 \mathrm{~Hz}$, $1 \mathrm{H}), 4.62-4.59(\mathrm{~m}, 2 \mathrm{H}), 4.59-4.55(\mathrm{~m}, 3 \mathrm{H}), 4.43-4.32(\mathrm{~m}, 4 \mathrm{H}), 4.23-4.14(\mathrm{~m}$, $2 \mathrm{H}), 4.14-4.09(\mathrm{~m}, 1 \mathrm{H}), 4.07(\mathrm{dd}, J=6.6,5.2 \mathrm{~Hz}, 1 \mathrm{H}), 3.97(\mathrm{dd}, J=6.5,4.3 \mathrm{~Hz}, 1 \mathrm{H})$, $3.78-3.66(\mathrm{~m}, 5 \mathrm{H}), 3.65-3.60(\mathrm{~m}, 2 \mathrm{H}), 3.46-3.42(\mathrm{~m}, 4 \mathrm{H}) \mathrm{ppm} ;{ }^{13} \mathrm{C} \mathrm{NMR}(126 \mathrm{MHz}$, $\left.\mathrm{CDCl}_{3}\right): \delta=138.4,138.3,138.3,138.0,135.8,134.9,128.4,128.4,128.3,127.8,127.8$ $127.7,127.7,127.6,127.6,127.5,127.5,117.7,116.4,102.4,97.9,84.0,83.5,80.5$, 80.2, 80.1, 80.1, 74.2, 73.3, 73.2, 72.6, 72.5, 72.4, 72.2, 70.0, 69.2, 55.1 ppm; HRESI-MS: $\mathrm{m} / \mathrm{z}$ : $789.3593\left([\mathrm{M}+\mathrm{Na}]^{+}\right.$, calcd. for $\mathrm{C}_{46} \mathrm{H}_{54} \mathrm{O}_{10} \mathrm{Na}^{+}$: 789.3609).

$(2 R, 3 R, 4 S, 5 R)-3,4-B$ is (benzyloxy)-5-(((2S,3S,4R,5R)-3,4-bis(benzyloxy)-5((benzyloxy)methyl)tetrahydrofuran-2-yl)oxy)-2-methoxytetrahydro-2H-pyran (3j)

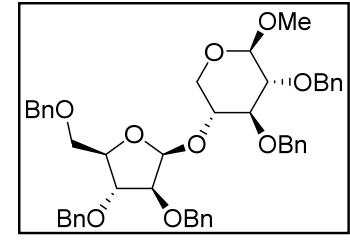

Prepared according to General Procedure B, 1c $(114 \mathrm{mg}$, $0.2 \mathrm{mmol}, \quad 1.0$ eq.) and 1-O-methyl-2,3-di-O-benzyl- $\beta$-Dxylopyranose $2 \mathbf{j}$ (138 mg, $0.4 \mathrm{mmol}, 2.0$ eq.) were converted to $3 \mathbf{j}$ at $40{ }^{\circ} \mathrm{C}$ in $48 \mathrm{~h}$, yielding the product as a colorless oil $(109 \mathrm{mg}$, $73 \%, \beta: \alpha 21: 1)$. The anomeric ratio was determined by integration of the arabinose anomeric signals of the $\beta$ - and $\alpha$-product: ${ }^{1} \mathrm{H}$ NMR $\left(600 \mathrm{MHz}, \mathrm{CDCl}_{3}\right): \delta=5.20\left(\mathrm{~d}, J=1.2 \mathrm{~Hz}, \mathrm{H}_{\beta}\right), 5.08\left(\mathrm{~d}, J=4.0 \mathrm{~Hz}, \mathrm{H}_{\alpha}\right) \mathrm{ppm}$, respecively. 
Following General Procedure C, the glycosylation induced by TMSOTf was performed, yielding the desired product (99\% NMR yield, $\beta: \alpha$ 10:1).

${ }^{1} \mathrm{H}$ NMR $\left(600 \mathrm{MHz}, \mathrm{CDCl}_{3}\right): \delta=7.42-7.22(\mathrm{~m}, 25 \mathrm{H}), 5.13(\mathrm{~d}, J=3.9 \mathrm{~Hz}, 1 \mathrm{H}), 4.92(\mathrm{~d}$, $J=11.0 \mathrm{~Hz}, 1 \mathrm{H}), 4.88(\mathrm{~s}, 2 \mathrm{H}), 4.75(\mathrm{~d}, J=11.0 \mathrm{~Hz}, 1 \mathrm{H}), 4.66(\mathrm{~d}, J=7.5 \mathrm{~Hz}, 2 \mathrm{H}), 4.65$ (s, 2H), $4.36(\mathrm{~d}, J=12.0 \mathrm{~Hz}, 1 \mathrm{H}), 4.29(\mathrm{~d}, J=7.5 \mathrm{~Hz}, 1 \mathrm{H}), 4.25(\mathrm{~d}, J=12.0 \mathrm{~Hz}, 1 \mathrm{H})$, $4.15-4.11(\mathrm{~m}, 1 \mathrm{H}), 4.11-4.09(\mathrm{~m}, 2 \mathrm{H}), 4.05(\mathrm{dd}, J=11.5,5.2 \mathrm{~Hz}, 1 \mathrm{H}), 3.91$ (ddd, $J=9.9,8.8,5.2 \mathrm{~Hz}, 1 \mathrm{H}), 3.63-3.59(\mathrm{~m}, 2 \mathrm{H}), 3.58(\mathrm{~s}, 3 \mathrm{H}), 3.46(\mathrm{dd}, J=10.0,5.4 \mathrm{~Hz}$, $1 \mathrm{H}), 3.44-3.39(\mathrm{~m}, 1 \mathrm{H}), 3.19(\mathrm{dd}, \mathrm{J}=11.6,9.9 \mathrm{~Hz}, 1 \mathrm{H}) \mathrm{ppm} ;{ }^{13} \mathrm{C}$ NMR $(126 \mathrm{MHz}$, $\left.\mathrm{CDCl}_{3}\right): \delta=139.0,138.7,138.4,138.2,137.9,128.6,128.5,128.4,128.4,128.3$, $128.3,128.1,128.0,128.0,128.0,127.9,127.8,127.7,127.7,127.7,127.5,127.5$, 105.4, 98.7, 84.3, 83.1, 82.5, 82.1, 80.6, 75.2, 74.9, 74.0, 73.0, 72.6, 72.4, 63.2, 57.1 ppm; HR-ESI-MS: m/z: $769.3329\left([\mathrm{M}+\mathrm{Na}]^{+}\right.$, calcd. for $\mathrm{C}_{46} \mathrm{H}_{50} \mathrm{O}_{9} \mathrm{Na}^{+}:$769.3347).

$(2 R, 3 R, 4 S, 5 R)-4,5-B$ is(benzyloxy)-3-(((2S,3S,4R,5R)-3,4-bis(benzyloxy)-5((benzyloxy)methyl)tetrahydrofuran-2-yl)oxy)-2-methoxytetrahydro-2H-pyran (3k)

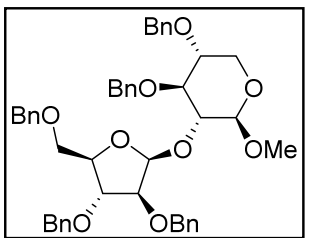

Prepared according to General Procedure B, 1c (114 mg, $0.2 \mathrm{mmol}$, $\quad 1.0$ eq.) and 1-O-methyl-3,4-di-O-benzyl- $\beta$-Dxylopyranose $\mathbf{2 k}$ (138 $\mathrm{mg}, 0.4 \mathrm{mmol}, 2.0$ eq.) were converted to $3 \mathbf{k}$ at $40{ }^{\circ} \mathrm{C}$ in $48 \mathrm{~h}$, yielding the product as a colorless oil $(109 \mathrm{mg}$, $73 \%, \beta: \alpha 10: 1)$. The anomeric ratio was determined by integration of the arabinose anomeric signals of the $\beta$ - and $\alpha$-product: ${ }^{1} \mathrm{H}$ NMR (600 $\left.\mathrm{MHz}_{\mathrm{CDCl}}\right): \delta=5.56\left(\mathrm{~s}, \mathrm{H}_{\alpha}\right), 5.45\left(\mathrm{~d}, J=4.5 \mathrm{~Hz}, \mathrm{H}_{\beta}\right)$ ppm, respectively.

Following General Procedure $\mathrm{C}$, the glycosylation induced by TMSOTf was performed, yielding the desired product (99\% NMR yield, $\beta: \alpha 10: 1)$.

${ }^{1} \mathrm{H}$ NMR $\left(600 \mathrm{MHz}, \mathrm{CDCl}_{3}\right): \delta=7.40-7.23(\mathrm{~m}, 23 \mathrm{H}), 7.21$ (qd, $J=4.6,3.7,1.7 \mathrm{~Hz}$, $2 \mathrm{H}), 5.48(\mathrm{~d}, J=4.5 \mathrm{~Hz}, 1 \mathrm{H}), 4.95(\mathrm{~d}, J=11.5 \mathrm{~Hz}, 1 \mathrm{H}), 4.84(\mathrm{~d}, J=11.5 \mathrm{~Hz}, 1 \mathrm{H}), 4.67$ (s, 2H), $4.66(\mathrm{~d}, J=2.4 \mathrm{~Hz}, 1 \mathrm{H}), 4.64(\mathrm{~d}, J=2.5 \mathrm{~Hz}, 2 \mathrm{H}), 4.60(\mathrm{~d}, J=11.6 \mathrm{~Hz}, 1 \mathrm{H})$, $4.56(\mathrm{~d}, J=12.0 \mathrm{~Hz}, 1 \mathrm{H}), 4.45(\mathrm{~d}, J=11.9 \mathrm{~Hz}, 1 \mathrm{H}), 4.31(\mathrm{dd}, J=4.8,2.0 \mathrm{~Hz}, 1 \mathrm{H}), 4.15$ $(\mathrm{td}, J=4.2,1.8 \mathrm{~Hz}, 2 \mathrm{H}), 4.08-4.04(\mathrm{~m}, 1 \mathrm{H}), 3.98-3.94(\mathrm{~m}, 1 \mathrm{H}), 3.76-3.72(\mathrm{~m}, 1 \mathrm{H})$, $3.67-3.62(\mathrm{~m}, 4 \mathrm{H}), 3.40(\mathrm{~s}, 3 \mathrm{H}), 3.26(\mathrm{ddt}, \mathrm{J}=12.5,6.7,5.3 \mathrm{~Hz}, 1 \mathrm{H}) \mathrm{ppm} ;{ }^{13} \mathrm{C}$ NMR $\left(126 \mathrm{MHz}, \mathrm{CDCl}_{3}\right): \delta=138.9,138.5,138.4,138.1,137.7,128.6,128.5,128.5,128.4$, $128.4,128.1,128.0,128.0,127.9,127.9,127.8,127.7,127.7127 .7,127.4,127.3$, 103.5, 101.4, 83.9, 83.8, 83.7, 80.4, 78.5, 78.4, 74.6, 73.4, 73.0, 72.8, 72.3, 72.2, 63.3, 56.4 ppm; HR-ESI-MS: m/z: $769.3331\left([\mathrm{M}+\mathrm{Na}]^{+}\right.$, calcd. for $\mathrm{C}_{46} \mathrm{H}_{50} \mathrm{O}_{9} \mathrm{Na}^{+}:$769.3347).

(2S,3S,4R,5R)-3,4-Bis(benzyloxy)-2-(((2S,3R,4S,5R)-4-(benzyloxy)-5((benzyloxy)methyl)-2-methoxytetrahydrofuran-3-yl)oxy)-5-((benzyloxy) methyl)tetrahydrofuran (3I)

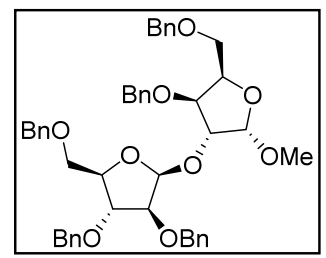

Prepared according to General Procedure B, 1c (114 mg, $0.2 \mathrm{mmol}, \quad 1.0$ eq.) and 1-O-methyl-3,5-di-O-benzyl- $\alpha$-Dxylofuranose $2 \mathrm{l}$ (138 $\mathrm{mg}, 0.4 \mathrm{mmol}, 2.0$ eq.) were converted to $\mathbf{3 l}$ at $40{ }^{\circ} \mathrm{C}$ in $18 \mathrm{~h}$, yielding the product as a colorless oil $(132 \mathrm{mg}$, $88 \%, \beta: \alpha>30: 1)$. The anomeric ratio was determined by integration 
of the arabinose anomeric signals of the $\beta$ - and $\alpha$-product: ${ }^{1} \mathrm{H}$ NMR $\left(600 \mathrm{MHz}, \mathrm{CDCl}_{3}\right)$ : $\delta=5.11\left(\mathrm{~d}, J=4.5 \mathrm{~Hz}, \mathrm{H}_{\beta}\right), 4.98\left(\mathrm{~m}, \mathrm{H}_{\alpha}\right) \mathrm{ppm}$, respectively.

Following General Procedure C, the glycosylation induced by TMSOTf was performed, yielding the desired product (90\% NMR yield, $\beta: \alpha$ 8:1).

${ }^{1} \mathrm{H}$ NMR $\left(600 \mathrm{MHz}, \mathrm{CDCl}_{3}\right): \delta=7.41-7.27(\mathrm{~m}, 23 \mathrm{H}), 7.17(\mathrm{dd}, J=6.7,2.9 \mathrm{~Hz}, 2 \mathrm{H})$, $5.17(\mathrm{~d}, J=4.5 \mathrm{~Hz}, 1 \mathrm{H}), 4.90(\mathrm{~d}, J=4.2 \mathrm{~Hz}, 1 \mathrm{H}), 4.74(\mathrm{t}, J=11.3 \mathrm{~Hz}, 2 \mathrm{H}), 4.69$ (d, $J=11.8 \mathrm{~Hz}, 1 \mathrm{H}), 4.66(\mathrm{~d}, J=12.2 \mathrm{~Hz}, 1 \mathrm{H}), 4.65-4.53(\mathrm{~m}, 6 \mathrm{H}), 4.41-4.36(\mathrm{~m}, 2 \mathrm{H})$, 4.21 (dq, $J=12.3,6.2 \mathrm{~Hz}, 2 \mathrm{H}$ ), 4.14 (ddd, $J=12.7,6.7,4.3 \mathrm{~Hz}, 2 \mathrm{H}$ ), $3.77-3.74(\mathrm{~m}$, $1 \mathrm{H}), 3.72$ (dd, $J=9.8,6.5 \mathrm{~Hz}, 1 \mathrm{H}), 3.67(\mathrm{dd}, J=9.9,5.6 \mathrm{~Hz}, 1 \mathrm{H}), 3.65-3.61(\mathrm{~m}, 1 \mathrm{H})$, 3.37 (s, 3H) ppm; ${ }^{13} \mathrm{C}$ NMR $\left(126 \mathrm{MHz}, \mathrm{CDCl}_{3}\right): \delta=138.3,138.3,138.2,138.1,137.8$, $128.5,128.4,128.4,127.9,127.8,127.8,127.7,127.7,127.7,127.6,127.5,102.2$, 100.8, 84.1, 83.8, 83.4, 80.9, 80.3, 75.0, 73.6, 73.3, 72.5, 72.5, 72.1, 69.6, 54.7 ppm; HR-ESI-MS: $m / z$ : $769.3328\left([M+N a]^{+}\right.$, calcd. for $\mathrm{C}_{46} \mathrm{H}_{50} \mathrm{O}_{9} \mathrm{Na}^{+}:$769.3347).

$(2 S, 3 S, 4 R, 5 R)-3,4-B i s($ benzyloxy)-2-((2R,3R,4S,5R)-4-(benzyloxy)-5((benzyloxy)methyl)-2-methoxytetrahydrofuran-3-yl)oxy)-5-((benzyloxy)methyl) tetrahydrofuran $(3 \mathrm{~m})$

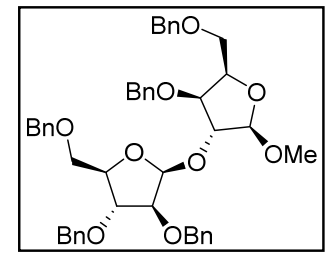

Prepared according to General Procedure B, 1c $(114 \mathrm{mg}$, $0.2 \mathrm{mmol}, \quad 1.0$ eq.) and 1-O-methyl-3,5-di-O-benzyl- $\beta$-Dxylofuranose $2 \mathrm{~m}$ (138 $\mathrm{mg}, 0.4 \mathrm{mmol}, 2.0$ eq.) were converted to $3 \mathrm{~m}$ at $40^{\circ} \mathrm{C}$ in $18 \mathrm{~h}$, yielding the product as a colorless oil $(128 \mathrm{mg}$, $86 \%, \beta: \alpha>30: 1)$. The anomeric ratio was determined by integration of the arabinose anomeric signals of the $\beta$ - and $\alpha$-product: ${ }^{1} \mathrm{H}$ NMR $\left(600 \mathrm{MHz}, \mathrm{CDCl}_{3}\right): \delta=4.87\left(\mathrm{~d}, J=4.4 \mathrm{~Hz}, \mathrm{H}_{\beta}\right), 4.85$ (d, J=1.3 Hz, $\left.\mathrm{H}_{\alpha}\right) \mathrm{ppm}$, respectively.

Following General Procedure C, the glycosylation induced by TMSOTf was performed, yielding the desired product (71\% NMR yield, $\beta: \alpha 2: 1)$.

${ }^{1} \mathrm{H}$ NMR $\left(600 \mathrm{MHz}, \mathrm{CDCl}_{3}\right): \delta=7.42-7.25(\mathrm{~m}, 25 \mathrm{H}), 5.04(\mathrm{~d}, J=1.5 \mathrm{~Hz}, 1 \mathrm{H}), 4.91(\mathrm{~d}$, $J=4.3 \mathrm{~Hz}, 1 \mathrm{H}), 4.71(\mathrm{~d}, J=11.9 \mathrm{~Hz}, 1 \mathrm{H}), 4.65(\mathrm{q}, J=5.5 \mathrm{~Hz}, 3 \mathrm{H}), 4.64(\mathrm{q}, J=3.7 \mathrm{~Hz}$, $2 \mathrm{H}), 4.62-4.60(\mathrm{~m}, 2 \mathrm{H}), 4.57(\mathrm{~d}, J=16.8 \mathrm{~Hz}, 1 \mathrm{H}), 4.54-4.48(\mathrm{~m}, 3 \mathrm{H}), 4.42(\mathrm{dt}$, $J=7.0,5.2 \mathrm{~Hz}, 1 \mathrm{H}), 4.22(\mathrm{dd}, J=2.6,1.6 \mathrm{~Hz}, 1 \mathrm{H}), 4.16(\mathrm{dt}, J=5.7,3.7 \mathrm{~Hz}, 2 \mathrm{H})$, $4.09-4.06(\mathrm{~m}, 1 \mathrm{H}), 3.88$ (dd, $J=5.8,2.5 \mathrm{~Hz}, 1 \mathrm{H}), 3.83$ (dd, $J=10.2,5.0 \mathrm{~Hz}, 1 \mathrm{H}), 3.76$ (dd, $J=10.2,7.1 \mathrm{~Hz}, 1 \mathrm{H}), 3.59(\mathrm{dq}, J=6.0,4.2 \mathrm{~Hz}, 2 \mathrm{H}), 3.39(\mathrm{~s}, 3 \mathrm{H}) \mathrm{ppm} ;{ }^{13} \mathrm{C}$ NMR $\left(126 \mathrm{MHz}_{\mathrm{CDCl}}\right): \delta=138.4,138.2,138.1,137.8,137.6,128.6,128.5,128.5,128.4$, $128.4,128.0,128.0,128.0,127.9,127.9$, 127.8, 127.8, 127.7, 127.7, 127.7, 108.2, 100.1, 84.9, 84.3, 82.9, 81.1, 80.5, 79.9, 73.5, 73.4, 72.6, 72.5, 72.3, 72.1, 69.8, 55.8 ppm; HR-ESI-MS: m/z: $769.3328\left([M+N a]^{+}\right.$, calcd. for $\mathrm{C}_{46} \mathrm{H}_{50} \mathrm{O}_{9} \mathrm{Na}^{+}:$769.3347). 
(3a' $R, 5^{\prime} R, 6$ ' $\left.S, 6 \mathrm{a}^{\prime} R\right)-6$ '-(((2S,3S,4R,5R)-3,4-Bis(benzyloxy)-5-((benzyloxy)methyl) tetrahydrofuran-2-yl)oxy)-5'-((R)-1,4-dioxaspiro[4.5]decan-2-yl)tetrahydrospiro [cyclohexane-1,2'-furo[2,3-d][1,3]dioxole] (3n)

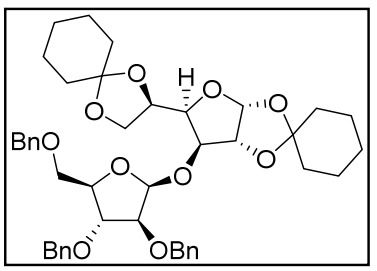

Prepared according to General Procedure B, 1c $(114 \mathrm{mg}$, $0.2 \mathrm{mmol}, \quad 1.0$ eq.) and 1,2:5,6-di-O-cyclohexylidene- $\alpha$-Dglucofuranose $2 \mathrm{n}$ ( $136 \mathrm{mg}, 0.4 \mathrm{mmol}, 2.0$ eq.) were converted to $3 \mathrm{n}$ at $40^{\circ} \mathrm{C}$ in $48 \mathrm{~h}$, yielding the product as a colorless oil (92 mg, 62\%, $\beta: \alpha 20: 1$ ). The anomeric ratio was determined by integration of the arabinose anomeric signals of the $\beta$ - and $\alpha$ product: ${ }^{1} \mathrm{H}$ NMR $\left(600 \mathrm{MHz}, \mathrm{CDCl}_{3}\right): \delta=5.20\left(\mathrm{~s}, \mathrm{H}_{\alpha}\right), 5.05\left(\mathrm{~d}, J=4.2 \mathrm{~Hz}, \mathrm{H}_{\beta}\right) \mathrm{ppm}$, respectively.

Following General Procedure $C$, the glycosylation induced by TMSOTf was performed, yielding the desired product (46\% NMR yield, $\beta: \alpha 2: 1$ ).

${ }^{1} \mathrm{H} \mathrm{NMR}\left(600 \mathrm{MHz}, \mathrm{CDCl}_{3}\right): \delta=7.40-7.26(\mathrm{~m}, 15 \mathrm{H}), 5.77(\mathrm{~d}, J=3.7 \mathrm{~Hz}, 1 \mathrm{H}), 5.07(\mathrm{~d}$, $J=4.2 \mathrm{~Hz}, 1 \mathrm{H}), 4.69(\mathrm{~d}, J=11.7 \mathrm{~Hz}, 1 \mathrm{H}), 4.66-4.59(\mathrm{~m}, 3 \mathrm{H}), 4.57-4.53(\mathrm{~m}, 2 \mathrm{H})$, $4.32(\mathrm{dd}, J=10.6,3.4 \mathrm{~Hz}, 2 \mathrm{H}), 4.31-4.27(\mathrm{~m}, 1 \mathrm{H}), 4.19-4.10(\mathrm{~m}, 3 \mathrm{H}), 4.09-4.03$ $(\mathrm{m}, 2 \mathrm{H}), 4.01(\mathrm{dd}, J=8.6,5.8 \mathrm{~Hz}, 1 \mathrm{H}), 3.69(\mathrm{dd}, J=10.4,6.8 \mathrm{~Hz}, 1 \mathrm{H}), 3.62$ (dd, $J=10.4,5.0 \mathrm{~Hz}, 1 \mathrm{H}), 1.77-1.25(\mathrm{~m}, 2 \mathrm{H}) \mathrm{ppm} ;{ }^{13} \mathrm{C} \mathrm{NMR}\left(126 \mathrm{MHz}, \mathrm{CDCl}_{3}\right)$ : $\delta=138.3,138.2,137.9,128.6,128.5,128.5,128.1,128.1,127.9,127.8,127.7,127.7$, 112.5, 109.6, 104.8, 98.2, 84.6, 82.7, 81.2, 81.1, 80.4, 78.0, 73.2, 72.9, 72.6, 72.3, 72.1, 66.8, 36.6, 36.5, 35.9, 35.1, 25.3, 25.0, 24.2, 24.0, 24.0, 23.7 ppm; HR-ESI-MS: $\mathrm{m} / \mathrm{z}: 765.3592\left([\mathrm{M}+\mathrm{Na}]^{+}\right.$, calcd. for $\mathrm{C}_{44} \mathrm{H}_{54} \mathrm{O}_{10} \mathrm{Na}^{+}:$765.3609).

(2S,3S,4R,5R)-3,4-Bis(benzyloxy)-2-(((2S,3S,4R,5R)-4-(benzyloxy)-5((benzyloxy)methyl)-2-methoxytetrahydrofuran-3-yl)oxy)-5-((benzyloxy) methyl)tetra-hydrofuran (30)

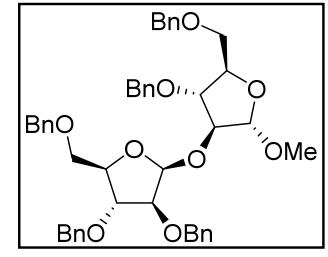

Prepared according to General Procedure B, 1c (114 mg, $0.2 \mathrm{mmol}, \quad 1.0$ eq.) and 1-O-methyl-3,5-di-O-benzyl- $\alpha$-Darabinofuranose 20 (138 $\mathrm{mg}, 0.4 \mathrm{mmol}, 2.0$ eq.) were converted to 30 at $40{ }^{\circ} \mathrm{C}$ in $48 \mathrm{~h}$, yielding the product as a colorless oil $(115 \mathrm{mg}$, $77 \%, \beta: \alpha 22: 1)$. The anomeric ratio was determined by integration of the arabinose anomeric signals of the $\beta$ - and $\alpha$-product: ${ }^{1} \mathrm{H}$ NMR $\left(600 \mathrm{MHz}, \mathrm{CDCl}_{3}\right): \delta=5.06\left(\mathrm{~d}, J=4.2 \mathrm{~Hz}, \mathrm{H}_{\beta}\right), 5.01\left(\mathrm{~s}, \mathrm{H}_{\alpha}\right) \mathrm{ppm}$, respectively.

Following General Procedure C, the glycosylation induced by TMSOTf was performed, yielding the desired product (30\% NMR yield, $\beta: \alpha 2: 1)$.

${ }^{1} \mathrm{H} \mathrm{NMR}\left(600 \mathrm{MHz}, \mathrm{CDCl}_{3}\right): \delta=7.39-7.20(\mathrm{~m}, 25 \mathrm{H}), 5.08(\mathrm{~d}, J=4.3 \mathrm{~Hz}, 1 \mathrm{H}), 4.91(\mathrm{~d}$, $J=1.1 \mathrm{~Hz}, 1 \mathrm{H}), 4.67(\mathrm{t}, J=12.5 \mathrm{~Hz}, 2 \mathrm{H}), 4.64-4.58(\mathrm{~m}, 3 \mathrm{H}), 4.54(\mathrm{~d}, J=1.0 \mathrm{~Hz}, 2 \mathrm{H})$, $4.48(\mathrm{~d}, J=11.9 \mathrm{~Hz}, 1 \mathrm{H}), 4.43(\mathrm{~d}, J=12.0 \mathrm{~Hz}, 1 \mathrm{H}), 4.37(\mathrm{~d}, J=12.0 \mathrm{~Hz}, 1 \mathrm{H}), 4.28(\mathrm{dt}$, $J=1.7,0.9 \mathrm{~Hz}, 1 \mathrm{H}), 4.23(\mathrm{td}, J=5.8,4.3 \mathrm{~Hz}, 1 \mathrm{H}), 4.14-4.11(\mathrm{~m}, 2 \mathrm{H}), 4.11-4.08(\mathrm{~m}$, $1 \mathrm{H}), 4.00(\mathrm{dd}, J=6.1,2.8 \mathrm{~Hz}, 1 \mathrm{H}), 3.58(\mathrm{dd}, J=5.0,2.5 \mathrm{~Hz}, 2 \mathrm{H}), 3.56-3.51(\mathrm{~m}, 2 \mathrm{H})$, 3.40 (s, 3H) ppm; ${ }^{13} \mathrm{C} \mathrm{NMR}\left(126 \mathrm{MHz}, \mathrm{CDCl}_{3}\right): \delta=138.3,138.2,138.1,137.8,128.6$, $128.5,128.5,128.4,128.1,128.1,127.9,127.8,127.8,127.8,127.7,127.7,107.1$, $100.5,86.1,84.2,84.1,83.1,81.5,80.2,73.4,73.2,72.6,72.5,72.3,72.2,70.3$, 
55.1 ppm; HR-ESI-MS: m/z: $769.3329\left([\mathrm{M}+\mathrm{Na}]^{+}\right.$, calcd. for $\mathrm{C}_{46} \mathrm{H}_{50} \mathrm{O}_{9} \mathrm{Na}^{+}$: 769.3347$)$; analytical data in agreement with the literature. ${ }^{16}$

\section{Methyl O-((2R,3S,4R,5R)-3,4-bis(benzyloxy)-5-((benzyloxy)methyl) tetrahydrofuran-2-yl)- $\mathrm{N}$-(tert-butoxycarbonyl)-L-threoninate (3b)}

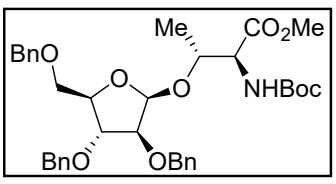

Prepared according to General Procedure B, 1c $(114 \mathrm{mg}$, $0.2 \mathrm{mmol}, 1.0$ eq.) and $N$-Boc-L-threonine methyl ester $\mathbf{2 b}$ (93 $\mathrm{mg}$, $0.4 \mathrm{mmol}, 2.0$ eq.) were converted to $3 \mathrm{~b}$ at $40{ }^{\circ} \mathrm{C}$ in $18 \mathrm{~h}$, yielding the product as a colorless oil (92 $\mathrm{mg}, 72 \%, \beta: \alpha 23: 1)$. The anomeric ratio was determined by integration of the arabinose anomeric signals of the $\beta$ - and $\alpha$-product: ${ }^{1} \mathrm{H}$ NMR $\left(600 \mathrm{MHz}, \mathrm{CDCl}_{3}\right): \delta=5.04\left(\mathrm{~s}, \mathrm{H}_{\alpha}\right), 4.92(\mathrm{~d}, J=4.7 \mathrm{~Hz}$, $\left.\mathrm{H}_{\beta}\right) \mathrm{ppm}$, respectively.

Following General Procedure $C$, the glycosylation induced by TMSOTf was performed, yielding the desired product (22\% NMR yield, $\beta: \alpha 3: 1)$.

${ }^{1} \mathrm{H}$ NMR $\left(600 \mathrm{MHz}, \mathrm{CDCl}_{3}\right): \delta=7.42-7.26(\mathrm{~m}, 13 \mathrm{H}), 7.25-7.21(\mathrm{~m}, 2 \mathrm{H}), 6.27(\mathrm{~d}$, $J=9.7 \mathrm{~Hz}, 1 \mathrm{H}), 4.94(\mathrm{~d}, J=4.9 \mathrm{~Hz}, 1 \mathrm{H}), 4.74(\mathrm{~d}, J=11.7 \mathrm{~Hz}, 1 \mathrm{H}), 4.64(\mathrm{~d}, J=3.0 \mathrm{~Hz}$, 2H), $4.59(\mathrm{q}, J=11.6 \mathrm{~Hz}, 2 \mathrm{H}), 4.51(\mathrm{~d}, J=11.6 \mathrm{~Hz}, 1 \mathrm{H}), 4.41-4.34(\mathrm{~m}, 2 \mathrm{H}), 4.30(\mathrm{t}$, $J=7.7 \mathrm{~Hz}, 1 \mathrm{H}), 4.04-4.00(\mathrm{~m}, 1 \mathrm{H}), 3.84(\mathrm{dt}, J=7.5,3.7 \mathrm{~Hz}, 1 \mathrm{H}), 3.65(\mathrm{~d}, J=1.0 \mathrm{~Hz}$, $3 \mathrm{H}), 3.50$ (dd, $J=10.8,3.3 \mathrm{~Hz}, 1 \mathrm{H}), 3.33$ (dd, $J=10.7,4.0 \mathrm{~Hz}, 1 \mathrm{H}), 1.42$ (d, $J=1.0 \mathrm{~Hz}, 9 \mathrm{H}), 1.28(\mathrm{~d}, J=6.3 \mathrm{~Hz}, 4 \mathrm{H}) \mathrm{ppm} ;{ }^{13} \mathrm{C}$ NMR $\left(126 \mathrm{MHz}, \mathrm{CDCl}_{3}\right): \delta=171.7$, 156.6, 138.4, 137.9, 137.7, 128.6, 128.5, 128.5, 128.4, 128.2, 128.2, 128.1, 127.8, 127.8, 99.7, 83.8, 80.5, 79.7, 79.2, 75.1, 73.2, 72.8, 72.5, 69.1, 58.3, 52.2, 28.5, 18.5 ppm; HR-ESI-MS: m/z: $636.3150\left([\mathrm{M}+\mathrm{H}]^{+}\right.$, calcd. for $\left.\mathrm{C}_{36} \mathrm{H}_{46} \mathrm{NO}_{9}{ }^{+}: 636.3167\right)$.

Methyl O-((2R,3S,4R,5R)-3,4-bis(benzyloxy)-5-((benzyloxy)methyl) tetrahydrofuran-2-yl)-N-(tert-butoxycarbonyl)-D-threoninate (3p)

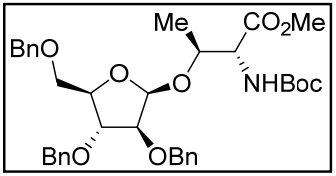

Prepared according to General Procedure B, 1c $(114 \mathrm{mg}$, $0.2 \mathrm{mmol}, 1.0 \mathrm{eq}$.) and $N$-Boc-D-threonine methyl ester $2 p$ (93 mg, $0.4 \mathrm{mmol}, 2.0$ eq.) were converted to $3 \mathrm{p}$ at $40^{\circ} \mathrm{C}$ in $18 \mathrm{~h}$, yielding the product as a colorless oil (107 $\mathrm{mg}, 85 \%, \beta: \alpha>30: 1)$.

The anomeric ratio was determined by integration of the arabinose anomeric signals of the $\beta$ - and $\alpha$-product: ${ }^{1} \mathrm{H}$ NMR $\left(600 \mathrm{MHz}, \mathrm{CDCl}_{3}\right): \delta=5.12\left(\mathrm{~s}, \mathrm{H}_{\alpha}\right), 5.00$ (d, $J=4.4 \mathrm{~Hz}, \mathrm{H}_{\beta}$ ) ppm, respectively.

Following General Procedure C, the glycosylation induced by TMSOTf was performed, yielding the desired product (95\% NMR yield, $\beta: \alpha 3: 1)$.

${ }^{1} \mathrm{H}$ NMR $\left(600 \mathrm{MHz}, \mathrm{CDCl}_{3}\right): \delta=7.45-7.21(\mathrm{~m}, 15 \mathrm{H}), 5.43(\mathrm{~d}, J=9.3 \mathrm{~Hz}, 1 \mathrm{H}), 5.01(\mathrm{~d}$, $J=4.4 \mathrm{~Hz}, 1 \mathrm{H}), 4.66(\mathrm{dd}, J=11.7,7.5 \mathrm{~Hz}, 2 \mathrm{H}), 4.57(\mathrm{~d}, J=14.5 \mathrm{~Hz}, 3 \mathrm{H}), 4.47$ (d, $J=11.7 \mathrm{~Hz}, 1 \mathrm{H}), 4.39(\mathrm{qd}, J=6.4,2.1 \mathrm{~Hz}, 1 \mathrm{H}), 4.25(\mathrm{dd}, J=9.4,2.2 \mathrm{~Hz}, 1 \mathrm{H}), 4.08$ (dt, $J=7.8,3.9 \mathrm{~Hz}, 2 \mathrm{H}), 4.05-3.99(\mathrm{~m}, 1 \mathrm{H}), 3.65(\mathrm{~s}, 3 \mathrm{H}), 3.58-3.51(\mathrm{~m}, 2 \mathrm{H}), 1.45(\mathrm{~s}$, $9 \mathrm{H}), 1.27(\mathrm{~d}, J=6.4 \mathrm{~Hz}, 3 \mathrm{H}) \mathrm{ppm} ;{ }^{13} \mathrm{C} \mathrm{NMR}\left(126 \mathrm{MHz} \mathrm{CDCl}_{3}\right): \delta=171.8,156.2$, 138.2, 138.0, 137.8, 128.5, 128.5, 128.4, 128.0, 127.9, 127.9, 127.8, 127.8, 127.7, 100.9 , 83.9, 82.9, 80.2, 80.0, 74.9, 73.4, 72.4, 72.2, 58.2, 52.4, 28.4, 19.3 ppm; HRESI-MS: $m / z: 636.3150\left([M+\mathrm{H}]^{+}\right.$, calcd. for $\left.\mathrm{C}_{36} \mathrm{H}_{46} \mathrm{NO}_{9} \mathrm{Na}^{+}: 636.3167\right)$. 


\section{Methyl O-((2R,3S,4R,5R)-3,4-bis(benzyloxy)-5-((benzyloxy)methyl)}

tetrahydrofuran-2-yl)-N-(tert-butoxycarbonyl)-L-serinate (3q)

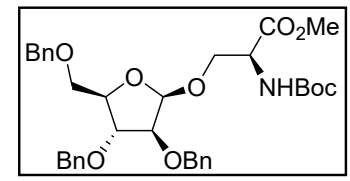

Prepared according to General Procedure B, 1c $(114 \mathrm{mg}$, $0.2 \mathrm{mmol}, 1.0$ eq.) and $\mathrm{N}$-Boc-L-serine methyl ester $2 \mathrm{q}(88 \mathrm{mg}$, $0.4 \mathrm{mmol}, 2.0$ eq.) were converted to $3 \mathrm{q}$ at $40{ }^{\circ} \mathrm{C}$ in $18 \mathrm{~h}$, yielding the product as a colorless oil $(104 \mathrm{mg}, 85 \%, \beta: \alpha 22: 1)$. The anomeric ratio was determined by integration of the arabinose anomeric signals of the $\beta$ - and $\alpha$-product: ${ }^{1} \mathrm{H}$ NMR $\left(600 \mathrm{MHz}, \mathrm{CDCl}_{3}\right): \delta=5.02\left(\mathrm{~s}, \mathrm{H}_{\alpha}\right), 4.77(\mathrm{~d}, J=4.4 \mathrm{~Hz}$, $\left.\mathrm{H}_{\beta}\right) \mathrm{ppm}$, respectively.

Following General Procedure C, the glycosylation induced by TMSOTf was performed, yielding the desired product (55\% NMR yield, $\beta: \alpha 3: 1)$.

${ }^{1} \mathrm{H}$ NMR $\left(600 \mathrm{MHz}, \mathrm{CDCl}_{3}\right): \delta=7.41-7.25(\mathrm{~m}, 15 \mathrm{H}), 5.86(\mathrm{~d}, J=8.7 \mathrm{~Hz}, 1 \mathrm{H}), 4.79(\mathrm{~d}$, $J=4.4 \mathrm{~Hz}, 1 \mathrm{H}), 4.68(\mathrm{~d}, J=11.7 \mathrm{~Hz}, 1 \mathrm{H}), 4.60(\mathrm{dd}, J=9.7,3.8 \mathrm{~Hz}, 3 \mathrm{H}), 4.58-4.54$ $(\mathrm{d}, J=11.7 \mathrm{~Hz}, 1 \mathrm{H}), 4.52-4.46(\mathrm{~m}, 1 \mathrm{H}), 4.16(\mathrm{t}, J=6.9 \mathrm{~Hz}, 1 \mathrm{H}), 4.09$ (dd, $J=10.4,3.3 \mathrm{~Hz}, 1 \mathrm{H}), 4.06(\mathrm{dd}, J=7.4,4.4 \mathrm{~Hz}, 1 \mathrm{H}), 4.04-4.00(\mathrm{~m}, 1 \mathrm{H}), 3.68$ (dd, $J=10.2,3.9 \mathrm{~Hz}, 1 \mathrm{H}), 3.67(\mathrm{~s}, 3 \mathrm{H}), 3.50$ (dd, $J=10.3,4.6 \mathrm{~Hz}, 1 \mathrm{H}), 3.43$ (dd, $J=10.3,5.6 \mathrm{~Hz}, 1 \mathrm{H}), 1.44$ (s, 9H) ppm; ${ }^{13} \mathrm{C} \mathrm{NMR}\left(126 \mathrm{MHz} \mathrm{CDCl}_{3}\right): \delta=171.1,155.7$, 138.2, 138.0, 137.7, 128.6, 128.5, 128.5, 128.4, 128.2, 128.1, 128.0, 127.9, 127.8, 127.8, 127.8, 127.7, 101.1, 84.2, 82.0, 80.0, 80.0, 73.2, 72.7, 72.6, 71.1, 68.5, 53.9, 52.4, $28.4 \mathrm{ppm}$; HR-ESI-MS: $\mathrm{m} / \mathrm{z}$ : $622.3003\left([\mathrm{M}+\mathrm{H}]^{+}\right.$, calcd. for $\mathrm{C}_{35} \mathrm{H}_{44} \mathrm{NO}_{9}{ }^{+}$: $622.3011)$.

1-(tert-Butyl) 2-methyl (2S,4R)-4-((2R,3S,4R,5R)-3,4-bis(benzyloxy)-5((benzyloxy)methyl)tetrahydrofuran-2-yl)oxy)pyrrolidine-1,2-dicarboxylate (3r)

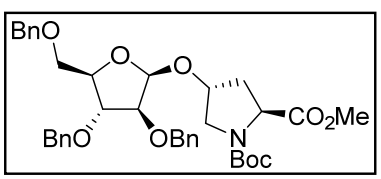

Prepared according to General Procedure B, 1c $(114 \mathrm{mg}$, $0.2 \mathrm{mmol}, 1.0$ eq.) and $N$-Boc-L-hydroxyproline methyl ester $2 \mathrm{r}$ (98 mg, $0.4 \mathrm{mmol}, 2.0$ eq.) were converted to $3 \mathrm{r}$ at $40{ }^{\circ} \mathrm{C}$ in $18 \mathrm{~h}$, yielding the product as a colorless oil $(109 \mathrm{mg}, 84 \%$, $\beta: \alpha>30: 1,2$ rotamers in NMR), which crystallized in the fridge after a few days. The anomeric ratio was determined by integration of the arabinose anomeric signals of the $\beta$ - and $\alpha$-product: ${ }^{1} \mathrm{H}$ NMR $\left(600 \mathrm{MHz}, \mathrm{CDCl}_{3}\right): \delta=5.03 \quad\left(\mathrm{~s}, \mathrm{H}_{\alpha}\right), 4.88$ (dd, $J=7.3,4.2 \mathrm{~Hz}, \mathrm{H}_{\beta}$ ) ppm, respectively.

Following General Procedure C, the glycosylation induced by TMSOTf was performed, yielding the desired product (72\% NMR yield, $\beta: \alpha 4: 1)$.

${ }^{1} \mathrm{H}$ NMR $\left(600 \mathrm{MHz}, \mathrm{CDCl}_{3}\right): \delta=7.40-7.23(\mathrm{~m}, 15 \mathrm{H}), 4.88(\mathrm{dd}, J=7.3,4.2 \mathrm{~Hz}, 1 \mathrm{H})$, $4.68(\mathrm{t}, J=11.6 \mathrm{~Hz}, 1 \mathrm{H}), 4.64-4.58(\mathrm{~m}, 2 \mathrm{H}), 4.58-4.51(\mathrm{~m}, 3 \mathrm{H}), 4.38-4.24(\mathrm{~m}, 2 \mathrm{H})$, $4.14-4.04(\mathrm{~m}, 3 \mathrm{H}), 3.75(\mathrm{~d}, J=8.3 \mathrm{~Hz}, 3 \mathrm{H}), 3.65-3.60(\mathrm{~m}, 1.6 \mathrm{H}), 3.51$ (dddd, $J=15.0,9.4,5.6,3.0 \mathrm{~Hz}, 2 \mathrm{H}), 3.42(\mathrm{dd}, J=11.3,4.1 \mathrm{~Hz}, 0.4 \mathrm{H}), 2.19(\mathrm{~m}, 1 \mathrm{H})$, $2.07-1.96(\mathrm{~m}, 1 \mathrm{H}), 1.48-1.41(\mathrm{~m}, 9 \mathrm{H}) \mathrm{ppm} ;{ }^{13} \mathrm{C} \mathrm{NMR}\left(126 \mathrm{MHz}, \mathrm{CDCl}_{3}\right): \delta=173.5$, 173.2, 154.3, 153.6, 138.3, 138.2, 138.1, 138.0, 137.8, 137.6, 128.6, 128.6, 128.5, $128.5,128.4,128.3,128.2,128.1,128.0,127.9,127.8,127.8,127.7,99.5,99.4,84.4$, 84.3, 83.3, 83.0, 80.3, 80.2, 80.1, 74.9, 74.3, 73.4, 72.9, 72.8, 72.6, 72.3, 72.1, 57.9, 57.5, 52.4, 52.3, 52.2, 52.1, 36.0, 35.0, 30.4, 29.8, 28.5, 28.4, 28.4 ppm; HR-ESI-MS: $\mathrm{m} / \mathrm{z}: 648.3156\left([\mathrm{M}+\mathrm{H}]^{+}\right.$, calcd. for $\left.\mathrm{C}_{37} \mathrm{H}_{46} \mathrm{NO}_{9}{ }^{+}: 648.3167\right)$. 
Crystals suitable for X-ray crystallographic analysis were obtained by slow evaporation from hexanes/Et $2 \mathrm{O}$.

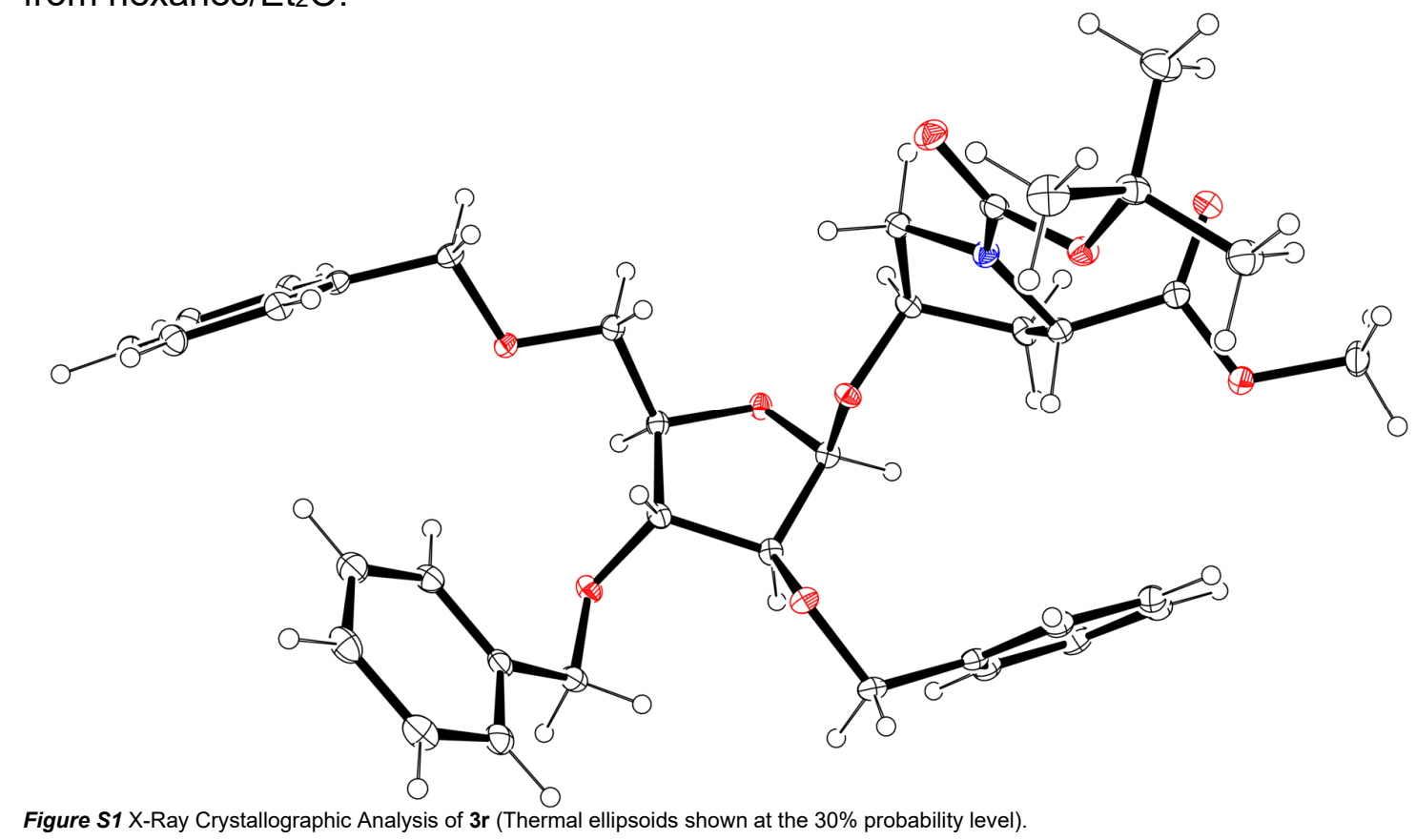

(3aR,5R,5aS,8aS,8bR)-5-(((2R,3S,4R,5R)-3,4-Bis(benzyloxy)-5-((S)-1(benzyloxy)ethyl)tetrahydrofuran-2-yl)oxy)methyl)-2,2,7,7tetramethyltetrahydro-5H-bis([1,3]dioxolo)[4,5-b:4',5'-d]pyran (6a)

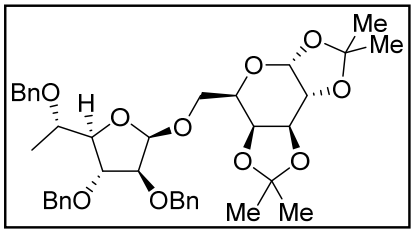

Prepared according to General Procedure B, 5a $(117 \mathrm{mg}$, $0.2 \mathrm{mmol}, \quad 1.0$ eq.) and 1,2:3,4-di-O-isopropylidene- $\alpha$-Dgalactopyranose $2 \mathrm{a}$ (104 $\mathrm{mg}, 0.4 \mathrm{mmol}, 2.0$ eq.) were converted to $6 \mathrm{a}$ at $40^{\circ} \mathrm{C}$ in $18 \mathrm{~h}$, yielding the product as a colorless oil (114 mg, 84\%, $\beta: \alpha 12: 1)$. The anomeric ratio was determined by integration of the arabinose anomeric signals of the $\beta$ - and $\alpha$-product: ${ }^{1} \mathrm{H}$ NMR $\left(600 \mathrm{MHz}, \mathrm{CDCl}_{3}\right): \delta=5.23\left(\mathrm{~d}, J=4.2 \mathrm{~Hz}, \mathrm{H}_{\beta}\right), 5.16\left(\mathrm{~d}, J=1.5 \mathrm{~Hz}, \mathrm{H}_{\alpha}\right) \mathrm{ppm}$, respectively.

Following General Procedure $\mathrm{C}$, the glycosylation induced by TMSOTf was performed, yielding the desired product (91\% NMR yield, $\beta: \alpha$ 9:1).

${ }^{1} \mathrm{H}$ NMR $\left(600 \mathrm{MHz}, \mathrm{CDCl}_{3}\right): \delta=7.46-7.42(\mathrm{~m}, 2 \mathrm{H}), 7.40-7.22(\mathrm{~m}, 13 \mathrm{H}), 5.58(\mathrm{~d}$, $J=5.0 \mathrm{~Hz}, 1 \mathrm{H}), 5.25(\mathrm{~d}, J=4.2 \mathrm{~Hz}, 1 \mathrm{H}), 4.81(\mathrm{dd}, J=11.7,7.4 \mathrm{~Hz}, 2 \mathrm{H}), 4.67(\mathrm{~s}, 2 \mathrm{H})$, $4.60(\mathrm{~d}, J=5.3 \mathrm{~Hz}, 1 \mathrm{H}), 4.60-4.57(\mathrm{~m}, 2 \mathrm{H}), 4.32$ (dd, J = 5.0, $2.3 \mathrm{~Hz}, 1 \mathrm{H}), 4.20$ (dd, $J=7.9,1.9 \mathrm{~Hz}, 1 \mathrm{H}), 4.16(\mathrm{t}, J=7.0 \mathrm{~Hz}, 1 \mathrm{H}), 4.08(\mathrm{dd}, J=7.3,4.2 \mathrm{~Hz}, 1 \mathrm{H}), 4.04(\mathrm{td}$, $J=5.9,1.8 \mathrm{~Hz}, 1 \mathrm{H}), 3.90(\mathrm{dd}, J=7.5,6.7 \mathrm{~Hz}, 1 \mathrm{H}), 3.80(\mathrm{~s}, 2 \mathrm{H}), 3.67(\mathrm{p}, J=6.3 \mathrm{~Hz}$, $1 \mathrm{H}), 1.44(\mathrm{~s}, 6 \mathrm{H}), 1.33(\mathrm{~s}, 3 \mathrm{H}), 1.32(\mathrm{~s}, 3 \mathrm{H}), 1.18(\mathrm{~d}, J=6.4 \mathrm{~Hz}, 3 \mathrm{H}) \mathrm{ppm} ;{ }^{13} \mathrm{C}$ NMR $\left(126 \mathrm{MHz}_{\mathrm{CDCl}}\right): \delta=139.1,138.3,138.1,128.4,128.4,128.4,128.2,128.0,127.9$, 127.8, 127.7, 127.4, 109.3, 108.6, 100.7, 96.4, 84.1, 84.0, 82.2, 77.5, 72.2, 71.9, 71.5, 71.2, 70.8, 70.6, 68.4, 66.1, 26.1, 26.1, 25.0, 24.5, 15.7 ppm; HR-ESI-MS: $\mathrm{m} / \mathrm{z}$ : $699.3124\left([\mathrm{M}+\mathrm{Na}]^{+}\right.$, calcd. for $\left.\mathrm{C}_{39} \mathrm{H}_{48} \mathrm{O}_{10} \mathrm{Na}^{+}: 699.3140\right)$. 
(3aR,5i,5aS,8aS,8bR)-5-((((2S,3R,4S,5S)-3,4-Bis(benzyloxy)-5-((R)-1,2-bis (benzyloxy)ethyl)tetrahydrofuran-2-yl)oxy)methyl)-2,2,7,7-tetramethyl tetrahydro-5H-bis([1,3]dioxolo)[4,5-b:4',5'-d]pyran (6b)

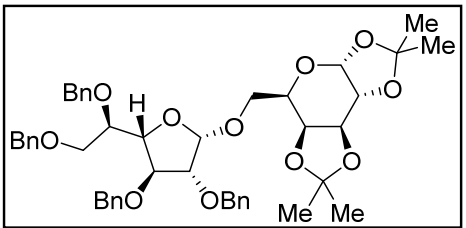

Prepared according to General Procedure B, 5b (138 mg, $0.2 \mathrm{mmol}, 1.0$ eq.) and 1,2:3,4-di-O-isopropylidene- $\alpha$-Dgalactopyranose $2 \mathrm{a}$ (104 $\mathrm{mg}, 0.4 \mathrm{mmol}, 2.0$ eq.) were converted to $6 \mathrm{~b}$ at $40{ }^{\circ} \mathrm{C}$ in $18 \mathrm{~h}$ using ent-4i $(10 \mathrm{mg}$, $0.01 \mathrm{mmol}, 0.05$ eq.), yielding the product as a colorless oil (122 $\mathrm{mg}, 78 \%, \beta: \alpha 1: 11)$. The anomeric ratio was determined by integration of the arabinose anomeric signals of the $\beta$ - and $\alpha$-product: ${ }^{1} \mathrm{H}$ NMR $\left(600 \mathrm{MHz}, \mathrm{CDCl}_{3}\right)$ : $\delta=5.20\left(\mathrm{~s}, \mathrm{H}_{\beta}\right), 5.05\left(\mathrm{~d}, J=4.3 \mathrm{~Hz}, \mathrm{H}_{\alpha}\right) \mathrm{ppm}$, respectively.

Following General Procedure $C$, the glycosylation induced by TMSOTf was performed, yielding the desired product (81\% NMR yield, $\beta: \alpha 1: 3)$.

${ }^{1} \mathrm{H}$ NMR $\left(600 \mathrm{MHz}, \mathrm{CDCl}_{3}\right): \delta=7.43-7.23(\mathrm{~m}, 20 \mathrm{H}), 5.55(\mathrm{~d}, J=5.0 \mathrm{~Hz}, 1 \mathrm{H}), 5.08(\mathrm{~d}$, $J=4.3 \mathrm{~Hz}, 1 \mathrm{H}), 4.76(\mathrm{dd}, J=11.7,5.8 \mathrm{~Hz}, 3 \mathrm{H}), 4.68(\mathrm{~d}, J=11.8 \mathrm{~Hz}, 1 \mathrm{H}), 4.61-4.56$ $(\mathrm{m}, 2 \mathrm{H}), 4.53(\mathrm{~d}, J=12.0 \mathrm{~Hz}, 1 \mathrm{H}), 4.51-4.47(\mathrm{~m}, 2 \mathrm{H}), 4.36(\mathrm{dd}, J=7.7,1.7 \mathrm{~Hz}, 2 \mathrm{H})$, $4.35-4.32(\mathrm{~m}, 1 \mathrm{H}), 4.12-4.08(\mathrm{~m}, 2 \mathrm{H}), 4.01(\mathrm{dd}, J=7.2,6.0 \mathrm{~Hz}, 1 \mathrm{H}), 3.90$ (dd, $J=10.0,6.1 \mathrm{~Hz}, 1 \mathrm{H}), 3.75(\mathrm{td}, J=6.2,4.1 \mathrm{~Hz}, 1 \mathrm{H}), 3.71-3.69(\mathrm{~m}, 1 \mathrm{H}), 3.67$ (dd, $J=6.6,3.7 \mathrm{~Hz}, 1 \mathrm{H}), 3.62(\mathrm{dd}, J=10.3,6.3 \mathrm{~Hz}, 1 \mathrm{H}), 1.52(\mathrm{~s}, 3 \mathrm{H}), 1.47(\mathrm{~s}, 3 \mathrm{H}), 1.35$ (s, $3 \mathrm{H}), 1.30(\mathrm{~s}, 3 \mathrm{H}) \mathrm{ppm} ;{ }^{13} \mathrm{C}$ NMR $\left(126 \mathrm{MHz} \mathrm{CDCl}_{3}\right): \delta=138.9,138.4,138.3,137.9$, $128.5,128.4,128.4,128.4,128.3,128.0,128.0,127.9,127.7,127.6,127.6,127.5$, 109.2, 108.6, 100.4, 96.4, 84.0, 81.0, 80.7, 79.5, 73.4, 73.3, 72.3, 71.9, 70.8, 70.7, 70.6, 66.0, 65.6, 26.2, 26.2, 25.0, 24.6 ppm; HR-ESI-MS: m/z: $805.3540\left([\mathrm{M}+\mathrm{Na}]^{+}\right.$, calcd. for $\mathrm{C}_{46} \mathrm{H}_{54} \mathrm{O}_{11} \mathrm{Na}^{+}$: 805.3558).

(3aR,5R,5aS,8aS,8bR)-5-((((2R,3S,4R,5R)-4-(Benzyloxy)-5-((benzyloxy)methyl)3-fluorotetrahydrofuran-2-yl)oxy)methyl)-2,2,7,7-tetramethyltetrahydro-5Hbis([1,3]dioxolo)[4,5-b:4',5'-d]pyran (6c)

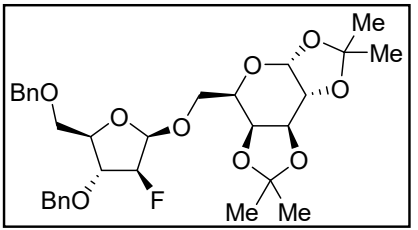

Prepared according to General Procedure B, 5c $(96 \mathrm{mg}$, $0.2 \mathrm{mmol}, 1.0$ eq.) and 1,2:3,4-di-O-isopropylidene- $\alpha$-Dgalactopyranose 2a (104 mg, $0.4 \mathrm{mmol}, 2.0$ eq.) were converted to $6 \mathrm{c}$ at $60{ }^{\circ} \mathrm{C}$ in $48 \mathrm{~h}$, yielding the product as a colorless oil (100 $\mathrm{mg}, 87 \%, \beta: \alpha$ 5:1). The anomeric ratio was determined by integration of the arabinose anomeric signals of the $\beta$ - and $\alpha$-product: ${ }^{1} \mathrm{H}$ NMR $\left(600 \mathrm{MHz} \mathrm{CDCl}_{3}\right): \delta=5.23\left(\mathrm{~d}, J=12.1 \mathrm{~Hz}, \mathrm{H}_{\alpha}\right), 5.19\left(\mathrm{~d}, J=4.3 \mathrm{~Hz}, \mathrm{H}_{\beta}\right) \mathrm{ppm}$, respectively.

Following General Procedure C, the glycosylation induced by TMSOTf was performed, yielding the desired product (90\% NMR yield, $\beta: \alpha 3: 1)$.

${ }^{1} \mathrm{H}$ NMR $\left(600 \mathrm{MHz}_{\mathrm{CDCl}}\right): \delta=7.40-7.25(\mathrm{~m}, 10 \mathrm{H}), 5.52(\mathrm{~d}, J=4.9 \mathrm{~Hz}, 1 \mathrm{H}), 5.20(\mathrm{~d}$, $J=4.3 \mathrm{~Hz}, 1 \mathrm{H}), 5.01(\mathrm{ddd}, J=52.7,6.1,4.3 \mathrm{~Hz}, 1 \mathrm{H}), 4.72-4.67(\mathrm{~m}, 1 \mathrm{H}), 4.62-4.57$ $(\mathrm{m}, 3 \mathrm{H}), 4.55(\mathrm{~d}, J=11.9 \mathrm{~Hz}, 1 \mathrm{H}), 4.30(\mathrm{dd}, J=5.0,2.4 \mathrm{~Hz}, 1 \mathrm{H}), 4.24-4.19(\mathrm{~m}, 2 \mathrm{H})$, $4.15(\mathrm{q}, J=5.9 \mathrm{~Hz}, 1 \mathrm{H}), 4.00-3.97(\mathrm{~m}, 1 \mathrm{H}), 3.88(\mathrm{dd}, J=10.9,5.6 \mathrm{~Hz}, 1 \mathrm{H}), 3.71$ (dd, $J=10.9,6.6 \mathrm{~Hz}, 1 \mathrm{H}), 3.65$ (dd, $J=10.2,6.5 \mathrm{~Hz}, 1 \mathrm{H}), 3.60$ (dd, $J=10.2,5.7 \mathrm{~Hz}, 1 \mathrm{H}$ ), $1.54(\mathrm{~s}, 3 \mathrm{H}), 1.44(\mathrm{~s}, 3 \mathrm{H}), 1.33(\mathrm{~s}, 4 \mathrm{H}), 1.32(\mathrm{~s}, 3 \mathrm{H}) \mathrm{ppm} ;{ }^{13} \mathrm{C}$ NMR $\left(126 \mathrm{MHz}, \mathrm{CDCl}_{3}\right)$ : 
$\delta=138.2,137.7,128.6,128.5,128.5,128.5,127.9,127.9,127.8,127.7,109.4,108.8$, $100.3\left(\mathrm{~d}, J_{\mathrm{CF}}=17.0 \mathrm{~Hz}\right), 96.4,95.3\left(\mathrm{~d}, J_{\mathrm{CF}}=199.5 \mathrm{~Hz}\right), 82.0\left(\mathrm{~d}, J_{\mathrm{CF}}=21.0 \mathrm{~Hz}\right), 80.0$, 80.0, 73.4, 72.3, 71.1, 70.7, 70.7, 67.5, 66.6, 26.2, 26.2, 25.1, 24.6 ppm; ${ }^{19} \mathrm{~F}$ NMR $\left(471 \mathrm{MHz}, \mathrm{CDCl}_{3}\right): \delta=-188.47(\mathrm{~d}, J=3.8 \mathrm{~Hz}) \mathrm{ppm}$; HR-ESI-MS: $\mathrm{m} / \mathrm{z}: 597.2458$ $\left([\mathrm{M}+\mathrm{Na}]^{+}\right.$, calcd. for $\mathrm{C}_{31} \mathrm{H}_{39} \mathrm{FO}_{9} \mathrm{Na}^{+}:$597.2470).

(3aR,5R,5aS,8aS,8bR)-5-((((2S,3R,4S,5S)-3,4-Bis(benzyloxy)-5-((benzyloxy) methyl)tetrahydrofuran-2-yl)oxy)methyl)-2,2,7,7-tetramethyltetrahydro-5Hbis([1,3]dioxolo)[4,5-b:4',5'-d]pyran (6d)

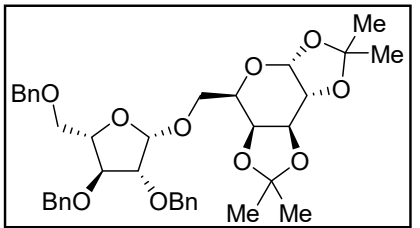

Prepared according to General Procedure B, 5d (114 mg, $0.2 \mathrm{mmol}, \quad 1.0$ eq.) and 1,2:3,4-di-O-isopropylidene- $\alpha$-Dgalactopyranose $2 \mathrm{a}$ (104 $\mathrm{mg}, 0.4 \mathrm{mmol}, 2.0$ eq.) were converted to $6 \mathrm{~d}$ at $40^{\circ} \mathrm{C}$ in $3 \mathrm{~h}$ using ent-4i $(10 \mathrm{mg}$, $0.01 \mathrm{mmol}, 0.05$ eq.), yielding the product as a colorless oil (110 $\mathrm{mg}, 83 \%, \beta: \alpha 20: 1)$. The anomeric ratio was determined by integration of the arabinose anomeric signals of the $\beta$ - and $\alpha$-product: ${ }^{1} \mathrm{H}$ NMR $\left(600 \mathrm{MHz}, \mathrm{CDCl}_{3}\right)$ : $\delta=5.21\left(\mathrm{~d}, J=1.4 \mathrm{~Hz}, \mathrm{H}_{\alpha}\right), 5.06\left(\mathrm{~d}, J=4.3 \mathrm{~Hz}, \mathrm{H}_{\beta}\right) \mathrm{ppm}$, respectively.

Following General Procedure $\mathrm{C}$, the glycosylation induced by TMSOTf was performed, yielding the desired product (99\% NMR yield, $\beta: \alpha$ 4:1).

${ }^{1} \mathrm{H}$ NMR $\left(600 \mathrm{MHz}, \mathrm{CDCl}_{3}\right): \delta=7.48-7.19(\mathrm{~m}, 15 \mathrm{H}), 5.55(\mathrm{~d}, J=5.0 \mathrm{~Hz}, 1 \mathrm{H}), 5.09(\mathrm{~d}$, $J=4.2 \mathrm{~Hz}, 1 \mathrm{H}), 4.72(\mathrm{dd}, J=19.7,11.8 \mathrm{~Hz}, 2 \mathrm{H}), 4.73(\mathrm{~d}, J=11.8 \mathrm{~Hz}, 1 \mathrm{H}), 4.70(\mathrm{~d}$, $J=11.7 \mathrm{~Hz}, 1 \mathrm{H}), 4.63(\mathrm{~d}, J=11.9 \mathrm{~Hz}, 1 \mathrm{H}), 4.61-4.54(\mathrm{~m}, 4 \mathrm{H}), 4.33$ (dd, $J=5.1,2.4 \mathrm{~Hz}, 1 \mathrm{H}), 4.30(\mathrm{dd}, J=8.0,2.0 \mathrm{~Hz}, 1 \mathrm{H}), 4.18-4.13(\mathrm{~m}, 2 \mathrm{H}), 4.11(\mathrm{p}$, $J=4.1,3.7 \mathrm{~Hz}, 1 \mathrm{H}), 4.04$ (ddd, $J=8.0,6.1,2.0 \mathrm{~Hz}, 1 \mathrm{H}), 3.85(\mathrm{dd}, J=10.0,6.2 \mathrm{~Hz}$, $1 \mathrm{H}), 3.66$ (dd, $J=10.0,8.0 \mathrm{~Hz}, 1 \mathrm{H}$ ), 3.60 (dddd, $J=18.9,9.1,4.5,2.4 \mathrm{~Hz}, 2 \mathrm{H}$ ), 1.54 (s, 3H), 1.47 (s, 3H), 1.35 (s, 3H), 1.32 (s, 3H) ppm; ${ }^{13} \mathrm{C}$ NMR (126 MHz, $\left.\mathrm{CDCl}_{3}\right)$ : $\delta=138.4,138.2,138.0,128.5,128.5,128.4,127.9,127.9,127.8,127.8,127.7,127.7$, 109.3, 108.6, 100.9, 96.4, 84.2, 83.5, 80.6, 73.5, 72.8, 72.3, 72.0, 70.9, 70.7, 65.9, 65.6, 26.2, 26.2, 25.0, 24.7 ppm; HR-ESI-MS: m/z: $685.2968\left([M+N a]^{+}\right.$, calcd. for

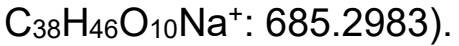

(3aR,5R,5aS,8aS,8bR)-5-((((2S,3R,4R,5R)-3,4-Bis(benzyloxy)-5-((benzyloxy) methyl)tetrahydrofuran-2-yl)oxy)methyl)-2,2,7,7-tetramethyltetrahydro-5Hbis([1,3]dioxolo)[4,5-b:4',5'-d]pyran (6e)

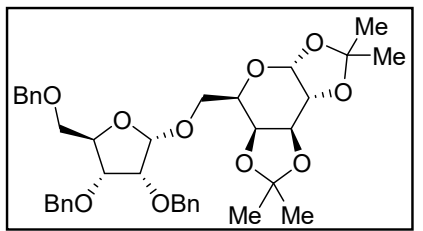

Prepared according to General Procedure B, 5e (114 mg, $0.2 \mathrm{mmol}, 1.0$ eq.) and 1,2:3,4-di-O-isopropylidene- $\alpha$-Dgalactopyranose $2 \mathrm{a}$ (104 $\mathrm{mg}, 0.4 \mathrm{mmol}, 2.0$ eq.) were converted to $6 \mathbf{e}$ at $40^{\circ} \mathrm{C}$ in $18 \mathrm{~h}$ using ent-4i $(10 \mathrm{mg}$, $0.01 \mathrm{mmol}, 0.05$ eq.), yielding the product as a colorless oil $(108 \mathrm{mg}, 82 \%, \beta: \alpha 1: 14)$. The anomeric ratio was determined by integration of the arabinose anomeric signals of the $\beta$ - and $\alpha$-product: ${ }^{1} \mathrm{H}$ NMR $\left(600 \mathrm{MHz}, \mathrm{CDCl}_{3}\right)$ : $\delta=5.29\left(\mathrm{~d}, J=1.1 \mathrm{~Hz}, \mathrm{H}_{\beta}\right), 5.13\left(\mathrm{~d}, J=4.2 \mathrm{~Hz}, \mathrm{H}_{\alpha}\right) \mathrm{ppm}$, respectively.

Following General Procedure C, the glycosylation induced by TMSOTf was performed, yielding the desired product (95\% NMR yield, $\beta: \alpha 1: 3)$. 
${ }^{1} \mathrm{H}$ NMR $\left(600 \mathrm{MHz}, \mathrm{CDCl}_{3}\right): \delta=7.42-7.16(\mathrm{~m}, 15 \mathrm{H}), 5.51(\mathrm{~d}, J=5.0 \mathrm{~Hz}, 1 \mathrm{H}), 5.14(\mathrm{~d}$, $J=4.2 \mathrm{~Hz}, 1 \mathrm{H}), 4.73(\mathrm{~d}, J=12.5 \mathrm{~Hz}, 2 \mathrm{H}), 4.62(\mathrm{~d}, J=12.3 \mathrm{~Hz}, 1 \mathrm{H}), 4.59-4.53(\mathrm{~m}$, $2 \mathrm{H}), 4.49(\mathrm{~d}, J=12.1 \mathrm{~Hz}, 1 \mathrm{H}), 4.42(\mathrm{~d}, \mathrm{~J}=12.1 \mathrm{~Hz}, 1 \mathrm{H}), 4.39(\mathrm{dd}, J=8.0,1.9 \mathrm{~Hz}, 1 \mathrm{H})$, 4.29 (dd, $J=5.0,2.3 \mathrm{~Hz}, 1 \mathrm{H}), 4.25(\mathrm{q}, J=3.9 \mathrm{~Hz}, 1 \mathrm{H}), 4.17-4.11(\mathrm{~m}, 1 \mathrm{H}), 3.92-3.84$ $(\mathrm{m}, 2 \mathrm{H}), 3.82-3.75(\mathrm{~m}, 2 \mathrm{H}), 3.45(\mathrm{dd}, J=10.6,3.9 \mathrm{~Hz}, 1 \mathrm{H}), 3.39(\mathrm{dd}, J=10.6,4.0 \mathrm{~Hz}$, $1 \mathrm{H}), 1.52(\mathrm{~s}, 3 \mathrm{H}), 1.44(\mathrm{~s}, 3 \mathrm{H}), 1.32(\mathrm{~s}, 3 \mathrm{H}), 1.29(\mathrm{~s}, 3 \mathrm{H}) \mathrm{ppm} ;{ }^{13} \mathrm{C}$ NMR $(126 \mathrm{MHz}$, $\left.\mathrm{CDCl}_{3}\right): \delta=138.6,138.2,138.1,128.5,128.5,128.4,128.1,127.9,127.8,127.7$, 127.6, 109.1, 108.6, 102.2, 96.4, 81.6, 77.6, 75.6, 73.5, 72.4, 72.3, 70.9, 70.7, 70.7, 70.0, 66.7, 66.0, 26.2, 26.2, 25.1, 24.6 ppm; HR-ESI-MS: $\mathrm{m} / \mathrm{z}: 685.2968\left([\mathrm{M}+\mathrm{Na}]^{+}\right.$, calcd. for $\mathrm{C}_{38} \mathrm{H}_{46} \mathrm{O}_{10} \mathrm{Na}^{+}$: 685.2983); analytical data in agreement with the literature. ${ }^{17}$

(3aR,5R,5aS,8aS,8bR)-5-(((2R,3R,4S,5R)-3,4-Bis(benzyloxy)-5-((benzyloxy) methyl)tetrahydrofuran-2-yl)oxy)methyl)-2,2,7,7-tetramethyltetrahydro-5Hbis([1,3]dioxolo)[4,5-b:4',5'-d]pyran (6f)

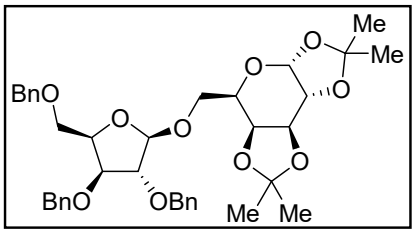

Prepared according to General Procedure B, 5f $(114 \mathrm{mg}$, $0.2 \mathrm{mmol}, 1.0$ eq.) and 1,2:3,4-di-O-isopropylidene- $\alpha$-Dgalactopyranose $2 \mathrm{a}$ (104 $\mathrm{mg}, 0.4 \mathrm{mmol}, 2.0 \mathrm{eq}$.) were converted to $6 \mathrm{f}$ at $40{ }^{\circ} \mathrm{C}$ in $18 \mathrm{~h}$, yielding the product as a colorless oil (109 mg, 83\%, $\beta: \alpha$ 13:1). The anomeric ratio was determined by integration of the arabinose anomeric signals of the $\beta$ - and $\alpha$-product: ${ }^{1} \mathrm{H}$ NMR $\left(600 \mathrm{MHz}, \mathrm{CDCl}_{3}\right): \delta=5.14\left(\mathrm{~d}, J=1.5 \mathrm{~Hz}, \mathrm{H}_{\beta}\right), 5.10\left(\mathrm{~d}, J=4.4 \mathrm{~Hz}, \mathrm{H}_{\alpha}\right) \mathrm{ppm}$, respectively.

Following General Procedure C, the glycosylation induced by TMSOTf was performed, yielding the desired product (99\% NMR yield, $\beta: \alpha 4: 1)$.

${ }^{1} \mathrm{H}$ NMR $\left(600 \mathrm{MHz}, \mathrm{CDCl}_{3}\right): \delta=7.42-7.25(\mathrm{~m}, 15 \mathrm{H}), 5.56(\mathrm{~d}, J=5.0 \mathrm{~Hz}, 1 \mathrm{H}), 5.17(\mathrm{~d}$, $J=1.5 \mathrm{~Hz}, 1 \mathrm{H}), 4.66(\mathrm{~d}, J=11.9 \mathrm{~Hz}, 1 \mathrm{H}), 4.62(\mathrm{~d}, J=12.0 \mathrm{~Hz}, 1 \mathrm{H}), 4.59-4.55(\mathrm{~m}$, $3 \mathrm{H}), 4.55-4.51(\mathrm{~m}, 1 \mathrm{H}), 4.50-4.44(\mathrm{~m}, 2 \mathrm{H}), 4.31(\mathrm{dd}, J=5.0,2.4 \mathrm{~Hz}, 1 \mathrm{H}), 4.18$ (dd, $J=7.9,1.8 \mathrm{~Hz}, 1 \mathrm{H}), 4.10-4.06(\mathrm{~m}, 2 \mathrm{H}), 3.99$ (ddd, $J=6.9,4.4,1.8 \mathrm{~Hz}, 1 \mathrm{H}), 3.96(\mathrm{dd}$, $J=10.9,4.3 \mathrm{~Hz}, 1 \mathrm{H}$ ), 3.83 (dd, $J=10.3,4.9 \mathrm{~Hz}, 1 \mathrm{H}), 3.76$ (dd, $J=10.4,7.1 \mathrm{~Hz}, 1 \mathrm{H}$ ), 3.70 (dd, $J=10.9,7.2 \mathrm{~Hz}, 1 \mathrm{H}), 1.52(\mathrm{~s}, 3 \mathrm{H}), 1.45(\mathrm{~s}, 3 \mathrm{H}), 1.34(\mathrm{~s}, 3 \mathrm{H}), 1.31$ (s, 3H) ppm; ${ }^{13} \mathrm{C}$ NMR $\left(126 \mathrm{MHz}, \mathrm{CDCl}_{3}\right): \delta=138.6,138.1,137.9,128.5,128.4,128.0$, 127.9, 127.8, 127.7, 127.6, 109.4, 108.6, 107.5, 96.4, 86.5, 82.1, 80.1, 73.5, 72.1, 71.9, 71.3, 70.8, 70.7, 70.0, 67.9, 67.4, 26.2, 26.1, 25.1, 24.5 ppm; HR-ESI-MS: $\mathrm{m} / \mathrm{z}$ : $685.2967\left([\mathrm{M}+\mathrm{Na}]^{+}\right.$, calcd. for $\mathrm{C}_{38} \mathrm{H}_{46} \mathrm{O}_{10} \mathrm{Na}^{+}:$685.2983). 


\section{Reaction Optimization}

\section{General Procedure D for Reaction Optimization}

The specified furanose phosphate $(0.018 \mathrm{mmol}, 1.0$ eq. $)$, the specified acceptor (0.036 mmol, 2.0 eq.), the specified catalyst $(0.0009 \mathrm{mmol}, 0.05$ eq.), 1,3,5-trimethoxybenzene $(3.0 \mathrm{mg}, 0.018 \mathrm{mmol}, 1.0 \mathrm{eq}$.$) , and 45 \mathrm{mg} 4 \AA$ molecular sieves were suspended in the specified solvent $(0.45 \mathrm{~mL})$ and the resulting mixture was stirred at $40{ }^{\circ} \mathrm{C}$ for the specified reaction time. The resulting mixture was filtered through a fritted syringe filter, eluted with $\mathrm{CH}_{2} \mathrm{Cl}_{2}$, and concentrated in vacuo. The product NMR yield and anomeric ratio were analyzed by ${ }^{1} \mathrm{H}$ NMR.

\section{Furanose Phosphate Optimization}

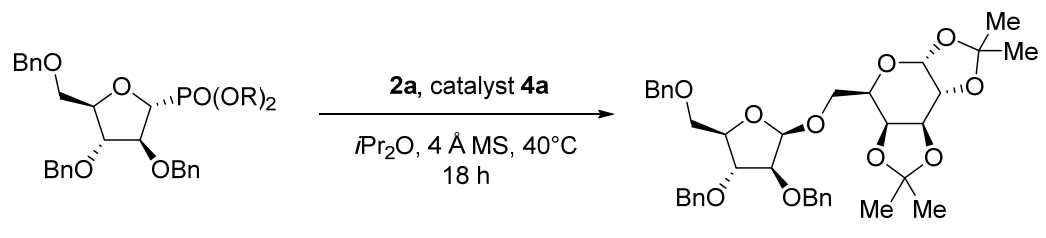

1

$3 \mathbf{a}$
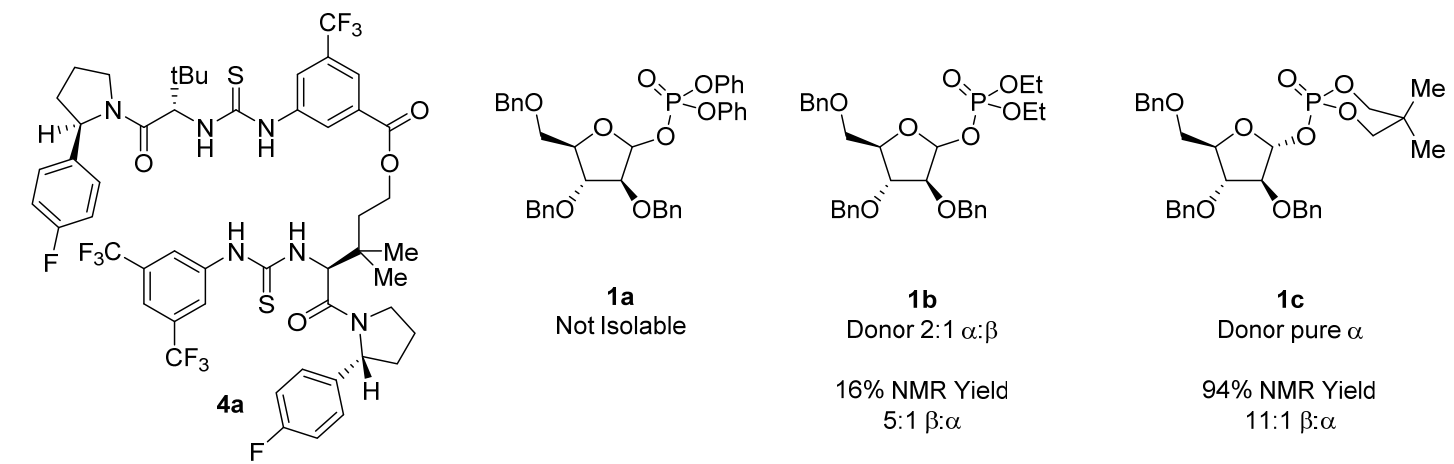

Scheme S2 Optimization of Arabinose Phosphate Donor.
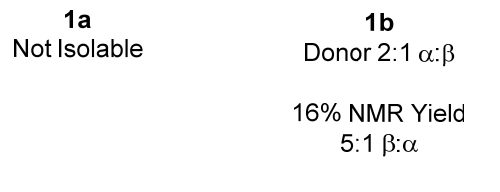

$$
\begin{gathered}
\text { 1c } \\
\text { Donor pure } \alpha \\
\text { 94\% NMR Yield } \\
\text { 11:1 } \beta: \alpha
\end{gathered}
$$




\section{Catalyst Optimization}
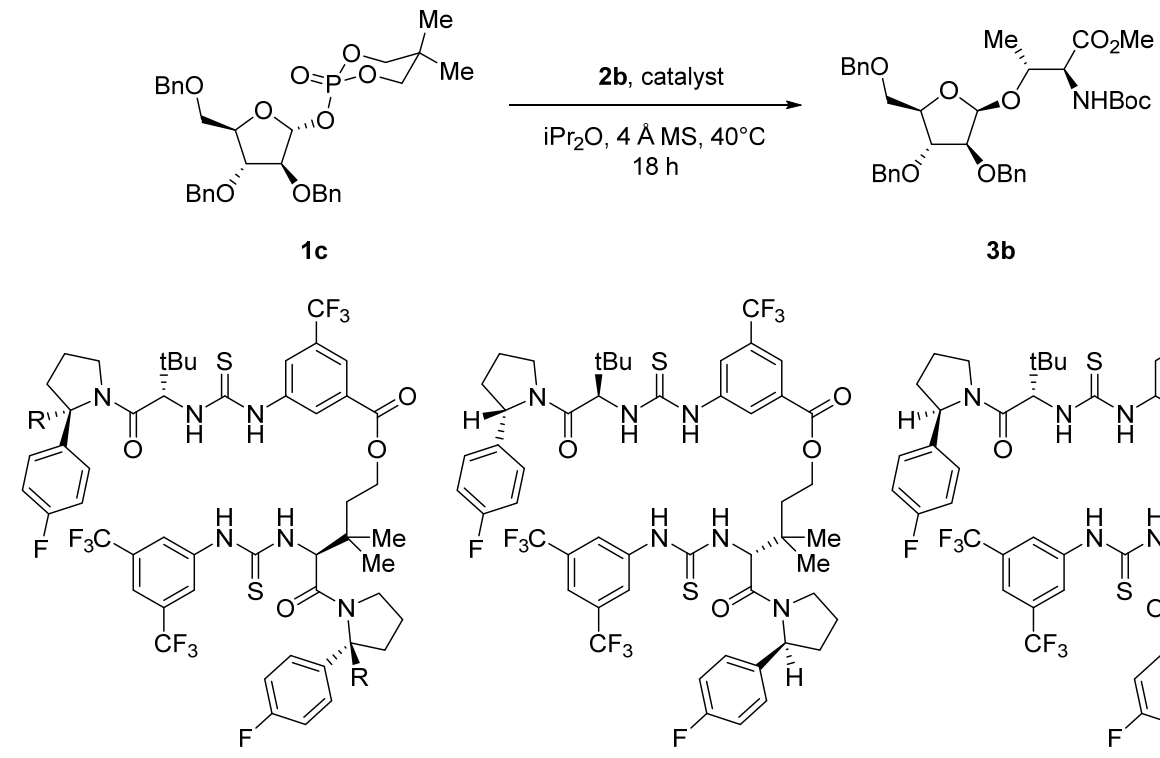

3b

$$
\begin{array}{cc}
\mathbf{4 a} & \mathbf{4 b} \\
\mathrm{R}=\mathrm{H} & \mathrm{R}=\mathrm{Me} \\
& \\
20 \% \text { Yield } & 16 \% \text { Yield } \\
11: 1 \beta: \alpha & 12: 1 \beta: \alpha
\end{array}
$$$$
\text { ent-4a }
$$$$
16 \% \text { Yield }
$$$$
\text { 2.4:1 } \beta: \alpha
$$
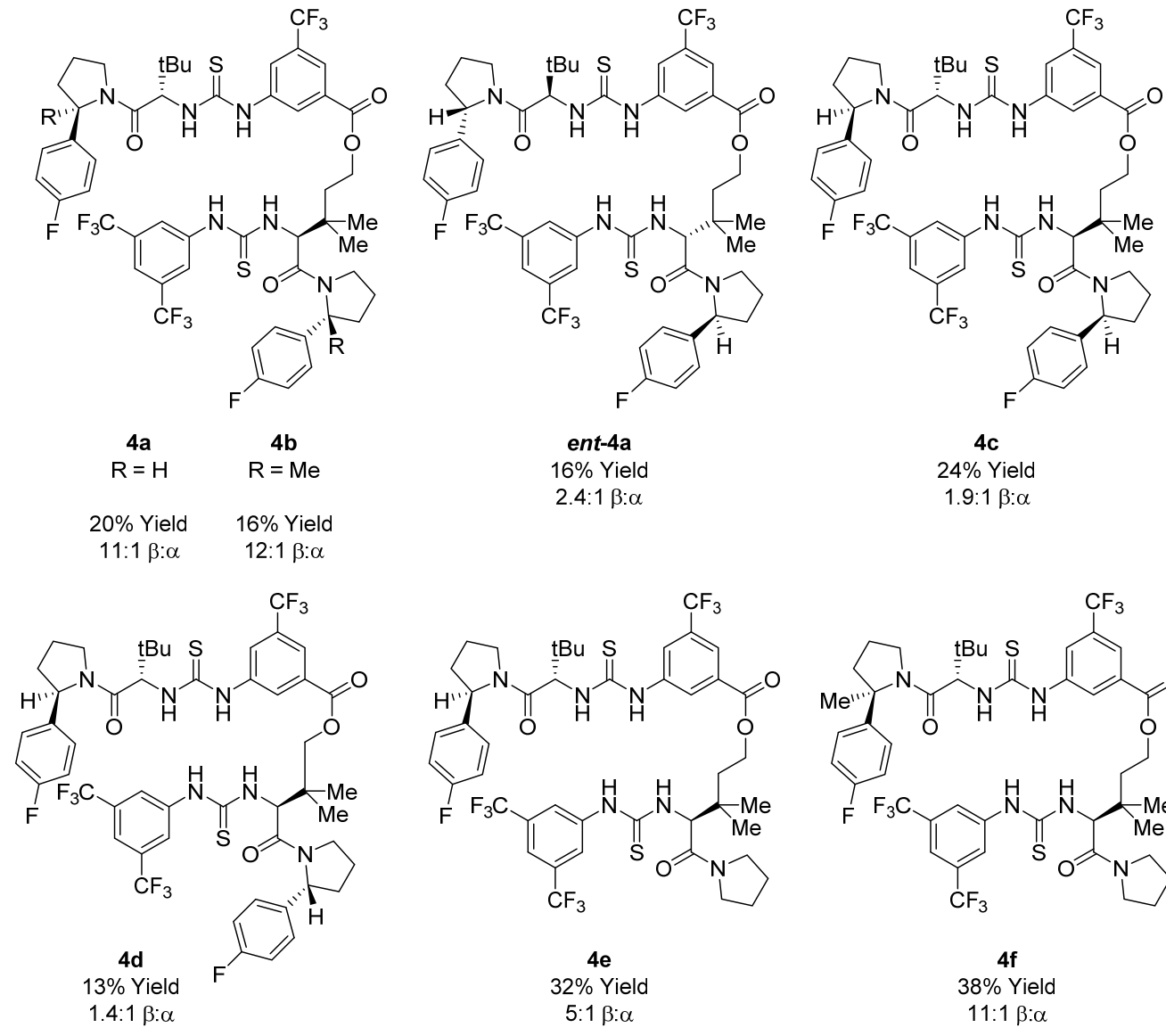

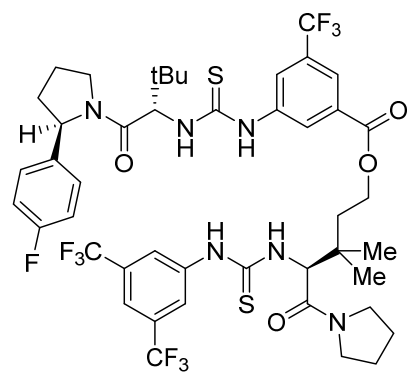

$$
\begin{gathered}
4 \text { e } \\
32 \% \text { Yield }
\end{gathered}
$$$$
5: 1 \beta: \alpha
$$

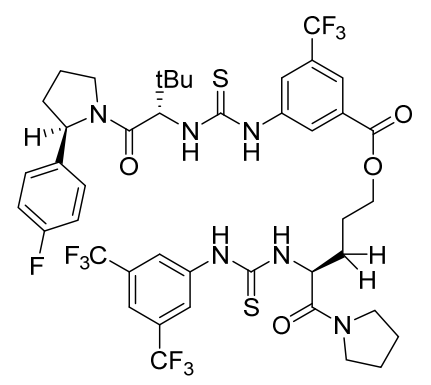

4g

$19 \%$ Yield

2:1 $\beta: \alpha$

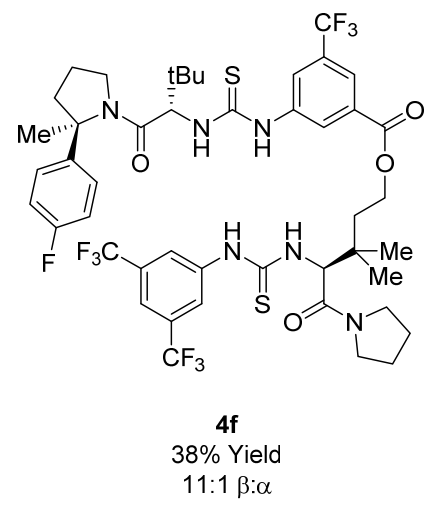

Scheme S3 Catalyst Scaffold Optimization. 


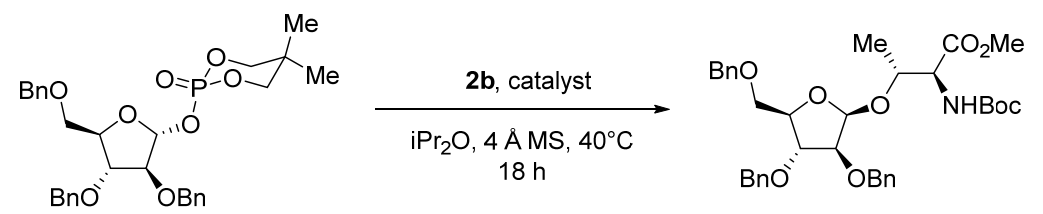

$1 c$
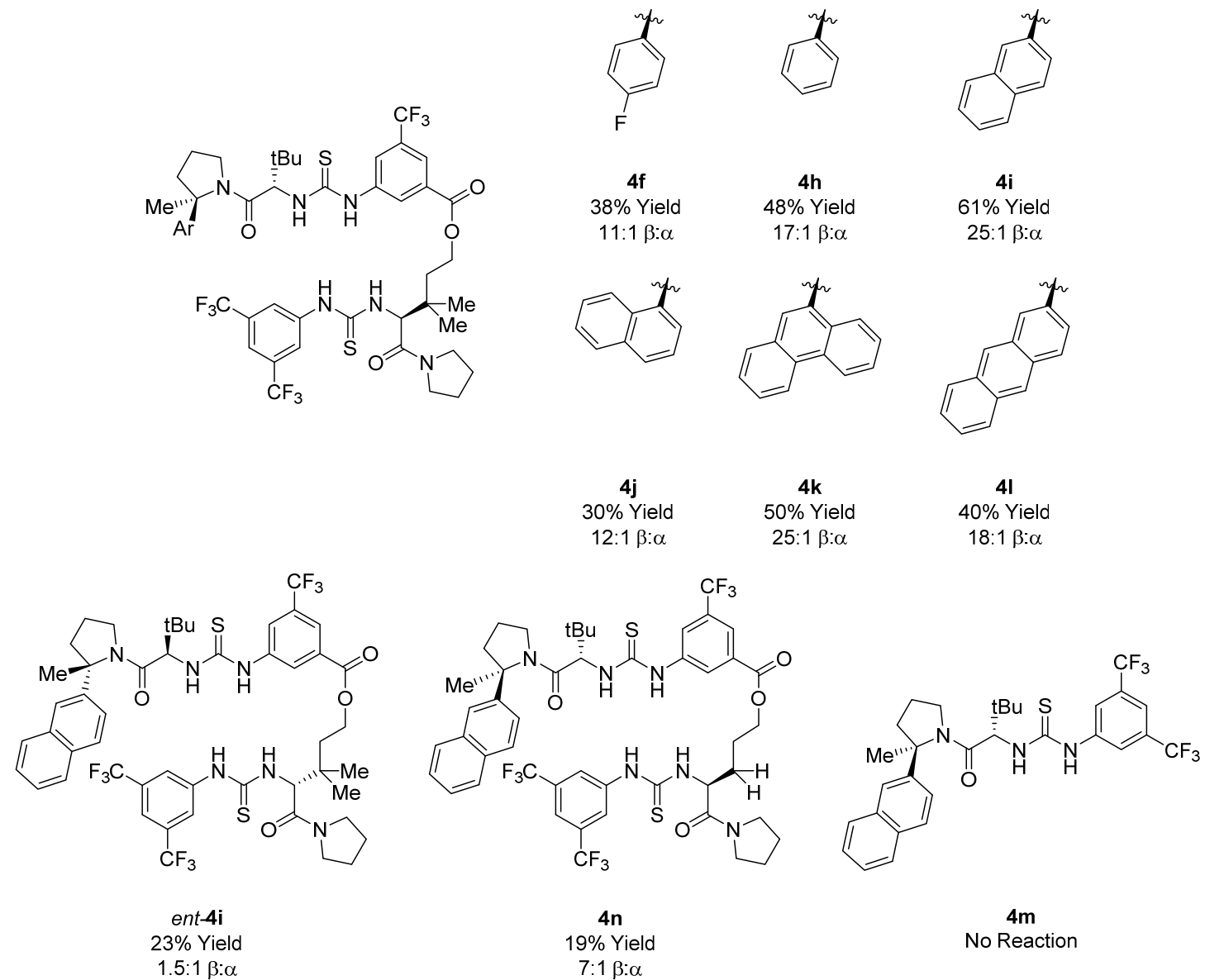

Scheme S4 Optimization of the Arylpyrrolidine Moiety on the Upper Catalyst Portion and the Linker Substitution. 


\section{Reaction Condition Optimization}

Table S1 Solvent Optimization for the Glycosylation of 1c and 1,2:3,4-Di-O-isopropylidene- $\alpha$-galactopyranose 2a.

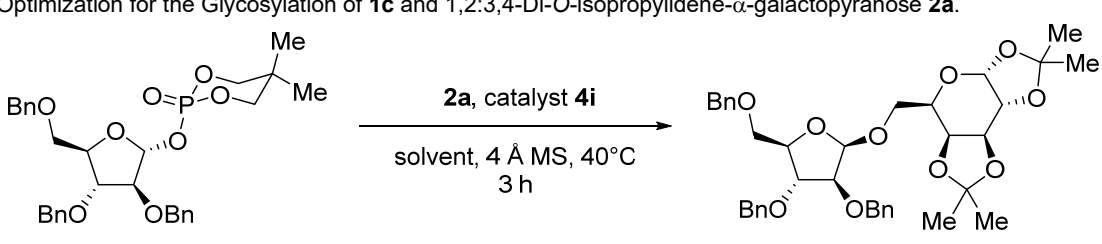

\begin{tabular}{cccc} 
1c & 3a & NMR Yield \\
Solvent & $\begin{array}{c}\text { Scale } \\
{[\mathrm{mmol}]}\end{array}$ & $\begin{array}{c}\text { Product } \\
\beta: \alpha\end{array}$ \\
\hline $\begin{array}{c}\text { i } \mathrm{Pr}_{2} \mathrm{O} \\
\mathrm{Pr}_{2} \mathrm{O} / \mathrm{CH}_{2} \mathrm{Cl}_{2} \\
(9: 1)\end{array}$ & 0.018 & quant. & $17: 1$ \\
$n \mathrm{Pr}_{2} \mathrm{O}$ & 0.018 & 91 & $21: 1$ \\
$n \mathrm{Pr}_{2} \mathrm{O} / \mathrm{CH}_{2} \mathrm{Cl}_{2}$ & 0.018 & quant. & $21: 1$ \\
$(9: 1)$ & 0.018 & 82 & $21: 1$ \\
$n \mathrm{Bu}_{2} \mathrm{O}$ & 0.018 & quant. & $23: 1$ \\
$n \mathrm{Bu}_{2} \mathrm{O} / \mathrm{CH}_{2} \mathrm{Cl}_{2}$ & 0.018 & 94 & $25: 1$ \\
$(9: 1)$ & & & $16: 1$ \\
$i \mathrm{Pr}_{2} \mathrm{O}$ & 0.2 & 87 & $19: 1$ \\
$n \mathrm{Pr}_{2} \mathrm{O}$ & 0.2 & 82 & $18: 1$ \\
$n \mathrm{Bu}_{2} \mathrm{O}$ & 0.2 & 90 & \\
\hline
\end{tabular}

Table S2 Solvent Optimization for the Glycosylation of 1c and N-Boc-L-Threonine Methyl Ester 2b.

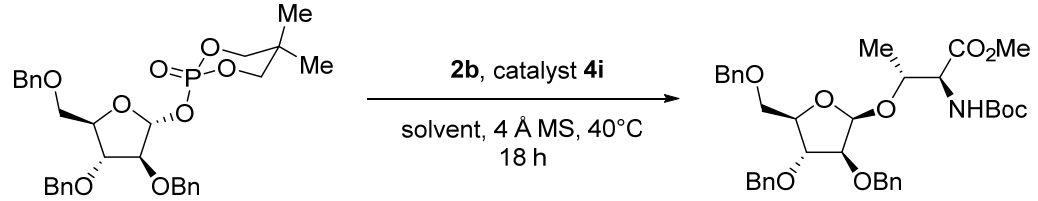

\begin{tabular}{|c|c|c|c|}
\hline Solvent & $\begin{array}{c}\text { Scale } \\
{[\mathrm{mmol}]}\end{array}$ & $\begin{array}{c}\text { NMR Yield } \\
{[\%]}\end{array}$ & $\begin{array}{c}\text { Product } \\
\beta: \alpha\end{array}$ \\
\hline $\mathrm{Pr}_{2} \mathrm{O}$ & 0.018 & 70 & $25: 1$ \\
\hline $\begin{array}{c}\mathrm{PPr}_{2} \mathrm{O} / \mathrm{CH}_{2} \mathrm{Cl}_{2} \\
(9: 1)\end{array}$ & 0.018 & 55 & $22: 1$ \\
\hline$n \mathrm{Pr}_{2} \mathrm{O}$ & 0.018 & 67 & $24: 1$ \\
\hline $\begin{array}{c}n \mathrm{Pr}_{2} \mathrm{O} / \mathrm{CH}_{2} \mathrm{Cl}_{2} \\
(9: 1)\end{array}$ & 0.018 & 51 & $23: 1$ \\
\hline$n \mathrm{Bu}_{2} \mathrm{O}$ & 0.018 & 70 & $23: 1$ \\
\hline $\begin{array}{c}n \mathrm{Bu}_{2} \mathrm{O} / \mathrm{CH}_{2} \mathrm{Cl}_{2} \\
(9: 1)\end{array}$ & 0.018 & 52 & $20: 1$ \\
\hline
\end{tabular}


Table S3 Reaction Condition Optimization for the Glycosylation of 1c and 1,2:3,4-Di-O-isopropylidene- $\alpha$-galactopyranose 2a.

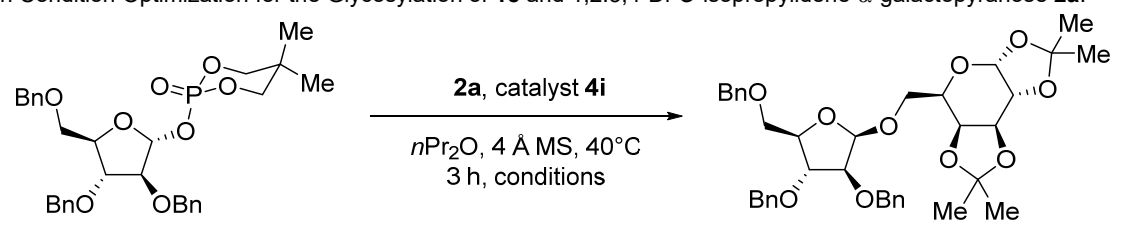

1c $3 a$

\begin{tabular}{cccccc}
\hline Entry & $\begin{array}{c}\text { Conc. } \\
{[\mathrm{M}]}\end{array}$ & Eq. Acceptor & $\begin{array}{c}\text { Cat. Loading } \\
{[\mathrm{mol} \%]}\end{array}$ & $\begin{array}{c}\text { NMR Yield } \\
{[\%]}\end{array}$ & $\begin{array}{c}\text { Product } \\
\beta: \alpha\end{array}$ \\
\hline 1 & 0.036 & 2 & 5 & quant. & $21: 1$ \\
2 & 0.072 & 2 & 5 & quant. & $15: 1$ \\
3 & 0.018 & 2 & 5 & 74 & $19: 1$ \\
4 & 0.036 & 1.5 & 5 & quant. & $14: 1$ \\
5 & 0.036 & 3 & 5 & quant. & $16: 1$ \\
6 & 0.036 & 2 & 10 & quant. & $14: 1$ \\
7 & 0.036 & 2 & 1 & 37 & $25: 1$ \\
\hline
\end{tabular}

Table S4 Reaction Condition Optimization for the Glycosylation of 1c and N-Boc-L-Threonine Methyl Ester 2b.

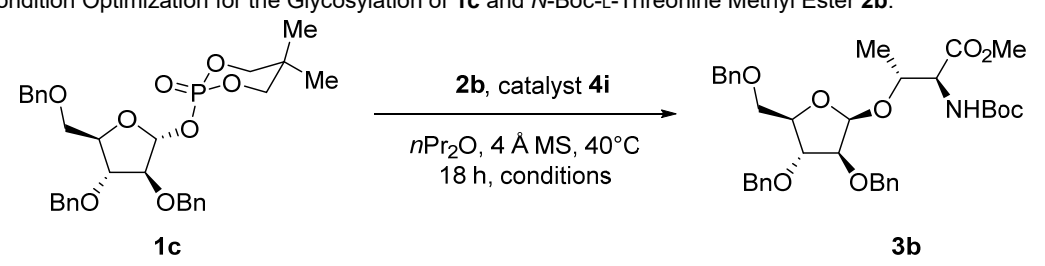

\begin{tabular}{cccccc}
\hline Entry & $\begin{array}{c}\text { Conc. } \\
{[\mathrm{M}]}\end{array}$ & Eq. Acceptor & $\begin{array}{c}\text { Cat. Loading } \\
{[\mathrm{mol} \%]}\end{array}$ & $\begin{array}{c}\text { NMR Yield } \\
{[\%]}\end{array}$ & $\begin{array}{c}\text { Product } \\
\beta: \alpha\end{array}$ \\
\hline 1 & 0.036 & 2 & 5 & 67 & $24: 1$ \\
2 & 0.072 & 2 & 5 & 70 & $19: 1$ \\
3 & 0.018 & 2 & 5 & 50 & $>30: 1$ \\
4 & 0.036 & 1.5 & 5 & 55 & $29: 1$ \\
5 & 0.036 & 3 & 5 & 75 & $24: 1$ \\
6 & 0.036 & 2 & 10 & 72 & $>30: 1$ \\
7 & 0.036 & 2 & 1 & 19 & $22: 1$ \\
\hline
\end{tabular}


Table S5 Solvent Batch Effect on the Glycosylation of 1c and 1,2:3,4-Di-O-isopropylidene- $\alpha$-galactopyranose 2a.

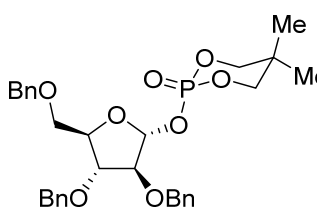

$1 \mathrm{c}$

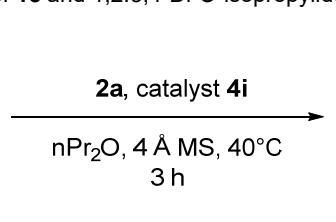

$3 \mathrm{~h}$

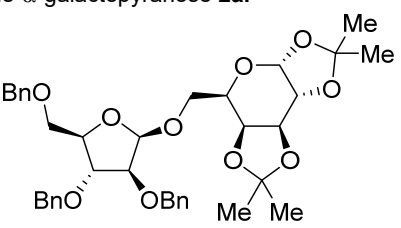

$3 a$

\begin{tabular}{ccccc}
\hline $\begin{array}{c}\text { Solvent } \\
\text { Batch }\end{array}$ & Supplier & $\begin{array}{c}\text { Scale } \\
{[\mathrm{mmol}]}\end{array}$ & $\begin{array}{c}\text { NMR Yield } \\
{[\%]}\end{array}$ & $\begin{array}{c}\text { Product } \\
\beta: \alpha\end{array}$ \\
\hline 1 & Sigma (Old) & 0.018 & 99 & $19: 1$ \\
2 & Sigma (New 1) & 0.018 & 94 & $21: 1$ \\
3 & Sigma (New 2) & 0.018 & 96 & $22: 1$ \\
4 & TCl (New) & 0.018 & $67^{\mathrm{a}}$ & $21: 1$ \\
5 & TCl (New, & 0.018 & 95 & $22: 1$ \\
6 & Neutralized) & & \\
6 & Sigma (New 2, $^{\mathrm{b}}$ & 0.018 & 99 & $25: 1$
\end{tabular}

a Significant formation of $n$-propyl glycoside was observed, which is likely a consequence of remaining $n$-propanol in the commercially available ether. ${ }^{b}$ The solvent was filtered over basic alumina prior to use in the catalyzed glycosylation reaction to remove residual acid. 


\section{Scope Limitations}

According to General Procedure B, the following acceptors were tested under the optimized reaction conditions with donor 1c, resulting in no product formation in all cases.
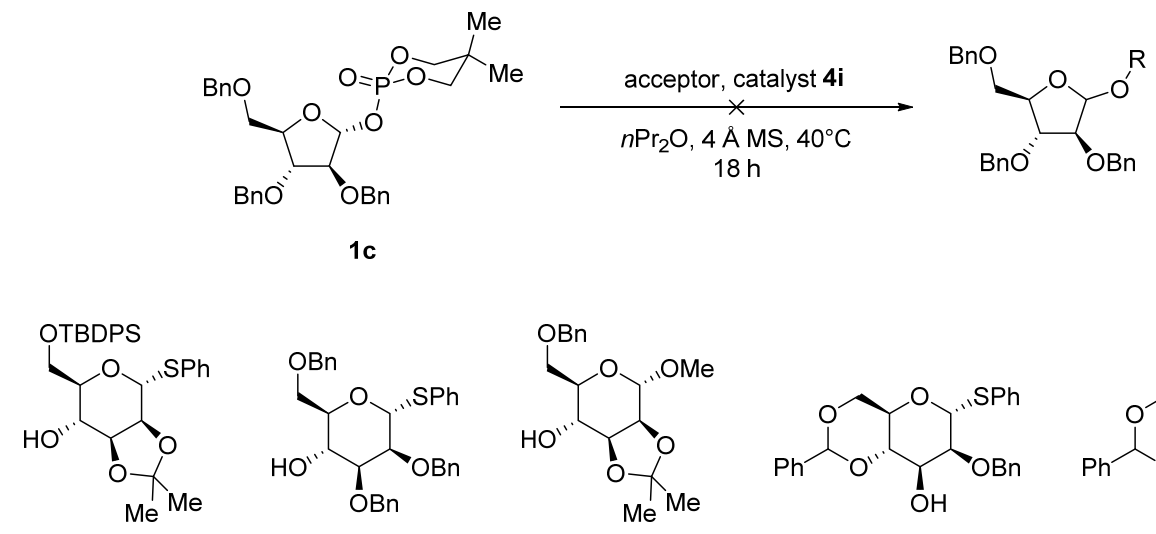<smiles>OC1C([Se]c2ccccc2)OC2CC(c3ccccc3)OC21</smiles><smiles>OC1C([SeH])OC2COC(c3ccccc3)OC2C1O</smiles>

Scheme S5 Acceptor Scope Limitations of the Developed Transformation.
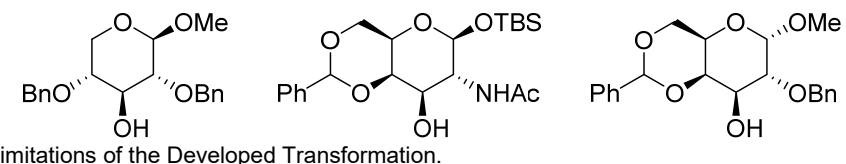

Additionally, 1-O-methyl-2,3,4-tri-O-acetyl- $\alpha$-D-glucose was tested. While the product was obtained in good NMR yield ( 95\%) and moderate $\alpha$-selectivity ( $\alpha: \beta \quad 2.5: 1)$, significant background reactivity was observed ( 95\% NMR yield, pure $\alpha)$ in the absence of catalyst. Thus, the transformation is only partially induced by catalyst $4 \mathbf{i}$.

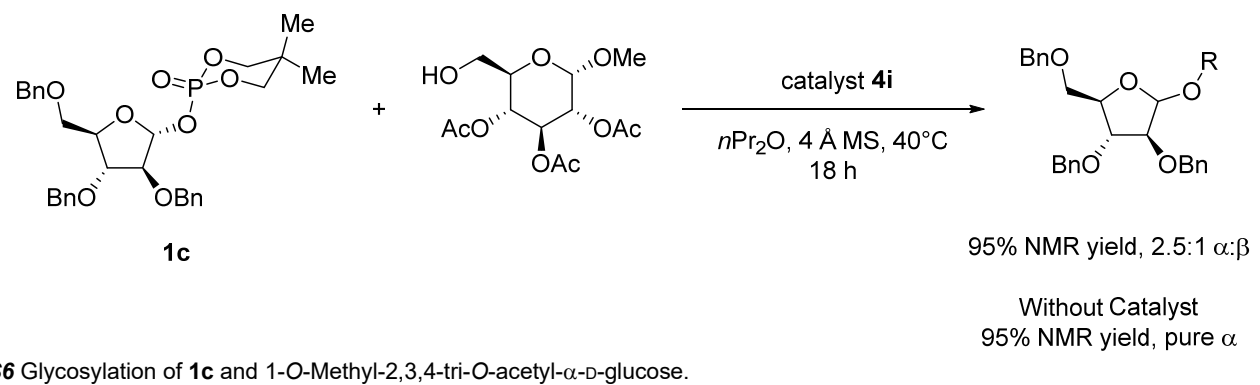

Scheme S6 Glycosylation of 1c and 1-O-Methyl-2,3,4-tri-O-acetyl- $\alpha$-D-glucose.

To test the protecting group compatibility of the developed method, two differently protected arabinose donors were used under the reaction conditions, leading to low formation of the desired product. 

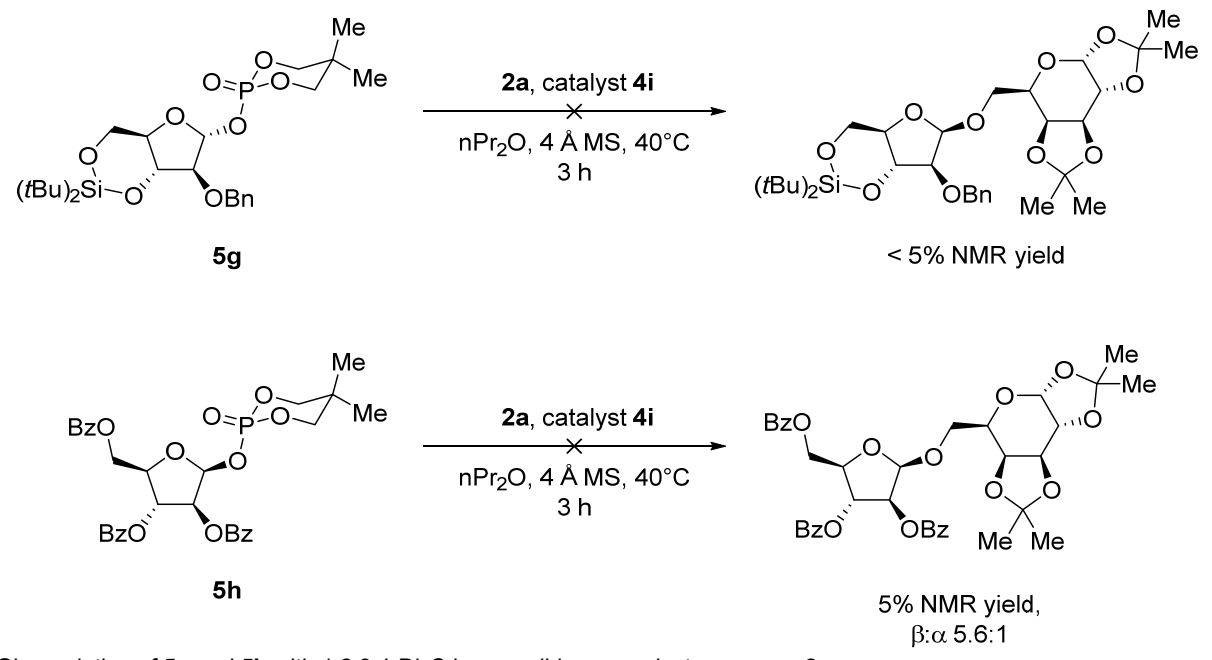

Scheme $\mathbf{S 7}$ Glycosylation of $\mathbf{5 g}$ and $\mathbf{5 h}$ with 1,2:3,4-Di-O-isopropylidene- $\alpha$-galactopyranose $\mathbf{2 a}$. 


\section{Mechanistic Investigations}

\section{Effect of Donor Anomeric Purity}

The donor in the specified anomeric purity $(0.018 \mathrm{mmol}, 1.0$ eq. $), 1,2: 3,4-$ di-Oisopropylidene- $\alpha$-D-galactopyranose $\quad 2 a \quad(0.036 \mathrm{mmol}, \quad 2.0 \mathrm{eq}$.$) , catalyst \mathbf{4 i}$ ( $0.0009 \mathrm{mmol}, 0.05$ eq.), 1,3,5-trimethoxybenzene ( $3.0 \mathrm{mg}, 0.018 \mathrm{mmol}, 1.0$ eq.) and $4 \AA$ molecular sieves were suspended in the specified solvent $(0.5 \mathrm{~mL})$ and the resulting mixture was stirred at $40^{\circ} \mathrm{C}$ for the specified reaction time. The resulting mixture was filtered through a syringe filter, eluted with $\mathrm{CH}_{2} \mathrm{Cl}_{2}$, and concentrated in vacuo. The product NMR yield and anomeric ratio were analyzed by ${ }^{1} \mathrm{H}$ NMR.
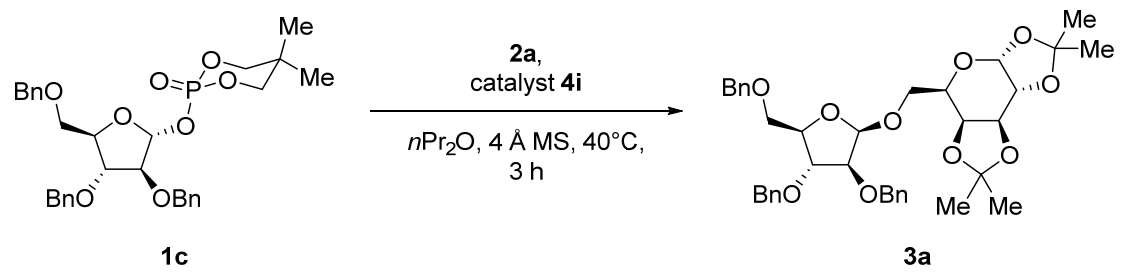

$\begin{array}{ll}\text { Starting from 8:1 } \alpha: \beta & 84 \%, 10: 1 \beta: \alpha \\ \text { Starting from pure } \alpha & 87 \%, 25: 1 \beta: \alpha\end{array}$

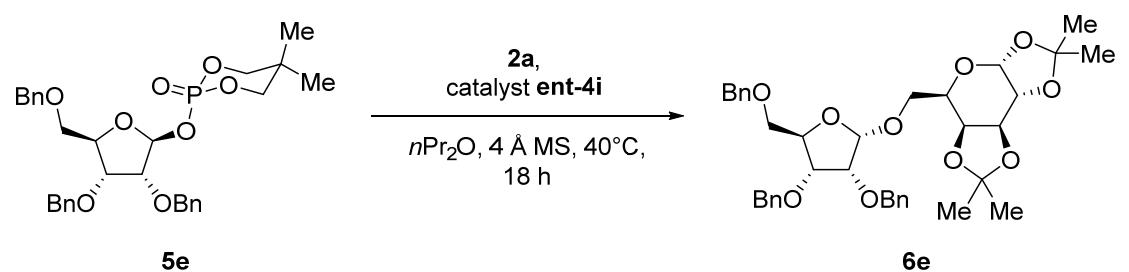

$5 e$

$6 e$

Starting from $1: 3 \alpha: \beta \quad 82 \%, 1: 8 \beta: \alpha$ Starting from pure $\beta \quad 82 \%, 1: 14 \beta: \alpha$

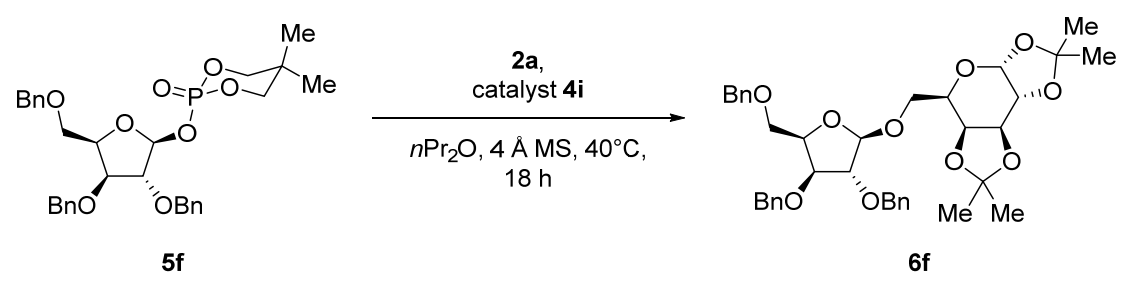

Starting from $1: 11 \alpha: \beta \quad 79 \%, 13: 1 \beta: \alpha$ Starting from pure $\beta \quad 83 \%, 13: 1 \beta: \alpha$ Scheme S8 Effect of Anomeric Purity on the Catalyzed Glycosylation of Arabinose Phosphate 1c (top), Ribose Phosphate 5e (middle), and Xylofuanose Phosphate $\mathbf{5 f}$ (bottom) with 1,2:3,4-Di-O-isopropylidene- $\alpha$-D-galactopyranose 2a.

Consistent with a stereospecific glycosylation event, decreased anomeric purity of the arabinose-phosphate 1c and ribose-phosphate $\mathbf{5 e}$ donors resulted in decreased anomeric selectivity of the formed glycosides, $3 \mathbf{a}$ and $\mathbf{6 e}$, respectively. In contrast, the xylofuranose glycosylation providing product $6 f$ proved to be independent of the starting material $\mathbf{5 f}$ composition, possibly suggesting a change in the glycosylation mechanism. 


\section{Multi-Pot Quench Kinetic Experiments}

\section{General Procedure E for Multi-Pot Quench Kinetic Experiments}

Multiple 0.5 dram vials were charged with the specified amount of the specified donor and $50 \mathrm{mg} 4 \AA$ molecular sieves and $0.5 \mathrm{~mL} n \mathrm{Pr}_{2} \mathrm{O}$ stock solution of the internal standard 1,3,5-trimethoxybenzene ( $3.0 \mathrm{mg}, 0.018 \mathrm{mmol}, 1.0$ eq.), and the specified amount of catalyst $\mathbf{4 a}$ or $\mathbf{4} \mathbf{i}$ and 1,2:3,4-di-O-isopropylidene- $\alpha$-D-galactopyranose $\mathbf{2} \mathbf{a}$ or $\mathrm{N}$-Boc-L-threonine methyl ester $\mathbf{2} \mathbf{b}$. The vials were sealed and premixed on a VWR Mini Vortex Mixer before being stirred at $40{ }^{\circ} \mathrm{C}$ for the specified reaction times. Every 60 min, one sample was diluted with $\mathrm{CH}_{2} \mathrm{Cl}_{2}$, the resulting solution was filtered over a syringe filter, eluted with $\mathrm{CH}_{2} \mathrm{Cl}_{2}$ and concentrated in vacuo. The $\alpha$-donor, $\beta$-donor, $\beta$ product and $\alpha$-product and the corresponding anomeric ratios were analyzed by ${ }^{1} \mathrm{HNMR}$ (in $\mathrm{CDCl}_{3}$ ) via integration against the internal standard 1,3,5trimethoxybenzene.

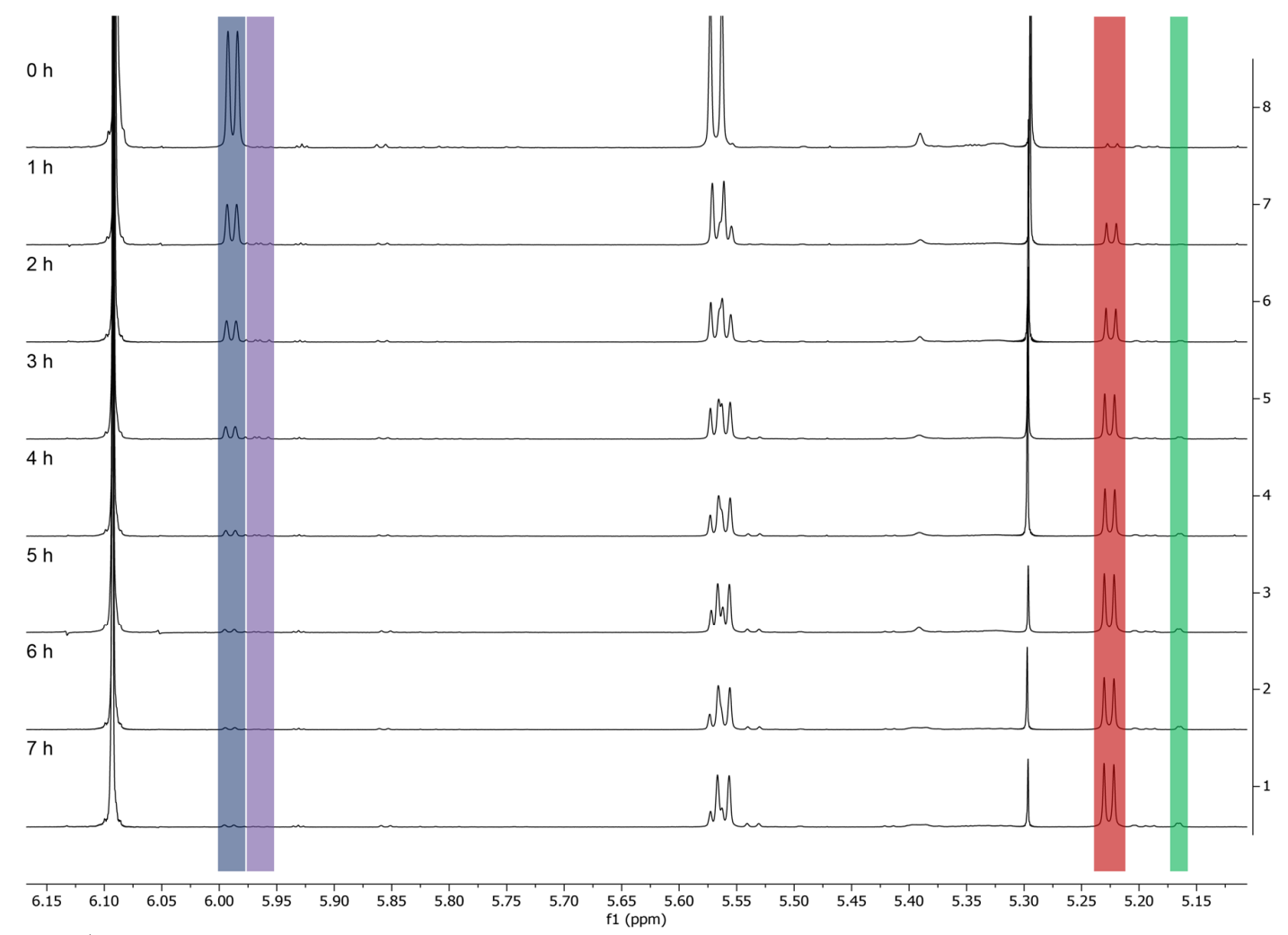

Figure S2 ${ }^{1} \mathrm{H}$ NMR Spectral Data for the Reaction of 1c with 1,2:3,4-Di-O-isopropylidene- $\alpha$-D-galactopyranose 2a Obtained by Multi-Pot Quench Kinetic Experiments (Blue: $\alpha-1 \mathbf{c}$; Purple: $\beta$-1c; Red: $\beta-3 \mathbf{a}$; Green: $\alpha-3 \mathbf{a}$ ). 


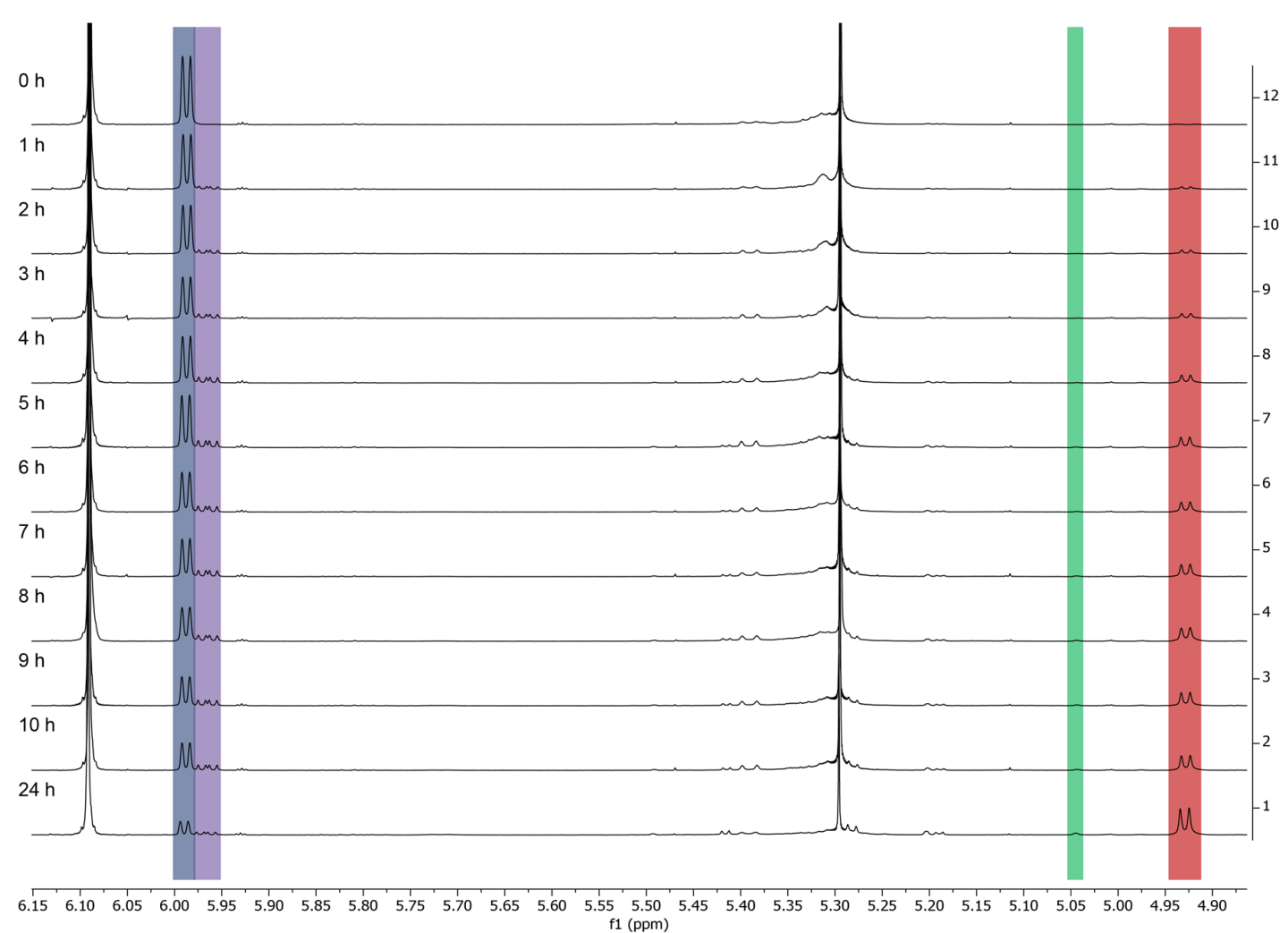

Figure S3 ${ }^{1} \mathrm{H}$ NMR Spectral Data for the Reaction of 1c with N-Boc-L-threonine Methyl Ester $\mathbf{2 b}$ Obtained by Multi-Pot Quench Kinetic Experiments (Blue: $\alpha-1 c$; Purple: $\beta-1 c$; Red: $\beta-3 b$; Green: $\alpha-3 b$ ). 


\section{Standard Reactions Conditions}

According to General Procedure E, the experiment was performed using 1c $(10.2 \mathrm{mg}$, $0.018 \mathrm{mmol}, 1.0$ eq.), catalyst $4 \mathbf{i}(0.94 \mathrm{mg}, 0.0009 \mathrm{mmol}, 0.05$ eq. $)$, and 1,2:3,4-di-Oisopropylidene- $\alpha$-D-galactopyranose $2 \mathrm{a}(9.4 \mathrm{mg}, 0.036 \mathrm{mmol}, 2.0$ eq.) or $\mathrm{N}$-Boc-Lthreonine methyl ester $\mathbf{2 b}(8.4 \mathrm{mg}, 0.036 \mathrm{mmol}, 2.0$ eq.). The reaction was monitored over $5 \mathrm{~h}$ for 1,2:3,4-di-O-isopropylidene- $\alpha$-D-galactopyranose $2 \mathrm{a}$ and $10 \mathrm{~h}$ for $\mathrm{N}$-BocL-threonine methyl ester $\mathbf{2 b}$.

\begin{tabular}{|c|c|c|c|c|c|c|c|c|c|}
\hline \multirow{2}{*}{$\begin{array}{c}\text { Time } \\
{[\mathrm{h}]}\end{array}$} & \multirow{2}{*}{$\begin{array}{c}\text { Std. } \\
{[\mathrm{mol} / \mathrm{L}]}\end{array}$} & \multicolumn{2}{|c|}{ Donor 1c } & \multicolumn{2}{|c|}{ Product $\mathbf{3 a}$} & \multicolumn{2}{|c|}{ Donor 1c } & \multicolumn{2}{|c|}{ Product 3a } \\
\hline & & $\begin{array}{c}\alpha \\
{[\mathrm{mol} / \mathrm{L}]}\end{array}$ & $\begin{array}{c}\beta \\
{[\mathrm{mol} / \mathrm{L}]}\end{array}$ & $\begin{array}{c}\beta \\
{[\mathrm{mol} / \mathrm{L}]}\end{array}$ & $\begin{array}{c}\alpha \\
{[\mathrm{mol} / \mathrm{L}]}\end{array}$ & $\alpha$ & $\beta$ & $\beta$ & $\alpha$ \\
\hline 0 & $3.60 * 10^{-2}$ & $2.88^{*} 10^{-2}$ & 0 & 0 & 0 & 1 & 0 & 1 & 0 \\
\hline 1 & $3.60 * 10^{-2}$ & $1.76^{*} 10^{-2}$ & $1.44^{*} 10^{-3}$ & $1.26 * 10^{-2}$ & $3.60 * 10^{-4}$ & 14 & 1 & 36 & 1 \\
\hline 2 & $3.60 * 10^{-2}$ & $7.20 * 10^{-3}$ & $1.08 * 10^{-3}$ & $2.16^{*} 10^{-2}$ & $7.20 * 10^{-4}$ & 7 & 1 & 35 & 1 \\
\hline 3 & $3.60 * 10^{-2}$ & $1.80 * 10^{-3}$ & $7.20 * 10^{-4}$ & $2.74^{*} 10^{-2}$ & $1.44^{*} 10^{-3}$ & 2 & 1 & 19 & 1 \\
\hline 4 & $3.60 * 10^{-2}$ & $1.08 * 10^{-3}$ & $3.60 * 10^{-4}$ & $2.95^{*} 10^{-2}$ & $1.80^{*} 10^{-3}$ & 2 & 1 & 17 & 1 \\
\hline 5 & $3.60 * 10^{-2}$ & 0 & 0 & $2.99 * 10^{-2}$ & $1.80 * 10^{-3}$ & 1 & 1 & 18 & 1 \\
\hline \multicolumn{10}{|c|}{$\begin{array}{l}\text { Table S7 Concentration Data and Anomeric Ratios for the Catalyzed Reaction of 1c with 1,2:3,4-Di-O-isopropylidene- } \alpha \text {-D-galactopyranose 2a Unc } \\
\text { Standard Reaction Conditions (2 } 2^{\text {nd }} \text { Run). }\end{array}$} \\
\hline Time & Std. & \multicolumn{2}{|c|}{ Donor 1c } & \multicolumn{2}{|c|}{ Product $3 \mathbf{a}$} & \multicolumn{2}{|c|}{ Donor 1c } & \multicolumn{2}{|c|}{ Product $3 a$} \\
\hline [h] & {$[\mathrm{mol} / \mathrm{L}]$} & $\begin{array}{c}\alpha \\
{[\mathrm{mol} / \mathrm{L}]}\end{array}$ & $\begin{array}{c}\beta \\
{[\mathrm{mol} / \mathrm{L}]}\end{array}$ & $\begin{array}{c}\beta \\
{[\mathrm{mol} / \mathrm{L}]}\end{array}$ & $\begin{array}{c}\alpha \\
{[\mathrm{mol} / \mathrm{L}]}\end{array}$ & $\alpha$ & $\beta$ & $\beta$ & $\alpha$ \\
\hline 0 & $3.60^{*} 10^{-2}$ & $3.28 * 10^{-2}$ & 0 & $3.96 * 10^{-4}$ & 0 & 1 & 0 & 1 & 0 \\
\hline 1 & $3.60 * 10^{-2}$ & $1.92 * 10^{-2}$ & $1.26 * 10^{-3}$ & $1.28 * 10^{-2}$ & $3.24 * 10^{-4}$ & 15 & 1 & 40 & 1 \\
\hline 2 & $3.60 * 10^{-2}$ & $6.55^{*} 10^{-3}$ & $1.80^{*} 10^{-3}$ & $2.29 * 10^{-2}$ & $7.56^{*} 10^{-4}$ & 4 & 1 & 31 & 1 \\
\hline 3 & $3.60 * 10^{-2}$ & $1.62 * 10^{-3}$ & $6.12^{*} 10^{-4}$ & $2.79 * 10^{-2}$ & $1.30 * 10^{-3}$ & 3 & 1 & 21 & 1 \\
\hline 4 & $3.60 * 10^{-2}$ & $3.24 * 10^{-4}$ & $1.80^{*} 10^{-4}$ & $2.94^{*} 10^{-2}$ & $1.62 * 10^{-3}$ & 2 & 1 & 18 & 1 \\
\hline 5 & $3.60^{*} 10^{-2}$ & 0 & 0 & $3.05^{*} 10^{-2}$ & $1.66 * 10^{-3}$ & 0 & 0 & 18 & 1 \\
\hline \multicolumn{10}{|c|}{$\begin{array}{l}\text { Table S8 Concentration Data and Anomeric Ratios for the Catalyzed Reaction of 1c with 1,2:3,4-Di-O-isopropylidene- } \alpha \text {-D-galactopyranose } 2 \text { a Und } \\
\text { Standard Reaction Conditions (Average). }\end{array}$} \\
\hline Time & Std. & \multicolumn{2}{|c|}{ Donor 1c } & \multicolumn{2}{|c|}{ Product 3a } & \multicolumn{2}{|c|}{ Donor 1c } & \multicolumn{2}{|c|}{ Product $3 a$} \\
\hline [h] & {$[\mathrm{mol} / \mathrm{L}]$} & $\begin{array}{c}\alpha \\
{[\mathrm{mol} / \mathrm{L}]}\end{array}$ & $\begin{array}{c}\beta \\
{[\mathrm{mol} / \mathrm{L}]}\end{array}$ & $\begin{array}{c}\beta \\
{[\mathrm{mol} / \mathrm{L}]}\end{array}$ & $\begin{array}{c}\alpha \\
{[\mathrm{mol} / \mathrm{L}]}\end{array}$ & $\alpha$ & $\beta$ & $\beta$ & $\alpha$ \\
\hline 0 & $3.60^{*} 10^{-2}$ & $3.08 * 10^{-2}$ & 0 & $1.98 * 10^{-4}$ & 0 & 1 & 0 & 1 & 0 \\
\hline 1 & $3.60^{*} 10^{-2}$ & $1.84 * 10^{-2}$ & $1.35^{*} 10^{-3}$ & $1.27^{*} 10^{-2}$ & $3.42 * 10^{-4}$ & 15 & 1 & 38 & 1 \\
\hline 2 & $3.60 * 10^{-2}$ & $6.88^{*} 10^{-3}$ & $1.44^{*} 10^{-3}$ & $2.22 * 10^{-2}$ & $7.38 * 10^{-4}$ & 6 & 1 & 33 & 1 \\
\hline 3 & $3.60 * 10^{-2}$ & $1.71 * 10^{-3}$ & $6.66^{*} 10^{-4}$ & $2.76^{*} 10^{-2}$ & $1.37^{*} 10^{-3}$ & 3 & 1 & 20 & 1 \\
\hline 4 & $3.60 * 10^{-2}$ & $7.02 * 10^{-4}$ & $2.70^{*} 10^{-4}$ & $2.95^{*} 10^{-2}$ & $1.71 * 10^{-3}$ & 2 & 1 & 18 & 1 \\
\hline 5 & $3.60 * 10^{-2}$ & 0 & 0 & $3.02 * 10^{-2}$ & $1.73^{*} 10^{-3}$ & 1 & 0 & 18 & 1 \\
\hline
\end{tabular}




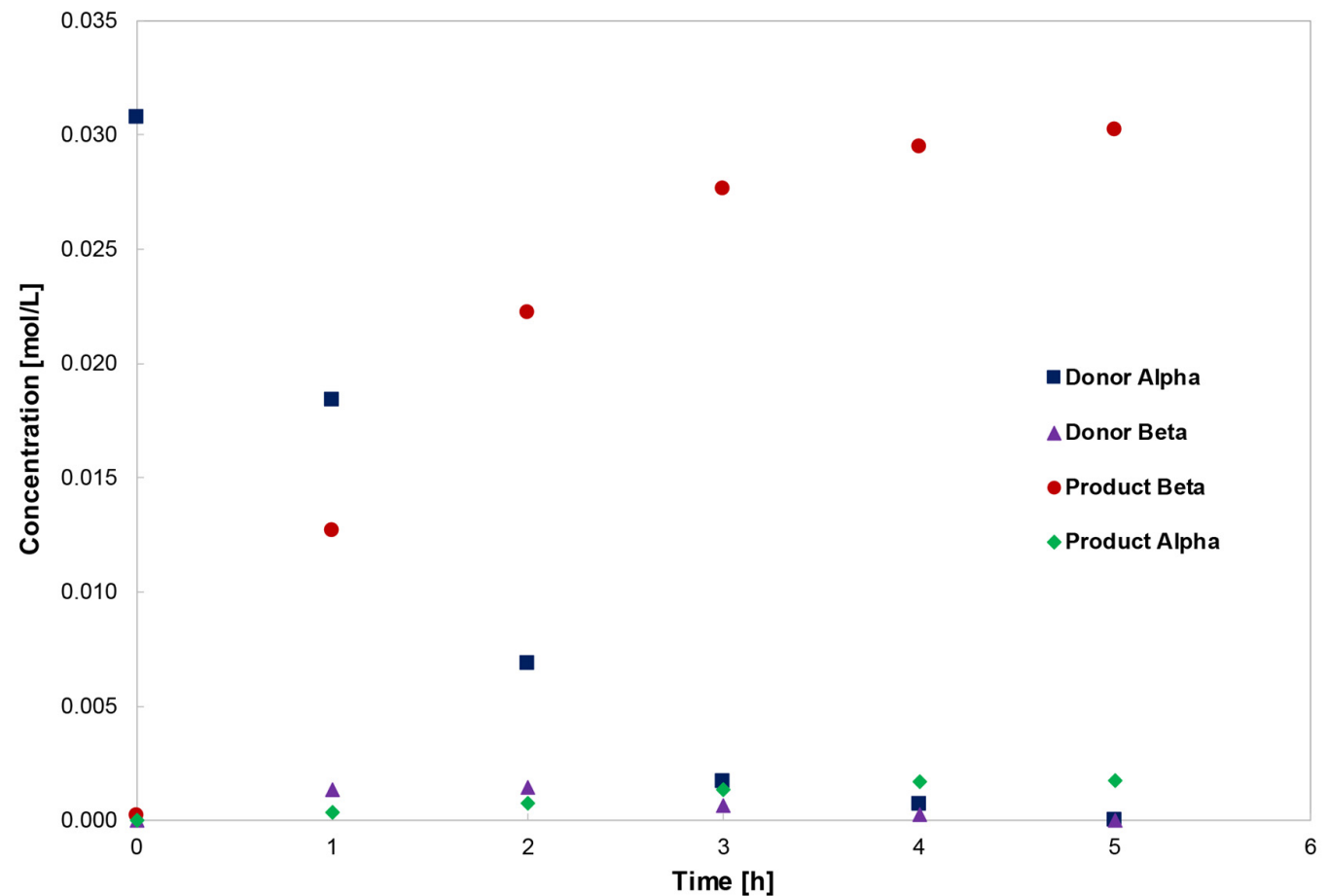

Figure S4 Averaged Kinetic Trace for the Reaction of 1c with 1,2:3,4-Di-O-isopropylidene- $\alpha$-D-galactopyranose 2a Under Standard Reaction Conditions (Blue Squares: $\alpha-1 c$; Purple Triangles: $\beta-1 \mathbf{c}$; Red Dots: $\beta-3 a$; Green Diamonds: $\alpha-3 a$ ).

Table S9 Concentrations and Anomeric Ratios for the Catalyzed Reaction 1c with 1,2:3,4-Di-O-isopropylidene- $\alpha$-D-galactopyranose 2a Under Standard Reaction Conditions (1 ${ }^{\text {st }}$ Run).

\begin{tabular}{cccccccccc}
\hline Time & Std. & \multicolumn{2}{c}{ Donor 1c } & \multicolumn{2}{c}{ Product 3a } & \multicolumn{2}{c}{ Donor 1c } & \multicolumn{2}{c}{ Product 3a } \\
{$[\mathrm{min}]$} & {$[\mathrm{mol} / \mathrm{L}]$} & $\begin{array}{c}\alpha \\
{[\mathrm{mol} / \mathrm{L}]}\end{array}$ & $\begin{array}{c}\beta \\
{[\mathrm{mol} / \mathrm{L}]}\end{array}$ & $\begin{array}{c}\beta \\
{[\mathrm{mol} / \mathrm{L}]}\end{array}$ & $\begin{array}{c}\alpha \\
{[\mathrm{mol} / \mathrm{L}]}\end{array}$ & $\alpha$ & $\beta$ & $\beta$ & $\alpha$ \\
\hline 0 & $3.60^{*} 10^{-2}$ & $3.01^{*} 10^{-2}$ & 0 & $8.64^{*} 10^{-4}$ & 0 & 1 & 0 & 1 & 0 \\
15 & $3.60^{*} 10^{-2}$ & $2.58^{*} 10^{-2}$ & $1.12^{*} 10^{-3}$ & $6.48^{*} 10^{-3}$ & $3.24^{*} 10^{-4}$ & 23 & 1 & 21 & 1 \\
30 & $3.60^{*} 10^{-2}$ & $2.00^{*} 10^{-2}$ & $1.01^{*} 10^{-3}$ & $1.04^{*} 10^{-2}$ & $5.04^{*} 10^{-4}$ & 20 & 1 & 21 & 1 \\
45 & $3.60^{*} 10^{-2}$ & $1.43^{*} 10^{-2}$ & $1.26^{*} 10^{-3}$ & $1.46^{*} 10^{-2}$ & $5.40^{*} 10^{-4}$ & 11 & 1 & 27 & 1 \\
60 & $3.60^{*} 10^{-2}$ & $1.13^{*} 10^{-2}$ & $1.15^{*} 10^{-3}$ & $1.83^{*} 10^{-2}$ & $6.84^{*} 10^{-3}$ & 10 & 1 & 26 & 1 \\
\hline
\end{tabular}

Table S10 Concentrations and Anomeric Ratios for the Catalyzed Reaction of 1c with 1,2:3,4-Di-O-isopropylidene- $\alpha$-D-galactopyranose 2a Under Standard Reaction Conditions (2 ${ }^{\text {nd }}$ Run).

\begin{tabular}{|c|c|c|c|c|c|c|c|c|c|}
\hline \multirow{2}{*}{$\begin{array}{l}\text { Time } \\
\text { [min] }\end{array}$} & \multirow{2}{*}{$\begin{array}{c}\text { Std. } \\
{[\mathrm{mol} / \mathrm{L}]}\end{array}$} & \multicolumn{2}{|c|}{ Donor 1c } & \multicolumn{2}{|c|}{ Product 3a } & \multicolumn{2}{|c|}{ Donor 1c } & \multicolumn{2}{|c|}{ Product 3a } \\
\hline & & $\begin{array}{c}\alpha \\
{[\mathrm{mol} / \mathrm{L}]}\end{array}$ & $\begin{array}{c}\beta \\
{[\mathrm{mol} / \mathrm{L}]}\end{array}$ & $\begin{array}{c}\beta \\
{[\mathrm{mol} / \mathrm{L}]}\end{array}$ & $\begin{array}{c}\alpha \\
{[\mathrm{mol} / \mathrm{L}]}\end{array}$ & $\alpha$ & $\beta$ & $\beta$ & $\alpha$ \\
\hline 0 & $3.60^{*} 10^{-2}$ & $3.05^{*} 10^{-2}$ & 0 & $8.28 * 10^{-4}$ & 0 & 1 & $\overline{0}$ & 1 & 0 \\
\hline 15 & $3.60 * 10^{-2}$ & $2.29 * 10^{-2}$ & $7.92 * 10^{-4}$ & $6.12^{*} 10^{-3}$ & $2.16^{*} 10^{-4}$ & 30 & 1 & 28 & 1 \\
\hline 30 & $3.60^{*} 10^{-2}$ & $2.07^{*} 10^{-2}$ & $9.00 * 10^{-4}$ & $1.03 * 10^{-2}$ & $3.96 * 10^{-4}$ & 23 & 1 & 25 & 1 \\
\hline 45 & $3.60^{*} 10^{-2}$ & $1.22 * 10^{-2}$ & $1.22 * 10^{-3}$ & $1.45^{*} 10^{-2}$ & $3.96^{*} 10^{-4}$ & 12 & 1 & 38 & 1 \\
\hline 60 & $3.60^{*} 10^{-2}$ & $1.04 * 10^{-2}$ & $1.04 * 10^{-3}$ & $1.81 * 10^{-2}$ & $6.12^{*} 10^{-4}$ & 9 & 1 & 30 & 1 \\
\hline
\end{tabular}

Table S11 Concentrations and Anomeric Ratios for the Catalyzed Reaction of 1c with 1,2:3,4-Di-O-isopropylidene- $\alpha$-D-galactopyranose 2a Under Table $\mathbf{S 1 1}$ Concentrations and Anomeric
Standard Reaction Conditions (Average)

\begin{tabular}{|c|c|c|c|c|c|c|c|c|c|}
\hline \multirow{2}{*}{$\begin{array}{l}\text { Time } \\
\text { [min] }\end{array}$} & \multirow{2}{*}{$\begin{array}{c}\text { Std. } \\
{[\mathrm{mol} / \mathrm{L}]}\end{array}$} & \multicolumn{2}{|c|}{ Donor 1c } & \multicolumn{2}{|c|}{ Product 3a } & \multicolumn{2}{|c|}{ Donor 1c } & \multicolumn{2}{|c|}{ Product 3a } \\
\hline & & $\begin{array}{c}\alpha \\
{[\mathrm{mol} / \mathrm{L}]}\end{array}$ & $\begin{array}{c}\beta \\
{[\mathrm{mol} / \mathrm{L}]}\end{array}$ & $\begin{array}{c}\beta \\
{[\mathrm{mol} / \mathrm{L}]}\end{array}$ & $\begin{array}{c}\alpha \\
{[\mathrm{mol} / \mathrm{L}]}\end{array}$ & $\alpha$ & $\beta$ & $\beta$ & $\alpha$ \\
\hline 0 & $3.60^{*} 10^{-2}$ & $3.03^{*} 10^{-2}$ & 0 & $8.46^{*} 10^{-4}$ & 0 & 1 & 0 & 0 & 0 \\
\hline 15 & $3.60^{*} 10^{-2}$ & $2.44^{*} 10^{-2}$ & $9.54^{*} 10^{-4}$ & $6.30^{*} 10^{-3}$ & $2.70^{*} 10^{-4}$ & 27 & 1 & 25 & 1 \\
\hline 30 & $3.60^{*} 10^{-2}$ & $2.03^{*} 10^{-2}$ & $9.54 * 10^{-4}$ & $1.04 * 10^{-2}$ & $4.50 * 10^{-4}$ & 22 & 1 & 23 & 1 \\
\hline 45 & $3.60 * 10^{-2}$ & $1.44^{*} 10^{-2}$ & $1.24^{*} 10^{-3}$ & $1.45^{*} 10^{-2}$ & $4.68 * 10^{-4}$ & 12 & 1 & 33 & 1 \\
\hline 60 & $3.60^{*} 10^{-2}$ & $1.05^{*} 10^{-2}$ & $1.10^{*} 10^{-3}$ & $1.82^{*} 10^{-2}$ & $6.48^{*} 10^{-4}$ & 10 & 1 & 28 & 1 \\
\hline
\end{tabular}




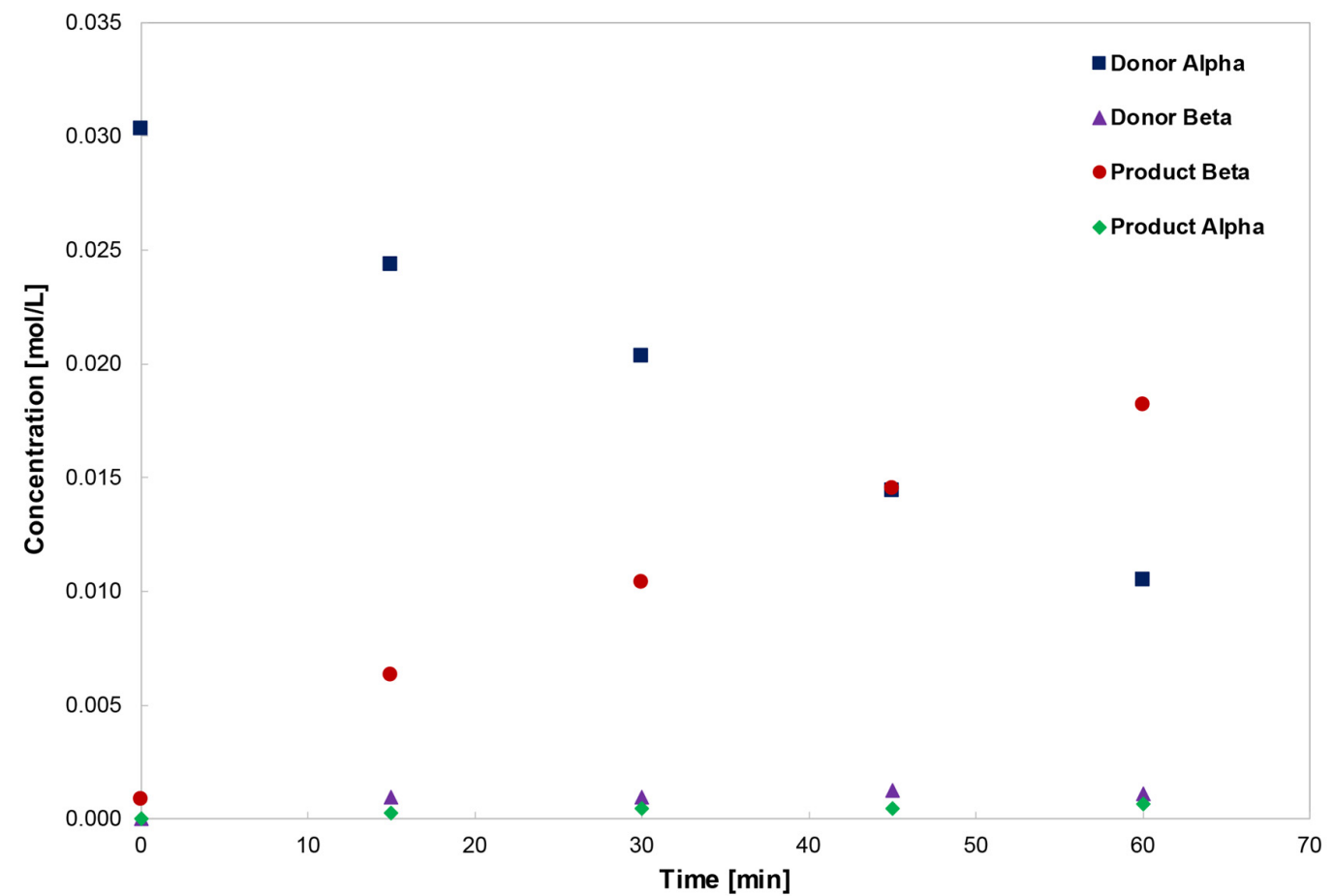

Figure S5 Substrate and Product Concentration as a Function of Time for the Reaction of 1c with 1,2:3,4-Di-O-isopropylidene- $\alpha$-D-galactopyranose 2a Under Standard Reaction Conditions (Blue Squares: $\alpha$-1c; Purple Triangles: $\beta$-1c; Red Dots: $\beta$-3a; Green Diamonds: $\alpha$-3a).

Table S12 Concentration Data and Anomeric Ratios for the Catalyzed Reaction of 1c with N-Boc-L-threonine Methyl Ester 2b Under Standard Reaction Conditions ( $1^{\text {st }}$ Run)

\begin{tabular}{|c|c|c|c|c|c|c|c|c|c|}
\hline \multirow{2}{*}{$\begin{array}{c}\text { Time } \\
{[\mathrm{h}]}\end{array}$} & \multirow{2}{*}{$\begin{array}{c}\text { Std. } \\
{[\mathrm{mol} / \mathrm{L}]}\end{array}$} & \multicolumn{2}{|c|}{ Donor 1c } & \multicolumn{2}{|c|}{ Product 3b } & \multicolumn{2}{|c|}{ Donor 1c } & \multicolumn{2}{|c|}{ Product $\mathbf{3 b}$} \\
\hline & & $\begin{array}{c}\alpha \\
{[\mathrm{mol} / \mathrm{L}]}\end{array}$ & $\begin{array}{c}\beta \\
{[\mathrm{mol} / \mathrm{L}]}\end{array}$ & $\begin{array}{c}\beta \\
{[\mathrm{mol} / \mathrm{L}]}\end{array}$ & $\begin{array}{c}\alpha \\
{[\mathrm{mol} / \mathrm{L}]}\end{array}$ & $\alpha$ & $\beta$ & $\beta$ & $\alpha$ \\
\hline$\overline{0}$ & $3.60^{*} 10^{-2}$ & $3.17^{*} 10^{-2}$ & $7.20^{*} 10^{-4}$ & 0 & 0 & $\overline{48}$ & 1 & 1 & $\overline{0}$ \\
\hline 1 & $3.60^{*} 10^{-2}$ & $2.81^{*} 10^{-2}$ & $2.52^{*} 10^{-3}$ & $2.88^{*} 10^{-3}$ & 0 & 11 & 1 & 31 & 1 \\
\hline 2 & $3.60^{*} 10^{-2}$ & $2.45^{*} 10^{-2}$ & $3.24^{*} 10^{-3}$ & $4.68^{*} 10^{-3}$ & $1.44^{*} 10^{-4}$ & 7 & 1 & 33 & 1 \\
\hline 3 & $3.60^{*} 10^{-2}$ & $2.20^{*} 10^{-2}$ & $3.96^{*} 10^{-3}$ & $6.12^{*} 10^{-3}$ & $1.80 * 10^{-4}$ & 5 & 1 & 36 & 1 \\
\hline 4 & $3.60^{*} 10^{-2}$ & $1.89^{*} 10^{-2}$ & $4.18^{*} 10^{-3}$ & $7.74 * 10^{-3}$ & $2.16^{*} 10^{-4}$ & 5 & 1 & 37 & 1 \\
\hline 5 & $3.60^{*} 10^{-2}$ & $1.82^{*} 10^{-2}$ & $4.90^{*} 10^{-3}$ & $9.94^{*} 10^{-3}$ & $3.60 * 10^{-4}$ & 4 & 1 & 27 & 1 \\
\hline 6 & $3.60^{*} 10^{-2}$ & $1.50^{*} 10^{-2}$ & $4.54^{*} 10^{-3}$ & $1.17^{*} 10^{-2}$ & $4.32 * 10^{-4}$ & 3 & 1 & 28 & 1 \\
\hline 7 & $3.60^{*} 10^{-2}$ & $1.27^{*} 10^{-2}$ & $4.43^{*} 10^{-3}$ & $1.20 * 10^{-2}$ & $4.32 * 10^{-4}$ & 3 & 1 & 27 & 1 \\
\hline 8 & $3.60^{*} 10^{-2}$ & $1.20^{*} 10^{-2}$ & $4.14^{*} 10^{-3}$ & $1.29 * 10^{-2}$ & $3.96 * 10^{-4}$ & 3 & 1 & 32 & 1 \\
\hline 9 & $3.60^{*} 10^{-2}$ & $1.04^{*} 10^{-2}$ & $3.89^{*} 10^{-3}$ & $1.48^{*} 10^{-2}$ & $6.48^{*} 10^{-4}$ & 3 & 1 & 23 & 1 \\
\hline 10 & $3.60^{*} 10^{-2}$ & $9.04 * 10^{-3}$ & $3.42^{*} 10^{-3}$ & $1.61^{*} 10^{-2}$ & $7.13^{*} 10^{-4}$ & 3 & 1 & 23 & 1 \\
\hline \multicolumn{10}{|c|}{$\begin{array}{l}\text { Table S13 Concentration Data and Anomeric Ratios for the Catalyzed Reaction of } 1 \mathbf{c} \text { with N-Boc-L-threonine Methyl Ester } 2 \mathbf{b} \text { Under Stand } \\
\text { Reaction Conditions }\left(2^{\text {nd }} \text { Run }\right) \text {. }\end{array}$} \\
\hline Time & Std. & \multicolumn{2}{|c|}{ Donor 1c } & \multicolumn{2}{|c|}{ Product $\mathbf{3 b}$} & \multicolumn{2}{|c|}{ Donor 1c } & \multicolumn{2}{|c|}{ Product 3b } \\
\hline [h] & {$[\mathrm{mol} / \mathrm{L}]$} & $\begin{array}{c}\alpha \\
{[\mathrm{mol} / \mathrm{L}]}\end{array}$ & $\begin{array}{c}\beta \\
{[\mathrm{mol} / \mathrm{L}]}\end{array}$ & $\begin{array}{c}\beta \\
{[\mathrm{mol} / \mathrm{L}]}\end{array}$ & $\begin{array}{c}\alpha \\
{[\mathrm{mol} / \mathrm{L}]}\end{array}$ & $\alpha$ & $\beta$ & $\beta$ & $\alpha$ \\
\hline$\overline{0}$ & $3.60^{*} 10^{-2}$ & $2.95^{*} 10^{-2}$ & 0 & $\overline{0}$ & 0 & 1 & 0 & 0 & 0 \\
\hline 1 & $3.60^{*} 10^{-2}$ & $2.43^{*} 10^{-2}$ & $1.55^{\star} 10^{-3}$ & $2.23^{*} 10^{-3}$ & 0 & 16 & 1 & 99 & 1 \\
\hline 2 & $3.60 * 10^{-2}$ & $2.30^{*} 10^{-2}$ & $2.74 * 10^{-3}$ & $3.64^{*} 10^{-3}$ & $1.44^{*} 10^{-4}$ & 8 & 1 & 28 & 1 \\
\hline 3 & $3.60^{*} 10^{-2}$ & $1.89^{*} 10^{-2}$ & $3.10^{*} 10^{-3}$ & $5.18^{*} 10^{-3}$ & $1.08 * 10^{-4}$ & 6 & 1 & 44 & 1 \\
\hline 4 & $3.60 * 10^{-2}$ & $1.73^{*} 10^{-2}$ & $4.21^{*} 10^{-3}$ & $7.13^{*} 10^{-3}$ & $3.60 * 10^{-4}$ & 4 & 1 & 20 & 1 \\
\hline 5 & $3.60^{*} 10^{-2}$ & $1.48^{*} 10^{-2}$ & $4.32^{*} 10^{-3}$ & $8.86^{*} 10^{-3}$ & $3.96^{*} 10^{-4}$ & 3 & 1 & 22 & 1 \\
\hline 6 & $3.60^{*} 10^{-2}$ & $1.34^{*} 10^{-2}$ & $4.10^{*} 10^{-3}$ & $9.72 * 10^{-3}$ & $5.04 * 10^{-4}$ & 3 & 1 & 19 & 1 \\
\hline 7 & $3.60^{*} 10^{-2}$ & $1.26^{*} 10^{-2}$ & $4.07^{*} 10^{-3}$ & $1.11 * 10^{-2}$ & $5.76 * 10^{-4}$ & 3 & 1 & 19 & 1 \\
\hline 8 & $3.60^{*} 10^{-2}$ & $1.08 * 10^{-2}$ & $3.89^{*} 10^{-3}$ & $1.20^{*} 10^{-2}$ & $5.40^{*} 10^{-4}$ & 3 & 1 & 22 & 1 \\
\hline 9 & $3.60^{*} 10^{-2}$ & $9.43^{*} 10^{-3}$ & $3.49^{*} 10^{-3}$ & $1.30 * 10^{-2}$ & $3.24^{*} 10^{-4}$ & 3 & 1 & 29 & 1 \\
\hline 10 & $3.60 * 10^{-2}$ & $9.47^{*} 10^{-3}$ & $3.71^{*} 10^{-3}$ & $1.37 * 10^{-2}$ & $5.04 * 10^{-4}$ & 3 & 1 & 23 & 1 \\
\hline
\end{tabular}


Table S14 Concentration Data and Anomeric Ratios for the Catalyzed Reaction of 1c with N-Boc-L-threonine Methyl Ester 2b Under Standard Reaction Conditions (Average).

\begin{tabular}{|c|c|c|c|c|c|c|c|c|c|}
\hline \multirow{2}{*}{$\begin{array}{c}\text { Time } \\
{[\mathrm{h}]}\end{array}$} & \multirow{2}{*}{$\begin{array}{c}\text { Std. } \\
{[\mathrm{mol} / \mathrm{L}]}\end{array}$} & \multicolumn{2}{|c|}{ Donor 1c } & \multicolumn{2}{|c|}{ Product 3b } & \multicolumn{2}{|c|}{ Donor 1c } & \multicolumn{2}{|c|}{ Product $\mathbf{3 b}$} \\
\hline & & $\begin{array}{c}\alpha \\
{[\mathrm{mol} / \mathrm{L}]}\end{array}$ & $\begin{array}{c}\beta \\
{[\mathrm{mol} / \mathrm{L}]}\end{array}$ & $\begin{array}{c}\beta \\
{[\mathrm{mol} / \mathrm{L}]}\end{array}$ & $\begin{array}{c}\alpha \\
{[\mathrm{mol} / \mathrm{L}]}\end{array}$ & $\alpha$ & $\beta$ & $\beta$ & $\alpha$ \\
\hline 0 & $3.60^{*} 10^{-2}$ & $3.06^{*} 10^{-2}$ & $3.60^{*} 10^{-4}$ & 0 & 0 & 74 & $\overline{1}$ & 0 & 0 \\
\hline 1 & $3.60^{*} 10^{-2}$ & $2.62^{*} 10^{-2}$ & $2.03^{*} 10^{-3}$ & $2.56^{*} 10^{-3}$ & 0 & 14 & 1 & 65 & 1 \\
\hline 2 & $3.60^{*} 10^{-2}$ & $2.38^{*} 10^{-2}$ & $2.99^{*} 10^{-3}$ & $4.16^{*} 10^{-3}$ & $1.44 * 10^{-4}$ & 8 & 1 & 31 & 1 \\
\hline 3 & $3.60^{*} 10^{-2}$ & $2.04^{*} 10^{-2}$ & $3.53^{*} 10^{-3}$ & $5.65^{*} 10^{-3}$ & $1.44 * 10^{-4}$ & 6 & 1 & 40 & 1 \\
\hline 4 & $3.60^{*} 10^{-2}$ & $1.81 * 10^{-2}$ & $4.19^{*} 10^{-3}$ & $7.42^{*} 10^{-3}$ & $2.88^{*} 10^{-4}$ & 5 & 1 & 29 & 1 \\
\hline 5 & $3.60^{*} 10^{-2}$ & $1.65^{*} 10^{-2}$ & $4.61^{*} 10^{-3}$ & $9.40^{*} 10^{-3}$ & $3.78 * 10^{-4}$ & 4 & 1 & 25 & 1 \\
\hline 6 & $3.60^{*} 10^{-2}$ & $1.42^{*} 10^{-2}$ & $4.32^{*} 10^{-3}$ & $1.07 * 10^{-2}$ & $4.68^{*} 10^{-4}$ & 3 & 1 & 24 & 1 \\
\hline 7 & $3.60^{*} 10^{-2}$ & $1.27^{*} 10^{-2}$ & $4.25^{\star} 10^{-3}$ & $1.15^{*} 10^{-2}$ & $5.04 * 10^{-4}$ & 3 & 1 & 23 & 1 \\
\hline 8 & $3.60 * 10^{-2}$ & $1.14^{*} 10^{-2}$ & $4.01^{*} 10^{-3}$ & $1.24 * 10^{-2}$ & $4.68 * 10^{-4}$ & 3 & 1 & 27 & 1 \\
\hline 9 & $3.60^{*} 10^{-2}$ & $9.94 * 10^{-3}$ & $3.69^{*} 10^{-3}$ & $1.39 * 10^{-2}$ & $4.86 * 10^{-4}$ & 3 & 1 & 26 & 1 \\
\hline 10 & $3.60 * 10^{-2}$ & $9.26^{*} 10^{-3}$ & $3.57^{*} 10^{-3}$ & $1.49 * 10^{-2}$ & $6.08^{*} 10^{-4}$ & 3 & 1 & 23 & 1 \\
\hline
\end{tabular}

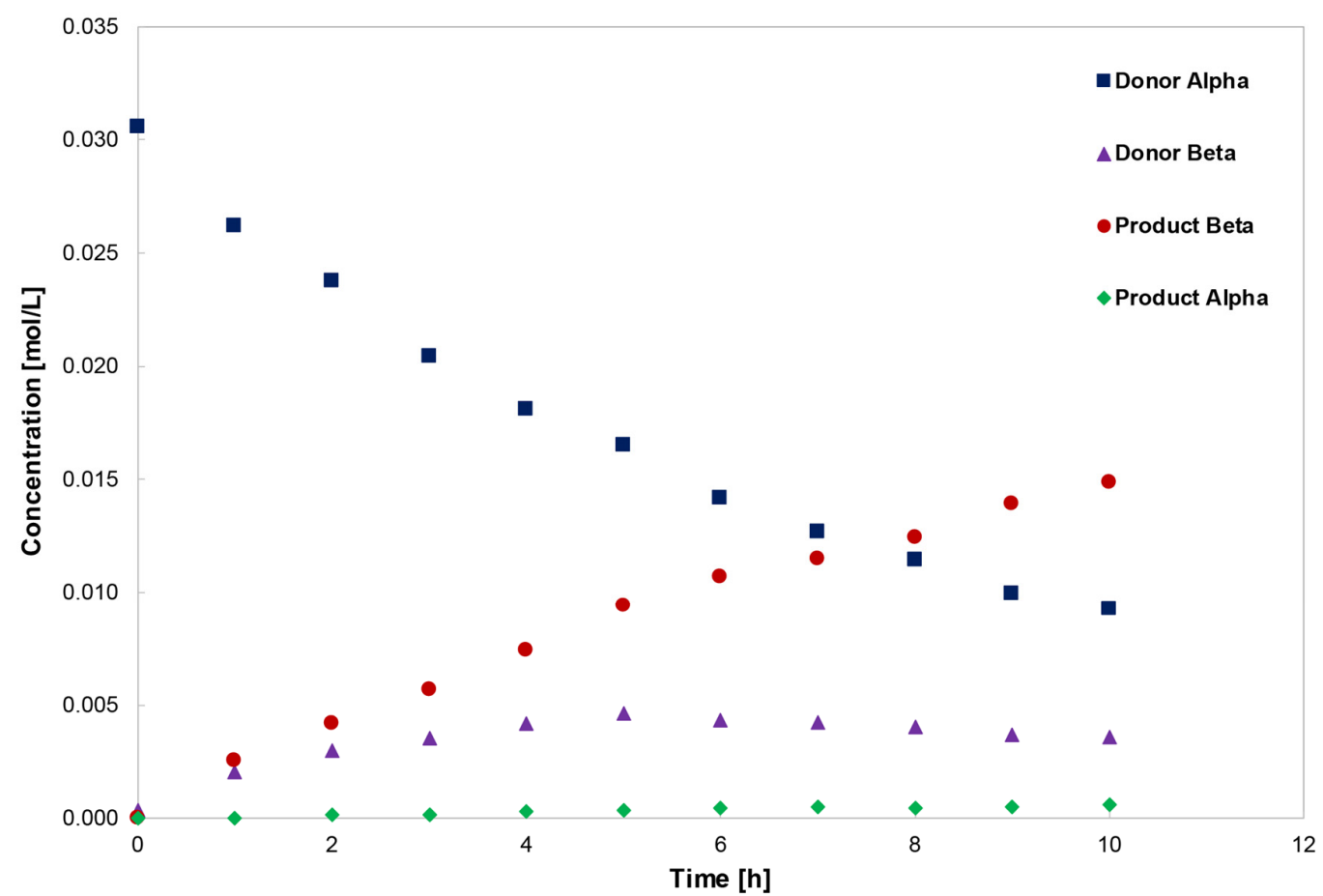

Figure S6 Averaged Kinetic Trace for the Reaction of 1c with N-Boc-L-threonine Methyl Ester 2I Under Standard Reaction Conditions (Blue Squares: $\alpha-1 \mathbf{c}$; Purple Triangles: $\beta-1 \mathbf{c}$; Red Dots: $\beta-3 \mathbf{b}$; Green Diamonds: $\alpha-3 \mathbf{b})$. 


\section{Acceptor Loading Effect}

According to General Procedure E, the experiment was performed using 1c $(10.2 \mathrm{mg}$, $0.018 \mathrm{mmol}, 1.0$ eq.), catalyst $4 \mathbf{i}(0.94 \mathrm{mg}, 0.0009 \mathrm{mmol}, 0.05$ eq.), and 1,2:3,4-di-Oisopropylidene- $\alpha$-D-galactopyranose $2 \mathrm{a}(4.7 \mathrm{mg}, 0.018 \mathrm{mmol}, 1.0 \mathrm{eq}$.$) or \mathrm{N}$-Boc-Lthreonine methyl ester $\mathbf{2 b}(4.2 \mathrm{mg}, 0.018 \mathrm{mmol}, 1.0 \mathrm{eq}$.). The reaction was monitored over $5 \mathrm{~h}$ for 1,2:3,4-di-O-isopropylidene- $\alpha$-D-galactopyranose $\mathbf{2 a}$ and $10 \mathrm{~h}$ for $\mathrm{N}$-BocL-threonine methyl ester $\mathbf{2 b}$.

Table S15 Concentration Data and Anomeric Ratios for the Catalyzed Reaction of 1c with 1 Equivalent of 1,2:3,4-Di-O-isopropylidene- $\alpha$-Dgalactopyranose $2 \mathbf{a}\left(1^{\text {st }}\right.$ Run).

\begin{tabular}{cccccccccc}
\hline $\begin{array}{c}\text { Time } \\
{[\mathrm{h}]}\end{array}$ & $\begin{array}{c}\text { Std. } \\
{[\mathrm{mol} / \mathrm{L}]}\end{array}$ & $\begin{array}{c}\alpha \\
{[\mathrm{mol} / \mathrm{L}]}\end{array}$ & $\begin{array}{c}\text { Donor 1c } \\
{[\mathrm{mol} / \mathrm{L}]}\end{array}$ & $\begin{array}{c}\beta \\
{[\mathrm{mol} / \mathrm{L}]}\end{array}$ & $\begin{array}{c}\alpha \\
{[\mathrm{mol} / \mathrm{L}]}\end{array}$ & $\alpha$ & $\beta$ & $\beta$ & $\alpha$ \\
\hline 0 & $3.60^{*} 10^{-2}$ & $3.09^{*} 10^{-2}$ & $2.52^{*} 10^{-4}$ & $7.92^{*} 10^{-4}$ & 0 & 1 & 0 & 99 & 1 \\
1 & $3.60^{*} 10^{-2}$ & $2.09^{*} 10^{-2}$ & $1.40^{*} 10^{-3}$ & $8.60^{*} 10^{-3}$ & $2.16^{*} 10^{-4}$ & 15 & 1 & 37 & 1 \\
2 & $3.60^{*} 10^{-2}$ & $1.26^{*} 10^{-2}$ & $1.80^{*} 10^{-3}$ & $1.57^{*} 10^{-2}$ & $5.40^{*} 10^{-4}$ & 7 & 1 & 29 & 1 \\
3 & $3.60^{*} 10^{-2}$ & $7.20^{*} 10^{-3}$ & $1.98^{*} 10^{-3}$ & $2.07^{*} 10^{-2}$ & $1.01^{*} 10^{-3}$ & 4 & 1 & 21 & 1 \\
4 & $3.60^{*} 10^{-2}$ & $3.67^{*} 10^{-3}$ & $1.22^{*} 10^{-3}$ & $2.42^{*} 10^{-3}$ & $1.37^{*} 10^{-3}$ & 3 & 1 & 18 & 1 \\
5 & $3.60^{*} 10^{-2}$ & $1.58^{*} 10^{-3}$ & $5.40^{*} 10^{-4}$ & $2.51^{*} 10^{-3}$ & $1.48^{*} 10^{-3}$ & 3 & 1 & 17 & 1 \\
6 & $3.60^{*} 10^{-2}$ & $1.30^{*} 10^{-3}$ & $3.60^{*} 10^{-4}$ & $2.71^{*} 10^{-2}$ & $1.62^{*} 10^{-3}$ & 3 & 1 & 17 & 1 \\
7 & $3.60^{*} 10^{-2}$ & $1.01^{*} 10^{-3}$ & $2.88^{*} 10^{-4}$ & $2.81^{*} 10^{-2}$ & $1.76^{*} 10^{-3}$ & 3 & 1 & 16 & 1 \\
\hline
\end{tabular}

Table S16 Concentration Data and Anomeric Ratios for the Catalyzed Reaction of 1c with 1 Equivalent of 1,2:3,4-Di-O-isopropylidene- $\alpha$-Dgalactopyranose $\mathbf{2 a}\left(2^{\text {nd }}\right.$ Run).

\begin{tabular}{cccccccccc}
\hline $\begin{array}{c}\text { Time } \\
{[\mathrm{h}]}\end{array}$ & $\begin{array}{c}\text { Std. } \\
{[\mathrm{mol} / \mathrm{L}]}\end{array}$ & $\begin{array}{c}\alpha \\
{[\mathrm{mol} / \mathrm{L}]}\end{array}$ & $\begin{array}{c}c \\
{[\mathrm{~mol} / \mathrm{L}]}\end{array}$ & $\begin{array}{c}\beta \\
{[\mathrm{mol} / \mathrm{L}]}\end{array}$ & $\begin{array}{c}\alpha \\
{[\mathrm{mol} / \mathrm{L}]}\end{array}$ & $\alpha$ & $\beta$ & $\beta$ & $\alpha$ \\
\hline 0 & $3.60^{*} 10^{-2}$ & $2.97^{*} 10^{-2}$ & 0 & $7.20^{*} 10^{-4}$ & 0 & 1 & 0 & 99 & 1 \\
1 & $3.60^{*} 10^{-2}$ & $2.11^{*} 10^{-2}$ & $1.19^{*} 10^{-3}$ & $7.74^{*} 10^{-3}$ & $1.80^{*} 10^{-4}$ & 18 & 1 & 47 & 1 \\
2 & $3.60^{*} 10^{-2}$ & $1.33^{*} 10^{-2}$ & $1.87^{*} 10^{-3}$ & $1.54^{*} 10^{-2}$ & $4.68^{*} 10^{-4}$ & 7 & 1 & 33 & 1 \\
3 & $3.60^{*} 10^{-2}$ & $7.78^{*} 10^{-3}$ & $2.09^{*} 10^{-3}$ & $1.94^{*} 10^{-2}$ & $9.00^{*} 10^{-4}$ & 5 & 1 & 22 & 1 \\
4 & $3.60^{*} 0^{-2}$ & $4.97^{*} 0^{-3}$ & $1.51^{*} 10^{-3}$ & $2.14^{*} 10^{-2}$ & $1.15^{*} 10^{-3}$ & 3 & 1 & 19 & 1 \\
5 & $3.60^{*} 10^{-2}$ & $2.88^{*} 10^{-3}$ & $1.51^{*} 10^{-4}$ & $2.40^{*} 10^{-2}$ & $1.44^{*} 10^{-3}$ & 2 & 1 & 17 & 1 \\
6 & $3.60^{*} 10^{-2}$ & $1.48^{*} 10^{-4}$ & $6.12^{*} 10^{-4}$ & $2.52^{*} 10^{-2}$ & $1.58^{*} 10^{-3}$ & 2 & 1 & 16 & 1 \\
7 & $3.60^{*} 10^{-2}$ & $7.56^{*} 10^{-4}$ & $2.52^{*} 10^{-4}$ & $2.53^{*} 10^{-2}$ & $1.58^{*} 10^{-3}$ & 3 & 1 & 16 & 1 \\
\hline
\end{tabular}

Table S17 Concentration Data and Anomeric Ratios for the Catalyzed Reaction of 1c with 1 Equivalent of 1,2:3,4-Di-O-isopropylidene- $\alpha$-Dgalactopyranose 2a (Average).

\begin{tabular}{|c|c|c|c|c|c|c|c|c|c|}
\hline \multirow{2}{*}{$\begin{array}{c}\text { Time } \\
{[\mathrm{h}]}\end{array}$} & \multirow{2}{*}{$\begin{array}{c}\text { Std. } \\
{[\mathrm{mol} / \mathrm{L}]}\end{array}$} & \multicolumn{2}{|c|}{ Donor 1c } & \multicolumn{2}{|c|}{ Product 3a } & \multicolumn{2}{|c|}{ Donor 1c } & \multicolumn{2}{|c|}{ Product 3a } \\
\hline & & $\begin{array}{c}\alpha \\
{[\mathrm{mol} / \mathrm{L}]}\end{array}$ & $\begin{array}{c}\beta \\
{[\mathrm{mol} / \mathrm{L}]}\end{array}$ & $\begin{array}{c}\beta \\
{[\mathrm{mol} / \mathrm{L}]}\end{array}$ & $\begin{array}{c}\alpha \\
{[\mathrm{mol} / \mathrm{L}]}\end{array}$ & $\alpha$ & $\beta$ & $\beta$ & $\alpha$ \\
\hline 0 & $3.60^{*} 10^{-2}$ & $3.03^{*} 10^{-2}$ & $1.26^{*} 10^{-4}$ & $7.56^{*} 10^{-4}$ & 0 & 1 & 0 & 99 & 1 \\
\hline 1 & $3.60^{*} 10^{-2}$ & $2.10^{*} 10^{-2}$ & $1.30^{*} 10^{-3}$ & $8.17^{*} 10^{-3}$ & $1.98 * 10^{-4}$ & 17 & 1 & 42 & 1 \\
\hline 2 & $3.60 * 10^{-2}$ & $1.30^{*} 10^{-3}$ & $1.84^{*} 10^{-3}$ & $1.55^{*} 10^{-2}$ & $5.04^{*} 10^{-4}$ & 7 & 1 & 31 & 1 \\
\hline 3 & $3.60 * 10^{-2}$ & $7.49 * 10^{-3}$ & $2.03^{*} 10^{-2}$ & $2.00 * 10^{-2}$ & $9.54 * 10^{-4}$ & 4 & 1 & 22 & 1 \\
\hline 4 & $3.60 * 10^{-2}$ & $4.32 * 10^{-3}$ & $1.37^{*} 10^{-2}$ & $2.28 * 10^{-2}$ & $1.26^{*} 10^{-3}$ & 3 & 1 & 19 & 1 \\
\hline 5 & $3.60 * 10^{-2}$ & $2.23^{*} 10^{-3}$ & $1.03^{*} 10^{-3}$ & $2.46^{*} 10^{-2}$ & $1.46^{*} 10^{-3}$ & 3 & 1 & 17 & 1 \\
\hline 6 & $3.60^{*} 10^{-2}$ & $1.39^{*} 10^{-3}$ & $4.86^{*} 10^{-4}$ & $2.61 * 10^{-2}$ & $1.60^{*} 10^{-3}$ & 3 & 1 & 17 & 1 \\
\hline 7 & $3.60^{*} 10^{-2}$ & $8.82 * 10^{-4}$ & $2.70 * 10^{-4}$ & $2.67 * 10^{-2}$ & $1.67^{*} 10^{-3}$ & 3 & 1 & 16 & 1 \\
\hline
\end{tabular}




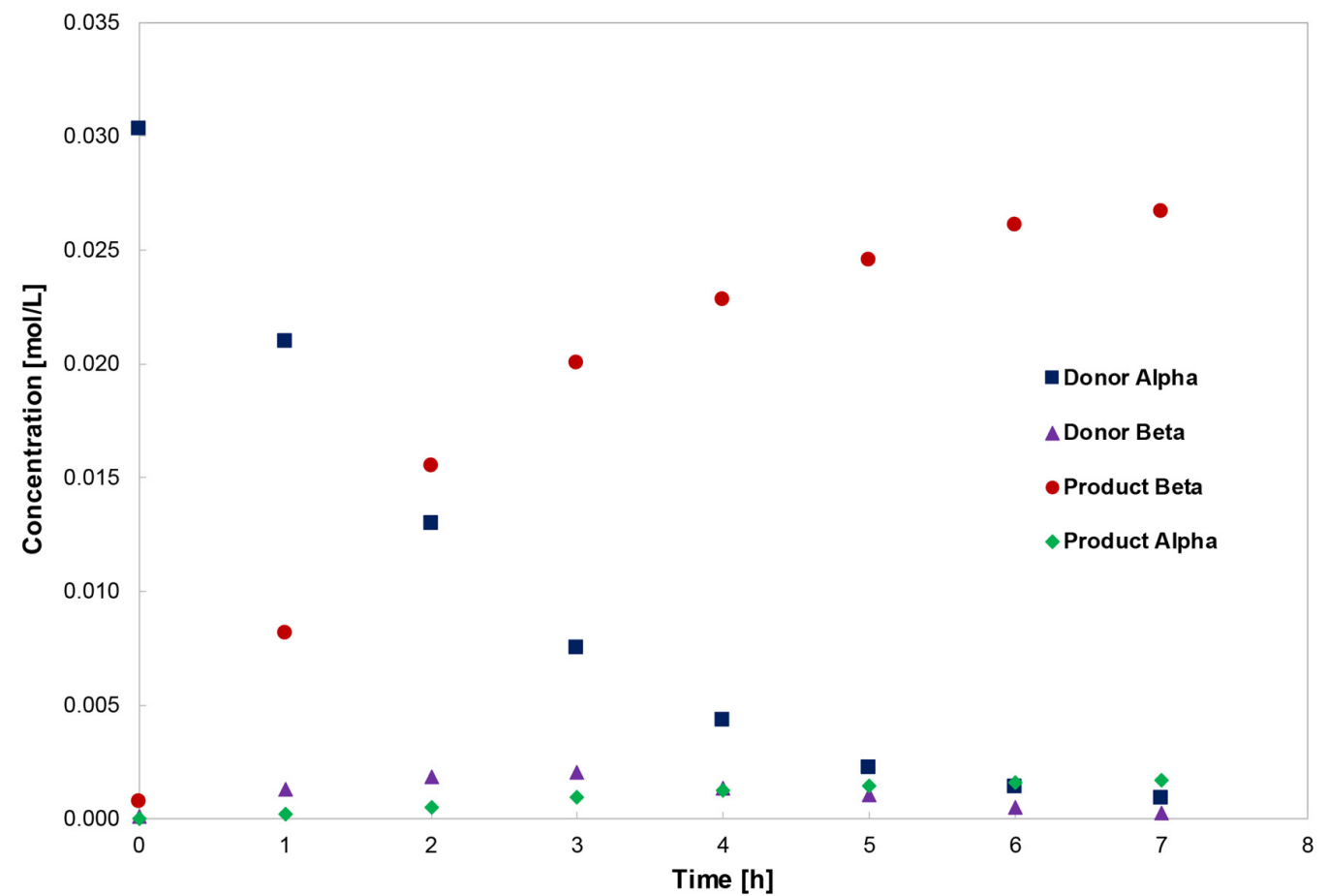

Figure S7 Averaged Kinetic Trace for the Reaction of 1c with 1 Equivalent 1,2:3,4-Di-O-isopropylidene- $\alpha$-D-galactopyranose 2a (Blue Squares: $\alpha$ 1c; Purple Triangles: $\beta-1 c$; Red Dots: $\beta-3 a$; Green Diamonds: $\alpha-3 a$ ).

Table S18 Concentration Data and Anomeric Ratios for the Catalyzed Reaction of 1c with 1 Equivalent N-Boc-L-threonine Methyl Ester 2b (1 $1^{\text {st }}$ Run).

\begin{tabular}{cccccccccc}
\hline $\begin{array}{c}\text { Time } \\
{[\mathrm{h}]}\end{array}$ & $\begin{array}{c}\text { Std. } \\
{[\mathrm{mol} / \mathrm{L}]}\end{array}$ & $\begin{array}{c}\alpha \\
{[\mathrm{mol} / \mathrm{L}]}\end{array}$ & $\begin{array}{c}\beta \\
{[\mathrm{mol} / \mathrm{L}]}\end{array}$ & $\begin{array}{c}\beta \\
{[\mathrm{mol} / \mathrm{L}]}\end{array}$ & $\begin{array}{c}\alpha \\
{[\mathrm{mol} / \mathrm{L}]}\end{array}$ & $\alpha$ & $\beta$ & $\beta$ & $\alpha$ \\
\hline 0 & $3.60^{*} 10^{-2}$ & $3.10^{*} 10^{-2}$ & 0 & 0 & 0 & 99 & 1 & 0 & 0 \\
1 & $3.60^{*} 10^{-2}$ & $2.71^{*} 10^{-2}$ & $2.09^{*} 10^{-3}$ & $1.44^{*} 10^{-3}$ & 0 & 13 & 1 & 99 & 1 \\
2 & $3.60^{*} 10^{-2}$ & $2.55^{*} 10^{-2}$ & $3.20^{*} 10^{-3}$ & $2.20^{*} 10^{-3}$ & $3.60^{*} 10^{-5}$ & 8 & 1 & 57 & 1 \\
3 & $3.60^{*} 10^{-2}$ & $2.36^{*} 10^{-2}$ & $3.78^{*} 10^{-3}$ & $3.28^{*} 10^{-3}$ & $1.08^{*} 10^{-4}$ & 6 & 1 & 33 & 1 \\
4 & $3.60^{*} 10^{-2}$ & $2.12^{*} 10^{-2}$ & $4.00^{*} 10^{-3}$ & $4.50^{*} 10^{-3}$ & $1.08^{*} 10^{-4}$ & 5 & 1 & 36 & 1 \\
5 & $3.60^{*} 10^{-2}$ & $2.07^{*} 10^{-2}$ & $4.25^{*} 10^{-3}$ & $5.29^{*} 10^{-3}$ & $1.08^{*} 10^{-4}$ & 5 & 1 & 50 & 1 \\
6 & $3.60^{*} 10^{-2}$ & $1.95^{*} 10^{-2}$ & $4.43^{*} 10^{-3}$ & $6.41^{*} 10^{-3}$ & $2.16^{*} 10^{-4}$ & 4 & 1 & 30 & 1 \\
7 & $3.60^{*} 10^{-2}$ & $1.74^{*} 10^{-2}$ & $4.39^{*} 10^{-3}$ & $7.31^{*} 10^{-3}$ & $2.52^{*} 10^{-4}$ & 4 & 1 & 28 & 1 \\
8 & $3.60^{*} 10^{-2}$ & $1.53^{*} 10^{-2}$ & $4.21^{*} 10^{-3}$ & $7.96^{*} 10^{-3}$ & $2.88^{*} 10^{-4}$ & 4 & 1 & 26 & 1 \\
9 & $3.60^{*} 10^{-2}$ & $1.47^{*} 10^{-2}$ & $4.32^{*} 10^{-3}$ & $8.71^{*} 10^{-3}$ & $3.60^{*} 10^{-4}$ & 3 & 1 & 25 & 1 \\
10 & $3.60^{*} 10^{-2}$ & $1.34^{*} 10^{-2}$ & $4.03^{*} 10^{-3}$ & $9.22^{*} 10^{-3}$ & $3.60^{*} 10^{-4}$ & 3 & 1 & 25 & 1 \\
24 & $3.60^{*} 10^{-2}$ & $7.02^{*} 10^{-3}$ & $2.34^{*} 10^{-3}$ & $1.74^{*} 10^{-2}$ & $7.92^{*} 10^{-4}$ & 3 & 1 & 22 & 1 \\
\hline
\end{tabular}

Table S19 Concentration Data and Anomeric Ratios for the Catalyzed Reaction of 1c with 1 Equivalent N-Boc-L-threonine Methyl Ester 2b (2 ${ }^{\text {nd }}$ Run).

\begin{tabular}{cccccccccc}
$\begin{array}{c}\text { Time } \\
\text { Th] }\end{array}$ & $\begin{array}{c}\text { Std. } \\
{[\mathrm{mol} / \mathrm{L}]}\end{array}$ & $\begin{array}{c}\alpha \\
{[\mathrm{mol} / \mathrm{L}]}\end{array}$ & $\begin{array}{c}\beta \\
{[\mathrm{mol} / \mathrm{L}]}\end{array}$ & $\begin{array}{c}\beta \\
{[\mathrm{mol} / \mathrm{L}]}\end{array}$ & $\begin{array}{c}\alpha \\
{[\mathrm{mol} / \mathrm{L}]}\end{array}$ & $\alpha$ & $\beta$ & $\beta$ & $\alpha$ \\
\hline 0 & $3.60^{*} 10^{-2}$ & $3.02^{*} 10^{-2}$ & 0 & 0 & 0 & 1 & 0 & 0 & 0 \\
1 & $3.60^{*} 10^{-2}$ & $2.62^{*} 10^{-2}$ & $1.84^{*} 10^{-3}$ & $1.30^{*} 10^{-3}$ & 0 & 14 & 1 & 1 & 0 \\
2 & $3.60^{*} 10^{-2}$ & $2.49^{*} 10^{-2}$ & $2.95^{*} 10^{-3}$ & $2.30^{*} 10^{-3}$ & $1.44^{*} 10^{-4}$ & 10 & 1 & 15 & 1 \\
3 & $3.60^{*} 10^{-2}$ & $2.19^{*} 10^{-2}$ & $3.74^{*} 10^{-3}$ & $3.17^{*} 10^{-3}$ & $1.08^{*} 10^{-4}$ & 7 & 1 & 31 & 1 \\
4 & $3.60^{*} 10^{-2}$ & $1.99^{*} 10^{-2}$ & $3.96^{*} 10^{-3}$ & $4.07^{*} 10^{-3}$ & $1.08^{*} 10^{-4}$ & 5 & 1 & 34 & 1 \\
5 & $3.60^{*} 10^{-2}$ & $1.88^{*} 10^{-2}$ & $4.50^{*} 10^{-3}$ & $5.11^{*} 10^{-3}$ & $2.88^{*} 10^{-4}$ & 4 & 1 & 18 & 1 \\
6 & $3.60^{*} 10^{-2}$ & $1.80^{*} 10^{-2}$ & $4.54^{*} 10^{-3}$ & $5.65^{*} 10^{-3}$ & $1.80^{*} 10^{-4}$ & 4 & 1 & 32 & 1 \\
7 & $3.60^{*} 10^{-2}$ & $1.59^{*} 10^{-2}$ & $4.46^{*} 10^{-3}$ & $6.16^{*} 10^{-3}$ & $2.16^{*} 10^{-4}$ & 4 & 1 & 30 & 1 \\
8 & $3.60^{*} 10^{-2}$ & $1.53^{*} 10^{-2}$ & $4.43^{*} 10^{-3}$ & $7.16^{*} 10^{-3}$ & $3.60^{*} 10^{-4}$ & 3 & 1 & 21 & 1 \\
9 & $3.60^{*} 10^{-2}$ & $1.41^{*} 10^{-2}$ & $4.46^{*} 10^{-3}$ & $7.78^{*} 10^{-3}$ & $3.24^{*} 10^{-4}$ & 3 & 1 & 23 & 1 \\
10 & $3.60^{*} 10^{-2}$ & $1.33^{*} 10^{-2}$ & $4.07^{*} 10^{-3}$ & $8.75^{*} 10^{-3}$ & $4.68^{*} 10^{-4}$ & 3 & 1 & 19 & 1 \\
24 & $3.60^{*} 10^{-2}$ & $6.05^{*} 10^{-3}$ & $1.87^{*} 10^{-3}$ & $1.50^{*} 10^{-2}$ & $6.84^{*} 10^{-4}$ & 3 & 1 & 22 & 1 \\
\hline Table S20 Concentration Data and Anomeric Ratios for the Catalyzed Reaction of 1c with 1Equivalent $N$-Boc-L-threonine Methyl Ester 2b (Average).
\end{tabular}




\begin{tabular}{|c|c|c|c|c|c|c|c|c|c|}
\hline \multirow{2}{*}{$\begin{array}{c}\text { Time } \\
{[\mathrm{h}]}\end{array}$} & \multirow{2}{*}{$\begin{array}{c}\text { Std. } \\
{[\mathrm{mol} / \mathrm{L}]}\end{array}$} & \multicolumn{2}{|c|}{ Donor 1c } & \multicolumn{2}{|c|}{ Product 3b } & \multicolumn{2}{|c|}{ Donor 1c } & \multicolumn{2}{|c|}{ Product $\mathbf{3 b}$} \\
\hline & & $\begin{array}{c}\alpha \\
{[\mathrm{mol} / \mathrm{L}]}\end{array}$ & $\begin{array}{c}\beta \\
{[\mathrm{mol} / \mathrm{L}]}\end{array}$ & $\begin{array}{c}\beta \\
{[\mathrm{mol} / \mathrm{L}]}\end{array}$ & $\begin{array}{c}\alpha \\
{[\mathrm{mol} / \mathrm{L}]}\end{array}$ & $\alpha$ & $\beta$ & $\beta$ & $\alpha$ \\
\hline 0 & $3.60^{*} 10^{-2}$ & $3.06^{*} 10^{-2}$ & 0 & 0 & 0 & 1 & 0 & 0 & 0 \\
\hline 1 & $3.60^{*} 10^{-2}$ & $2.66^{*} 10^{-2}$ & $1.96 * 10^{-3}$ & $1.37^{*} 10^{-3}$ & 0 & 14 & 1 & 1 & 0 \\
\hline 2 & $3.60 * 10^{-2}$ & $2.52^{*} 10^{-2}$ & $3.08^{*} 10^{-3}$ & $2.25^{*} 10^{-3}$ & $9.00 * 10^{-5}$ & 9 & 1 & 36 & 1 \\
\hline 3 & $3.60 * 10^{-2}$ & $2.28^{*} 10^{-2}$ & $3.76 * 10^{-3}$ & $3.22^{*} 10^{-3}$ & $1.08 * 10^{-4}$ & 7 & 1 & 32 & 1 \\
\hline 4 & $3.60 * 10^{-2}$ & $2.05^{\star} 10^{-2}$ & $3.98 * 10^{-3}$ & $4.28 * 10^{-3}$ & $1.08 * 10^{-4}$ & 6 & 1 & 36 & 1 \\
\hline 5 & $3.60^{*} 10^{-2}$ & $1.97^{*} 10^{-2}$ & $4.37^{*} 10^{-3}$ & $5.20^{*} 10^{-3}$ & $1.98^{*} 10^{-4}$ & 6 & 1 & 34 & 1 \\
\hline 6 & $3.60^{*} 10^{-2}$ & $1.88^{*} 10^{-2}$ & $4.48^{*} 10^{-3}$ & $6.03^{*} 10^{-3}$ & $1.98^{*} 10^{-4}$ & 5 & 1 & 31 & 1 \\
\hline 7 & $3.60 * 10^{-2}$ & $1.67^{*} 10^{-2}$ & $4.43^{*} 10^{-3}$ & $6.73^{*} 10^{-3}$ & $2.34^{*} 10^{-4}$ & 5 & 1 & 29 & 1 \\
\hline 8 & $3.60 * 10^{-2}$ & $1.53^{*} 10^{-2}$ & $4.32 * 10^{-3}$ & $7.56^{*} 10^{-3}$ & $3.24 * 10^{-4}$ & 3 & 1 & 24 & 1 \\
\hline 9 & $3.60 * 10^{-2}$ & $1.44^{*} 10^{-2}$ & $4.39 * 10^{-3}$ & $8.24^{*} 10^{-3}$ & $3.42 * 10^{-4}$ & 3 & 1 & 24 & 1 \\
\hline 10 & $3.60^{*} 10^{-2}$ & $1.33^{*} 10^{-2}$ & $4.05^{*} 10^{-3}$ & $8.98^{*} 10^{-3}$ & $4.14^{*} 10^{-4}$ & 3 & 1 & 22 & 1 \\
\hline 24 & $3.60^{*} 10^{-2}$ & $6.53^{*} 10^{-3}$ & $2.11^{*} 10^{-3}$ & $1.62 * 10^{-2}$ & $7.38^{*} 10^{-4}$ & 3 & 1 & 22 & 1 \\
\hline
\end{tabular}

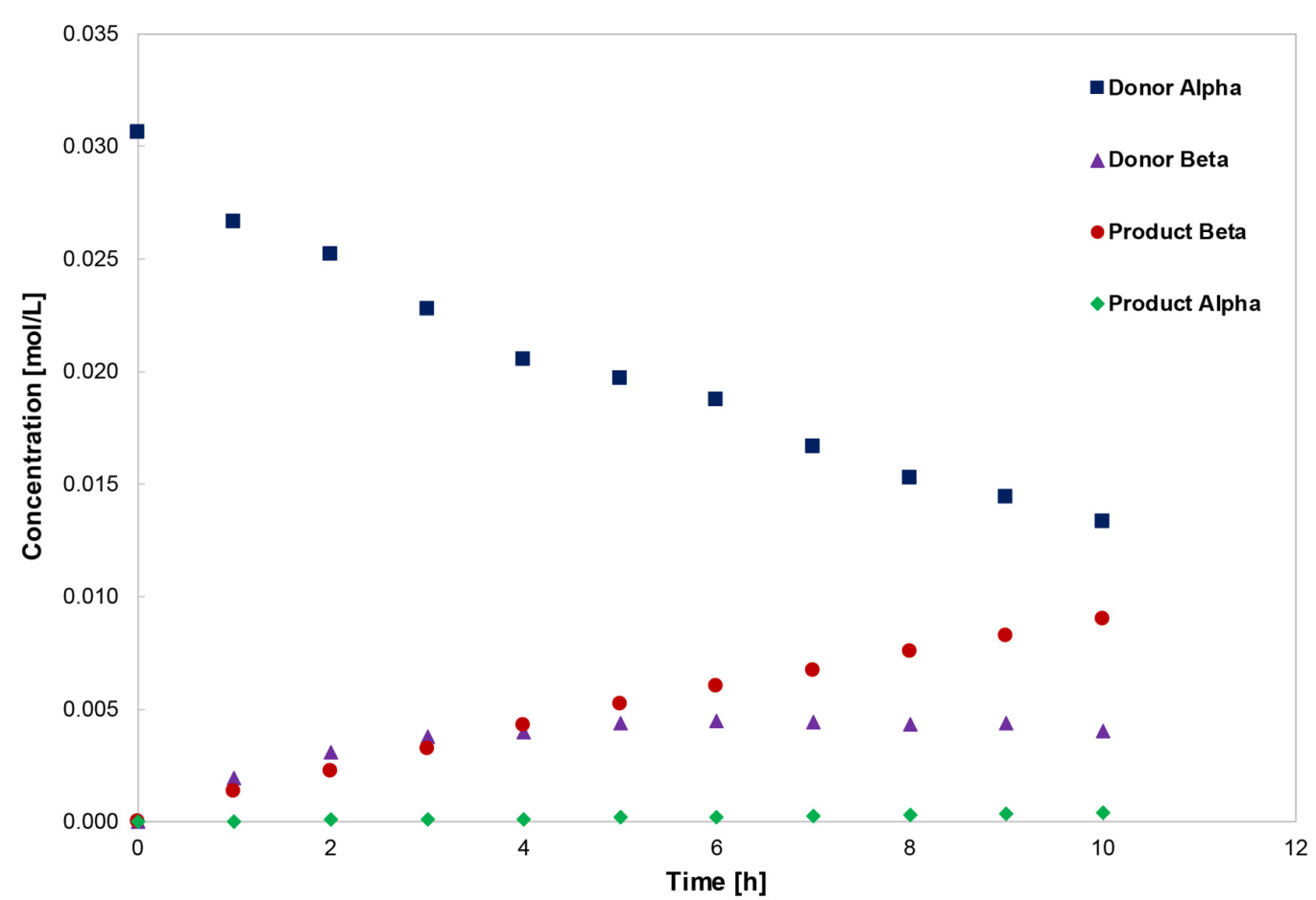

Figure S8 Averaged Kinetic Trace for the Reaction of 1c with 1 Equivalent $N$-Boc-L-threonine Methyl Ester 2l (Blue Squares: $\alpha-1 c ;$ Purple Triangles: $\beta$-1c; Red Dots: $\beta-3 b$; Green Diamonds: $\alpha-3 b)$. 


\section{Catalyst Loading Effect}

According to General Procedure E, the experiment was performed using 1c $(10.2 \mathrm{mg}$, $0.018 \mathrm{mmol}, 1.0$ eq.), catalyst $4 \mathbf{i}(0.47 \mathrm{mg}, 0.00045 \mathrm{mmol}, 0.025 \mathrm{eq}$.), and 1,2:3,4-diO-isopropylidene- $\alpha$-D-galactopyranose $\mathbf{2 a}(9.4 \mathrm{mg}, 0.036 \mathrm{mmol}, 2.0 \mathrm{eq}$.) or $\mathrm{N}$-Boc-Lthreonine methyl ester $\mathbf{2 b}(8.4 \mathrm{mg}, 0.036 \mathrm{mmol}, 2.0$ eq.). The reaction was monitored over $5 \mathrm{~h}$ for 1,2:3,4-di-O-isopropylidene- $\alpha$-D-galactopyranose $2 \mathrm{a}$ and $10 \mathrm{~h}$ for $\mathrm{N}$-BocL-threonine methyl ester $\mathbf{2 b}$.

\begin{tabular}{|c|c|c|c|c|c|c|c|c|c|}
\hline \multirow{2}{*}{$\begin{array}{c}\text { Time } \\
{[\mathrm{h}]}\end{array}$} & \multirow{2}{*}{$\begin{array}{c}\text { Std. } \\
{[\mathrm{mol} / \mathrm{L}]}\end{array}$} & \multicolumn{2}{|c|}{ Donor 1c } & \multicolumn{2}{|c|}{ Product $\mathbf{3 a}$} & \multicolumn{2}{|c|}{ Donor 1c } & \multicolumn{2}{|c|}{ Product 3a } \\
\hline & & $\begin{array}{c}\alpha \\
{[\mathrm{mol} / \mathrm{L}]}\end{array}$ & $\begin{array}{c}\beta \\
{[\mathrm{mol} / \mathrm{L}]}\end{array}$ & $\begin{array}{c}\beta \\
{[\mathrm{mol} / \mathrm{L}]}\end{array}$ & $\begin{array}{c}\alpha \\
{[\mathrm{mol} / \mathrm{L}]}\end{array}$ & $\alpha$ & $\beta$ & $\beta$ & $\alpha$ \\
\hline 0 & $3.60^{*} 10^{-2}$ & $3.01^{*} 10^{-2}$ & 0 & 0 & 0 & 1 & 0 & 0 & 0 \\
\hline 1 & $3.60 * 10^{-2}$ & $2.15^{*} 10^{-2}$ & $8.28 * 10^{-4}$ & $7.92 * 10^{-3}$ & $2.16 * 10^{-4}$ & 17 & 1 & 37 & 1 \\
\hline 2 & $3.60 * 10^{-2}$ & $1.27^{*} 10^{-2}$ & $9.72 * 10^{-4}$ & $1.54^{*} 10^{-2}$ & $4.32 * 10^{-4}$ & 13 & 1 & 35 & 1 \\
\hline 3 & $3.60 * 10^{-2}$ & $7.27^{*} 10^{-3}$ & $1.30 * 10^{-3}$ & $2.18^{*} 10^{-2}$ & $7.92 * 10^{-4}$ & 6 & 1 & 28 & 1 \\
\hline 4 & $3.60 * 10^{-2}$ & $3.10^{*} 10^{-3}$ & $1.04 * 10^{-3}$ & $2.54^{*} 10^{-3}$ & $9.72 * 10^{-4}$ & 3 & 1 & 26 & 1 \\
\hline 5 & $3.60 * 10^{-2}$ & $9.72 * 10^{-4}$ & $6.84 * 10^{-4}$ & $2.70^{*} 10^{-3}$ & $1.37^{*} 10^{-3}$ & 1 & 1 & 20 & 1 \\
\hline 6 & $3.60^{*} 10^{-2}$ & $4.32 * 10^{-4}$ & $2.16^{*} 10^{-4}$ & $2.73^{*} 10^{-2}$ & $1.33^{*} 10^{-3}$ & 1 & 1 & 20 & 1 \\
\hline 7 & $3.60 * 10^{-2}$ & 0 & $2.88 * 10^{-4}$ & $2.89^{*} 10^{-2}$ & $1.69 * 10^{-3}$ & 0 & 0 & 17 & 1 \\
\hline
\end{tabular}

Table S22 Concentration Data and Anomeric Ratios for the Catalyzed Reaction of 1c with 1,2:3,4-Di-O-isopropylidene- $\alpha$-D-galactopyranose 2a Using $2.5 \mathrm{~mol} \%$ of $4 \mathbf{i}\left(2^{\text {nd }} R\right.$ Run).

\begin{tabular}{|c|c|c|c|c|c|c|c|c|c|}
\hline \multirow{2}{*}{$\begin{array}{c}\text { Time } \\
{[\mathrm{h}]}\end{array}$} & \multirow{2}{*}{$\begin{array}{c}\text { Std. } \\
{[\mathrm{mol} / \mathrm{L}]}\end{array}$} & \multicolumn{2}{|c|}{ Donor 1c } & \multicolumn{2}{|c|}{ Product 3a } & \multicolumn{2}{|c|}{ Donor 1c } & \multicolumn{2}{|c|}{ Product 3a } \\
\hline & & $\begin{array}{c}\alpha \\
{[\mathrm{mol} / \mathrm{L}]}\end{array}$ & $\begin{array}{c}\beta \\
{[\mathrm{mol} / \mathrm{L}]}\end{array}$ & $\begin{array}{c}\beta \\
{[\mathrm{mol} / \mathrm{L}]}\end{array}$ & $\begin{array}{c}\alpha \\
{[\mathrm{mol} / \mathrm{L}]}\end{array}$ & $\alpha$ & $\beta$ & $\beta$ & $\alpha$ \\
\hline 0 & $3.60 * 10^{-2}$ & $3.39^{*} 10^{-2}$ & 0 & 0 & 0 & 1 & 0 & 0 & 0 \\
\hline 1 & $3.60 * 10^{-2}$ & $2.30 * 10^{-2}$ & $1.19^{*} 10^{-4}$ & $8.21^{*} 10^{-3}$ & $2.16^{*} 10^{-4}$ & 38 & 1 & 38 & 1 \\
\hline 2 & $3.60 * 10^{-2}$ & $1.45^{\star} 10^{-2}$ & $8.64^{*} 10^{-4}$ & $1.46^{*} 10^{-2}$ & $3.24 * 10^{-4}$ & 17 & 1 & 45 & 1 \\
\hline 3 & $3.60^{*} 10^{-2}$ & $4.14^{*} 10^{-3}$ & $1.15^{*} 10^{-3}$ & $2.42^{*} 10^{-2}$ & $1.04 * 10^{-3}$ & 4 & 1 & 23 & 1 \\
\hline 4 & $3.60 * 10^{-2}$ & $2.56^{*} 10^{-3}$ & $7.92^{*} 10^{-4}$ & $2.49^{*} 10^{-2}$ & $1.01^{*} 10^{-3}$ & 3 & 1 & 24 & 1 \\
\hline 5 & $3.60 * 10^{-2}$ & $2.16^{*} 10^{-3}$ & $7.92^{*} 10^{-4}$ & $2.70^{*} 10^{-2}$ & $1.19^{*} 10^{-3}$ & 3 & 1 & 23 & 1 \\
\hline 6 & $3.60 * 10^{-2}$ & $1.01 * 10^{-3}$ & $6.12^{*} 10^{-4}$ & $2.82^{*} 10^{-2}$ & $1.33^{*} 10^{-3}$ & 2 & 1 & 21 & 1 \\
\hline 7 & $3.60^{*} 10^{-2}$ & $3.24^{*} 10^{-4}$ & $3.60^{*} 10^{-4}$ & $2.84^{*} 10^{-2}$ & $1.62^{*} 10^{-3}$ & 1 & 1 & 18 & 1 \\
\hline \multicolumn{10}{|c|}{$\begin{array}{l}\text { Table } \mathbf{S 2 3} \text { Concentration Data and Anomeric Ratios for the Catalyzed Reaction of } 1 \mathbf{c} \text { with 1,2:3,4-Di-O-isopropylidene- } \alpha \text {-D-galactopyranose } \\
\text { Using } 2.5 \text { mol\% of } 4 \mathbf{i} \text { (Average). }\end{array}$} \\
\hline \multirow{2}{*}{$\begin{array}{c}\text { Time } \\
{[\mathrm{h}]}\end{array}$} & Std. & \multicolumn{2}{|c|}{ Donor 1c } & \multicolumn{2}{|c|}{ Product $\mathbf{3 a}$} & \multicolumn{2}{|c|}{ Donor 1c } & \multicolumn{2}{|c|}{ Product $\mathbf{3 a}$} \\
\hline & {$[\mathrm{mol} / \mathrm{L}]$} & $\begin{array}{c}\alpha \\
{[\mathrm{mol} / \mathrm{L}]}\end{array}$ & $\begin{array}{c}\beta \\
{[\mathrm{mol} / \mathrm{L}]}\end{array}$ & $\begin{array}{c}\beta \\
{[\mathrm{mol} / \mathrm{L}]}\end{array}$ & $\begin{array}{c}\alpha \\
{[\mathrm{mol} / \mathrm{L}]}\end{array}$ & $\alpha$ & $\beta$ & $\beta$ & $\alpha$ \\
\hline 0 & $3.60 * 10^{-2}$ & $3.20^{*} 10^{-2}$ & 0 & 0 & 0 & 1 & 0 & 0 & 0 \\
\hline 1 & $3.60 * 10^{-2}$ & $2.22 * 10^{-2}$ & $7.20^{*} 10^{-4}$ & $8.06^{*} 10^{-3}$ & $2.16^{*} 10^{-4}$ & 28 & 1 & 38 & 1 \\
\hline 2 & $3.60^{*} 10^{-2}$ & $1.36^{*} 10^{-2}$ & $9.18^{*} 10^{-4}$ & $1.50^{*} 10^{-2}$ & $3.78^{*} 10^{-4}$ & 15 & 1 & 40 & 1 \\
\hline 3 & $3.60 * 10^{-2}$ & $5.71^{*} 10^{-3}$ & $1.22^{*} 10^{-3}$ & $2.30^{*} 10^{-2}$ & $9.18^{*} 10^{-4}$ & 5 & 1 & 26 & 1 \\
\hline 4 & $3.60 * 10^{-2}$ & $2.83^{*} 10^{-3}$ & $9.18^{*} 10^{-4}$ & $2.52^{*} 10^{-2}$ & $9.90^{*} 10^{-4}$ & 3 & 1 & 25 & 1 \\
\hline 5 & $3.60 * 10^{-2}$ & $1.57^{*} 10^{-3}$ & $7.38^{*} 10^{-4}$ & $2.70^{*} 10^{-2}$ & $1.28 * 10^{-3}$ & 2 & 1 & 22 & 1 \\
\hline 6 & $3.60 * 10^{-2}$ & $7.20^{*} 10^{-4}$ & $4.14^{*} 10^{-4}$ & $2.77^{*} 10^{-2}$ & $1.33^{*} 10^{-3}$ & 2 & 1 & 21 & 1 \\
\hline 7 & $3.60 * 10^{-2}$ & $1.62 * 10^{-4}$ & $3.24 * 10^{-4}$ & $2.86 * 10^{-2}$ & $1.66^{*} 10^{-3}$ & 1 & 1 & 18 & 1 \\
\hline
\end{tabular}




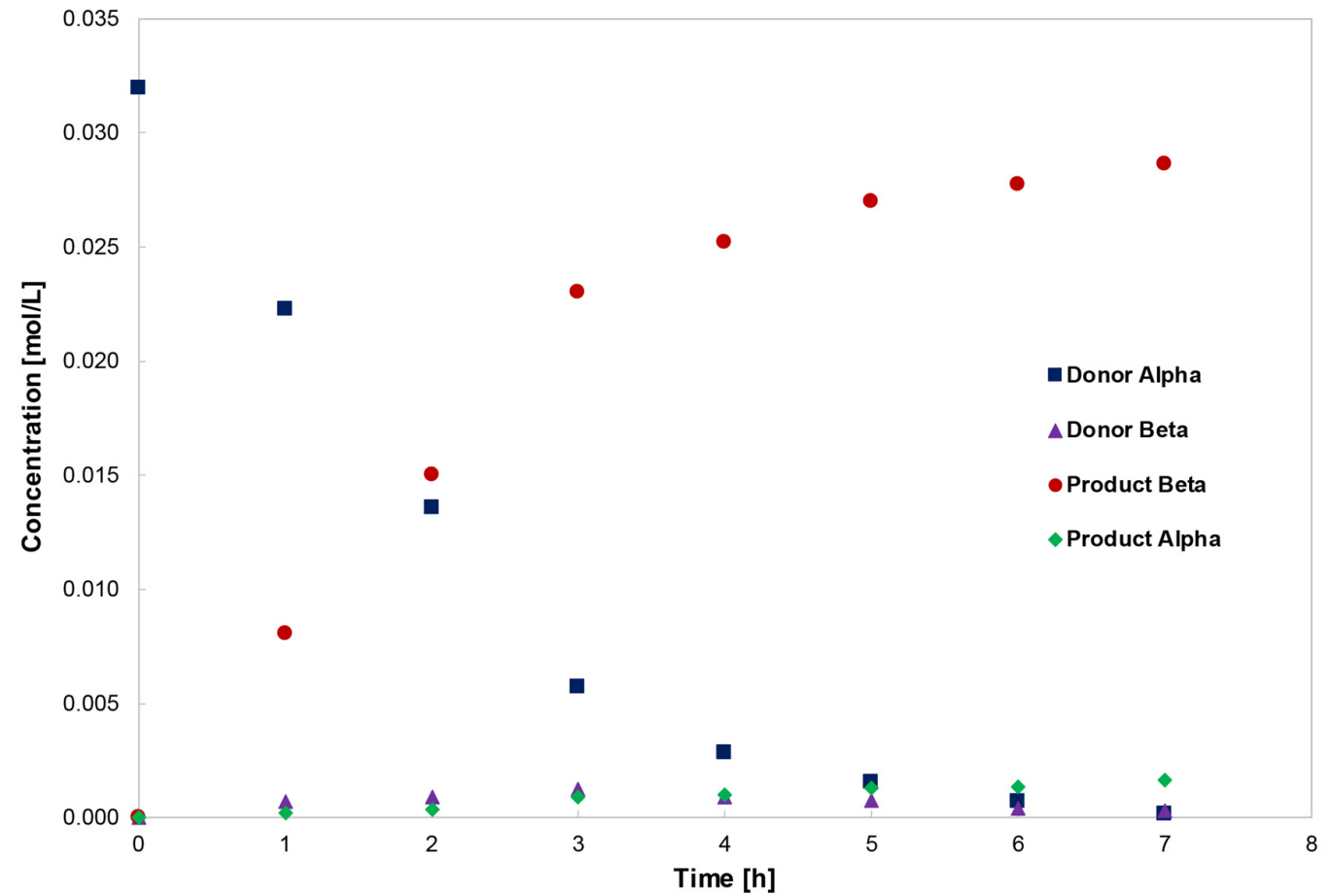

Figure S9 Averaged Kinetic Trace for the Reaction of 1c with 1,2:3,4-Di-O-isopropylidene- $\alpha$-D-galactopyranose 2a Using 2.5 mol\% of $4 \mathbf{i}$ (Blue Squares: $\alpha-1 \mathrm{c}$; Purple Triangles: $\beta-1 \mathrm{c}$; Red Dots: $\beta-3 a$; Green Diamonds: $\alpha-3 a)$.

Table S24 Concentration Data and Anomeric Ratios for the Catalyzed Reaction of 1c with N-Boc-L-threonine Methyl Ester $\mathbf{2 b}$ Using 2.5 mol\% of $\mathbf{4 i}$ (1 $1^{\text {st }}$ Run).

\begin{tabular}{cccccccccc}
\hline $\begin{array}{c}\text { Time } \\
{[\mathrm{h}]}\end{array}$ & Std. & \multicolumn{2}{c}{ Donor 1c } & \multicolumn{2}{c}{ Product 3b } & \multicolumn{2}{c}{ Donor 1c } & \multicolumn{2}{c}{ Product 3b } \\
& {$[\mathrm{mol} / \mathrm{L}]$} & $\begin{array}{c}\alpha \\
{[\mathrm{mol} / \mathrm{L}]}\end{array}$ & $\begin{array}{c}\beta \\
{[\mathrm{mol} / \mathrm{L}]}\end{array}$ & $\begin{array}{c}\beta \\
{[\mathrm{mol} / \mathrm{L}]}\end{array}$ & $\begin{array}{c}\alpha \\
{[\mathrm{mol} / \mathrm{L}]}\end{array}$ & $\alpha$ & $\beta$ & $\beta$ & $\alpha$ \\
\hline 0 & $3.60^{*} 10^{-2}$ & $3.07^{*} 10^{-2}$ & 0 & 0 & 0 & 1 & 0 & 0 & 0 \\
1 & $3.60^{*} 10^{-2}$ & $2.97^{*} 10^{-2}$ & $1.12^{*} 10^{-3}$ & $1.04^{*} 10^{-3}$ & 0 & 26 & 1 & 1 & 0 \\
2 & $3.60^{*} 10^{-2}$ & $2.53^{*} 10^{-2}$ & $1.44^{*} 10^{-3}$ & $1.80^{*} 10^{-3}$ & $3.60^{*} 10^{-5}$ & 19 & 1 & 37 & 1 \\
3 & $3.60^{*} 10^{-2}$ & $2.61^{*} 10^{-2}$ & $1.98^{*} 10^{-3}$ & $2.84^{*} 10^{-3}$ & $1.08^{*} 10^{-4}$ & 13 & 1 & 29 & 1 \\
4 & $3.60^{*} 10^{-2}$ & $2.43^{*} 10^{-2}$ & $2.41^{*} 10^{-3}$ & $3.53^{*} 10^{-3}$ & $1.44^{*} 10^{-4}$ & 10 & 1 & 25 & 1 \\
5 & $3.60^{*} 10^{-2}$ & $2.28^{*} 10^{-2}$ & $3.06^{*} 10^{-3}$ & $4.43^{*} 10^{-3}$ & $1.80^{*} 10^{-4}$ & 7 & 1 & 23 & 1 \\
6 & $3.60^{*} 10^{-2}$ & $2.14^{*} 10^{-2}$ & $3.42^{*} 10^{-3}$ & $5.72^{*} 10^{-3}$ & $3.96^{*} 10^{-4}$ & 6 & 1 & 15 & 1 \\
7 & $3.60^{*} 10^{-2}$ & $1.99^{*} 10^{-2}$ & $3.42^{*} 10^{-3}$ & $5.87^{*} 10^{-3}$ & $3.60^{*} 10^{-4}$ & 6 & 1 & 16 & 1 \\
8 & $3.60^{*} 10^{-2}$ & $1.84^{*} 10^{-2}$ & $3.92^{*} 10^{-3}$ & $6.73^{*} 10^{-3}$ & $4.32^{*} 10^{-4}$ & 5 & 1 & 16 & 1 \\
9 & $3.60^{*} 10^{-2}$ & $1.78^{*} 10^{-2}$ & $4.10^{*} 10^{-3}$ & $7.34^{*} 10^{-3}$ & $5.40^{*} 10^{-4}$ & 4 & 1 & 13 & 1 \\
10 & $3.60^{*} 10^{-2}$ & $1.72^{*} 10^{-2}$ & $4.21^{*} 10^{-3}$ & $7.99^{*} 10^{-3}$ & $4.32^{*} 10^{-4}$ & 4 & 1 & 19 & 1 \\
24 & $3.60^{*} 10^{-2}$ & $8.21^{*} 10^{-3}$ & $2.81^{*} 10^{-3}$ & $1.53^{*} 10^{-2}$ & $7.56^{*} 10^{-4}$ & 3 & 1 & 20 & 1 \\
\hline
\end{tabular}

Table S25 Concentration Data and Anomeric Ratios for the Catalyzed Reaction of 1c with N-Boc-L-threonine Methyl Ester $2 \mathrm{~b}$ Using 2.5 mol\% of $4 \mathrm{i}$ (2 $2^{\text {nd }}$ Run).

\begin{tabular}{|c|c|c|c|c|c|c|c|c|c|}
\hline \multirow{2}{*}{$\begin{array}{c}\text { Time } \\
{[\mathrm{h}]}\end{array}$} & \multirow{2}{*}{$\begin{array}{c}\text { Std. } \\
{[\mathrm{mol} / \mathrm{L}]}\end{array}$} & \multicolumn{2}{|c|}{ Donor 1c } & \multicolumn{2}{|c|}{ Product $\mathbf{3 b}$} & \multicolumn{2}{|c|}{ Donor 1c } & \multicolumn{2}{|c|}{ Product 3b } \\
\hline & & $\begin{array}{c}\alpha \\
{[\mathrm{mol} / \mathrm{L}]}\end{array}$ & $\begin{array}{c}\beta \\
{[\mathrm{mol} / \mathrm{L}]}\end{array}$ & $\begin{array}{c}\beta \\
{[\mathrm{mol} / \mathrm{L}]}\end{array}$ & $\begin{array}{c}\alpha \\
{[\mathrm{mol} / \mathrm{L}]}\end{array}$ & $\alpha$ & $\beta$ & $\beta$ & $\alpha$ \\
\hline 0 & $3.60 * 10^{-2}$ & $3.22 * 10^{-2}$ & 0 & 0 & 0 & $\overline{1}$ & 0 & $\overline{0}$ & 0 \\
\hline 1 & $3.60 * 10^{-2}$ & $2.94^{*} 10^{-2}$ & $1.12^{*} 10^{-3}$ & $9.72^{*} 10^{-4}$ & 0 & 26 & 1 & 1 & 0 \\
\hline 2 & $3.60 * 10^{-2}$ & $2.66^{*} 10^{-2}$ & $1.55^{*} 10^{-3}$ & $1.84^{*} 10^{-3}$ & $3.60 * 10^{-5}$ & 17 & 1 & 26 & 1 \\
\hline 3 & $3.60^{*} 10^{-2}$ & $2.48^{*} 10^{-2}$ & $1.84^{*} 10^{-3}$ & $2.92^{*} 10^{-3}$ & $1.44^{*} 10^{-4}$ & 14 & 1 & 17 & 1 \\
\hline 4 & $3.60 * 10^{-2}$ & $2.24^{*} 10^{-2}$ & $2.38^{*} 10^{-3}$ & $3.71 * 10^{-3}$ & $1.80 * 10^{-4}$ & 9 & 1 & 14 & 1 \\
\hline 5 & $3.60 * 10^{-2}$ & $2.28 * 10^{-2}$ & $2.63^{*} 10^{-3}$ & $4.61 * 10^{-3}$ & $1.08 * 10^{-4}$ & 9 & 1 & 9 & 1 \\
\hline 6 & $3.60 * 10^{-2}$ & $2.11^{*} 10^{-2}$ & $3.24^{*} 10^{-3}$ & $5.08^{*} 10^{-3}$ & $1.08 * 10^{-4}$ & 6 & 1 & 9 & 1 \\
\hline 7 & $3.60 * 10^{-2}$ & $1.81 * 10^{-2}$ & $3.35^{*} 10^{-3}$ & $6.19^{*} 10^{-3}$ & $2.88^{*} 10^{-4}$ & 5 & 1 & 6 & 1 \\
\hline 8 & $3.60^{*} 10^{-2}$ & $1.84^{*} 10^{-2}$ & $4.18^{*} 10^{-3}$ & $7.06^{*} 10^{-3}$ & $1.80 * 10^{-4}$ & 4 & 1 & 5 & 1 \\
\hline 9 & $3.60 * 10^{-2}$ & $1.72 * 10^{-2}$ & $4.28^{*} 10^{-3}$ & $8.39 * 10^{-3}$ & $3.24 * 10^{-4}$ & 4 & 1 & 4 & 1 \\
\hline 10 & $3.60^{*} 10^{-2}$ & $1.56^{*} 10^{-2}$ & $4.21^{*} 10^{-3}$ & $8.17^{*} 10^{-3}$ & $4.68 * 10^{-4}$ & 4 & 1 & 4 & 1 \\
\hline 24 & $3.60^{*} 10^{-2}$ & $8.53^{*} 10^{-3}$ & $3.10^{*} 10^{-3}$ & $1.66^{*} 10^{-2}$ & $7.92 * 10^{-4}$ & 3 & 1 & 3 & 1 \\
\hline
\end{tabular}


Table S26 Concentration Data and Anomeric Ratios for the Catalyzed Reaction of 1c with N-Boc-L-threonine Methyl Ester $\mathbf{2 b}$ Using 2.5 mol\% of $\mathbf{4 i}$ (Average).

\begin{tabular}{|c|c|c|c|c|c|c|c|c|c|}
\hline \multirow{2}{*}{$\begin{array}{c}\text { Time } \\
{[\mathrm{h}]}\end{array}$} & \multirow{2}{*}{$\begin{array}{c}\text { Std. } \\
{[\mathrm{mol} / \mathrm{L}]}\end{array}$} & \multicolumn{2}{|c|}{ Donor 1c } & \multicolumn{2}{|c|}{ Product 3b } & \multicolumn{2}{|c|}{ Donor 1c } & \multicolumn{2}{|c|}{ Product $\mathbf{3 b}$} \\
\hline & & $\begin{array}{c}\alpha \\
{[\mathrm{mol} / \mathrm{L}]}\end{array}$ & $\begin{array}{c}\beta \\
{[\mathrm{mol} / \mathrm{L}]}\end{array}$ & $\begin{array}{c}\beta \\
{[\mathrm{mol} / \mathrm{L}]}\end{array}$ & $\begin{array}{c}\alpha \\
{[\mathrm{mol} / \mathrm{L}]}\end{array}$ & $\alpha$ & $\beta$ & $\beta$ & $\alpha$ \\
\hline 0 & $3.60^{*} 10^{-2}$ & $3.14^{*} 10^{-2}$ & 0 & 0 & 0 & 1 & 0 & 0 & 0 \\
\hline 1 & $3.60 * 10^{-2}$ & $2.96^{*} 10^{-2}$ & $1.12^{*} 10^{-3}$ & $1.01^{*} 10^{-3}$ & 0 & 26 & 1 & 1 & 0 \\
\hline 2 & $3.60^{*} 10^{-2}$ & $2.60^{*} 10^{-2}$ & $1.49 * 10^{-3}$ & $1.82^{*} 10^{-3}$ & $3.60 * 10^{-5}$ & 18 & 1 & 36 & 1 \\
\hline 3 & $3.60^{*} 10^{-2}$ & $2.55^{*} 10^{-2}$ & $1.91 * 10^{-3}$ & $2.88^{*} 10^{-3}$ & $1.26 * 10^{-4}$ & 14 & 1 & 24 & 1 \\
\hline 4 & $3.60 * 10^{-2}$ & $2.33^{*} 10^{-2}$ & $2.39 * 10^{-3}$ & $3.62^{*} 10^{-3}$ & $1.62 * 10^{-4}$ & 10 & 1 & 22 & 1 \\
\hline 5 & $3.60 * 10^{-2}$ & $2.28^{*} 10^{-2}$ & $2.84^{*} 10^{-3}$ & $4.52^{*} 10^{-3}$ & $1.44^{*} 10^{-4}$ & 8 & 1 & 32 & 1 \\
\hline 6 & $3.60^{*} 10^{-2}$ & $2.13^{*} 10^{-2}$ & $3.33^{*} 10^{-3}$ & $5.40^{*} 10^{-3}$ & $2.52^{*} 10^{-4}$ & 6 & 1 & 25 & 1 \\
\hline 7 & $3.60^{*} 10^{-2}$ & $1.90^{*} 10^{-2}$ & $3.38^{*} 10^{-3}$ & $6.03^{*} 10^{-3}$ & $3.24 * 10^{-4}$ & 6 & 1 & 19 & 1 \\
\hline 8 & $3.60 * 10^{-2}$ & $1.84^{*} 10^{-2}$ & $4.05^{\star} 10^{-3}$ & $6.89 * 10^{-3}$ & $3.06^{*} 10^{-4}$ & 5 & 1 & 28 & 1 \\
\hline 9 & $3.60^{*} 10^{-2}$ & $1.75^{*} 10^{-2}$ & $4.19^{*} 10^{-3}$ & $7.87^{*} 10^{-3}$ & $4.32 * 10^{-4}$ & 4 & 1 & 20 & 1 \\
\hline 10 & $3.60^{*} 10^{-2}$ & $1.64^{*} 10^{-2}$ & $4.21 * 10^{-3}$ & $8.08^{*} 10^{-3}$ & $4.50 * 10^{-4}$ & 4 & 1 & 19 & 1 \\
\hline 24 & $3.60 * 10^{-2}$ & $8.73^{*} 10^{-3}$ & $2.95^{*} 10^{-3}$ & $1.59^{*} 10^{-2}$ & $7.74 * 10^{-4}$ & 3 & 1 & 21 & 1 \\
\hline
\end{tabular}

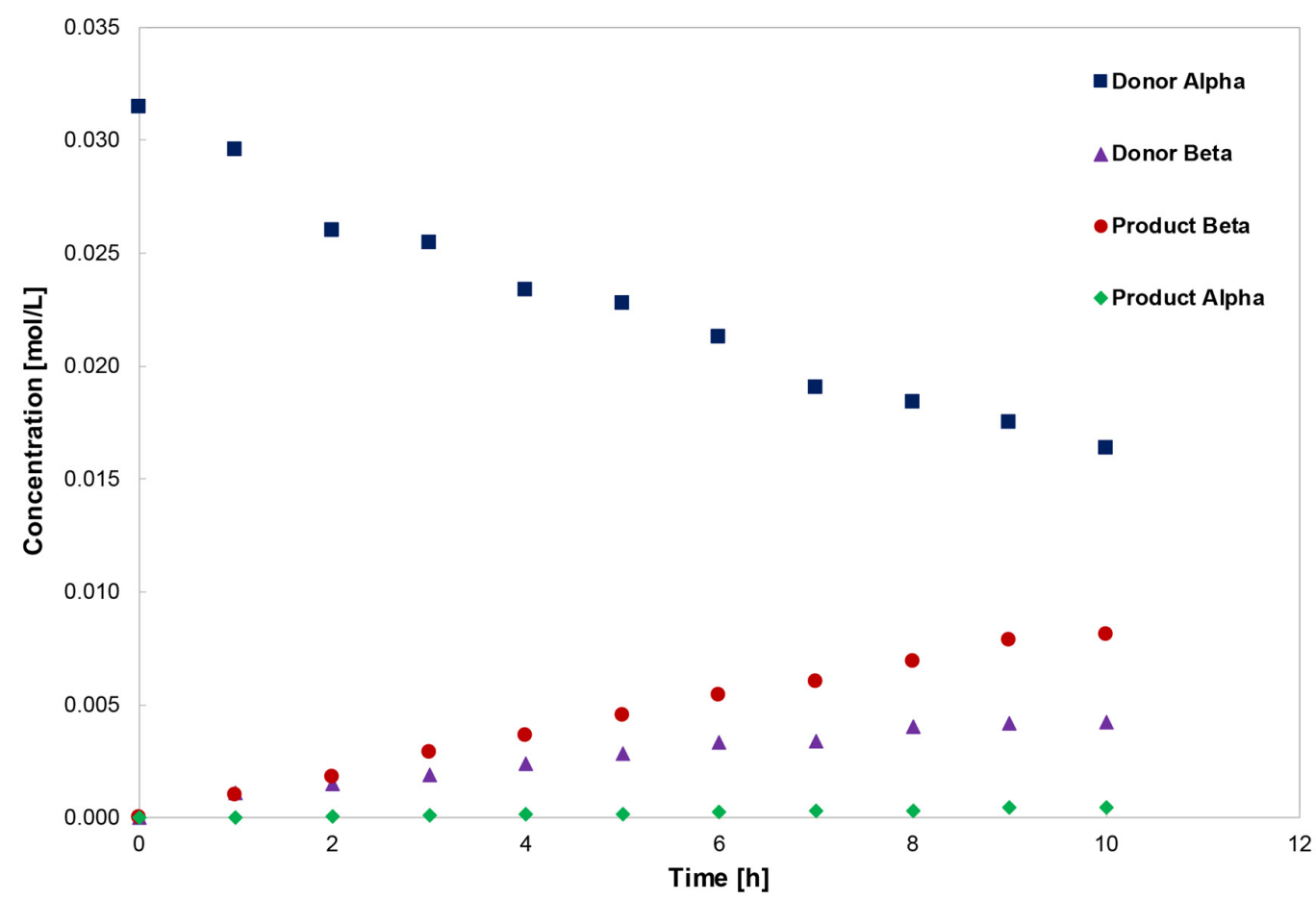

Figure S10 Averaged Kinetic Trace for the Reaction of 1c with N-Boc-L-threonine Methyl Ester 2I Using 2.5 mol\% of 4i (Blue Squares: $\alpha-1 c$; Purple Triangles: $\beta$-1c; Red Dots: $\beta$-3b; Green Diamonds: $\alpha-3 \mathbf{b})$. 


\section{Donor Loading Effect}

According to General Procedure E, the experiment was performed using 1c $(5.1 \mathrm{mg}$, $0.009 \mathrm{mmol}, 0.5$ eq.), catalyst $4 \mathbf{i}(0.94 \mathrm{mg}, 0.00045 \mathrm{mmol}, 0.025$ eq.), and 1,2:3,4-diO-isopropylidene- $\alpha$-D-galactopyranose $\mathbf{2 a}(9.4 \mathrm{mg}, 0.036 \mathrm{mmol}, 2.0 \mathrm{eq}$.) or $\mathrm{N}$-Boc-Lthreonine methyl ester $\mathbf{2 b}(8.4 \mathrm{mg}, 0.036 \mathrm{mmol}, 2.0$ eq.). The reaction was monitored over $5 \mathrm{~h}$ for 1,2:3,4-di-O-isopropylidene- $\alpha$-D-galactopyranose $2 \mathrm{a}$ and $10 \mathrm{~h}$ for $\mathrm{N}$-BocL-threonine methyl ester $\mathbf{2 b}$.

\begin{tabular}{|c|c|c|c|c|c|c|c|c|c|}
\hline \multirow{2}{*}{$\begin{array}{c}\text { Time } \\
{[\mathrm{h}]}\end{array}$} & \multirow{2}{*}{$\begin{array}{c}\text { Std. } \\
{[\mathrm{mol} / \mathrm{L}]}\end{array}$} & \multicolumn{2}{|c|}{ Donor 1c } & \multicolumn{2}{|c|}{ Product 3a } & \multicolumn{2}{|c|}{ Donor 1c } & \multicolumn{2}{|c|}{ Product 3a } \\
\hline & & $\begin{array}{c}\alpha \\
{[\mathrm{mol} / \mathrm{L}]}\end{array}$ & $\begin{array}{c}\beta \\
{[\mathrm{mol} / \mathrm{L}]}\end{array}$ & $\begin{array}{c}\beta \\
{[\mathrm{mol} / \mathrm{L}]}\end{array}$ & $\begin{array}{c}\alpha \\
{[\mathrm{mol} / \mathrm{L}]}\end{array}$ & $\alpha$ & $\beta$ & $\beta$ & $\alpha$ \\
\hline 0 & $3.60 * 10^{-2}$ & $1.67^{*} 10^{-2}$ & 0 & 0 & 0 & 1 & 0 & 0 & 0 \\
\hline 1 & $3.60 * 10^{-2}$ & $3.20 * 10^{-3}$ & $7.92 * 10^{-4}$ & $1.39 * 10^{-2}$ & $5.40^{*} 10^{-4}$ & 4 & 1 & 26 & 1 \\
\hline 2 & $3.60 * 10^{-2}$ & $3.60 * 10^{-4}$ & $3.24 * 10^{-4}$ & $1.54^{*} 10^{-2}$ & $7.20^{*} 10^{-4}$ & 1 & 1 & 21 & 1 \\
\hline 3 & $3.60 * 10^{-2}$ & 0 & 0 & $1.53^{*} 10^{-2}$ & $9.00 * 10^{-4}$ & 0 & 0 & 17 & 1 \\
\hline 4 & $3.60 * 10^{-2}$ & 0 & 0 & $1.52 * 10^{-3}$ & $7.92 * 10^{-4}$ & 0 & 0 & 19 & 1 \\
\hline 5 & $3.60 * 10^{-2}$ & 0 & 0 & $1.51 * 10^{-2}$ & $9.00^{*} 10^{-3}$ & 0 & 0 & 17 & 1 \\
\hline \multicolumn{10}{|c|}{$\begin{array}{l}\text { Table } S 28 \text { Concentration Data and Anomeric Ratios for the Catalyzed Reaction of } 0.5 \text { eq. 1c with } 1,2: 3,4-D i-O-i s o p r o p y l i d e n e-\alpha-D-g a l a c t o p y r a n o s \\
2 a\left(2^{\text {nd }} \text { Run). }\right.\end{array}$} \\
\hline Time & Std. & \multicolumn{2}{|c|}{ Donor 1c } & \multicolumn{2}{|c|}{ Product 3a } & \multicolumn{2}{|c|}{ Donor 1c } & \multicolumn{2}{|c|}{ Product 3a } \\
\hline [h] & {$[\mathrm{mol} / \mathrm{L}]$} & $\begin{array}{c}\alpha \\
{[\mathrm{mol} / \mathrm{L}]}\end{array}$ & $\begin{array}{c}\beta \\
{[\mathrm{mol} / \mathrm{L}]}\end{array}$ & $\begin{array}{c}\beta \\
{[\mathrm{mol} / \mathrm{L}]}\end{array}$ & $\begin{array}{c}\alpha \\
{[\mathrm{mol} / \mathrm{L}]}\end{array}$ & $\alpha$ & $\beta$ & $\beta$ & $\alpha$ \\
\hline 0 & $3.60 * 10^{-2}$ & $1.38^{*} 10^{-2}$ & 0 & 0 & 0 & 1 & 0 & 0 & 0 \\
\hline 1 & $3.60 * 10^{-2}$ & $2.81^{*} 10^{-3}$ & $5.76^{*} 10^{-4}$ & $1.25^{*} 10^{-2}$ & $5.04^{*} 10^{-4}$ & 5 & 1 & 25 & 1 \\
\hline 2 & $3.60 * 10^{-2}$ & $3.24^{*} 10^{-4}$ & $2.16^{*} 10^{-4}$ & $1.50 * 10^{-2}$ & $7.56^{*} 10^{-4}$ & 2 & 1 & 20 & 1 \\
\hline 3 & $3.60 * 10^{-2}$ & 0 & 0 & $1.60 * 10^{-2}$ & $6.84^{*} 10^{-4}$ & 0 & 0 & 24 & 1 \\
\hline 4 & $3.60 * 10^{-2}$ & 0 & 0 & $1.50 * 10^{-2}$ & $7.56^{*} 10^{-4}$ & 0 & 0 & 20 & 1 \\
\hline 5 & $3.60 * 10^{-2}$ & 0 & 0 & $1.52 * 10^{-2}$ & $7.92 * 10^{-4}$ & 0 & 0 & 18 & 1 \\
\hline \multicolumn{10}{|c|}{$\begin{array}{l}\text { Table S29 Concentration Data and Anomeric Ratios for the Catalyzed Reaction of } 0.5 \text { eq. 1c with 1,2:3,4-Di-O-isopropylidene- } \alpha \text {-D-galactopyrano } \\
\text { 2a (Average). }\end{array}$} \\
\hline Time & Std. & \multicolumn{2}{|c|}{ Donor 1c } & \multicolumn{2}{|c|}{ Product 3a } & \multicolumn{2}{|c|}{ Donor 1c } & \multicolumn{2}{|c|}{ Product 3a } \\
\hline [h] & {$[\mathrm{mol} / \mathrm{L}]$} & $\begin{array}{c}\alpha \\
{[\mathrm{mol} / \mathrm{L}]}\end{array}$ & $\begin{array}{c}\beta \\
{[\mathrm{mol} / \mathrm{L}]}\end{array}$ & $\begin{array}{c}\beta \\
{[\mathrm{mol} / \mathrm{L}]}\end{array}$ & $\begin{array}{c}\alpha \\
{[\mathrm{mol} / \mathrm{L}]}\end{array}$ & $\alpha$ & $\beta$ & $\beta$ & $\alpha$ \\
\hline 0 & $3.60 * 10^{-2}$ & $1.53^{*} 10^{-2}$ & 0 & 0 & 0 & 1 & 0 & 0 & 0 \\
\hline 1 & $3.60 * 10^{-2}$ & $3.01 * 10^{-3}$ & $6.84 * 10^{-4}$ & $1.32 * 10^{-2}$ & $5.22 * 10^{-4}$ & 5 & 1 & 26 & 1 \\
\hline 2 & $3.60 * 10^{-2}$ & $3.42^{*} 10^{-4}$ & $2.70^{*} 10^{-4}$ & $1.52^{*} 10^{-2}$ & $7.38^{*} 10^{-4}$ & 2 & 1 & 21 & 1 \\
\hline 3 & $3.60 * 10^{-2}$ & 0 & 0 & $1.57^{*} 10^{-2}$ & $7.92 * 10^{-4}$ & 0 & 0 & 21 & 1 \\
\hline 4 & $3.60 * 10^{-2}$ & 0 & 0 & $1.51 * 10^{-2}$ & $7.74^{*} 10^{-4}$ & 0 & 0 & 20 & 1 \\
\hline 5 & $3.60 * 10^{-2}$ & 0 & 0 & $1.52 * 10^{-2}$ & $8.46^{*} 10^{-3}$ & 0 & 0 & 18 & 1 \\
\hline
\end{tabular}




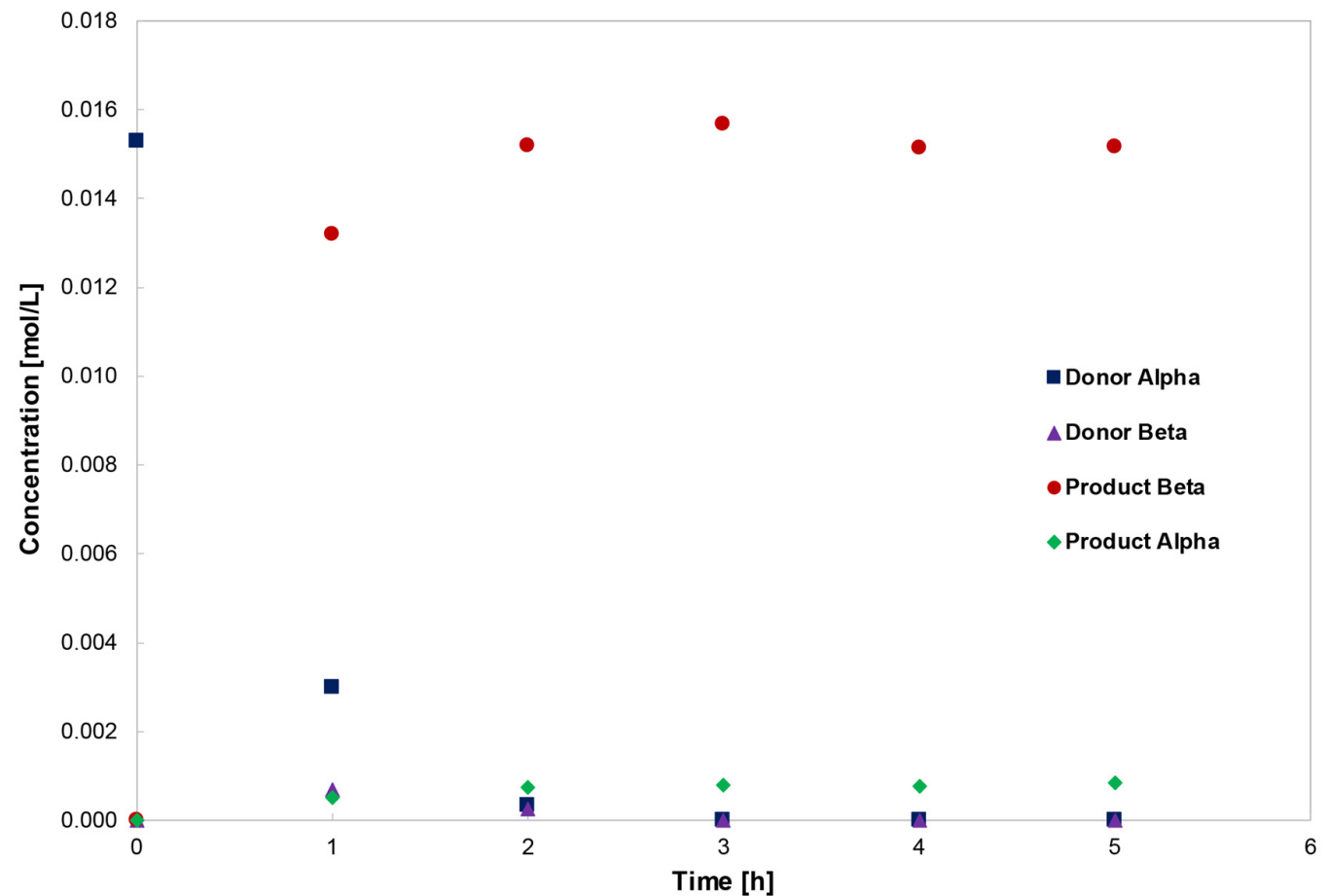

Figure S11 Averaged Kinetic Trace for the Reaction of 0.5 eq. 1c with 1,2:3,4-Di-O-isopropylidene- $\alpha$-D-galactopyranose 2a (Blue Squares: $\alpha$-1c Purple Triangles: $\beta-1 c$; Red Dots: $\beta-3 a$; Green Diamonds: $\alpha-3 a)$.

\begin{tabular}{|c|c|c|c|c|c|c|c|c|c|}
\hline \multirow{2}{*}{$\begin{array}{l}\text { Time } \\
\text { [min] }\end{array}$} & \multirow{2}{*}{$\begin{array}{c}\text { Std. } \\
{[\mathrm{mol} / \mathrm{L}]}\end{array}$} & \multicolumn{2}{|c|}{ Donor 1c } & \multicolumn{2}{|c|}{ Product $3 \mathbf{a}$} & \multicolumn{2}{|c|}{ Donor 1c } & \multicolumn{2}{|c|}{ Product 3a } \\
\hline & & $\begin{array}{c}\alpha \\
{[\mathrm{mol} / \mathrm{L}]}\end{array}$ & $\begin{array}{c}\beta \\
{[\mathrm{mol} / \mathrm{L}]}\end{array}$ & $\begin{array}{c}\beta \\
{[\mathrm{mol} / \mathrm{L}]}\end{array}$ & $\begin{array}{c}\alpha \\
{[\mathrm{mol} / \mathrm{L}]}\end{array}$ & $\alpha$ & $\beta$ & $\beta$ & $\alpha$ \\
\hline 0 & $3.60^{*} 10^{-2}$ & $1.47^{*} 10^{-2}$ & 0 & $9.36^{*} 10^{-4}$ & 0 & 1 & 0 & 1 & 0 \\
\hline 15 & $3.60 * 10^{-2}$ & $9.61^{*} 10^{-3}$ & $3.60 * 10^{-4}$ & $5.47^{*} 10^{-3}$ & $1.08 * 10^{-4}$ & 27 & 1 & 58 & 1 \\
\hline 30 & $3.60 * 10^{-2}$ & $7.56^{*} 10^{-3}$ & $5.04^{*} 10^{-4}$ & $8.32 * 10^{-3}$ & $2.88 * 10^{-4}$ & 16 & 1 & 30 & 1 \\
\hline 45 & $3.60^{*} 10^{-2}$ & $3.78^{*} 10^{-3}$ & $5.40^{*} 10^{-4}$ & $1.15^{*} 10^{-2}$ & $4.32 * 10^{-4}$ & 7 & 1 & 28 & 1 \\
\hline 60 & $3.60 * 10^{-2}$ & $2.05^{*} 10^{-3}$ & $4.32 * 10^{-4}$ & $1.29 * 10^{-2}$ & $5.40 * 10^{-3}$ & 5 & 1 & 24 & 1 \\
\hline
\end{tabular}

Table S31 Concentrations and Anomeric Ratios for the Catalyzed Reaction of 0.5 eq. 1c with 1,2:3,4-Di-O-isopropylidene- $\alpha$-D-galactopyranose 2a ( $2^{\text {nd }}$ Run).

\begin{tabular}{|c|c|c|c|c|c|c|c|c|c|}
\hline \multirow{2}{*}{$\begin{array}{l}\text { Time } \\
\text { [min] }\end{array}$} & \multirow{2}{*}{$\begin{array}{c}\text { Std. } \\
{[\mathrm{mol} / \mathrm{L}]}\end{array}$} & \multicolumn{2}{|c|}{ Donor 1c } & \multicolumn{2}{|c|}{ Product 3a } & \multicolumn{2}{|c|}{ Donor 1c } & \multicolumn{2}{|c|}{ Product $\mathbf{3 a}$} \\
\hline & & $\begin{array}{c}\alpha \\
{[\mathrm{mol} / \mathrm{L}]}\end{array}$ & $\begin{array}{c}\beta \\
{[\mathrm{mol} / \mathrm{L}]}\end{array}$ & $\begin{array}{c}\beta \\
{[\mathrm{mol} / \mathrm{L}]}\end{array}$ & $\begin{array}{c}\alpha \\
{[\mathrm{mol} / \mathrm{L}]}\end{array}$ & $\alpha$ & $\beta$ & $\beta$ & $\alpha$ \\
\hline 0 & $3.60^{*} 10^{-2}$ & $1.32^{*} 10^{-2}$ & 0 & $9.36^{*} 10^{-4}$ & 0 & 1 & 0 & 1 & 0 \\
\hline 15 & $3.60 * 10^{-2}$ & $1.03^{*} 10^{-2}$ & $2.88 * 10^{-4}$ & $5.47^{*} 10^{-3}$ & $1.44 * 10^{-4}$ & 34 & 1 & 35 & 1 \\
\hline 30 & $3.60 * 10^{-2}$ & $6.70^{*} 10^{-3}$ & $4.68 * 10^{-4}$ & $8.42 * 10^{-3}$ & $2.52 * 10^{-4}$ & 15 & 1 & 34 & 1 \\
\hline 45 & $3.60^{*} 10^{-2}$ & $3.74^{*} 10^{-3}$ & $4.68^{*} 10^{-4}$ & $1.12^{*} 10^{-2}$ & $3.96 * 10^{-4}$ & 8 & 1 & 30 & 1 \\
\hline 60 & $3.60 * 10^{-2}$ & $2.12^{*} 10^{-3}$ & $3.96 * 10^{-4}$ & $1.28 * 10^{-2}$ & $5.04 * 10^{-4}$ & 5 & 1 & 26 & 1 \\
\hline
\end{tabular}

Table S32 Concentrations and Anomeric Ratios for the Catalyzed Reaction of 0.5 eq. 1c with 1,2:3,4-Di-O-isopropylidene- $\alpha$-D-galactopyranose 2a (Average).

\begin{tabular}{|c|c|c|c|c|c|c|c|c|c|}
\hline \multirow{2}{*}{$\begin{array}{l}\text { Time } \\
\text { [min] }\end{array}$} & \multirow{2}{*}{$\begin{array}{c}\text { Std. } \\
{[\mathrm{mol} / \mathrm{L}]}\end{array}$} & \multicolumn{2}{|c|}{ Donor 1c } & \multicolumn{2}{|c|}{ Product 3a } & \multicolumn{2}{|c|}{ Donor 1c } & \multicolumn{2}{|c|}{ Product 3a } \\
\hline & & $\begin{array}{c}\alpha \\
{[\mathrm{mol} / \mathrm{L}]}\end{array}$ & $\begin{array}{c}\beta \\
{[\mathrm{mol} / \mathrm{L}]}\end{array}$ & $\begin{array}{c}\beta \\
{[\mathrm{mol} / \mathrm{L}]}\end{array}$ & $\begin{array}{c}\alpha \\
{[\mathrm{mol} / \mathrm{L}]}\end{array}$ & $\alpha$ & $\beta$ & $\beta$ & $\alpha$ \\
\hline$\overline{0}$ & $3.60^{*} 10^{-2}$ & $1.40^{*} 10^{-2}$ & $\overline{0}$ & $9.36^{*} 10^{-4}$ & $\overline{0}$ & 1 & 0 & 0 & 0 \\
\hline 15 & $3.60 * 10^{-2}$ & $9.94^{*} 10^{-3}$ & $3.24^{*} 10^{-4}$ & $5.47^{*} 10^{-3}$ & $1.26^{*} 10^{-4}$ & 31 & 1 & 47 & 1 \\
\hline 30 & $3.60 * 10^{-2}$ & $7.13^{*} 10^{-3}$ & $4.86^{*} 10^{-4}$ & $8.37^{*} 10^{-3}$ & $2.70^{*} 10^{-4}$ & 16 & 1 & 32 & 1 \\
\hline 45 & $3.60 * 10^{-2}$ & $3.76^{*} 10^{-3}$ & $5.04^{*} 10^{-4}$ & $1.14^{*} 10^{-2}$ & $4.14^{*} 10^{-4}$ & 8 & 1 & 29 & 1 \\
\hline 60 & $3.60^{*} 10^{-2}$ & $2.09^{*} 10^{-3}$ & $4.14^{*} 10^{-4}$ & $1.29^{*} 10^{-2}$ & $5.22^{*} 10^{-4}$ & 5 & 1 & 25 & 1 \\
\hline
\end{tabular}




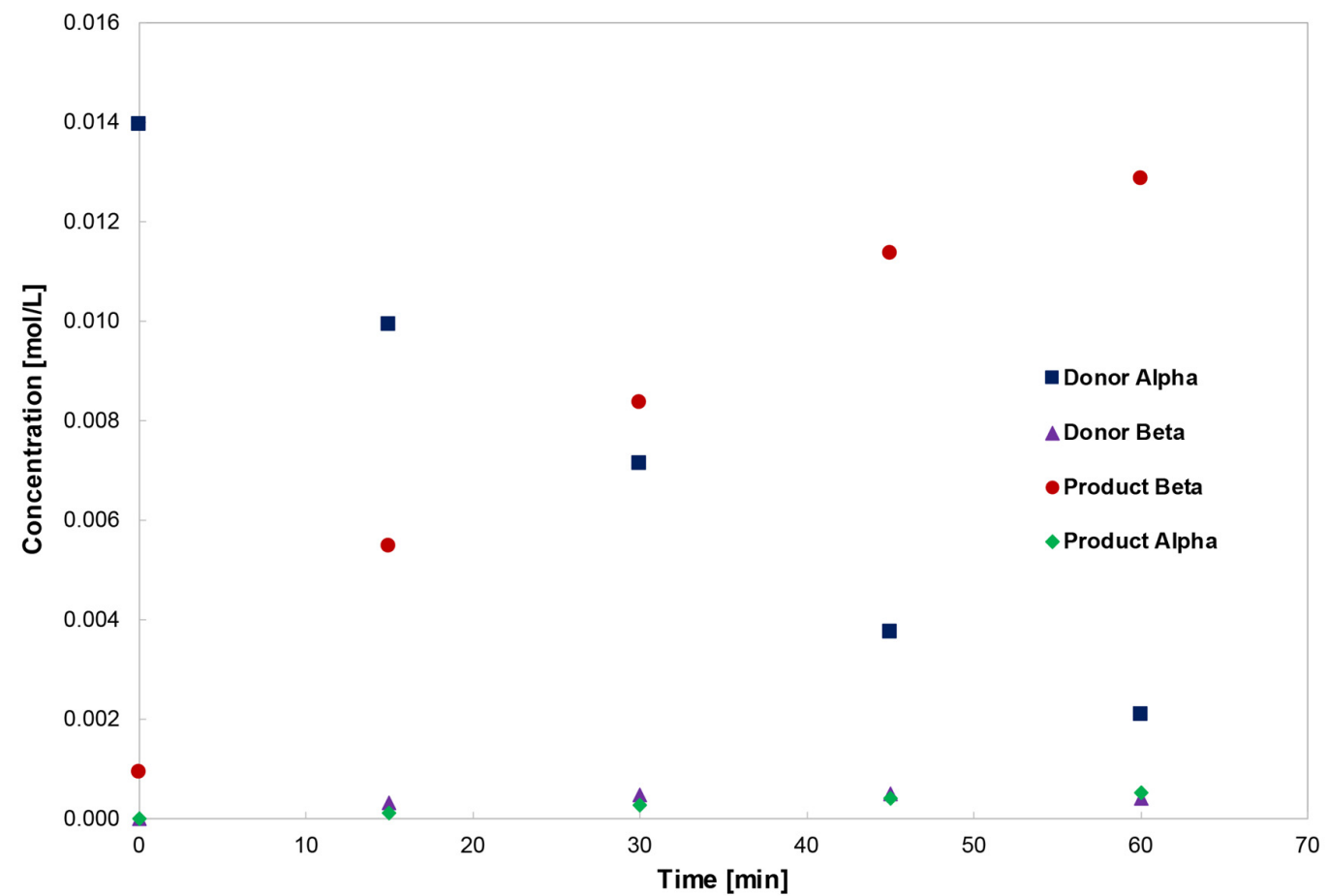

Figure S12 Substrate and Product Concentration as a Function of Time for the Reaction of 0.5 eq. 1c with 1,2:3,4-Di-O-isopropylidene- $\alpha$-Dgalactopyranose 2a (Blue Squares: $\alpha-1 \mathrm{c}$; Purple Triangles: $\beta-1 \mathrm{c}$; Red Dots: $\beta-3 a$; Green Diamonds: $\alpha-3 a)$.

\begin{tabular}{|c|c|c|c|c|c|c|c|c|c|}
\hline \multirow{2}{*}{$\begin{array}{c}\text { Time } \\
{[\mathrm{h}]}\end{array}$} & \multirow{2}{*}{$\begin{array}{c}\text { Std. } \\
{[\mathrm{mol} / \mathrm{L}]}\end{array}$} & \multicolumn{2}{|c|}{ Donor 1c } & \multicolumn{2}{|c|}{ Product 3b } & \multicolumn{2}{|c|}{ Donor 1c } & \multicolumn{2}{|c|}{ Product $\mathbf{3 b}$} \\
\hline & & $\begin{array}{c}\alpha \\
{[\mathrm{mol} / \mathrm{L}]}\end{array}$ & $\begin{array}{c}\beta \\
{[\mathrm{mol} / \mathrm{L}]}\end{array}$ & $\begin{array}{c}\beta \\
{[\mathrm{mol} / \mathrm{L}]}\end{array}$ & $\begin{array}{c}\alpha \\
{[\mathrm{mol} / \mathrm{L}]}\end{array}$ & $\alpha$ & $\beta$ & $\beta$ & $\alpha$ \\
\hline 0 & $3.60^{*} 10^{-2}$ & $1.52 * 10^{-2}$ & 0 & 0 & 0 & 1 & 0 & 0 & 0 \\
\hline 1 & $3.60^{*} 10^{-2}$ & $1.11^{*} 10^{-2}$ & $1.08^{*} 10^{-3}$ & $2.23^{*} 10^{-3}$ & $7.20^{*} 10^{-5}$ & 10 & 1 & 25 & 1 \\
\hline 2 & $3.60 * 10^{-2}$ & $9.25^{\star} 10^{-3}$ & $1.73^{*} 10^{-3}$ & $3.35^{*} 10^{-3}$ & $3.60^{*} 10^{-5}$ & 5 & 1 & 40 & 1 \\
\hline 3 & $3.60^{*} 10^{-2}$ & $8.06^{*} 10^{-3}$ & $2.12^{*} 10^{-3}$ & $5.08^{*} 10^{-3}$ & $3.60^{*} 10^{-5}$ & 4 & 1 & 40 & 1 \\
\hline 4 & $3.60 * 10^{-2}$ & $6.05^{*} 10^{-3}$ & $2.05^{*} 10^{-3}$ & $5.94^{*} 10^{-3}$ & $1.44^{*} 10^{-4}$ & 3 & 1 & 40 & 1 \\
\hline 5 & $3.60 * 10^{-2}$ & $4.32^{*} 10^{-3}$ & $1.91^{*} 10^{-3}$ & $6.80^{*} 10^{-3}$ & $1.44^{*} 10^{-4}$ & 2 & 1 & 46 & 1 \\
\hline 6 & $3.60^{*} 10^{-2}$ & $3.24^{*} 10^{-3}$ & $1.44^{*} 10^{-3}$ & $7.34^{*} 10^{-3}$ & $2.52^{*} 10^{-4}$ & 2 & 1 & 31 & 1 \\
\hline 7 & $3.60^{*} 10^{-2}$ & $2.63^{*} 10^{-3}$ & $1.22 * 10^{-3}$ & $8.39^{*} 10^{-3}$ & $3.60^{*} 10^{-4}$ & 2 & 1 & 24 & 1 \\
\hline 8 & $3.60 * 10^{-2}$ & $2.45^{\star} 10^{-3}$ & $1.04^{*} 10^{-3}$ & $9.50 * 10^{-3}$ & $2.52 * 10^{-4}$ & 2 & 1 & 34 & 1 \\
\hline 9 & $3.60 * 10^{-2}$ & $2.12^{*} 10^{-3}$ & $1.01 * 10^{-3}$ & $1.05^{*} 10^{-2}$ & $5.04 * 10^{-4}$ & 2 & 1 & 21 & 1 \\
\hline 10 & $3.60 * 10^{-2}$ & $1.66^{*} 10^{-3}$ & $7.20^{*} 10^{-4}$ & $1.09^{*} 10^{-2}$ & $3.96 * 10^{-4}$ & 2 & 1 & 27 & 1 \\
\hline \multirow{2}{*}{\multicolumn{10}{|c|}{$\begin{array}{l}\text { Table S34 Concentration Data and Anomeric Ratios for the Catalyzed Reaction of } 0.5 \text { eq. 1c with N-Boc-L-threonine Methyl Ester } 2 \mathbf{b}\left(2^{\text {nd }} \text { Run }\right. \\
\text { Time } \quad \text { Std. }\end{array}$}} \\
\hline & & & & & & & & & \\
\hline [h] & & $\begin{array}{c}\alpha \\
{[\mathrm{mol} / \mathrm{L}]}\end{array}$ & $\begin{array}{c}\beta \\
{[\mathrm{mol} / \mathrm{L}]}\end{array}$ & $\begin{array}{c}\beta \\
{[\mathrm{mol} / \mathrm{L}]}\end{array}$ & $\begin{array}{c}\alpha \\
{[\mathrm{mol} / \mathrm{L}]}\end{array}$ & $\alpha$ & $\beta$ & $\beta$ & $\alpha$ \\
\hline 0 & $3.60 * 10^{-2}$ & $1.54^{*} 10^{-2}$ & 0 & 0 & 0 & 1 & 0 & 0 & 0 \\
\hline 1 & $3.60^{*} 10^{-2}$ & $1.19^{*} 10^{-2}$ & $9.36^{*} 10^{-4}$ & $2.09^{*} 10^{-3}$ & 0 & 12 & 1 & 1 & 0 \\
\hline 2 & $3.60 * 10^{-2}$ & $9.11^{*} 10^{-3}$ & $1.55^{*} 10^{-3}$ & $3.67^{*} 10^{-3}$ & $3.60^{*} 10^{-5}$ & 6 & 1 & 71 & 1 \\
\hline 3 & $3.60 * 10^{-2}$ & $8.17^{*} 10^{-3}$ & $1.7310^{-3}$ & $4.86^{*} 10^{-3}$ & $3.60^{*} 10^{-5}$ & 5 & 1 & 50 & 1 \\
\hline 4 & $3.60 * 10^{-2}$ & $6.66^{*} 10^{-3}$ & $1.69 * 10^{-3}$ & $6.08^{*} 10^{-3}$ & $1.80^{*} 10^{-4}$ & 4 & 1 & 33 & 1 \\
\hline 5 & $3.60 * 10^{-2}$ & $5.83^{*} 10^{-3}$ & $1.73^{*} 10^{-3}$ & $7.34^{*} 10^{-3}$ & $2.88^{*} 10^{-4}$ & 3 & 1 & 25 & 1 \\
\hline 6 & $3.60 * 10^{-2}$ & $4.07^{*} 10^{-3}$ & $1.19^{*} 10^{-3}$ & $8.35^{*} 10^{-3}$ & $3.96 * 10^{-4}$ & 3 & 1 & 22 & 1 \\
\hline 7 & $3.60 * 10^{-2}$ & $3.49^{*} 10^{-3}$ & $1.15^{*} 10^{-3}$ & $9.04 * 10^{-3}$ & $2.88^{*} 10^{-4}$ & 3 & 1 & 30 & 1 \\
\hline 8 & $3.60 * 10^{-2}$ & $2.66^{*} 10^{-3}$ & $9.00 * 10^{-4}$ & $9.97^{*} 10^{-3}$ & $3.60^{*} 10^{-4}$ & 3 & 1 & 28 & 1 \\
\hline 9 & $3.60^{*} 10^{-2}$ & $2.52^{*} 10^{-3}$ & $9.36^{*} 10^{-4}$ & $1.05^{*} 10^{-2}$ & $4.32 * 10^{-4}$ & 3 & 1 & 24 & 1 \\
\hline 10 & $3.60 * 10^{-2}$ & $1.73^{*} 10^{-3}$ & $6.48^{*} 10^{-4}$ & $1.04 * 10^{-2}$ & $5.04 * 10^{-4}$ & 3 & 1 & 21 & 1 \\
\hline
\end{tabular}




\begin{tabular}{|c|c|c|c|c|c|c|c|c|c|}
\hline \multirow{2}{*}{$\begin{array}{c}\text { Time } \\
{[\mathrm{h}]}\end{array}$} & \multirow{2}{*}{$\begin{array}{c}\text { Std. } \\
{[\mathrm{mol} / \mathrm{L}]}\end{array}$} & \multicolumn{2}{|c|}{ Donor 1c } & \multicolumn{2}{|c|}{ Product 3b } & \multicolumn{2}{|c|}{ Donor 1c } & \multicolumn{2}{|c|}{ Product 3b } \\
\hline & & $\begin{array}{c}\alpha \\
{[\mathrm{mol} / \mathrm{L}]}\end{array}$ & $\begin{array}{c}\beta \\
{[\mathrm{mol} / \mathrm{L}]}\end{array}$ & $\begin{array}{c}\beta \\
{[\mathrm{mol} / \mathrm{L}]}\end{array}$ & $\begin{array}{c}\alpha \\
{[\mathrm{mol} / / \mathrm{L}]}\end{array}$ & $\alpha$ & $\beta$ & $\beta$ & $\alpha$ \\
\hline 0 & $3.60 * 10^{-2}$ & $1.53^{*} 10^{-2}$ & 0 & 0 & 0 & 1 & 0 & 0 & 0 \\
\hline 1 & $3.60 * 10^{-2}$ & $1.15^{*} 10^{-2}$ & $1.01 * 10^{-3}$ & $2.16^{*} 10^{-3}$ & $3.60 * 10^{-5}$ & 11 & 1 & 62 & 0 \\
\hline 2 & $3.60 * 10^{-2}$ & $9.18^{*} 10^{-3}$ & $1.64^{*} 10^{-3}$ & $3.51^{*} 10^{-3}$ & $3.60 * 10^{-5}$ & 6 & 1 & 56 & 1 \\
\hline 3 & $3.60 * 10^{-2}$ & $8.12^{*} 10^{-3}$ & $1.93^{*} 10^{-3}$ & $4.97^{*} 10^{-3}$ & $3.60 * 10^{-5}$ & 5 & 1 & 45 & 1 \\
\hline 4 & $3.60 * 10^{-2}$ & $6.35^{*} 10^{-3}$ & $1.87^{*} 10^{-3}$ & $6.01 * 10^{-3}$ & $1.62 * 10^{-4}$ & 4 & 1 & 37 & 1 \\
\hline 5 & $3.60 * 10^{-2}$ & $5.08^{*} 10^{-3}$ & $1.82^{*} 10^{-3}$ & $7.07^{*} 10^{-3}$ & $2.16^{*} 10^{-4}$ & 3 & 1 & 36 & 1 \\
\hline 6 & $3.60 * 10^{-2}$ & $3.65^{\star} 10^{-3}$ & $1.31 * 10^{-3}$ & $7.85^{\star} 10^{-3}$ & $3.24 * 10^{-4}$ & 3 & 1 & 27 & 1 \\
\hline 7 & $3.60 * 10^{-2}$ & $3.06^{*} 10^{-3}$ & $1.19^{*} 10^{-3}$ & $8.71^{*} 10^{-3}$ & $3.24 * 10^{-4}$ & 3 & 1 & 27 & 1 \\
\hline 8 & $3.60 * 10^{-2}$ & $2.56^{*} 10^{-3}$ & $9.72 * 10^{-4}$ & $9.74 * 10^{-3}$ & $3.06 * 10^{-4}$ & 3 & 1 & 31 & 1 \\
\hline 9 & $3.60 * 10^{-2}$ & $2.32^{*} 10^{-3}$ & $9.72^{*} 10^{-4}$ & $1.05^{*} 10^{-2}$ & $4.68 * 10^{-4}$ & 3 & 1 & 23 & 1 \\
\hline 10 & $3.60 * 10^{-2}$ & $1.69^{*} 10^{-3}$ & $6.84^{*} 10^{-4}$ & $1.07^{*} 10^{-2}$ & $4.50 * 10^{-4}$ & 3 & 1 & 24 & 1 \\
\hline
\end{tabular}

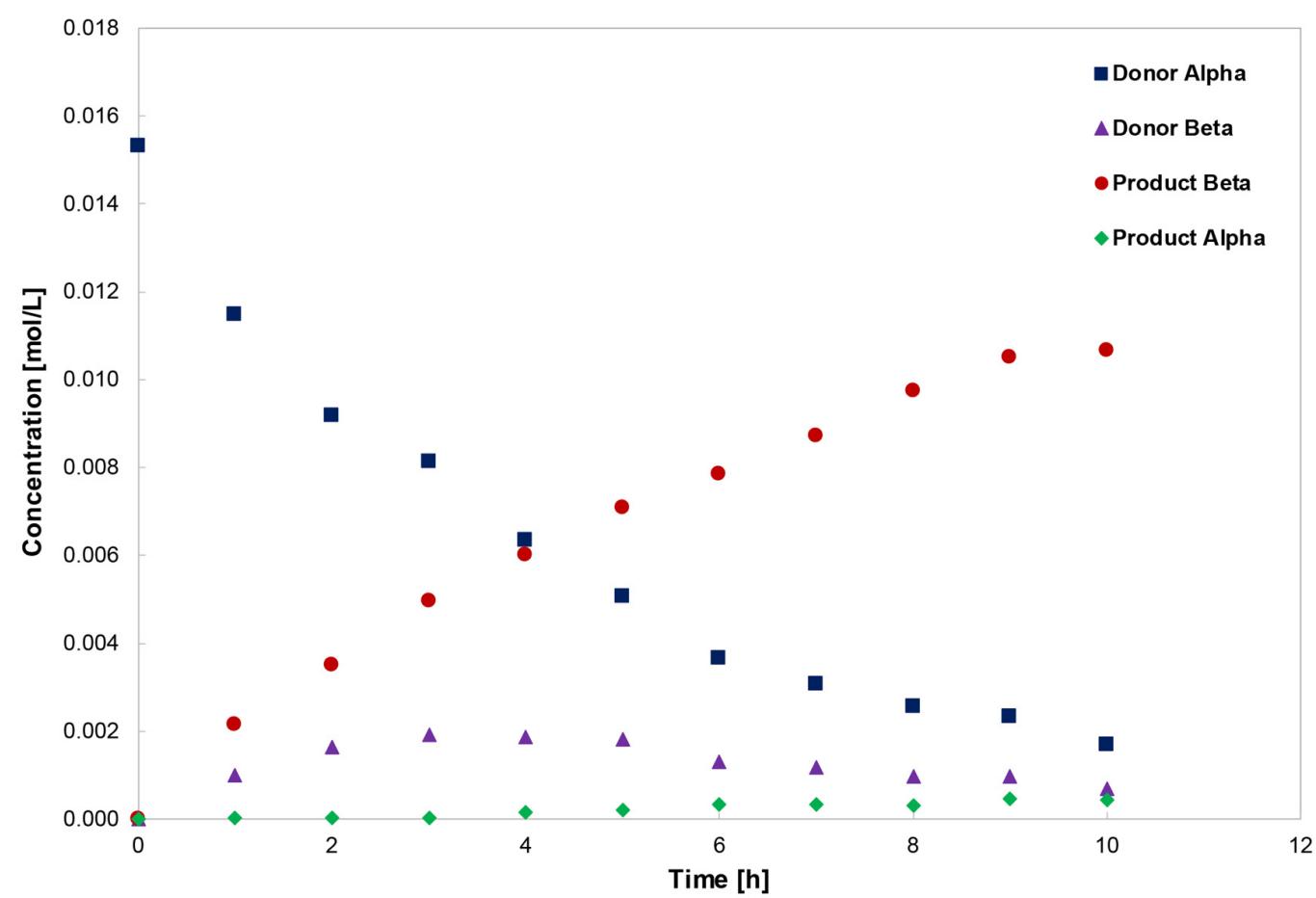

Figure S13 Averaged Kinetic Trace for the Reaction of 0.5 eq. 1c with N-Boc-L-threonine Methyl Ester $2 b$ (Blue Squares: $\alpha-1 c$; Purple Triangles: $\beta$ 1c; Red Dots: $\beta$-3b; Green Diamonds: $\alpha-3 \mathbf{b})$. 


\section{Non-optimal Catalyst Effect}

According to General Procedure E, the experiment was performed using 1c $(10.2 \mathrm{mg}$, $0.018 \mathrm{mmol}, 1.0$ eq.), catalyst $4 \mathrm{a}(1.00 \mathrm{mg}, 0.0009 \mathrm{mmol}, 0.05 \mathrm{eq}$.), and 1,2:3,4-di-Oisopropylidene- $\alpha$-D-galactopyranose $2 \mathrm{a}(9.4 \mathrm{mg}, 0.036 \mathrm{mmol}, 2.0$ eq.) or $\mathrm{N}$-Boc-Lthreonine methyl ester $\mathbf{2 b}(8.4 \mathrm{mg}, 0.036 \mathrm{mmol}, 2.0$ eq.). The reaction was monitored over $5 \mathrm{~h}$ for 1,2:3,4-di-O-isopropylidene- $\alpha$-D-galactopyranose $2 \mathrm{a}$ and $10 \mathrm{~h}$ for $\mathrm{N}$-BocL-threonine methyl ester $\mathbf{2 b}$.

\begin{tabular}{|c|c|c|c|c|c|c|c|c|c|}
\hline \multirow{2}{*}{$\begin{array}{c}\text { Time } \\
{[\mathrm{h}]}\end{array}$} & \multirow{2}{*}{$\begin{array}{c}\text { Std. } \\
{[\mathrm{mol} / \mathrm{L}]}\end{array}$} & \multicolumn{2}{|c|}{ Donor 1c } & \multicolumn{2}{|c|}{ Product $\mathbf{3 a}$} & \multicolumn{2}{|c|}{ Donor 1c } & \multicolumn{2}{|c|}{ Product $3 a$} \\
\hline & & $\begin{array}{c}\alpha \\
{[\mathrm{mol} / \mathrm{L}]}\end{array}$ & $\begin{array}{c}\beta \\
{[\mathrm{mol} / \mathrm{L}]}\end{array}$ & $\begin{array}{c}\beta \\
{[\mathrm{mol} / \mathrm{L}]}\end{array}$ & $\begin{array}{c}\alpha \\
{[\mathrm{mol} / \mathrm{L}]}\end{array}$ & $\alpha$ & $\beta$ & $\beta$ & $\alpha$ \\
\hline 0 & $3.60^{*} 10^{-2}$ & $3.25^{*} 10^{-2}$ & $6.12^{*} 10^{-4}$ & 0 & 0 & 1 & 0 & 0 & 0 \\
\hline 1 & $3.60 * 10^{-2}$ & $2.92 * 10^{-2}$ & $1.94^{*} 10^{-3}$ & $3.10^{*} 10^{-3}$ & $5.40 * 10^{-4}$ & 15 & 1 & 6 & 1 \\
\hline 2 & $3.60 * 10^{-2}$ & $2.69 * 10^{-2}$ & $1.19^{*} 10^{-3}$ & $4.79 * 10^{-2}$ & $7.92 * 10^{-4}$ & 22 & 1 & 6 & 1 \\
\hline 3 & $3.60 * 10^{-2}$ & $2.55^{*} 10^{-2}$ & $1.44^{*} 10^{-3}$ & $6.52 * 10^{-3}$ & $1.08 * 10^{-3}$ & 18 & 1 & 6 & 1 \\
\hline 4 & $3.60 * 10^{-2}$ & $2.27^{*} 10^{-2}$ & $1.51 * 10^{-3}$ & $7.81 * 10^{-3}$ & $1.40^{*} 10^{-3}$ & 15 & 1 & 6 & 1 \\
\hline 5 & $3.60 * 10^{-2}$ & $1.99 * 10^{-2}$ & $1.48^{*} 10^{-3}$ & $9.97^{*} 10^{-3}$ & $1.91 * 10^{-3}$ & 13 & 1 & 5 & 1 \\
\hline 6 & $3.60 * 10^{-2}$ & $1.83^{*} 10^{-2}$ & $1.84^{*} 10^{-3}$ & $1.12^{*} 10^{-2}$ & $2.16^{*} 10^{-3}$ & 10 & 1 & 5 & 1 \\
\hline 7 & $3.60 * 10^{-2}$ & $1.47^{*} 10^{-2}$ & $1.58 * 10^{-3}$ & $1.28 * 10^{-2}$ & $2.48^{*} 10^{-3}$ & 9 & 1 & 5 & 1 \\
\hline
\end{tabular}

Table S37 Concentration Data and Anomeric Ratios for the Catalyzed Reaction of 1c with 1,2:3,4-Di-O-isopropylidene- $\alpha$-D-galactopyranose 2a Catalyzed by 4 a $\left(2^{\text {nd }}\right.$ Run)

\begin{tabular}{cccccccccc}
\hline $\begin{array}{c}\text { Time } \\
{[\mathrm{h}]}\end{array}$ & $\begin{array}{c}\text { Std. } \\
{[\mathrm{mol} / \mathrm{L}]}\end{array}$ & $\begin{array}{c}\alpha \\
{[\mathrm{mol} / \mathrm{L}]}\end{array}$ & $\begin{array}{c}\beta \\
{[\mathrm{mol} / \mathrm{L}]}\end{array}$ & $\begin{array}{c}\beta \\
{[\mathrm{mol} / \mathrm{L}]}\end{array}$ & $\begin{array}{c}\alpha \\
{[\mathrm{mol} / \mathrm{L}]}\end{array}$ & $\alpha$ & $\beta$ & $\beta$ & $\alpha$ \\
\hline 0 & $3.60^{*} 10^{-2}$ & $3.07^{*} 10^{-2}$ & 0 & 0 & 0 & 1 & 0 & 0 & 0 \\
1 & $3.60^{*} 10^{-2}$ & $2.70^{*} 10^{-2}$ & $1.37^{*} 10^{-3}$ & $2.30^{*} 10^{-3}$ & $2.16^{*} 10^{-4}$ & 20 & 1 & 10 & 1 \\
2 & $3.60^{*} 10^{-2}$ & $2.59^{*} 10^{-2}$ & $1.12^{*} 10^{-3}$ & $4.07^{*} 10^{-3}$ & $4.32^{*} 10^{-4}$ & 23 & 1 & 9 & 1 \\
3 & $3.60^{*} 10^{-2}$ & $2.42^{*} 10^{-2}$ & $1.73^{*} 10^{-3}$ & $6.16^{*} 10^{-3}$ & $7.20^{*} 10^{-4}$ & 14 & 1 & 9 & 1 \\
4 & $3.60^{*} 10^{-2}$ & $1.92^{*} 10^{-2}$ & $1.15^{*} 10^{-3}$ & $7.27^{*} 10^{-3}$ & $9.00^{*} 10^{-4}$ & 17 & 1 & 8 & 1 \\
5 & $3.60^{*} 10^{-2}$ & $1.90^{*} 10^{-2}$ & $1.48^{*} 10^{-3}$ & $9.29^{*} 10^{-3}$ & $1.30^{*} 10^{-3}$ & 13 & 1 & 7 & 1 \\
6 & $3.60^{*} 10^{-2}$ & $1.83^{*} 10^{-2}$ & $1.80^{*} 10^{-3}$ & $1.16^{*} 10^{-2}$ & $1.87^{*} 10^{-3}$ & 10 & 1 & 6 & 1 \\
7 & $3.60^{*} 10^{-2}$ & $1.61^{*} 10^{-2}$ & $3.31^{*} 10^{-3}$ & $1.33^{*} 10^{-2}$ & $2.45^{*} 10^{-3}$ & 5 & 1 & 5 & 1 \\
\hline
\end{tabular}
Table S38 Concentration Data and Anomeric Ratios for the Catalyzed Reaction of 1c with 1,2:3,4-Di-O-isopropylidene- $\alpha$-D-galactopyranose 2a
Catalyzed by 4 a (Average).

\begin{tabular}{cccccccccc}
\hline $\begin{array}{c}\text { Time } \\
{[\mathrm{h}]}\end{array}$ & $\begin{array}{c}\text { Std. } \\
{[\mathrm{mol} / \mathrm{L}]}\end{array}$ & $\begin{array}{c}\alpha \\
{[\mathrm{mol} / \mathrm{L}]}\end{array}$ & $\begin{array}{c}c \\
{[\mathrm{~mol} / \mathrm{L}]}\end{array}$ & $\begin{array}{c}c \\
{[\mathrm{~mol} / \mathrm{L}]}\end{array}$ & $\begin{array}{c}\alpha \\
{[\mathrm{mol} / \mathrm{L}]}\end{array}$ & $\alpha$ & $\beta$ & $\beta$ & $\alpha$ \\
\hline 0 & $3.60^{*} 10^{-2}$ & $3.16^{*} 10^{-2}$ & $3.06^{*} 10^{-4}$ & 0 & 0 & 27 & 1 & 0 & 0 \\
1 & $3.60^{*} 10^{-2}$ & $2.81^{*} 10^{-2}$ & $1.66^{*} 10^{-3}$ & $2.70^{*} 10^{-3}$ & $3.78^{*} 10^{-4}$ & 18 & 1 & 8 & 1 \\
2 & $3.60^{*} 10^{-2}$ & $2.64^{*} 10^{-2}$ & $1.15^{*} 10^{-3}$ & $4.43^{*} 10^{-3}$ & $6.12^{*} 10^{-4}$ & 23 & 1 & 8 & 1 \\
3 & $3.60^{*} 10^{-2}$ & $2.49^{*} 10^{-2}$ & $1.58^{*} 10^{-3}$ & $6.34^{*} 10^{-3}$ & $9.00^{*} 10^{-4}$ & 16 & 1 & 8 & 1 \\
4 & $3.60^{*} 10^{-2}$ & $2.09^{*} 10^{-2}$ & $1.33^{*} 10^{-3}$ & $7.54^{*} 10^{-3}$ & $1.15^{*} 10^{-3}$ & 16 & 1 & 7 & 1 \\
5 & $3.60^{*} 10^{-2}$ & $1.94^{*} 10^{-2}$ & $1.48^{*} 10^{-3}$ & $9.63^{*} 10^{-3}$ & $1.60^{*} 10^{-3}$ & 13 & 1 & 6 & 1 \\
6 & $3.60^{*} 10^{-2}$ & $1.83^{*} 10^{-2}$ & $1.82^{*} 10^{-3}$ & $1.14^{*} 10^{-2}$ & $2.02^{*} 10^{-3}$ & 10 & 1 & 6 & 1 \\
7 & $3.60^{*} 10^{-2}$ & $1.54^{*} 10^{-2}$ & $2.45^{*} 10^{-3}$ & $1.31^{*} 10^{-2}$ & $2.47^{*} 10^{-3}$ & 7 & 1 & 5 & 1 \\
\hline
\end{tabular}




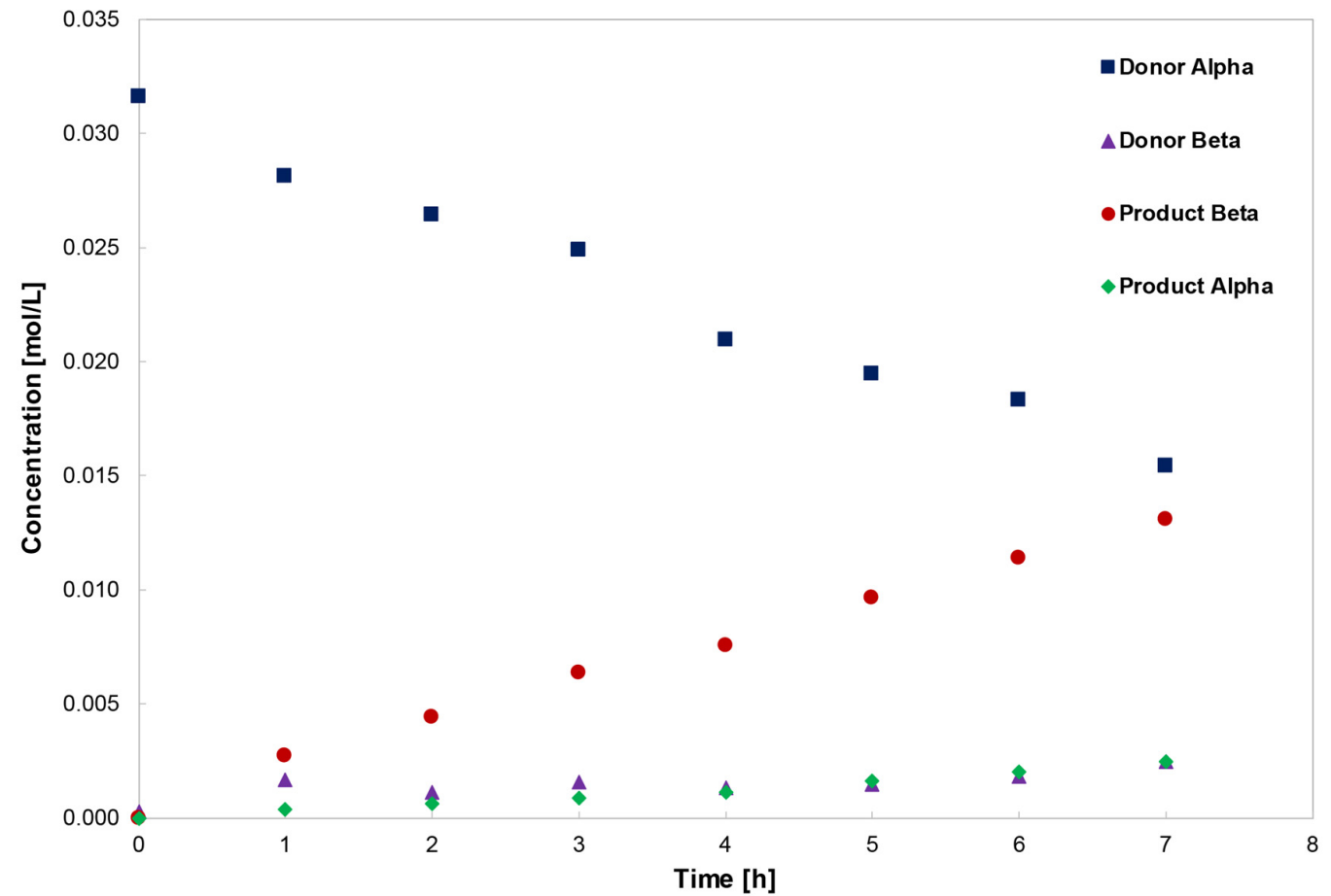

Figure S14 Averaged Kinetic Trace for the Reaction of 1c with 1,2:3,4-Di-O-isopropylidene- $\alpha$-D-galactopyranose 2a Catalyzed by 4a (Blue Squares: $\alpha-1 c$; Purple Triangles: $\beta-1 c$; Red Dots: $\beta-3 a$; Green Diamonds: $\alpha-3 a)$.

Table S39 Concentration Data and Anomeric Ratios for the Catalyzed Reaction of 1c with N-Boc-L-threonine Methyl Ester $\mathbf{2 b}$ Catalyzed by $4 \mathbf{a}$ (1 ${ }^{\text {st }}$ Run).

\begin{tabular}{|c|c|c|c|c|c|c|c|c|c|}
\hline \multirow{2}{*}{$\begin{array}{c}\text { Time } \\
{[\mathrm{h}]}\end{array}$} & \multirow{2}{*}{$\begin{array}{c}\text { Std. } \\
{[\mathrm{mol} / \mathrm{L}]}\end{array}$} & \multicolumn{2}{|c|}{ Donor 1c } & \multicolumn{2}{|c|}{ Product 3b } & \multicolumn{2}{|c|}{ Donor 1c } & \multicolumn{2}{|c|}{ Product 3b } \\
\hline & & $\begin{array}{c}\alpha \\
{[\mathrm{mol} / \mathrm{L}]}\end{array}$ & $\begin{array}{c}\beta \\
{[\mathrm{mol} / \mathrm{L}]}\end{array}$ & $\begin{array}{c}\beta \\
{[\mathrm{mol} / \mathrm{L}]}\end{array}$ & $\begin{array}{c}\alpha \\
{[\mathrm{mol} / \mathrm{L}]}\end{array}$ & $\alpha$ & $\beta$ & $\beta$ & $\alpha$ \\
\hline 0 & $3.60^{*} 10^{-2}$ & $2.80^{*} 10^{-2}$ & 0 & 0 & 0 & 1 & 0 & 0 & 0 \\
\hline 1 & $3.60 * 10^{-2}$ & $2.96 * 10^{-2}$ & $1.62 * 10^{-3}$ & 0 & 0 & 18 & 1 & 0 & 0 \\
\hline 2 & $3.60 * 10^{-2}$ & $2.79 * 10^{-2}$ & $2.34^{*} 10^{-3}$ & $7.20 * 10^{-4}$ & $7.20 * 10^{-5}$ & 12 & 1 & 18 & 1 \\
\hline 3 & $3.60^{*} 10^{-2}$ & $2.82 * 10^{-2}$ & $3.02 * 10^{-3}$ & $9.36 * 10^{-4}$ & $1.08 * 10^{-4}$ & 9 & 1 & 9 & 1 \\
\hline 4 & $3.60 * 10^{-2}$ & $2.52 * 10^{-2}$ & $3.31 * 10^{-3}$ & $1.08 * 10^{-3}$ & $7.20 * 10^{-5}$ & 8 & 1 & 13 & 1 \\
\hline 5 & $3.60 * 10^{-2}$ & $2.49^{*} 10^{-2}$ & $3.85^{*} 10^{-3}$ & $1.44^{*} 10^{-3}$ & $3.96 * 10^{-4}$ & 6 & 1 & 4 & 1 \\
\hline 6 & $3.60^{*} 10^{-2}$ & $2.23^{*} 10^{-2}$ & $3.67^{*} 10^{-3}$ & $1.76^{*} 10^{-3}$ & $2.16^{*} 10^{-4}$ & 6 & 1 & 8 & 1 \\
\hline 7 & $3.60^{*} 10^{-2}$ & $2.27^{*} 10^{-2}$ & $4.54^{*} 10^{-3}$ & $2.02 * 10^{-3}$ & $6.48 * 10^{-4}$ & 5 & 1 & 3 & 1 \\
\hline 8 & $3.60 * 10^{-2}$ & $2.28 * 10^{-2}$ & $4.57^{*} 10^{-3}$ & $1.94^{*} 10^{-3}$ & $2.16^{*} 10^{-4}$ & 5 & 1 & 3 & 1 \\
\hline 9 & $3.60^{*} 10^{-2}$ & $2.30^{*} 10^{-2}$ & $4.25^{\star} 10^{-3}$ & $2.20^{*} 10^{-3}$ & $3.24 * 10^{-4}$ & 5 & 1 & 7 & 1 \\
\hline 10 & $3.60 * 10^{-2}$ & $2.21 * 10^{-2}$ & $4.68 * 10^{-3}$ & $2.45^{*} 10^{-3}$ & $3.24 * 10^{-4}$ & 5 & 1 & 8 & 1 \\
\hline 24 & $3.60^{*} 10^{-2}$ & $1.57^{*} 10^{-2}$ & $5.18^{*} 10^{-3}$ & $4.72^{*} 10^{-2}$ & $6.12^{*} 10^{-4}$ & 3 & 1 & 8 & 1 \\
\hline \multicolumn{10}{|c|}{$\begin{array}{l}\text { Table S40 Concentration Data and Anomeric Ratios for the Catalyzed Reaction of 1c with N-Boc-L-threonine Methyl Ester } 2 \text { I Catalyzed by } 4 \text { a (2 } \\
\text { Run). }\end{array}$} \\
\hline Time & Std. & \multicolumn{2}{|c|}{ Donor 1c } & \multicolumn{2}{|c|}{ Product $\mathbf{3 b}$} & \multicolumn{2}{|c|}{ Donor 1c } & \multicolumn{2}{|c|}{ Product $3 b$} \\
\hline [h] & {$[\mathrm{mol} / \mathrm{L}]$} & $\begin{array}{c}\alpha \\
{[\mathrm{mol} / \mathrm{L}]}\end{array}$ & $\begin{array}{c}\beta \\
{[\mathrm{mol} / \mathrm{L}]}\end{array}$ & $\begin{array}{c}\beta \\
{[\mathrm{mol} / \mathrm{L}]}\end{array}$ & $\begin{array}{c}\alpha \\
{[\mathrm{mol} / \mathrm{L}]}\end{array}$ & $\alpha$ & $\beta$ & $\beta$ & $\alpha$ \\
\hline 0 & $3.60^{*} 10^{-2}$ & $3.33^{*} 10^{-2}$ & 0 & 0 & 0 & 1 & 0 & 0 & 0 \\
\hline 1 & $3.60 * 10^{-2}$ & $3.00 * 10^{-2}$ & $1.04^{*} 10^{-3}$ & 0 & 0 & 28 & 1 & 0 & 0 \\
\hline 2 & $3.60 * 10^{-2}$ & $2.71^{*} 10^{-2}$ & $1.84^{*} 10^{-3}$ & $7.92 * 10^{-4}$ & $1.08 * 10^{-4}$ & 15 & 1 & 8 & 1 \\
\hline 3 & $3.60 * 10^{-2}$ & $2.67^{*} 10^{-2}$ & $2.45^{*} 10^{-3}$ & $1.01 * 10^{-3}$ & $1.08 * 10^{-4}$ & 11 & 1 & 11 & 1 \\
\hline 4 & $3.60^{*} 10^{-2}$ & $2.79 * 10^{-2}$ & $3.17^{*} 10^{-3}$ & $1.12^{*} 10^{-3}$ & $1.44^{*} 10^{-4}$ & 9 & 1 & 9 & 1 \\
\hline 5 & $3.60^{*} 10^{-2}$ & $2.72 * 10^{-2}$ & $3.42 * 10^{-3}$ & $1.51 * 10^{-3}$ & $2.88 * 10^{-4}$ & 8 & 1 & 5 & 1 \\
\hline 6 & $3.60 * 10^{-2}$ & $2.52 * 10^{-2}$ & $3.74 * 10^{-3}$ & $1.76^{*} 10^{-3}$ & $2.52 * 10^{-4}$ & 7 & 1 & 7 & 1 \\
\hline 7 & $3.60 * 10^{-2}$ & $2.49^{*} 10^{-2}$ & $3.74 * 10^{-3}$ & $1.76^{*} 10^{-3}$ & $7.20 * 10^{-5}$ & 7 & 1 & 20 & 1 \\
\hline 8 & $3.60^{*} 10^{-2}$ & $2.39 * 10^{-2}$ & $4.00 * 10^{-3}$ & $1.80 * 10^{-3}$ & $2.16^{*} 10^{-4}$ & 6 & 1 & 9 & 1 \\
\hline 9 & $3.60^{*} 10^{-2}$ & $2.40^{*} 10^{-2}$ & $4.54^{*} 10^{-3}$ & $2.27^{*} 10^{-3}$ & $3.96 * 10^{-4}$ & 5 & 1 & 6 & 1 \\
\hline 10 & $3.60 * 10^{-2}$ & $2.30^{*} 10^{-2}$ & $5.08 * 10^{-3}$ & $2.52^{*} 10^{-3}$ & $3.96 * 10^{-4}$ & 5 & 1 & 7 & 1 \\
\hline 24 & $3.60 * 10^{-2}$ & $1.61 * 10^{-2}$ & $4.54^{*} 10^{-3}$ & $4.57^{*} 10^{-3}$ & $6.84 * 10^{-4}$ & 4 & 1 & 7 & 1 \\
\hline
\end{tabular}


Table S41 Concentration Data and Anomeric Ratios for the Catalyzed Reaction of 1c with N-Boc-L-threonine Methyl Ester $2 \mathrm{l}$ Catalyzed by 4a (Average).

\begin{tabular}{|c|c|c|c|c|c|c|c|c|c|}
\hline \multirow{2}{*}{$\begin{array}{c}\text { Time } \\
{[\mathrm{h}]}\end{array}$} & \multirow{2}{*}{$\begin{array}{c}\text { Std. } \\
{[\mathrm{mol} / \mathrm{L}]}\end{array}$} & \multicolumn{2}{|c|}{ Donor 1c } & \multicolumn{2}{|c|}{ Product 3b } & \multicolumn{2}{|c|}{ Donor 1c } & \multicolumn{2}{|c|}{ Product $\mathbf{3 b}$} \\
\hline & & $\begin{array}{c}\alpha \\
{[\mathrm{mol} / \mathrm{L}]}\end{array}$ & $\begin{array}{c}\beta \\
{[\mathrm{mol} / \mathrm{L}]}\end{array}$ & $\begin{array}{c}\beta \\
{[\mathrm{mol} / \mathrm{L}]}\end{array}$ & $\begin{array}{c}\alpha \\
{[\mathrm{mol} / \mathrm{L}]}\end{array}$ & $\alpha$ & $\beta$ & $\beta$ & $\alpha$ \\
\hline 0 & $3.60^{*} 10^{-2}$ & $3.07^{*} 10^{-2}$ & 0 & $\overline{0}$ & 0 & 1 & 0 & 0 & 0 \\
\hline 1 & $3.60 * 10^{-2}$ & $2.98^{*} 10^{-2}$ & $1.33^{*} 10^{-3}$ & 0 & 0 & 23 & 1 & 0 & 0 \\
\hline 2 & $3.60^{*} 10^{-2}$ & $2.75^{\star} 10^{-2}$ & $2.09^{*} 10^{-3}$ & $7.56^{*} 10^{-4}$ & $9.00 * 10^{-5}$ & 14 & 1 & 13 & 1 \\
\hline 3 & $3.60^{*} 10^{-2}$ & $2.75^{*} 10^{-2}$ & $2.74^{*} 10^{-3}$ & $9.72 * 10^{-4}$ & $1.08 * 10^{-4}$ & 10 & 1 & 10 & 1 \\
\hline 4 & $3.60 * 10^{-2}$ & $2.65^{*} 10^{-2}$ & $3.24^{*} 10^{-3}$ & $1.10^{*} 10^{-3}$ & $1.08 * 10^{-4}$ & 9 & 1 & 11 & 1 \\
\hline 5 & $3.60 * 10^{-2}$ & $2.60^{*} 10^{-2}$ & $3.64^{*} 10^{-3}$ & $1.48^{*} 10^{-3}$ & $3.42^{*} 10^{-4}$ & 7 & 1 & 5 & 1 \\
\hline 6 & $3.60^{*} 10^{-2}$ & $2.37^{*} 10^{-2}$ & $3.71 * 10^{-3}$ & $1.76 * 10^{-3}$ & $2.34^{*} 10^{-4}$ & 7 & 1 & 8 & 1 \\
\hline 7 & $3.60^{*} 10^{-2}$ & $2.38^{*} 10^{-2}$ & $4.14^{*} 10^{-3}$ & $1.89^{*} 10^{-3}$ & $3.60 * 10^{-4}$ & 6 & 1 & 12 & 1 \\
\hline 8 & $3.60 * 10^{-2}$ & $2.33^{*} 10^{-2}$ & $4.28 * 10^{-3}$ & $1.87^{*} 10^{-3}$ & $2.16^{*} 10^{-4}$ & 6 & 1 & 6 & 1 \\
\hline 9 & $3.60^{*} 10^{-2}$ & $2.35^{*} 10^{-2}$ & $4.39^{*} 10^{-3}$ & $2.23^{*} 10^{-3}$ & $3.60 * 10^{-4}$ & 5 & 1 & 7 & 1 \\
\hline 10 & $3.60^{*} 10^{-2}$ & $2.25^{*} 10^{-2}$ & $4.88^{*} 10^{-3}$ & $2.48^{*} 10^{-3}$ & $3.60 * 10^{-4}$ & 5 & 1 & 8 & 1 \\
\hline 24 & $3.60 * 10^{-2}$ & $1.59^{*} 10^{-2}$ & $4.86^{*} 10^{-3}$ & $4.64^{*} 10^{-3}$ & $6.48^{*} 10^{-4}$ & 4 & 1 & 8 & 1 \\
\hline
\end{tabular}

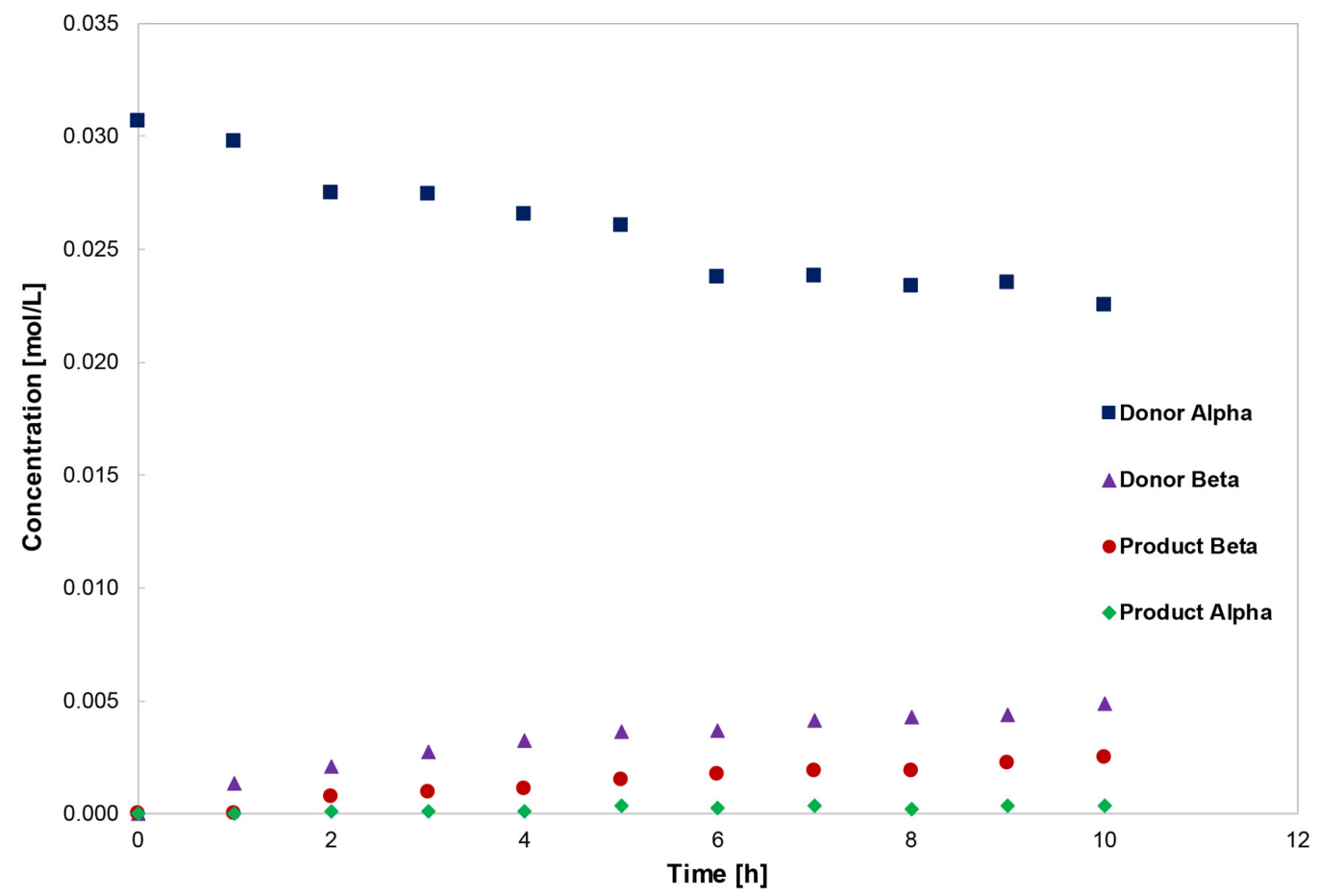

Figure S15 Averaged Kinetic Trace for the Reaction of 1c with N-Boc-L-threonine Methyl Ester 2l Catalyzed by 4a (Blue Squares: $\alpha-1 c$; Purple Triangles: $\beta$-1c; Red Dots: $\beta$-3b; Green Diamonds: $\alpha-3 b)$. 


\section{Component Reaction Order for the Product Formation}

To determine the molecularity of the transition state for the product-forming reaction, the kinetic traces of all described experiments were superimposed. The resulting graph illustrated a rate-determining step ordered in catalyst and nucleophile. Additionally, no influence of the donor concentration on the rate of product formation was observed.

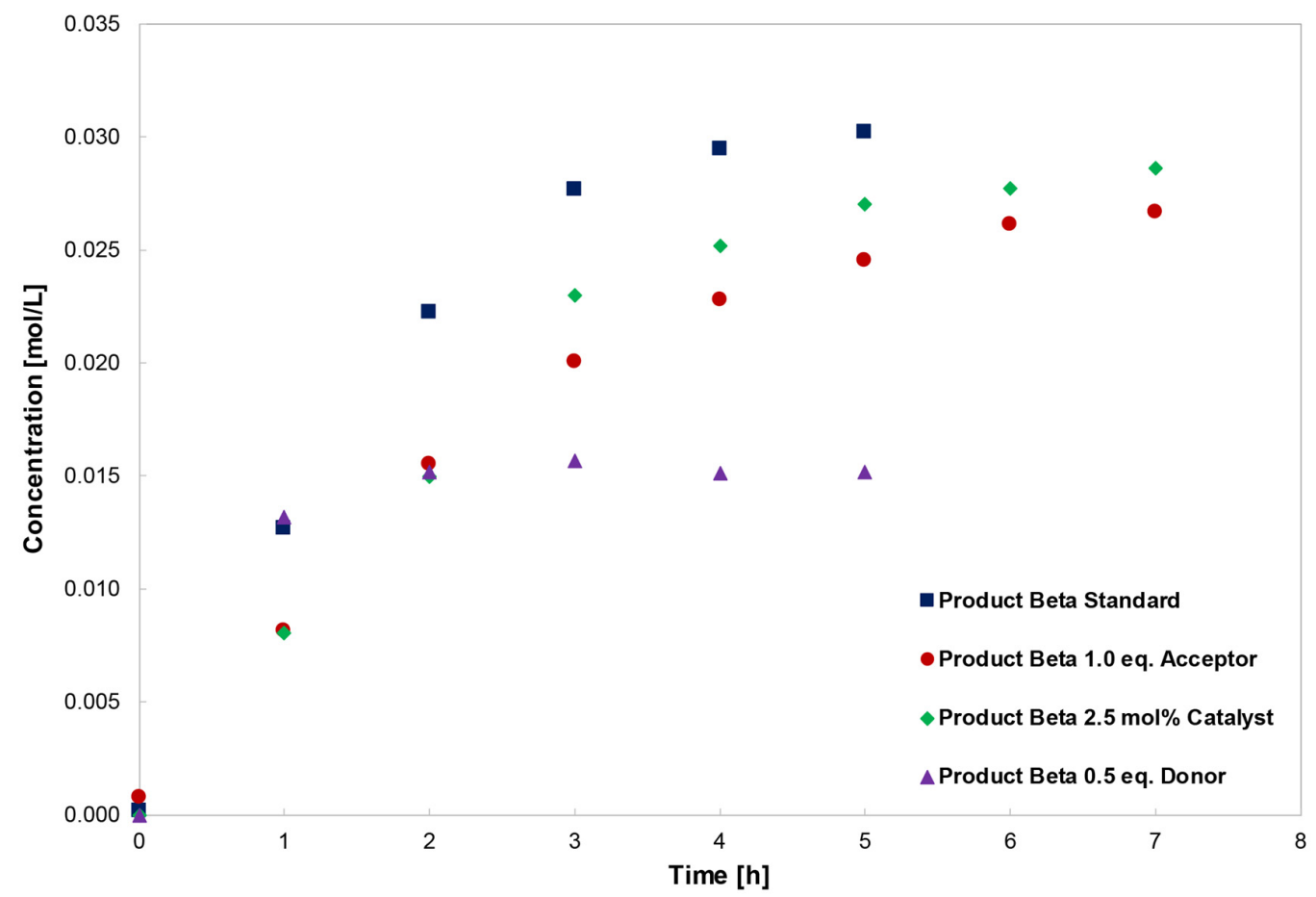

Figure S16 Overlaid Kinetic Traces of Product Formation for the Reaction of 1c with 1,2:3,4-Di-O-isopropylidene- $\alpha$-D-galactopyranose 2a Under the Described Reaction Conditions (Blue Squares: $\beta$-3a Under Standard Reaction Conditions; Red Dots: $\beta$-3a with 1.0 eq. Acceptor; Green Diamonds: $\beta-3 a$ with 2.5 mol\% Catalyst 4i; Purple Triangles: $\beta$-3a with 0.5 eq. Donor).

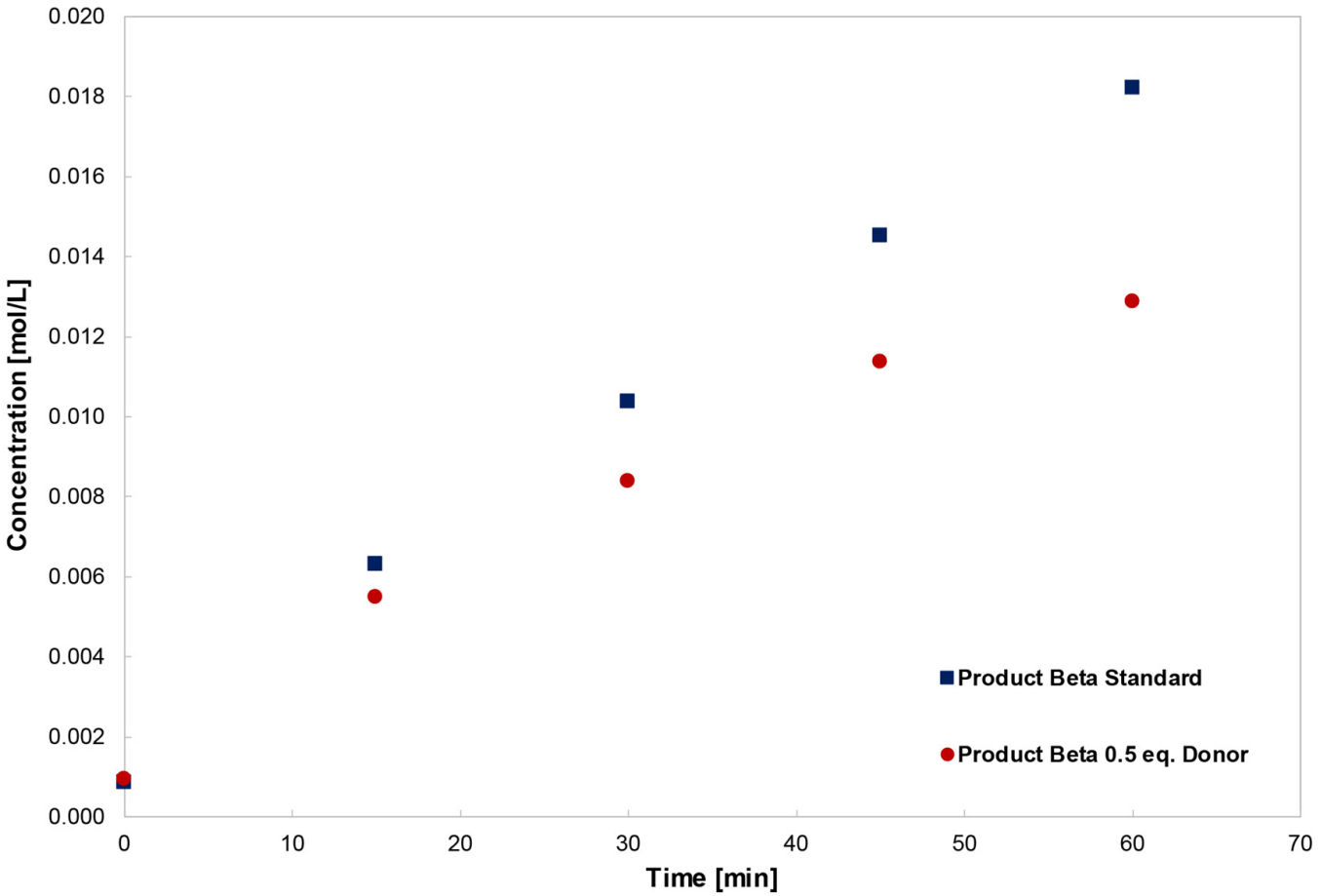

Figure S17 Overlaid Traces of Product Formation for the Reaction of 1c with 1,2:3,4-Di-O-isopropylidene- $\alpha$-D-galactopyranose 2a Under the Described Reaction Conditions (Blue Squares: $\beta$-3a Under Standard Reaction Conditions; Red Dots: $\beta-3 a$ with 0.5 eq. Donor). 


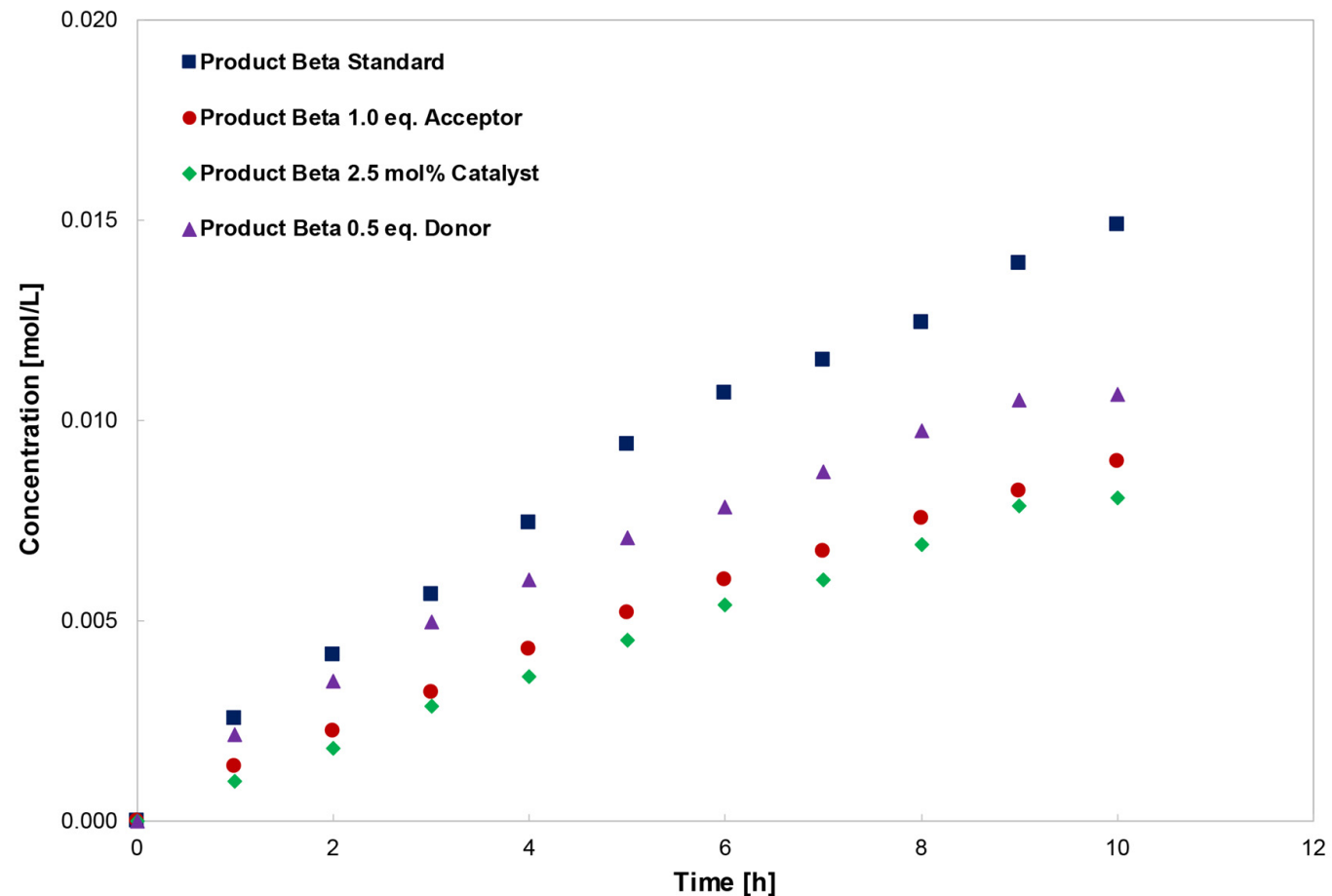

Figure S18 Overlaid Kinetic Traces of Product Formation for the Reaction of 1c with N-Boc-L-threonine Methyl Ester 2b Under the Described Reaction Conditions (Blue Squares: $\beta$-3b Under Standard Reaction Conditions; Red Dots: $\beta-3 \mathbf{b}$ with 1.0 eq. Acceptor; Green Diamonds: $\beta$-3b with $2.5 \mathrm{~mol} \%$ Catalyst $4 \mathbf{4}$; Purple Triangles: $\beta$-3b with 0.5 eq. Donor). 


\section{Component Reaction Order for the Epimerization}

To determine the molecularity of the transition state for the epimerization process, the kinetic traces of all described experiments were superimposed. The resulting graph illustrated a rate-determining step ordered in catalyst and donor with the absence of an acceptor order.

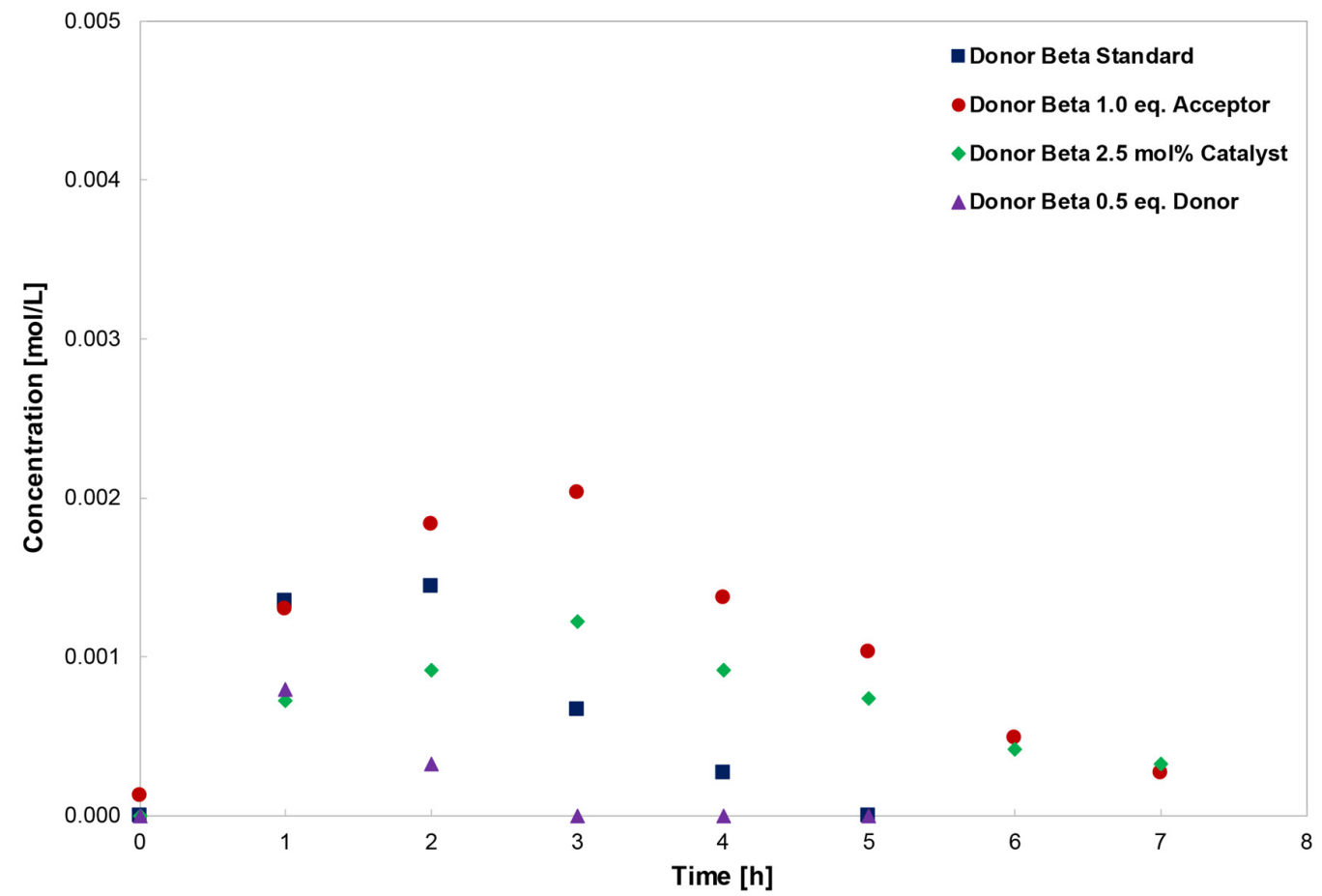

Figure S19 Overlaid Kinetic Traces of Epimerization for the Reaction of 1c with 1,2:3,4-Di-O-isopropylidene- $\alpha$-D-galactopyranose 2a Under the Described Reaction Conditions (Blue Squares: $\beta$-1c Under Standard Reaction Conditions; Red Dots: $\beta$-1c with 1.0 eq. Acceptor; Green Diamonds: $\beta-1 \mathrm{c}$ with $2.5 \mathrm{~mol} \%$ Catalyst $4 \mathrm{i}$; Purple Trianqles: $\beta-1 \mathrm{c}$ with 0.5 eq. Donor).

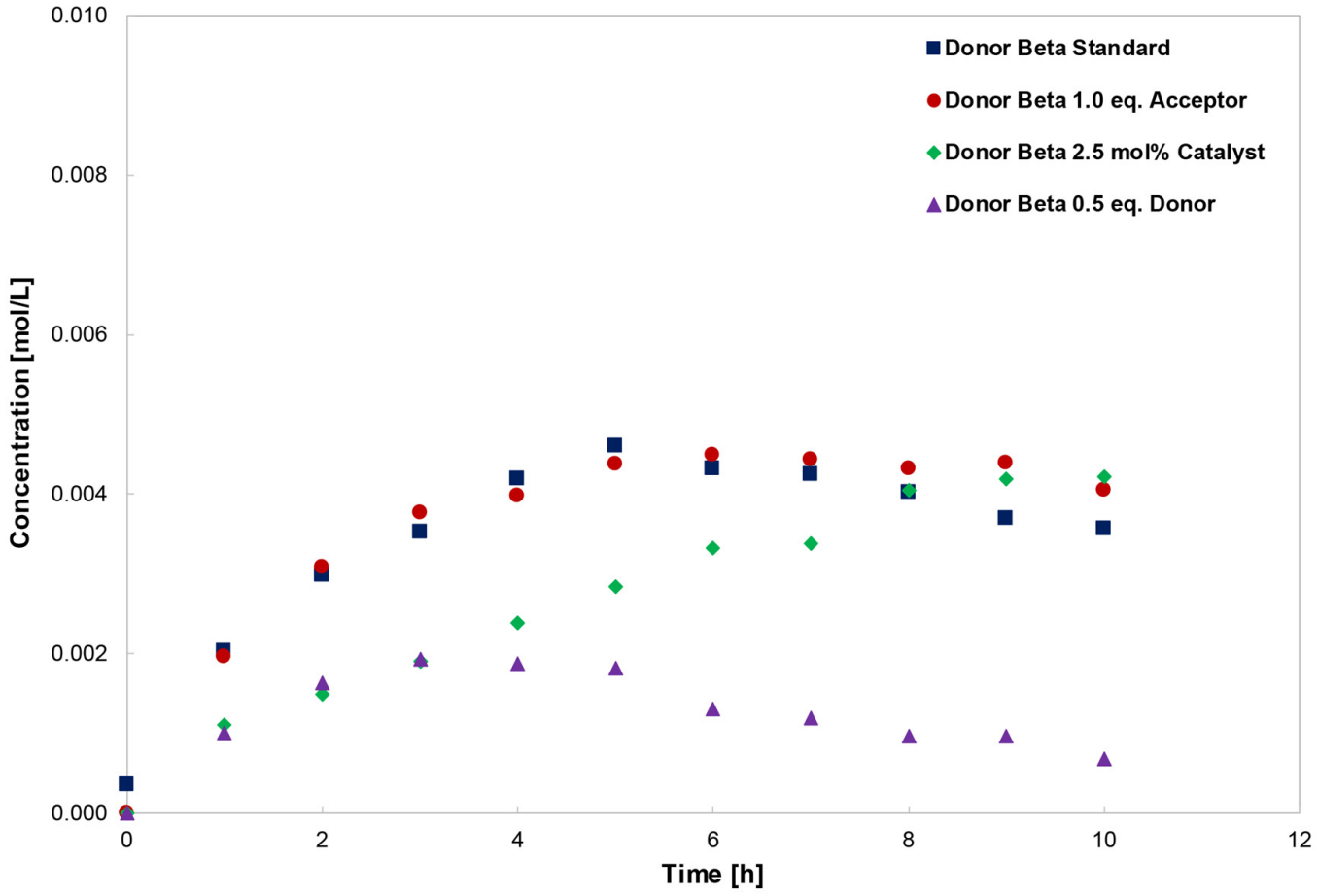

Figure S20 Overlaid Kinetic Traces of Epimerization for the Reaction of 1c with N-Boc-L-threonine Methyl Ester 2b Under the Described Reaction Conditions (Blue Squares: $\beta-1 c$ Under Standard Reaction Conditions; Red Dots: $\beta-1 c$ with 1.0 eq. Acceptor; Green Diamonds: $\beta-1 c$ with 2.5 mol\% Catalyst 4i; Purple Triangles: $\beta$-1c with 0.5 eq. Donor). 


\section{Xylofuranose Kinetics under Standard Reaction Conditions}

According to General Procedure E, the experiment was performed using $\mathbf{5 f}(10.2 \mathrm{mg}$, $0.018 \mathrm{mmol}, 1.0$ eq.), catalyst $4 \mathbf{i}(0.94 \mathrm{mg}, 0.0009 \mathrm{mmol}, 0.05$ eq. $)$, and 1,2:3,4-di-Oisopropylidene- $\alpha$-D-galactopyranose $2 \mathrm{a}(9.4 \mathrm{mg}, 0.036 \mathrm{mmol}, 2.0 \mathrm{eq}$.$) The reaction$ was monitored over $10 \mathrm{~h}$.

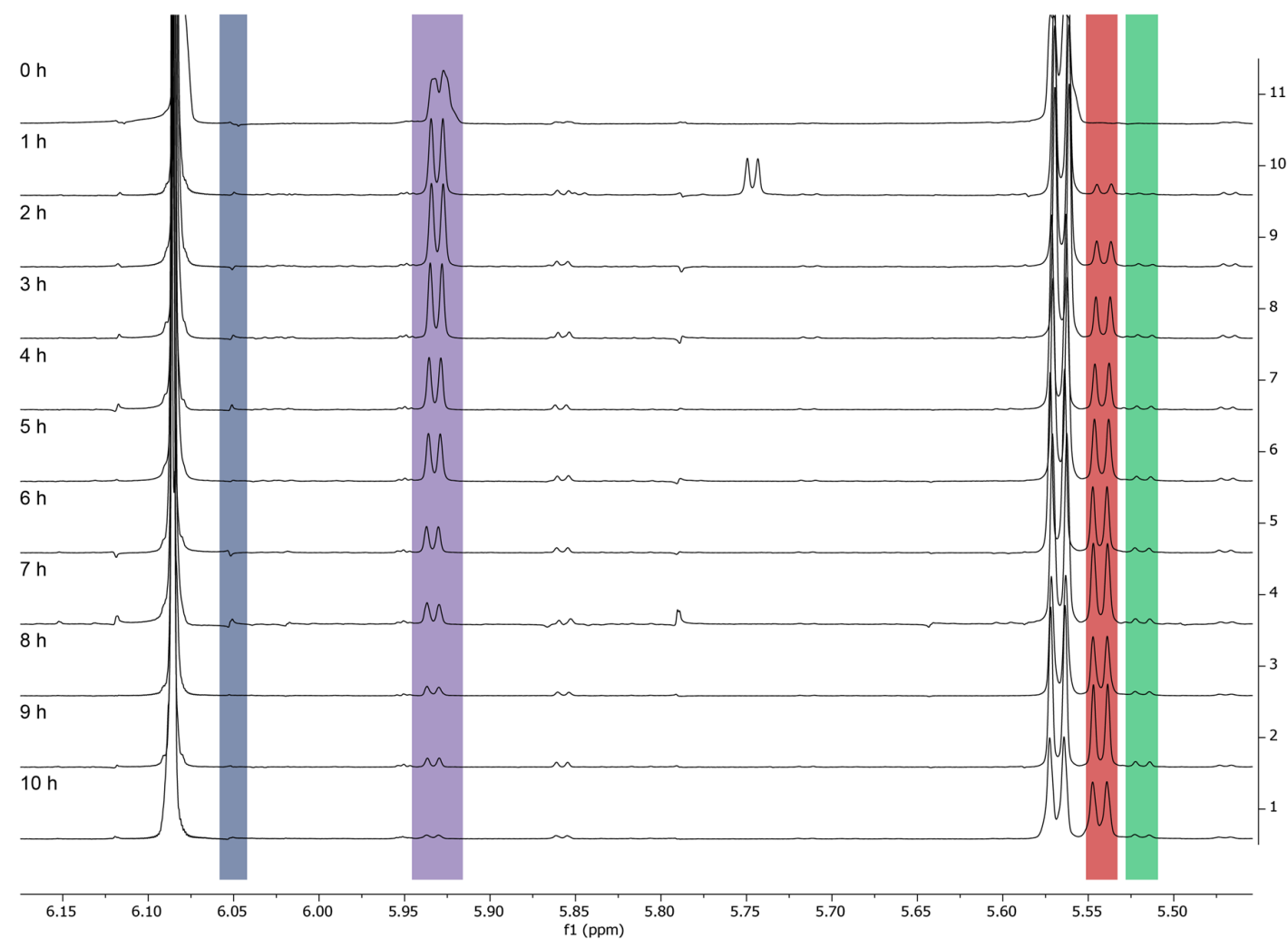

Figure S21 ${ }^{1} \mathrm{H}$ NMR Spectral Data for the Reaction of $\mathbf{5 f}$ with 1,2:3,4-Di-O-isopropylidene- $\alpha$-galactopyranose 2a Obtained by Multi-Pot Quench Kinetic Experiments (Blue: $\alpha-5 f$; Purple: $\beta-5 f ;$ Red: $\beta-6 f ;$ Green: $\alpha-6 f)$.

Table S42 Concentration Data and Anomeric Ratios for the Catalyzed Reaction of $\mathbf{5 f}$ with 1,2:3,4-Di-O-isopropylidene- $\alpha$-D-galactopyranose 2a Under Standard Reaction Conditions ( $1^{\text {st }}$ Run).

\begin{tabular}{|c|c|c|c|c|c|c|c|c|c|}
\hline \multirow{2}{*}{$\begin{array}{c}\text { Time } \\
{[\mathrm{h}]}\end{array}$} & \multirow{2}{*}{$\begin{array}{c}\text { Std. } \\
{[\mathrm{mol} / \mathrm{L}]}\end{array}$} & \multicolumn{2}{|c|}{ Donor $5 f$} & \multicolumn{2}{|c|}{ Product $\mathbf{6 f}$} & \multicolumn{2}{|c|}{ Donor $5 f$} & \multicolumn{2}{|c|}{ Product $6 f$} \\
\hline & & $\begin{array}{c}\alpha \\
{[\mathrm{mol} / \mathrm{L}]}\end{array}$ & $\begin{array}{c}\beta \\
{[\mathrm{mol} / \mathrm{L}]}\end{array}$ & $\begin{array}{c}\beta \\
{[\mathrm{mol} / \mathrm{L}]}\end{array}$ & $\begin{array}{c}\alpha \\
{[\mathrm{mol} / \mathrm{L}]}\end{array}$ & $\alpha$ & $\beta$ & $\beta$ & $\alpha$ \\
\hline$\overline{0}$ & $3.60^{*} 10^{-2}$ & $2.52^{*} 10^{-4}$ & $2.79^{*} 10^{-2}$ & 0 & 0 & 1 & $\overline{99}$ & 0 & $\overline{0}$ \\
\hline 1 & $3.60^{*} 10^{-2}$ & $7.20^{*} 10^{-4}$ & $2.57^{*} 10^{-2}$ & $4.03^{*} 10^{-3}$ & $7.92 * 10^{-4}$ & 1 & 37 & 5 & 1 \\
\hline 2 & $3.60 * 10^{-2}$ & $6.48^{*} 10^{-4}$ & $2.39 * 10^{-2}$ & $7.52^{*} 10^{-3}$ & $9.36^{*} 10^{-4}$ & 1 & 36 & 8 & 1 \\
\hline 3 & $3.60^{*} 10^{-2}$ & $5.04^{*} 10^{-4}$ & $1.86 * 10^{-2}$ & $1.07^{*} 10^{-2}$ & $9.72 * 10^{-4}$ & 1 & 36 & 11 & 1 \\
\hline 4 & $3.60 * 10^{-2}$ & $6.48^{*} 10^{-4}$ & $1.59 * 10^{-2}$ & $1.43^{*} 10^{-2}$ & $1.22 * 10^{-3}$ & 1 & 25 & 12 & 1 \\
\hline 5 & $3.60 * 10^{-2}$ & $5.04 * 10^{-4}$ & $1.37^{*} 10^{-2}$ & $1.83^{*} 10^{-2}$ & $1.51 * 10^{-3}$ & 1 & 28 & 12 & 1 \\
\hline 6 & $3.60^{*} 10^{-2}$ & $4.32 * 10^{-4}$ & $7.74^{*} 10^{-3}$ & $2.02 * 10^{-2}$ & $1.55^{*} 10^{-3}$ & 1 & 18 & 13 & 1 \\
\hline 7 & $3.60 * 10^{-2}$ & $3.24^{*} 10^{-4}$ & $5.47^{*} 10^{-3}$ & $2.18^{*} 10^{-2}$ & $1.51^{*} 10^{-3}$ & 1 & 17 & 14 & 1 \\
\hline 8 & $3.60^{*} 10^{-2}$ & $3.60^{*} 10^{-5}$ & $3.67^{*} 10^{-3}$ & $2.46^{*} 10^{-2}$ & $1.91^{*} 10^{-3}$ & 1 & 17 & 14 & 1 \\
\hline 9 & $3.60 * 10^{-2}$ & $1.08^{*} 10^{-4}$ & $2.74^{*} 10^{-3}$ & $2.47^{*} 10^{-2}$ & $1.76^{*} 10^{-3}$ & 1 & 23 & 14 & 1 \\
\hline 10 & $3.60^{*} 10^{-2}$ & $1.08^{*} 10^{-4}$ & $1.58 * 10^{-3}$ & $2.67^{*} 10^{-2}$ & $1.91 * 10^{-3}$ & 1 & 14 & 14 & 1 \\
\hline
\end{tabular}


Table S43 Concentration Data and Anomeric Ratios for the Catalyzed Reaction of $\mathbf{5 f}$ with 1,2:3,4-Di-O-isopropylidene- $\alpha$-D-galactopyranose 2a Under Standard Reaction Conditions ( $2^{\text {nd }}$ Run).

\begin{tabular}{|c|c|c|c|c|c|c|c|c|c|}
\hline \multirow{2}{*}{$\begin{array}{c}\text { Time } \\
\text { [h] }\end{array}$} & \multirow{2}{*}{$\begin{array}{c}\text { Std. } \\
{[\mathrm{mol} / \mathrm{L}]}\end{array}$} & \multicolumn{2}{|c|}{ Donor $5 f$} & \multicolumn{2}{|c|}{ Product 6f } & \multicolumn{2}{|c|}{ Donor $\mathbf{5 f}$} & \multicolumn{2}{|c|}{ Product 6f } \\
\hline & & $\begin{array}{c}\alpha \\
{[\mathrm{mol} / \mathrm{L}]}\end{array}$ & $\begin{array}{c}\beta \\
{[\mathrm{mol} / \mathrm{L}]}\end{array}$ & $\begin{array}{c}\beta \\
{[\mathrm{mol} / \mathrm{L}]}\end{array}$ & $\begin{array}{c}\alpha \\
{[\mathrm{mol} / \mathrm{L}]}\end{array}$ & $\alpha$ & $\beta$ & $\beta$ & $\alpha$ \\
\hline 0 & $3.60^{*} 10^{-2}$ & 0 & $2.98 * 10^{-2}$ & 0 & 0 & 0 & 1 & 0 & 0 \\
\hline 1 & $3.60^{*} 10^{-2}$ & $3.24^{*} 10^{-4}$ & $2.78^{*} 10^{-2}$ & $3.24^{*} 10^{-3}$ & $7.56^{*} 10^{-4}$ & 1 & 82 & 4 & 1 \\
\hline 2 & $3.60 * 10^{-2}$ & $3.24^{*} 10^{-4}$ & $2.19^{*} 10^{-2}$ & $7.92^{*} 10^{-3}$ & $7.20^{*} 10^{-4}$ & 1 & 68 & 11 & 1 \\
\hline 3 & $3.60 * 10^{-2}$ & $2.88^{*} 10^{-4}$ & $1.93^{*} 10^{-2}$ & $1.10^{*} 10^{-2}$ & $9.36^{*} 10^{-4}$ & 1 & 67 & 12 & 1 \\
\hline 4 & $3.60 * 10^{-2}$ & $6.84^{*} 10^{-4}$ & $1.47^{*} 10^{-2}$ & $1.48^{*} 10^{-2}$ & $1.30 * 10^{-3}$ & 1 & 22 & 12 & 1 \\
\hline 5 & $3.60^{*} 10^{-2}$ & $4.68 * 10^{-4}$ & $1.11^{*} 10^{-2}$ & $1.73^{*} 10^{-2}$ & $1.44^{*} 10^{-3}$ & 1 & 23 & 12 & 1 \\
\hline 6 & $3.60 * 10^{-2}$ & $4.68 * 10^{-4}$ & $8.89^{*} 10^{-3}$ & $1.86 * 10^{-2}$ & $1.22 * 10^{-2}$ & 1 & 19 & 15 & 1 \\
\hline 7 & $3.60 * 10^{-2}$ & $5.40^{*} 10^{-4}$ & $4.25^{*} 10^{-3}$ & $2.22 * 10^{-2}$ & $1.73^{*} 10^{-2}$ & 1 & 8 & 13 & 1 \\
\hline 8 & $3.60^{*} 10^{-2}$ & $5.76^{*} 10^{-4}$ & $3.64^{*} 10^{-3}$ & $2.39 * 10^{-2}$ & $1.91 * 10^{-2}$ & 1 & 7 & 13 & 1 \\
\hline 9 & $3.60^{*} 10^{-2}$ & $5.40^{*} 10^{-4}$ & $3.24^{*} 10^{-3}$ & $2.61^{*} 10^{-2}$ & $2.30^{*} 10^{-3}$ & 1 & 6 & 11 & 1 \\
\hline 10 & $3.60 * 10^{-2}$ & $1.80^{*} 10^{-4}$ & $1.33^{*} 10^{-3}$ & $2.80^{*} 10^{-2}$ & $1.94 * 10^{-3}$ & 1 & 8 & 15 & 1 \\
\hline \multicolumn{10}{|c|}{$\begin{array}{l}\text { Table S44 Concentration Data and Anomeric Ratios for the Catalyzed Reaction of } \mathbf{5 f} \text { with 1,2:3,4-Di-O-isopropylidene- } \alpha \text {-D-galactopyranose } \\
\text { Under Standard Reaction Conditions (Average). }\end{array}$} \\
\hline \multirow{2}{*}{$\begin{array}{c}\text { Time } \\
\text { [h] }\end{array}$} & \multirow{2}{*}{$\begin{array}{c}\text { Std. } \\
{[\mathrm{mol} / \mathrm{L}]}\end{array}$} & \multicolumn{2}{|c|}{ Donor $5 f$} & \multicolumn{2}{|c|}{ Product $6 \mathbf{f}$} & \multicolumn{2}{|c|}{ Donor $\mathbf{5 f}$} & \multicolumn{2}{|c|}{ Product 6f } \\
\hline & & $\begin{array}{c}\alpha \\
{[\mathrm{mol} / \mathrm{L}]}\end{array}$ & $\begin{array}{c}\beta \\
{[\mathrm{mol} / \mathrm{L}]}\end{array}$ & $\begin{array}{c}\beta \\
{[\mathrm{mol} / \mathrm{L}]}\end{array}$ & $\begin{array}{c}\alpha \\
{[\mathrm{mol} / \mathrm{L}]}\end{array}$ & $\alpha$ & $\beta$ & $\beta$ & $\alpha$ \\
\hline 0 & $3.60^{*} 10^{-2}$ & $1.26^{*} 10^{-4}$ & $2.89 * 10^{-2}$ & 0 & 0 & 1 & $>99$ & 0 & 0 \\
\hline 1 & $3.60 * 10^{-2}$ & $5.22 * 10^{-4}$ & $2.67^{*} 10^{-2}$ & $3.64^{*} 10^{-3}$ & $7.74^{*} 10^{-4}$ & 1 & 60 & 5 & 1 \\
\hline 2 & $3.60 * 10^{-2}$ & $4.86^{*} 10^{-4}$ & $2.29 * 10^{-2}$ & $7.72^{*} 10^{-3}$ & $8.28 * 10^{-4}$ & 1 & 52 & 10 & 1 \\
\hline 3 & $3.60 * 10^{-2}$ & $3.96 * 10^{-4}$ & $1.90 * 10^{-2}$ & $1.08 * 10^{-2}$ & $9.54^{*} 10^{-4}$ & 1 & 52 & 12 & 1 \\
\hline 4 & $3.60^{*} 10^{-2}$ & $6.66^{*} 10^{-4}$ & $1.53^{*} 10^{-2}$ & $1.45^{*} 10^{-2}$ & $1.26^{*} 10^{-3}$ & 1 & 24 & 12 & 1 \\
\hline 5 & $3.60 * 10^{-2}$ & $4.86^{*} 10^{-4}$ & $1.24 * 10^{-2}$ & $1.78 * 10^{-2}$ & $1.48^{*} 10^{-3}$ & 1 & 26 & 12 & 1 \\
\hline 6 & $3.60 * 10^{-2}$ & $4.50 * 10^{-4}$ & $8.32^{*} 10^{-3}$ & $1.94 * 10^{-2}$ & $1.39 * 10^{-3}$ & 1 & 19 & 14 & 1 \\
\hline 7 & $3.60^{*} 10^{-2}$ & $4.32 * 10^{-4}$ & $4.86^{*} 10^{-3}$ & $2.20^{*} 10^{-2}$ & $1.62 * 10^{-3}$ & 1 & 13 & 14 & 1 \\
\hline 8 & $3.60^{*} 10^{-2}$ & $3.06^{*} 10^{-4}$ & $3.65^{*} 10^{-3}$ & $2.42^{*} 10^{-2}$ & $1.91 * 10^{-3}$ & 1 & 12 & 14 & 1 \\
\hline 9 & $3.60 * 10^{-2}$ & $3.24^{*} 10^{-4}$ & $2.99 * 10^{-3}$ & $2.54^{*} 10^{-2}$ & $2.03^{*} 10^{-3}$ & 1 & 15 & 13 & 1 \\
\hline 10 & $3.60 * 10^{-2}$ & $1.44^{*} 10^{-4}$ & $1.46^{*} 10^{-3}$ & $2.74 * 10^{-2}$ & $1.93 * 10^{-3}$ & 1 & 11 & 15 & 1 \\
\hline
\end{tabular}

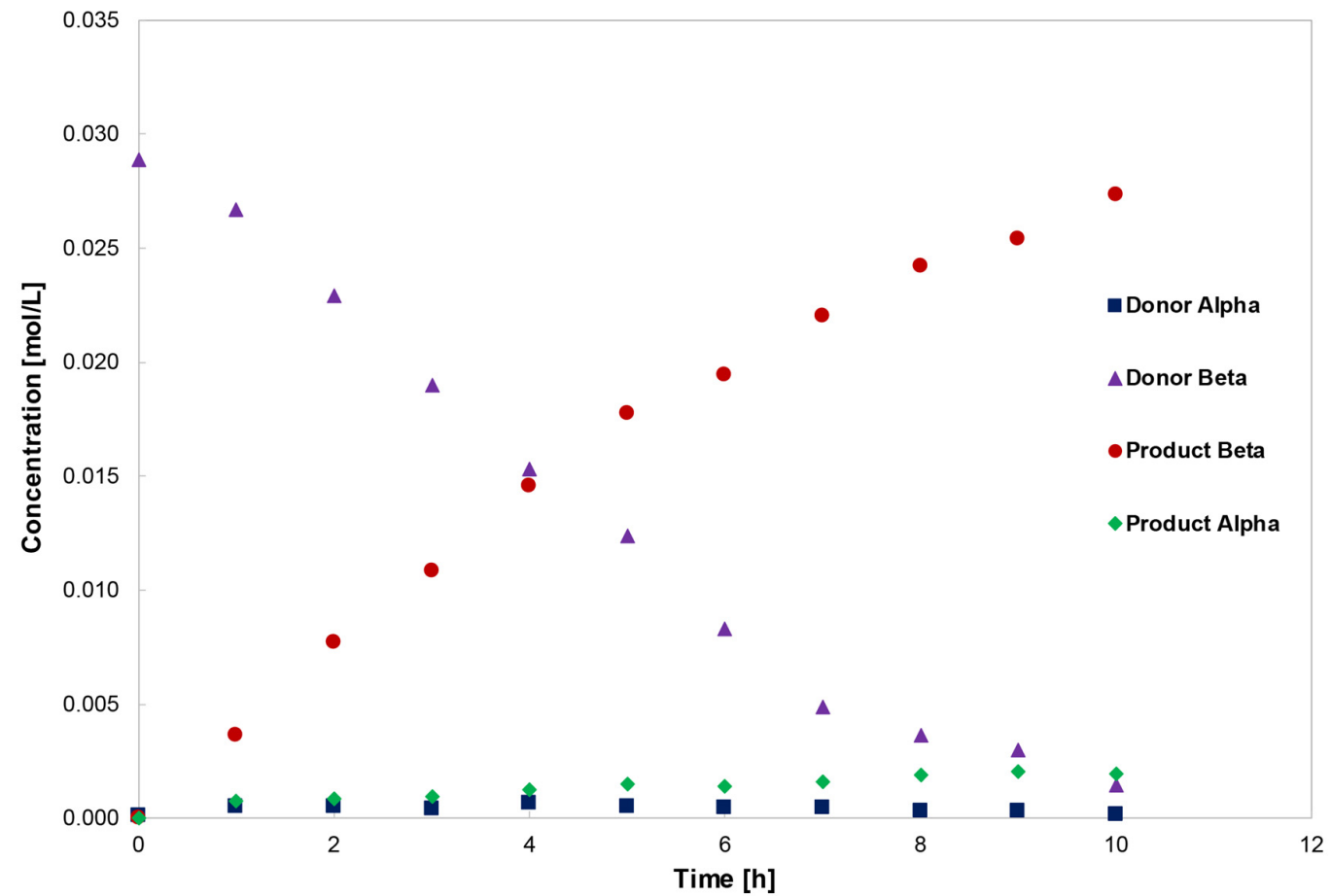

Figure S22 Average Kinetic Trace for the Reaction of $\mathbf{5 f}$ with 1,2:3,4-Di-O-isopropylidene- $\alpha$-D-galactopyranose 2a Under Standard Reaction Conditions (Blue Squares: $\alpha-5 f$; Purple Triangles: $\beta-5 f$; Red Dots: $\beta-6 \mathbf{6}$; Green Diamonds: $\alpha-6 f$ ). 


\section{Catalyst-Induced Donor Solubility and Resting State Determination}

\section{Procedure for Catalyst-Induced Donor Solubility Determination by ${ }^{31} \mathrm{P}$ NMR}

To an NMR tube containing 1c (10.2 mg, $0.018 \mathrm{mmol}, 1.0$ eq.) was added a stock solution of $n \mathrm{Pr}_{2} \mathrm{O}(0.8 \mathrm{~mL})$, containing the internal standard triphenylphopshine $\left(4.7 \mathrm{mg}, 0.018 \mathrm{mmol}, 1.0\right.$ eq.) and a ${ }^{31} \mathrm{P}$ NMR spectrum was recorded at room temperature after vigorous mixing (locking and shimming performed with a separate NMR tube containing $n \mathrm{Pr}_{2} \mathrm{O}(0.7 \mathrm{~mL})$ and $\left.\mathrm{C}_{6} \mathrm{D}_{6}(0.1 \mathrm{~mL})\right)$. To the resulting heterogenous mixture, a stock solution of $4 \mathbf{i}\left(3.7 \mathrm{mg}, 0.0036 \mathrm{mmol}, 0.2 \mathrm{eq}\right.$.) in $n \mathrm{Pr}_{2} \mathrm{O}$ $(0.2 \mathrm{~mL})$ was added, and a ${ }^{31} \mathrm{P}$ NMR spectrum was recorded at room temperature after vigorous mixing. Subsequently, a stock solution of 1,2:3,4-di-O-isopropylidene- $\alpha$-Dgalactopyranose $2 \mathrm{a}(9.4 \mathrm{mg}, 0.018 \mathrm{mmol}, 1.0$ eq. $)$ in $n \mathrm{Pr}_{2} \mathrm{O}(0.1 \mathrm{~mL})$ was added, and the sample was analyzed by ${ }^{31} \mathrm{P}$ NMR at room temperature after vigorous mixing.

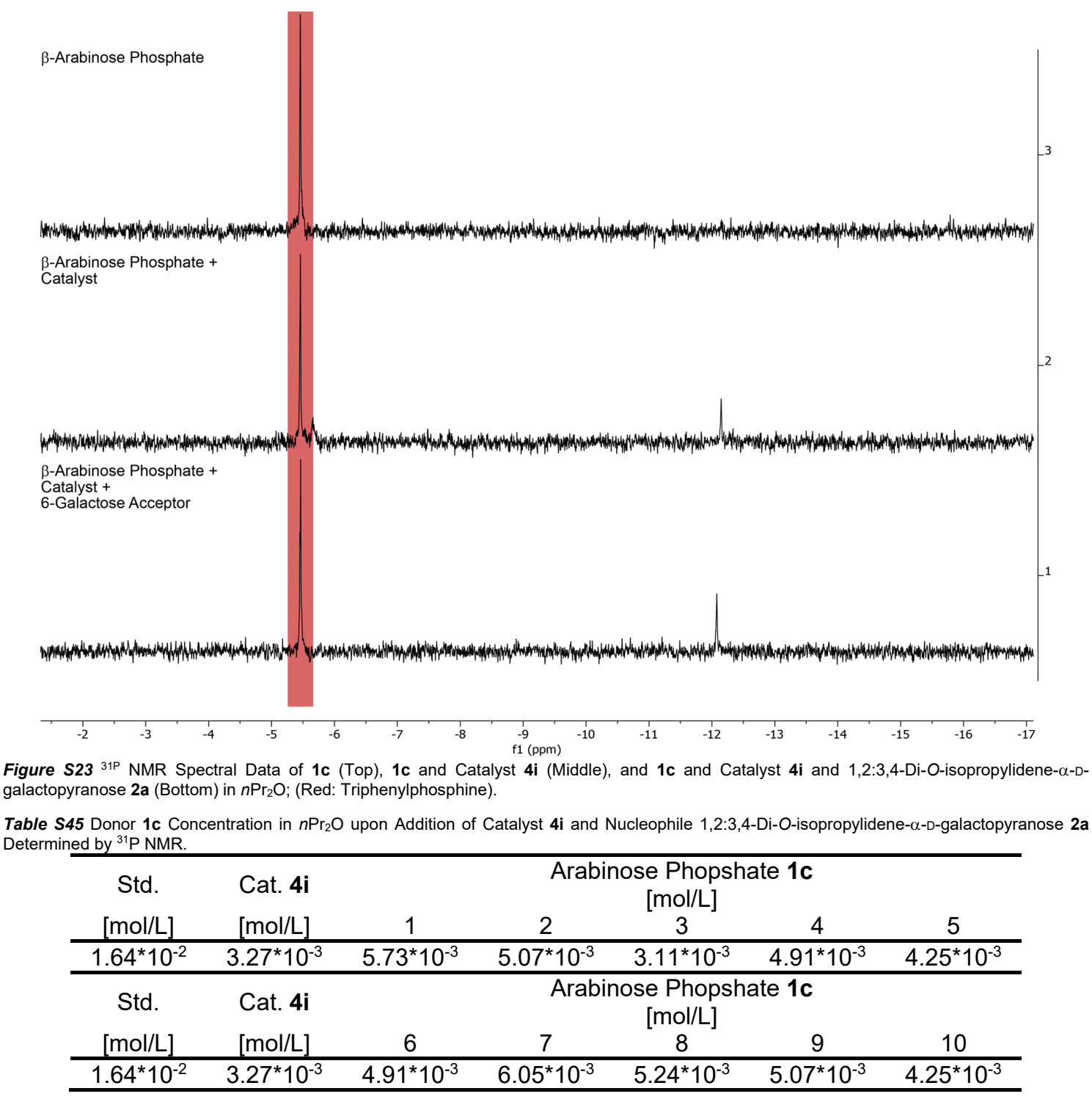


Table S46 Average Donor 1c Concentration in $n \mathrm{Pr}_{2} \mathrm{O}$ upon Addition of Catalyst $4 \mathbf{i}$ and Acceptor 2a, and Donor to Catalyst Ratio Determined by ${ }^{31} \mathrm{P}$ NMR.

\begin{tabular}{cc}
\hline $\begin{array}{c}\text { Arabinose Phosphate 1c } \\
{[\mathrm{mol} / \mathrm{L}]}\end{array}$ & $\begin{array}{c}\text { Arabinose Phosphate : Catalyst } \\
\text { Ratio }\end{array}$ \\
\hline $4.86^{*} 10^{-3} \pm 8.31^{*} 10^{-4}$ & $1.49 \pm 0.25$ \\
\hline
\end{tabular}

\section{Procedure for Resting-State Determination under Standard Reaction Conditions}

To an NMR tube containing 1c $(10.2 \mathrm{mg}, 0.018 \mathrm{mmol}, 1.0$ eq.) and $100 \mathrm{mg} 4 \AA$ molecular sieves was added a stock solution of $n \mathrm{Pr}_{2} \mathrm{O}(0.8 \mathrm{~mL})$ containing the internal standard, triphenylphosphine (4.7 mg, $0.018 \mathrm{mmol}, 1.0$ eq.), and a ${ }^{31} \mathrm{P}$ NMR spectrum was recorded at $40^{\circ} \mathrm{C}$ after vigorous mixing (locking and shimming performed with a separate NMR tube containing $n \operatorname{Pr}_{2} \mathrm{O}(0.7 \mathrm{~mL})$ and $\left.\mathrm{C}_{6} \mathrm{D}_{6}(0.1 \mathrm{~mL})\right)$. To the resulting heterogenous mixture a stock solution of $4 \mathbf{i}\left(1.9 \mathrm{mg}, 0.0018 \mathrm{mmol}, 0.1\right.$ eq.) in $n \operatorname{Pr}_{2} \mathrm{O}$ $(0.1 \mathrm{~mL})$ and a stock solution of 1,2:3,4-di-O-isopropylidene- $\alpha$-D-galactopyranose $\mathbf{2 a}$ (9.4 mg, $0.018 \mathrm{mmol}, 1.0$ eq.) in $n \mathrm{Pr}_{2} \mathrm{O}(0.1 \mathrm{~mL})$ were added, and a ${ }^{31} \mathrm{P}$ NMR spectrum was recorded every 6 minutes over $1 \mathrm{~h}$ at $40^{\circ} \mathrm{C}$.

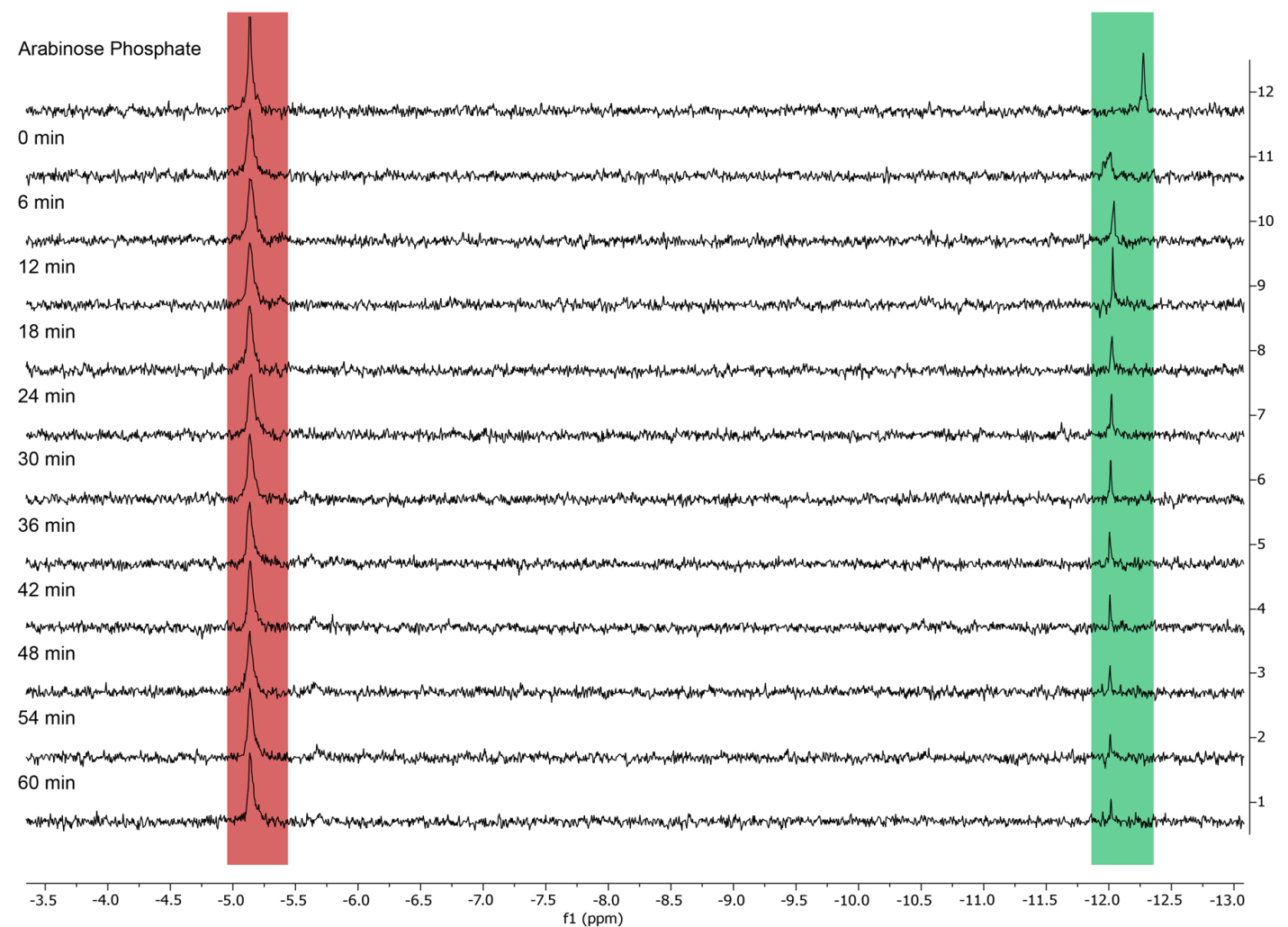

Figure S24 ${ }^{31 P}$ NMR Spectral Data of 1c (Top Spectrum), 1c, Catalyst 4i and 1,2:3,4-Di-O-isopropylidene- $\alpha$-D-galactopyranose 2a over 60 min in $n \mathrm{Pr}_{2} \mathrm{O}$ at $40{ }^{\circ} \mathrm{C}$ Containing $4 \AA$ MS (Red: Triphenylphosphine; Green: $\alpha-1 \mathrm{c}$ ).

Table S47 Donor 1c and Free Phosphoric Acid Concentration in $n \mathrm{Pr}_{2} \mathrm{O}$ Containing $4 \AA$ MS upon Addition of Catalyst 4i and 1,2:3,4-Di-Oisopropylidene- $\alpha$-D-galactopyranose $2 \mathrm{a}$ Determined by ${ }^{31} \mathrm{P}$ NMR over $60 \mathrm{~min}$.

\begin{tabular}{ccccccc}
\hline & Donor & $0 \mathrm{~min}$ & \multicolumn{4}{c}{ Concentration [mol/L] } \\
$6 \mathrm{~min}$ & $12 \mathrm{~min}$ & $18 \mathrm{~min}$ & $24 \mathrm{~min}$ \\
\hline $\begin{array}{c}\text { Arabinose Phos. } \\
\text { 1c }\end{array}$ & $6.12^{*} 10^{-3}$ & $5.04^{*} 10^{-3}$ & $3.96^{*} 10^{-3}$ & $4.14^{*} 10^{-3}$ & $3.42^{*} 10^{-3}$ & $4.14^{*} 10^{-3}$ \\
\hline & $30 \mathrm{~min}$ & $36 \mathrm{~min}$ & $42 \mathrm{~min}$ & $48 \mathrm{~min}$ & $54 \mathrm{~min}$ & $60 \mathrm{~min}$ \\
\hline $\begin{array}{c}\text { Arabinose Phos. } \\
\text { 1c }\end{array}$ & $3.24^{*} 10^{-3}$ & $2.88^{*} 10^{-3}$ & $2.34^{*} 10^{-3}$ & $1.98^{*} 10^{-3}$ & $1.44^{*} 10^{-3}$ & $1.62^{*} 10^{-3}$ \\
\hline
\end{tabular}


Table S48 Conversion to Product 3a, Hydrolysis, Donor 1c and Product Anomeric Ratio after 60 min in $n \mathrm{Pr}_{2} \mathrm{O}$ Containing $4 \AA \mathrm{MS}$ at $40{ }^{\circ} \mathrm{C}$ Determined by ${ }^{1} \mathrm{H}$ NMR.

\begin{tabular}{cccc}
\hline $\begin{array}{c}\text { Conv. to Product 3a } \\
\text { by }{ }^{1} \mathrm{H} \text { NMR }\end{array}$ & $\begin{array}{c}\text { Donor 1c } \\
\alpha: \beta\end{array}$ & $\begin{array}{c}\text { Product 3a } \\
\alpha: \beta\end{array}$ & $\begin{array}{c}\text { Hydrolysis } \\
\text { by }{ }^{1} \mathrm{H} \text { NMR }\end{array}$ \\
\hline $43 \%$ & $18: 1$ & $1: 20$ & $<5 \%$ \\
\hline
\end{tabular}

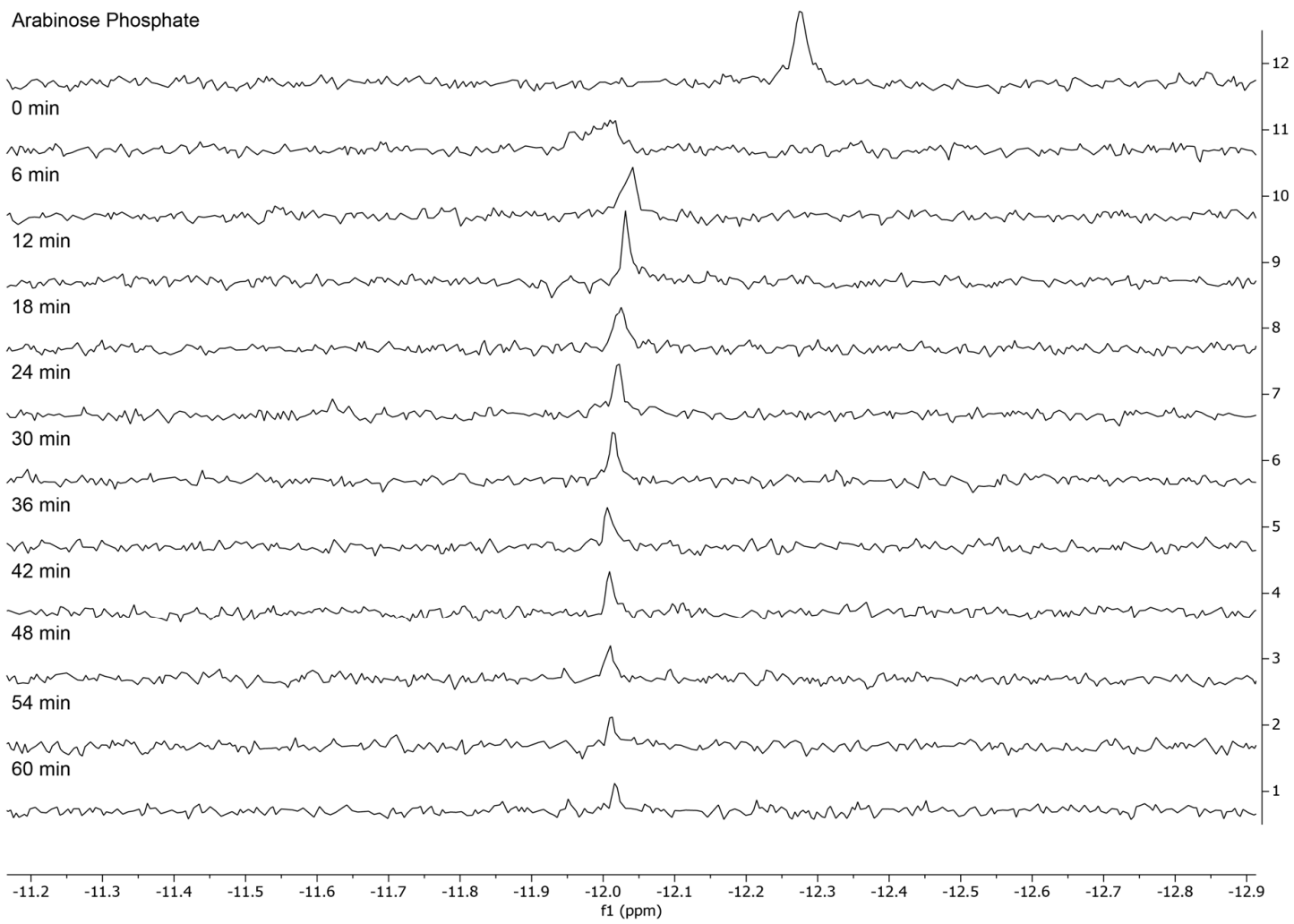

Figure S25 ${ }^{31} \mathrm{P}$ NMR Shift of Arabinose Phosphate in Solutions of 1c (Top Spectrum), and 1c, catalyst 4i and 1,2:3,4-Di-O-isopropylidene- $\alpha$-Dgalactopyranose $2 \mathrm{a}$ over 60 min in $n \mathrm{Pr}_{2} \mathrm{O}$ Containing $4 \AA \mathrm{MS}$. 


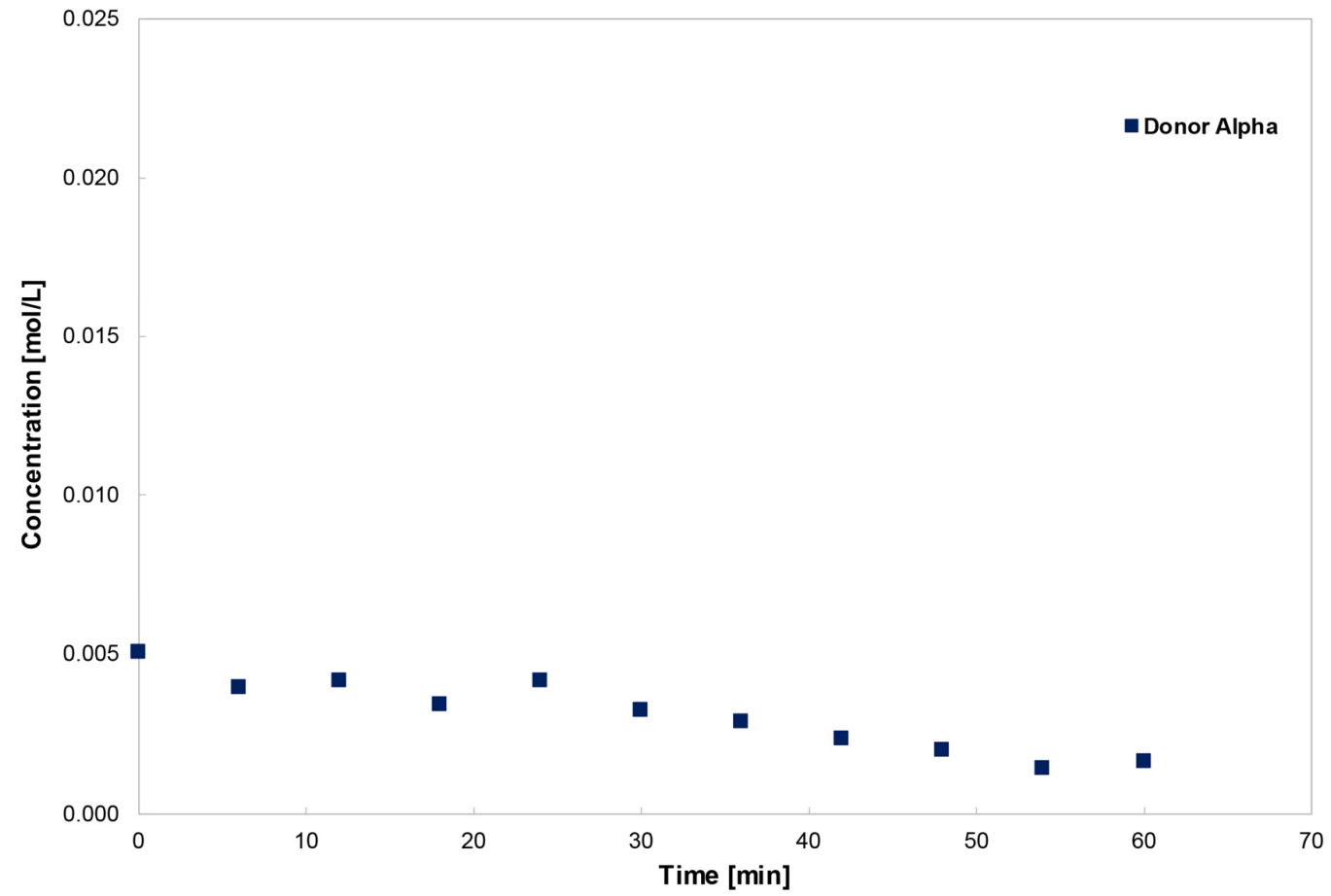

Figure S26 Concentration of $\alpha-1 \mathrm{c}$ over $60 \mathrm{~min}$ in $n \mathrm{Pr}_{2} \mathrm{O}$ Containing $4 \AA$ MS at $40{ }^{\circ} \mathrm{C}$ Determined by ${ }^{31} \mathrm{P}$ NMR (Blue Squares: $\left.\alpha-1 \mathrm{c}\right)$. 


\section{Procedure for Resting State Determination without Molecular Sieves}

To an NMR tube containing 1c $(10.2 \mathrm{mg}, 0.018 \mathrm{mmol}, 1.0$ eq.) was added a stock solution of $n \mathrm{Pr}_{2} \mathrm{O}(0.8 \mathrm{~mL})$ containing the internal standard, triphenylphosphine (4.7 mg, $0.018 \mathrm{mmol}, 1.0$ eq.), and a ${ }^{31} \mathrm{P}$ NMR spectrum was recorded at $40{ }^{\circ} \mathrm{C}$ after vigorous mixing (locking and shimming performed with a separate NMR tube containing $n \mathrm{Pr}_{2} \mathrm{O}(0.7 \mathrm{~mL})$ and $\left.\mathrm{C}_{6} \mathrm{D}_{6}(0.1 \mathrm{~mL})\right)$. To the resulting heterogenous mixture a stock solution of $4 \mathbf{i}(1.9 \mathrm{mg}, 0.0018 \mathrm{mmol}, 0.1$ eq. $)$ in $n \mathrm{Pr}_{2} \mathrm{O}(0.1 \mathrm{~mL})$ and a stock solution of 1,2:3,4-di-O-isopropylidene- $\alpha$-D-galactopyranose $2 \mathrm{a}(9.4 \mathrm{mg}, 0.018 \mathrm{mmol}$, 1.0 eq.) in $n \mathrm{Pr}_{2} \mathrm{O}(0.1 \mathrm{~mL})$ were added, and a ${ }^{31} \mathrm{P}$ NMR spectrum was recorded every 6 minutes over $1 \mathrm{~h}$ at $40^{\circ} \mathrm{C}$.

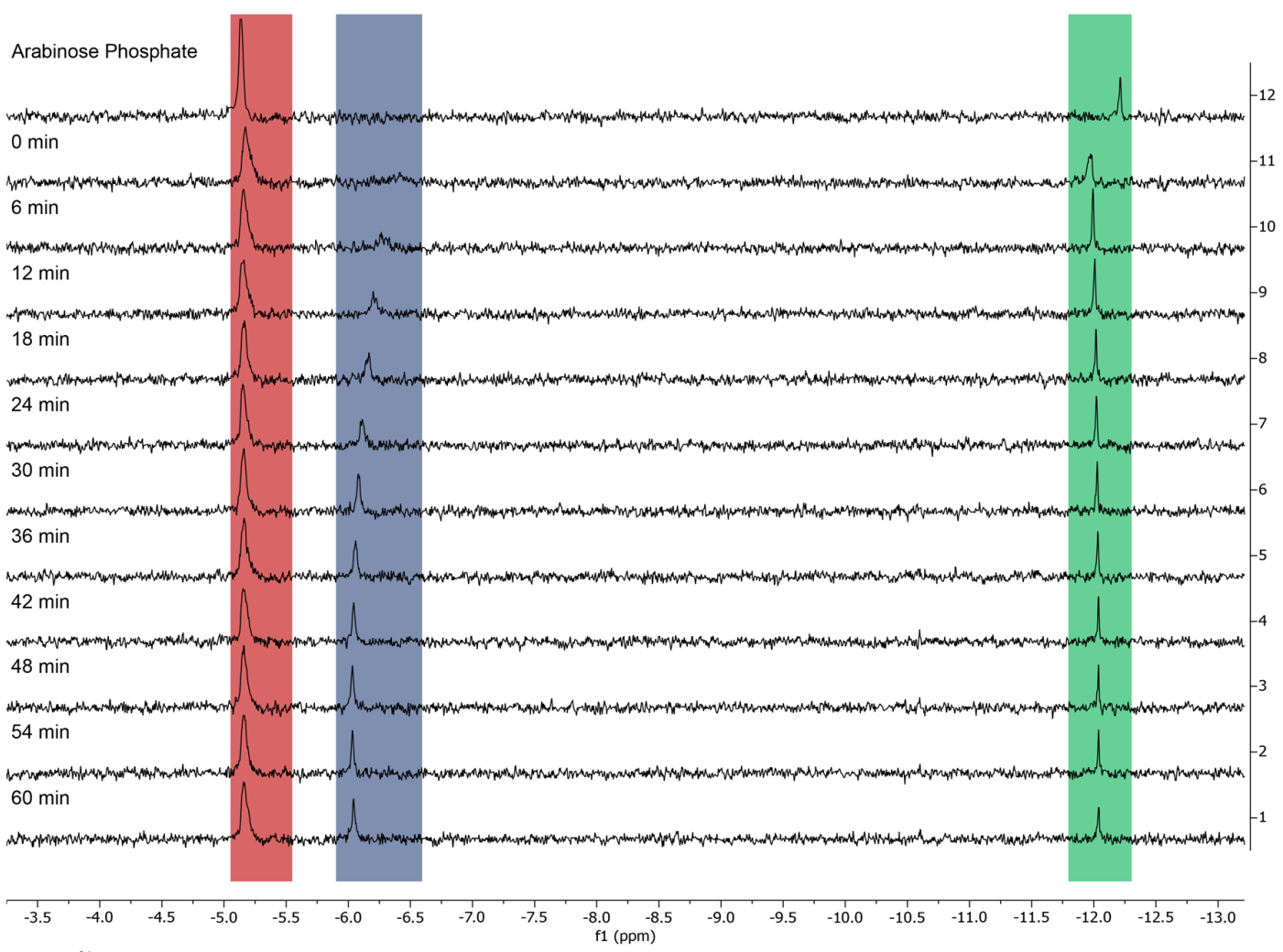

Figure S27 ${ }^{31} \mathrm{P}$ NMR Spectral Data of 1c (Top Spectrum), 1c, Catalyst 4i and 1,2:3,4-Di-O-isopropylidene- $\alpha$-D-galactopyranose $2 \mathrm{a}$ over 60 min in $n \mathrm{Pr}_{2} \mathrm{O}$ at $40{ }^{\circ} \mathrm{C}$; (Red: Triphenylphosphine; Blue: Phosphoric Acid; Green: $\alpha-1 \mathrm{c}$ ).

Table S49 Donor 1c and Free Phosphoric Acid Concentration in $n \mathrm{Pr}_{2} \mathrm{O}$ upon Addition of Catalyst 4i and 1,2:3,4-Di-O-isopropylidene- $\alpha$-Dgalactopyranose 2a Determined by ${ }^{31} \mathrm{P}$ NMR over $60 \mathrm{~min}$.

\begin{tabular}{|c|c|c|c|c|c|c|}
\hline & \multicolumn{6}{|c|}{ Concentration [mol/L] } \\
\hline & Donor & $0 \mathrm{~min}$ & $6 \mathrm{~min}$ & $12 \min$ & $18 \mathrm{~min}$ & $24 \min$ \\
\hline $\begin{array}{c}\text { Arabinose Phos. } \\
\text { 1c }\end{array}$ & $3.24 * 10^{-3}$ & $5.76^{*} 10^{-3}$ & $5.22 * 10^{-3}$ & $5.94 * 10^{-3}$ & $4.68 * 10^{-3}$ & $4.68 * 10^{-3}$ \\
\hline \multirow[t]{3}{*}{ Phosphoric Acid } & 0 & 0 & $3.06 * 10^{-3}$ & $4.86 * 10^{-3}$ & $5.22 * 10^{-3}$ & $5.94 * 10^{-3}$ \\
\hline & \multicolumn{6}{|c|}{ Concentration [mol/L] } \\
\hline & $30 \mathrm{~min}$ & $36 \min$ & $42 \min$ & $48 \min$ & $54 \mathrm{~min}$ & $60 \mathrm{~min}$ \\
\hline $\begin{array}{c}\text { Arabinose Phos. } \\
\text { 1c }\end{array}$ & $4.86^{*} 10^{-3}$ & $3.42^{*} 10^{-3}$ & $4.68 * 10^{-3}$ & $4.32 * 10^{-3}$ & $4.32 * 10^{-3}$ & $3.60 * 10^{-3}$ \\
\hline Phosphoric Acid & $7.38 * 10^{-3}$ & $7.02^{*} 10^{-3}$ & $6.48^{*} 10^{-3}$ & $7.74 * 10^{-3}$ & $6.30 * 10^{-3}$ & $7.56 * 10^{-3}$ \\
\hline
\end{tabular}

Table S50 Conversion to Product 3a, Hydrolysis, Donor 1c and Product 3a Anomeric Ratio after 60 min in $n \mathrm{Pr}_{2} \mathrm{O}$ at $40{ }^{\circ} \mathrm{C}$ Determined by ${ }^{1} \mathrm{H}$ NMR and Conversion to Free Phopshoric Acid after $60 \mathrm{~min}$ in $n \mathrm{Pr}_{2} \mathrm{O}$ at $40{ }^{\circ} \mathrm{C}$ Determined by ${ }^{31} \mathrm{P}$ NMR.

\begin{tabular}{ccccc}
\hline $\begin{array}{c}\text { Conv. to Product 3a } \\
\text { by }{ }^{1} \mathrm{H} \text { NMR }\end{array}$ & $\begin{array}{c}\text { Donor 1c } \\
\alpha: \beta\end{array}$ & $\begin{array}{c}\text { Product 3a } \\
\alpha: \beta\end{array}$ & $\begin{array}{c}\text { Hydrolysis } \\
\text { by }{ }^{1} \mathrm{H} \text { NMR }\end{array}$ & $\begin{array}{c}\text { Conv. to free Phosphoric Acid } \\
\text { by }{ }^{31} \mathrm{P} \text { NMR }\end{array}$ \\
\hline $31 \%$ & $5: 1$ & $1: 10$ & $26 \%$ & $42 \%$ \\
\hline
\end{tabular}




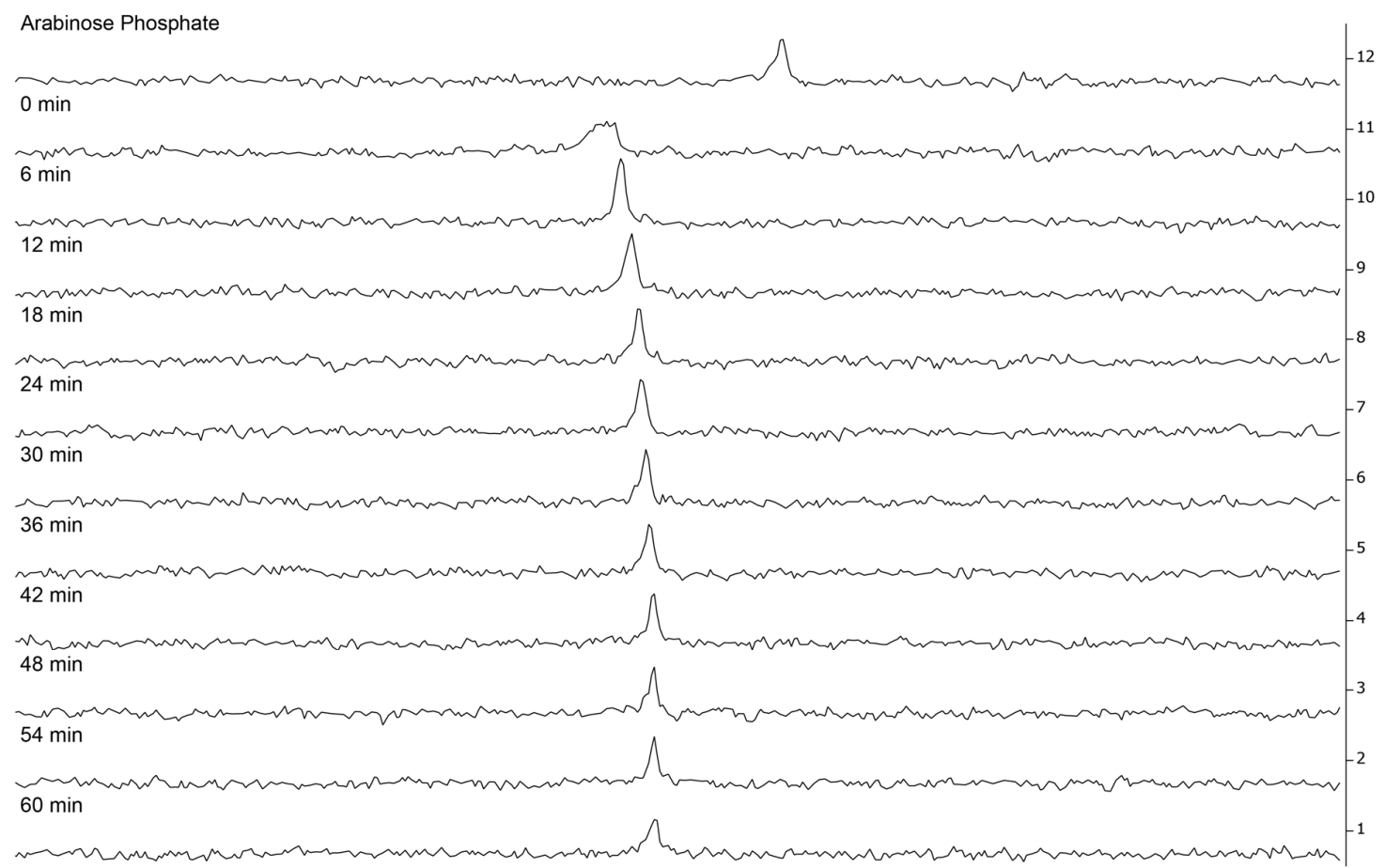

\begin{tabular}{llllllllllllllllll|l}
\hline-11.2 & -11.3 & -11.4 & -11.5 & -11.6 & -11.7 & -11.8 & -11.9 & -12.0 & -12.1 & -12.2 & -12.3 & -12.4 & -12.5 & -12.6 & -12.7 & -12.8 & -12.9 \\
\hline
\end{tabular}

Figure S28 ${ }^{31 P}$ NMR Shift of Arabinose Phosphate in Solutions of 1c (Top Spectrum), and 1c, Catalyst 4i and 1,2:3,4-Di-O-isopropylidene- $\alpha$-Dgalactopyranose 2 a over $60 \min$ in $n \mathrm{Pr}_{2} \mathrm{O}$.

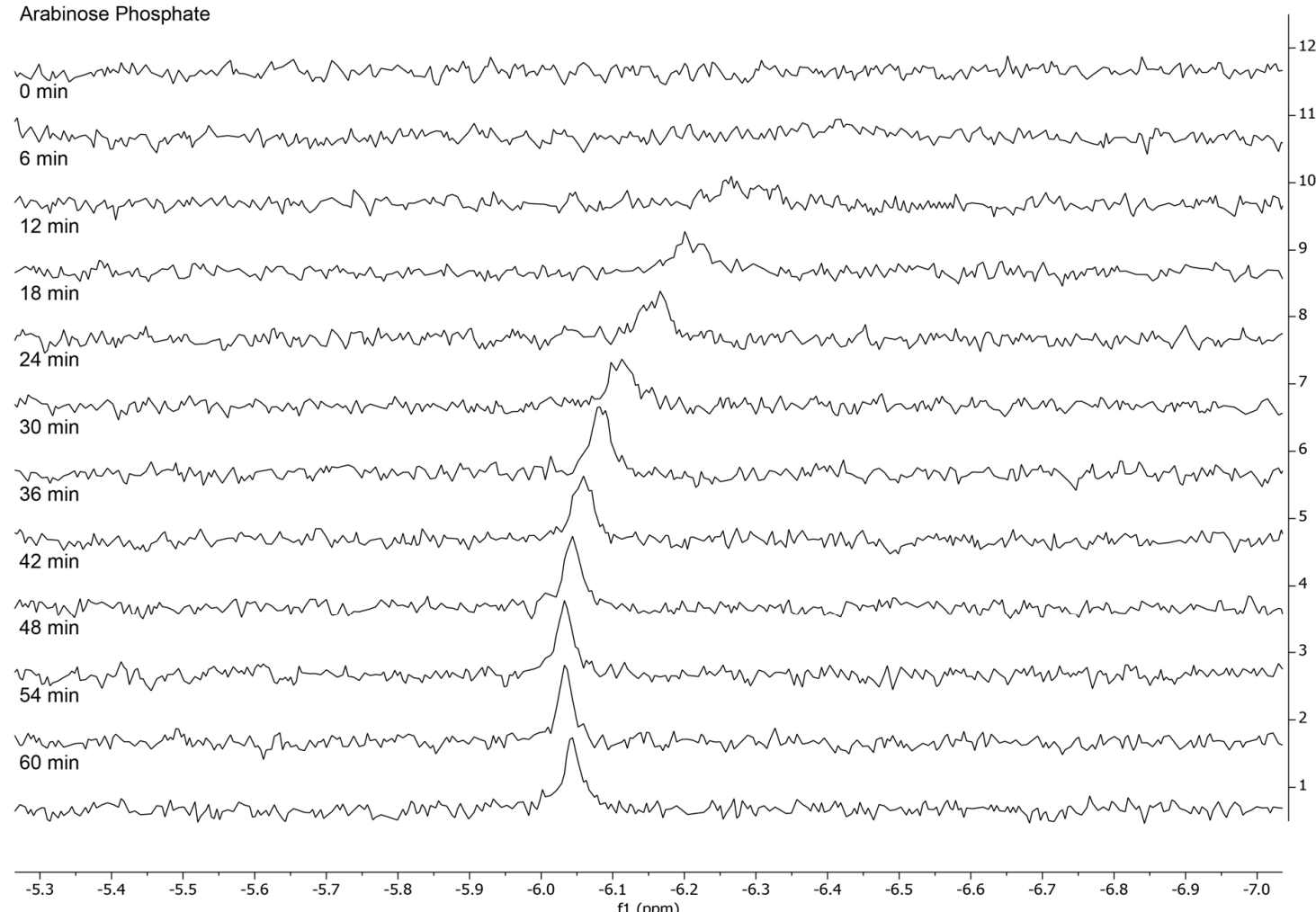

Figure S29 ${ }^{31 \mathrm{P}}$ NMR Shift of Free Phosphoric Acid in Solutions of 1c (Top Spectrum), and 1C, Catalyst 4i and 1,2:3,4-Di-O-isopropylidene- $\alpha$-Dgalactopyranose 2 a over 60 min in $n \mathrm{Pr}_{2} \mathrm{O}$. 


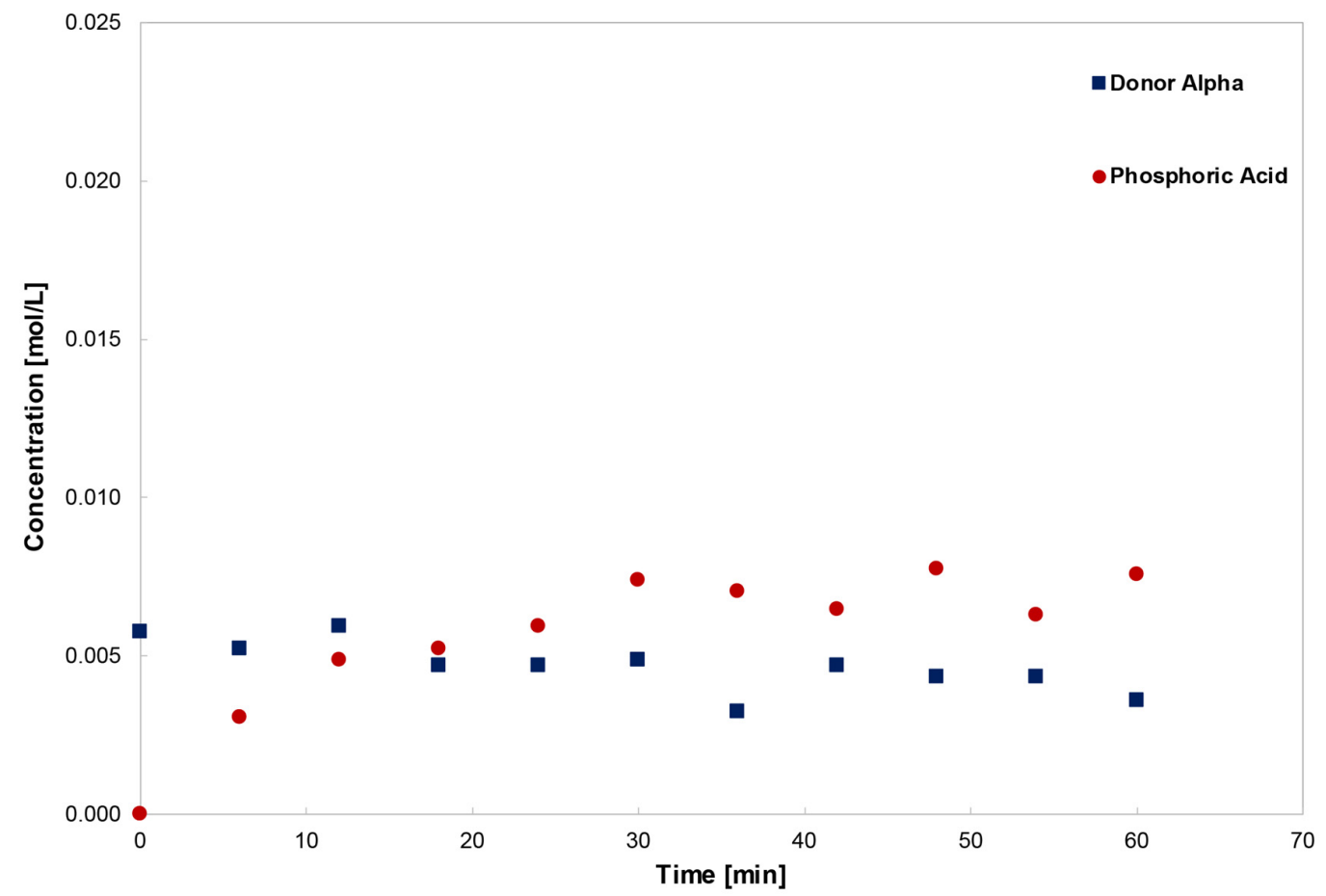

Figure S30 Concentration of $\alpha-1 \mathrm{c}$ and Free Phosphoric Acid over 60 min in $n \mathrm{Pr}_{2} \mathrm{O}$ at $40{ }^{\circ} \mathrm{C}$ Determined by ${ }^{31} \mathrm{P}$ NMR (Blue Squares: $\alpha-1 \mathrm{c}$; Red Dots: Free Phosphoric Acid).

\section{Discussion}

To test the hypothesis of a donor-catalyst-complex resting state, the ${ }^{31} \mathrm{P}$ NMR shift of arabinose phosphate 1c was analyzed in the presence and absence of catalyst $\mathbf{4 i}$ and 6-galactose acceptor $\mathbf{2 a}$. At room temperature, arabinose phosphate $\mathbf{1 c}$ is insoluble in $n \mathrm{Pr}_{2} \mathrm{O}$, with no observable signal in the ${ }^{31} \mathrm{P} \mathrm{NMR}$ spectrum. Addition of $20 \mathrm{~mol} \%$ catalyst $\mathbf{4} \mathbf{i}$ immediately produced a signal at $-12.0 \mathrm{ppm}$, likely corresponding to catalyst-bound arabinose phosphate (see Figure S23). Unfortunately, conducting the reaction at room temperature did not lead to product formation. At $40{ }^{\circ} \mathrm{C}$, low concentrations of solubilized arabinose phosphate 1c were observed at $-12.3 \mathrm{ppm}$ in the absence of catalyst. Addition of catalyst $4 \mathbf{i}$ resulted in a persistent downfield shift to $-12.0 \mathrm{ppm}$ (see Figure S24), consistent with formation of the catalyst-donor complex (see Figure S23). Monitoring the glycosylation reaction-with 6-gal $2 \mathbf{a}$ as the acceptor-over the course of $1 \mathrm{~h}$ resulted in product formation (Table S47, 43\% NMR yield, $\beta: \alpha 20: 1)$. The chemical shift for the arabinose phosphate remained constant over the course of the reaction confirming that catalyst-bound donor is the resting state of the catalytic reaction.

Further mechanistic investigations were carried out on the epimerization process. To study the effect of free phosphoric acid on the reaction, the ${ }^{31} \mathrm{P}$ NMR kinetic experiments were repeated in the absence of molecular sieves. Phosphoric acid was formed over the course of the reaction, appearing at $-6.2 \mathrm{ppm}$ in the ${ }^{31} \mathrm{P} N M R$ spectrum (Figure S27). This signal underwent a strong downfield shift as the reaction proceeded, concomitant with a corresponding upfield shift of the arabinose phosphate signal. This observation suggests competitive binding of free phosphoric acid to the bis-thiourea catalyst. We observed decreased anomeric purity of both the donor 1c and the disaccharide product $3 \mathbf{a}$ after 1 hour of reaction time (see Table S49, 31\% NMR yield, 10:1 $\beta: \alpha$ ), providing evidence for phosphoric acid-induced acceleration of 
the epimerization. Significant quantities of hydrolyzed donor were seen in these experiments, further highlighting the essential role of molecular sieves. 


\section{X-ray Crystallography}

A crystal was mounted on a diffractometer and data was collected at $100 \mathrm{~K}$. The intensities of the reflections were collected by means of a Bruker APEX DUO CCD diffractometer ( $\mathrm{Cuk}_{\alpha}$ radiation, $\lambda=1.54178 \AA$ ), and equipped with an Oxford Cryosystems nitrogen flow apparatus. The collection method involved $1.0^{\circ}$ scans in $\omega$ at $-30^{\circ},-55^{\circ},-80^{\circ}, 30^{\circ}, 55^{\circ}, 80^{\circ}$ and $115^{\circ}$ in $2 \theta$. Data integration down to $0.84 \AA$ resolution was carried out using SAINT V8.37 $\mathrm{A}^{18}$ with reflection spot size optimization. Absorption corrections were made with the program SADABS ${ }^{18}$. The structure was solved by the Intrinsic Phasing methods and refined by least-squares methods again $F^{2}$ using SHELXT-2014 20,21 and SHELXL-2014 20,21 with OLEX 2 interface. ${ }^{19}$ Nonhydrogen atoms were refined anisotropically, and hydrogen atoms were allowed to ride on the respective atoms. Crystal data as well as details of data collection and refinement are summarized in Table S49, and geometric parameters are shown in Table 2. The Ortep plots produced with SHELXL-2014 program, and the other drawings were produced with Accelrys DS Visualizer 2.0..$^{22,23}$

\section{X-Ray Crystallographic Data for $3 r$}

Table S51 Experimental details.

\begin{tabular}{|c|c|}
\hline & JBM-5-118 \\
\hline \multicolumn{2}{|l|}{ Crystal data } \\
\hline Chemical formula & $\mathrm{C}_{74} \mathrm{H}_{90} \mathrm{~N}_{2} \mathrm{O}_{18}$ \\
\hline$M_{\mathrm{r}}$ & 1295.47 \\
\hline Crystal system, space group & Monoclinic, $P 2_{1}$ \\
\hline Temperature $(\mathrm{K})$ & 100 \\
\hline$a, b, c(\AA)$ & $11.04683(14), 9.43212(12), 16.2660(2)$ \\
\hline$\beta\left(^{\circ}\right)$ & $96.9247(12)$ \\
\hline$V\left(\AA^{3}\right)$ & $1682.48(4)$ \\
\hline$Z$ & 1 \\
\hline Radiation type & $\mathrm{Cu} K \alpha$ \\
\hline$\mu\left(\mathrm{mm}^{-1}\right)$ & 0.75 \\
\hline Crystal size $(\mathrm{mm})$ & $0.18 \times 0.08 \times 0.04$ \\
\hline \multicolumn{2}{|l|}{ Data collection } \\
\hline Diffractometer & Bruker D8 goniometer with CCD area detector \\
\hline Absorption correction & $\begin{array}{l}\text { Multi-scan } \\
\text { SADABS }\end{array}$ \\
\hline$T_{\min }, T_{\max }$ & $0.688,0.864$ \\
\hline $\begin{array}{l}\text { No. of measured, independent and } \\
\text { observed }[I>2 \sigma(I)] \text { reflections }\end{array}$ & $38199,5734,5541$ \\
\hline$R_{\text {int }}$ & 0.082 \\
\hline$(\sin \theta / \lambda)_{\max }\left(\AA^{-1}\right)$ & 0.595 \\
\hline Refinement & \\
\hline
\end{tabular}




\begin{tabular}{|l|l|}
\hline$R\left[F^{2}>2 \sigma\left(F^{2}\right)\right], w R\left(F^{2}\right), S$ & $0.034,0.091,1.07$ \\
\hline No. of reflections & 5734 \\
\hline No. of parameters & 428 \\
\hline No. of restraints & 1 \\
\hline H-atom treatment & H-atom parameters constrained \\
\hline$\Delta \rho_{\text {max }}, \Delta \rho_{\text {min }}\left(\mathrm{e} \AA^{-3}\right)$ & $0.20,-0.20$ \\
\hline Absolute structure & Flack x determined using 2442 quotients $[(\mathrm{I}+)-(\mathrm{I}-)] /[(\mathrm{I}+)+(\mathrm{I}-)]^{24}$ \\
\hline Absolute structure parameter & $-0.13(11)$ \\
\hline
\end{tabular}

Computer programs: SAINT 8.37A, ${ }^{18}$ SHELXT2014, ${ }^{20,21}$ SHELXL2014, ${ }^{20,21}$ Bruker SHELXTL. ${ }^{20,21}$

Table S52 Geometric parameters ( $\AA$, ०).

\begin{tabular}{|c|c|c|c|}
\hline $\mathrm{O} 1-\mathrm{C} 5$ & $1.418(3)$ & $\mathrm{C} 8-\mathrm{C} 9$ & $1.389(4)$ \\
\hline $\mathrm{O} 1-\mathrm{C} 2$ & $1.454(3)$ & $\mathrm{C} 9-\mathrm{C} 10$ & $1.387(4)$ \\
\hline $\mathrm{O} 2-\mathrm{C} 1$ & $1.418(3)$ & $\mathrm{C} 10-\mathrm{C} 11$ & $1.384(4)$ \\
\hline $\mathrm{O} 2-\mathrm{C} 6$ & $1.436(3)$ & $\mathrm{C} 11-\mathrm{C} 12$ & $1.392(4)$ \\
\hline $\mathrm{O} 3-\mathrm{C} 13$ & $1.426(3)$ & $\mathrm{C} 13-\mathrm{C} 14$ & $1.519(3)$ \\
\hline $\mathrm{O} 3-\mathrm{C} 3$ & $1.433(3)$ & $\mathrm{C} 14-\mathrm{C} 15$ & $1.387(4)$ \\
\hline $\mathrm{O} 4-\mathrm{C} 4$ & $1.409(3)$ & $\mathrm{C} 14-\mathrm{C} 19$ & $1.394(4)$ \\
\hline $\mathrm{O} 4-\mathrm{C} 20$ & $1.437(3)$ & $\mathrm{C} 15-\mathrm{C} 16$ & $1.392(4)$ \\
\hline $\mathrm{O} 5-\mathrm{C} 5$ & $1.407(3)$ & $\mathrm{C} 16-\mathrm{C} 17$ & $1.383(4)$ \\
\hline $\mathrm{O} 5-\mathrm{C} 28$ & $1.441(3)$ & $\mathrm{C} 17-\mathrm{C} 18$ & $1.383(4)$ \\
\hline $\mathrm{O} 6-\mathrm{C} 31$ & $1.341(3)$ & $\mathrm{C} 18-\mathrm{C} 19$ & $1.397(4)$ \\
\hline $\mathrm{O} 6-\mathrm{C} 32$ & $1.451(3)$ & $\mathrm{C} 20-\mathrm{C} 21$ & $1.511(3)$ \\
\hline $\mathrm{O} 7-\mathrm{C} 31$ & $1.198(3)$ & $\mathrm{C} 21-\mathrm{C} 22$ & $1.394(3)$ \\
\hline $\mathrm{O} 8-\mathrm{C} 33$ & $1.213(3)$ & $\mathrm{C} 21-\mathrm{C} 26$ & $1.395(3)$ \\
\hline O9-C33 & $1.352(3)$ & $\mathrm{C} 22-\mathrm{C} 23$ & $1.386(4)$ \\
\hline $\mathrm{O} 9-\mathrm{C} 34$ & $1.483(3)$ & $\mathrm{C} 23-\mathrm{C} 24$ & $1.384(4)$ \\
\hline $\mathrm{N} 1-\mathrm{C} 33$ & $1.352(3)$ & $\mathrm{C} 24-\mathrm{C} 25$ & $1.401(4)$ \\
\hline $\mathrm{N} 1-\mathrm{C} 30$ & $1.455(3)$ & $\mathrm{C} 25-\mathrm{C} 26$ & $1.385(4)$ \\
\hline $\mathrm{N} 1-\mathrm{C} 27$ & $1.471(3)$ & $\mathrm{C} 27-\mathrm{C} 28$ & $1.513(3)$ \\
\hline $\mathrm{C} 1-\mathrm{C} 2$ & $1.518(3)$ & $\mathrm{C} 28-\mathrm{C} 29$ & $1.531(3)$ \\
\hline $\mathrm{C} 2-\mathrm{C} 3$ & $1.530(3)$ & $\mathrm{C} 29-\mathrm{C} 30$ & $1.542(3)$ \\
\hline $\mathrm{C} 3-\mathrm{C} 4$ & $1.532(3)$ & $\mathrm{C} 30-\mathrm{C} 31$ & $1.521(3)$ \\
\hline $\mathrm{C} 4-\mathrm{C} 5$ & $1.531(3)$ & C34-C35 & $1.520(4)$ \\
\hline $\mathrm{C} 6-\mathrm{C} 7$ & $1.496(3)$ & C34-C37 & $1.520(4)$ \\
\hline $\mathrm{C} 7-\mathrm{C} 8$ & $1.394(3)$ & $\mathrm{C} 34-\mathrm{C} 36$ & $1.527(4)$ \\
\hline $\mathrm{C} 7-\mathrm{C} 12$ & $1.397(3)$ & & \\
\hline $\mathrm{C} 5-\mathrm{O} 1-\mathrm{C} 2$ & $108.98(17)$ & $\mathrm{C} 19-\mathrm{C} 14-\mathrm{C} 13$ & $119.1(2)$ \\
\hline $\mathrm{C} 1-\mathrm{O} 2-\mathrm{C} 6$ & $112.37(18)$ & $\mathrm{C} 14-\mathrm{C} 15-\mathrm{C} 16$ & $120.2(2)$ \\
\hline $\mathrm{C} 13-\mathrm{O} 3-\mathrm{C} 3$ & $114.22(18)$ & $\mathrm{C} 17-\mathrm{C} 16-\mathrm{C} 15$ & $120.5(3)$ \\
\hline
\end{tabular}




\begin{tabular}{|c|c|c|c|}
\hline $\mathrm{C} 4-\mathrm{O} 4-\mathrm{C} 20$ & $114.63(17)$ & $\mathrm{C} 18-\mathrm{C} 17-\mathrm{C} 16$ & $119.7(2)$ \\
\hline $\mathrm{C} 5-\mathrm{O} 5-\mathrm{C} 28$ & $112.51(17)$ & $\mathrm{C} 17-\mathrm{C} 18-\mathrm{C} 19$ & $120.1(2)$ \\
\hline $\mathrm{C} 31-\mathrm{O} 6-\mathrm{C} 32$ & $115.97(19)$ & $\mathrm{C} 14-\mathrm{C} 19-\mathrm{C} 18$ & $120.2(3)$ \\
\hline $\mathrm{C} 33-\mathrm{O} 9-\mathrm{C} 34$ & $119.49(19)$ & $\mathrm{O} 4-\mathrm{C} 20-\mathrm{C} 21$ & $113.64(19)$ \\
\hline $\mathrm{C} 33-\mathrm{N} 1-\mathrm{C} 30$ & $126.0(2)$ & $\mathrm{C} 22-\mathrm{C} 21-\mathrm{C} 26$ & $119.0(2)$ \\
\hline $\mathrm{C} 33-\mathrm{N} 1-\mathrm{C} 27$ & $120.7(2)$ & $\mathrm{C} 22-\mathrm{C} 21-\mathrm{C} 20$ & $121.1(2)$ \\
\hline $\mathrm{C} 30-\mathrm{N} 1-\mathrm{C} 27$ & $113.29(19)$ & $\mathrm{C} 26-\mathrm{C} 21-\mathrm{C} 20$ & $119.9(2)$ \\
\hline $\mathrm{O} 2-\mathrm{C} 1-\mathrm{C} 2$ & $106.08(19)$ & $\mathrm{C} 23-\mathrm{C} 22-\mathrm{C} 21$ & $120.4(2)$ \\
\hline $\mathrm{O} 1-\mathrm{C} 2-\mathrm{C} 1$ & $109.17(19)$ & $\mathrm{C} 24-\mathrm{C} 23-\mathrm{C} 22$ & $120.5(2)$ \\
\hline $\mathrm{O} 1-\mathrm{C} 2-\mathrm{C} 3$ & $106.71(18)$ & $\mathrm{C} 23-\mathrm{C} 24-\mathrm{C} 25$ & $119.5(2)$ \\
\hline $\mathrm{C} 1-\mathrm{C} 2-\mathrm{C} 3$ & $115.13(19)$ & $\mathrm{C} 26-\mathrm{C} 25-\mathrm{C} 24$ & $119.8(2)$ \\
\hline $\mathrm{O} 3-\mathrm{C} 3-\mathrm{C} 2$ & $107.46(18)$ & $\mathrm{C} 25-\mathrm{C} 26-\mathrm{C} 21$ & $120.7(2)$ \\
\hline $\mathrm{O} 3-\mathrm{C} 3-\mathrm{C} 4$ & $113.08(19)$ & $\mathrm{N} 1-\mathrm{C} 27-\mathrm{C} 28$ & $103.4(2)$ \\
\hline $\mathrm{C} 2-\mathrm{C} 3-\mathrm{C} 4$ & $103.86(18)$ & $\mathrm{O} 5-\mathrm{C} 28-\mathrm{C} 27$ & $107.87(18)$ \\
\hline $\mathrm{O} 4-\mathrm{C} 4-\mathrm{C} 5$ & $115.16(19)$ & $\mathrm{O} 5-\mathrm{C} 28-\mathrm{C} 29$ & $110.7(2)$ \\
\hline $\mathrm{O} 4-\mathrm{C} 4-\mathrm{C} 3$ & $111.67(18)$ & $\mathrm{C} 27-\mathrm{C} 28-\mathrm{C} 29$ & $104.08(19)$ \\
\hline $\mathrm{C} 5-\mathrm{C} 4-\mathrm{C} 3$ & $102.97(19)$ & $\mathrm{C} 28-\mathrm{C} 29-\mathrm{C} 30$ & $103.60(19)$ \\
\hline $\mathrm{O} 5-\mathrm{C} 5-\mathrm{O} 1$ & $111.74(19)$ & $\mathrm{N} 1-\mathrm{C} 30-\mathrm{C} 31$ & $112.23(19)$ \\
\hline $\mathrm{O} 5-\mathrm{C} 5-\mathrm{C} 4$ & $108.53(18)$ & $\mathrm{N} 1-\mathrm{C} 30-\mathrm{C} 29$ & $102.82(19)$ \\
\hline $\mathrm{O} 1-\mathrm{C} 5-\mathrm{C} 4$ & $103.90(19)$ & $\mathrm{C} 31-\mathrm{C} 30-\mathrm{C} 29$ & $110.17(19)$ \\
\hline $\mathrm{O} 2-\mathrm{C} 6-\mathrm{C} 7$ & $107.11(18)$ & $\mathrm{O} 7-\mathrm{C} 31-\mathrm{O} 6$ & $124.5(2)$ \\
\hline $\mathrm{C} 8-\mathrm{C} 7-\mathrm{C} 12$ & $118.8(2)$ & $\mathrm{O} 7-\mathrm{C} 31-\mathrm{C} 30$ & $125.4(2)$ \\
\hline $\mathrm{C} 8-\mathrm{C} 7-\mathrm{C} 6$ & $120.5(2)$ & $\mathrm{O} 6-\mathrm{C} 31-\mathrm{C} 30$ & $110.04(19)$ \\
\hline $\mathrm{C} 12-\mathrm{C} 7-\mathrm{C} 6$ & $120.6(2)$ & $\mathrm{O} 8-\mathrm{C} 33-\mathrm{N} 1$ & $123.4(2)$ \\
\hline $\mathrm{C} 9-\mathrm{C} 8-\mathrm{C} 7$ & $120.7(2)$ & $\mathrm{O} 8-\mathrm{C} 33-\mathrm{O} 9$ & $126.4(2)$ \\
\hline $\mathrm{C} 10-\mathrm{C} 9-\mathrm{C} 8$ & $120.1(2)$ & $\mathrm{N} 1-\mathrm{C} 33-\mathrm{O} 9$ & $110.2(2)$ \\
\hline $\mathrm{C} 11-\mathrm{C} 10-\mathrm{C} 9$ & $119.7(2)$ & $\mathrm{O} 9-\mathrm{C} 34-\mathrm{C} 35$ & $110.1(2)$ \\
\hline $\mathrm{C} 10-\mathrm{C} 11-\mathrm{C} 12$ & $120.4(2)$ & $\mathrm{O} 9-\mathrm{C} 34-\mathrm{C} 37$ & $102.78(19)$ \\
\hline $\mathrm{C} 11-\mathrm{C} 12-\mathrm{C} 7$ & $120.3(2)$ & $\mathrm{C} 35-\mathrm{C} 34-\mathrm{C} 37$ & $110.8(2)$ \\
\hline $\mathrm{O} 3-\mathrm{C} 13-\mathrm{C} 14$ & $113.6(2)$ & $\mathrm{O} 9-\mathrm{C} 34-\mathrm{C} 36$ & $109.1(2)$ \\
\hline $\mathrm{C} 15-\mathrm{C} 14-\mathrm{C} 19$ & $119.3(2)$ & $\mathrm{C} 35-\mathrm{C} 34-\mathrm{C} 36$ & $112.8(2)$ \\
\hline $\mathrm{C} 15-\mathrm{C} 14-\mathrm{C} 13$ & $121.5(2)$ & $\mathrm{C} 37-\mathrm{C} 34-\mathrm{C} 36$ & $110.8(2)$ \\
\hline $\mathrm{C} 6-\mathrm{O} 2-\mathrm{C} 1-\mathrm{C} 2$ & $-178.52(19)$ & $\mathrm{C} 15-\mathrm{C} 14-\mathrm{C} 19-\mathrm{C} 18$ & $2.1(4)$ \\
\hline $\mathrm{C} 5-\mathrm{O} 1-\mathrm{C} 2-\mathrm{C} 1$ & $107.5(2)$ & $\mathrm{C} 13-\mathrm{C} 14-\mathrm{C} 19-\mathrm{C} 18$ & $-174.4(2)$ \\
\hline $\mathrm{C} 5-\mathrm{O} 1-\mathrm{C} 2-\mathrm{C} 3$ & $-17.5(2)$ & $\mathrm{C} 17-\mathrm{C} 18-\mathrm{C} 19-\mathrm{C} 14$ & $-1.3(4)$ \\
\hline $\mathrm{O} 2-\mathrm{C} 1-\mathrm{C} 2-\mathrm{O} 1$ & $169.59(17)$ & $\mathrm{C} 4-\mathrm{O} 4-\mathrm{C} 20-\mathrm{C} 21$ & $65.9(3)$ \\
\hline $\mathrm{O} 2-\mathrm{C} 1-\mathrm{C} 2-\mathrm{C} 3$ & $-70.5(2)$ & $\mathrm{O} 4-\mathrm{C} 20-\mathrm{C} 21-\mathrm{C} 22$ & $-97.8(3)$ \\
\hline $\mathrm{C} 13-\mathrm{O} 3-\mathrm{C} 3-\mathrm{C} 2$ & $-174.61(18)$ & $\mathrm{O} 4-\mathrm{C} 20-\mathrm{C} 21-\mathrm{C} 26$ & $83.1(3)$ \\
\hline $\mathrm{C} 13-\mathrm{O} 3-\mathrm{C} 3-\mathrm{C} 4$ & $71.4(2)$ & $\mathrm{C} 26-\mathrm{C} 21-\mathrm{C} 22-\mathrm{C} 23$ & $-1.2(4)$ \\
\hline
\end{tabular}




\begin{tabular}{|c|c|c|c|}
\hline $\mathrm{O} 1-\mathrm{C} 2-\mathrm{C} 3-\mathrm{O} 3$ & $-126.48(18)$ & $\mathrm{C} 20-\mathrm{C} 21-\mathrm{C} 22-\mathrm{C} 23$ & $179.8(2)$ \\
\hline $\mathrm{C} 1-\mathrm{C} 2-\mathrm{C} 3-\mathrm{O} 3$ & $112.2(2)$ & $\mathrm{C} 21-\mathrm{C} 22-\mathrm{C} 23-\mathrm{C} 24$ & $1.6(4)$ \\
\hline $\mathrm{O} 1-\mathrm{C} 2-\mathrm{C} 3-\mathrm{C} 4$ & $-6.4(2)$ & $\mathrm{C} 22-\mathrm{C} 23-\mathrm{C} 24-\mathrm{C} 25$ & $-0.7(4)$ \\
\hline $\mathrm{C} 1-\mathrm{C} 2-\mathrm{C} 3-\mathrm{C} 4$ & $-127.7(2)$ & $\mathrm{C} 23-\mathrm{C} 24-\mathrm{C} 25-\mathrm{C} 26$ & $-0.5(4)$ \\
\hline $\mathrm{C} 20-\mathrm{O} 4-\mathrm{C} 4-\mathrm{C} 5$ & $-100.5(2)$ & $\mathrm{C} 24-\mathrm{C} 25-\mathrm{C} 26-\mathrm{C} 21$ & $0.9(4)$ \\
\hline $\mathrm{C} 20-\mathrm{O} 4-\mathrm{C} 4-\mathrm{C} 3$ & $142.5(2)$ & $\mathrm{C} 22-\mathrm{C} 21-\mathrm{C} 26-\mathrm{C} 25$ & $-0.1(4)$ \\
\hline $\mathrm{O} 3-\mathrm{C} 3-\mathrm{C} 4-\mathrm{O} 4$ & $-94.0(2)$ & $\mathrm{C} 20-\mathrm{C} 21-\mathrm{C} 26-\mathrm{C} 25$ & $179.0(2)$ \\
\hline $\mathrm{C} 2-\mathrm{C} 3-\mathrm{C} 4-\mathrm{O} 4$ & $149.80(19)$ & $\mathrm{C} 33-\mathrm{N} 1-\mathrm{C} 27-\mathrm{C} 28$ & $-169.5(2)$ \\
\hline $\mathrm{O} 3-\mathrm{C} 3-\mathrm{C} 4-\mathrm{C} 5$ & $141.84(19)$ & $\mathrm{C} 30-\mathrm{N} 1-\mathrm{C} 27-\mathrm{C} 28$ & $11.3(3)$ \\
\hline $\mathrm{C} 2-\mathrm{C} 3-\mathrm{C} 4-\mathrm{C} 5$ & $25.7(2)$ & $\mathrm{C} 5-\mathrm{O} 5-\mathrm{C} 28-\mathrm{C} 27$ & $162.05(19)$ \\
\hline $\mathrm{C} 28-\mathrm{O} 5-\mathrm{C} 5-\mathrm{O} 1$ & $-70.3(2)$ & $\mathrm{C} 5-\mathrm{O} 5-\mathrm{C} 28-\mathrm{C} 29$ & $-84.7(2)$ \\
\hline $\mathrm{C} 28-\mathrm{O} 5-\mathrm{C} 5-\mathrm{C} 4$ & $175.73(18)$ & $\mathrm{N} 1-\mathrm{C} 27-\mathrm{C} 28-\mathrm{O} 5$ & $88.5(2)$ \\
\hline $\mathrm{C} 2-\mathrm{O} 1-\mathrm{C} 5-\mathrm{O} 5$ & $-82.8(2)$ & $\mathrm{N} 1-\mathrm{C} 27-\mathrm{C} 28-\mathrm{C} 29$ & $-29.1(2)$ \\
\hline $\mathrm{C} 2-\mathrm{O} 1-\mathrm{C} 5-\mathrm{C} 4$ & $34.0(2)$ & $\mathrm{O} 5-\mathrm{C} 28-\mathrm{C} 29-\mathrm{C} 30$ & $-79.3(2)$ \\
\hline $\mathrm{O} 4-\mathrm{C} 4-\mathrm{C} 5-\mathrm{O} 5$ & $-39.5(3)$ & $\mathrm{C} 27-\mathrm{C} 28-\mathrm{C} 29-\mathrm{C} 30$ & $36.4(2)$ \\
\hline $\mathrm{C} 3-\mathrm{C} 4-\mathrm{C} 5-\mathrm{O} 5$ & $82.3(2)$ & $\mathrm{C} 33-\mathrm{N} 1-\mathrm{C} 30-\mathrm{C} 31$ & $-49.6(3)$ \\
\hline $\mathrm{O} 4-\mathrm{C} 4-\mathrm{C} 5-\mathrm{O} 1$ & $-158.51(18)$ & $\mathrm{C} 27-\mathrm{N} 1-\mathrm{C} 30-\mathrm{C} 31$ & $129.6(2)$ \\
\hline $\mathrm{C} 3-\mathrm{C} 4-\mathrm{C} 5-\mathrm{O} 1$ & $-36.7(2)$ & $\mathrm{C} 33-\mathrm{N} 1-\mathrm{C} 30-\mathrm{C} 29$ & $-167.9(2)$ \\
\hline $\mathrm{C} 1-\mathrm{O} 2-\mathrm{C} 6-\mathrm{C} 7$ & $177.02(19)$ & $\mathrm{C} 27-\mathrm{N} 1-\mathrm{C} 30-\mathrm{C} 29$ & $11.2(3)$ \\
\hline $\mathrm{O} 2-\mathrm{C} 6-\mathrm{C} 7-\mathrm{C} 8$ & $-74.5(3)$ & $\mathrm{C} 28-\mathrm{C} 29-\mathrm{C} 30-\mathrm{N} 1$ & $-28.8(2)$ \\
\hline $\mathrm{O} 2-\mathrm{C} 6-\mathrm{C} 7-\mathrm{C} 12$ & $102.1(2)$ & $\mathrm{C} 28-\mathrm{C} 29-\mathrm{C} 30-\mathrm{C} 31$ & $-148.6(2)$ \\
\hline $\mathrm{C} 12-\mathrm{C} 7-\mathrm{C} 8-\mathrm{C} 9$ & $-2.6(4)$ & $\mathrm{C} 32-\mathrm{O} 6-\mathrm{C} 31-\mathrm{O} 7$ & $-0.5(3)$ \\
\hline $\mathrm{C} 6-\mathrm{C} 7-\mathrm{C} 8-\mathrm{C} 9$ & $174.0(2)$ & $\mathrm{C} 32-\mathrm{O} 6-\mathrm{C} 31-\mathrm{C} 30$ & $175.88(19)$ \\
\hline $\mathrm{C} 7-\mathrm{C} 8-\mathrm{C} 9-\mathrm{C} 10$ & $1.2(4)$ & $\mathrm{N} 1-\mathrm{C} 30-\mathrm{C} 31-\mathrm{O} 7$ & $-39.8(3)$ \\
\hline $\mathrm{C} 8-\mathrm{C} 9-\mathrm{C} 10-\mathrm{C} 11$ & $0.9(4)$ & $\mathrm{C} 29-\mathrm{C} 30-\mathrm{C} 31-\mathrm{O} 7$ & $74.1(3)$ \\
\hline $\mathrm{C} 9-\mathrm{C} 10-\mathrm{C} 11-\mathrm{C} 12$ & $-1.6(4)$ & $\mathrm{N} 1-\mathrm{C} 30-\mathrm{C} 31-\mathrm{O} 6$ & $143.8(2)$ \\
\hline $\mathrm{C} 10-\mathrm{C} 11-\mathrm{C} 12-\mathrm{C} 7$ & $0.2(4)$ & $\mathrm{C} 29-\mathrm{C} 30-\mathrm{C} 31-\mathrm{O} 6$ & $-102.2(2)$ \\
\hline $\mathrm{C} 8-\mathrm{C} 7-\mathrm{C} 12-\mathrm{C} 11$ & $1.9(4)$ & $\mathrm{C} 30-\mathrm{N} 1-\mathrm{C} 33-\mathrm{O} 8$ & $176.1(2)$ \\
\hline $\mathrm{C} 6-\mathrm{C} 7-\mathrm{C} 12-\mathrm{C} 11$ & $-174.7(2)$ & $\mathrm{C} 27-\mathrm{N} 1-\mathrm{C} 33-\mathrm{O} 8$ & $-3.0(4)$ \\
\hline $\mathrm{C} 3-\mathrm{O} 3-\mathrm{C} 13-\mathrm{C} 14$ & $76.8(3)$ & $\mathrm{C} 30-\mathrm{N} 1-\mathrm{C} 33-\mathrm{O} 9$ & $-4.3(3)$ \\
\hline $\mathrm{O} 3-\mathrm{C} 13-\mathrm{C} 14-\mathrm{C} 15$ & $21.1(3)$ & $\mathrm{C} 27-\mathrm{N} 1-\mathrm{C} 33-\mathrm{O} 9$ & $176.7(2)$ \\
\hline $\mathrm{O} 3-\mathrm{C} 13-\mathrm{C} 14-\mathrm{C} 19$ & $-162.4(2)$ & $\mathrm{C} 34-\mathrm{O} 9-\mathrm{C} 33-\mathrm{O} 8$ & $-17.5(4)$ \\
\hline $\mathrm{C} 19-\mathrm{C} 14-\mathrm{C} 15-\mathrm{C} 16$ & $-1.3(4)$ & $\mathrm{C} 34-\mathrm{O} 9-\mathrm{C} 33-\mathrm{N} 1$ & $162.85(19)$ \\
\hline $\mathrm{C} 13-\mathrm{C} 14-\mathrm{C} 15-\mathrm{C} 16$ & $175.1(2)$ & $\mathrm{C} 33-\mathrm{O} 9-\mathrm{C} 34-\mathrm{C} 35$ & $70.6(3)$ \\
\hline $\mathrm{C} 14-\mathrm{C} 15-\mathrm{C} 16-\mathrm{C} 17$ & $-0.3(4)$ & $\mathrm{C} 33-\mathrm{O} 9-\mathrm{C} 34-\mathrm{C} 37$ & $-171.4(2)$ \\
\hline $\mathrm{C} 15-\mathrm{C} 16-\mathrm{C} 17-\mathrm{C} 18$ & $1.1(4)$ & $\mathrm{C} 33-\mathrm{O} 9-\mathrm{C} 34-\mathrm{C} 36$ & $-53.7(3)$ \\
\hline $\mathrm{C} 16-\mathrm{C} 17-\mathrm{C} 18-\mathrm{C} 19$ & $-0.3(4)$ & & \\
\hline
\end{tabular}




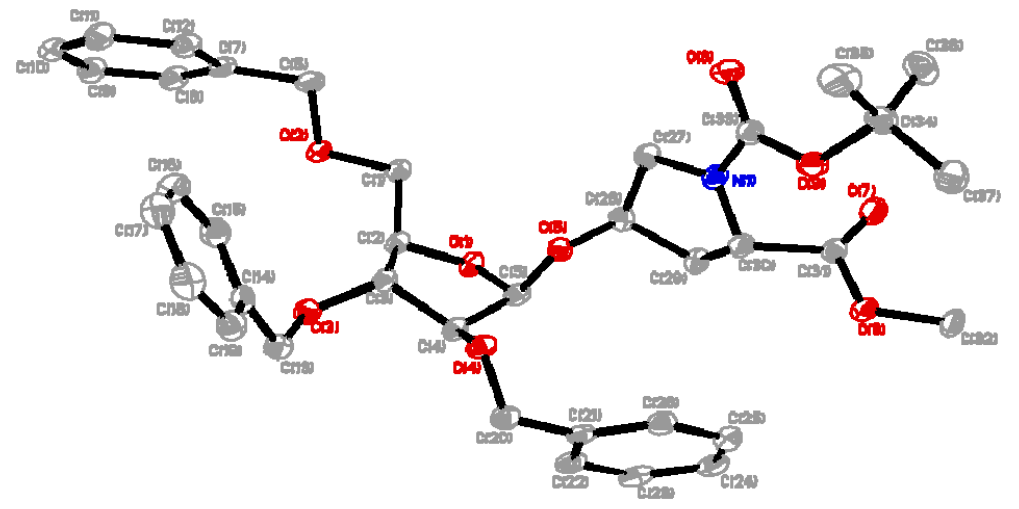

Figure S31 Perspective Views Showing 50\% Probability Displacement.

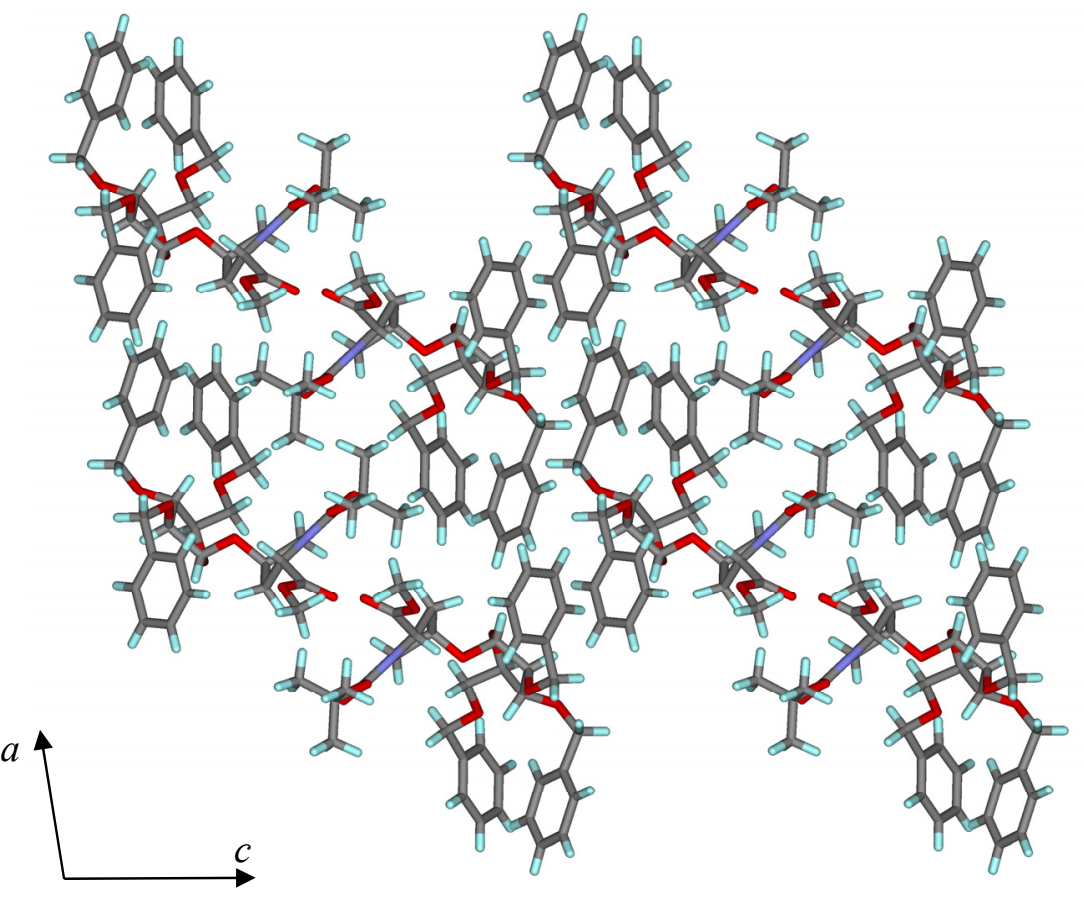

Figure S32 Three-Dimensional Supramolecular Architecture Viewed Along the b-Axis Direction. 
NMR Spectra of Key Compounds

${ }^{1} \mathrm{H}$ NMR $\left(600 \mathrm{MHz}, \mathrm{CDCl}_{3}\right)$
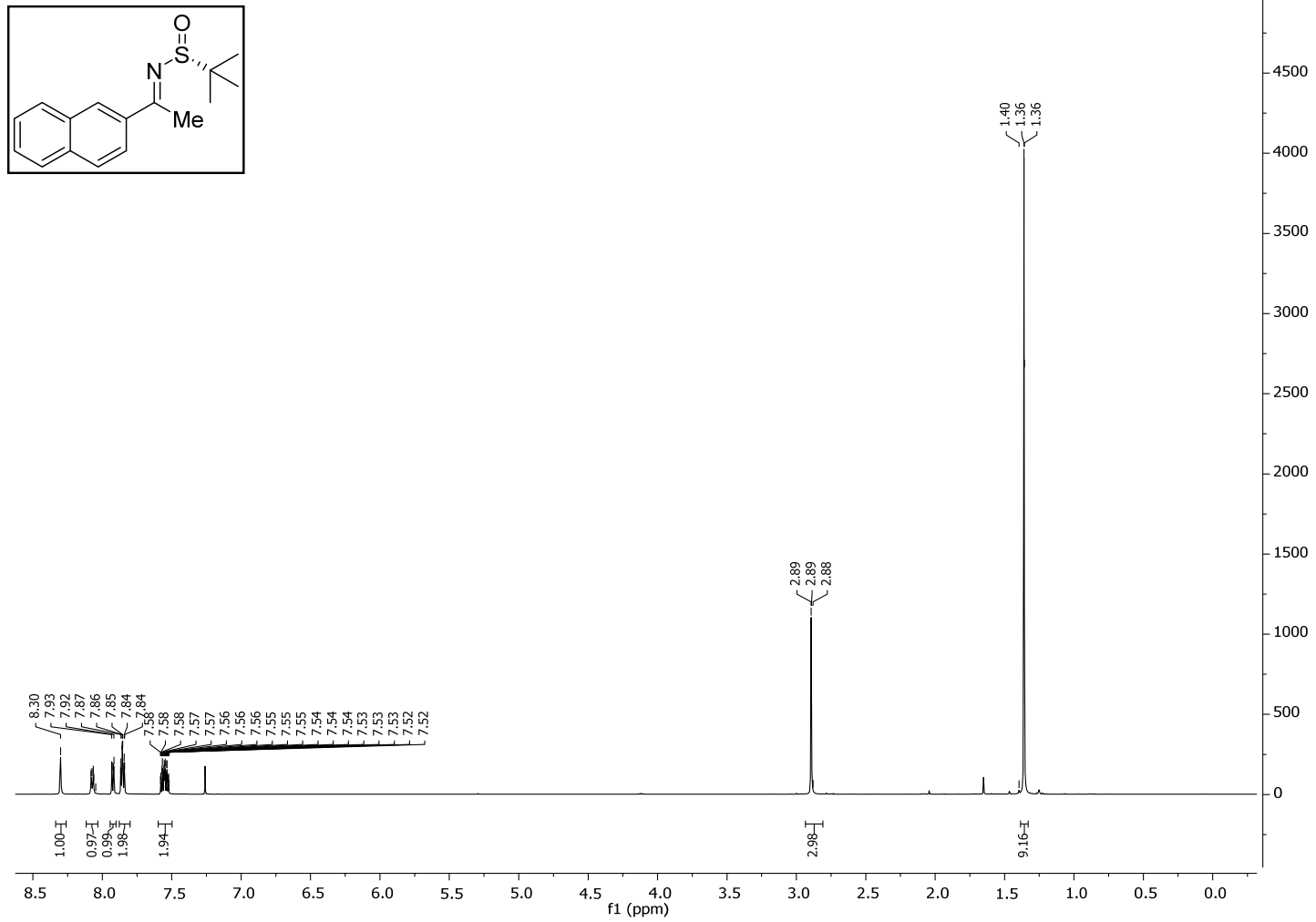

${ }^{13} \mathrm{C}$ NMR $\left(126 \mathrm{MHz}, \mathrm{CDCl}_{3}\right)$

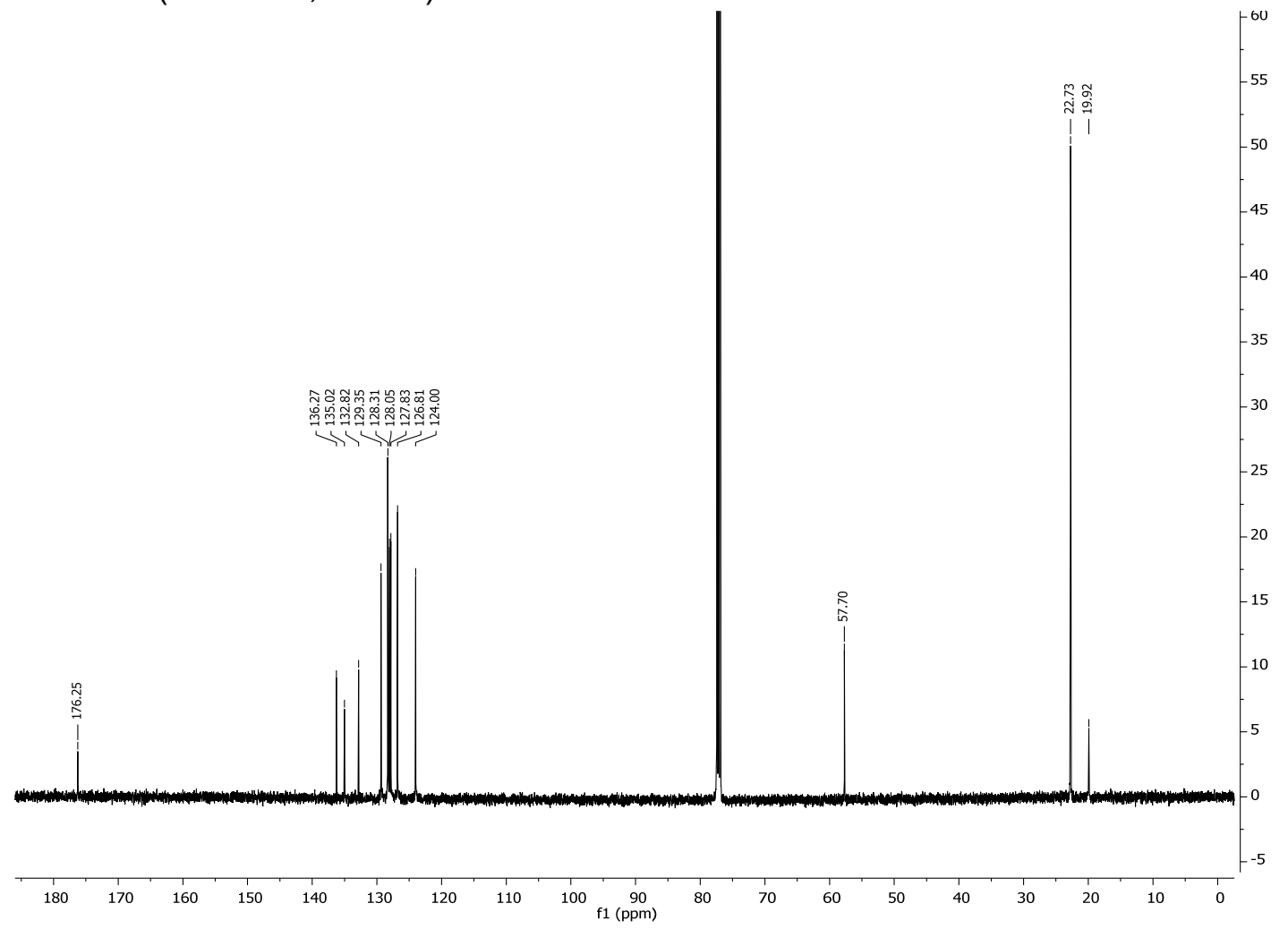


${ }^{1} \mathrm{H}$ NMR $\left(600 \mathrm{MHz}, \mathrm{CDCl}_{3}\right)$
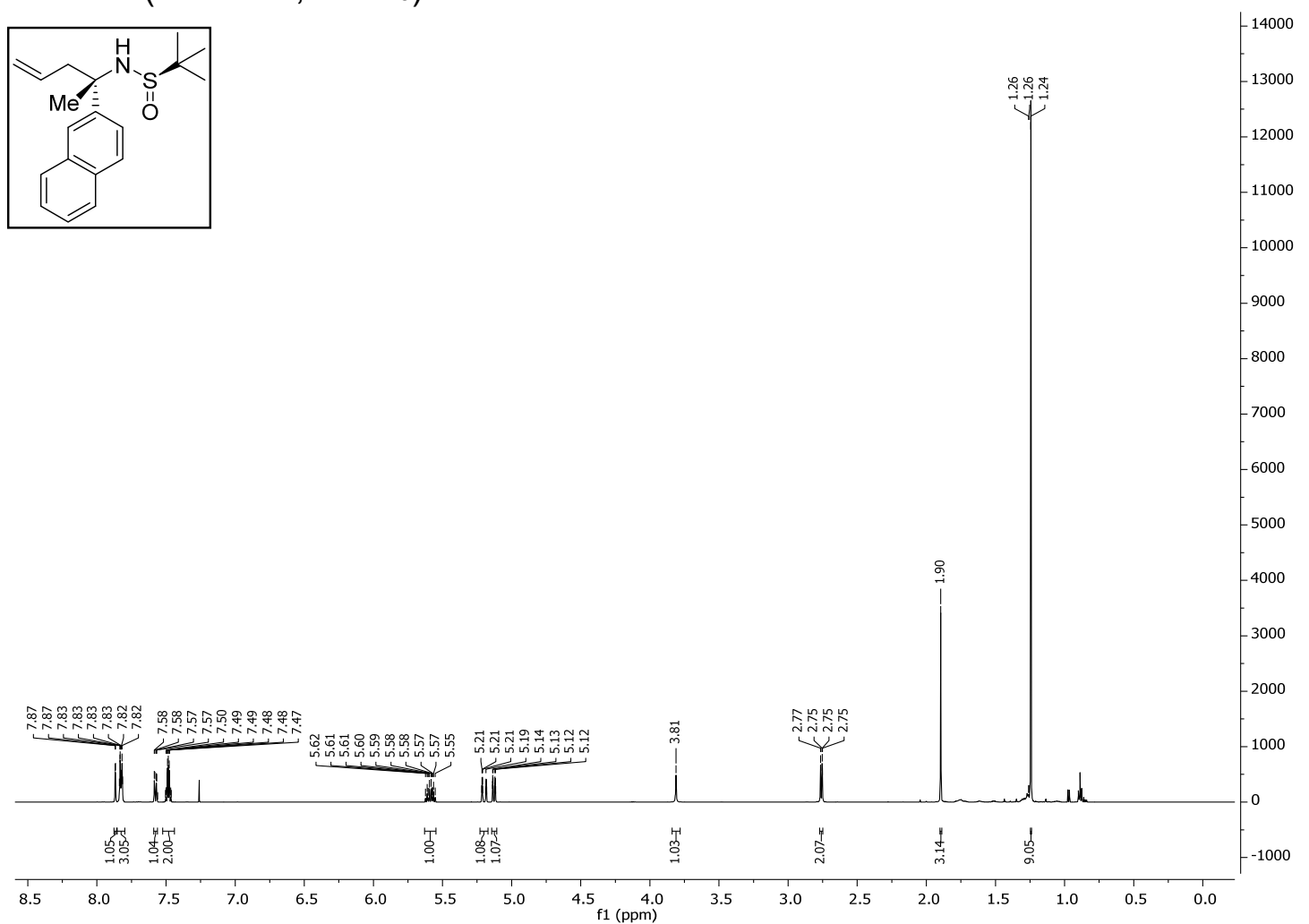

$\left.{ }^{13} \mathrm{C} \mathrm{NMR} \mathrm{(126} \mathrm{MHz,} \mathrm{CDCl}_{3}\right)$

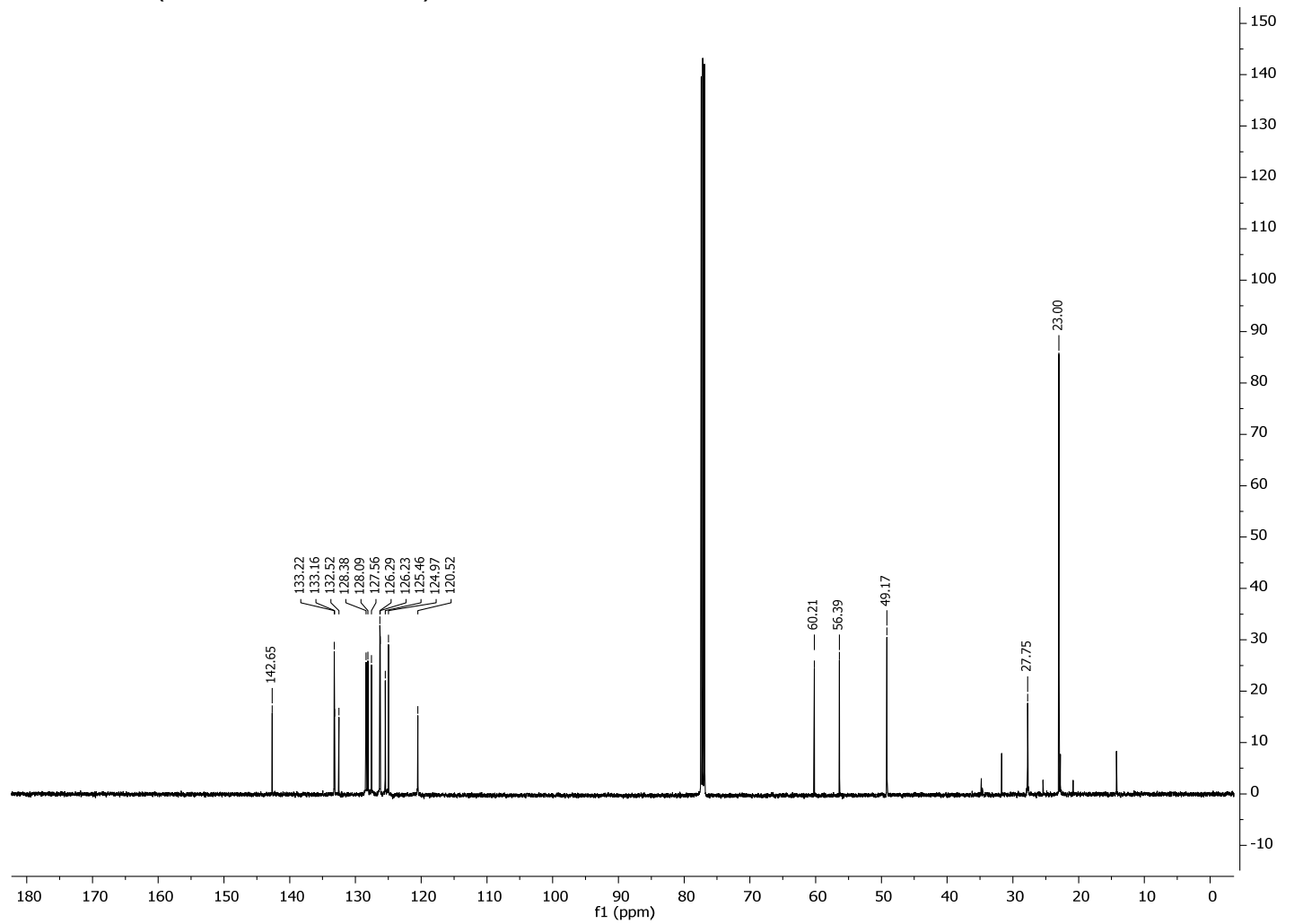


${ }^{1} \mathrm{H}$ NMR $\left(600 \mathrm{MHz}, \mathrm{CDCl}_{3}\right)$
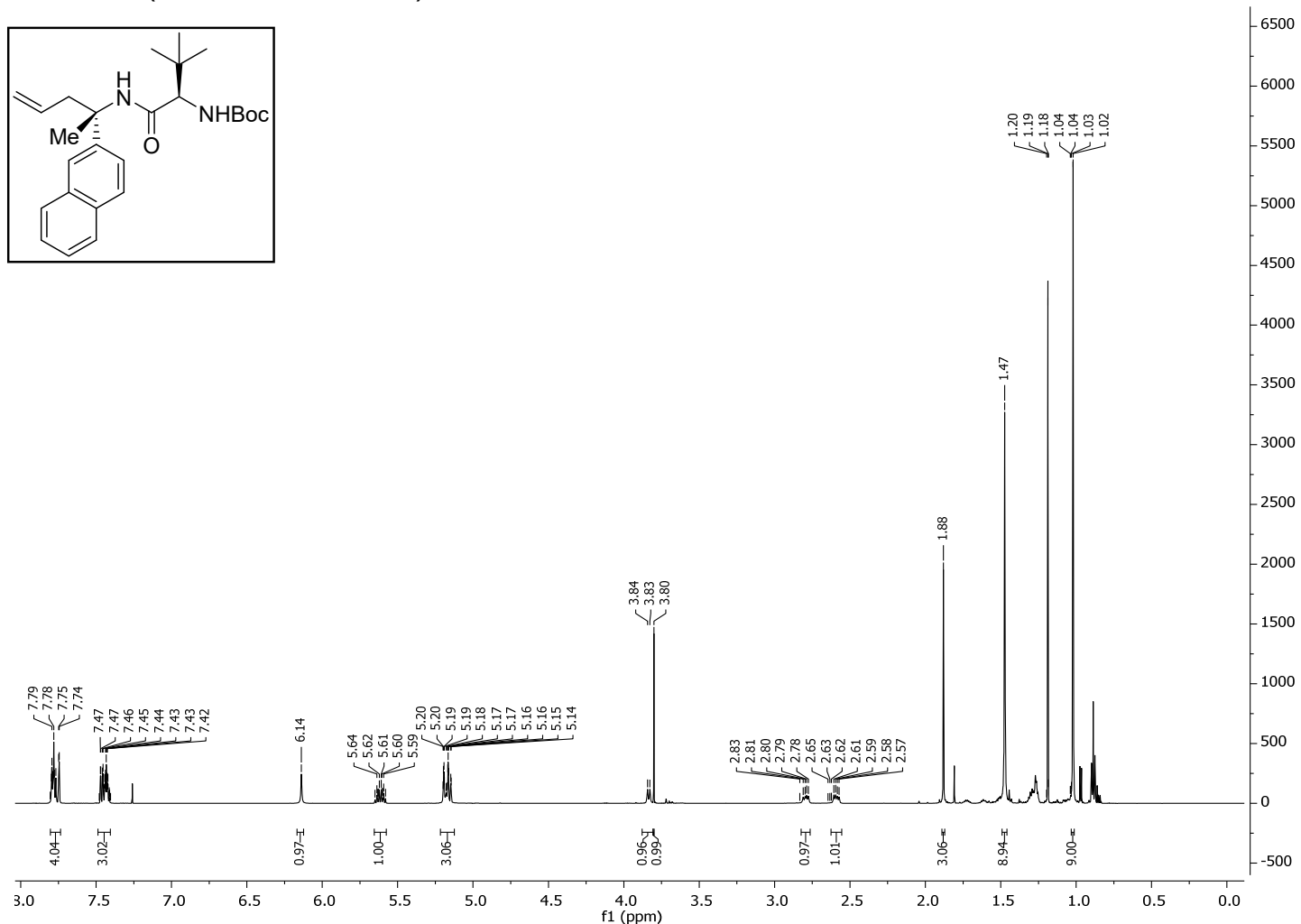

$\left.{ }^{13} \mathrm{C} \mathrm{NMR} \mathrm{(126} \mathrm{MHz,} \mathrm{CDCl}_{3}\right)$

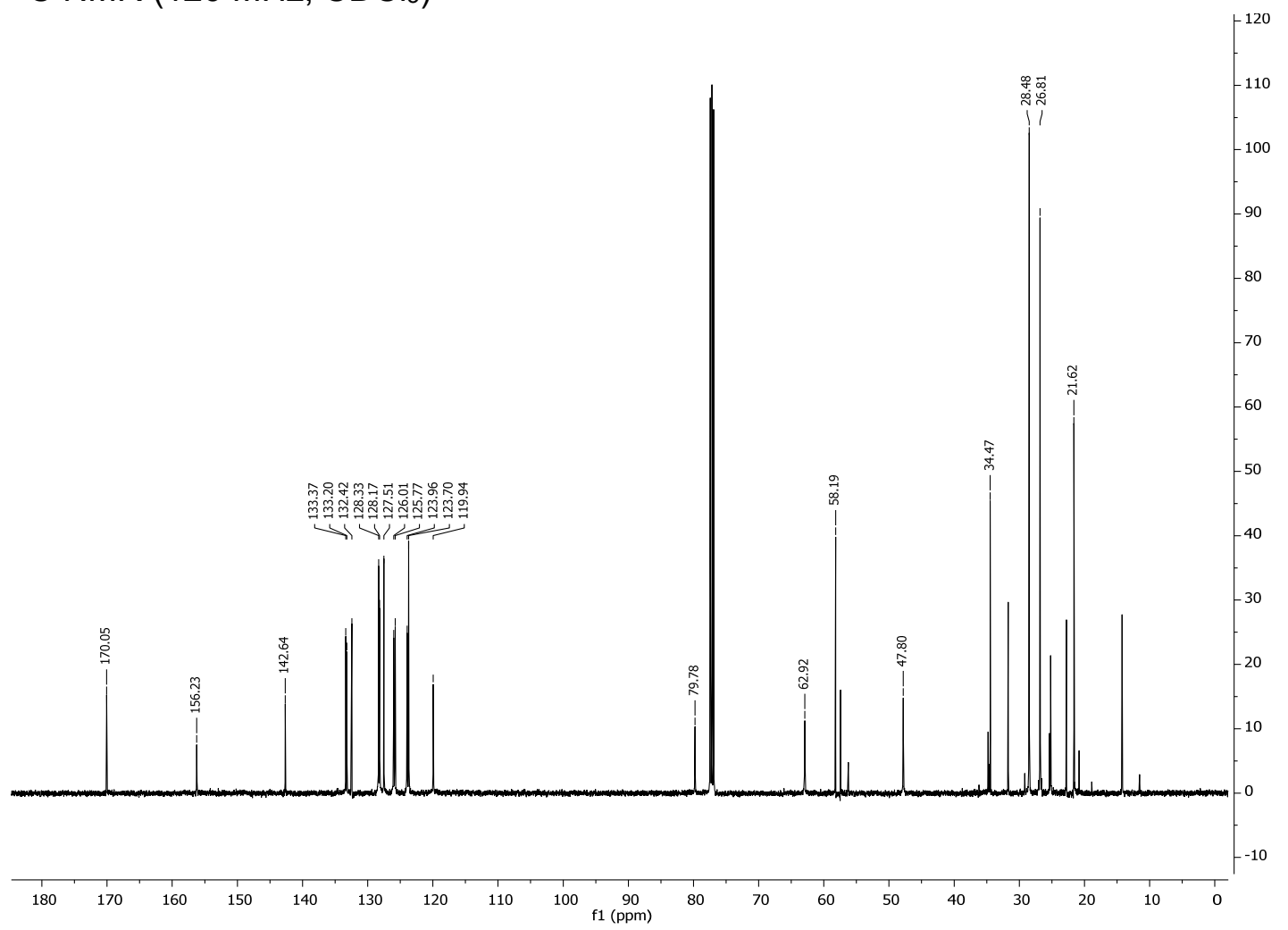


${ }^{1} \mathrm{H} \mathrm{NMR}\left(600 \mathrm{MHz}, \mathrm{CDCl}_{3}\right)$
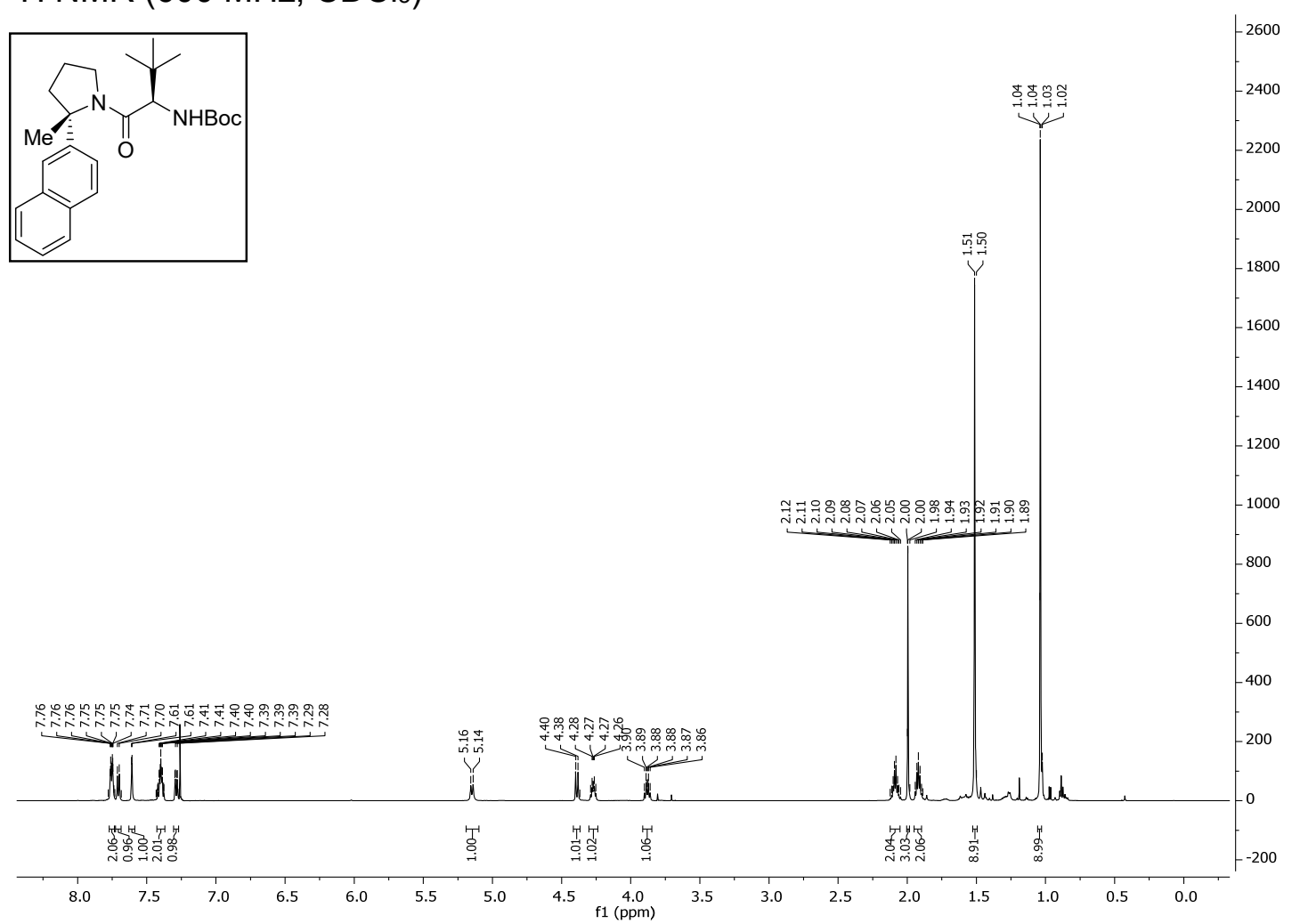

$\left.{ }^{13} \mathrm{C} \mathrm{NMR} \mathrm{(126} \mathrm{MHz,} \mathrm{CDCl}_{3}\right)$

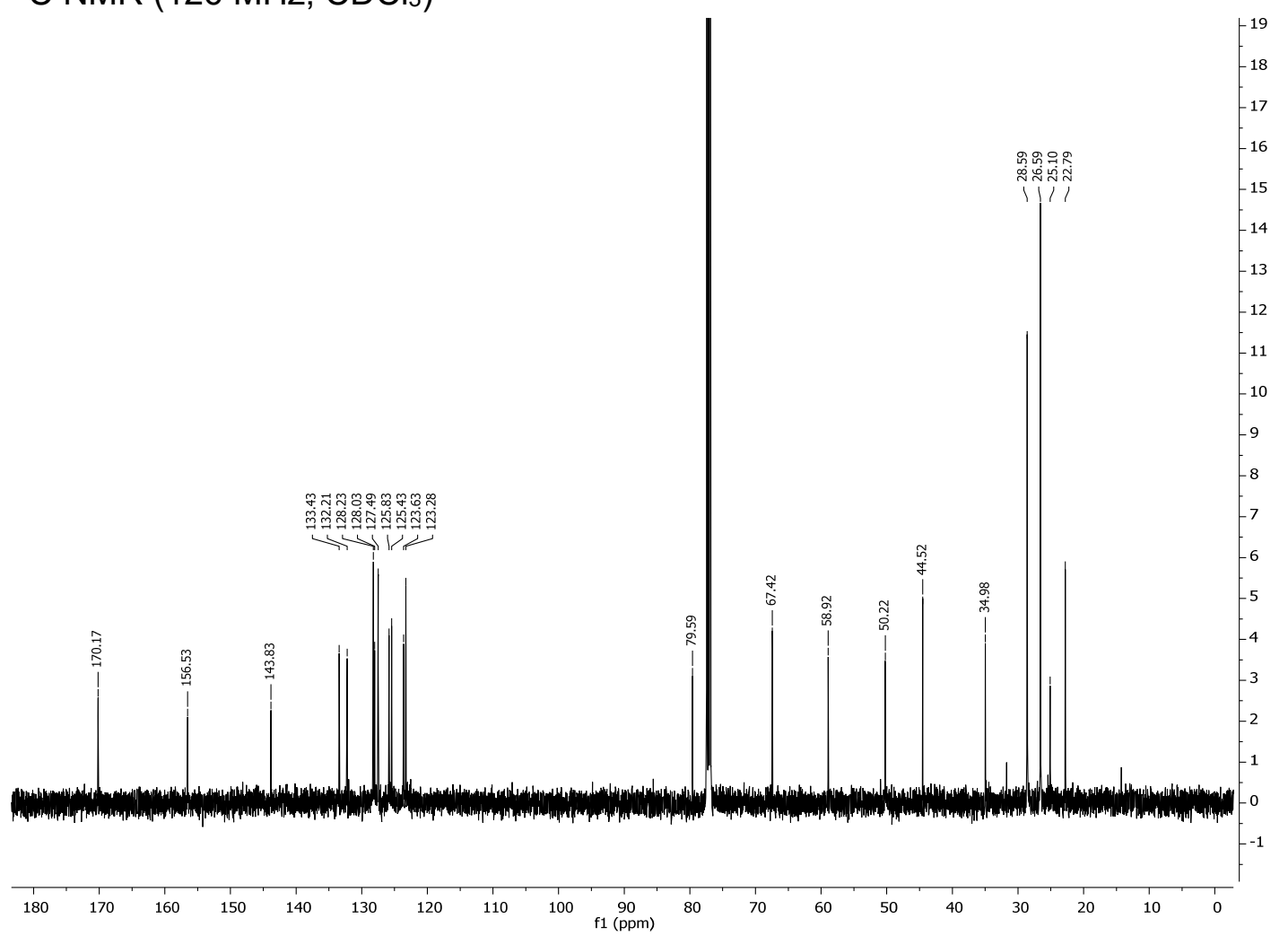


${ }^{1} \mathrm{H} \mathrm{NMR}\left(600 \mathrm{MHz}, \mathrm{CDCl}_{3}\right)$
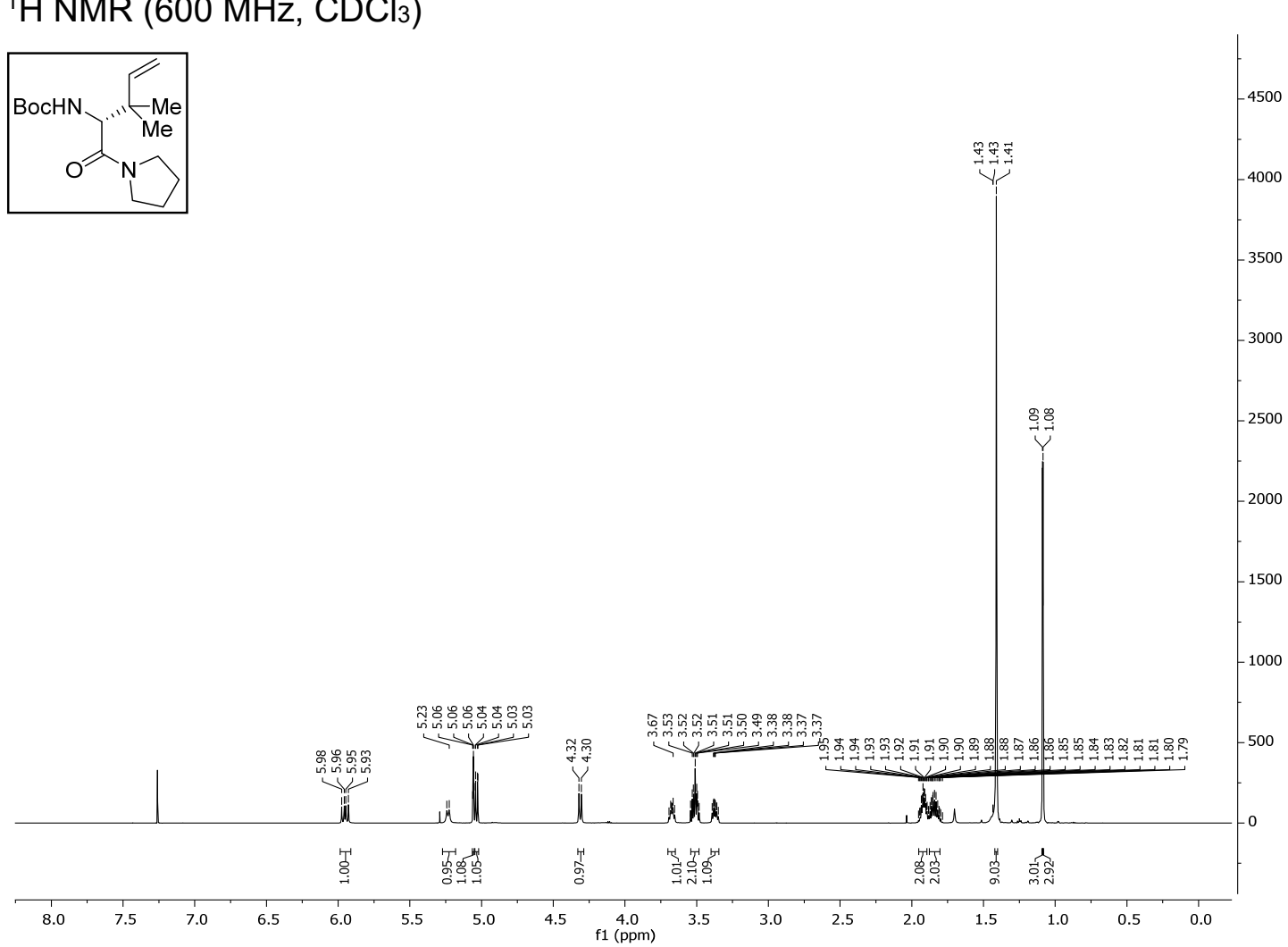

$\left.{ }^{13} \mathrm{C} \mathrm{NMR} \mathrm{(126} \mathrm{MHz,} \mathrm{CDCl}_{3}\right)$

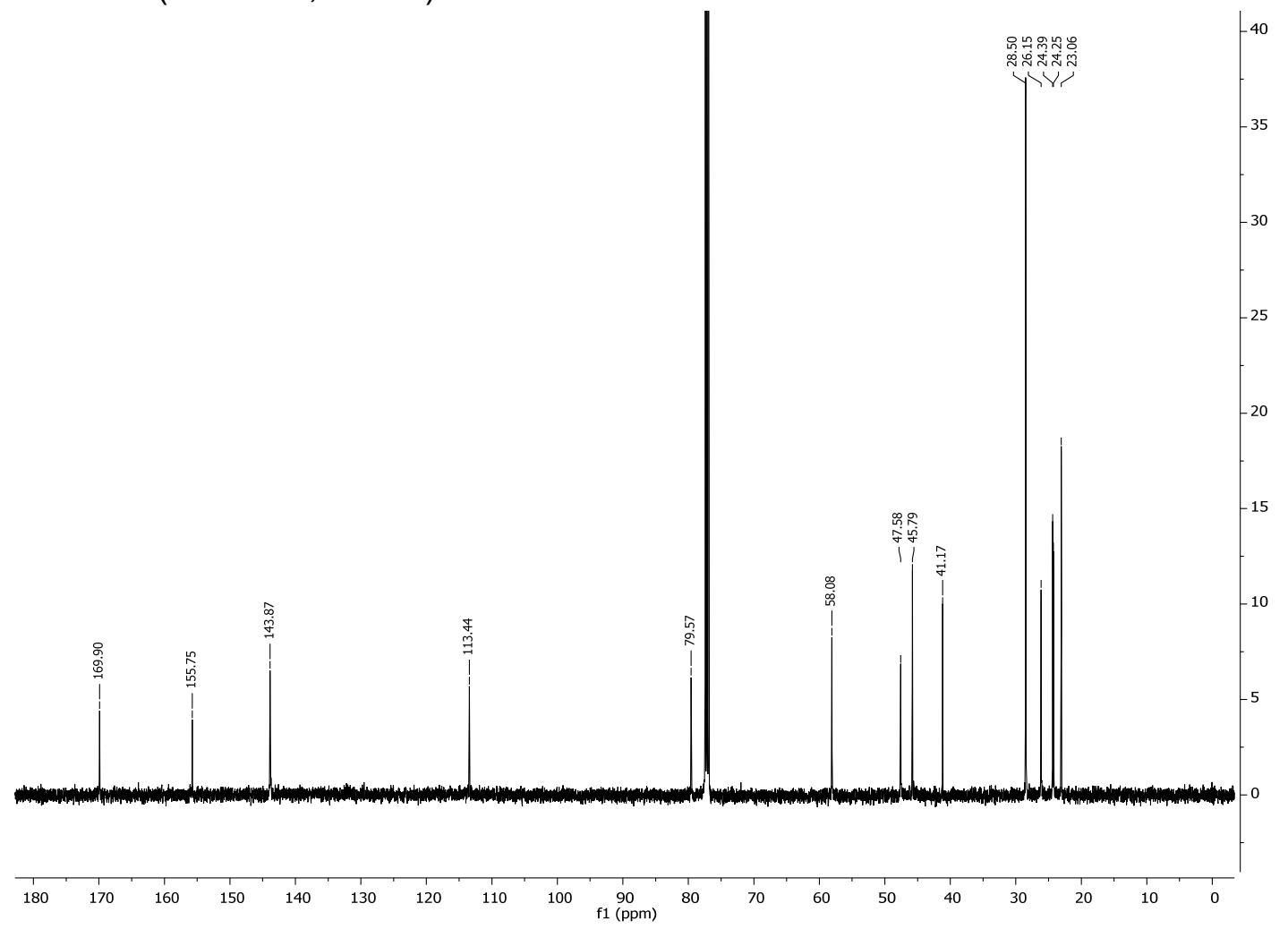


${ }^{1} \mathrm{H}$ NMR $\left(600 \mathrm{MHz}, \mathrm{CDCl}_{3}\right)$
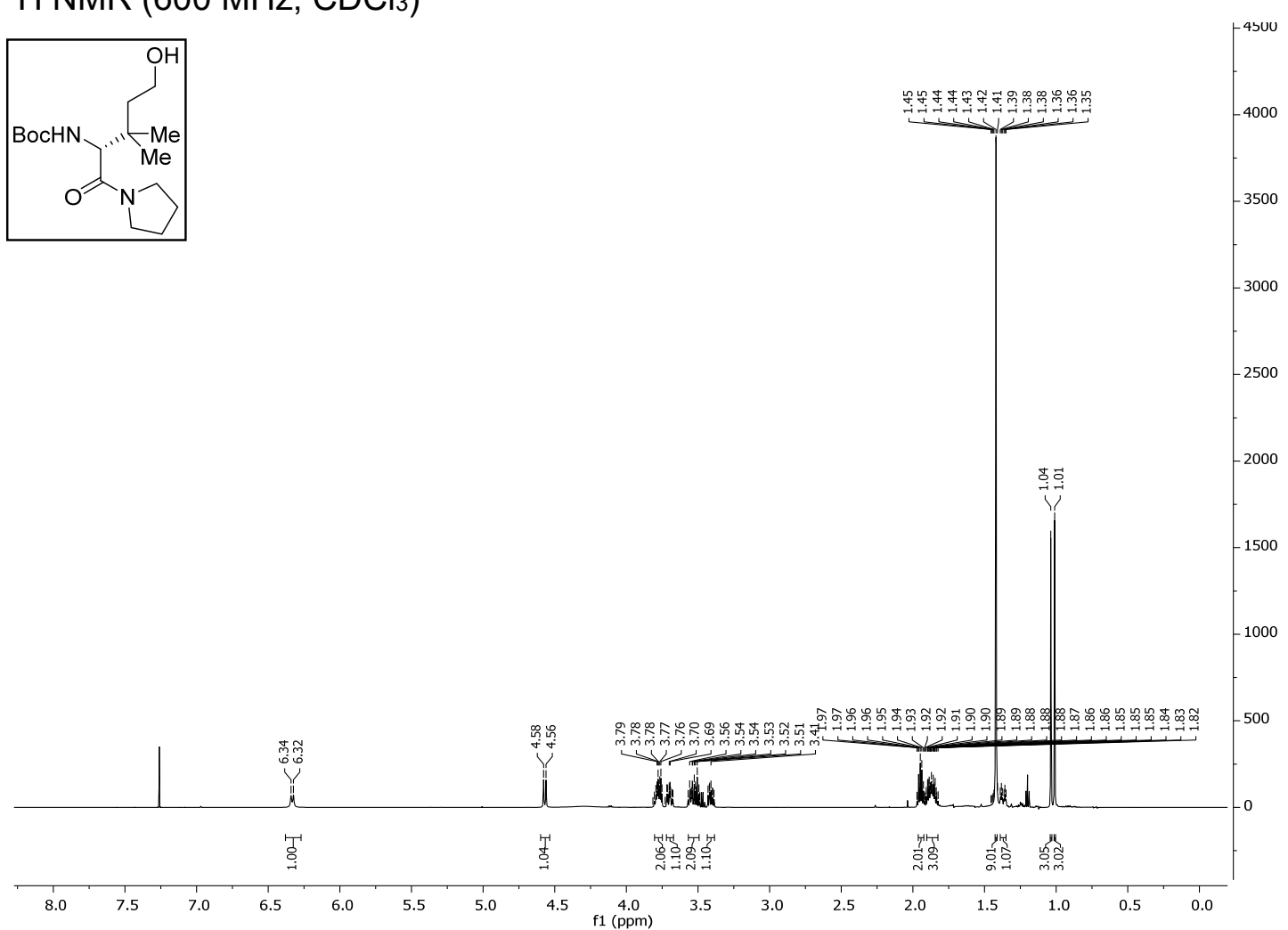

${ }^{13} \mathrm{C}$ NMR $\left(126 \mathrm{MHz}, \mathrm{CDCl}_{3}\right)$

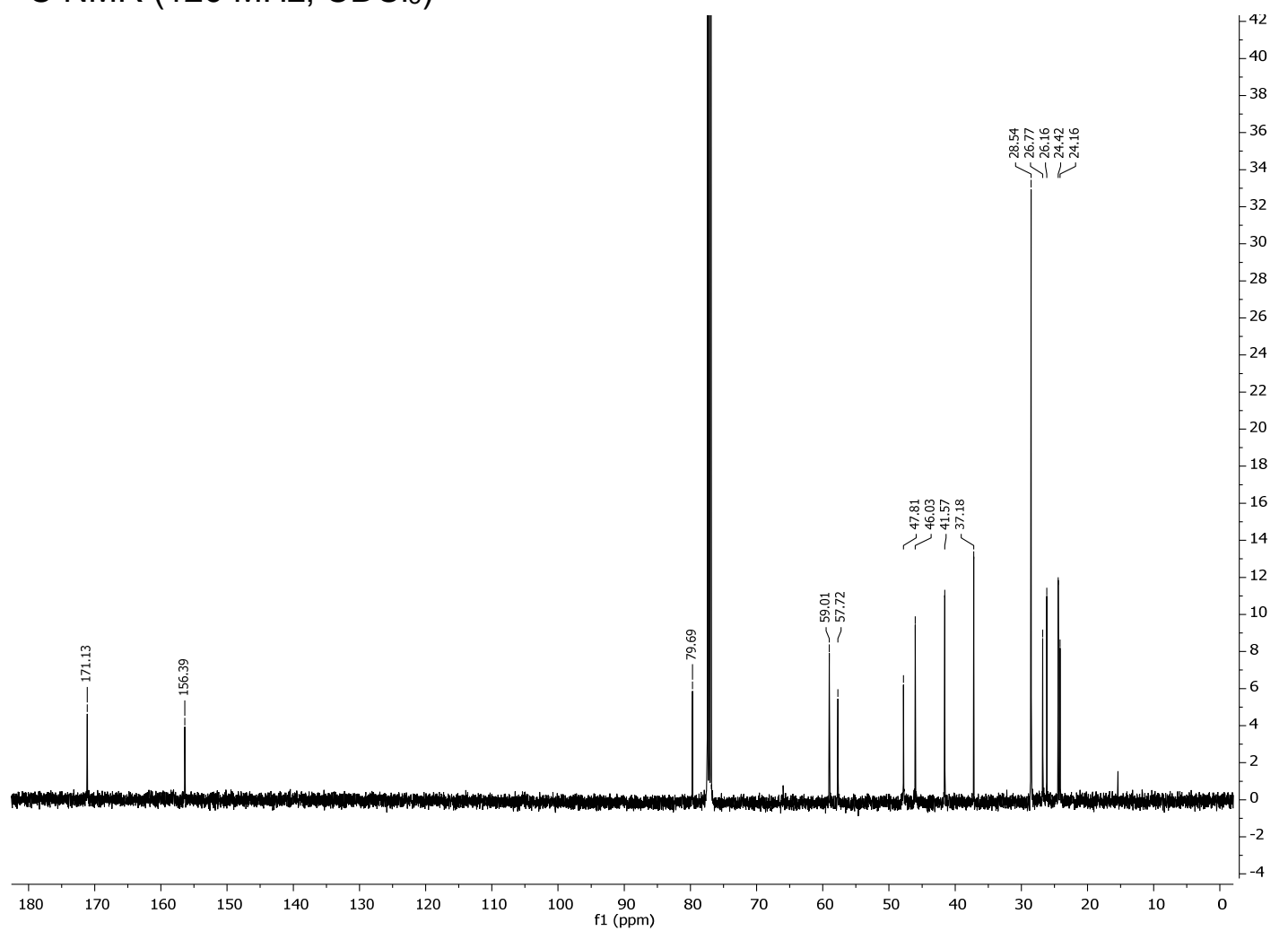


${ }^{1} \mathrm{H}$ NMR $\left(600 \mathrm{MHz}, \mathrm{CDCl}_{3}\right)$
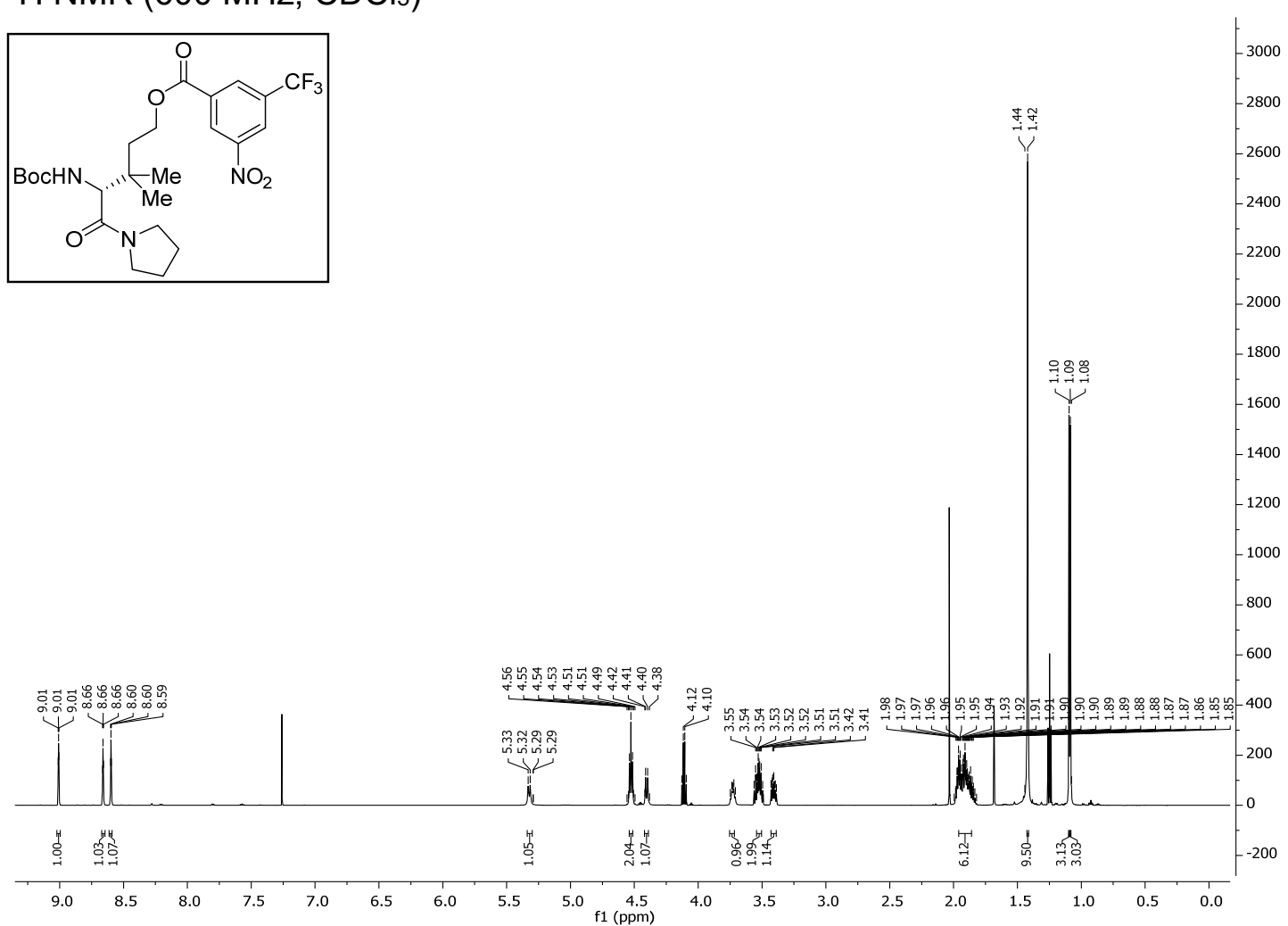

${ }^{13} \mathrm{C}$ NMR $\left(126 \mathrm{MHz}, \mathrm{CDCl}_{3}\right)$

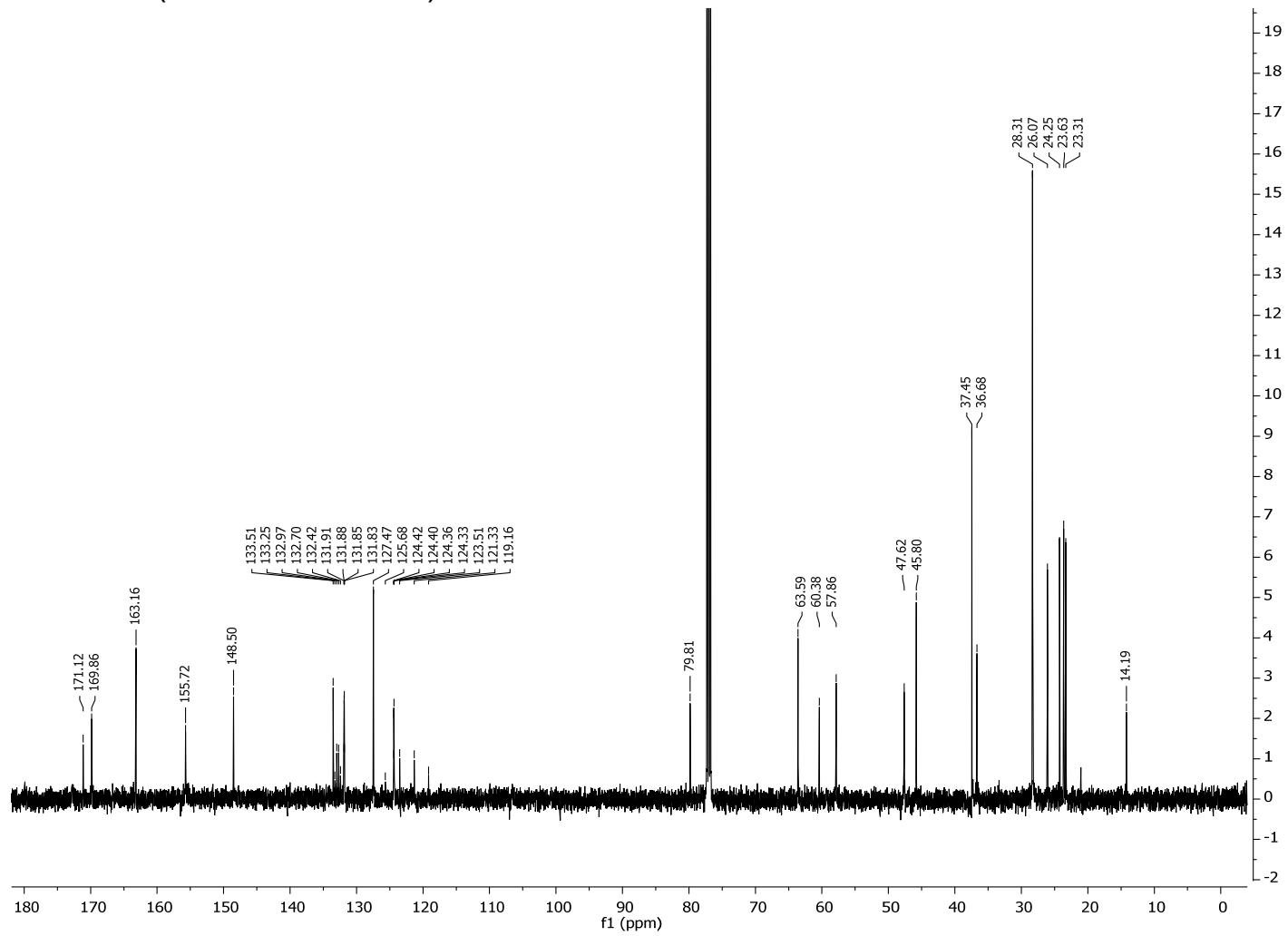


${ }^{19} \mathrm{~F} \mathrm{NMR}\left(471 \mathrm{MHz}, \mathrm{CDCl}_{3}\right)$

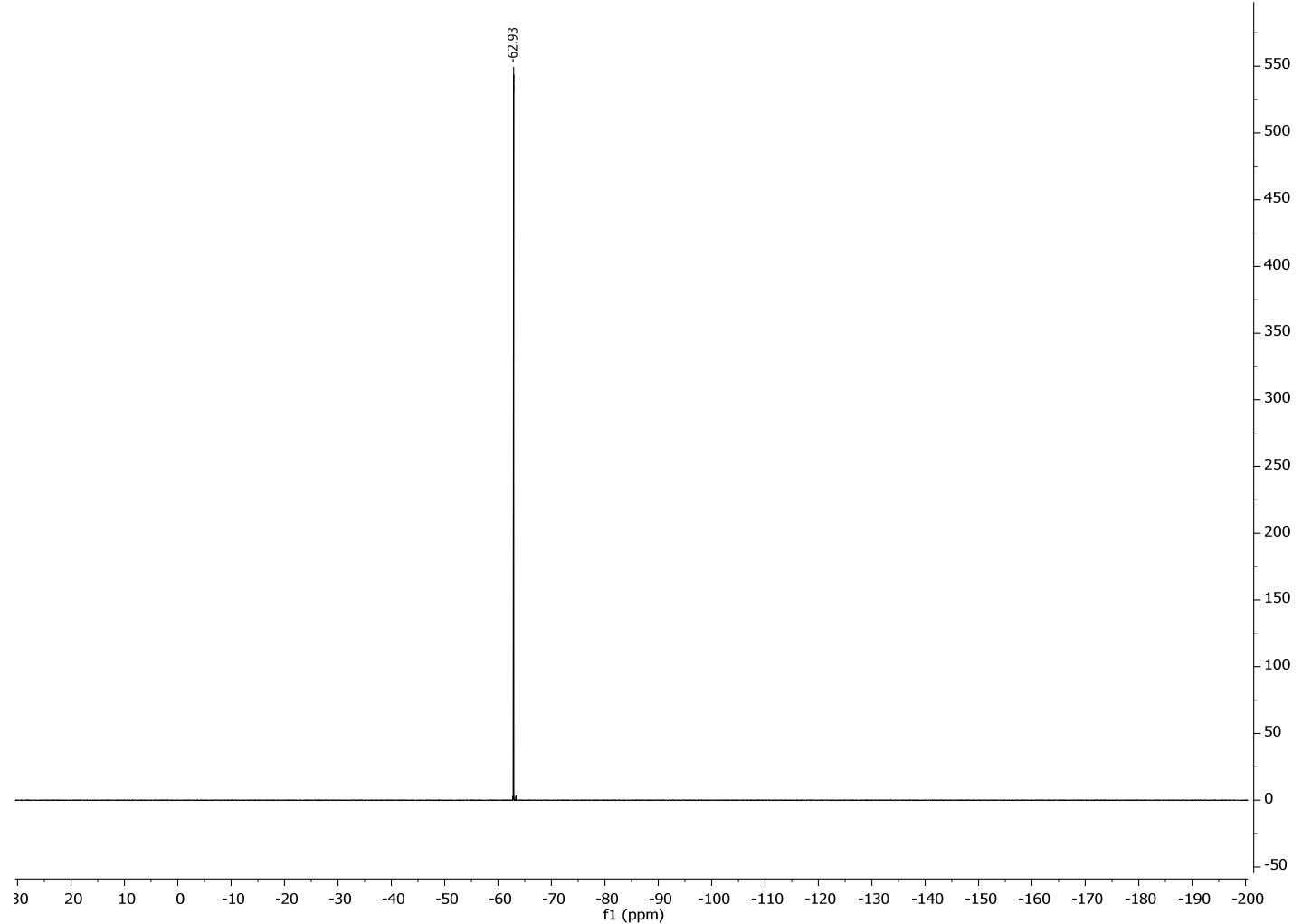


${ }^{1} \mathrm{H}$ NMR $\left(600 \mathrm{MHz}, \mathrm{CDCl}_{3}\right)$
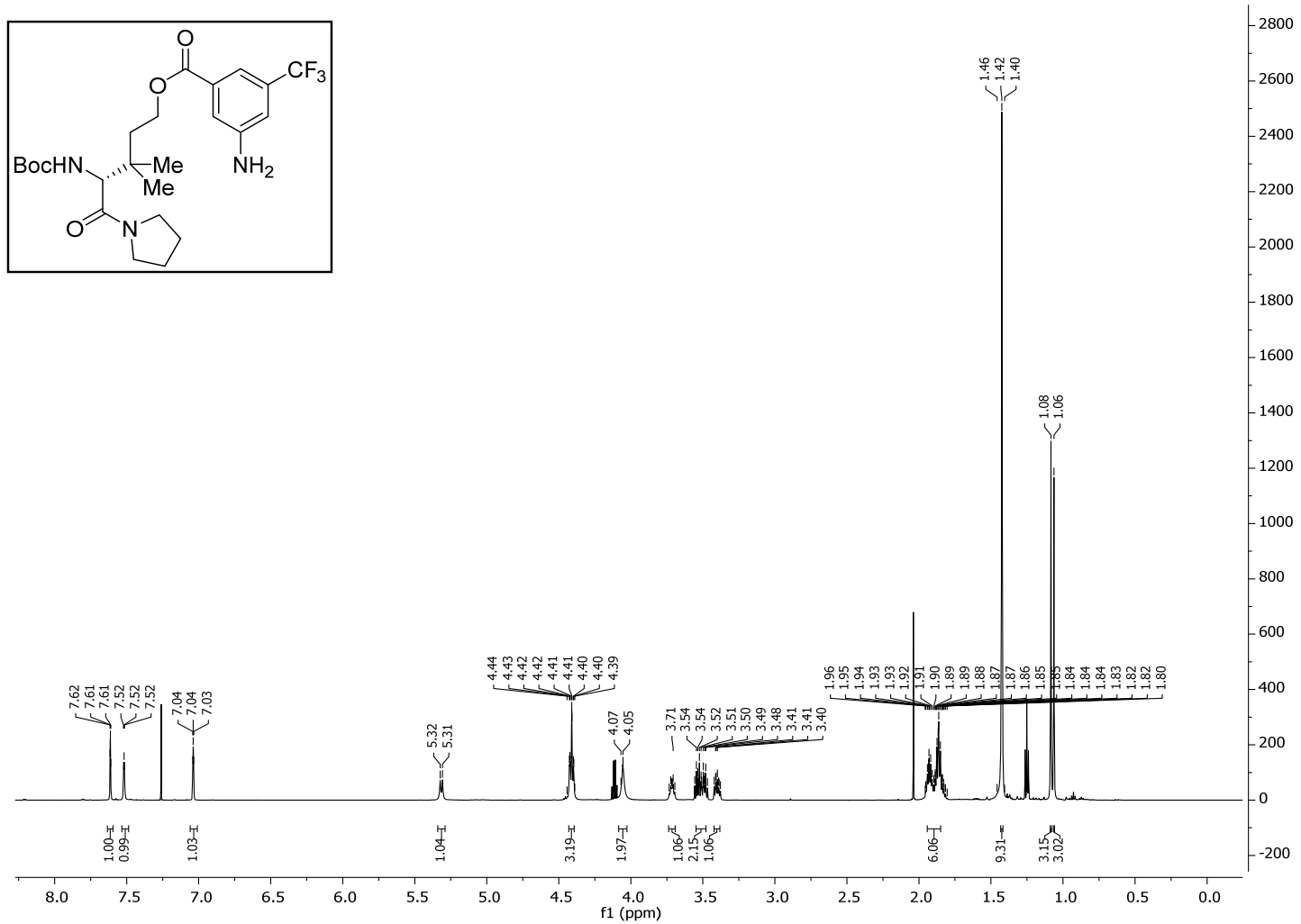

$\left.{ }^{13} \mathrm{C} \mathrm{NMR} \mathrm{(126} \mathrm{MHz,} \mathrm{CDCl}_{3}\right)$

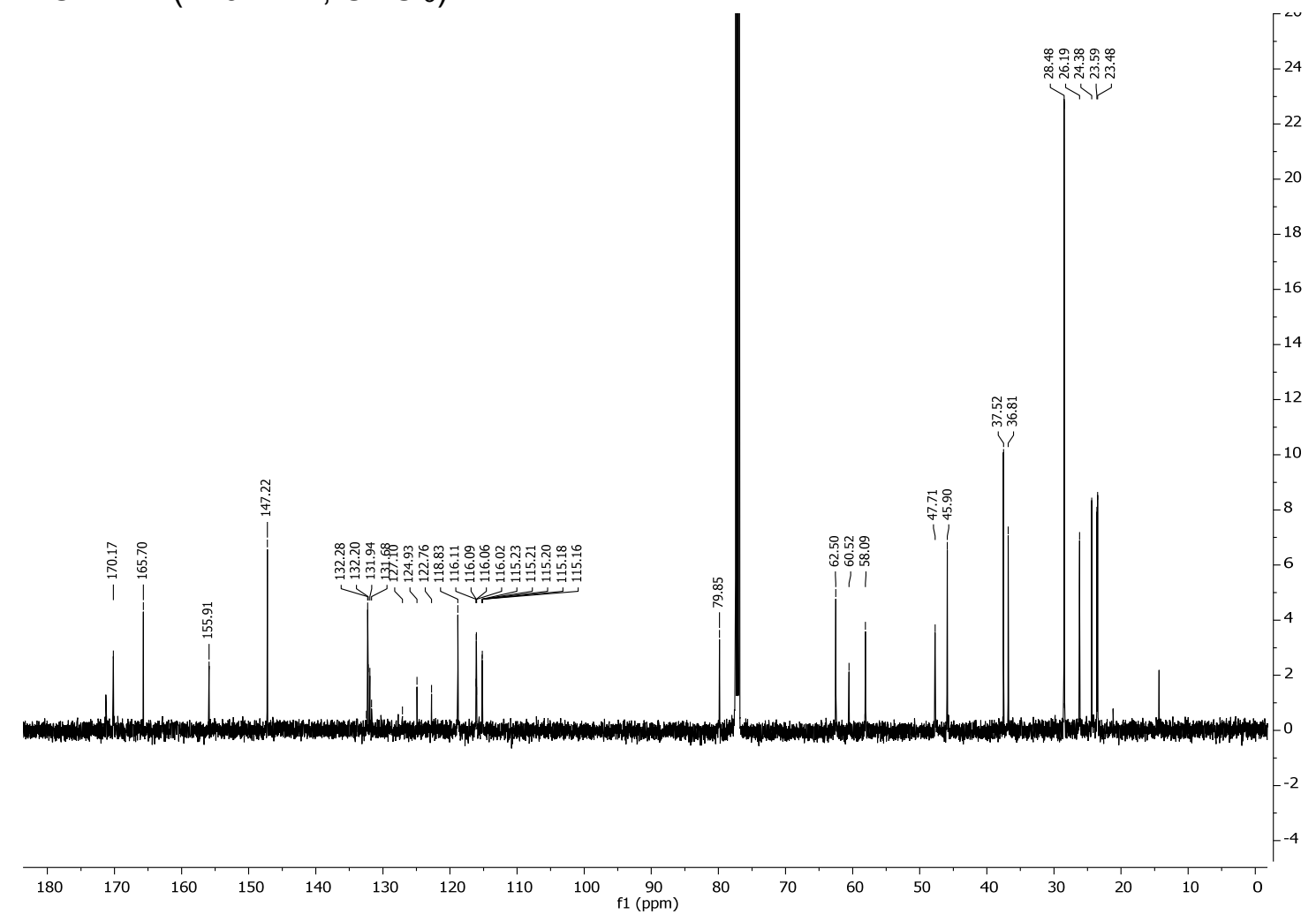


${ }^{19} \mathrm{~F} \mathrm{NMR}\left(471 \mathrm{MHz}, \mathrm{CDCl}_{3}\right)$

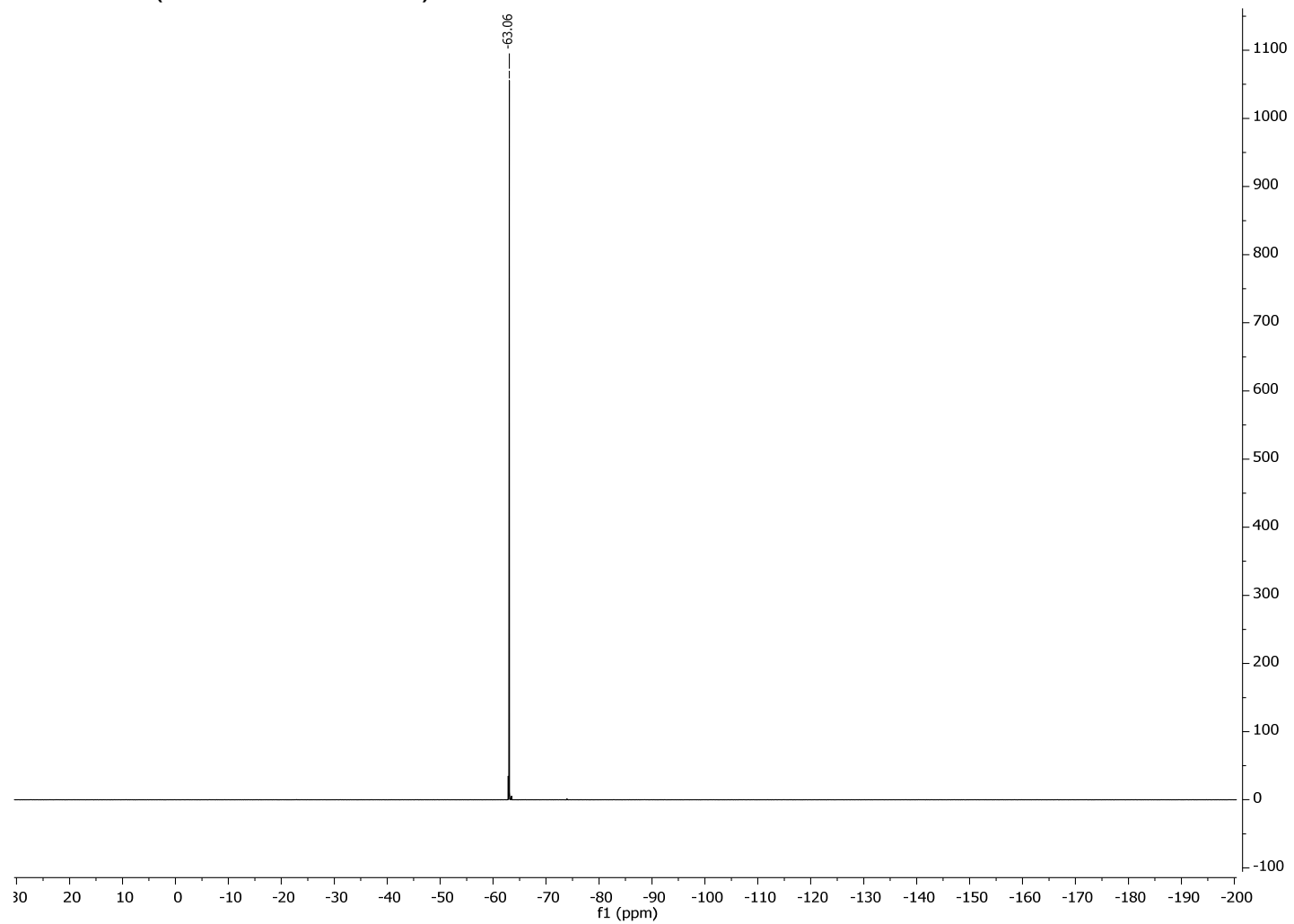


${ }^{1} \mathrm{H} \mathrm{NMR}\left(600 \mathrm{MHz}, \mathrm{CDCl}_{3}\right)$
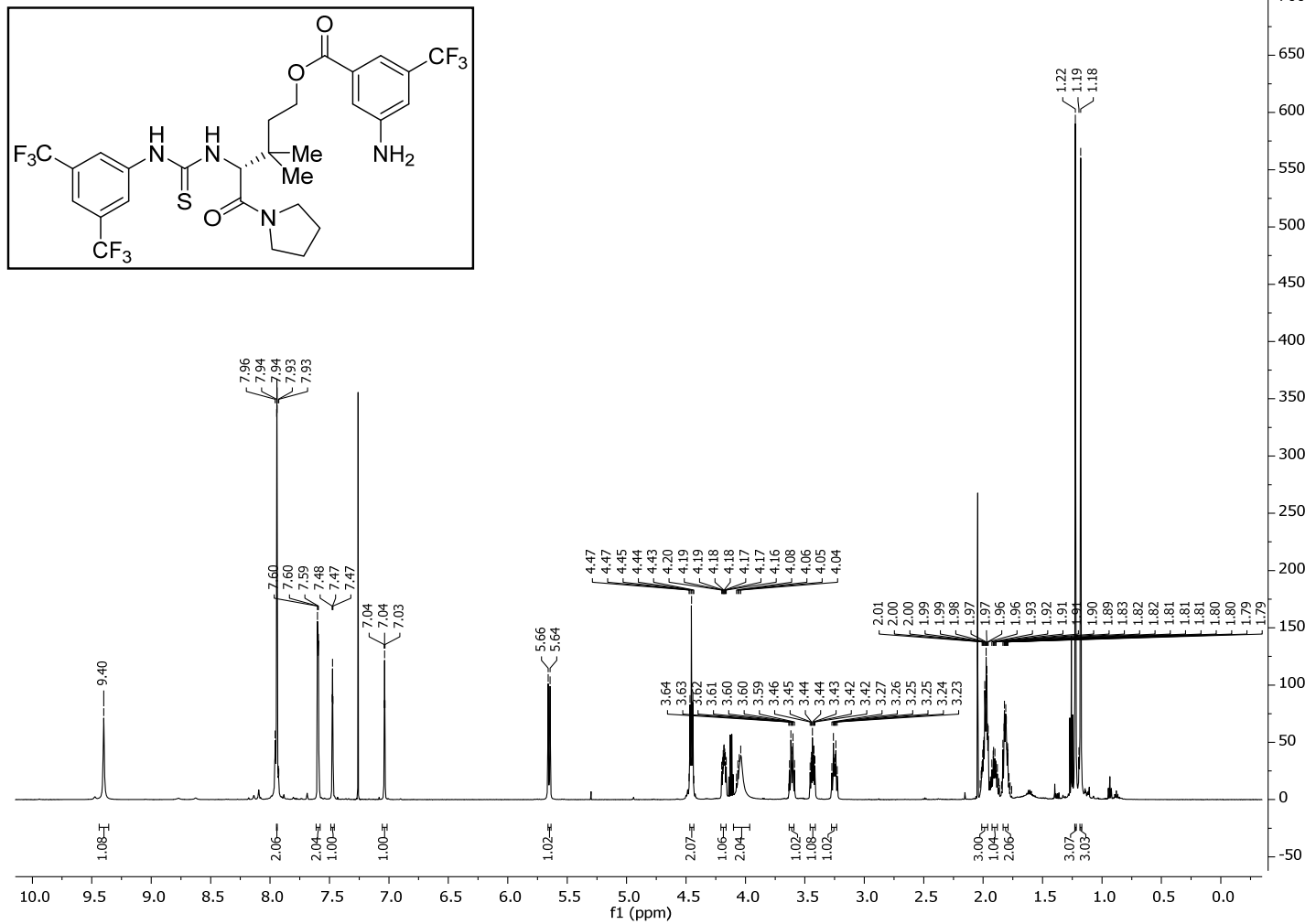

$\left.{ }^{13} \mathrm{C} \mathrm{NMR} \mathrm{(126} \mathrm{MHz,} \mathrm{CDCl}_{3}\right)$

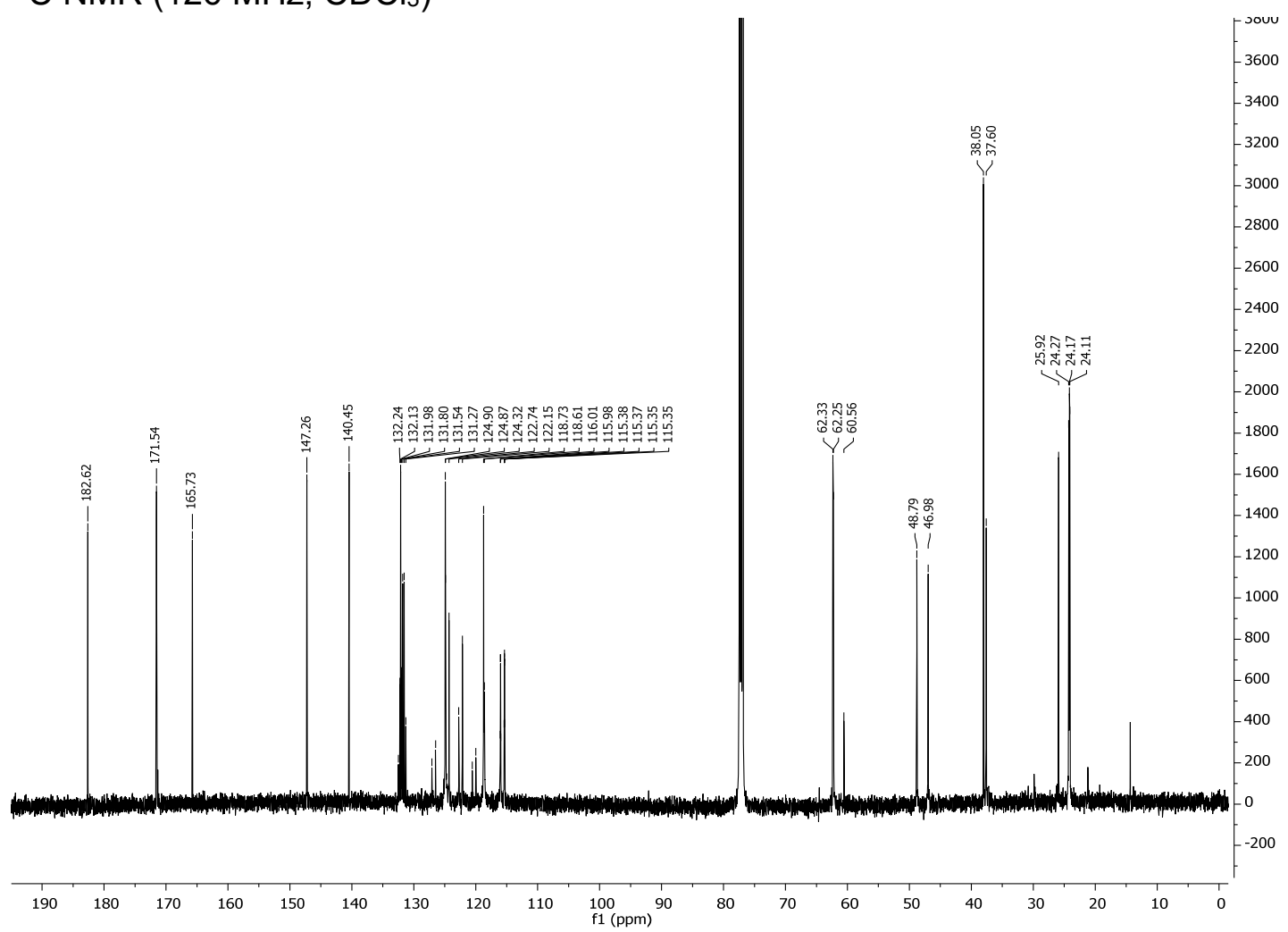


${ }^{19} \mathrm{~F} \mathrm{NMR}\left(471 \mathrm{MHz}, \mathrm{CDCl}_{3}\right)$

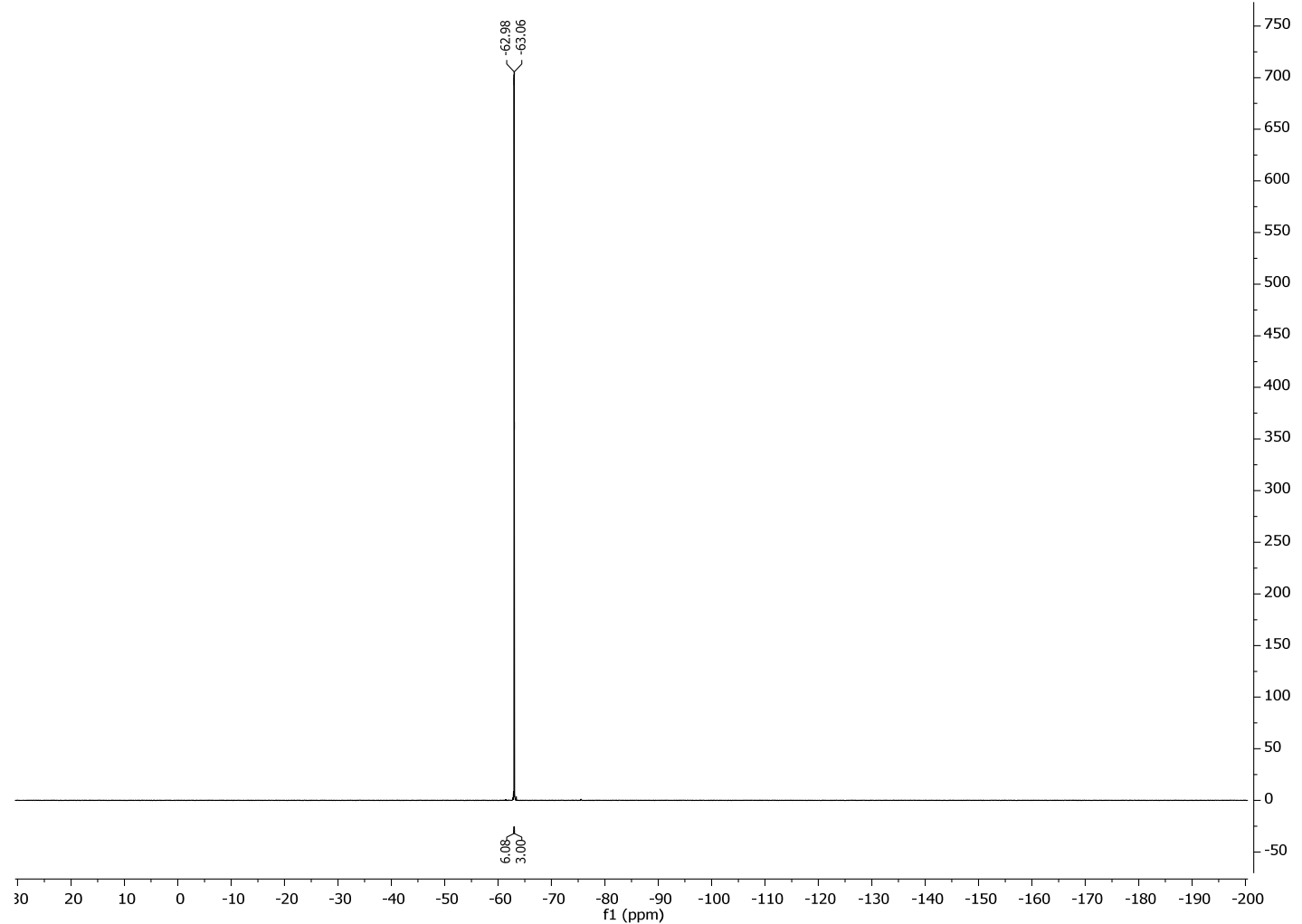


${ }^{1} \mathrm{H}$ NMR $\left(600 \mathrm{MHz}, \mathrm{CDCl}_{3}\right)$
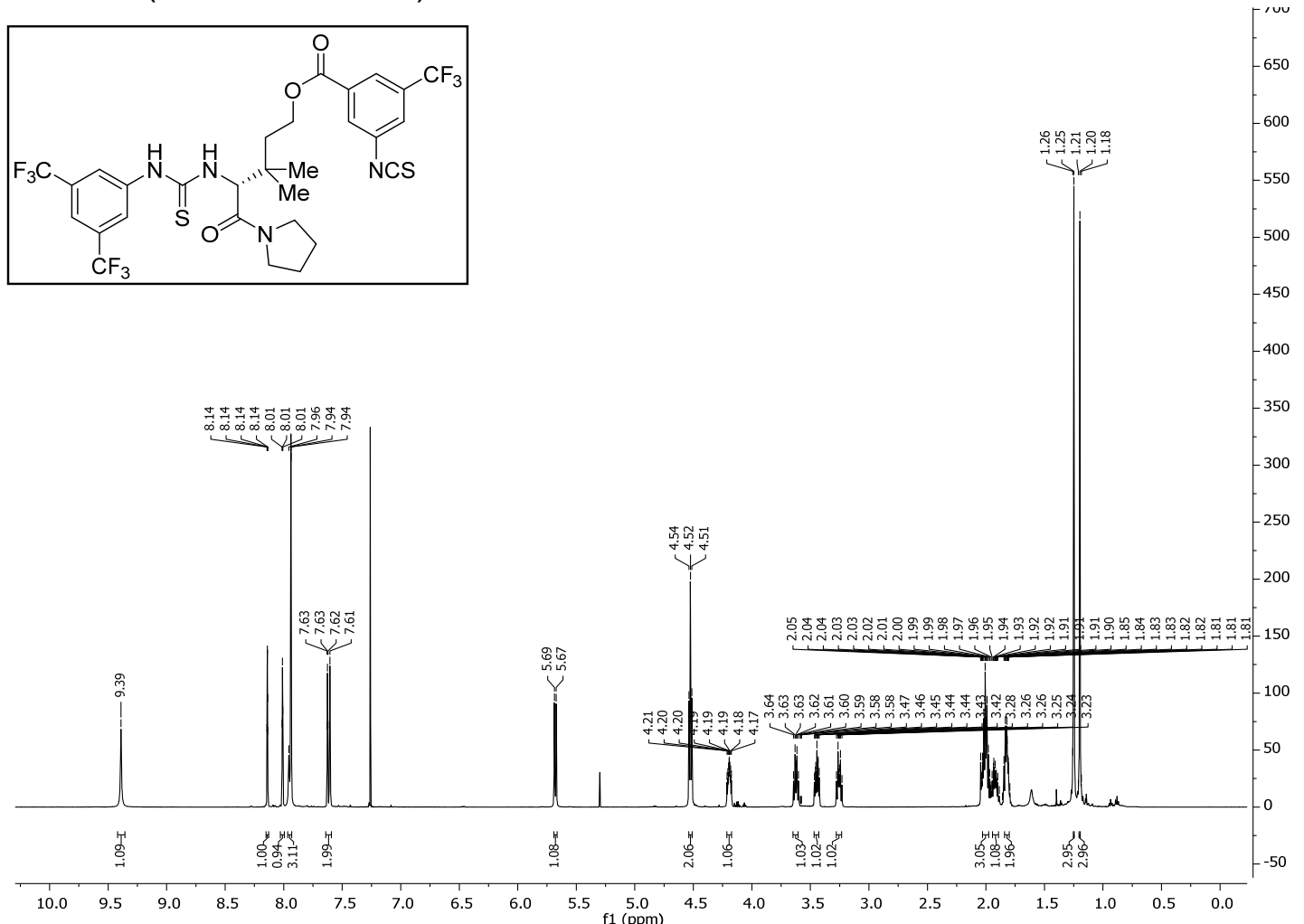

${ }^{13} \mathrm{C} \mathrm{NMR}\left(126 \mathrm{MHz}, \mathrm{CDCl}_{3}\right)$

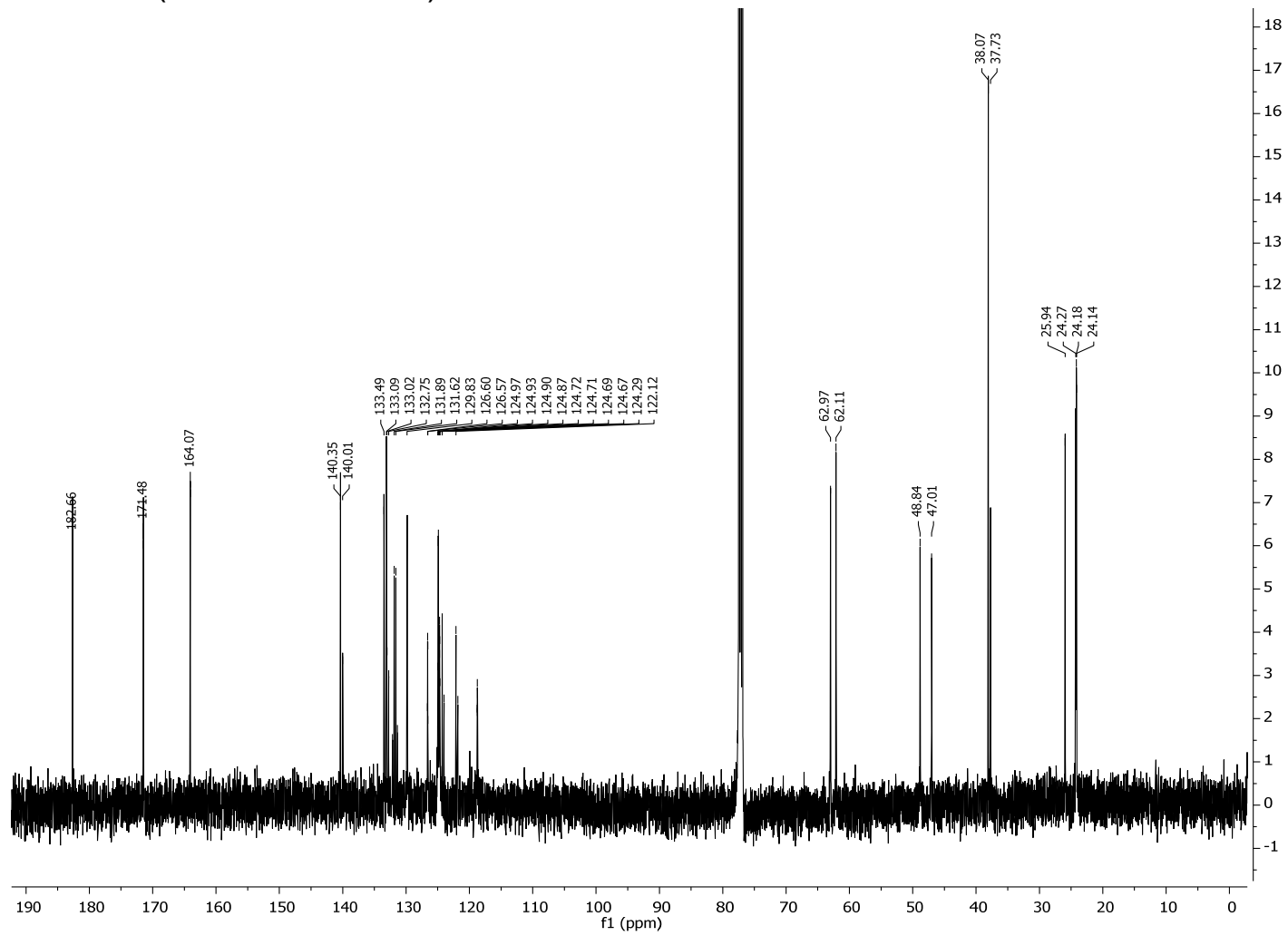


${ }^{19} \mathrm{~F} \mathrm{NMR}\left(471 \mathrm{MHz}, \mathrm{CDCl}_{3}\right)$

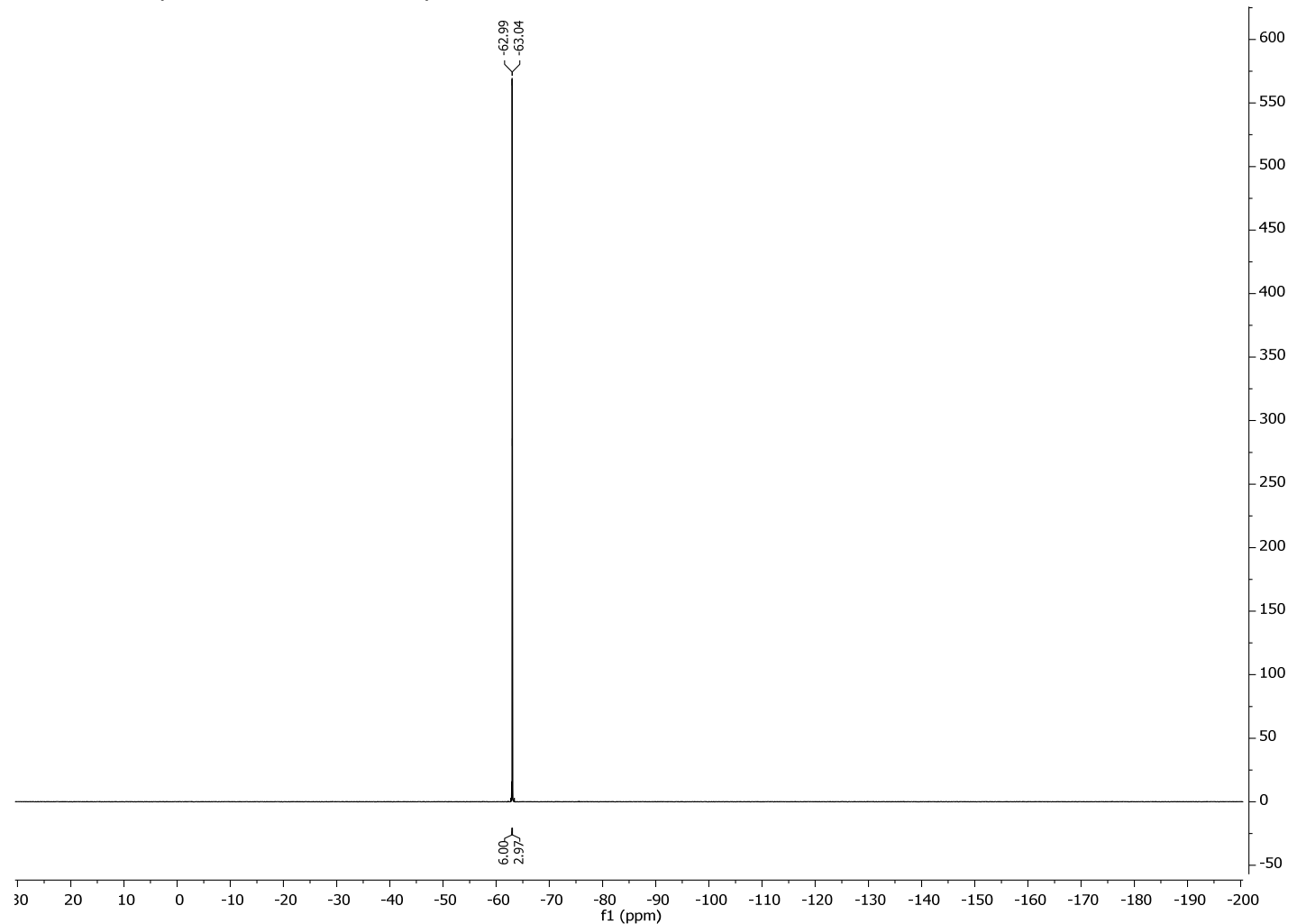


${ }^{1} \mathrm{H}$ NMR $\left(600 \mathrm{MHz}, \mathrm{CDCl}_{3}\right)$
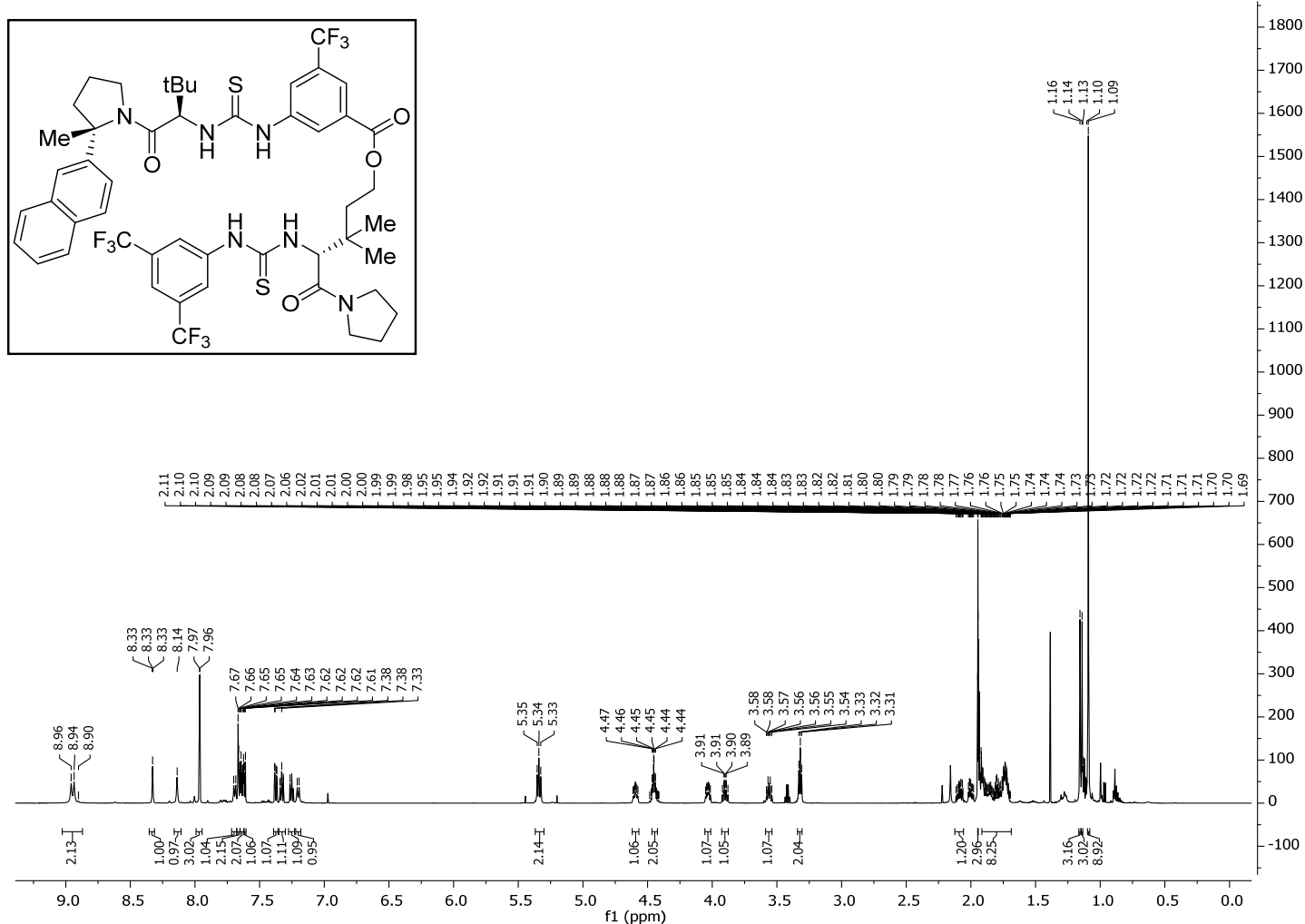

$\left.{ }^{13} \mathrm{C} \mathrm{NMR} \mathrm{(126} \mathrm{MHz,} \mathrm{CDCl}_{3}\right)$

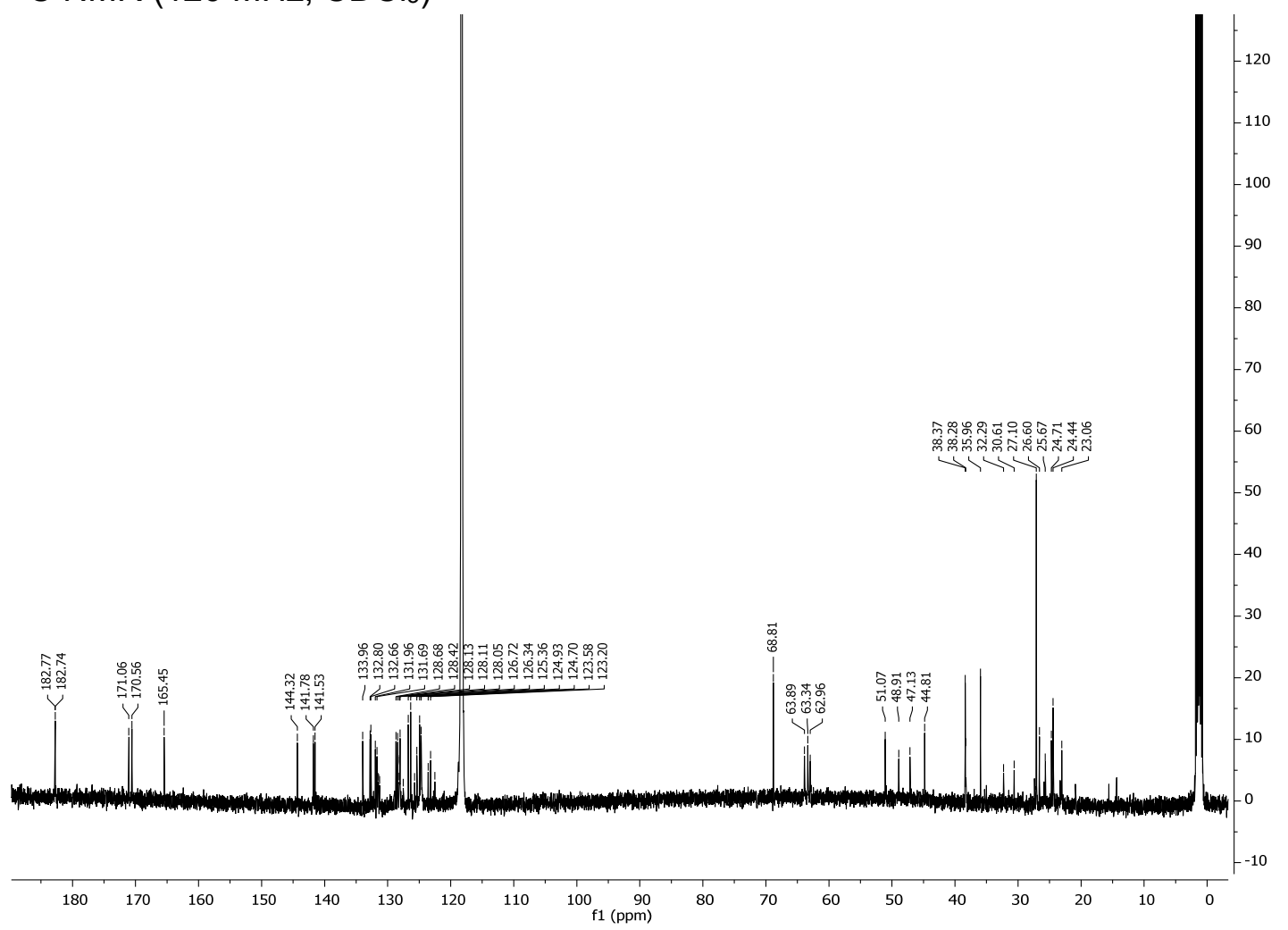


${ }^{19} \mathrm{~F} \mathrm{NMR}\left(471 \mathrm{MHz}, \mathrm{CDCl}_{3}\right)$

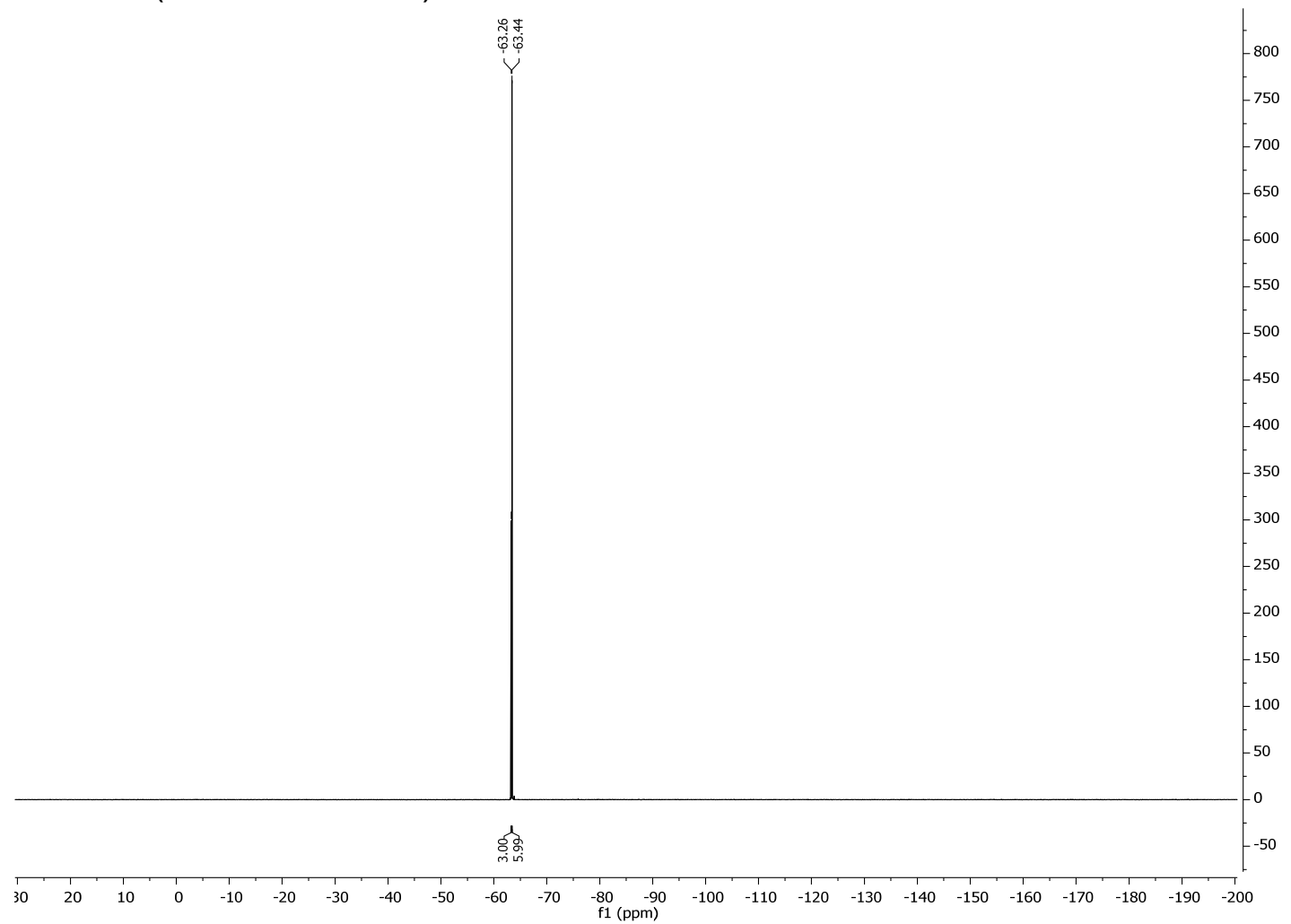


${ }^{1} \mathrm{H}$ NMR $\left(600 \mathrm{MHz}, \mathrm{CDCl}_{3}\right)$
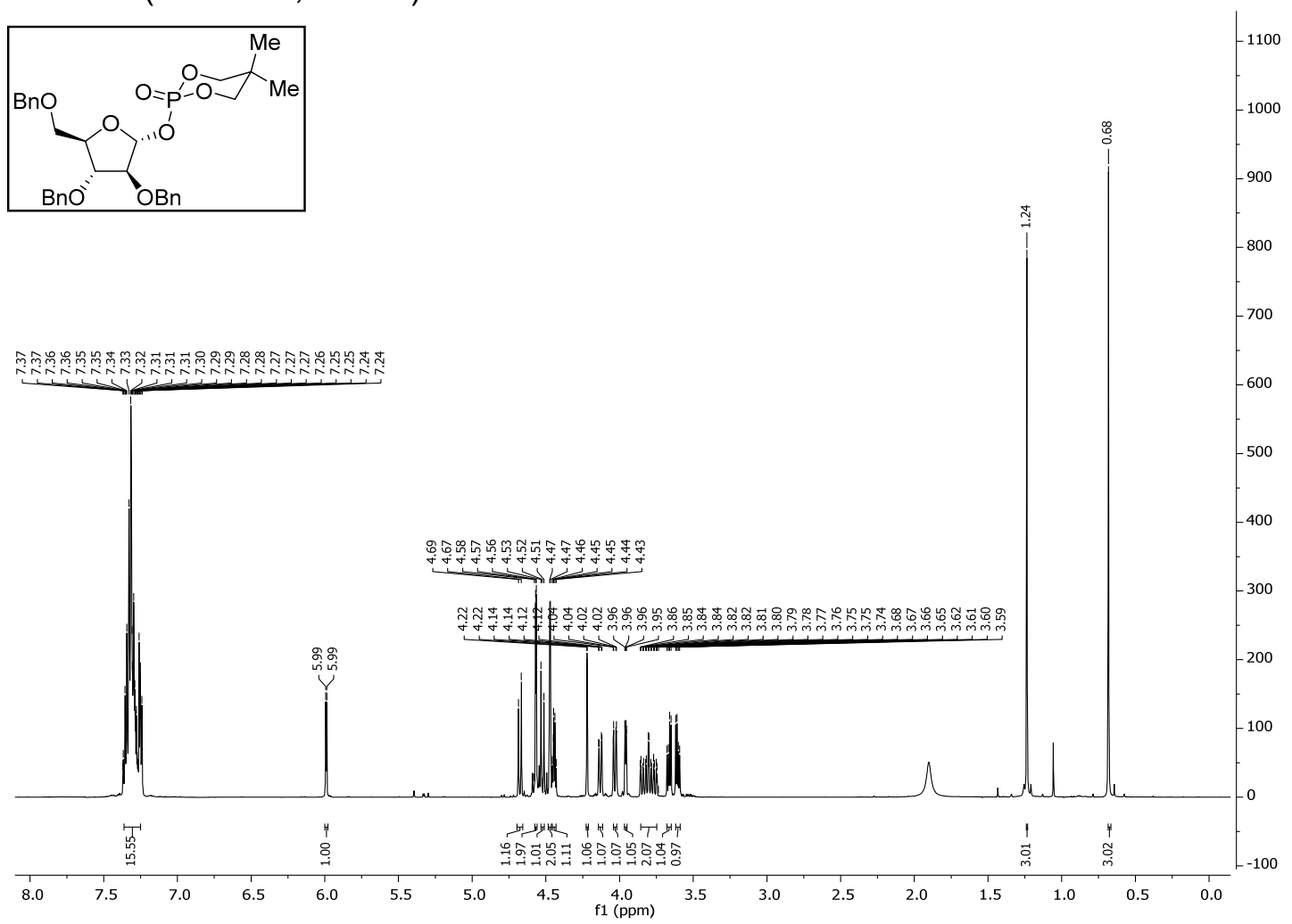

${ }^{13} \mathrm{C}$ NMR (126 MHz, $\left.\mathrm{CDCl}_{3}\right)$

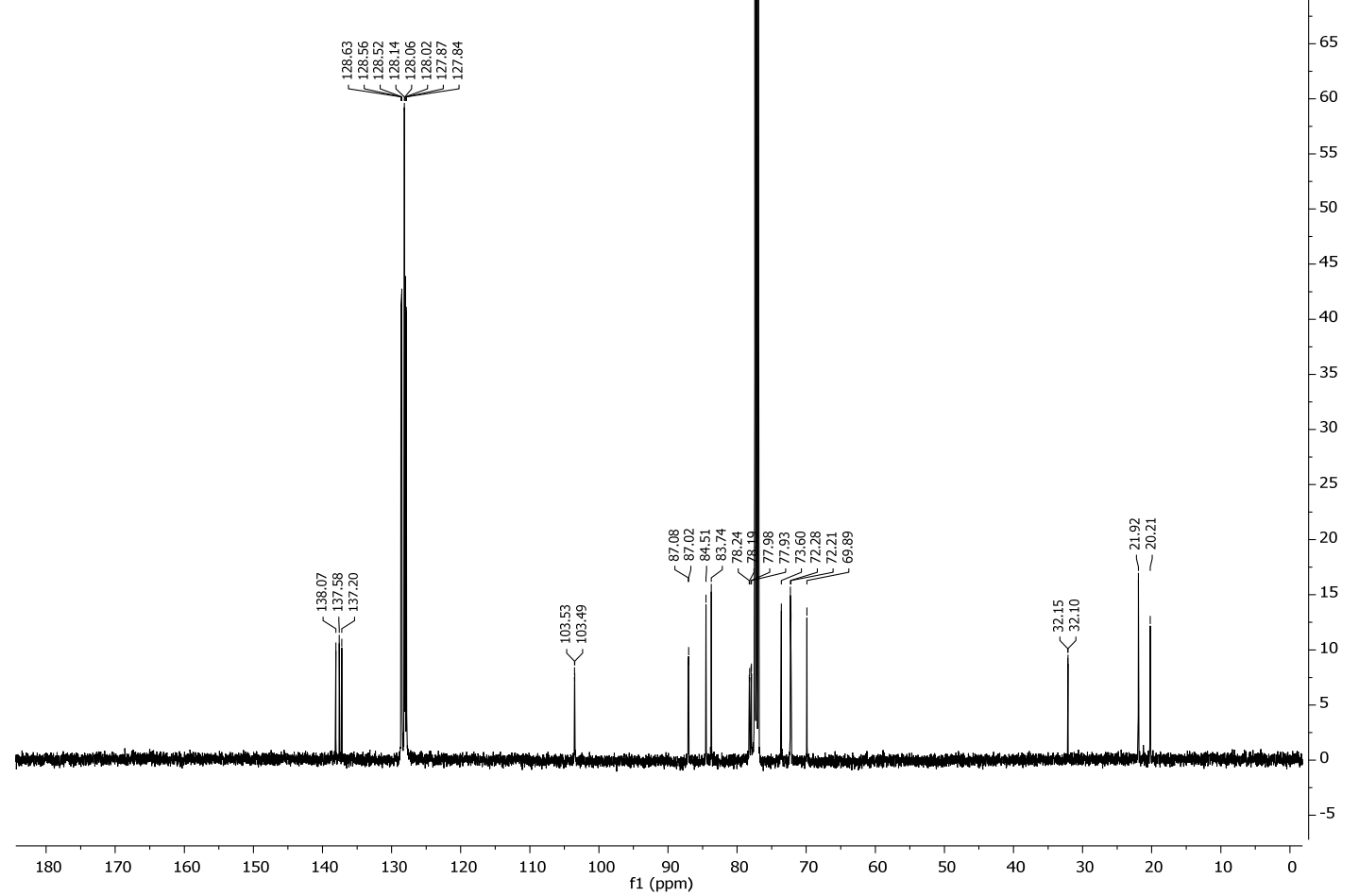


${ }^{31} \mathrm{P}$ NMR $\left(162 \mathrm{MHz}, \mathrm{CDCl}_{3}\right)$

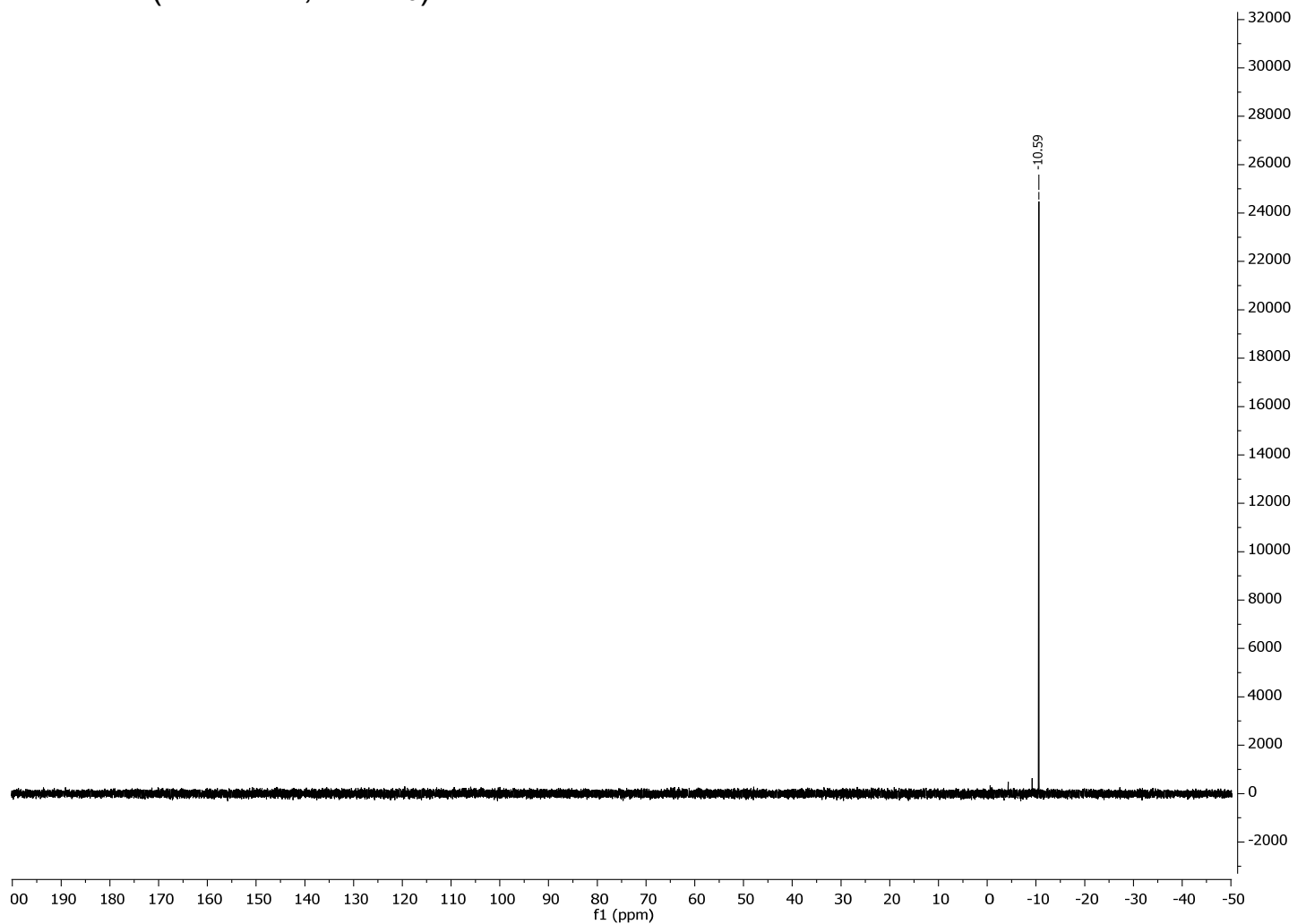


${ }^{1} \mathrm{H} \mathrm{NMR}\left(600 \mathrm{MHz}, \mathrm{CDCl}_{3}\right)$

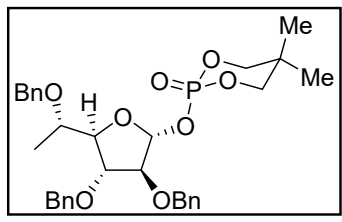

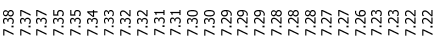

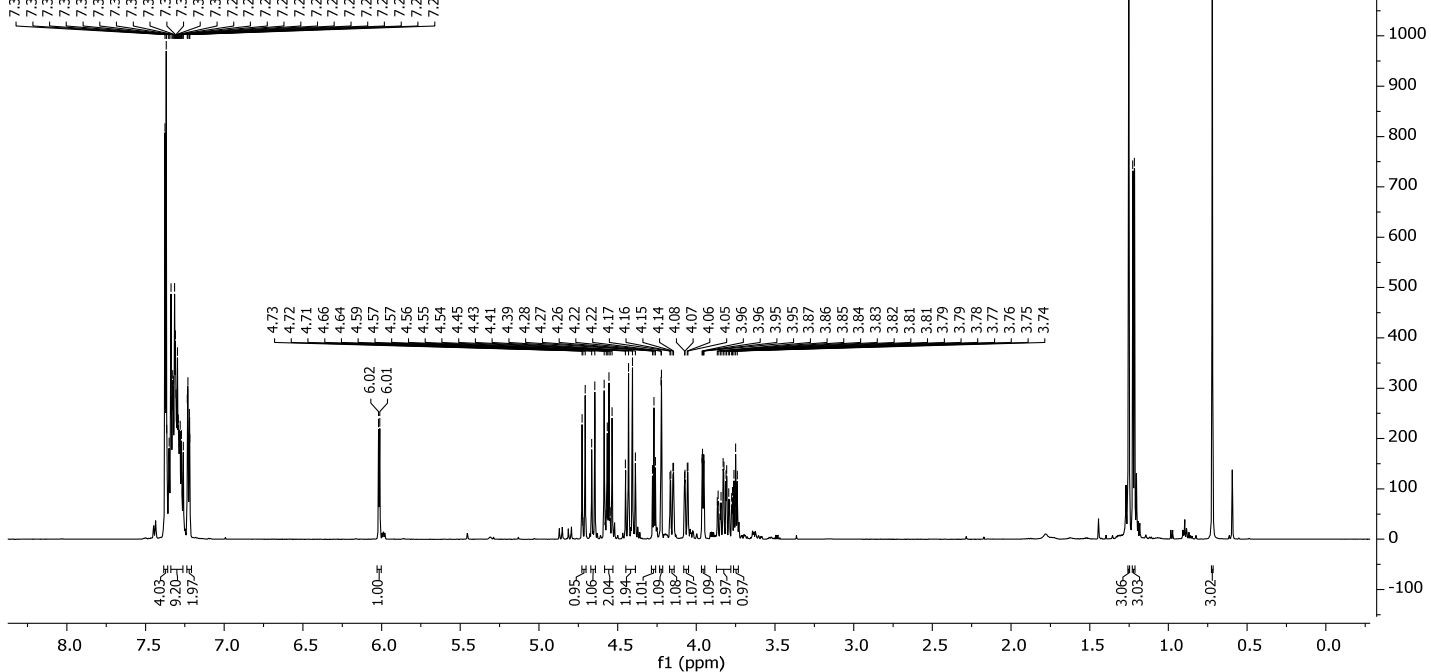

${ }^{13} \mathrm{C} \mathrm{NMR}\left(126 \mathrm{MHz}, \mathrm{CDCl}_{3}\right)$

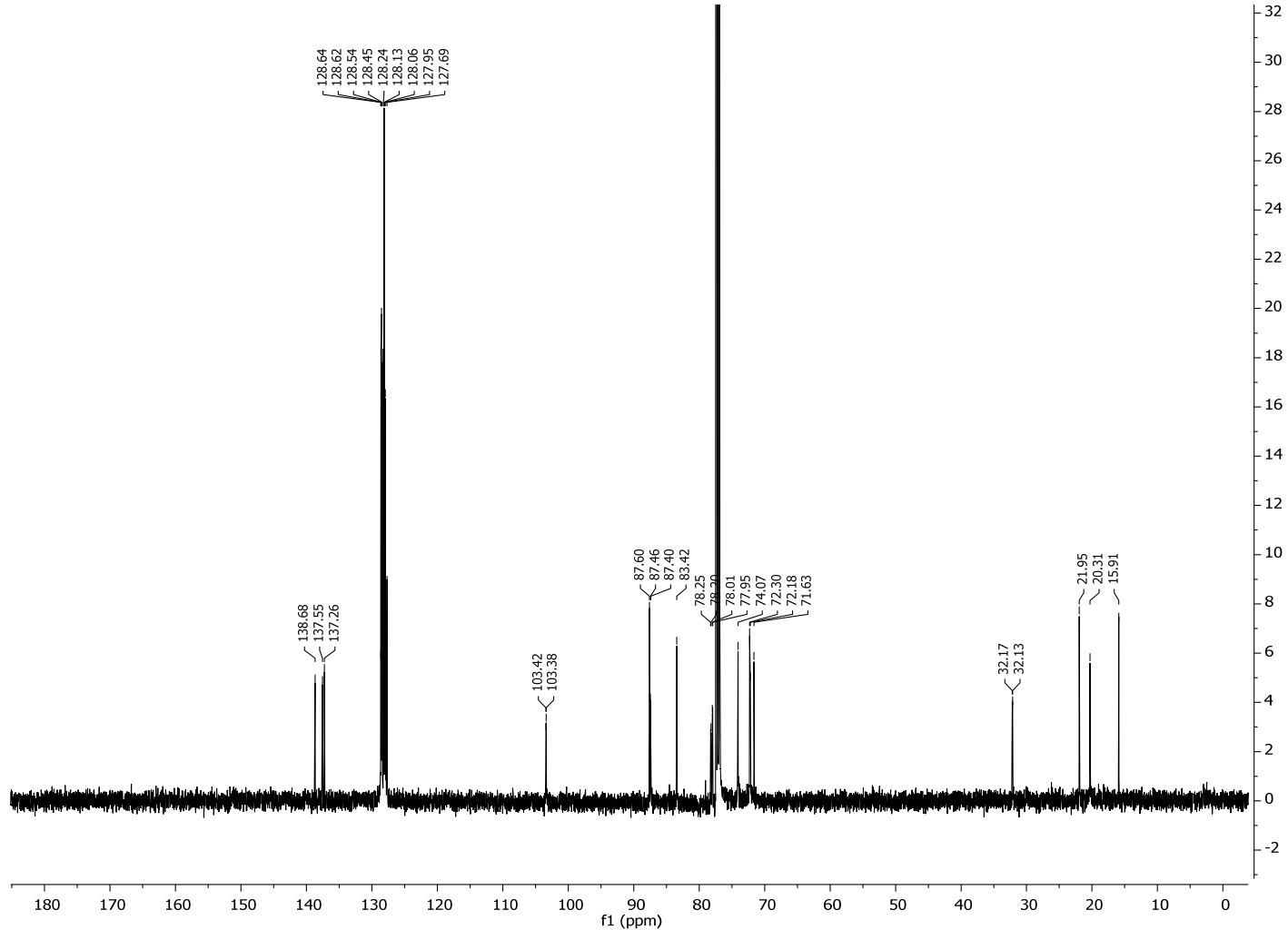


${ }^{31} \mathrm{P}$ NMR $\left(162 \mathrm{MHz}, \mathrm{CDCl}_{3}\right)$

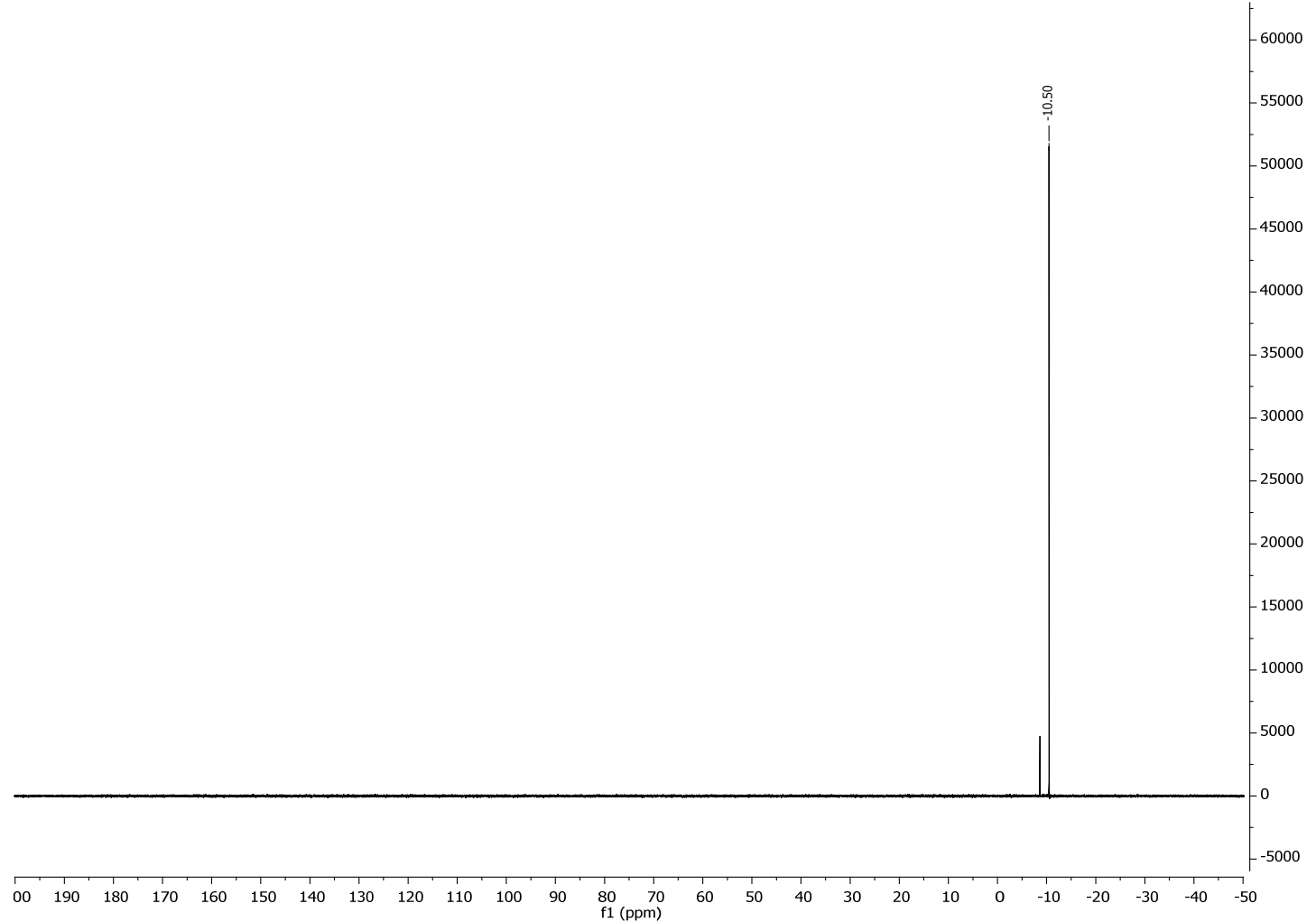


${ }^{1} \mathrm{H}$ NMR $\left(600 \mathrm{MHz}, \mathrm{CDCl}_{3}\right)$

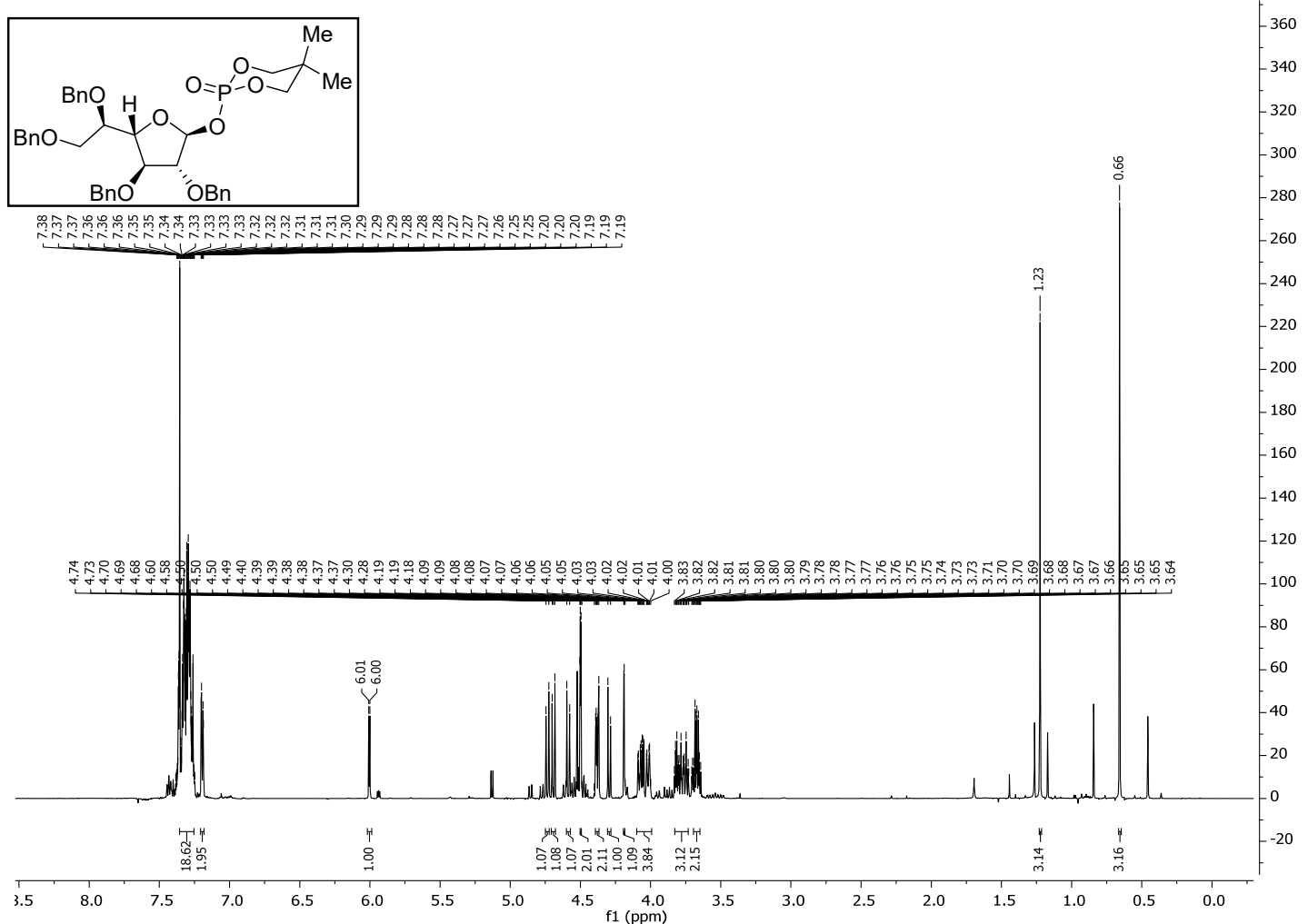

${ }^{13} \mathrm{C}$ NMR (126 MHz, $\left.\mathrm{CDCl}_{3}\right)$

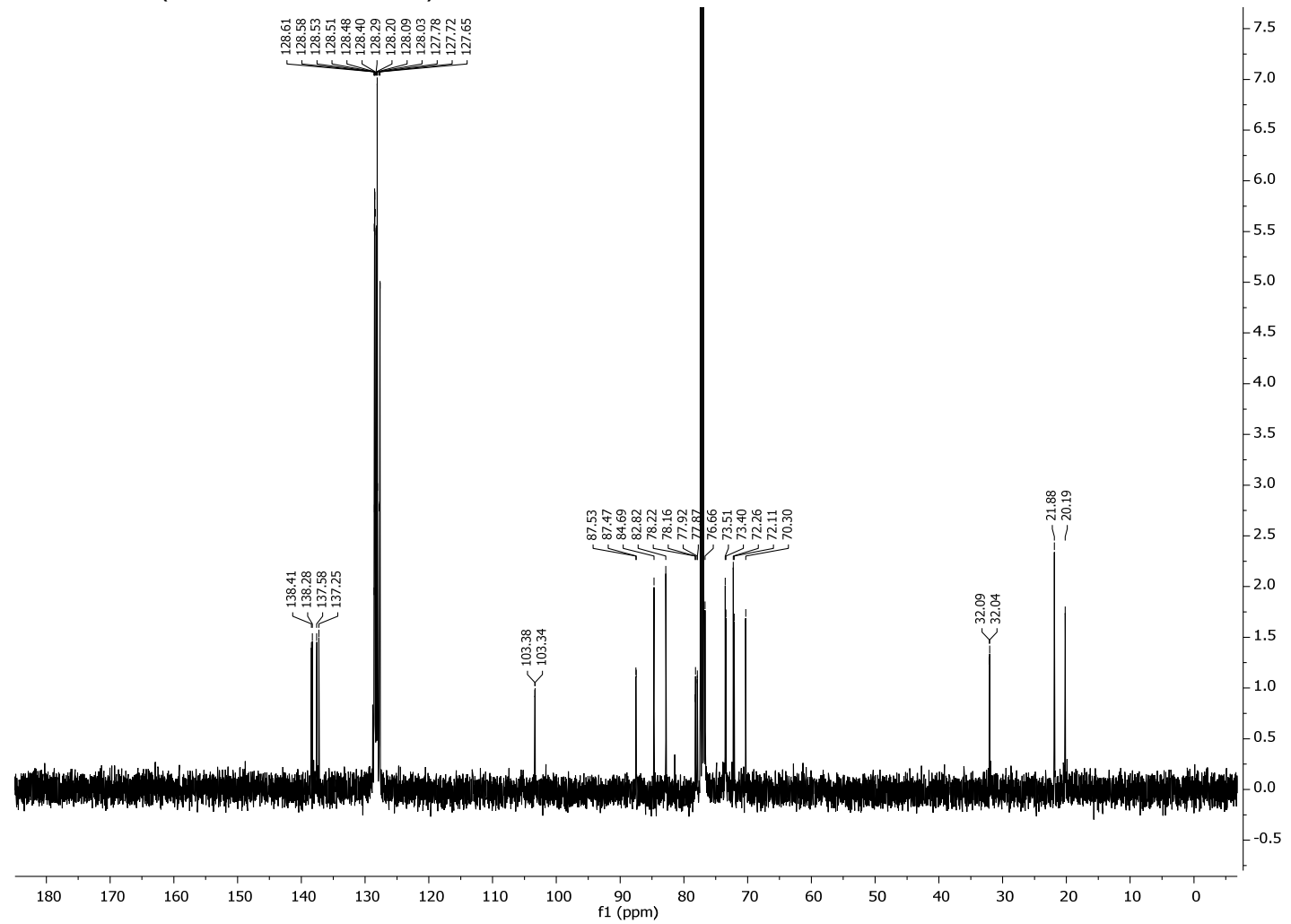


${ }^{31} \mathrm{P}$ NMR $\left(162 \mathrm{MHz}, \mathrm{CDCl}_{3}\right)$

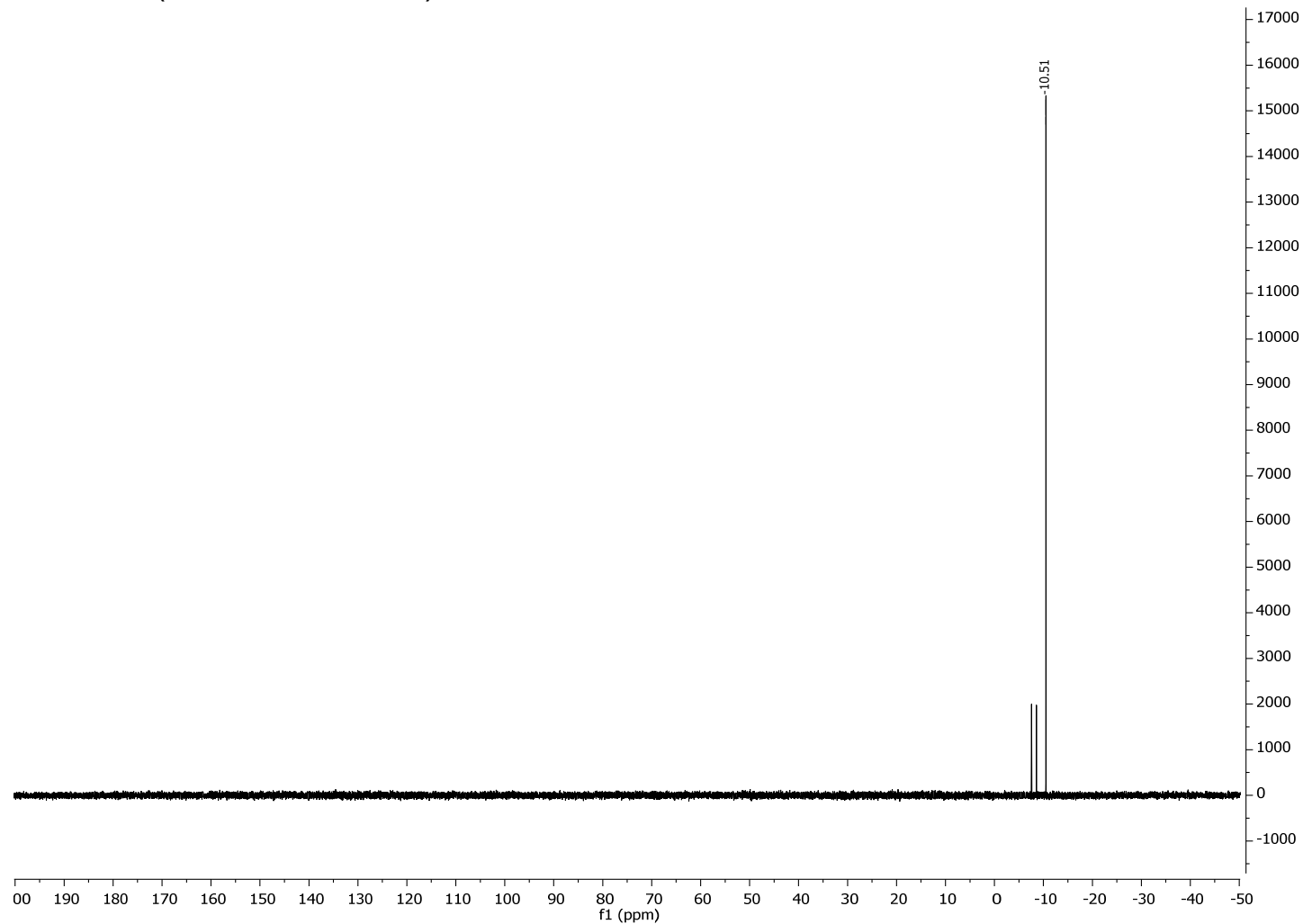


${ }^{1} \mathrm{H} \mathrm{NMR}\left(600 \mathrm{MHz}, \mathrm{CDCl}_{3}\right)$
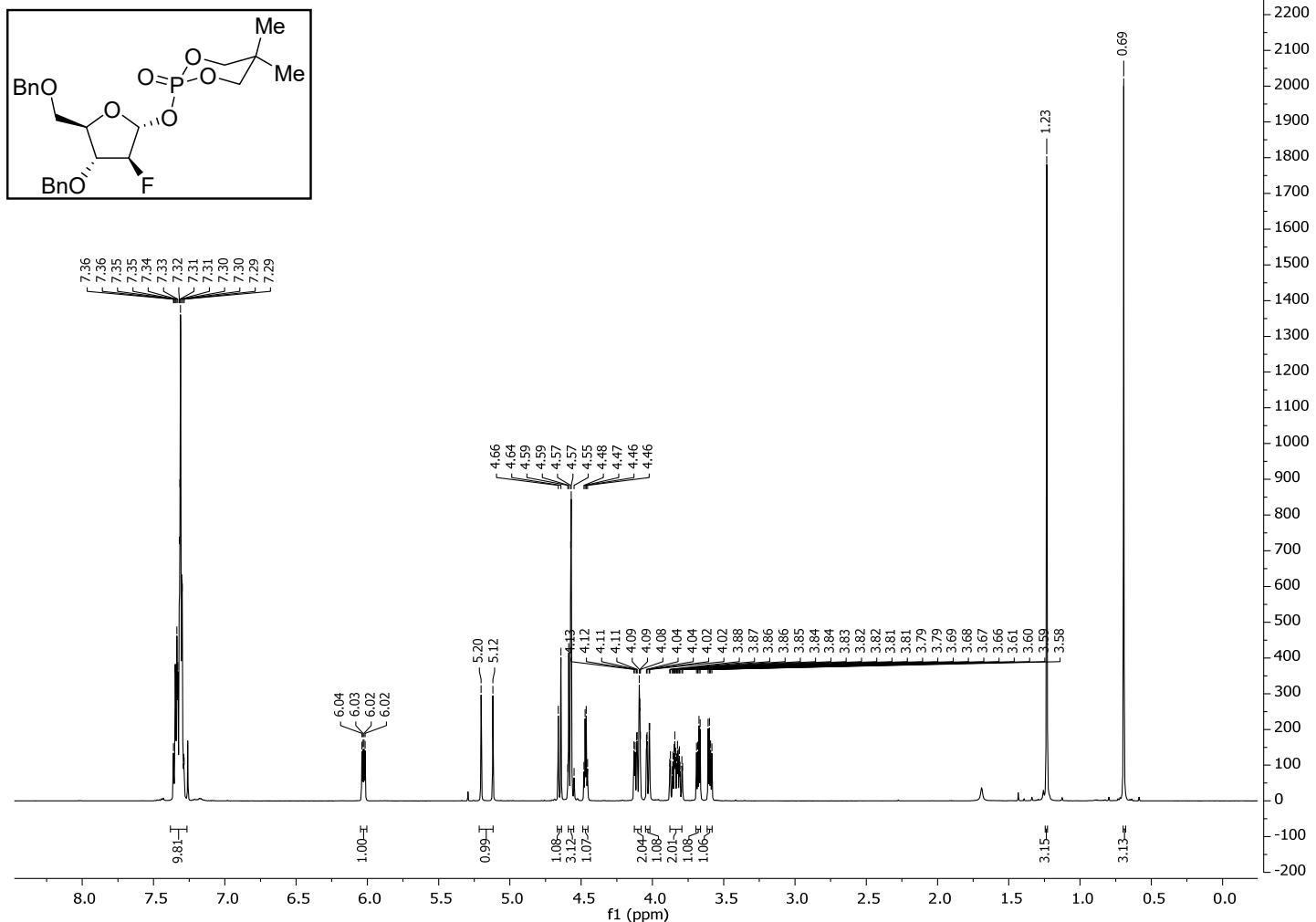

${ }^{13} \mathrm{C}$ NMR $\left(126 \mathrm{MHz}, \mathrm{CDCl}_{3}\right)$

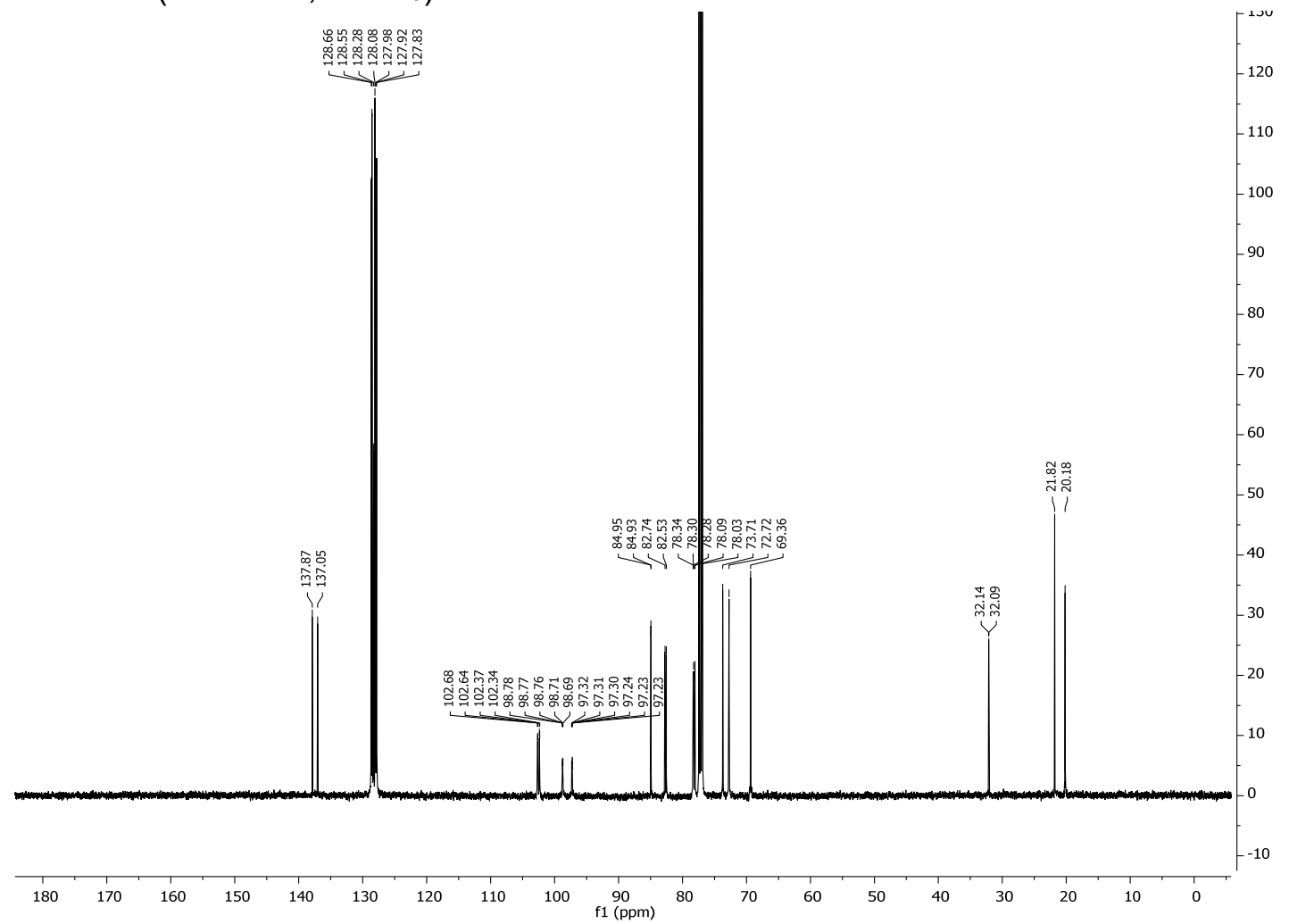


${ }^{31} \mathrm{P}$ NMR $\left(162 \mathrm{MHz}, \mathrm{CDCl}_{3}\right)$

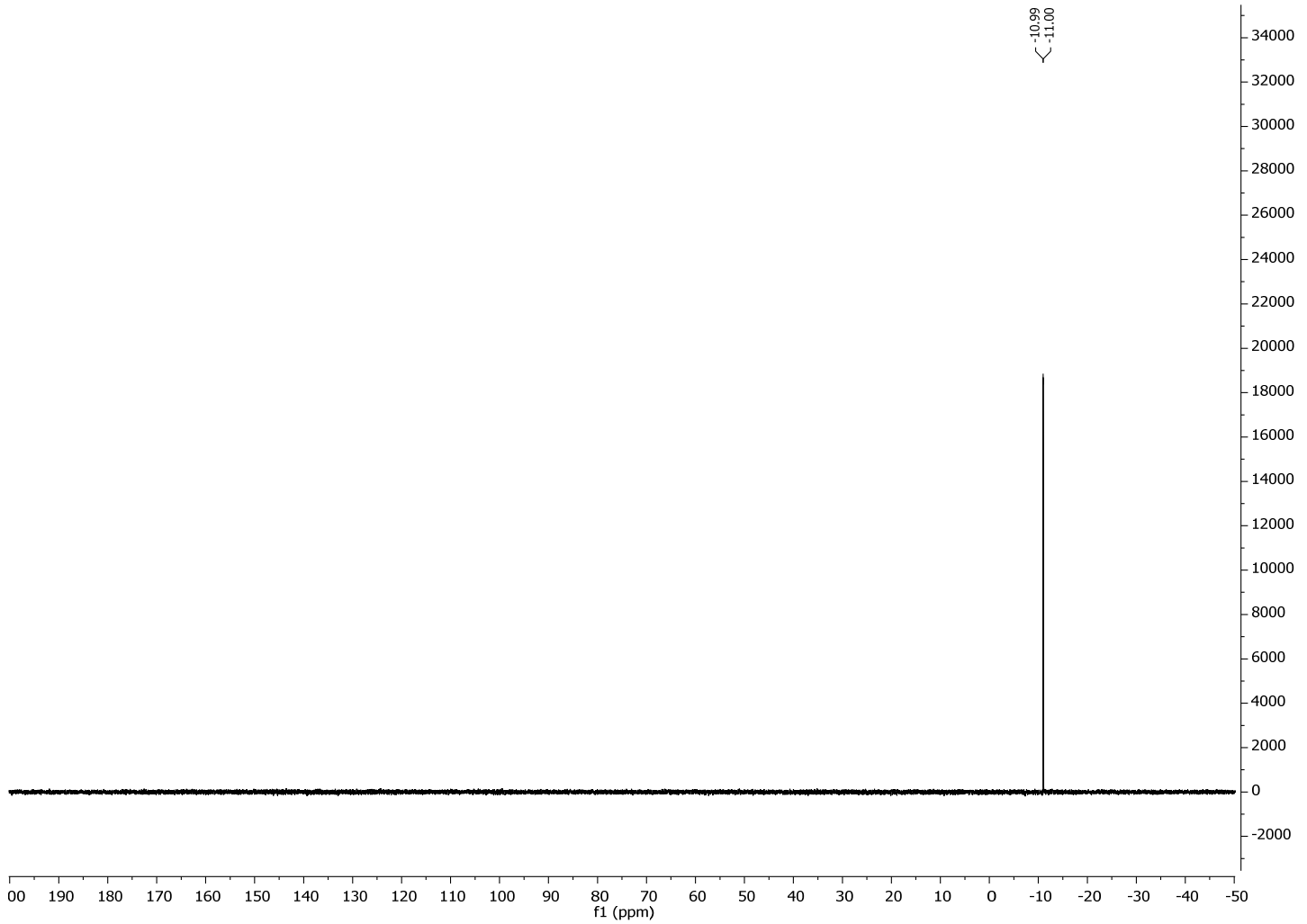

${ }^{19} \mathrm{~F}$ NMR (471 MHz, $\left.\mathrm{CDCl}_{3}\right)$

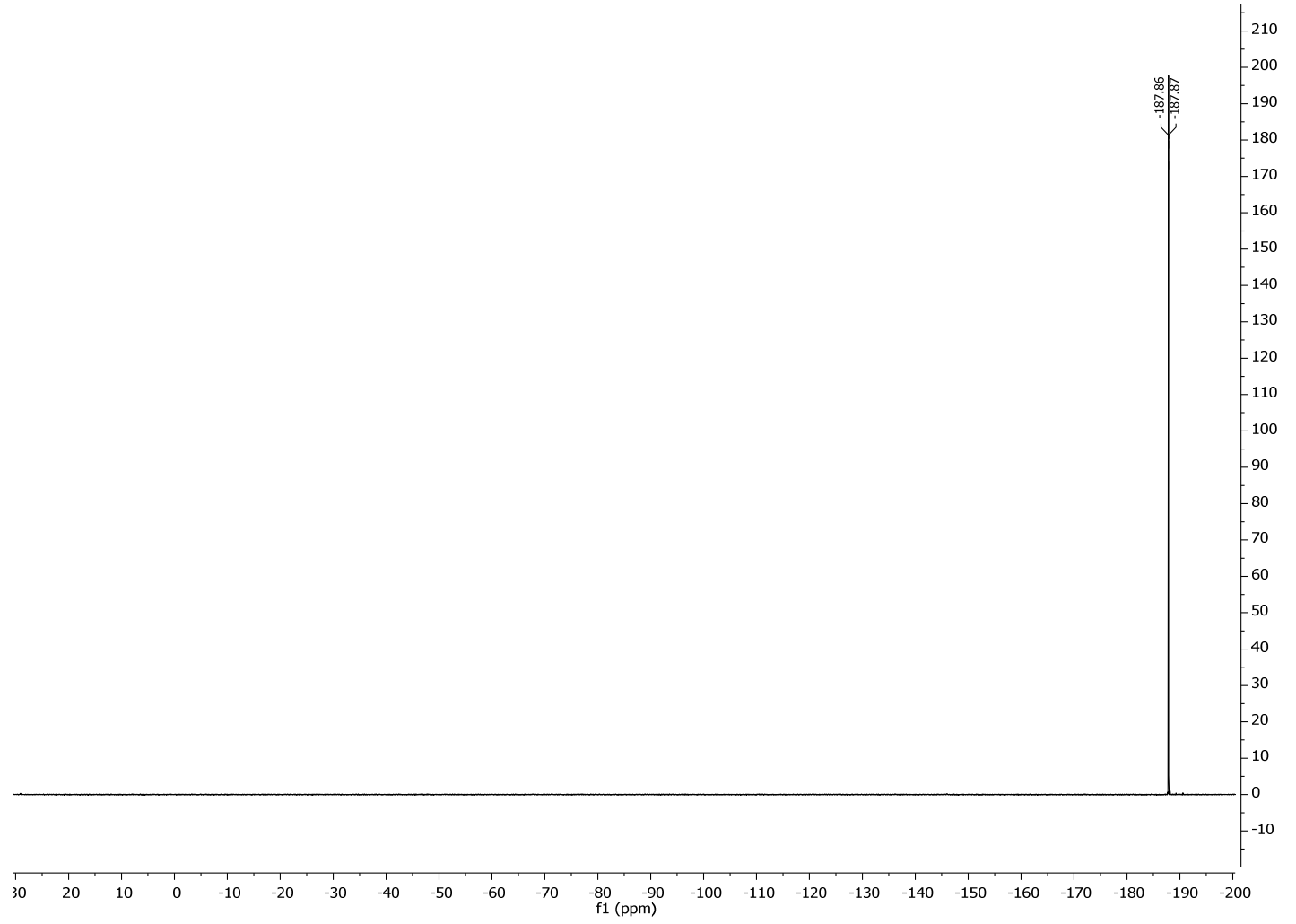


${ }^{1} \mathrm{H}$ NMR $\left(600 \mathrm{MHz}, \mathrm{CDCl}_{3}\right)$
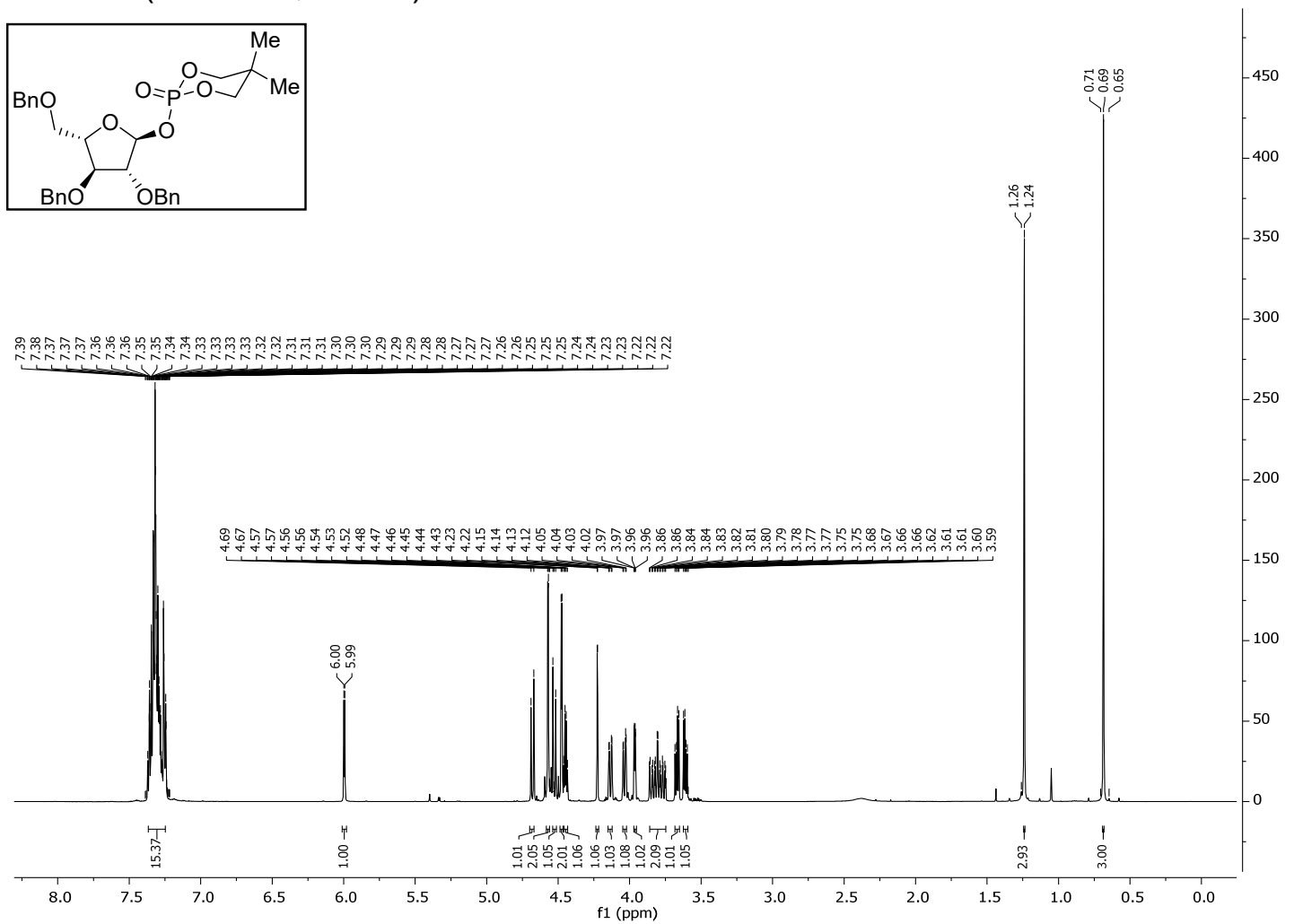

$\left.{ }^{13} \mathrm{C} \mathrm{NMR} \mathrm{(126} \mathrm{MHz,} \mathrm{CDCl}_{3}\right)$

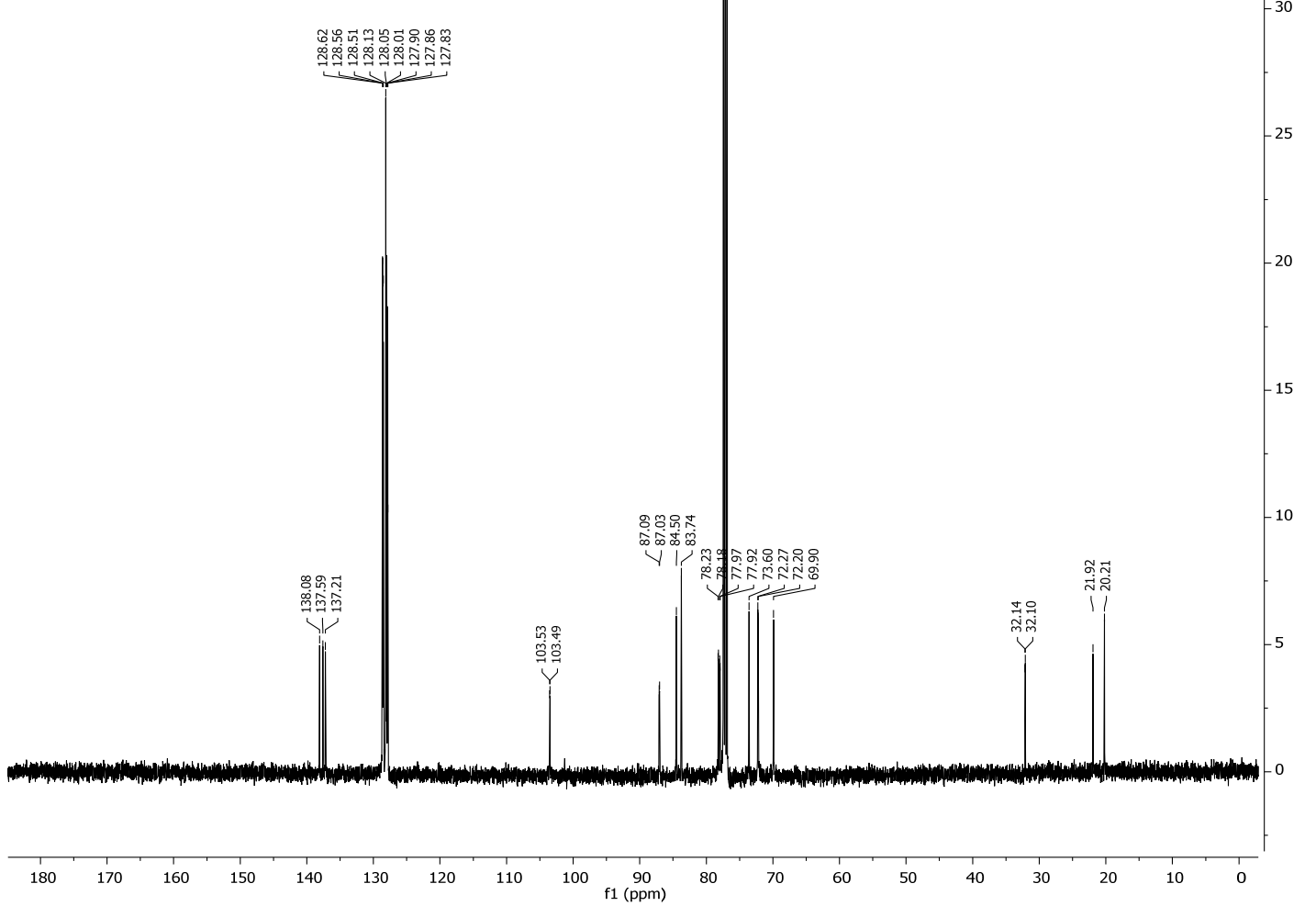


${ }^{31} \mathrm{P}$ NMR $\left(162 \mathrm{MHz}, \mathrm{CDCl}_{3}\right)$

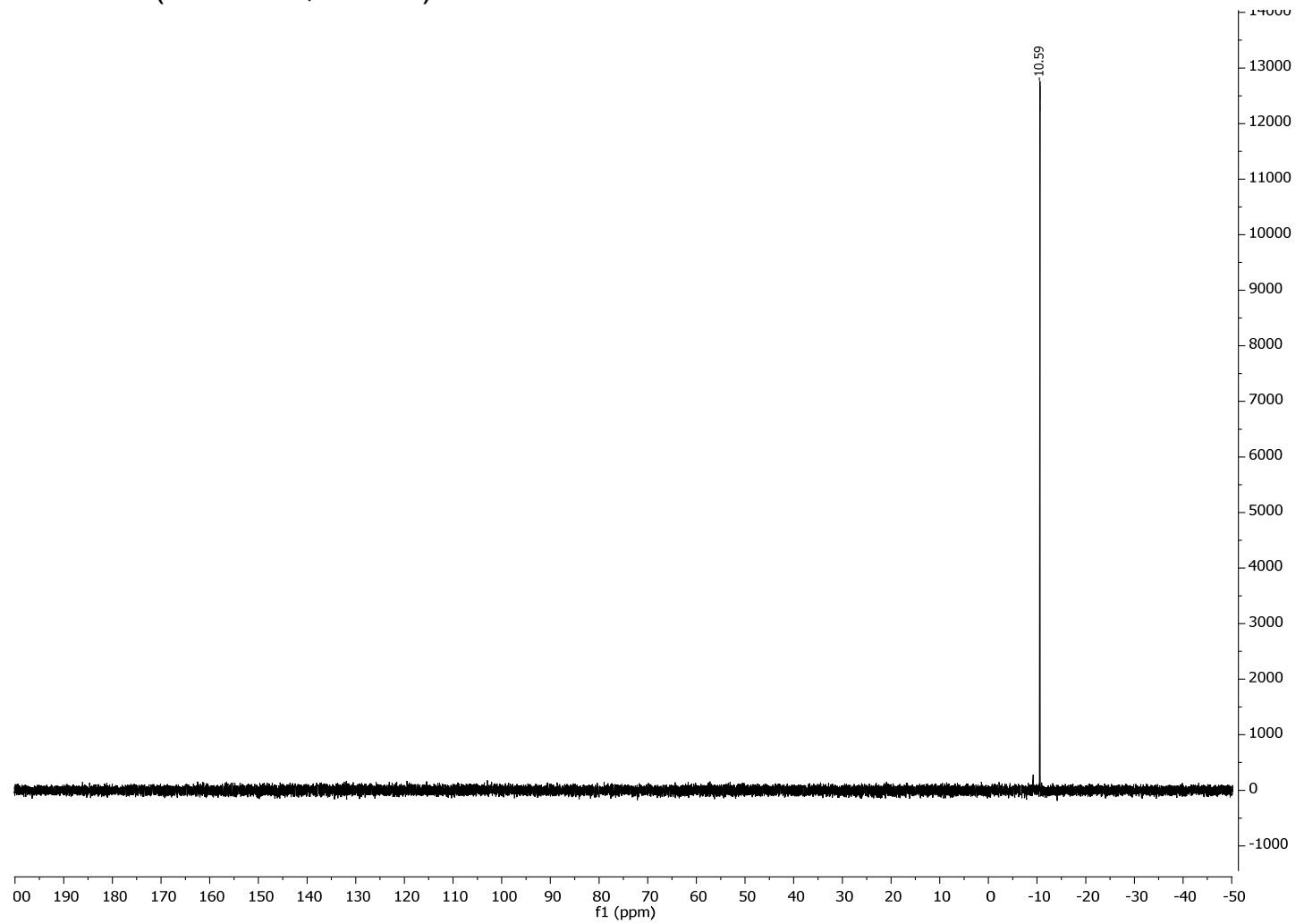


${ }^{1} \mathrm{H}$ NMR $\left(600 \mathrm{MHz}, \mathrm{CDCl}_{3}\right)$

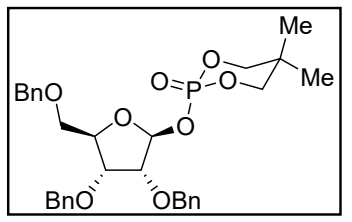

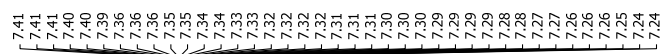

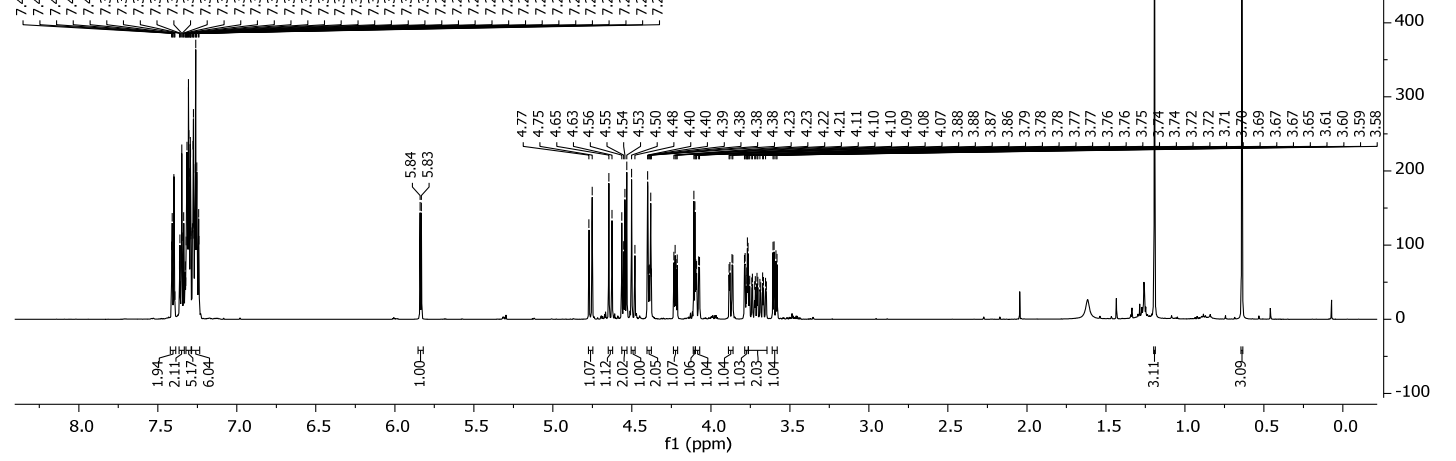

${ }^{13} \mathrm{C}$ NMR $\left(126 \mathrm{MHz}, \mathrm{CDCl}_{3}\right)$

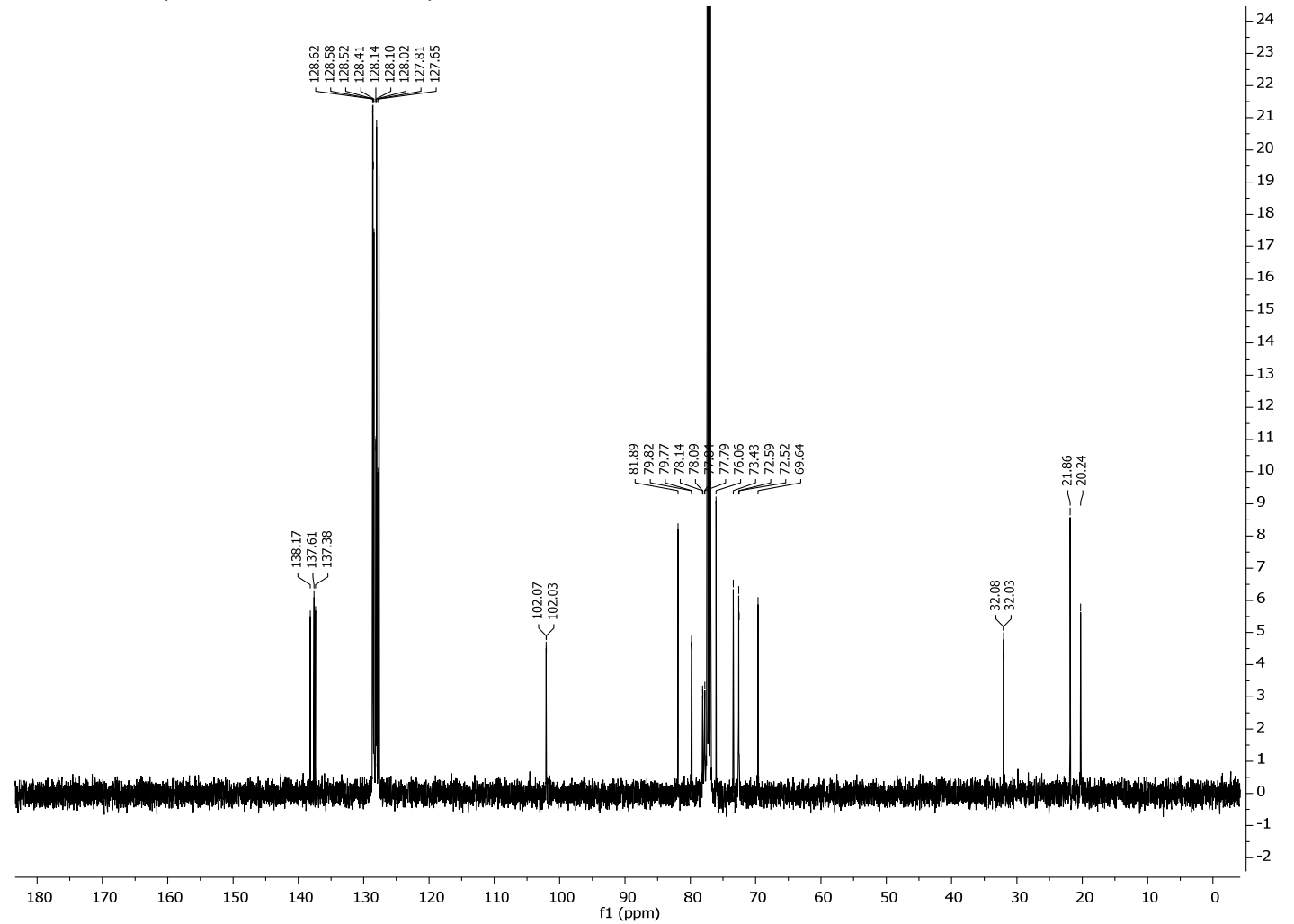


${ }^{31} \mathrm{P}$ NMR $\left(162 \mathrm{MHz}, \mathrm{CDCl}_{3}\right)$

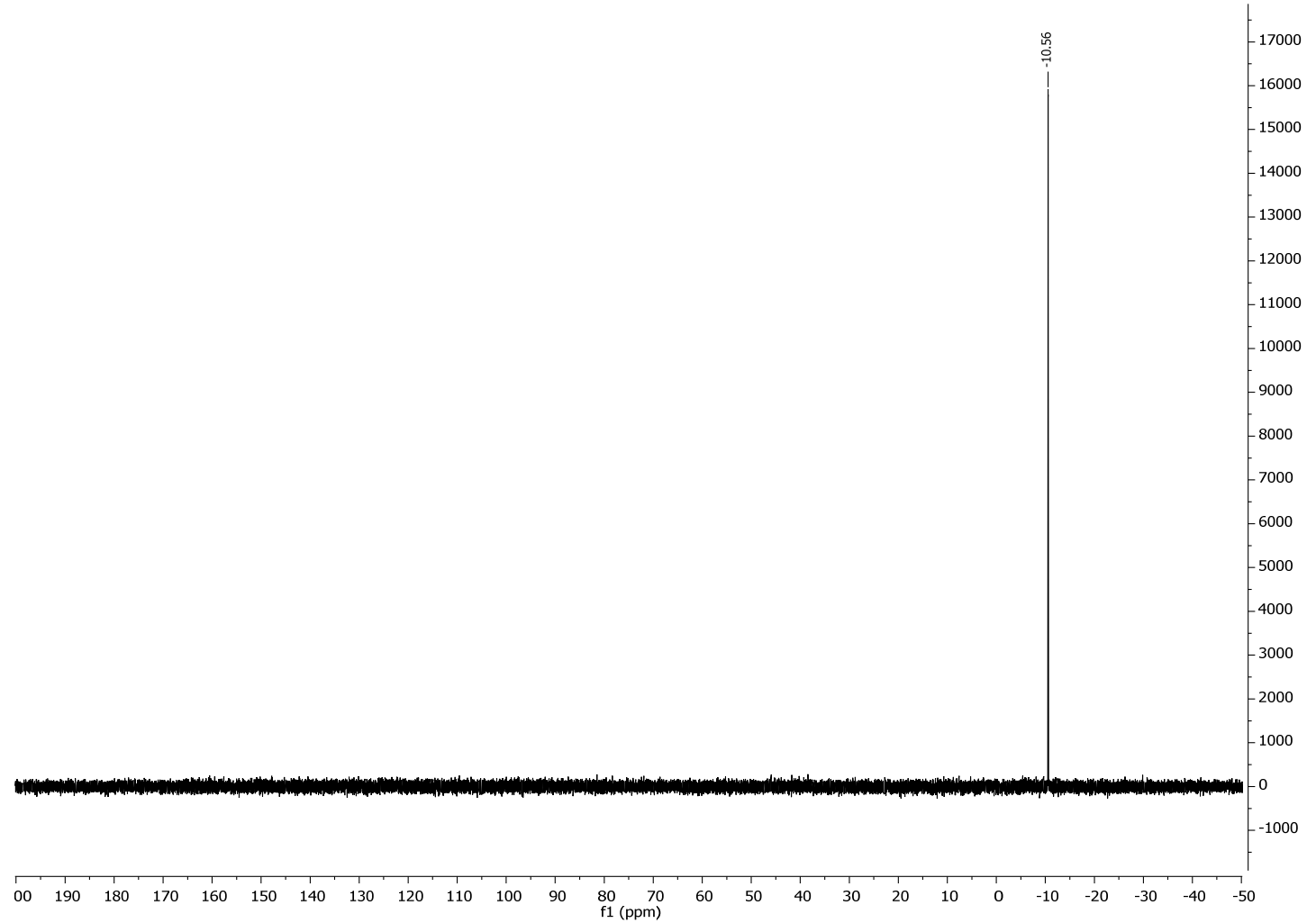


${ }^{1} \mathrm{H}$ NMR $\left(600 \mathrm{MHz}, \mathrm{CDCl}_{3}\right)$

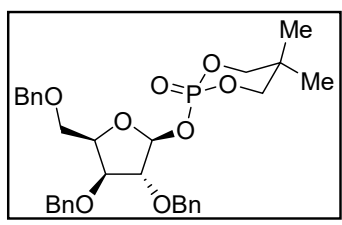

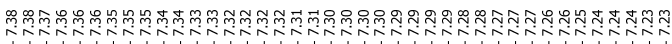

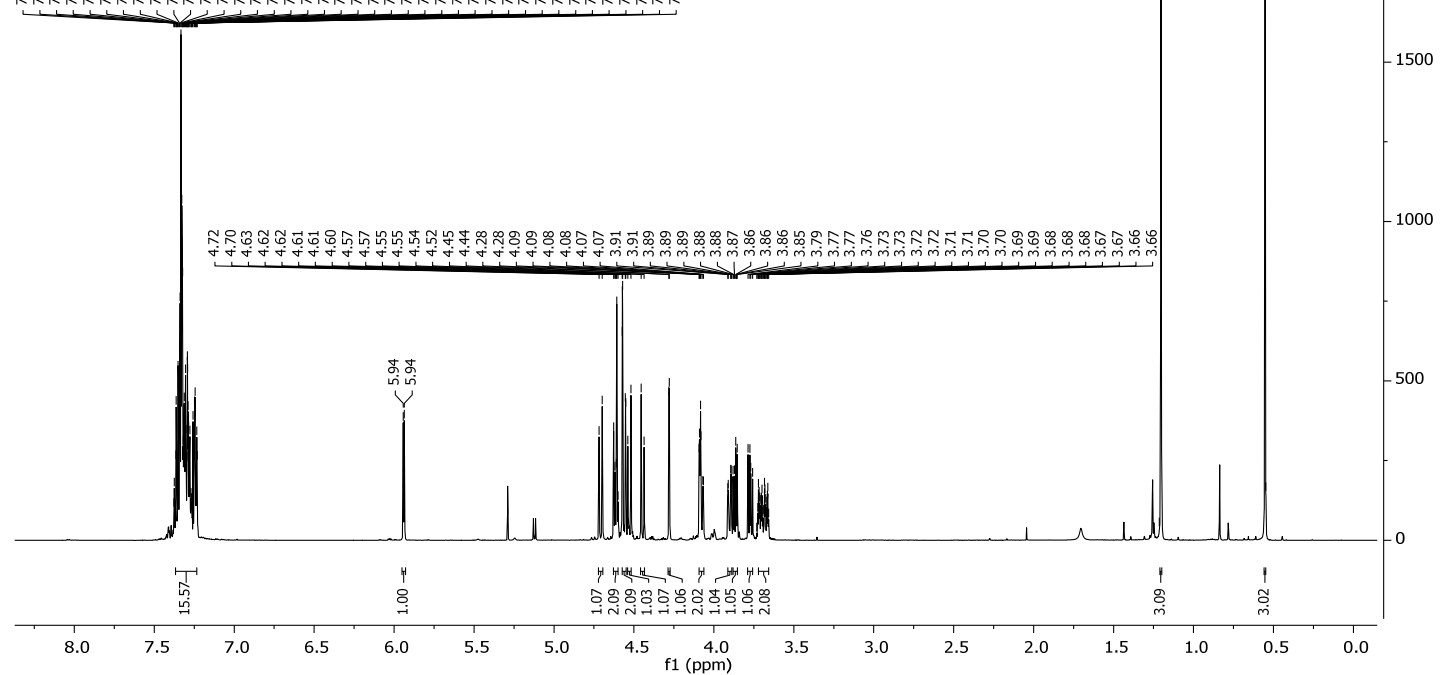

$\left.{ }^{13} \mathrm{C} \mathrm{NMR} \mathrm{(126} \mathrm{MHz,} \mathrm{CDCl}_{3}\right)$

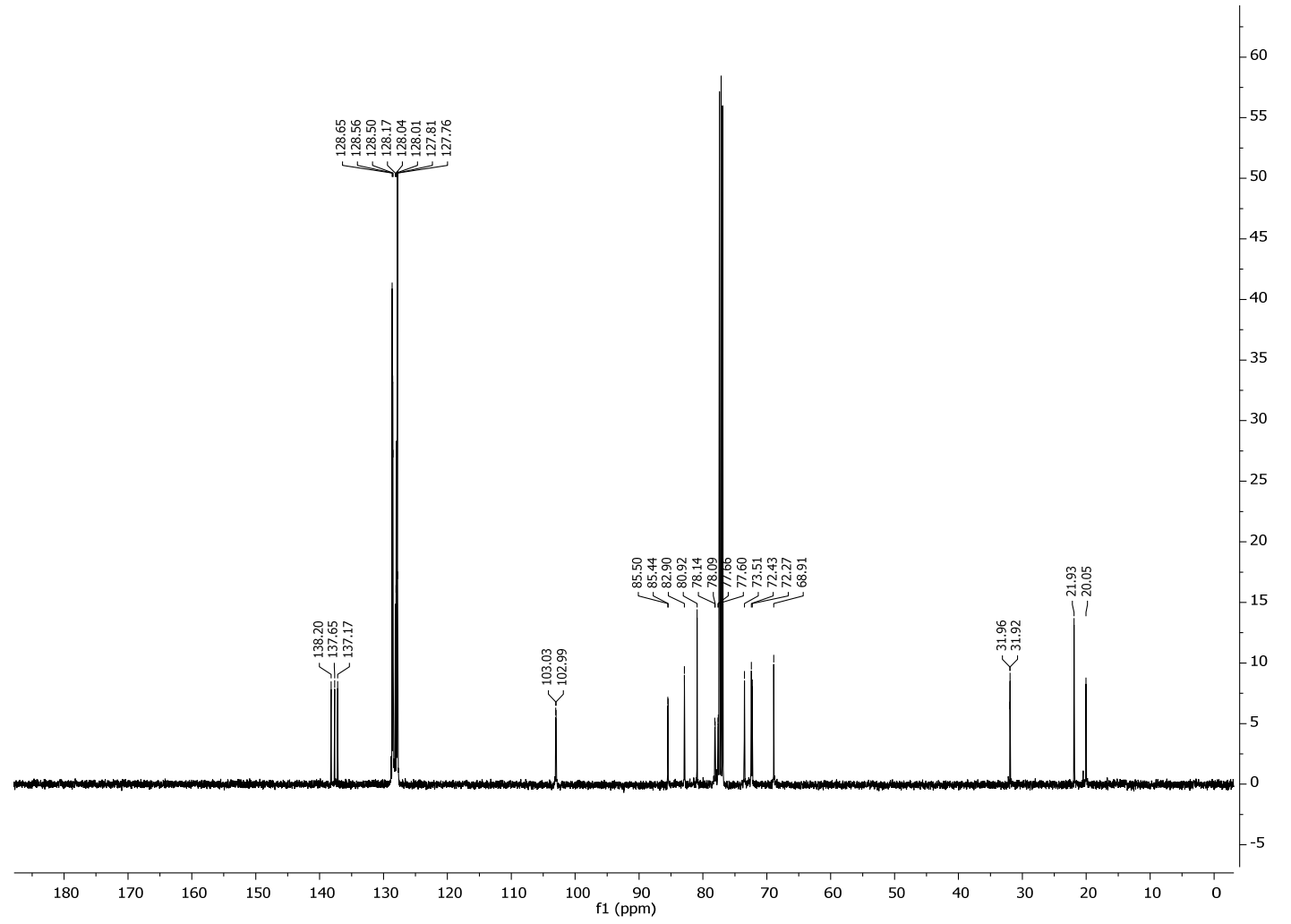


${ }^{31} \mathrm{P}$ NMR (162 MHz, $\left.\mathrm{CDCl}_{3}\right)$

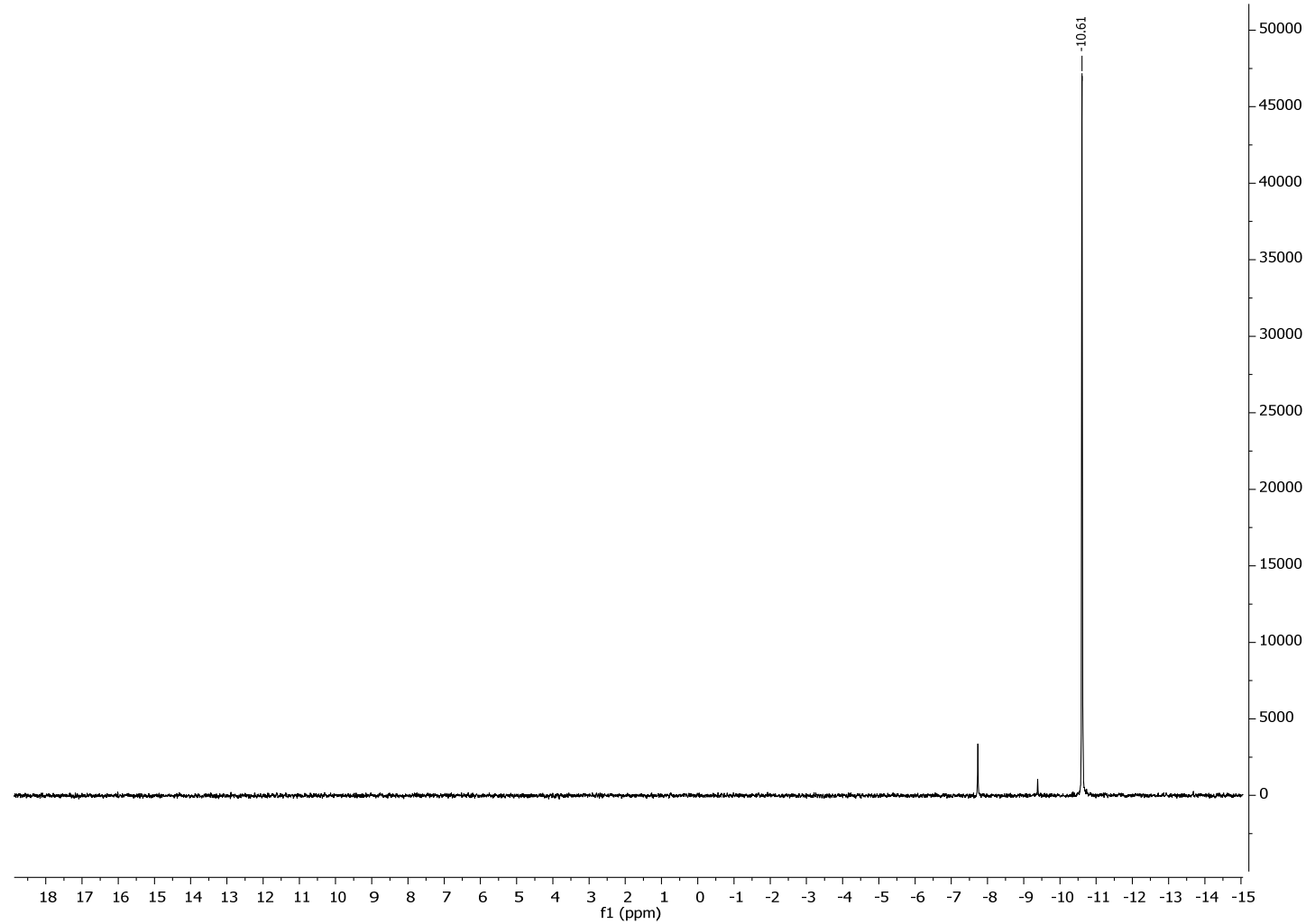


${ }^{1} \mathrm{H}$ NMR $\left(600 \mathrm{MHz}, \mathrm{CDCl}_{3}\right)$
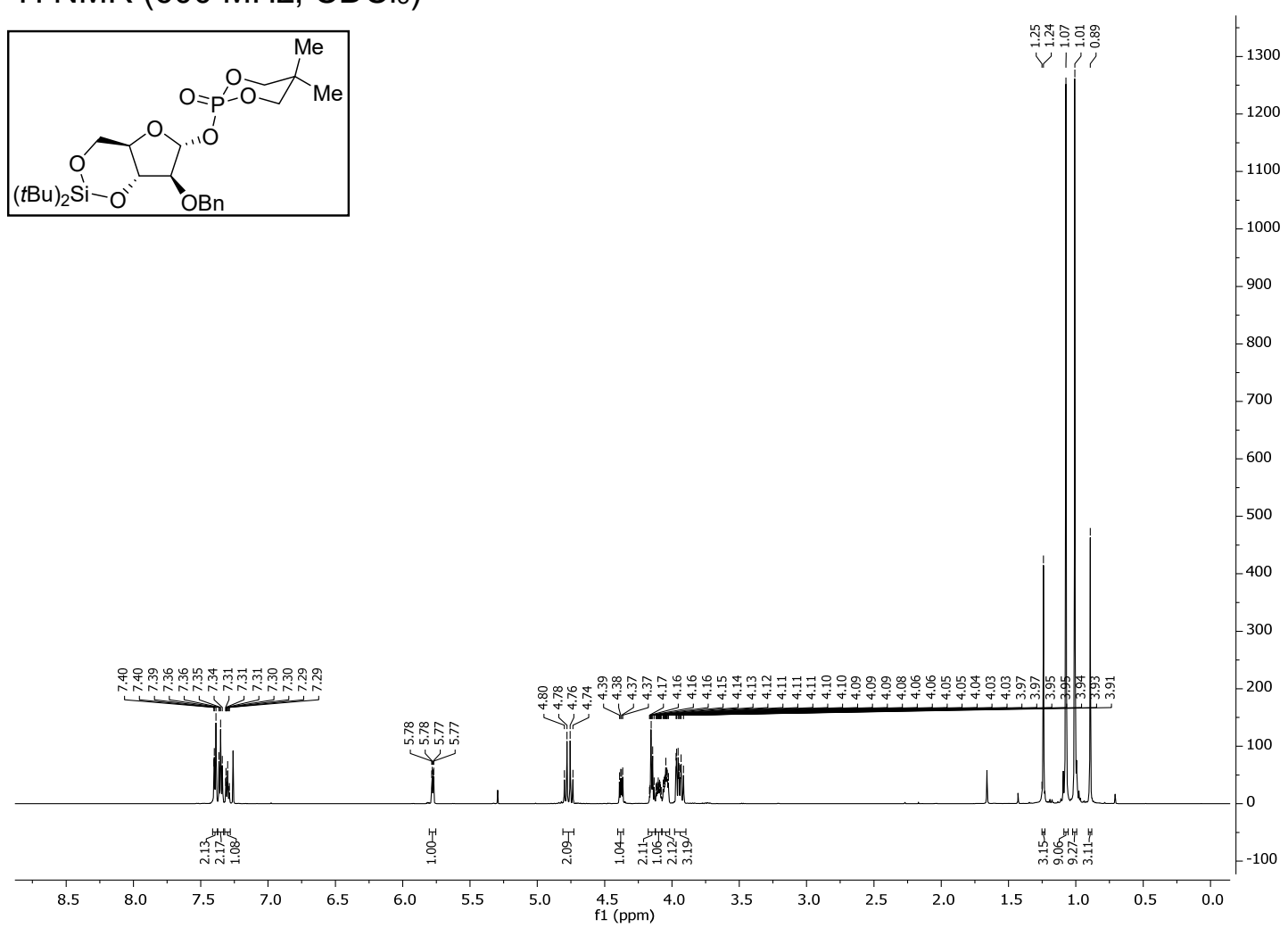

$\left.{ }^{13} \mathrm{C} \mathrm{NMR} \mathrm{(126} \mathrm{MHz,} \mathrm{CDCl}_{3}\right)$

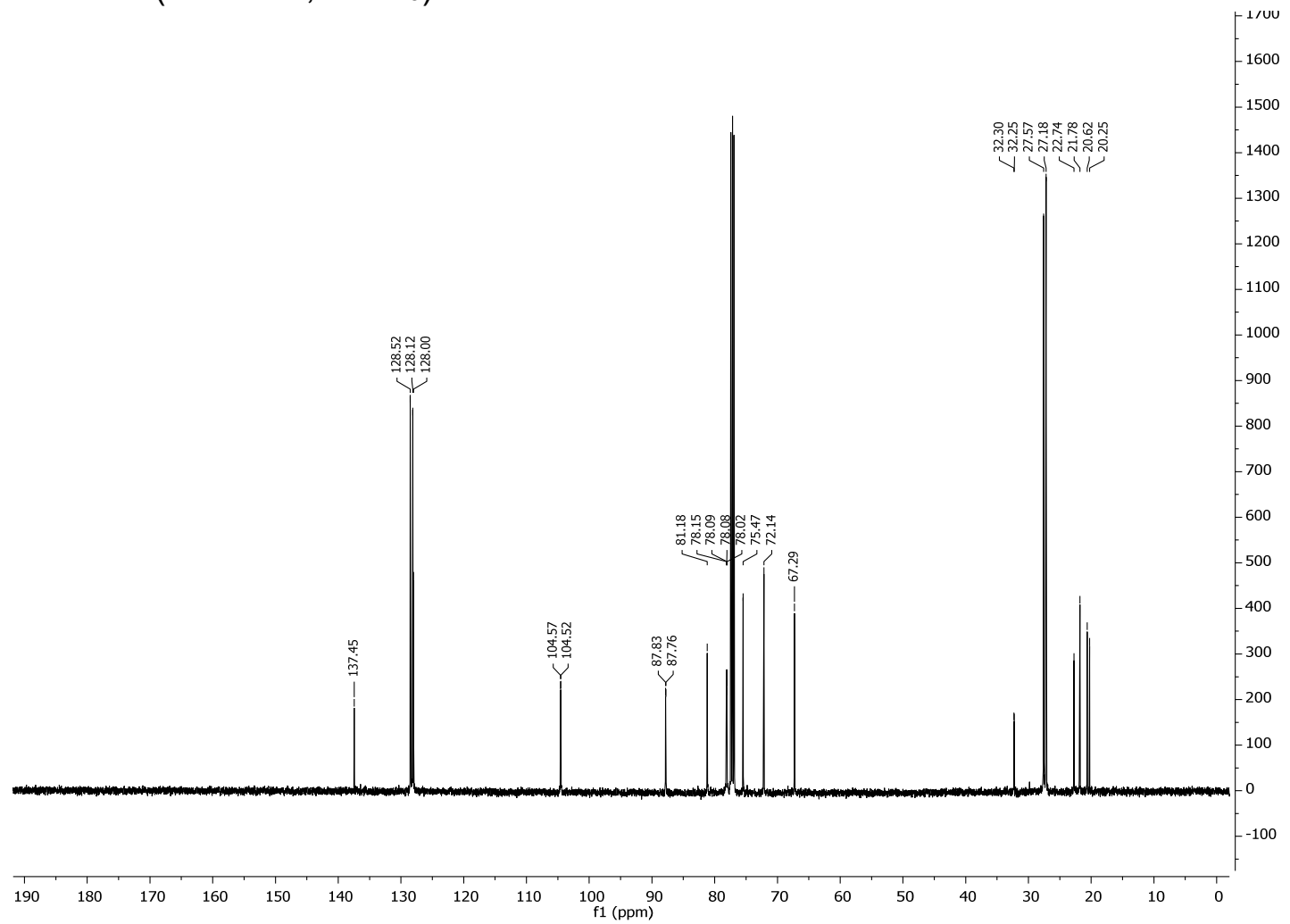


${ }^{31} \mathrm{P}$ NMR $\left(162 \mathrm{MHz}, \mathrm{CDCl}_{3}\right)$

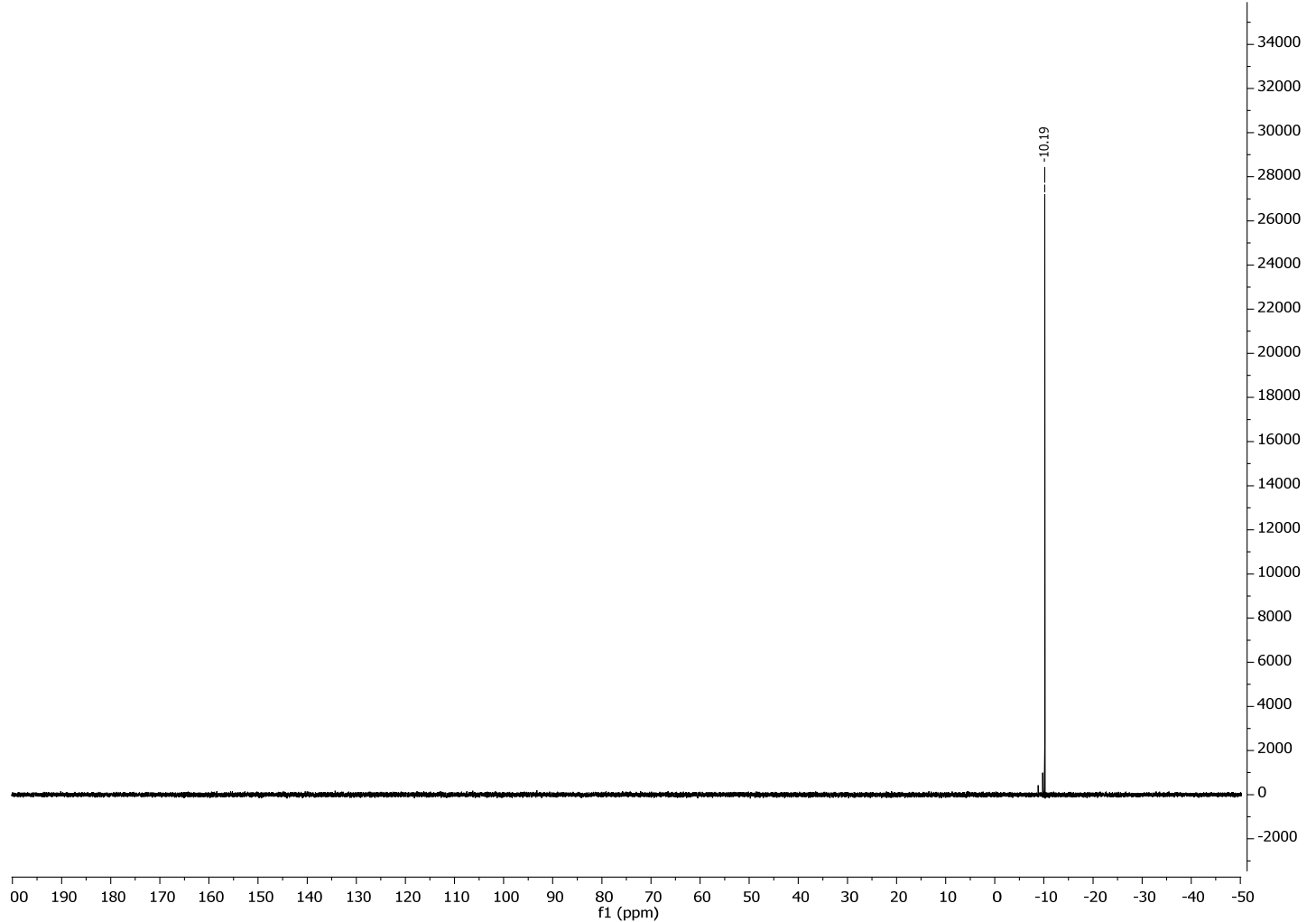


${ }^{1} \mathrm{H}$ NMR $\left(600 \mathrm{MHz}, \mathrm{CDCl}_{3}\right)$
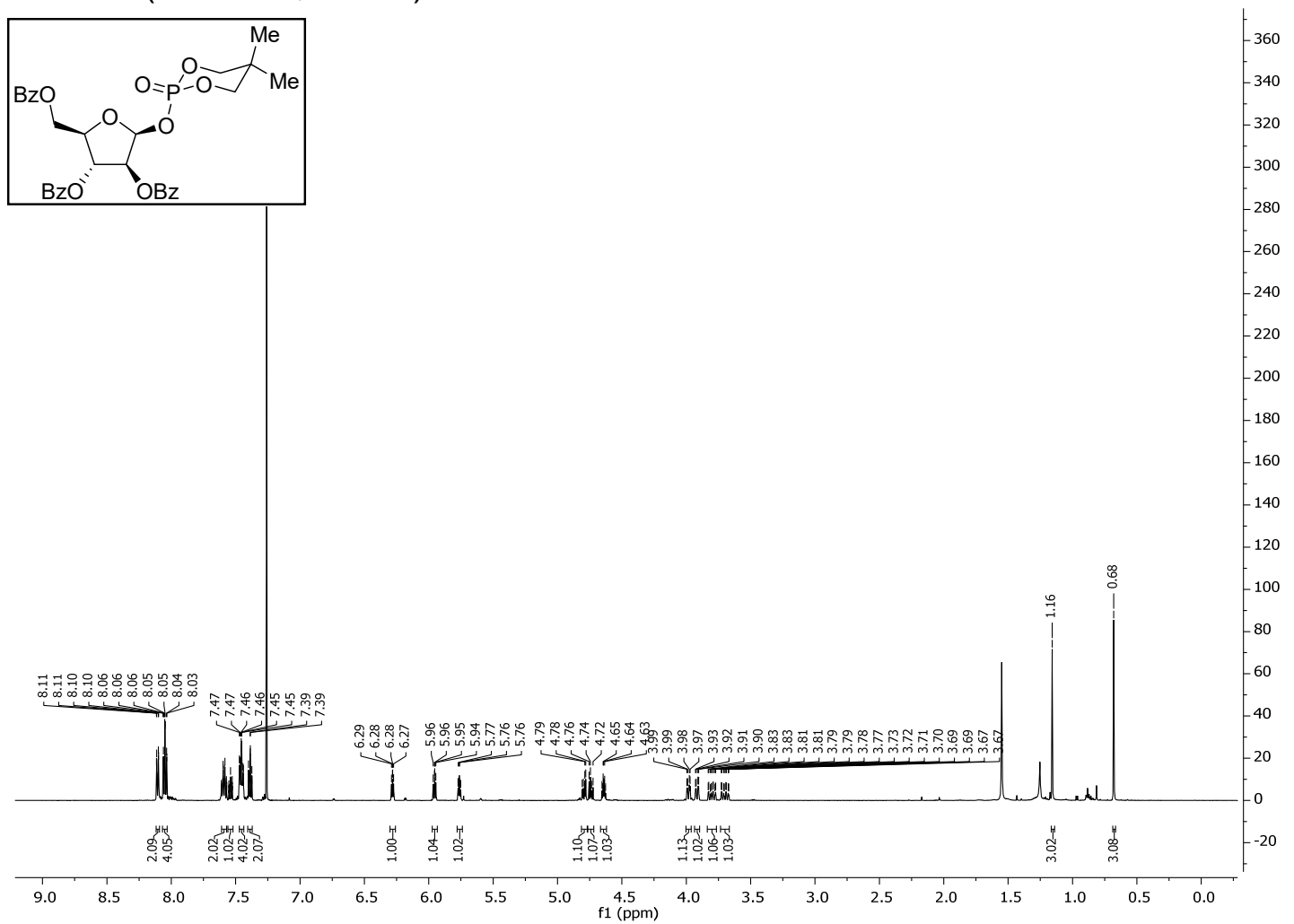

$\left.{ }^{13} \mathrm{C} \mathrm{NMR} \mathrm{(126} \mathrm{MHz,} \mathrm{CDCl}_{3}\right)$

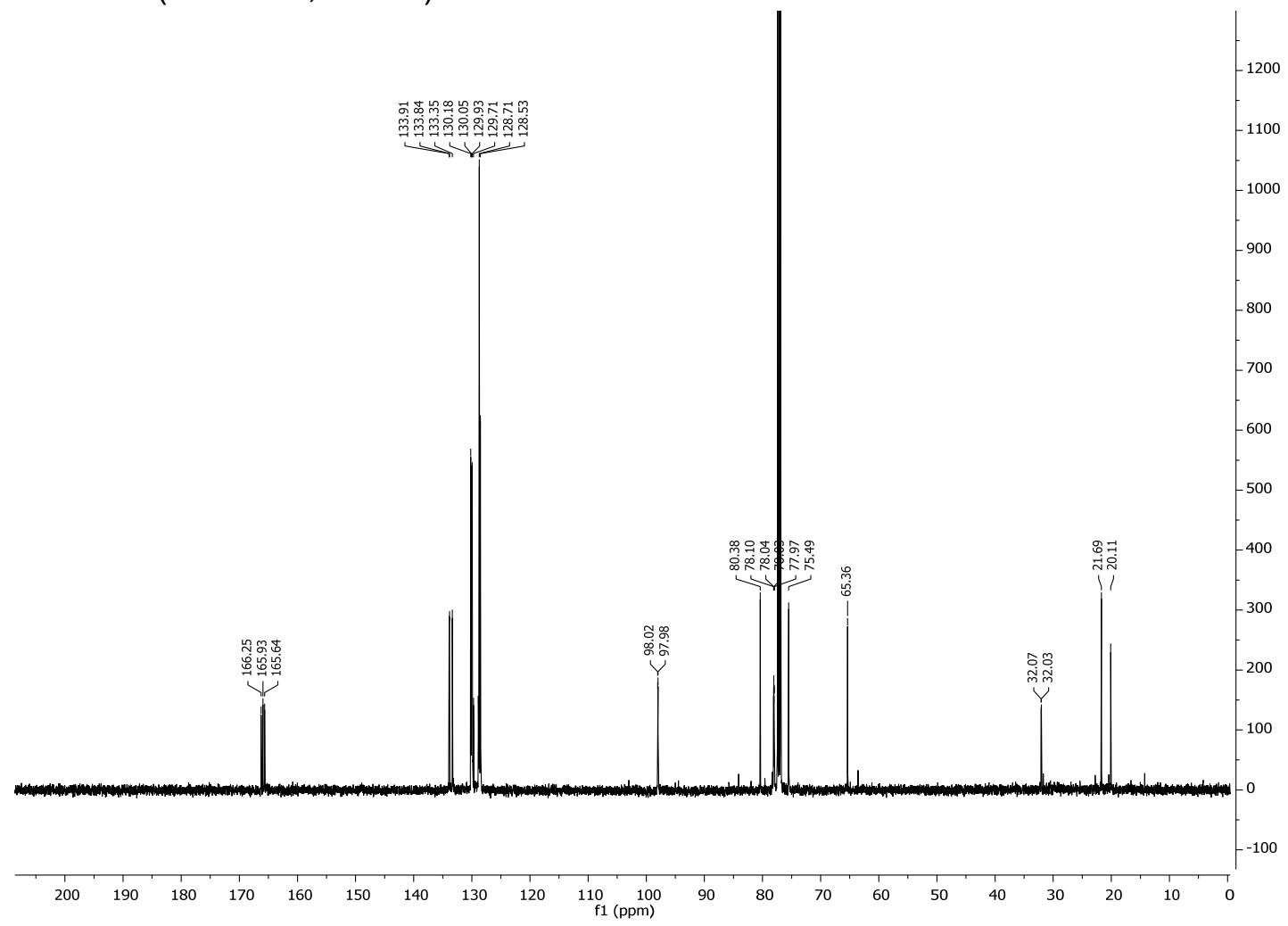


${ }^{31} \mathrm{P}$ NMR $\left(162 \mathrm{MHz}, \mathrm{CDCl}_{3}\right)$

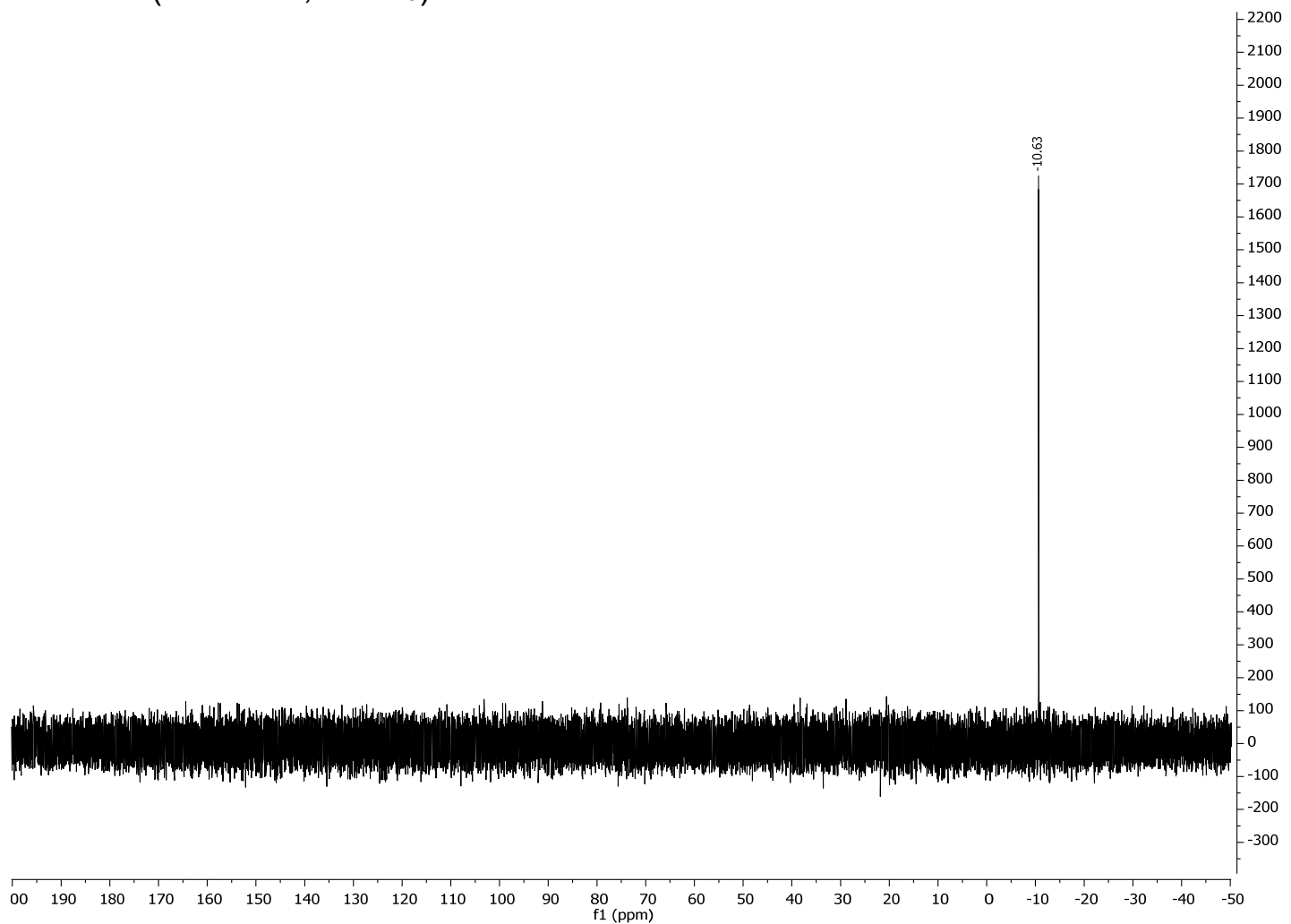


${ }^{1} \mathrm{H}$ NMR $\left(600 \mathrm{MHz}, \mathrm{CDCl}_{3}\right)$
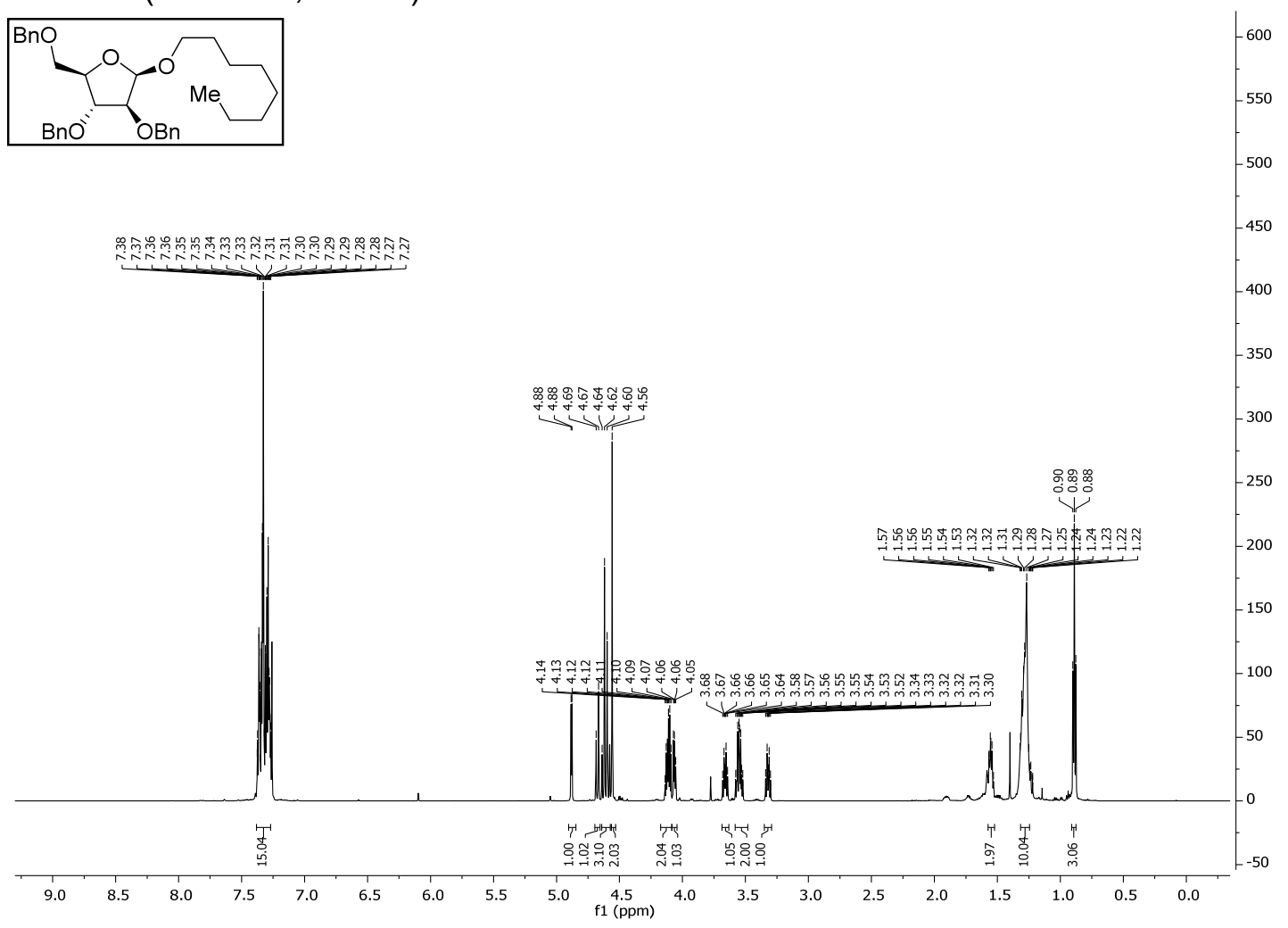

${ }^{13} \mathrm{C}$ NMR $\left(126 \mathrm{MHz}, \mathrm{CDCl}_{3}\right)$

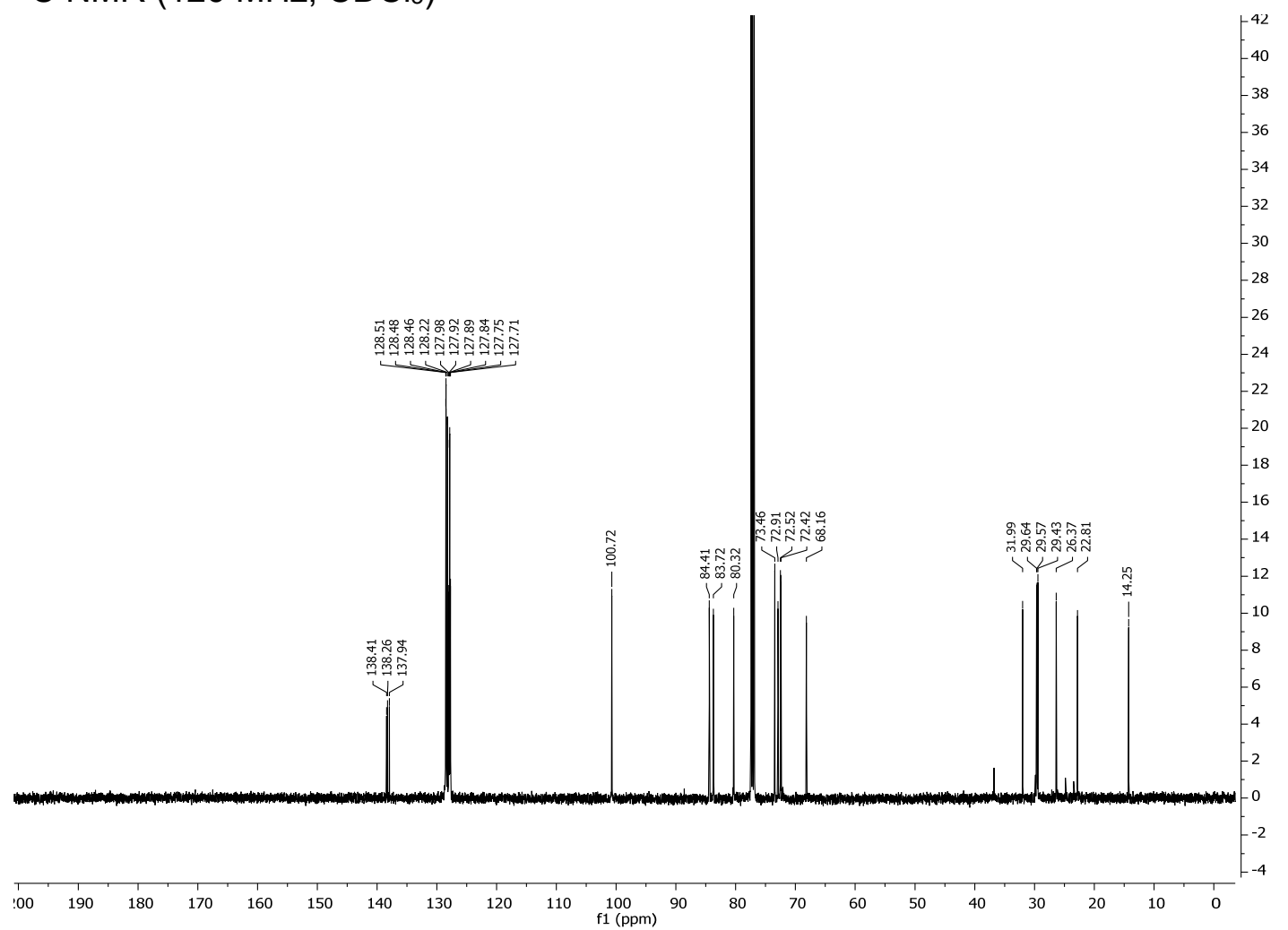


${ }^{1} \mathrm{H} \mathrm{NMR}\left(600 \mathrm{MHz}, \mathrm{CDCl}_{3}\right)$

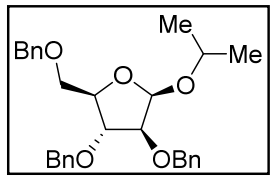

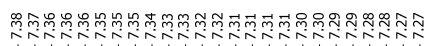

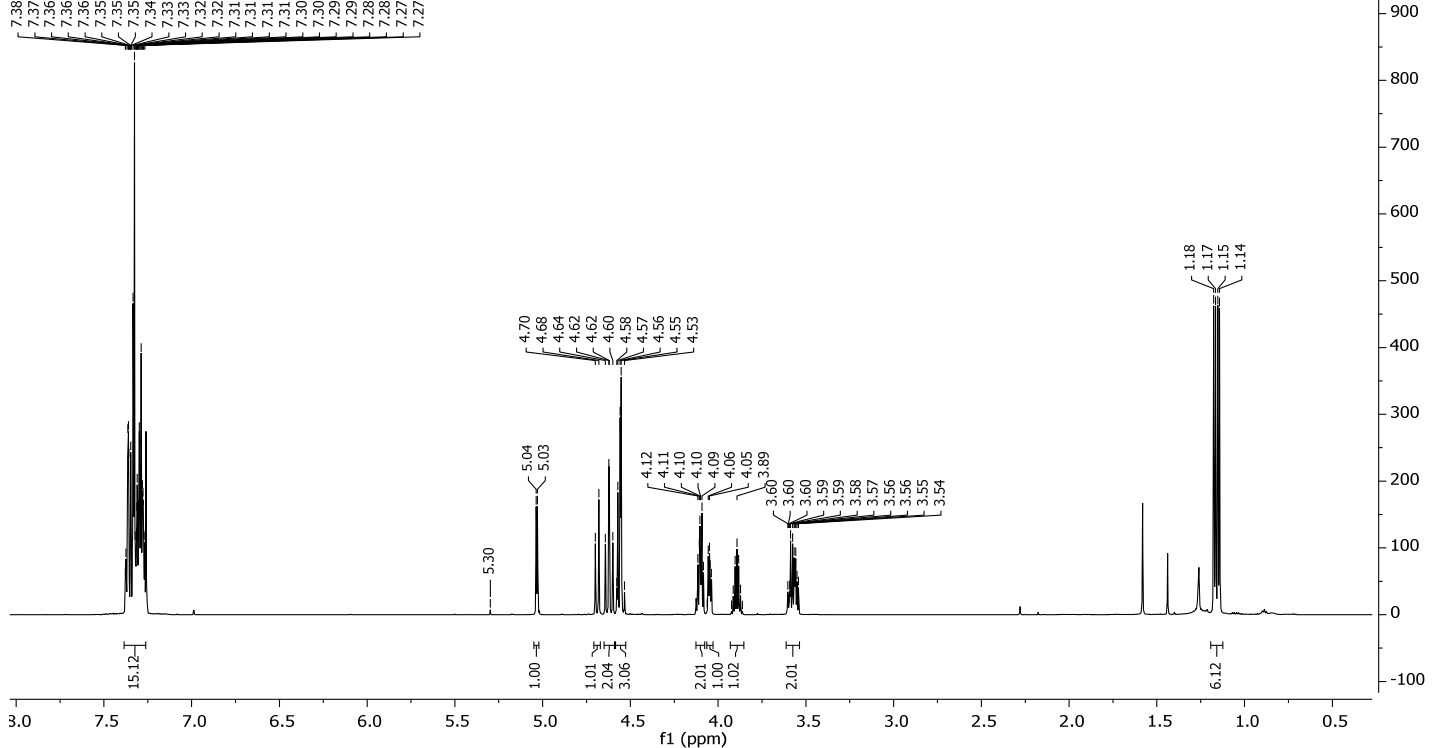

${ }^{13} \mathrm{C} \mathrm{NMR}\left(126 \mathrm{MHz}, \mathrm{CDCl}_{3}\right)$

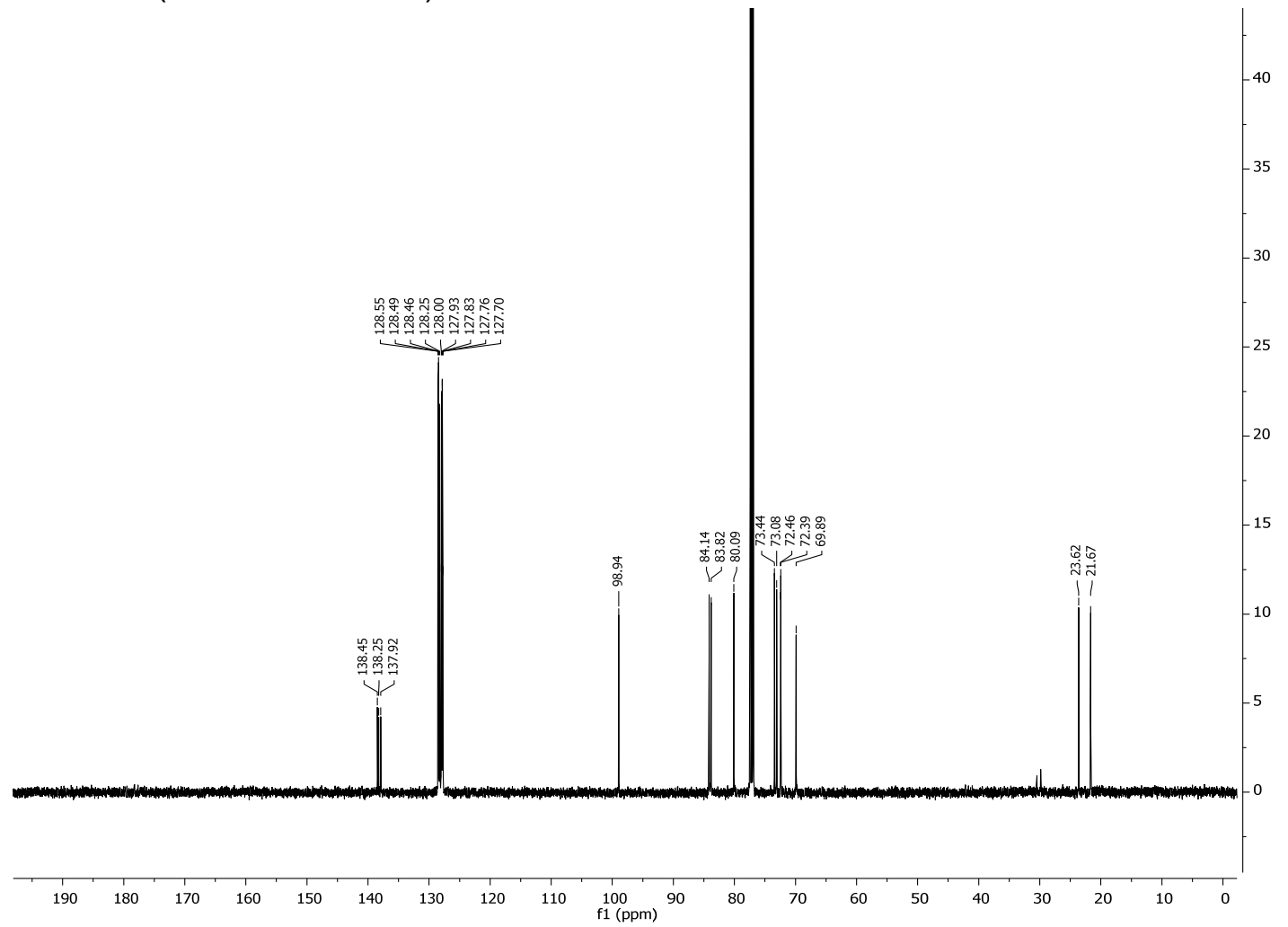


${ }^{1} \mathrm{H}$ NMR $\left(600 \mathrm{MHz}, \mathrm{CDCl}_{3}\right)$

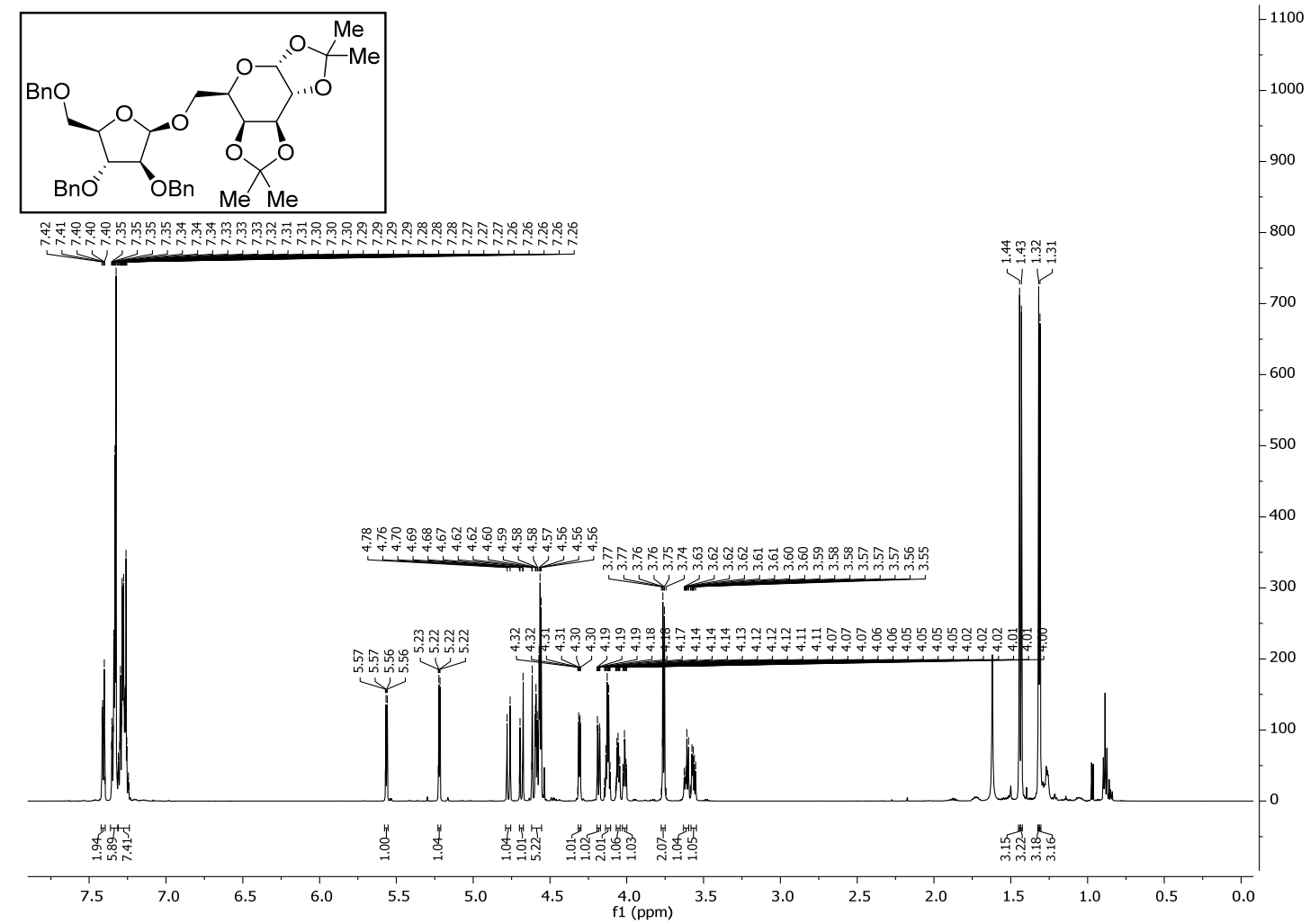

${ }^{13} \mathrm{C}$ NMR $\left(126 \mathrm{MHz}, \mathrm{CDCl}_{3}\right)$

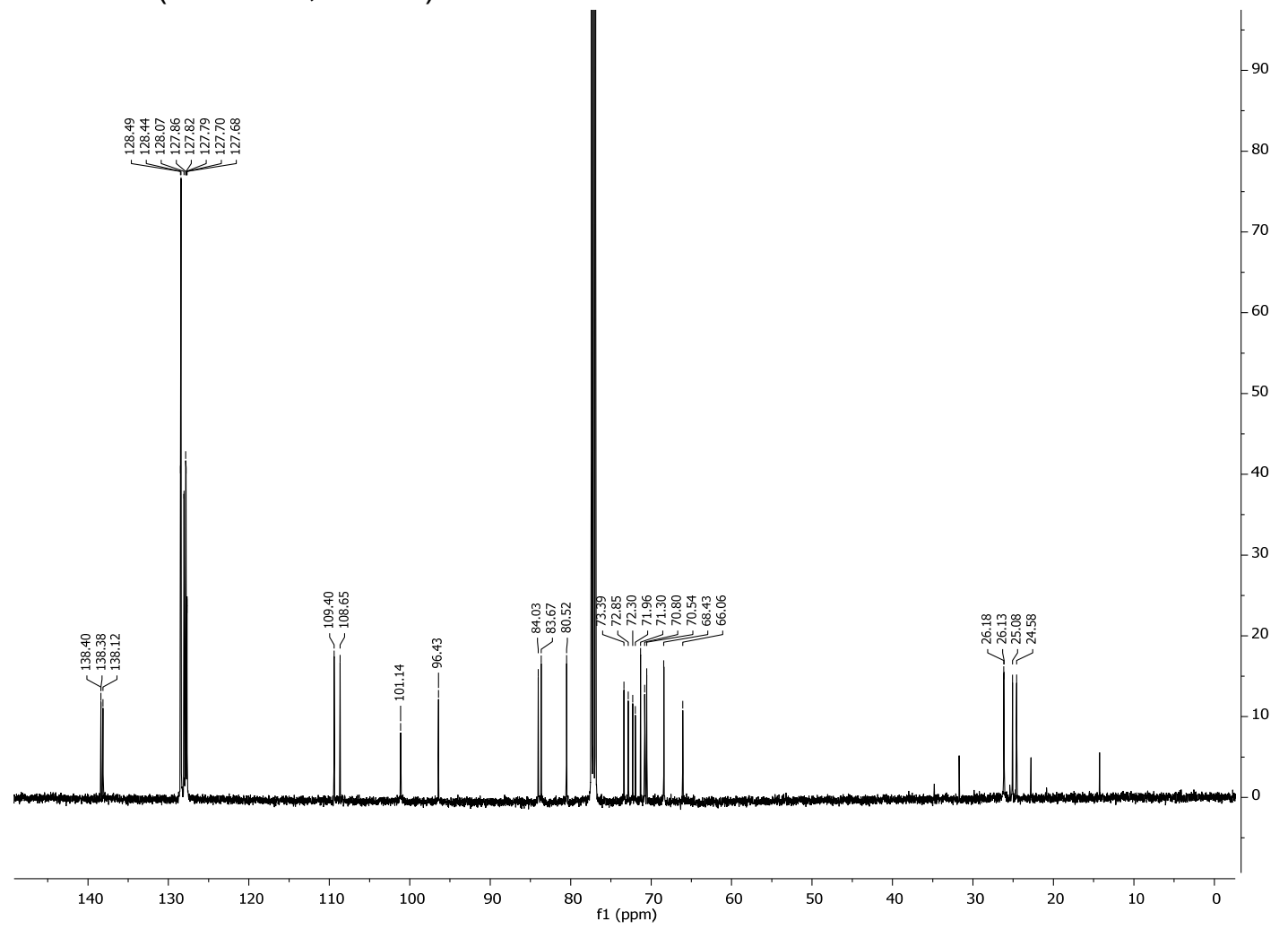


${ }^{1} \mathrm{H}$ NMR (600 MHz, $\left.\mathrm{CDCl}_{3}\right)$
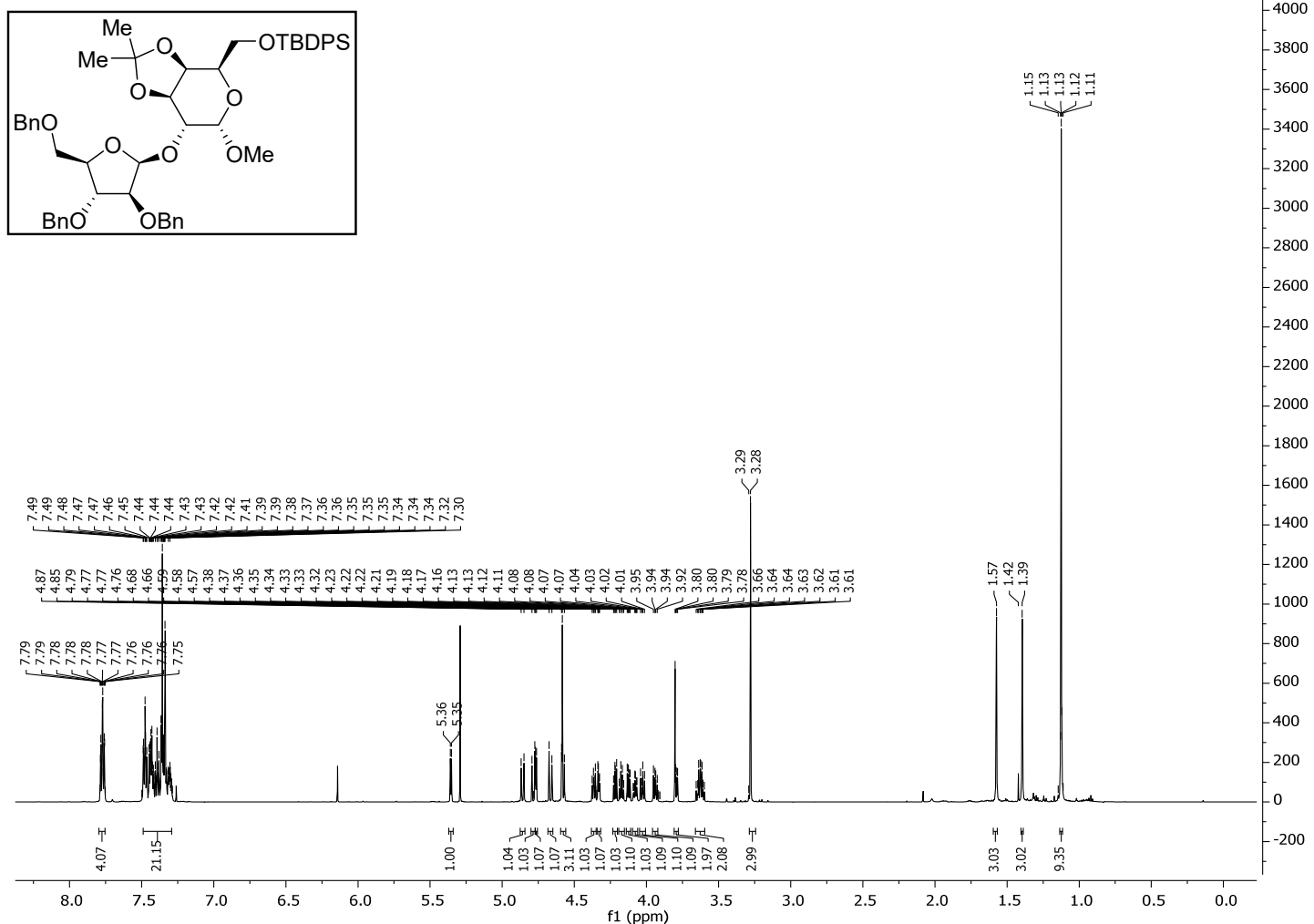

${ }^{13} \mathrm{C}$ NMR $\left(126 \mathrm{MHz}, \mathrm{CDCl}_{3}\right)$

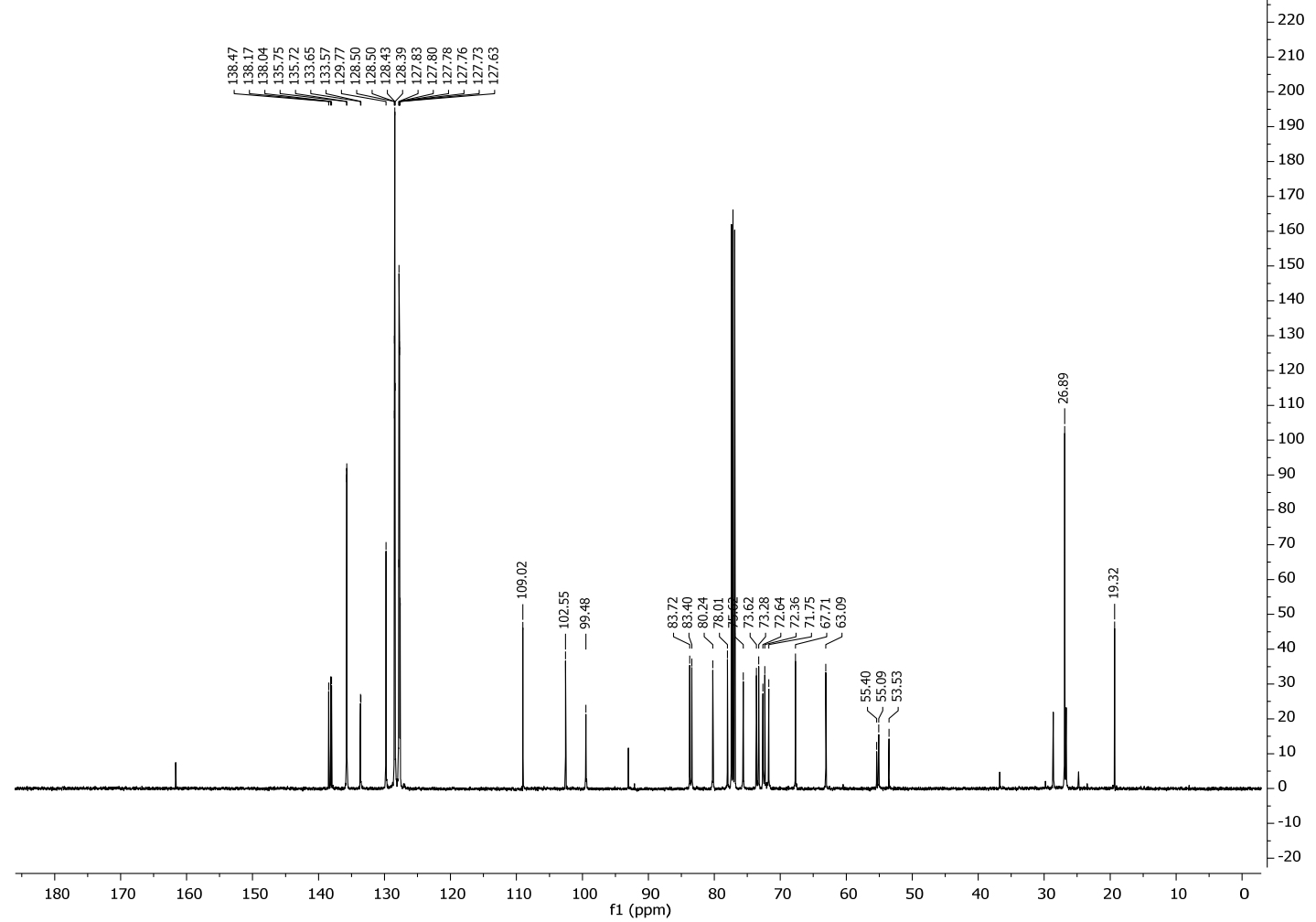


${ }^{1} \mathrm{H}$ NMR (600 MHz, $\left.\mathrm{CDCl}_{3}\right)$
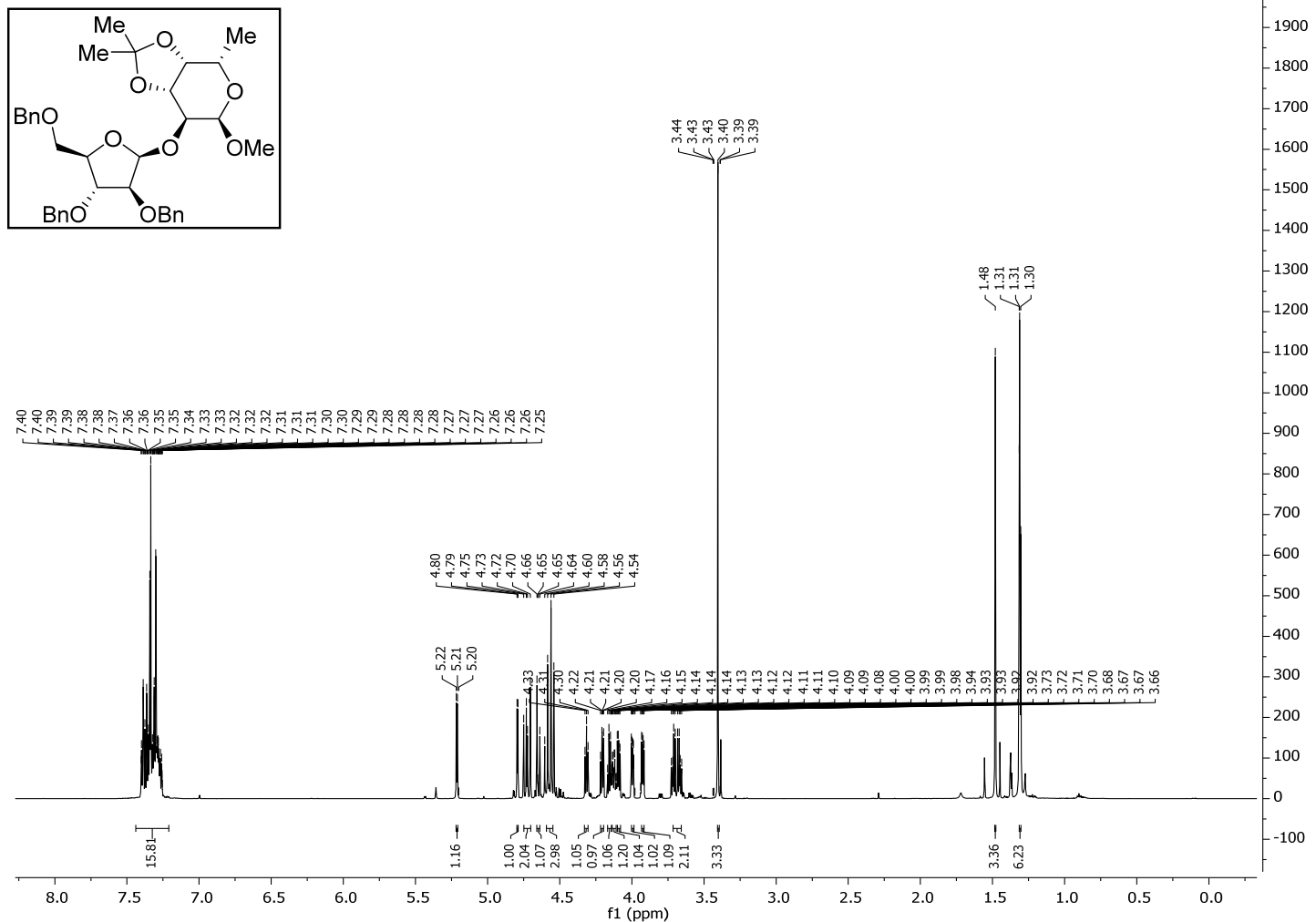

${ }^{13} \mathrm{C}$ NMR (126 MHz, $\left.\mathrm{CDCl}_{3}\right)$

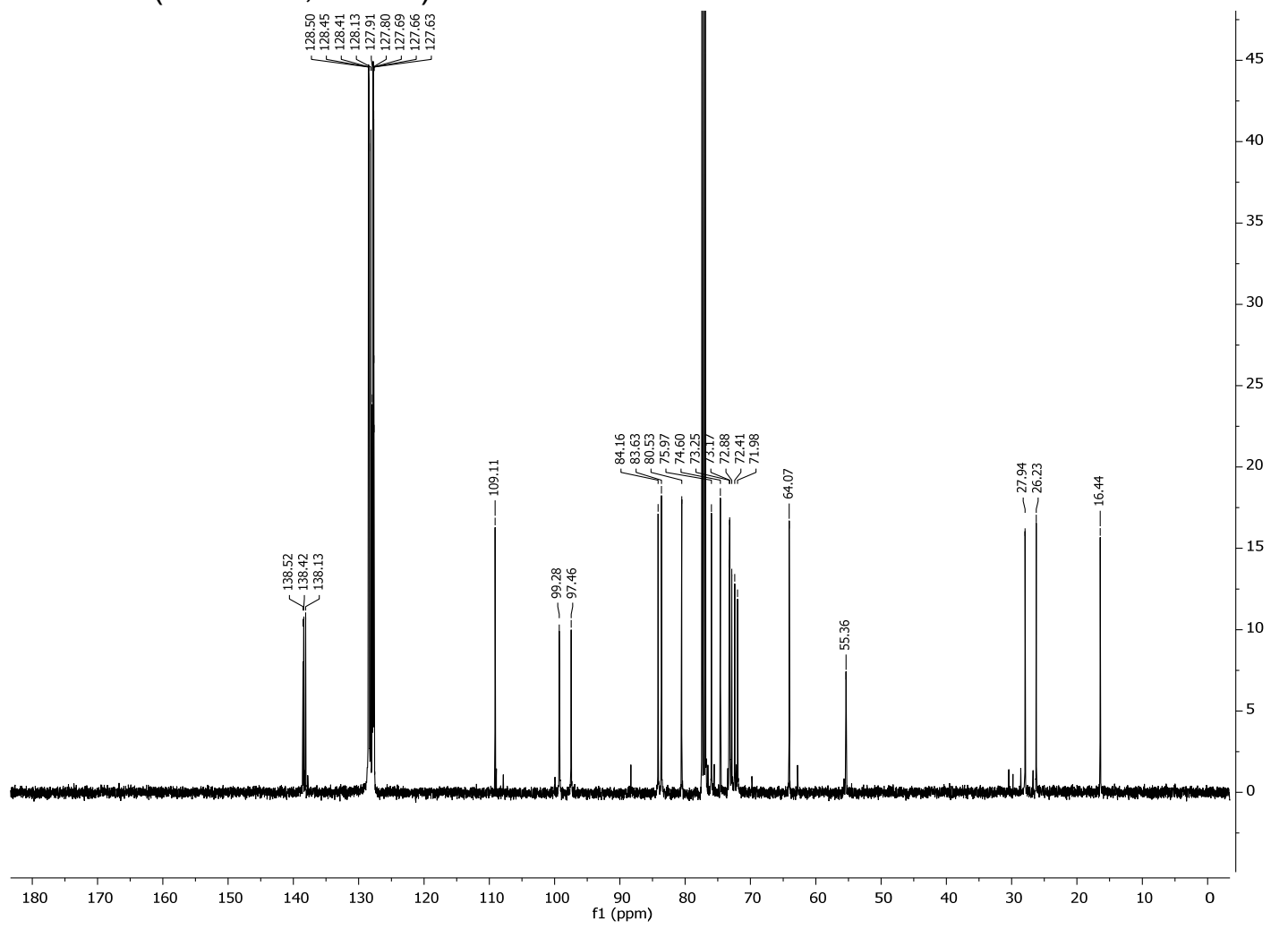


${ }^{1} \mathrm{H}$ NMR $\left(600 \mathrm{MHz}, \mathrm{CDCl}_{3}\right)$
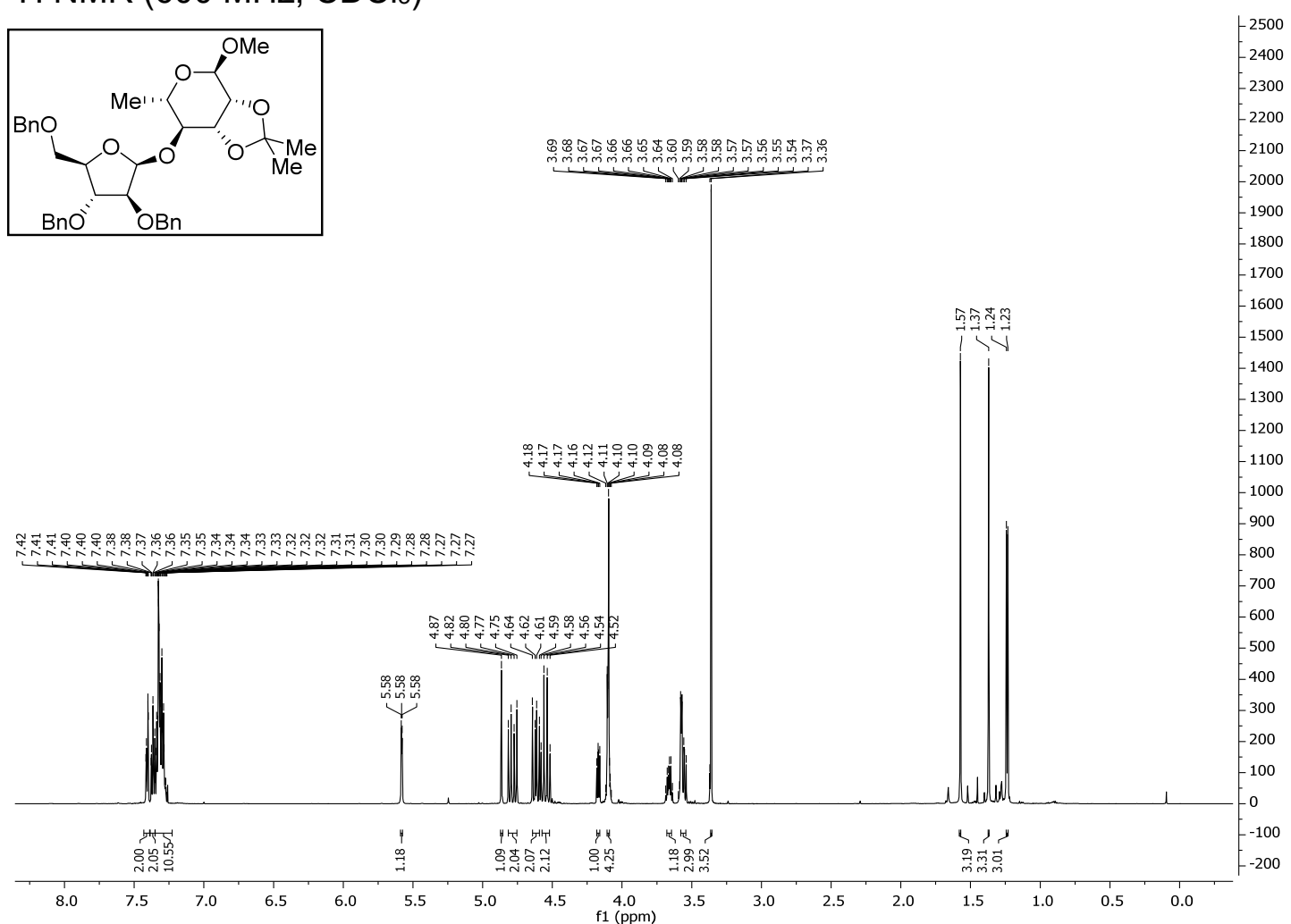

$\left.{ }^{13} \mathrm{C} \mathrm{NMR} \mathrm{(126} \mathrm{MHz,} \mathrm{CDCl}_{3}\right)$

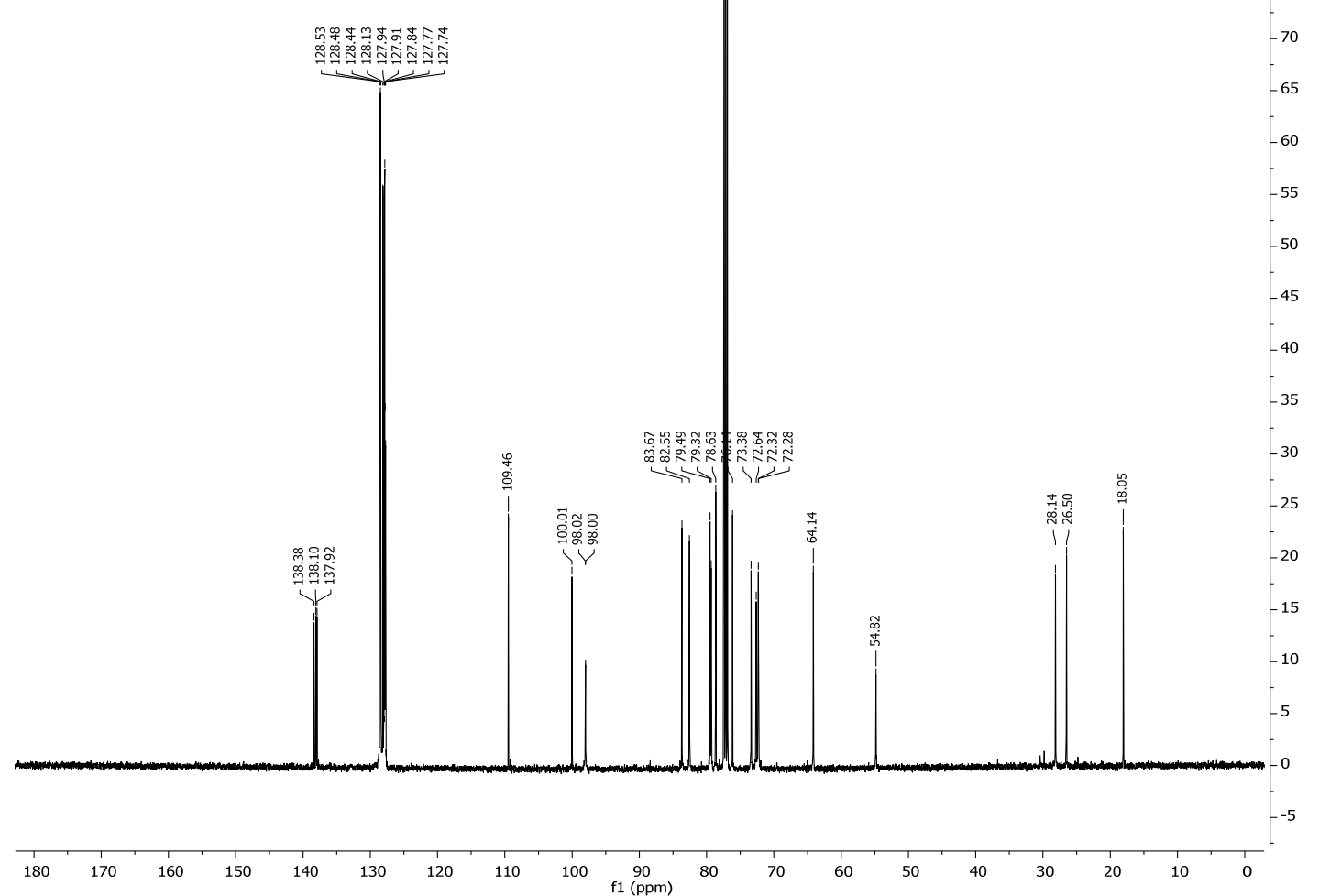


${ }^{1} \mathrm{H}$ NMR $\left(600 \mathrm{MHz}, \mathrm{CDCl}_{3}\right)$
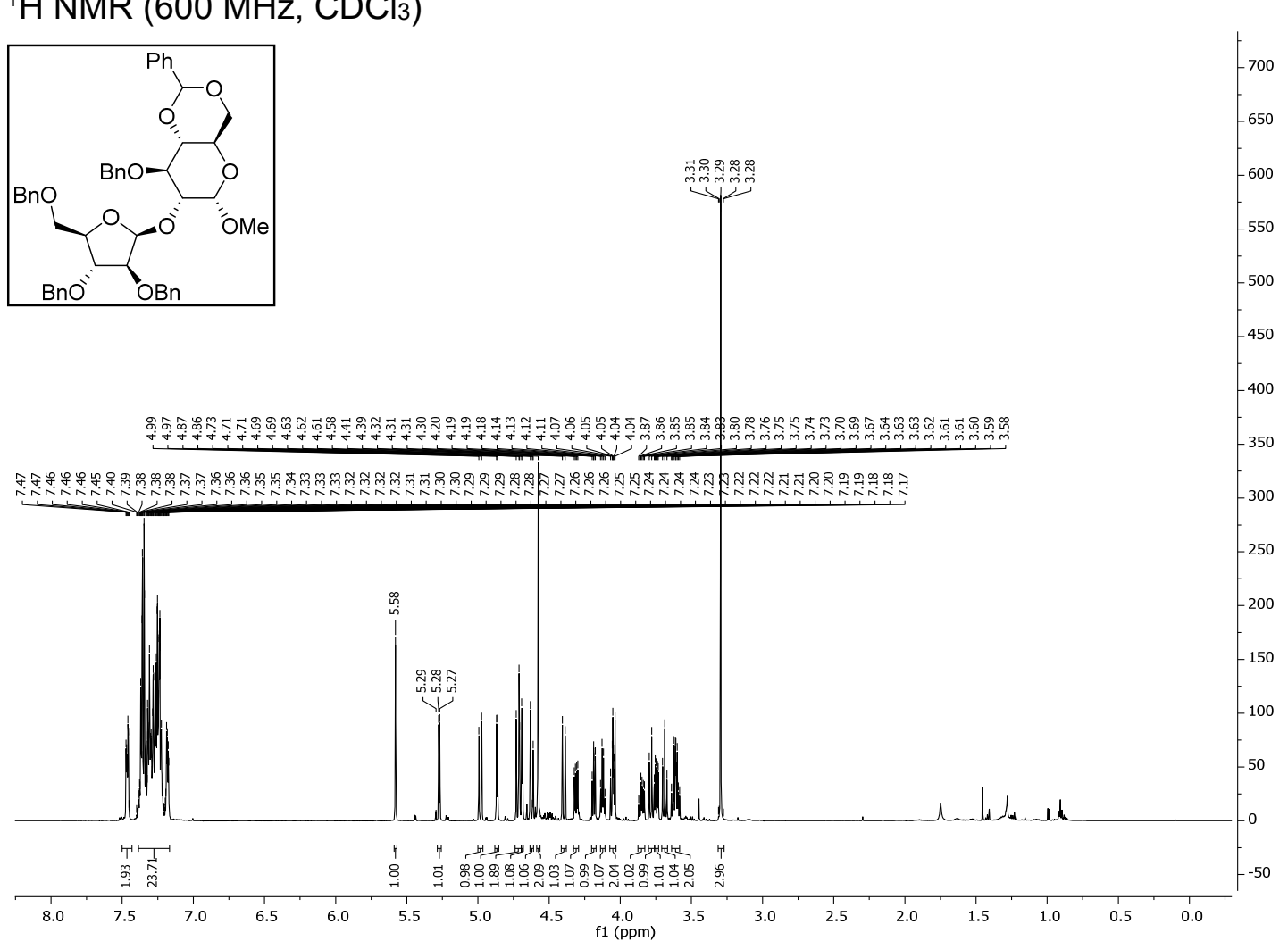

$\left.{ }^{13} \mathrm{C} \mathrm{NMR} \mathrm{(126} \mathrm{MHz,} \mathrm{CDCl}_{3}\right)$

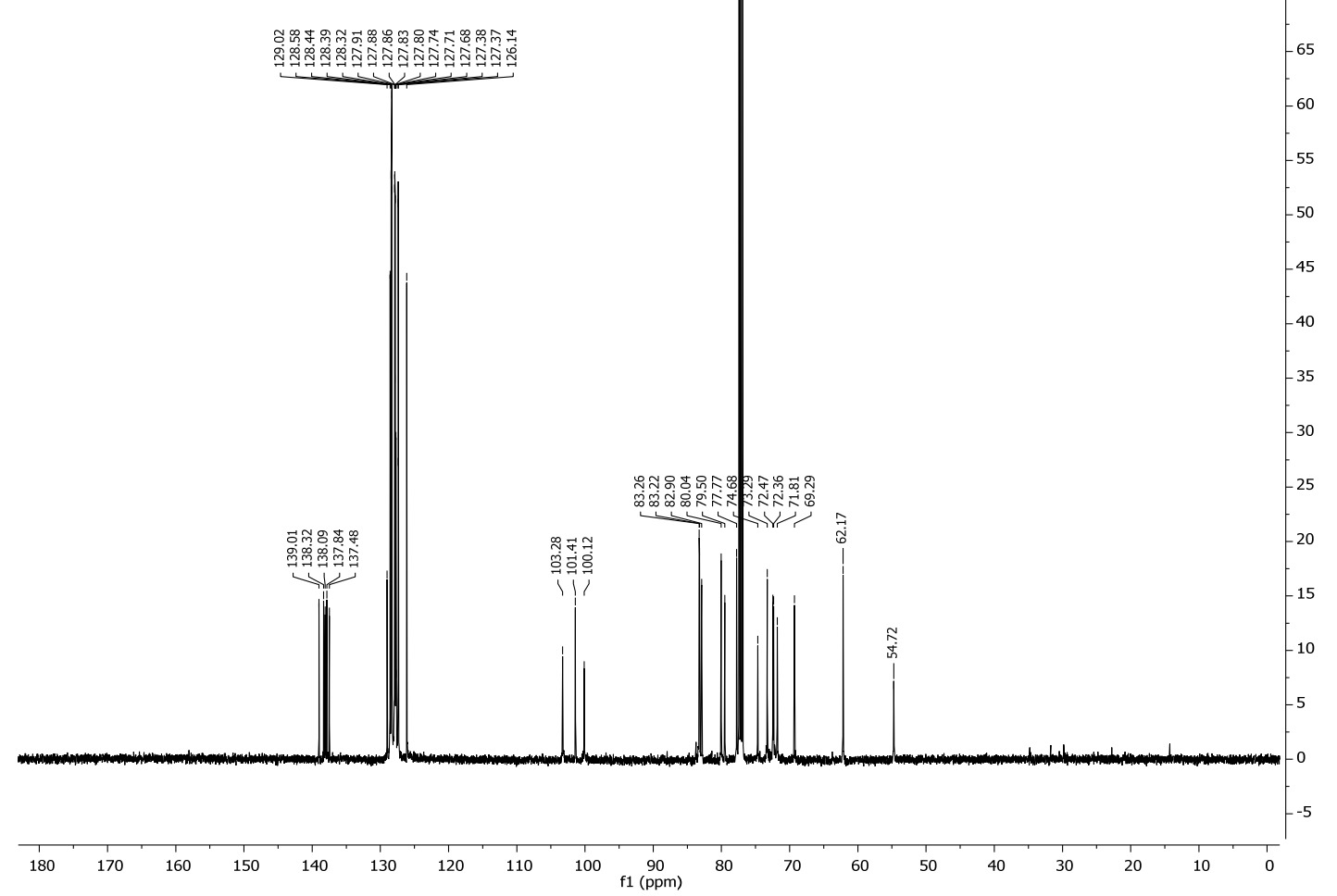


${ }^{1} \mathrm{H}$ NMR (600 MHz, $\mathrm{CDCl}_{3}$ )

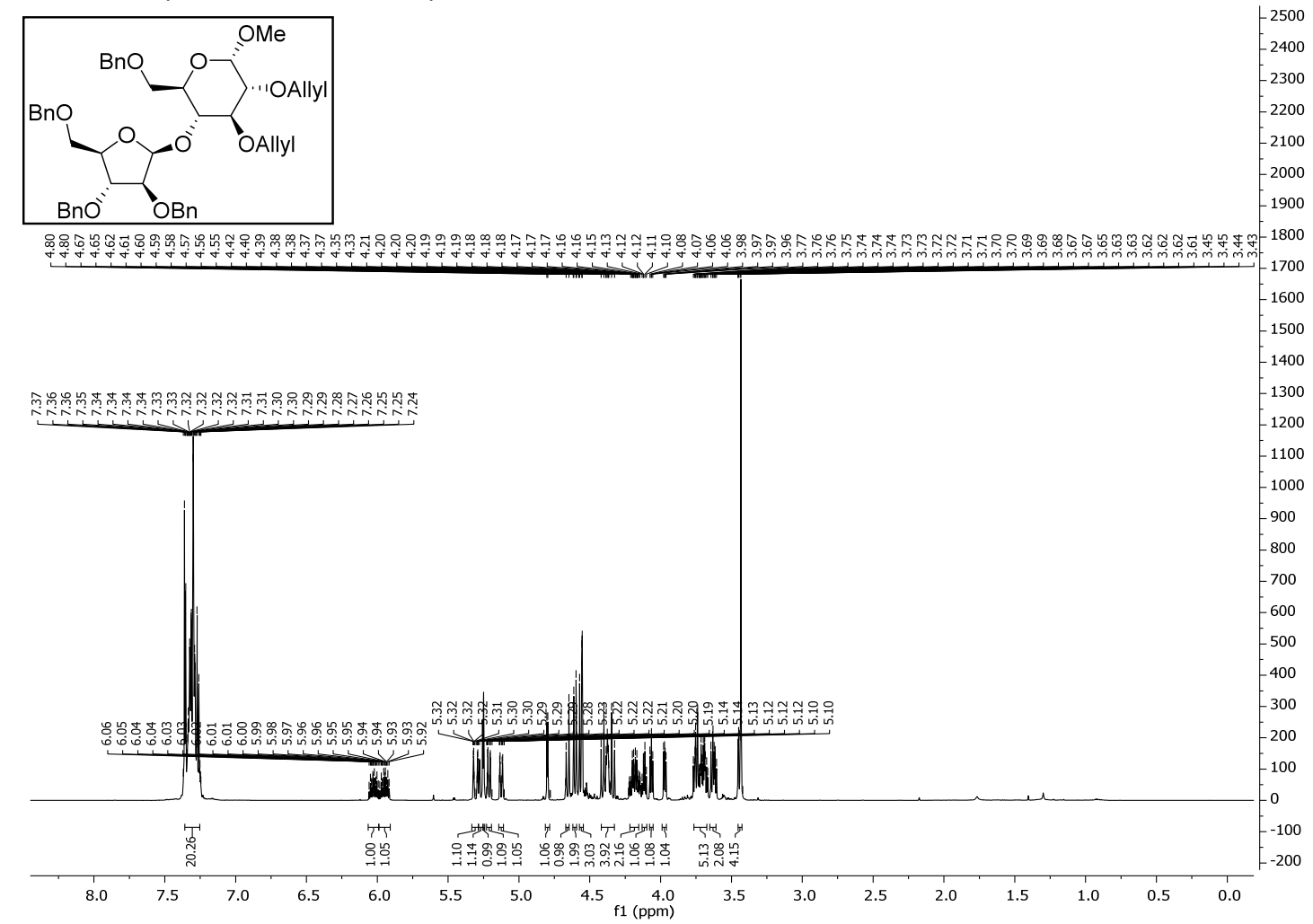

${ }^{13} \mathrm{C}$ NMR (126 MHz, $\left.\mathrm{CDCl}_{3}\right)$

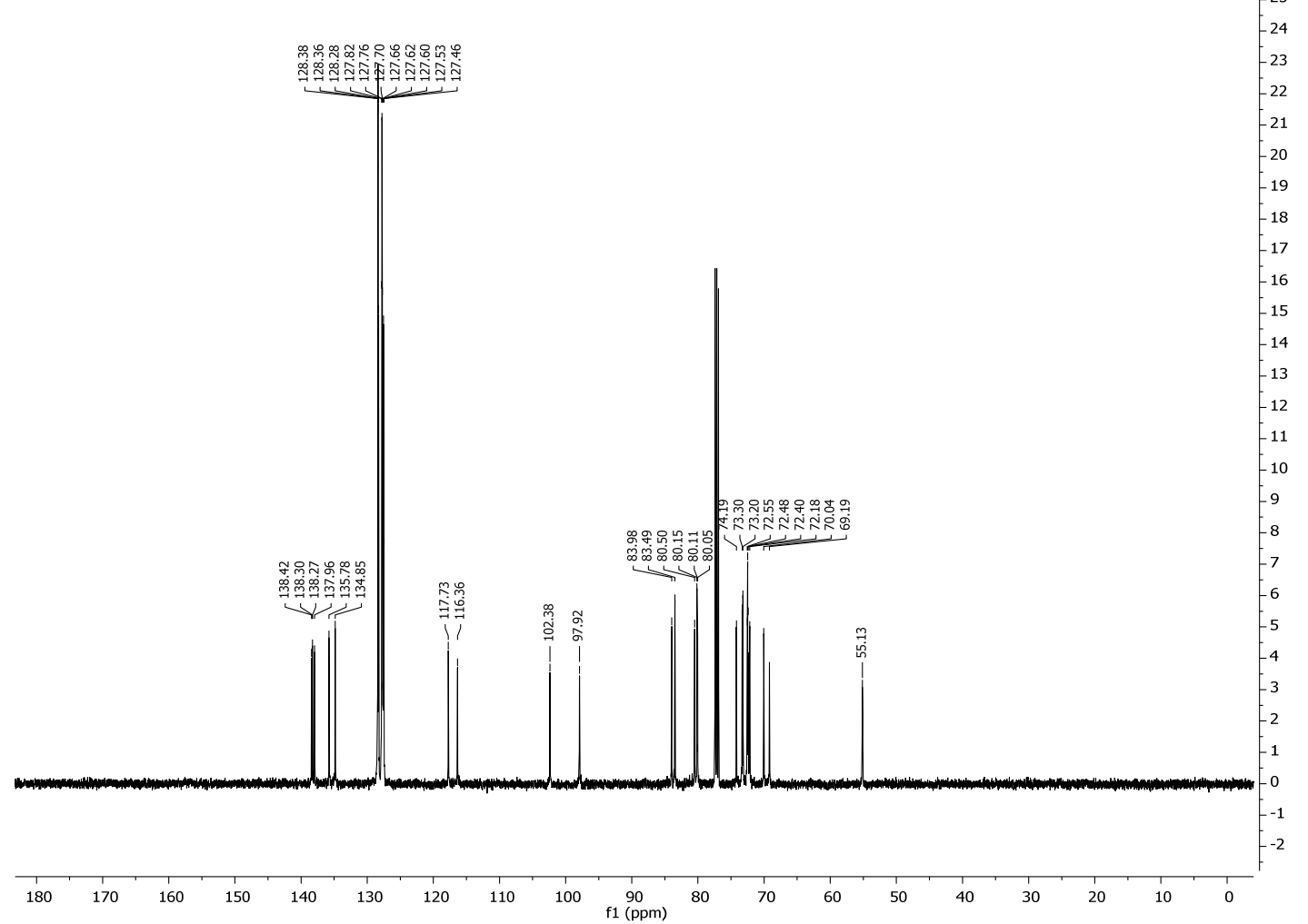


${ }^{1} \mathrm{H}$ NMR (600 MHz, $\left.\mathrm{CDCl}_{3}\right)$
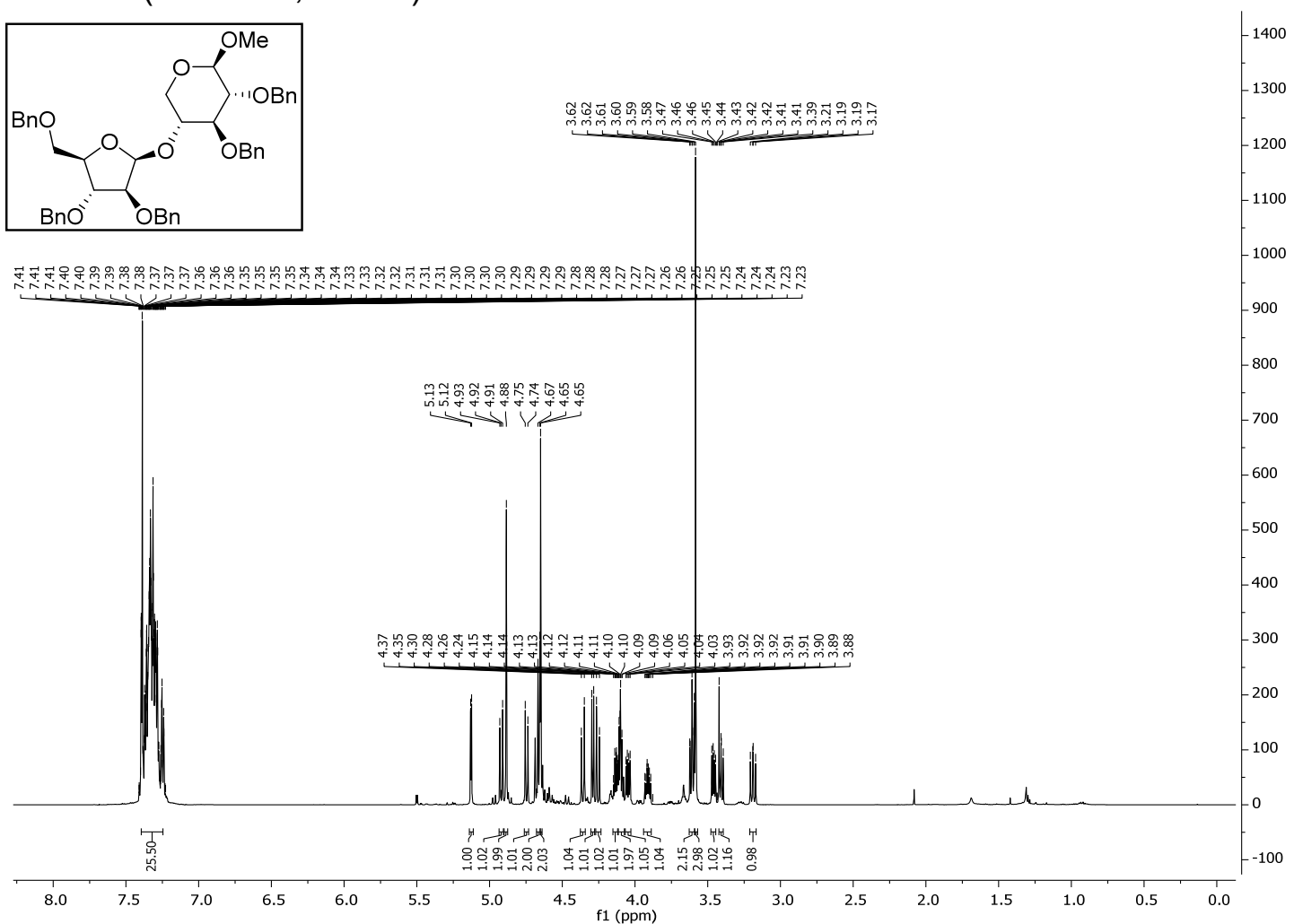

${ }^{13} \mathrm{C}$ NMR $\left(126 \mathrm{MHz}, \mathrm{CDCl}_{3}\right)$

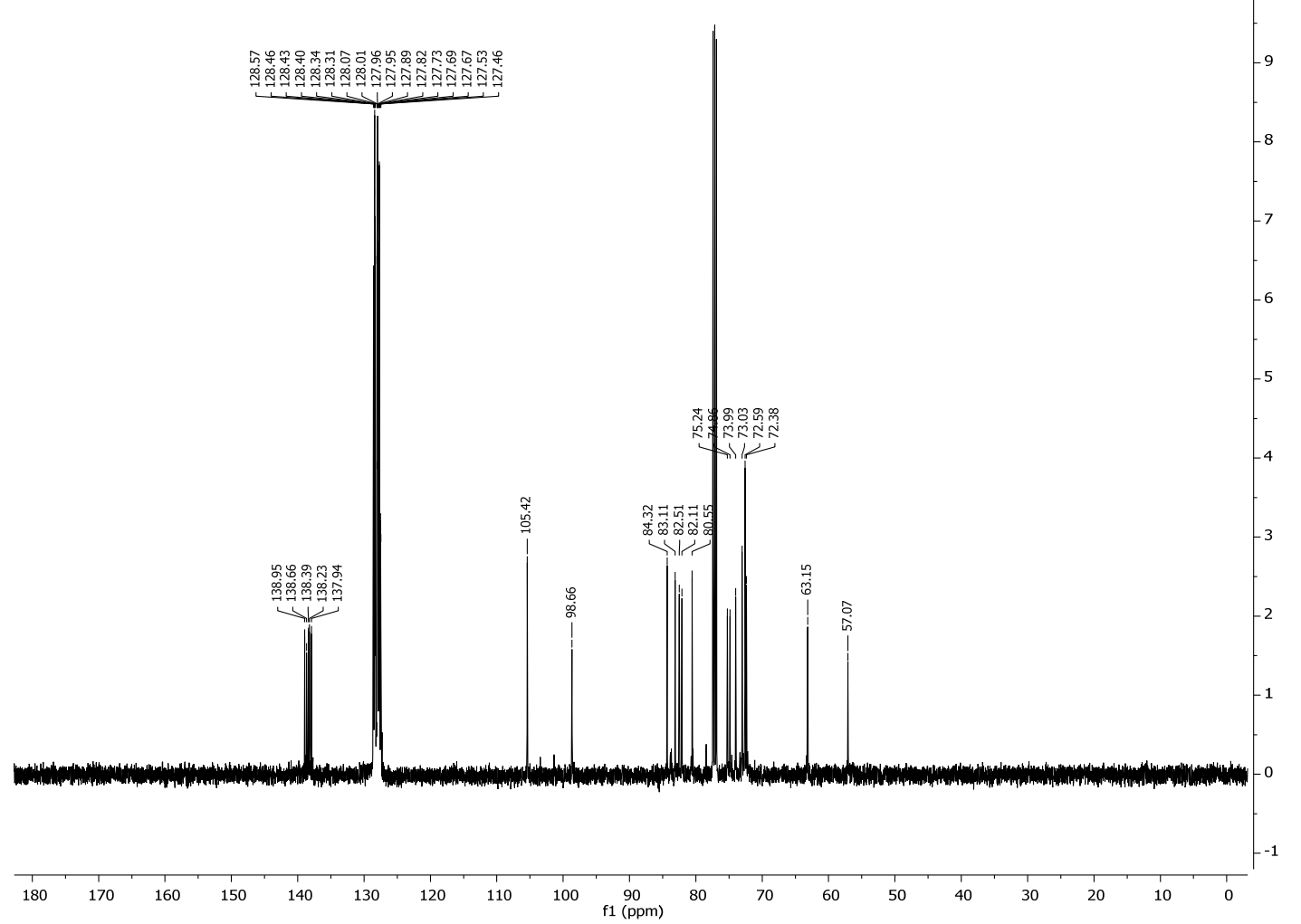


${ }^{1} \mathrm{H}$ NMR (600 MHz, $\mathrm{CDCl}_{3}$ )

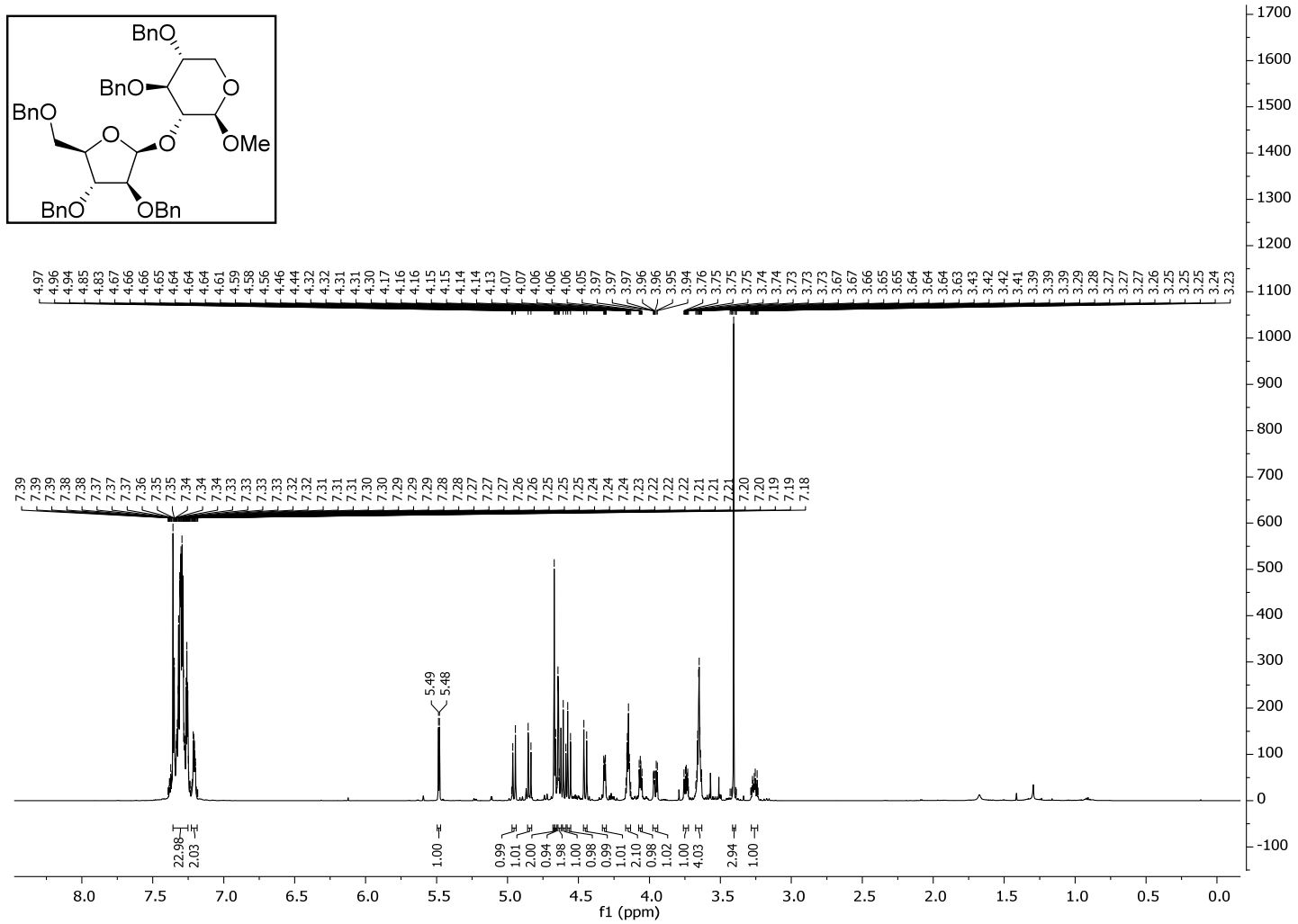

${ }^{13} \mathrm{C}$ NMR $\left(126 \mathrm{MHz}, \mathrm{CDCl}_{3}\right)$

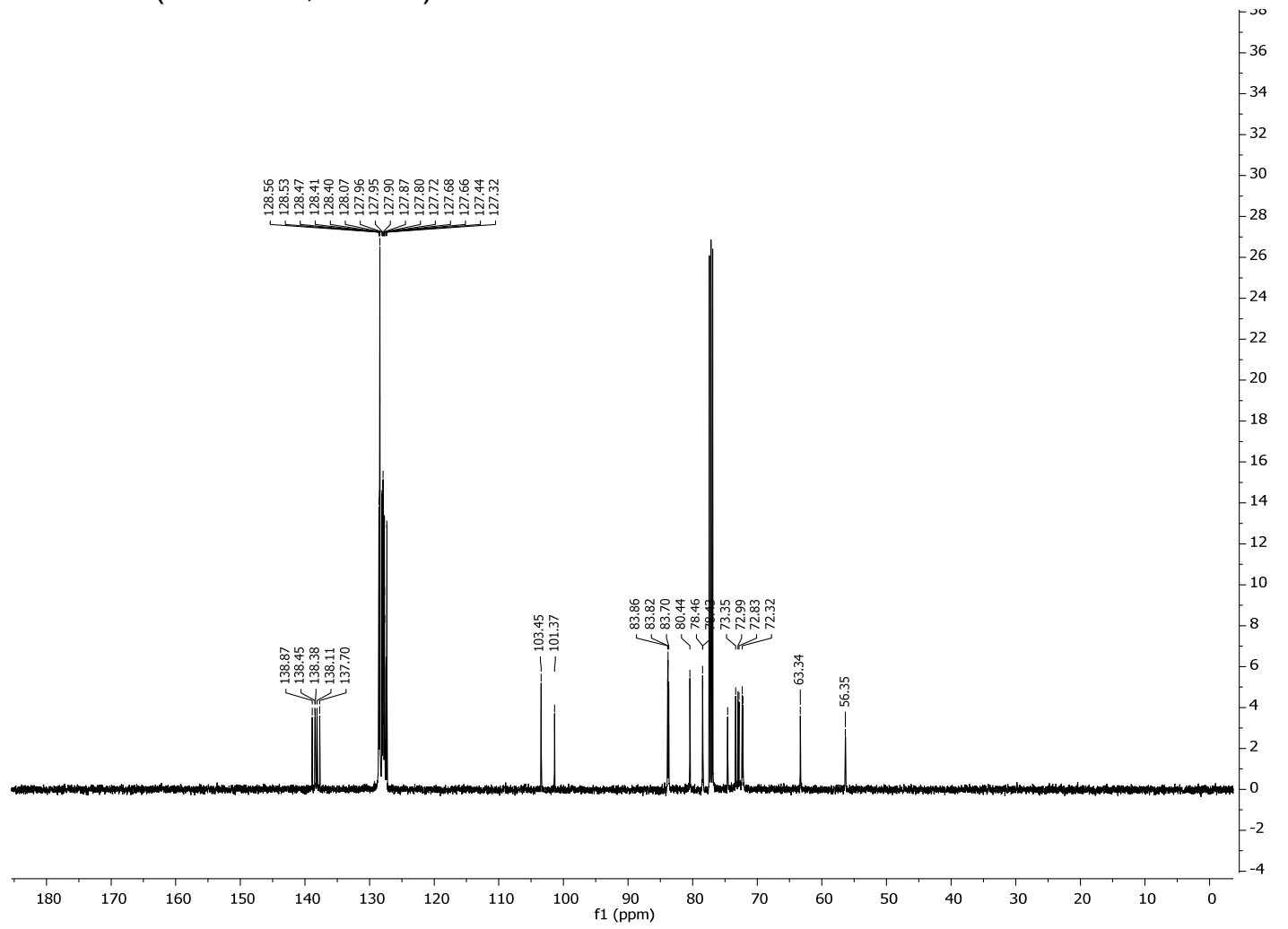


${ }^{1} \mathrm{H}$ NMR (600 MHz, $\left.\mathrm{CDCl}_{3}\right)$
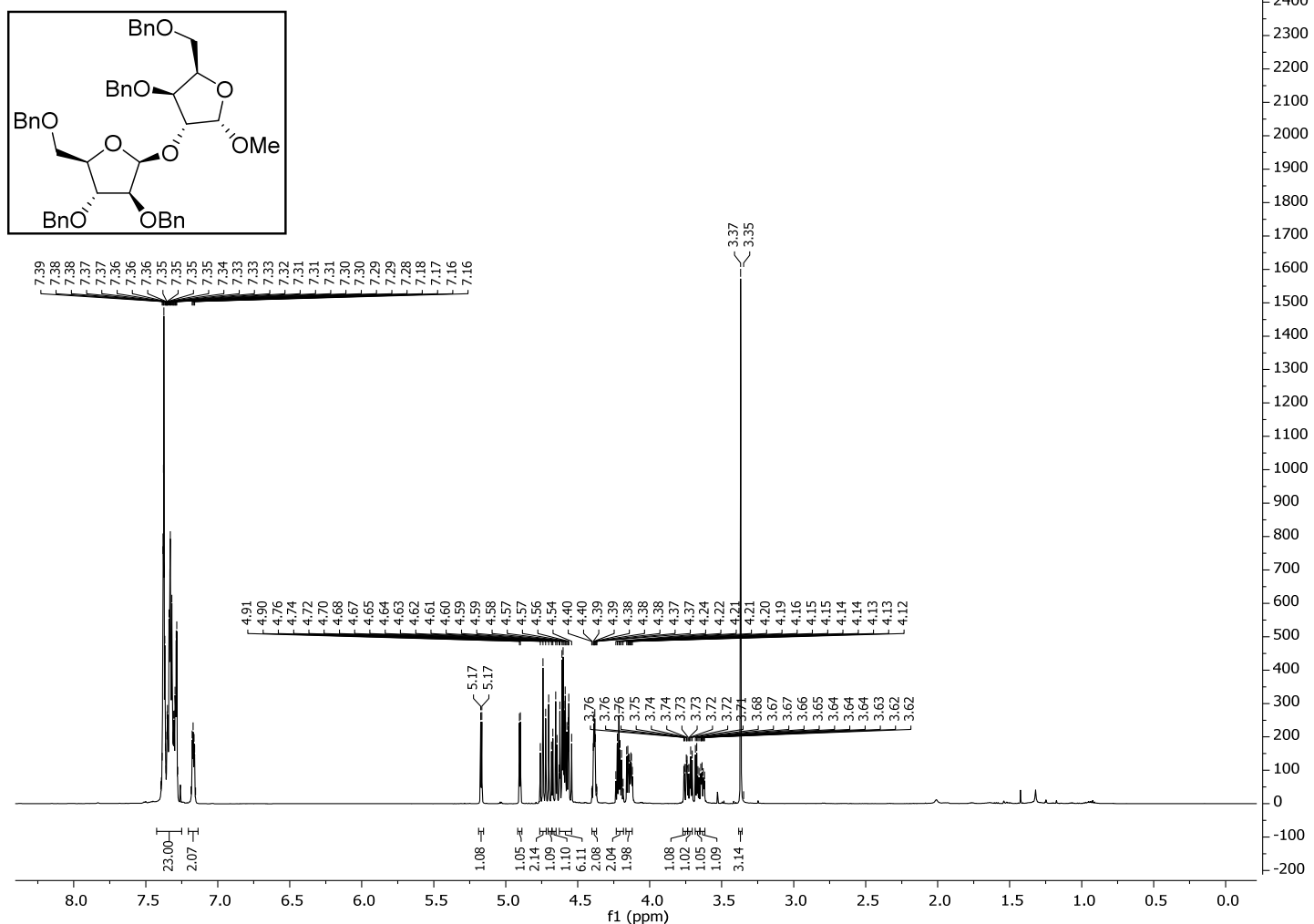

${ }^{13} \mathrm{C}$ NMR $\left(126 \mathrm{MHz}, \mathrm{CDCl}_{3}\right)$

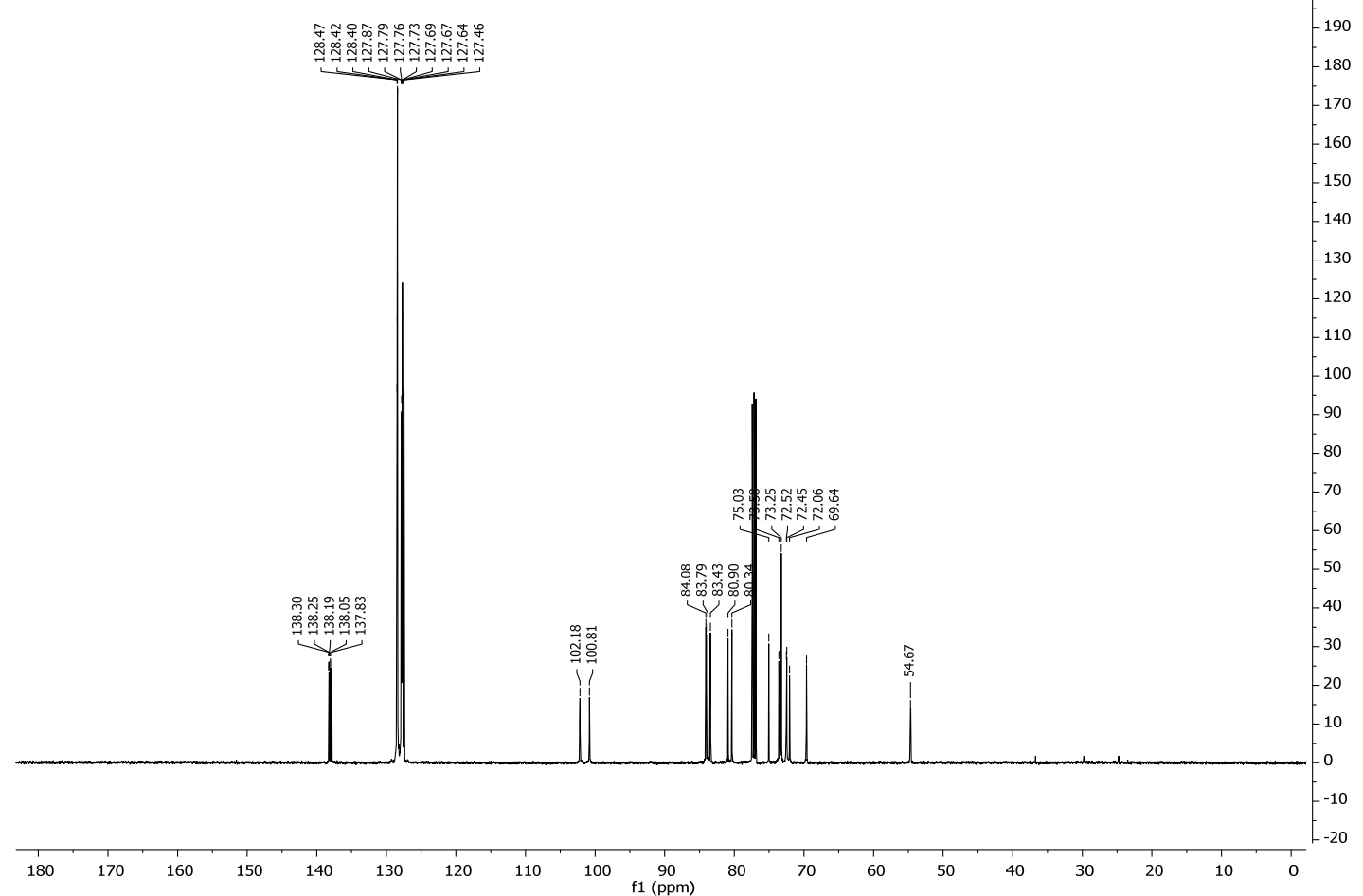


${ }^{1} \mathrm{H}$ NMR $\left(600 \mathrm{MHz}, \mathrm{CDCl}_{3}\right)$

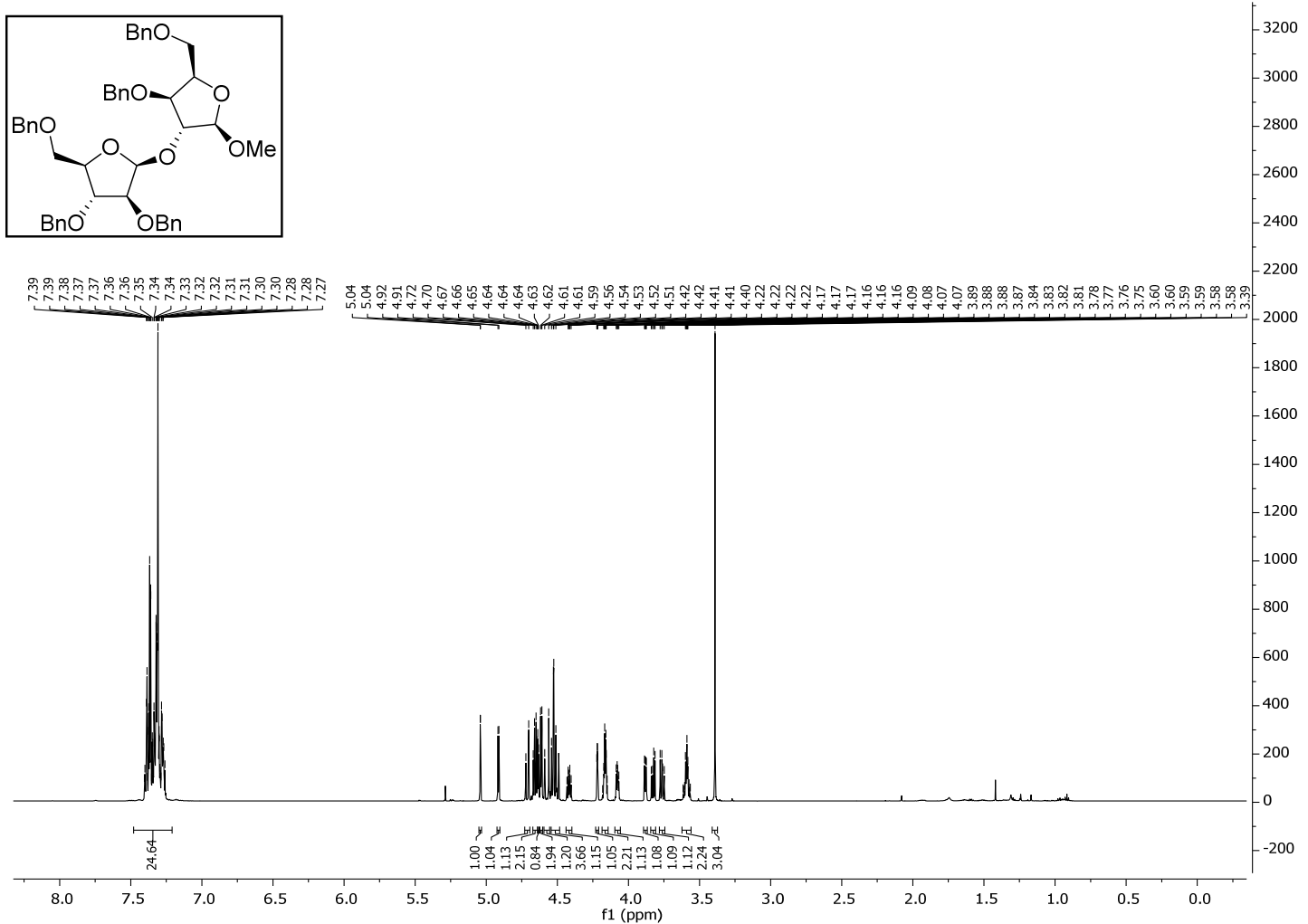

${ }^{13} \mathrm{C}$ NMR $\left(126 \mathrm{MHz}, \mathrm{CDCl}_{3}\right)$

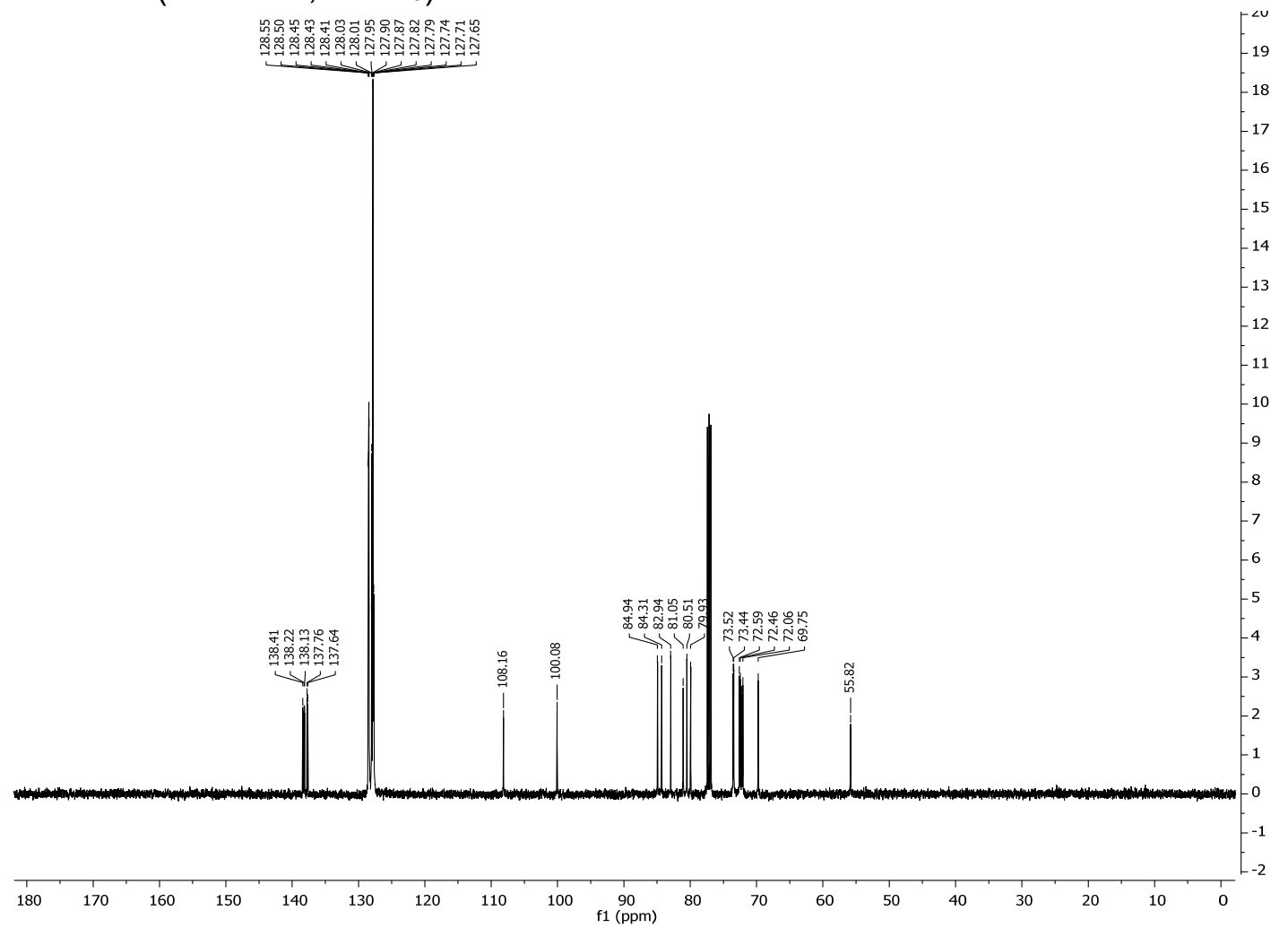


${ }^{1} \mathrm{H}$ NMR $\left(600 \mathrm{MHz}, \mathrm{CDCl}_{3}\right)$

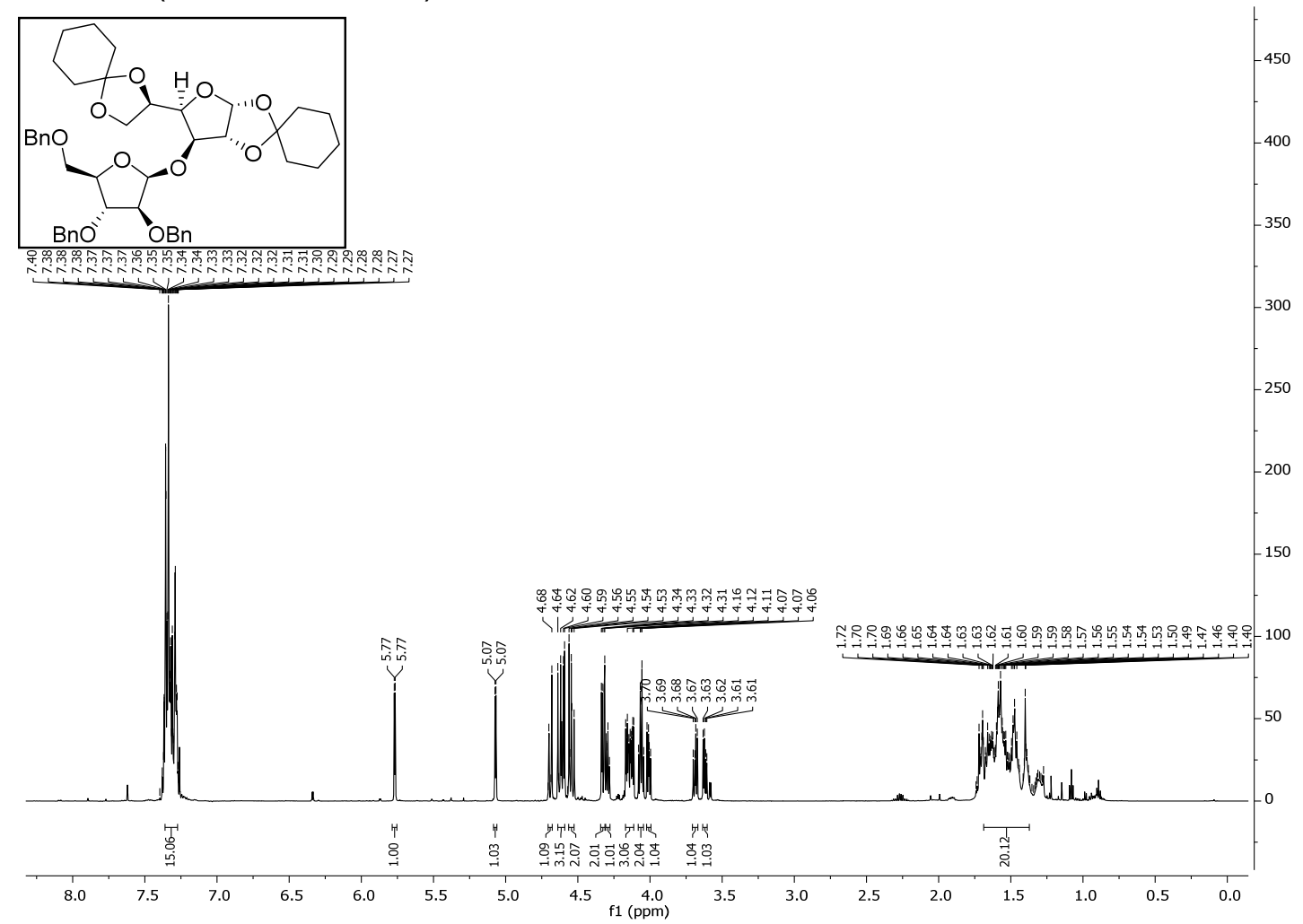

${ }^{13} \mathrm{C}$ NMR $\left(126 \mathrm{MHz}, \mathrm{CDCl}_{3}\right)$

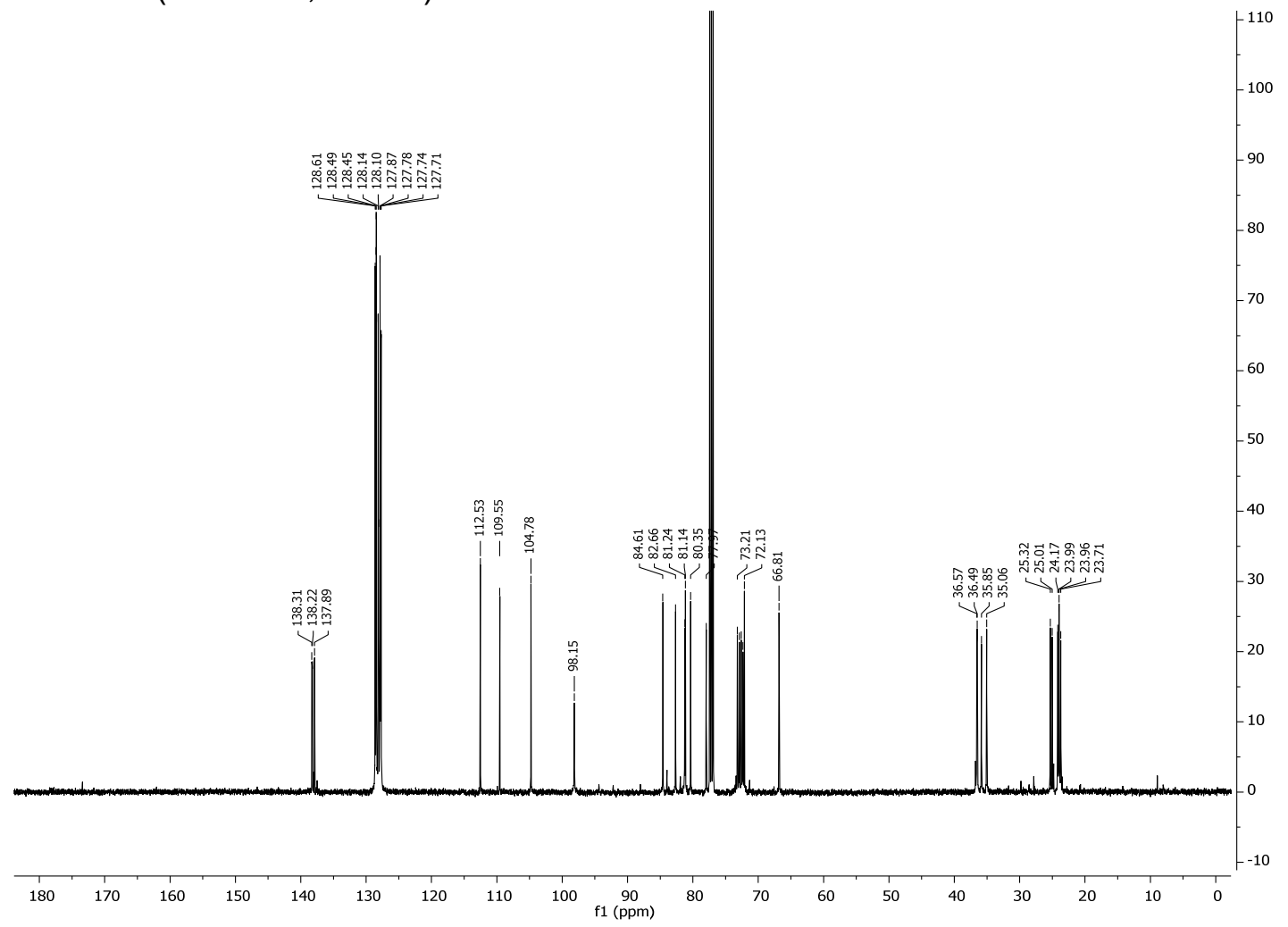


${ }^{1} \mathrm{H}$ NMR $\left(600 \mathrm{MHz}, \mathrm{CDCl}_{3}\right)$

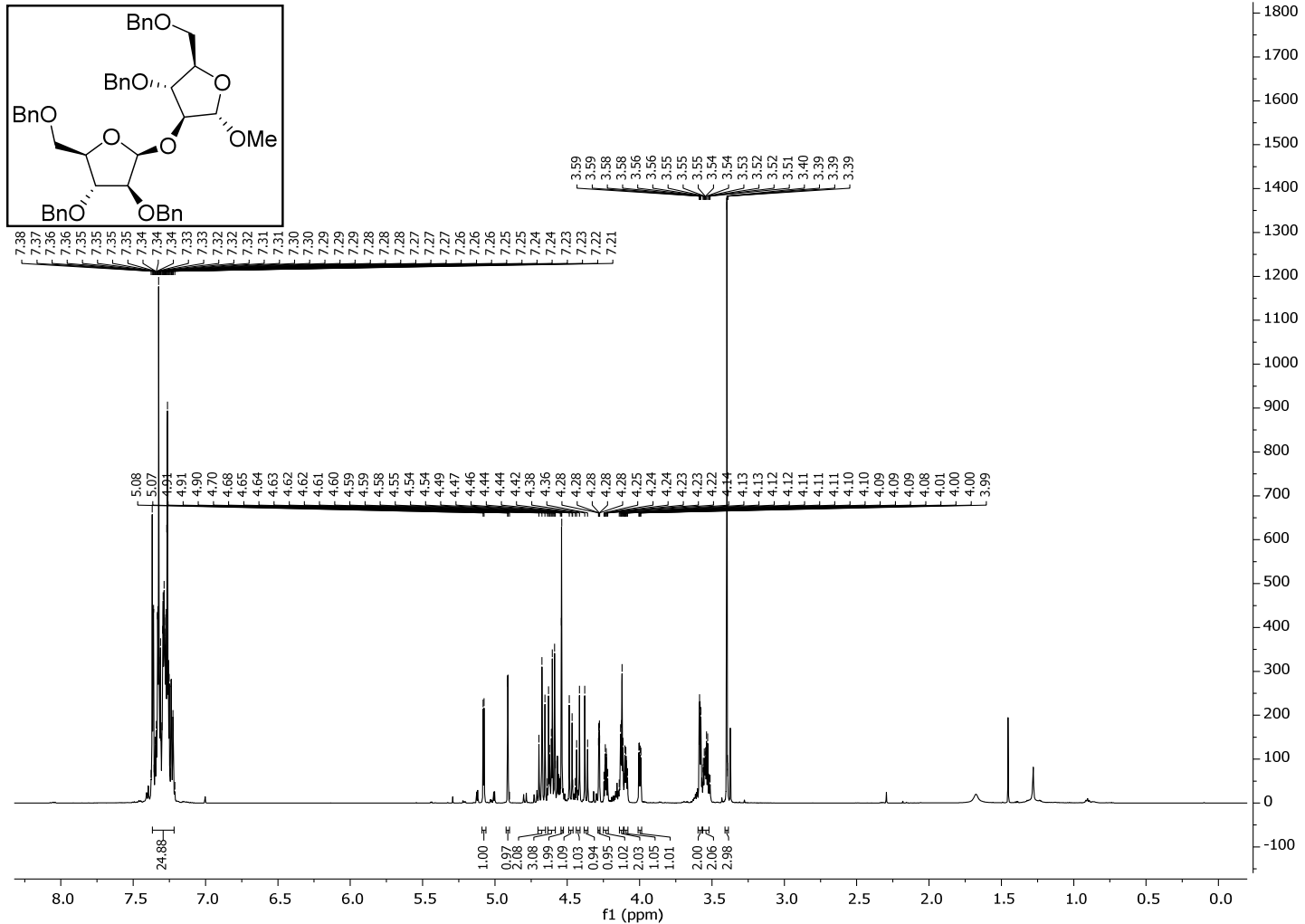

${ }^{13} \mathrm{C}$ NMR $\left(126 \mathrm{MHz}, \mathrm{CDCl}_{3}\right)$

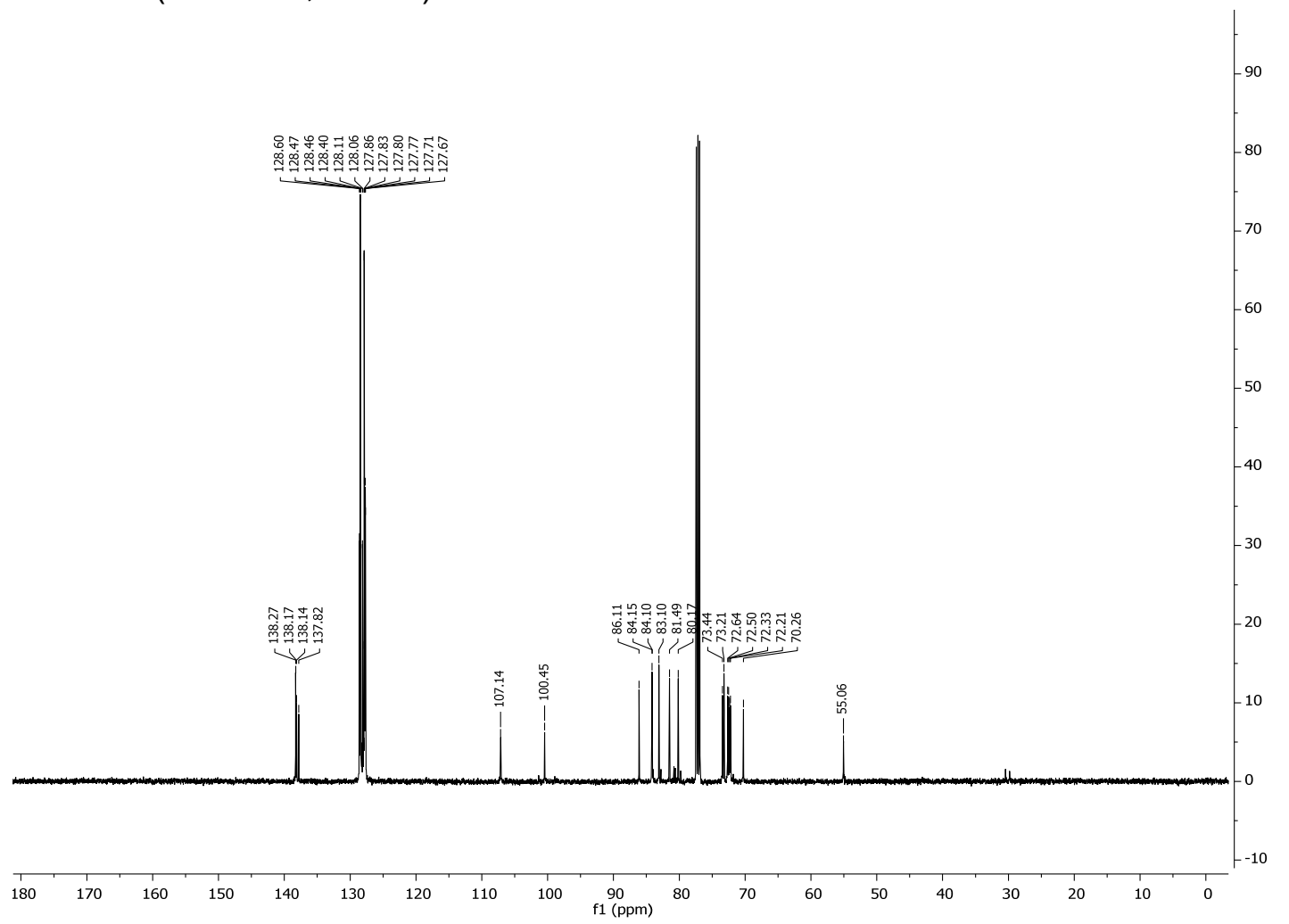


${ }^{1} \mathrm{H}$ NMR $\left(600 \mathrm{MHz}, \mathrm{CDCl}_{3}\right)$
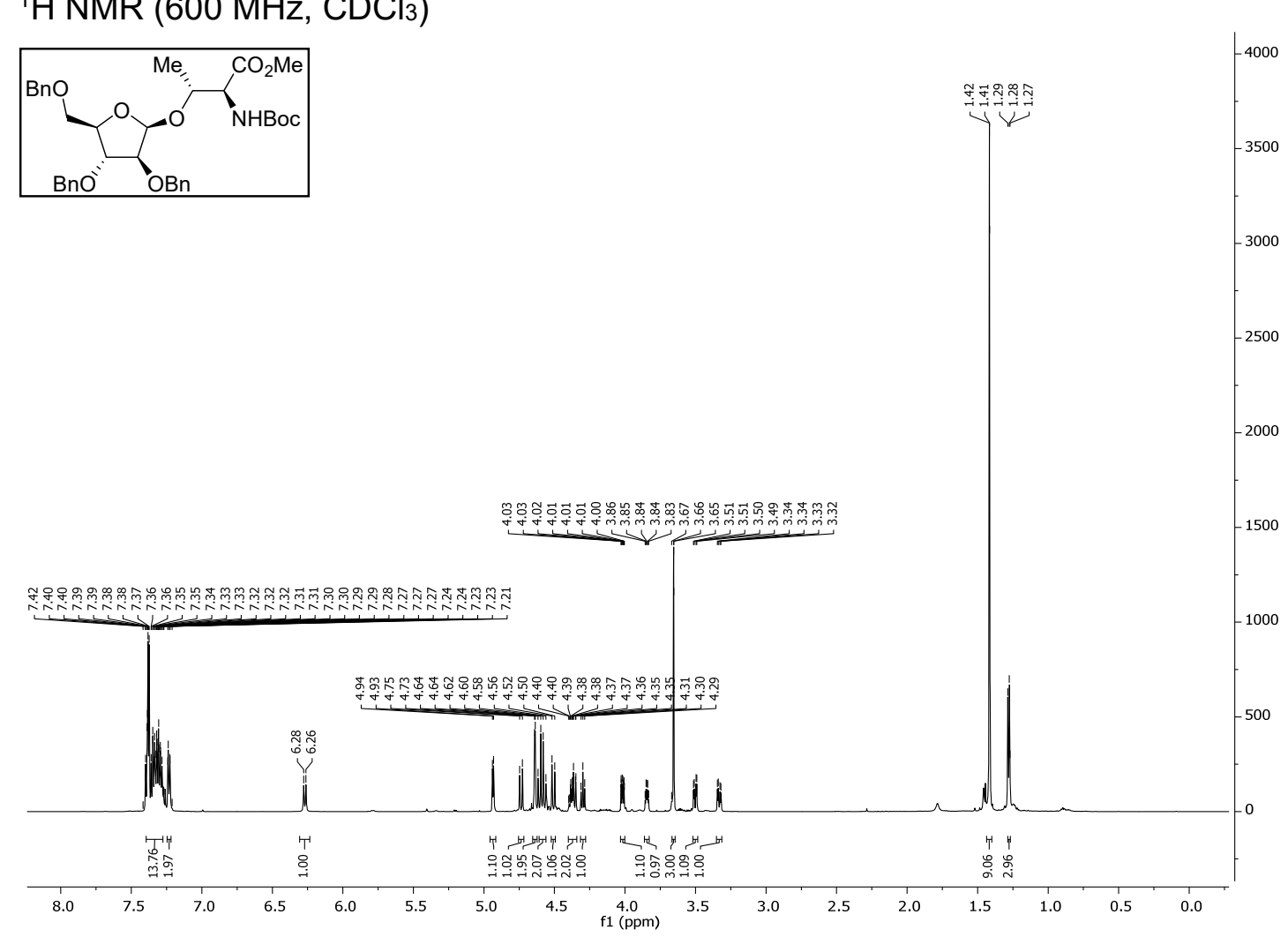

${ }^{13} \mathrm{C}$ NMR (126 MHz, $\mathrm{CDCl}_{3}$ )

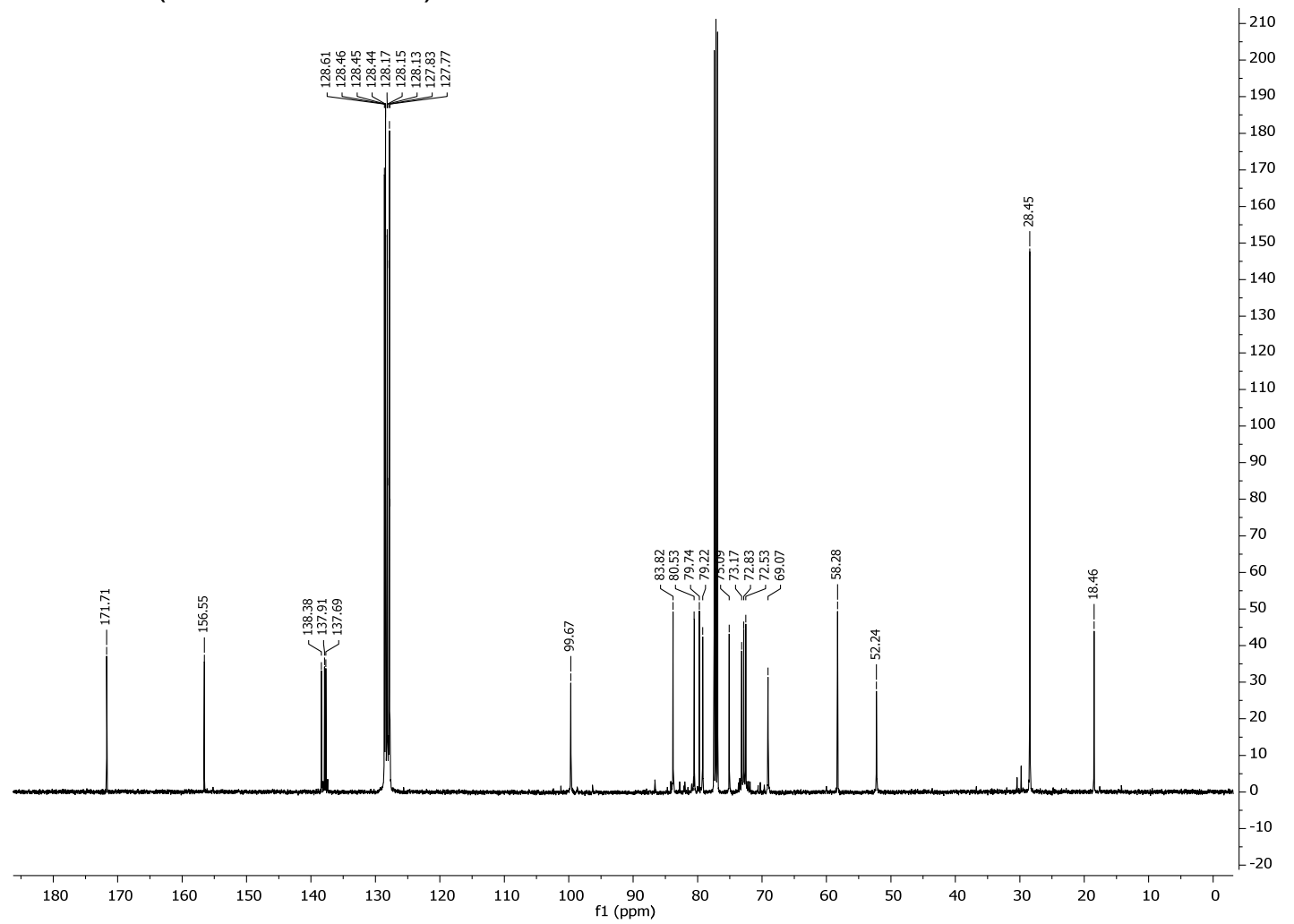


${ }^{1} \mathrm{H}$ NMR $\left(600 \mathrm{MHz}, \mathrm{CDCl}_{3}\right)$

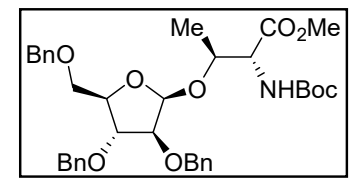

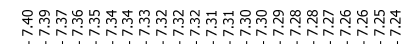

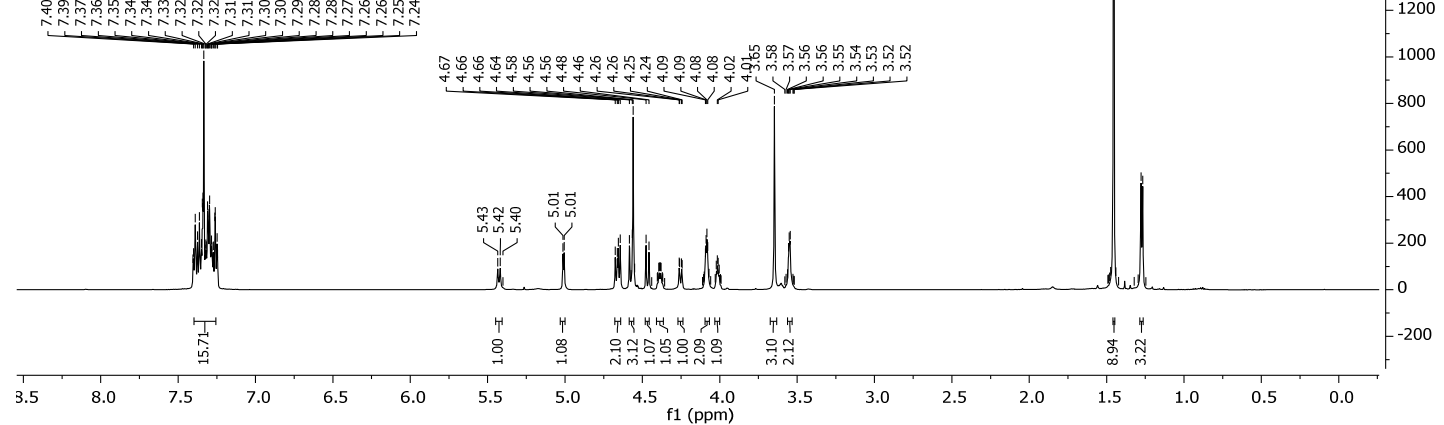

${ }^{13} \mathrm{C}$ NMR $\left(126 \mathrm{MHz}, \mathrm{CDCl}_{3}\right)$

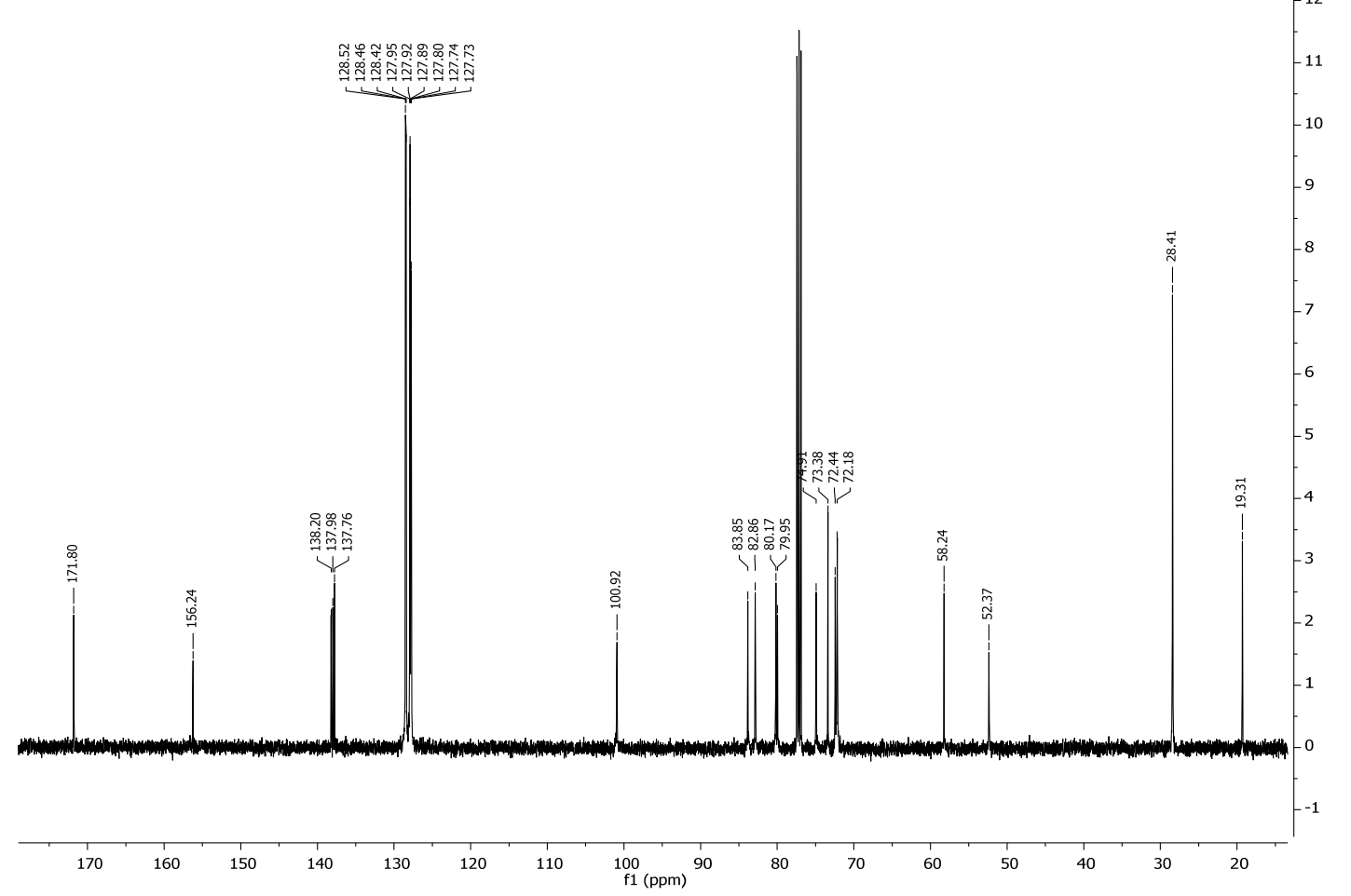


${ }^{1} \mathrm{H}$ NMR (600 MHz, $\left.\mathrm{CDCl}_{3}\right)$
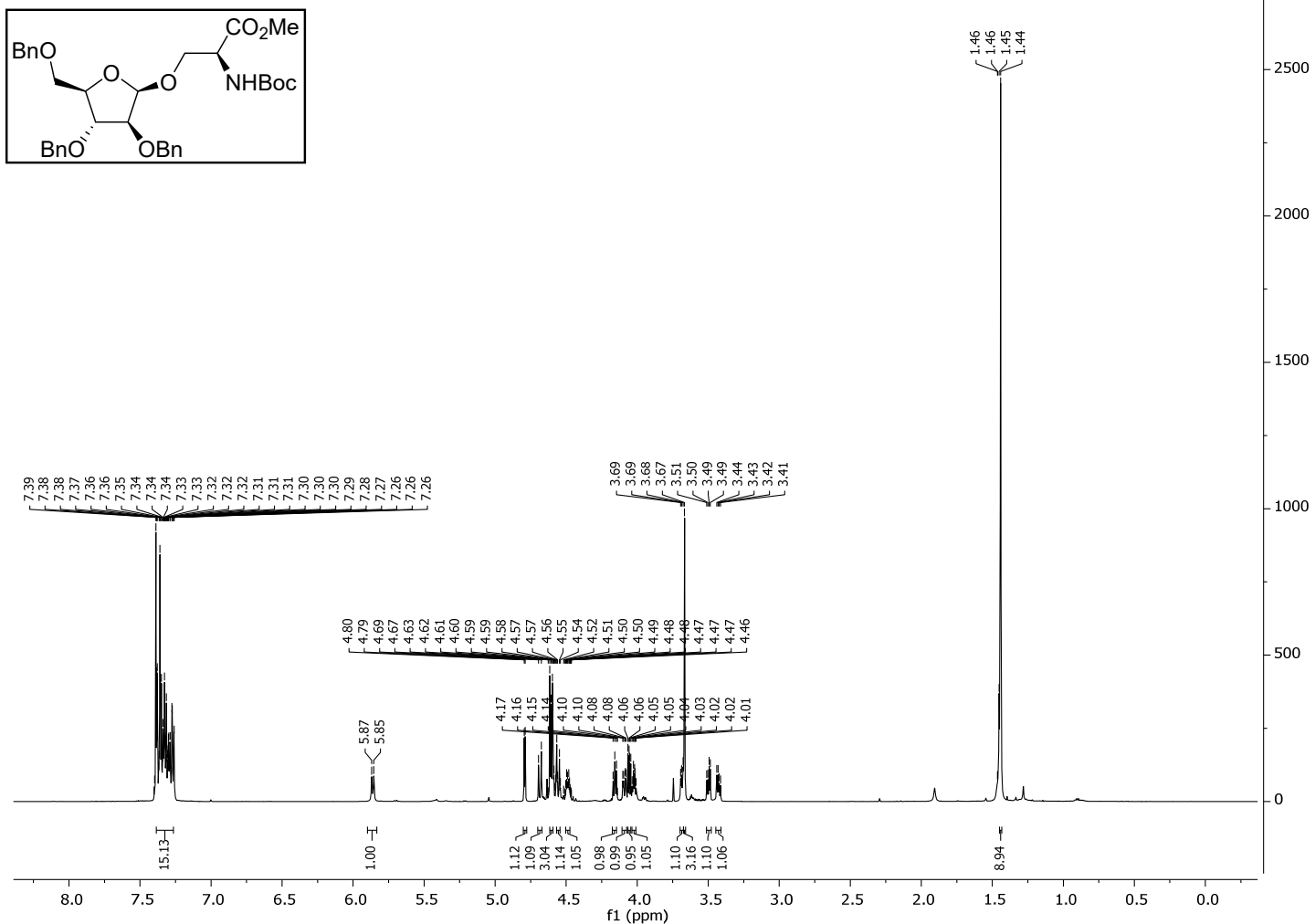

${ }^{13} \mathrm{C}$ NMR $\left(126 \mathrm{MHz}, \mathrm{CDCl}_{3}\right)$

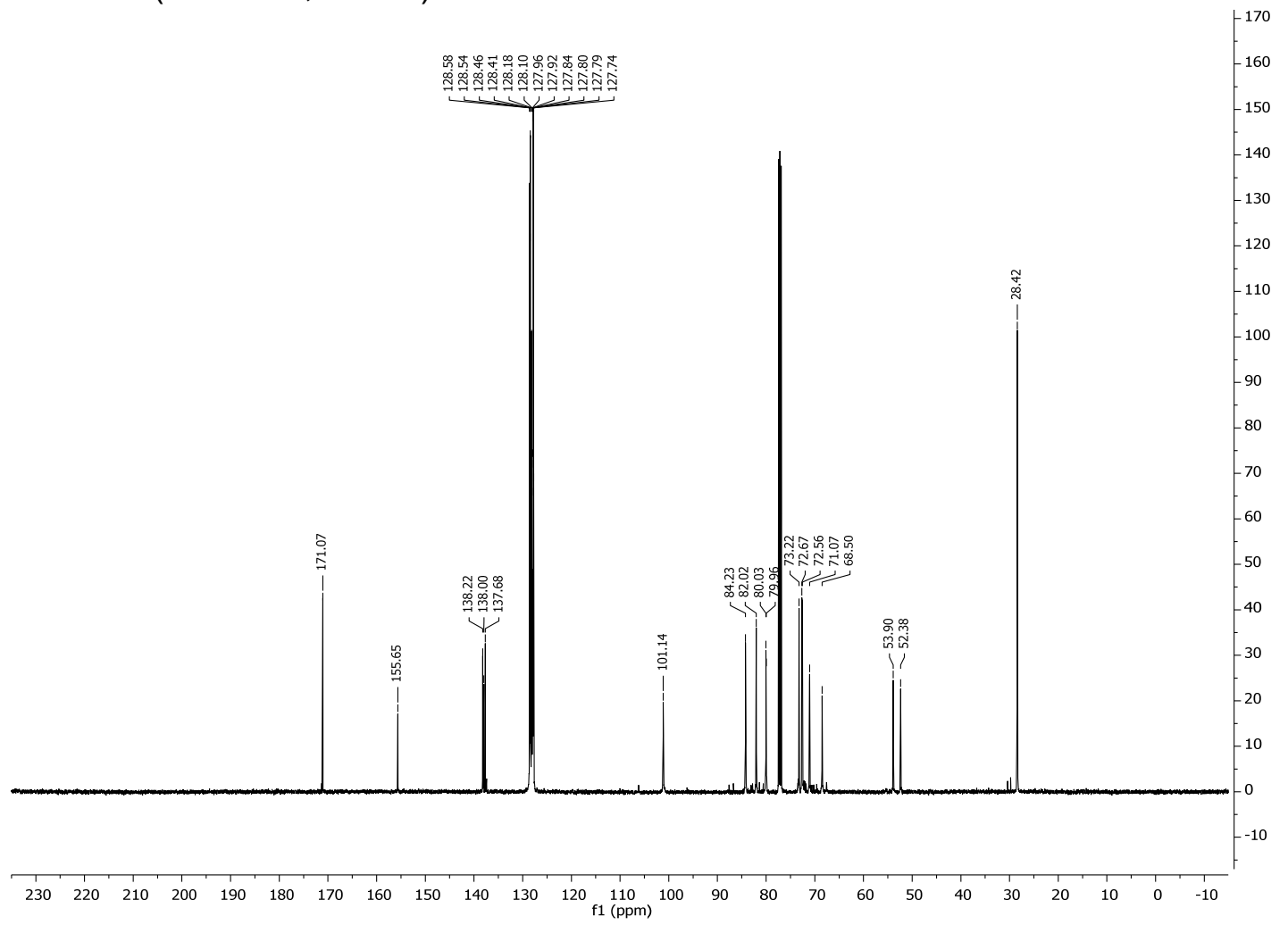


${ }^{1} \mathrm{H}$ NMR $\left(600 \mathrm{MHz}, \mathrm{CDCl}_{3}\right)$

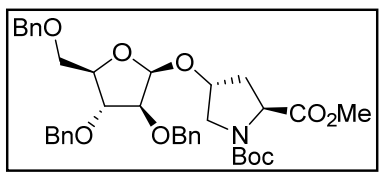

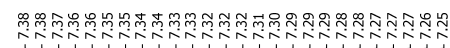

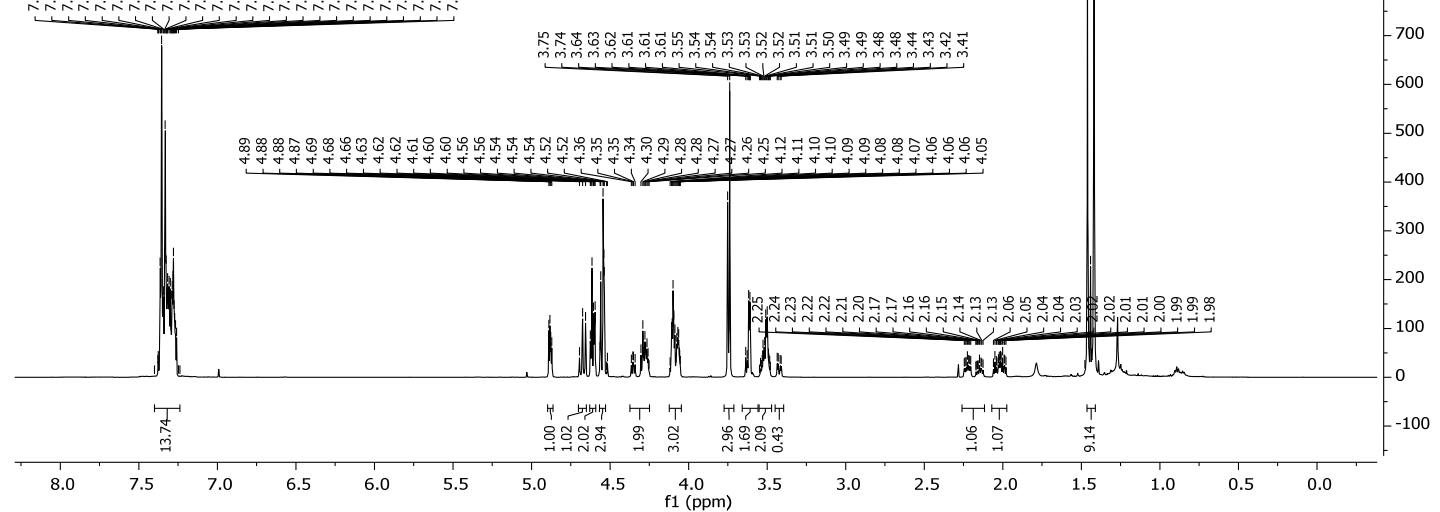

$\left.{ }^{13} \mathrm{C} \mathrm{NMR} \mathrm{(126} \mathrm{MHz,} \mathrm{CDCl}_{3}\right)$

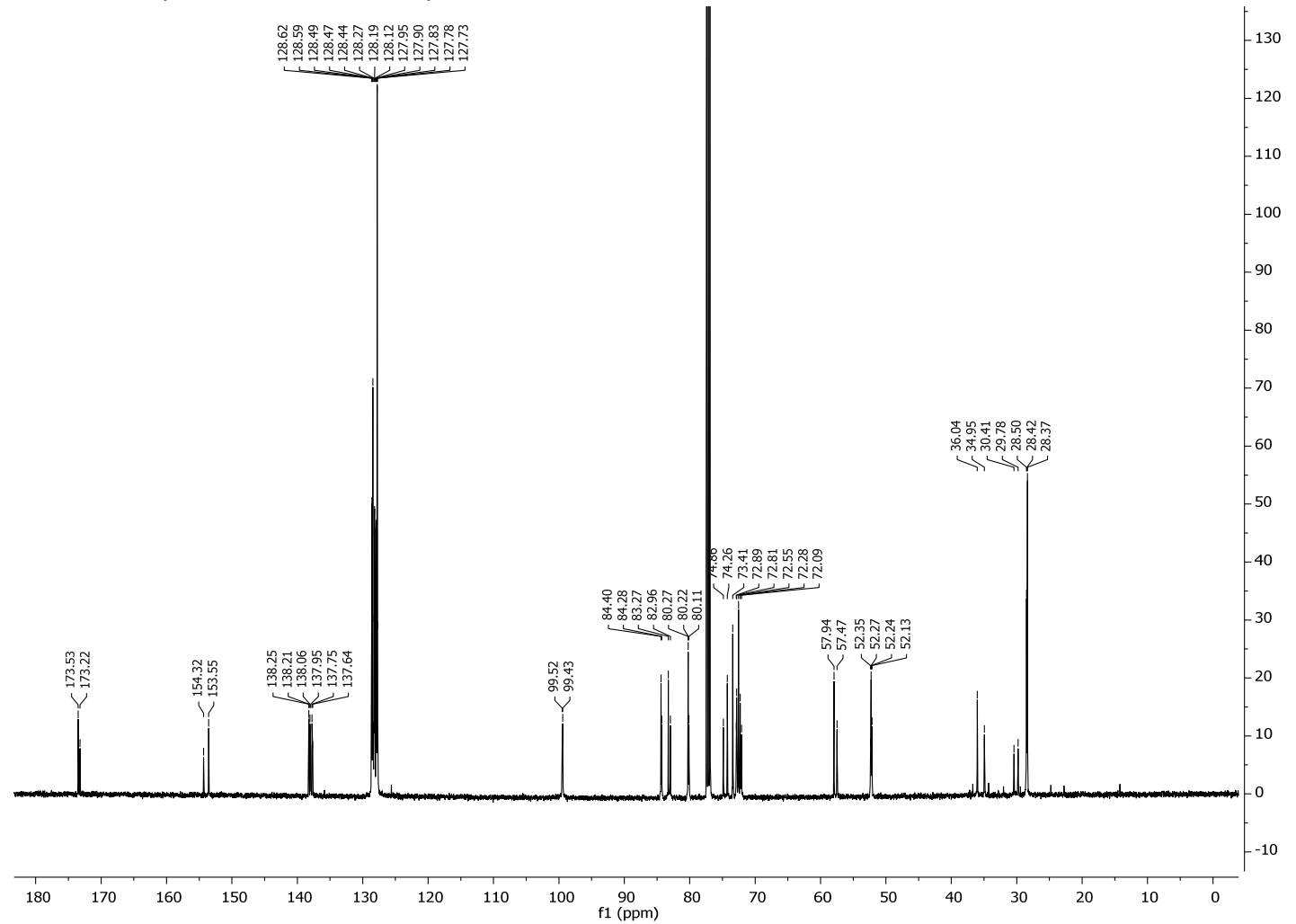


${ }^{1} \mathrm{H}$ NMR $\left(600 \mathrm{MHz}, \mathrm{CDCl}_{3}\right)$
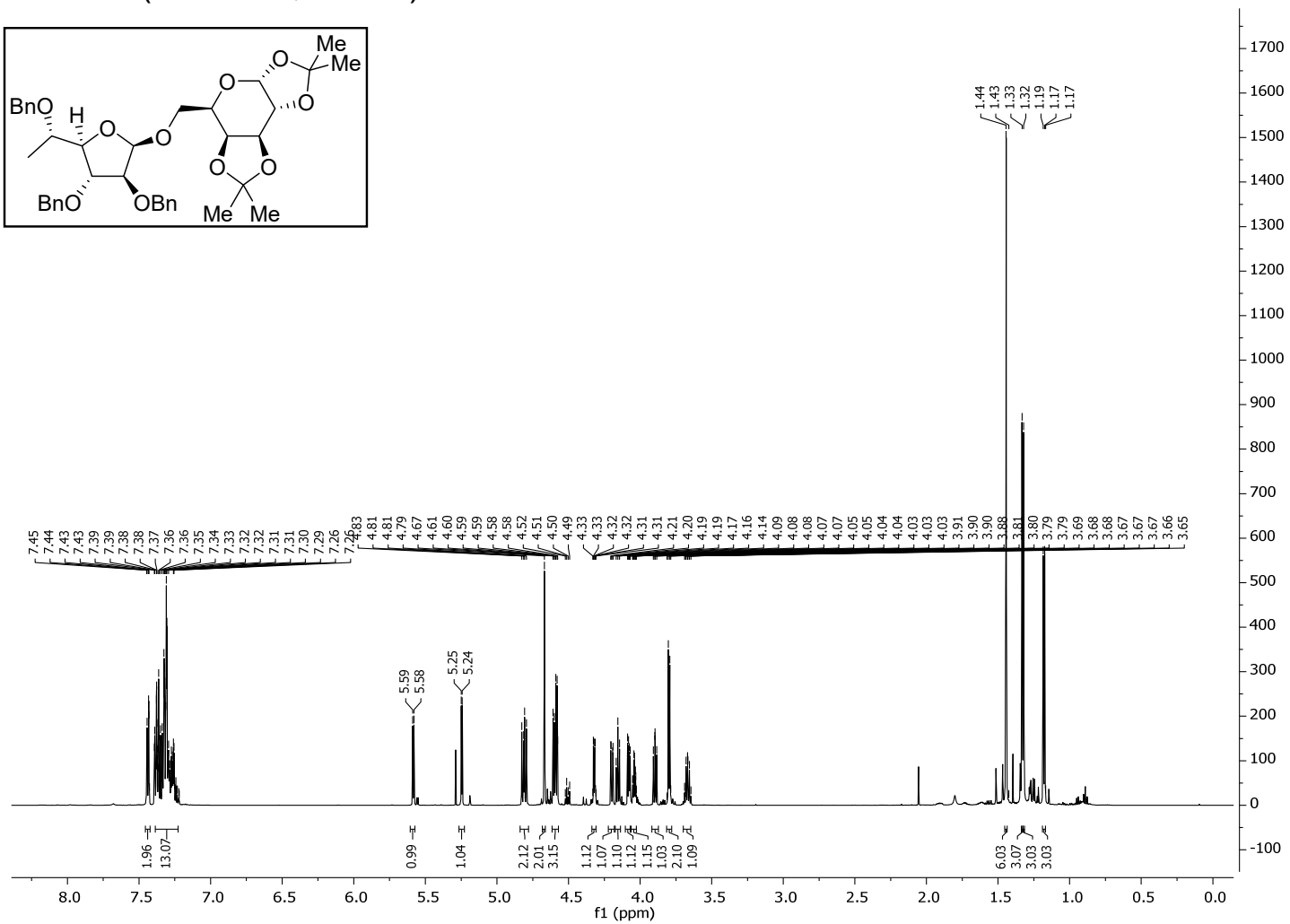

$\left.{ }^{13} \mathrm{C} \mathrm{NMR} \mathrm{(126} \mathrm{MHz,} \mathrm{CDCl}_{3}\right)$

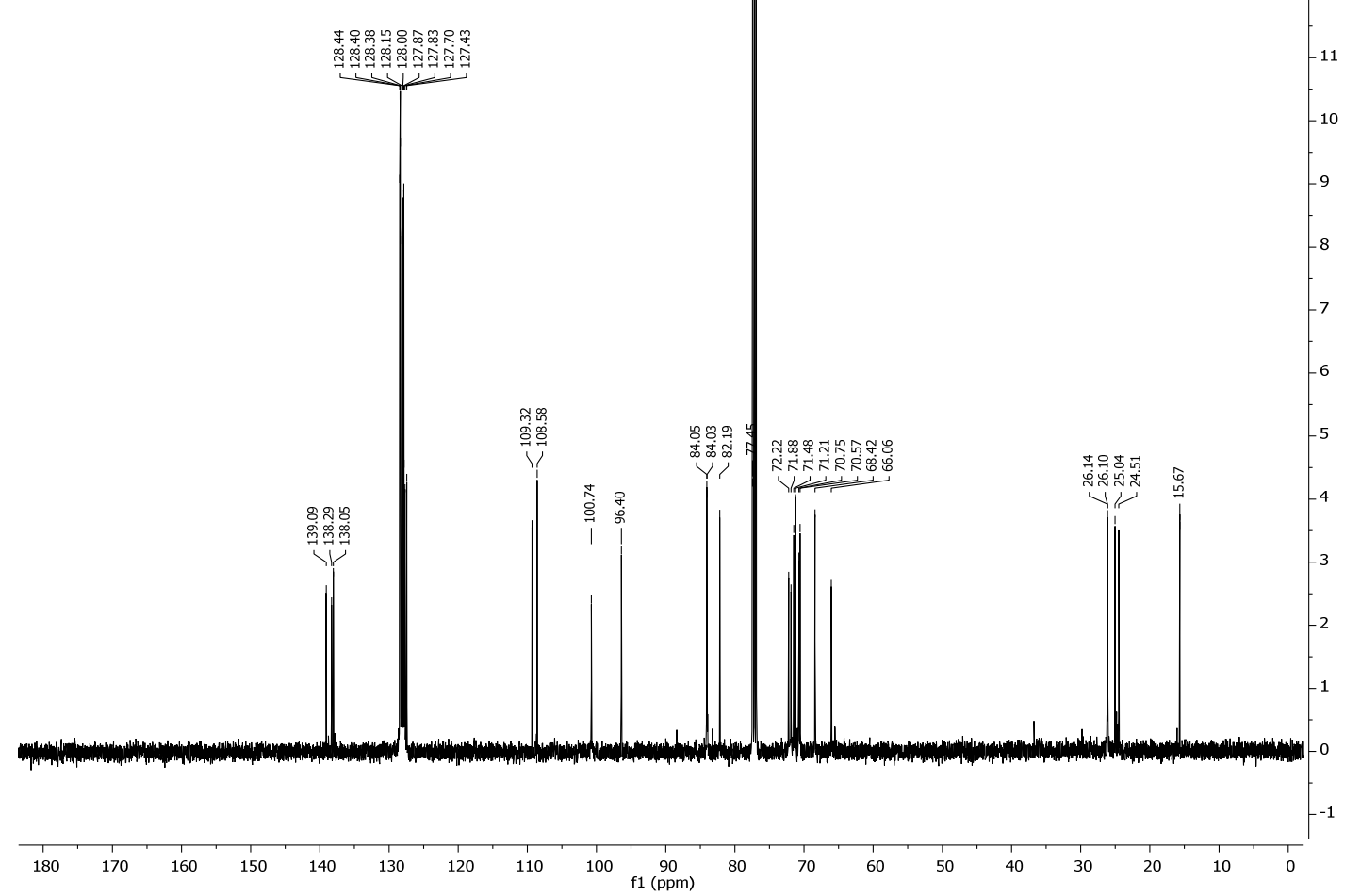


${ }^{1} \mathrm{H}$ NMR (600 MHz, $\left.\mathrm{CDCl}_{3}\right)$

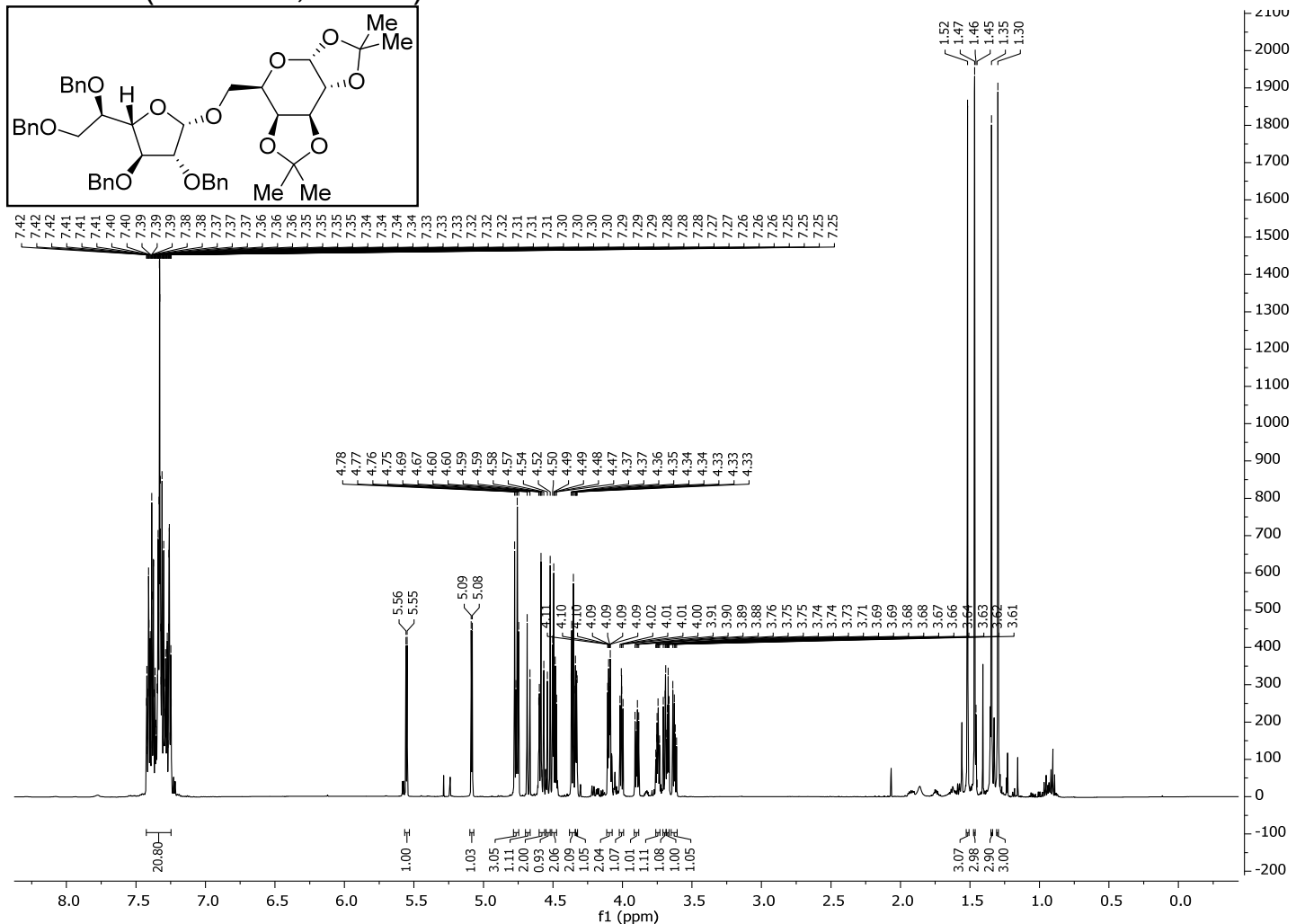

${ }^{13} \mathrm{C}$ NMR $\left(126 \mathrm{MHz}, \mathrm{CDCl}_{3}\right)$

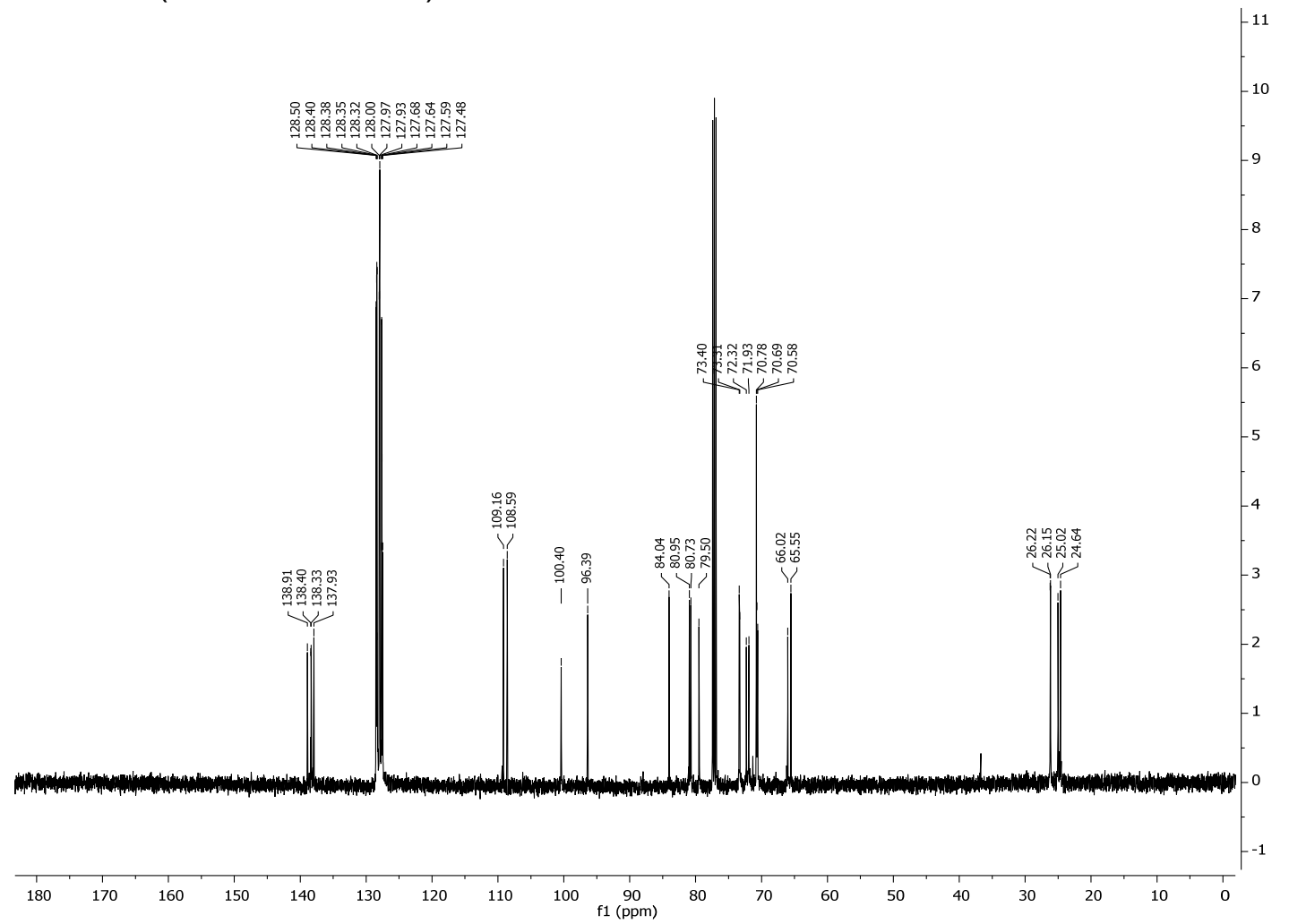


${ }^{1} \mathrm{H}$ NMR $\left(600 \mathrm{MHz}, \mathrm{CDCl}_{3}\right)$
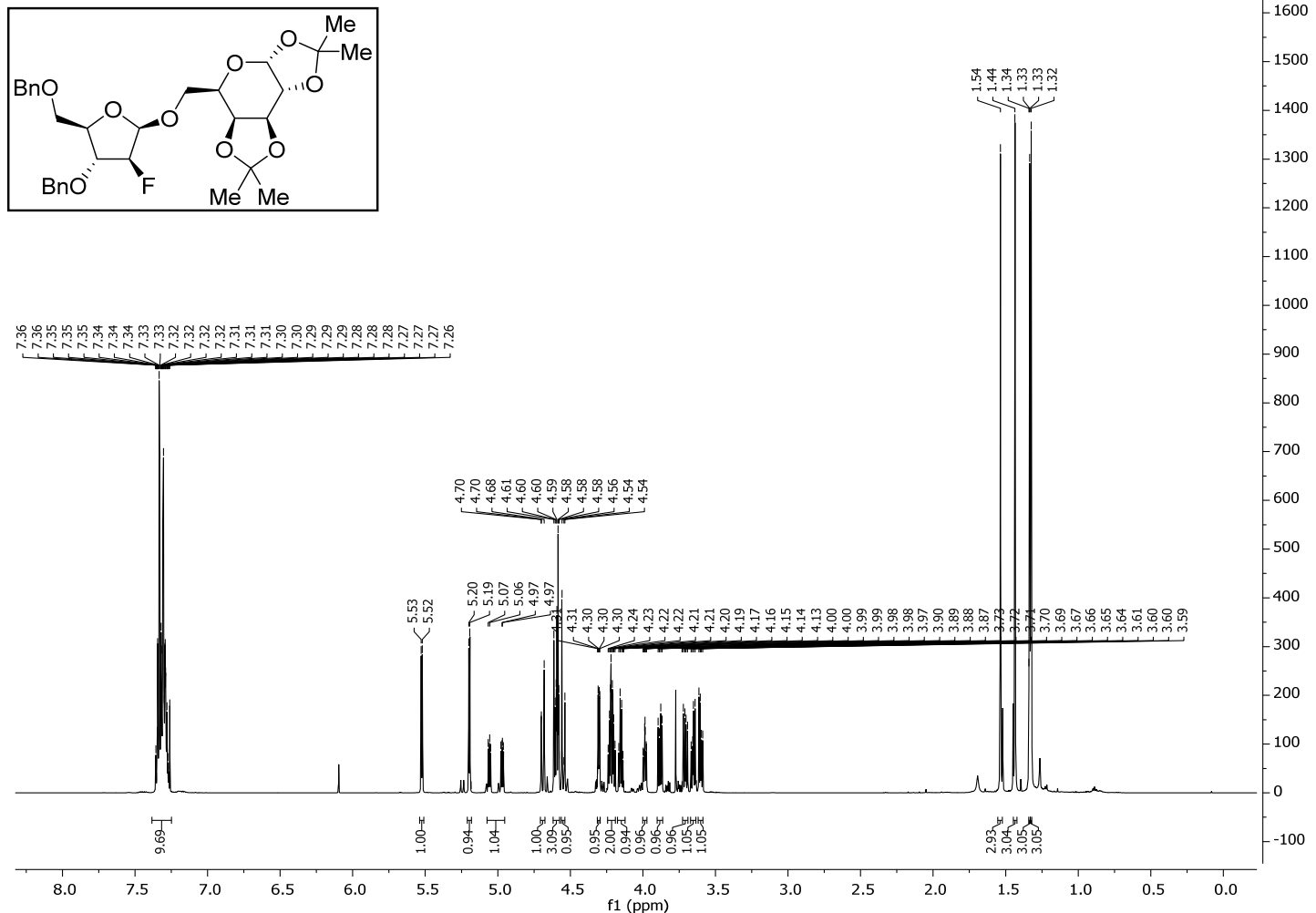

${ }^{13} \mathrm{C}$ NMR $\left(126 \mathrm{MHz}, \mathrm{CDCl}_{3}\right)$

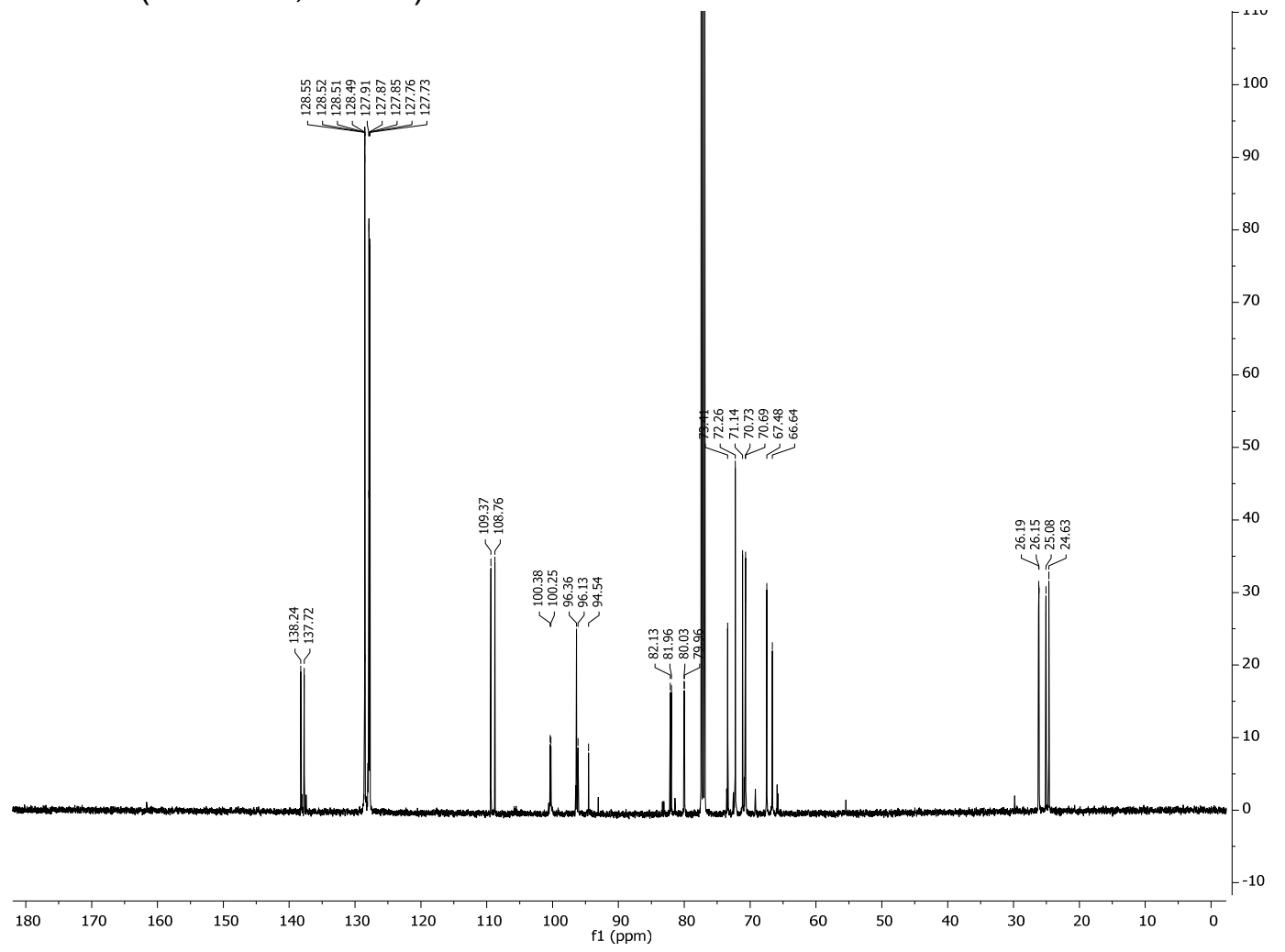


${ }^{19} \mathrm{~F} \mathrm{NMR}\left(471 \mathrm{MHz}, \mathrm{CDCl}_{3}\right)$

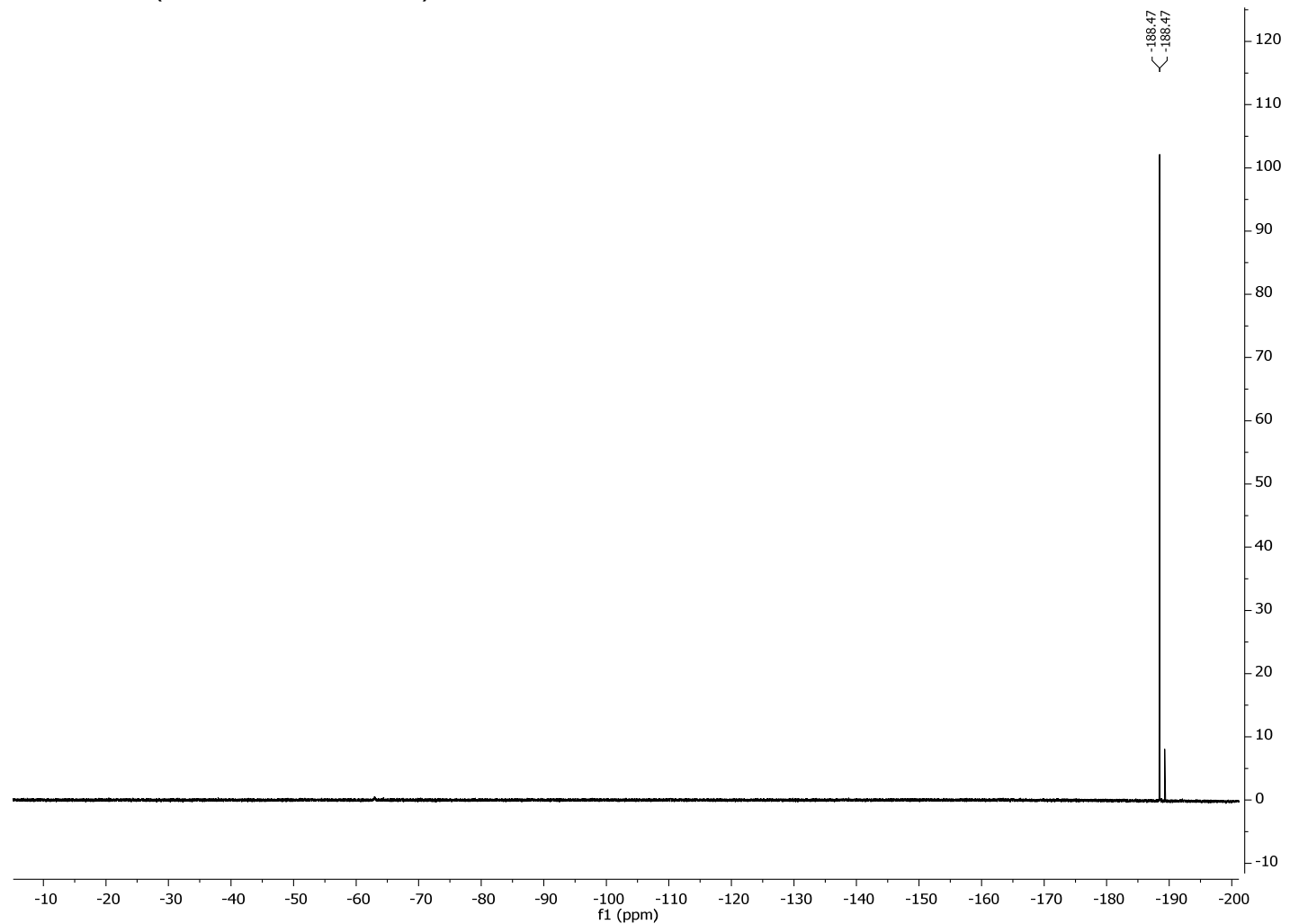


${ }^{1} \mathrm{H}$ NMR $\left(600 \mathrm{MHz}, \mathrm{CDCl}_{3}\right)$
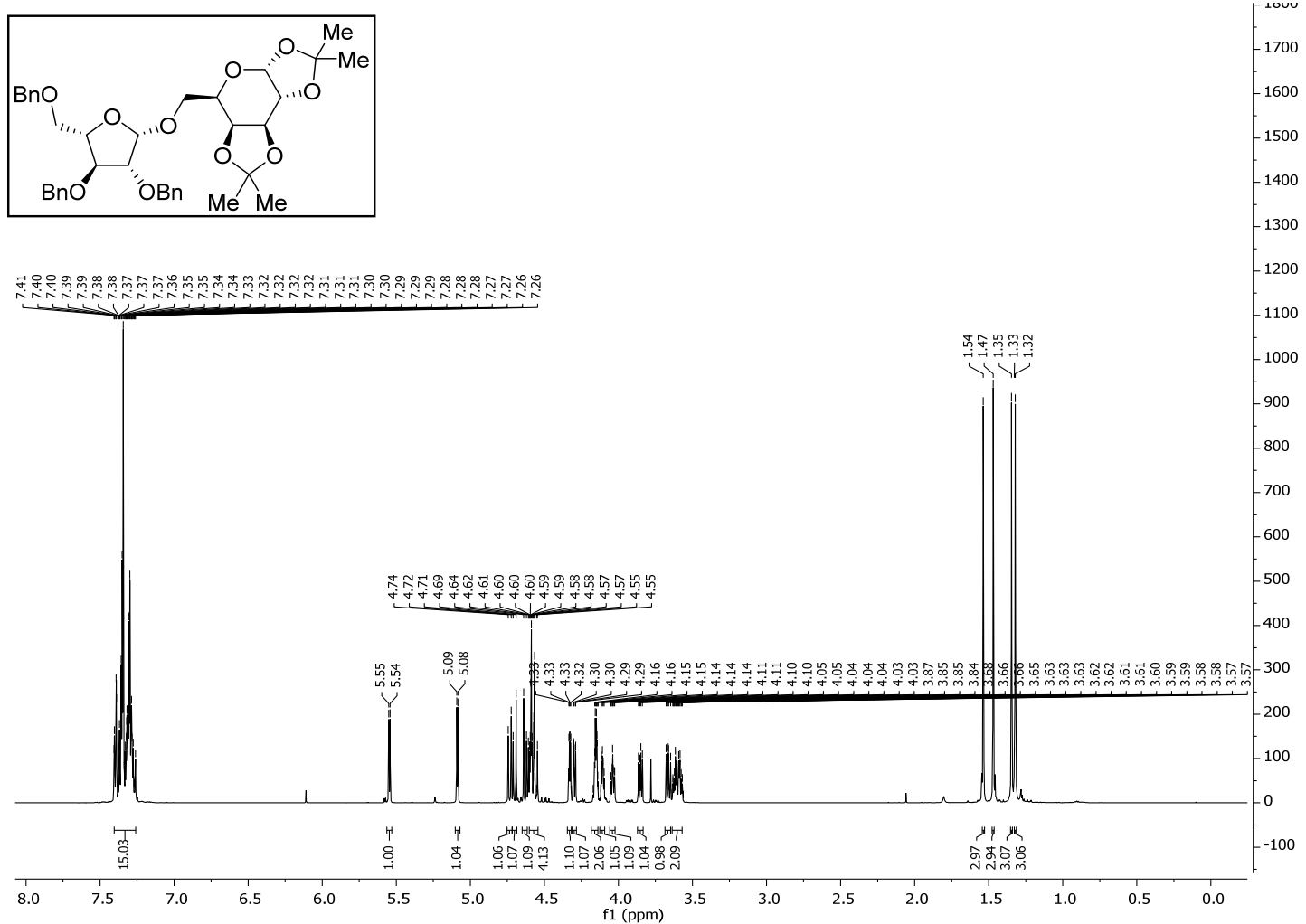

$\left.{ }^{13} \mathrm{C} \mathrm{NMR} \mathrm{(126} \mathrm{MHz,} \mathrm{CDCl}_{3}\right)$

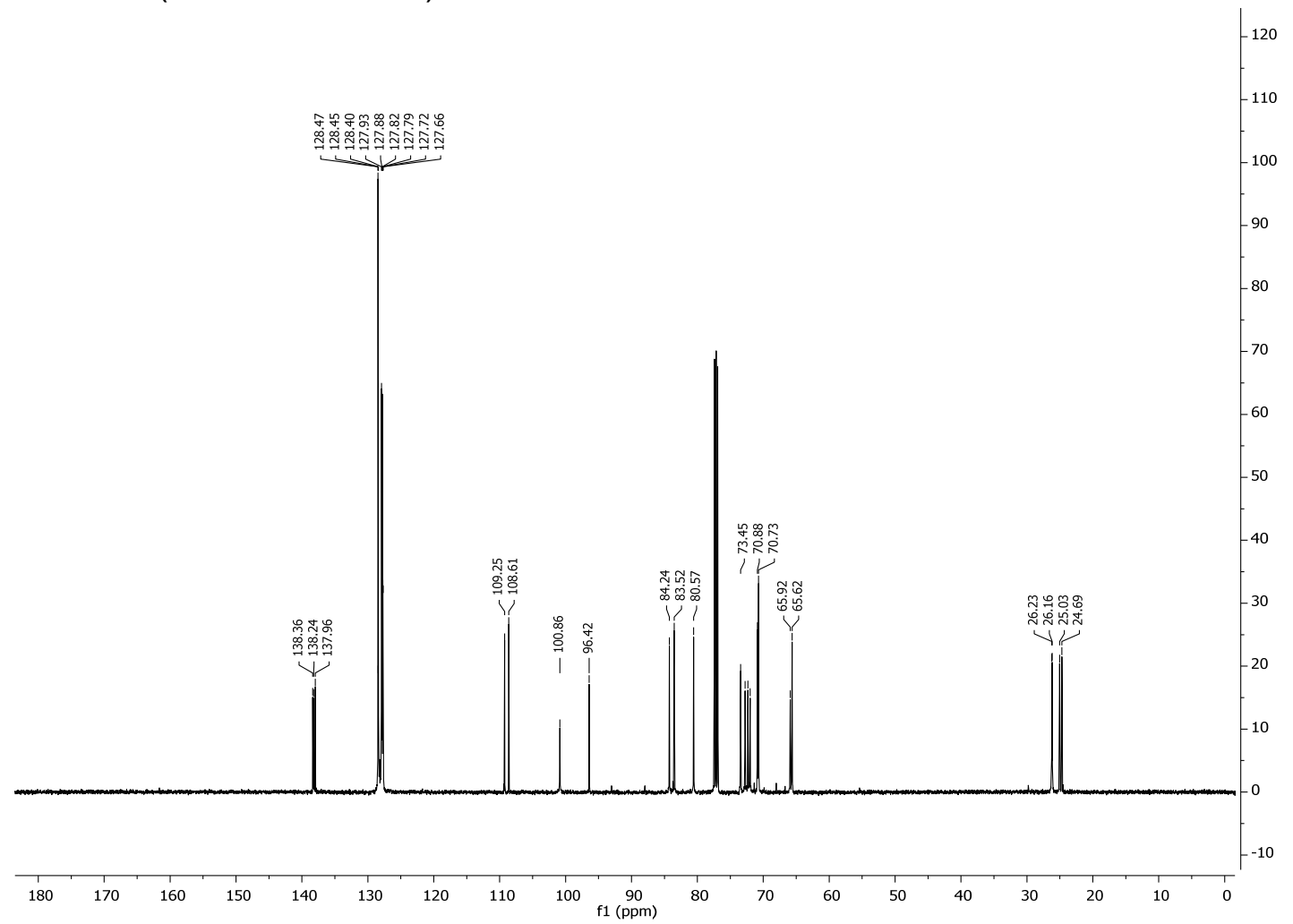


${ }^{1} \mathrm{H}$ NMR $\left(600 \mathrm{MHz}, \mathrm{CDCl}_{3}\right)$
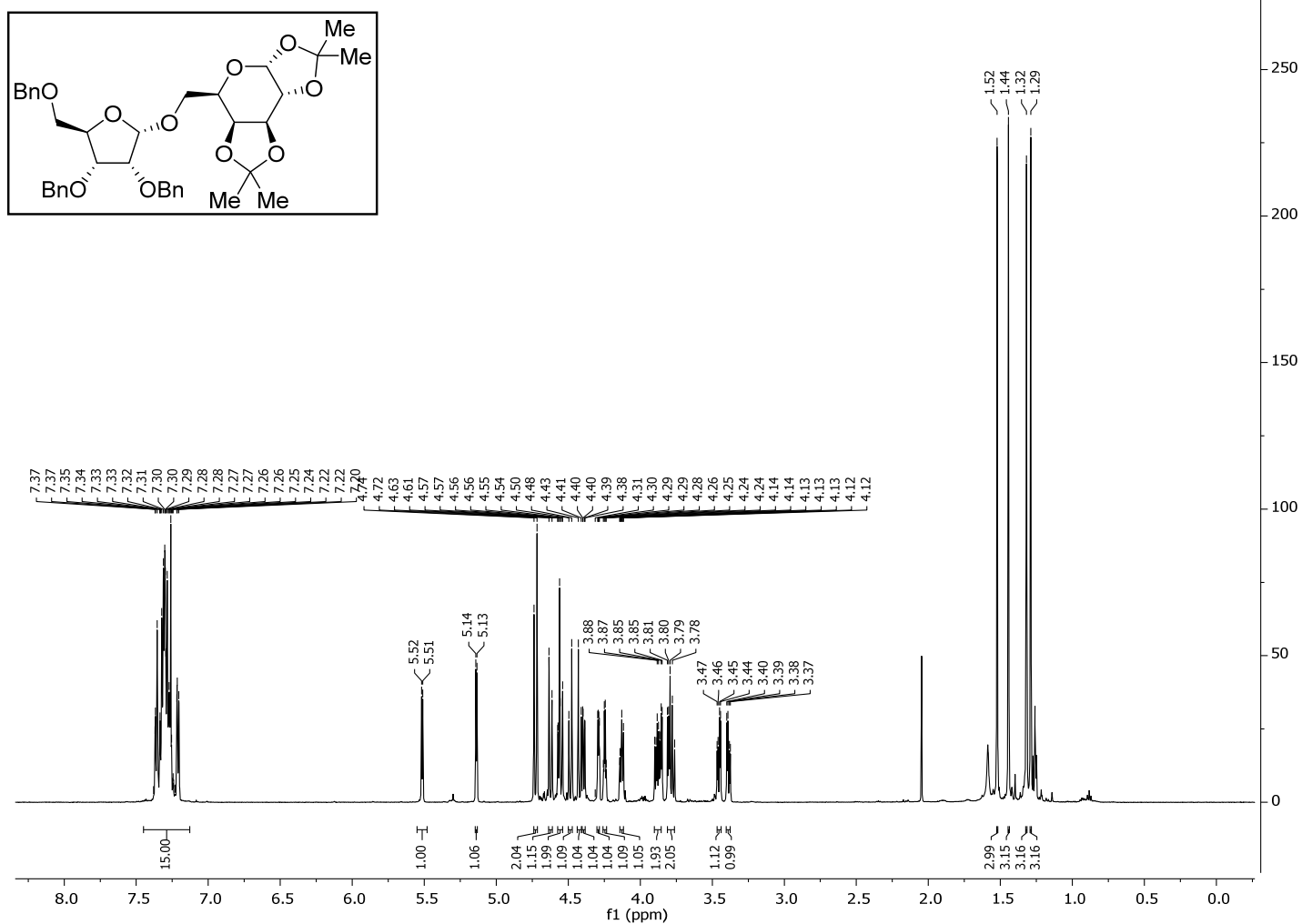

${ }^{13} \mathrm{C}$ NMR $\left(126 \mathrm{MHz}, \mathrm{CDCl}_{3}\right)$

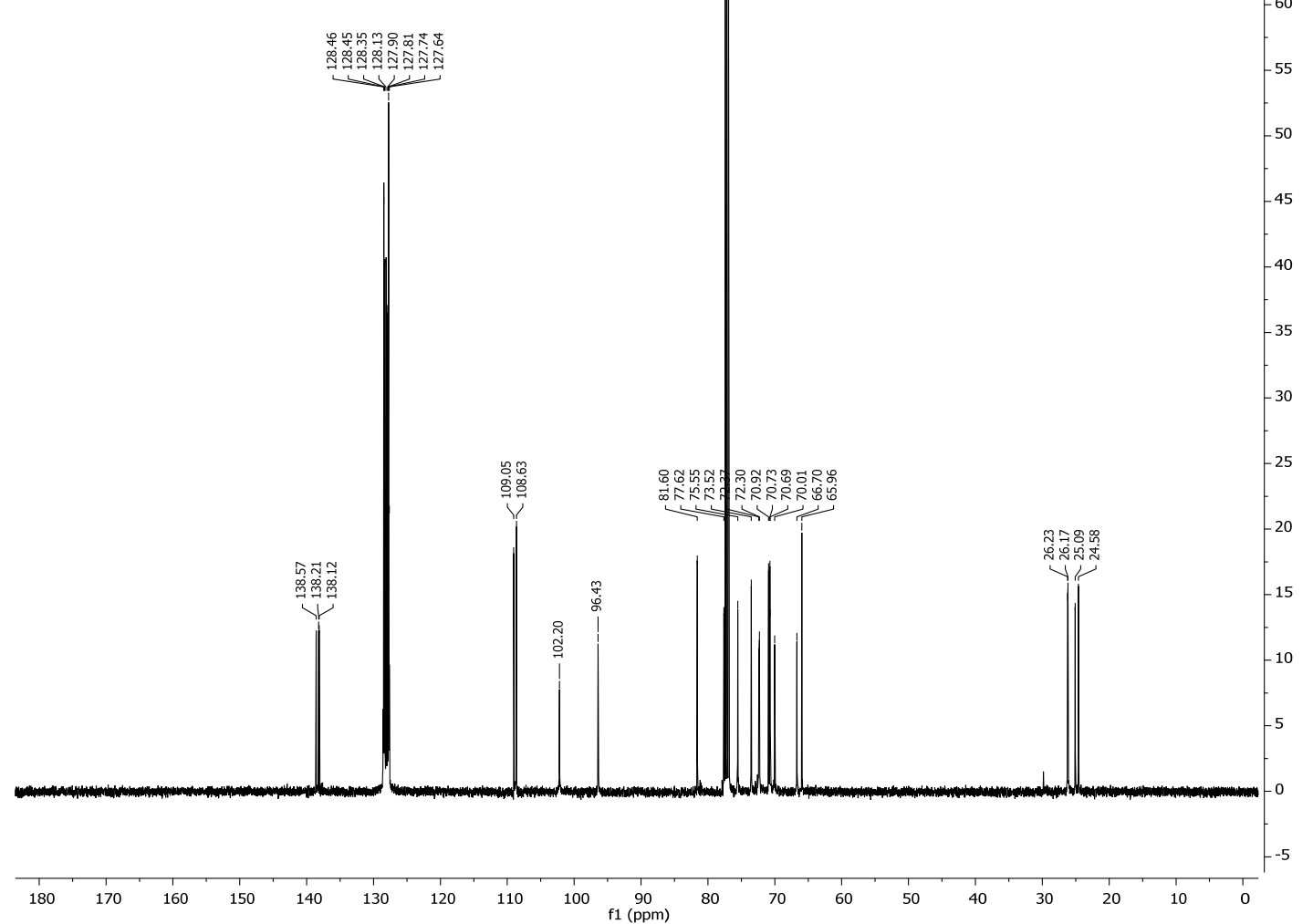


${ }^{1} \mathrm{H}$ NMR $\left(600 \mathrm{MHz}, \mathrm{CDCl}_{3}\right)$
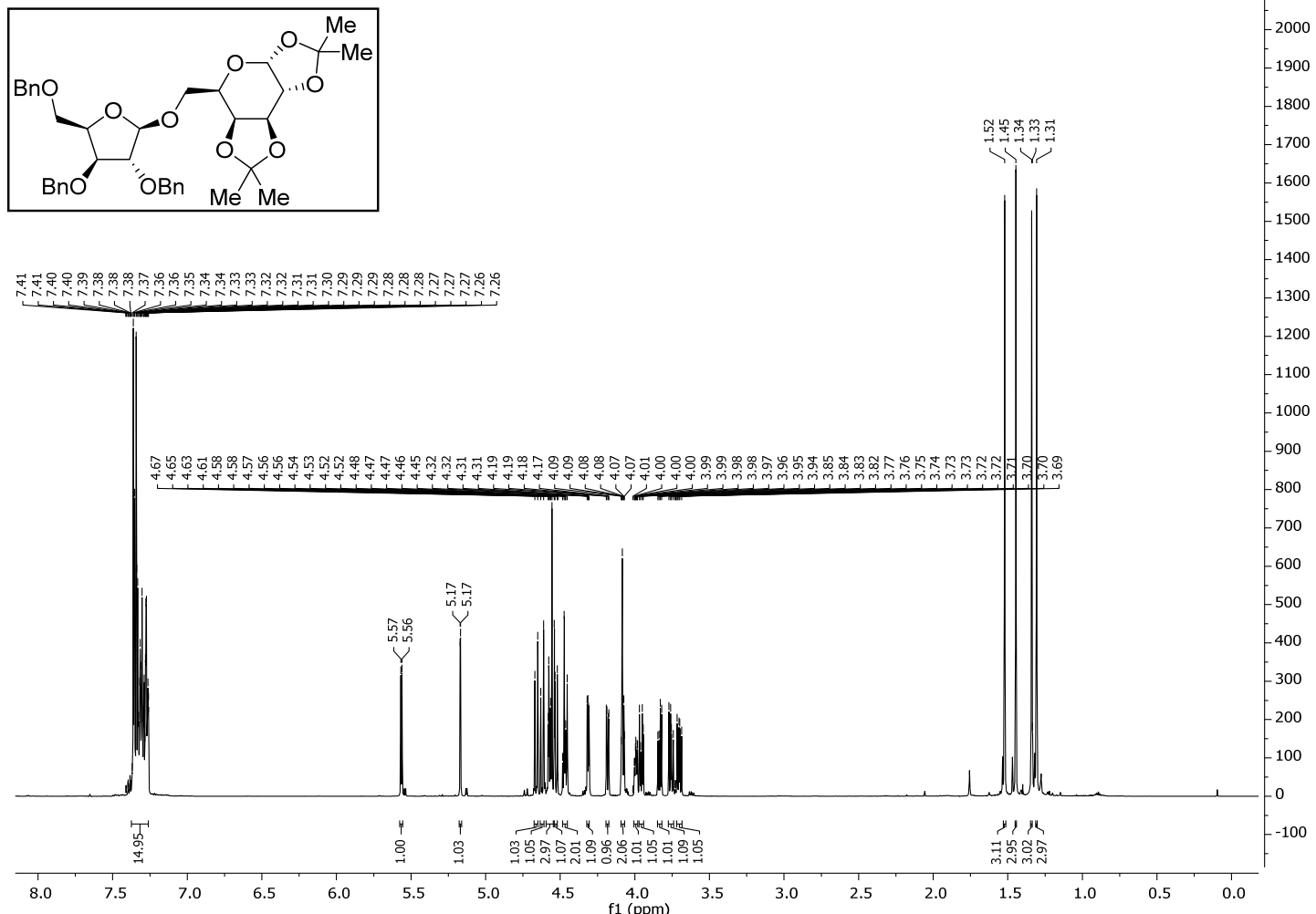

$\left.{ }^{13} \mathrm{C} \mathrm{NMR} \mathrm{(126} \mathrm{MHz,} \mathrm{CDCl}_{3}\right)$

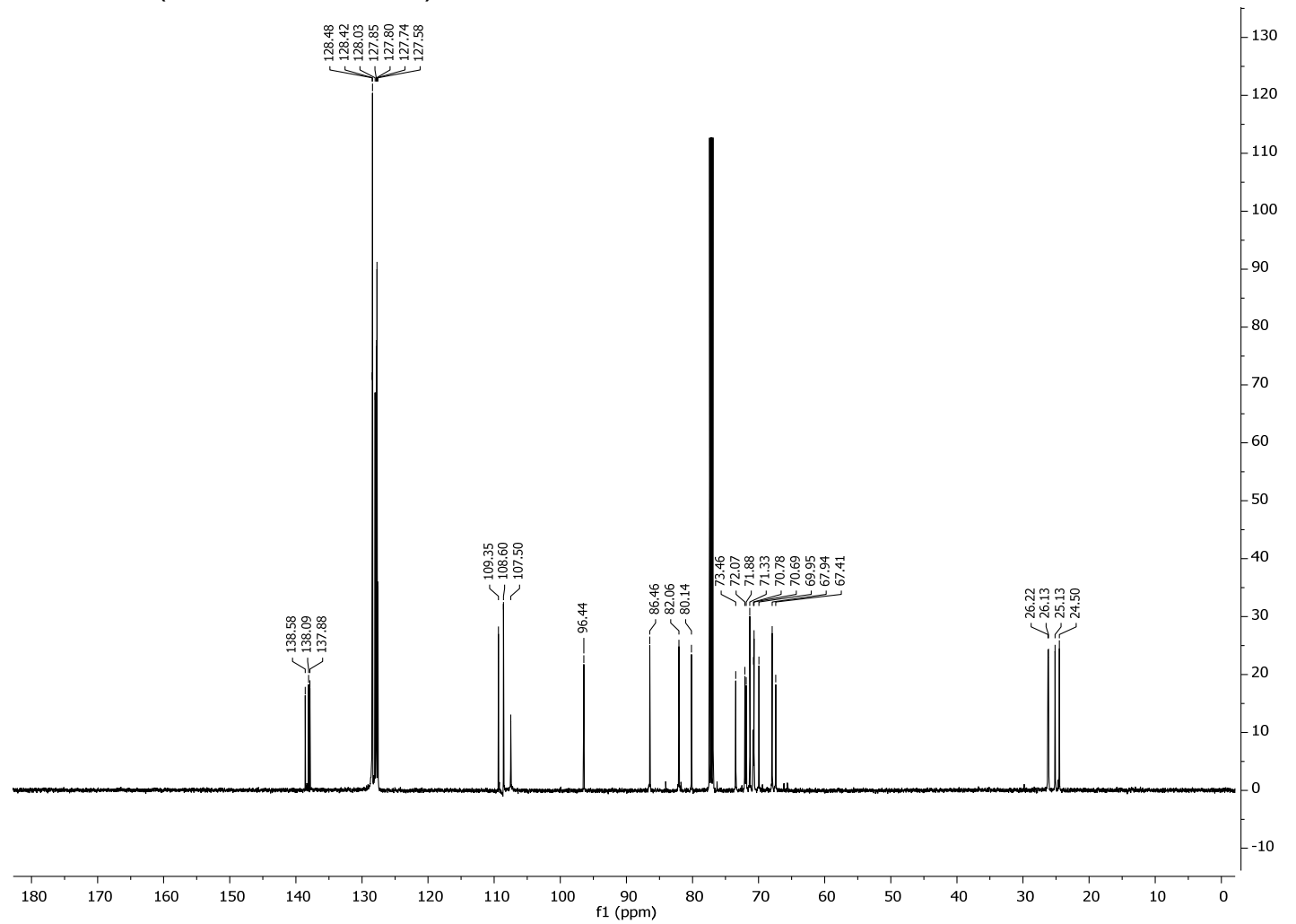




\section{References}

[1] Rossi, F.; Corcella, F.; Saverio Caldarelli, F.; Heidempergher, F.; Marchionni, C.; Auguadro, M.; Cattaneo, M.; Ceriani, L.; Visentin, G.; Ventrella, G.;

Pinciroli, V.; Ramella, G.; Candiani, I.; Bedeschi, A.; Tomasi, A.; Kline, B. J.;

Martinez, C. A.; Yazbeck, D.; Kucera, D. J. Process Research and

Development and Scale-up of 4,4-Difluoro-3,3-dimethylproline Derivative. Org. Process Res. Dev. 2008, 12 (2), 322-338.

[2] Wysocki, R. J.; Siddiqui, M. A.; Barchi, J. J.; Driscoll, J. S.; Marquez, V. E. A more Expedient Approach to the Synthesis of Anti-HIV-Active 2,3-Dideoxy-2fluoro- $\beta$-D-threo-pentofuranosyl Nucleosides. Synthesis 1991, 11, 1005-1008.

[3] Larsen, C. H.; Ridgway, B. H.; Shaw, J. T.; Smith, D. M.; Woerpel, K. A. Stereoselective C-Glycosylation Reactions of Ribose Derivatives: Electronic Effects of Five-Membered Ring Oxocarbenium lons. J. Am. Chem. Soc. 2005, 127 (31), 10879-10884.

[4] Pozsgay, V.; Coxon, B.; Yeh, H. Synthesis of di- to penta-saccharides related to the O-specific polysaccharide of Shigella dysenteriae type 1, and their nuclear magnetic resonance study. Bioorg. Med. Chem. 1993, 1 (4), 237-257.

[5] Baer, H. H.; Mateo, F. H.; Siemsen, L. A facile formation of 2,5-anhydro sugars by ring contraction in methyl hexopyranosides 2-triflates under conditions of nucleophilic displacement. Carbohydr. Res. 1989, 187 (1), 6792.

[6] Zhang, Y. K.; Sanchez-Ayala, M. A.; Sternberg, P. W.; Srinivasan, J.; Schroeder, F. C. Improved Synthesis for Modular Ascarosides Uncovers Biological Activity. Org. Lett. 2017, 19 (11), 2837-2840.

[7] Akthar, T.; Eriksson, L.; Cumpstey, I. Unsual synthesis of carbohydrate secsec ether-linked pseudodisaccharides. Carbohydr. Res. 2008, 343 (12), 20942100.

[8] Miethchen, R.; Fehring, V. Chirale Kronenether mit integrierten 1,4verbrückten d-Glucopyranose-Baustein. Synthesis 1998, 1, 94-98.

[9] Petráková, E.; Kovác, P. Synthesis of new methyl O-acetyl- $\alpha$ - and - $\beta$-Dxylopyranosides. Carbohydr. Res. 1982, 101 (1), 141-147.

[10] Shi, Z.-D.; Yang, B.-H.; Wu, Y.-L. A stereospecific synthesis of L-deoxyribose, L-ribose and L-ribosides. Tetrahedron 2002, 58 (16), 3287-3296.

[11] Dahlmann, O.; Garegg, P. J.; Mayer, H.; Schramek, S. Synthesis of the Three 3-C-Hydroxymethylpentoses with the D-ribo-, D-xylo- and L-lyxo-

Configurations. Identification of the Latter with a Monosaccharide Isolated from Phase I Coxiella burnetii Lipopolysaccharide. Acta Chem. Scand. B 1986, 40, 15-20.

[12] Kim, J.; Weledji, Y. N.; Greenberg, M. M. Independent Generation and Characterization of a C2'-Oxidized Abasic Site in Chemically Synthesized Oligonucelotides. J. Org. Chem. 2004, 69 (18), 6100-6104.

[13] Tsuji, H.; Yamamoto, H. Hydroxy-Directed Amidation of Carboxylic Esters Using a Tantalum Oxide Catalyst. J. Am. Chem. Soc. 2016, 138 (43), 1421814221.

[14] Kennedy, C. R.; Lehnherr, D.; Rajapaksa, N. S.; Ford, D. D.; Park, Y.; Jacobsen E. N. Mechanism-Guided Development of a Highly Active Bisthiourea Catalyst for Anion-Abstraction Catalysis. J. Am. Chem. Soc. 2016, 138 (41), 13525-13528. 
[15] Lehnherr, D.; Ford, D. F.; Bendelsmith, A. J.; Kennedy, C. R.; Jacobsen, E. N. Conformational Control of Chiral Amido-Thiourea Catalysts Enables Improved Activity and Enantioselectivity. Org. Lett. 2016, 18 (13), 3214-3217.

[16] Islam, M.; Gayatri, G.; Hotha, S. Influence of Steric Crowding on Diastereoselective Arabinofuranosylations. J. Org. Chem. 2015, 80 (16), 7937-7945.

[17] Oka, N.; Kajino, R.; Takeuchi, K.; Nagakawa, H.; Ando, K. a-Selective Ribofuranosylation of Alcohols with Ribofuranosyl lodides and Triphenylphosphine Oxide. J. Org. Chem. 2014, 79 (16), 7656-7664.

[18] Bruker AXS APEX3, Bruker AXS, Madison, Wisconsin, 2015.

[19] Dolomanov, O. V.; Bourhis, L. J.; Gildea, R. J.; Howard, J. A. K., Puschmann, $\mathrm{H}$. OLEX2: a complete structure solution, refinement and analysis program. J. Appl. Cryst. 2009, 42, 339-341.

[20] Sheldrick, G. M. SHELXT - Integrated space-group and crystal-structure determination. Acta Cryst. 2015, A71, 3-8.

[21] Sheldrick, G. M. Crystal structure refinement with SHELXL. Acta Cryst. 2015, C71, 3-8.

[22] Accelrys DS Visualizer v2.0.1, Accelrys Software. Inc., 2007.

[23] Spek, A. L., PLATON, A Multipurpose Crystallographic Tool, Utrecht University, Utrecht, The Netherlands, 2010.

[24] Parsons, S.; Flack, H. D.; Wagner, T. Use of intensity quotients and differences in absolute structure refinement. Acta Cryst. 2013, B69, 249-259. 